*ak RMIS View/Frint Document Cover Sheet tow

This document was retrieved from the Documentation and Records Manaqement (DRM) ISEARCH System. It is intended for Information only and may not be the most recent or updated version. Contact a Document Service Center (see Hanford Info for locations) if you need additional retrieval information.

Accession \#: D196069128

Document \#: SD-CP-CSWD-016

Title/Desc:

PFP MICON DCS COMPUTER SOFTWARE DOCUMENTATION [VOL

$1 \mathrm{THRU} 4]$

Pages: 515 


\begin{tabular}{|c|c|c|c|}
\hline \multirow{3}{*}{$\begin{array}{l}\text { 2. ECN Category } \\
\text { (mark one) } \\
\text { Suppl emental } \\
\text { Direct Revision } \\
\text { Change ECN } \\
\text { Temporary } \\
\text { Standby } \\
\text { Supersedure } \\
\text { Cancel/Noid }\end{array}$} & $\begin{array}{l}\text { 3. Originator's Name, Organization, MSIN, } \\
\text { and Telephone No. } \\
\text { GR Silvan, 15510,T4-20,373-2943 }\end{array}$ & $\begin{array}{l}\text { 3a. Usa Required? } \\
\text { [1] Yes [X] No }\end{array}$ & $\begin{array}{l}\text { 4. Date } \\
3-13-96\end{array}$ \\
\hline & $\begin{array}{l}\text { 5. Project Title/No./Work Order No. } \\
\text { B610 }\end{array}$ & $\begin{array}{l}\text { 6. Bldg./Sys./Fac. Mo. } \\
234-5 Z / 93 A\end{array}$ & $\begin{array}{l}\text { 7. Approval Designator } \\
\text { N/A }\end{array}$ \\
\hline & 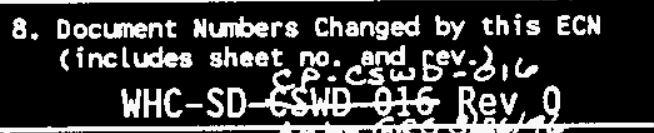 & $\begin{array}{l}\text { 9. Related ECN No(s). } \\
\qquad N / A\end{array}$ & $\begin{array}{l}\text { 10. Related PO No. } \\
\text { N/A } \\
\end{array}$ \\
\hline $\begin{array}{l}\text { 11a. Modification Work } \\
\text { [] Yes (fill out Blk. } \\
11 \mathrm{~b}) \\
\text { [X] No (MA B(ks. 11b, } \\
11 \mathrm{c}, 11 \mathrm{~d})\end{array}$ & \multicolumn{2}{|c|}{\begin{tabular}{l|l} 
11b. Hork Packsge & Dotle. Hodification Work Complete \\
N/A & N/A \\
& Cog. Engineer Signature \& Date
\end{tabular}} & $\begin{array}{l}\text { 11d. Restored to Original Condi- } \\
\text { tion (Tellp. or Stendby ECN only) } \\
\text { N/A } \\
\text { Cog. Engineer Signature \& Date }\end{array}$ \\
\hline \multicolumn{4}{|c|}{ 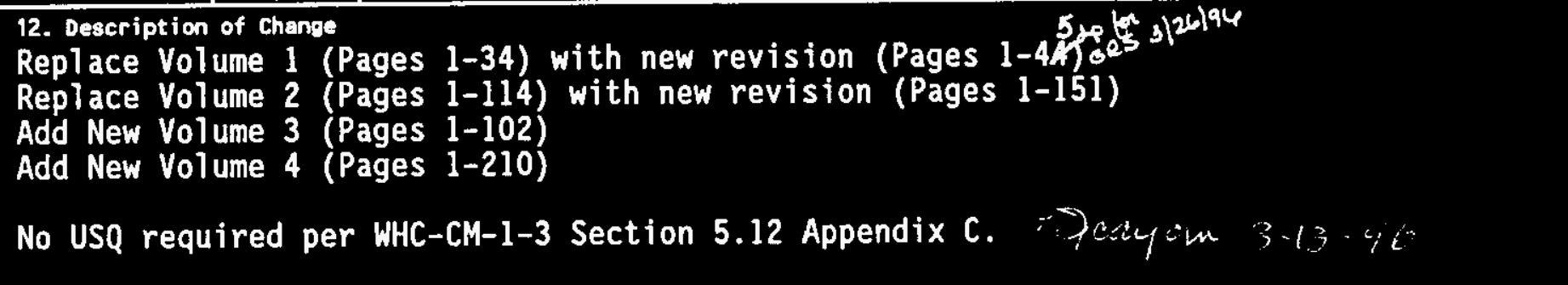 } \\
\hline \multicolumn{4}{|c|}{$\begin{array}{l}\text { 13a. Justification (mark one) } \\
\begin{array}{lllllll}\text { Criteria change } & {[]} & \text { Desion Improvenent } & {[\mathrm{X}]} & \text { Environmental } & {[]} & \text { Facility Deactivation } \\
\text { As-found } & {[]} & \text { Facil itate Const } & {[]} & \text { Const. Error/Omission } & {[]} & \text { Design Error/Omission }\end{array}\end{array}$} \\
\hline \multicolumn{4}{|c|}{$\begin{array}{l}\text { 13b. Justification Details } \\
\text { Volumes } 1 \text { \& } 2 \text { updated to reflect control changes in the Low Level Waste Treatment } \\
\text { Facility. } \\
\text { Volume } 1 \text { updated and Volumes } 3 \& 4 \text { added to incorporate project } B 610 \text { control additions } \\
\text { in Closed Loop Cooling and } 2736-\mathrm{B} \text { HVAC control. }\end{array}$} \\
\hline \multicolumn{4}{|c|}{$\begin{array}{l}\text { 14. Distribution (include name, MstM, and no. of copies) } \\
\text { See AHached Distribution Sheet }\end{array}$} \\
\hline
\end{tabular}

A-7900-013-2 (11/94) GEF095 


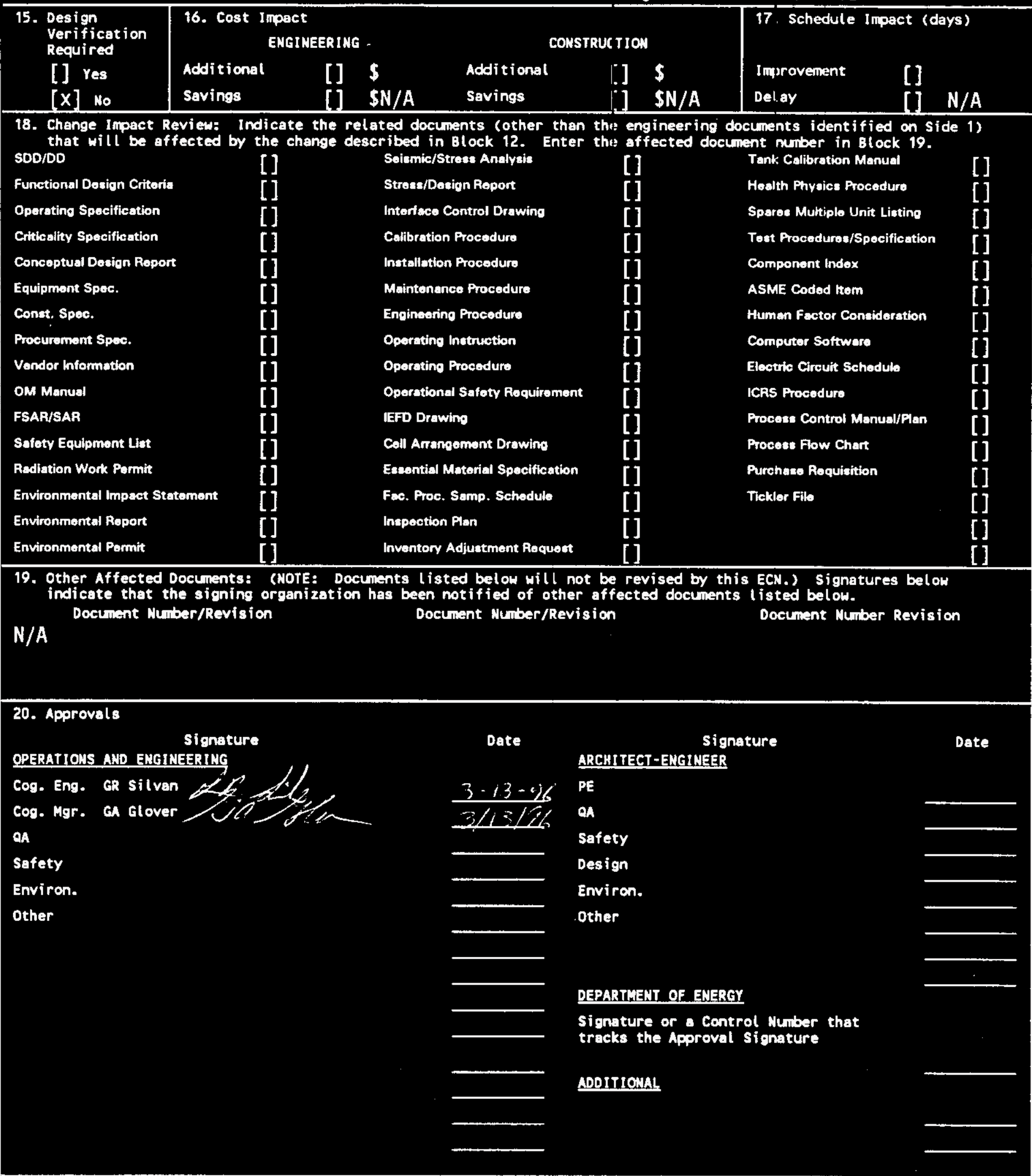




\title{
PFP MICON DCS Computer Software
} Documentation

\author{
6. R. Silvan
}

Westinghouse Hanford Company, Richland, WA 99352

U.S. Department of Energy Contract DE-AC06-87RL10930
EDT/ECN: 191441
UC: $\mathbf{5 0 6}$
Org Code: 15510
Charge Code: K6034
B\&R Code: EW7003000
Total Pages: 509

Key Words: MICON, MICON View, LLWTF, CLC, 2736-ZB, HVAC

Abstract: This document contains the complete printout of the MICON A/S system configuration used in the Plutonium Finishing Plant. The document is divided into several volumes. Volume 1 covers the workstation display and configuration. A11 other volumes contain the controller configurations, or programs.

TRADEMARK DISCLAIMER. Reference herein to any specific commercial product, process, or service by trade nene, trademark, manufacturer, or otheruise, does not necessarily constitute or inply its endorsentent, recomendation, or favoring by the United States Govermient or any agency thereof or its contractors or subcontractors.

Printed in the United States of America. To obtain copies of this document, contact: WHC/BCS Document Control Services, P.0. Box 1970, Mailstop H6-08, Richland uA 99352, Phone (509) 372-2420; Fax (509) 376-4989.
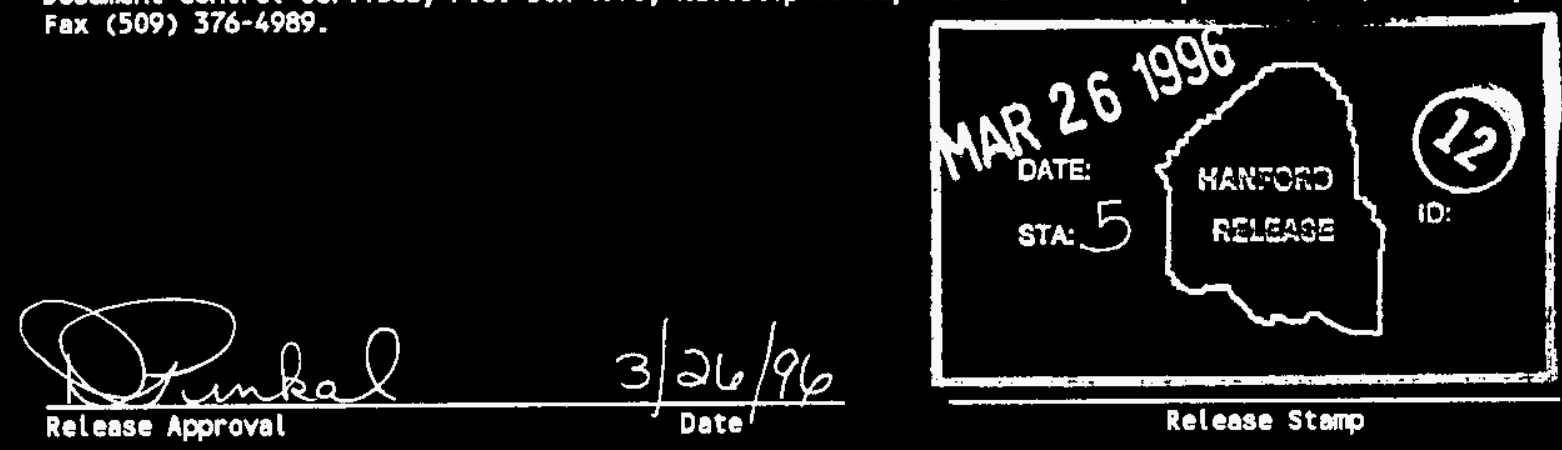

Release Stailp

\section{Approved for Public Release}




\section{RECORD OF REVISION}

(2) Title

PFP MICON DCS Computer Software Documentation

CHANGE CONTROL RECORD

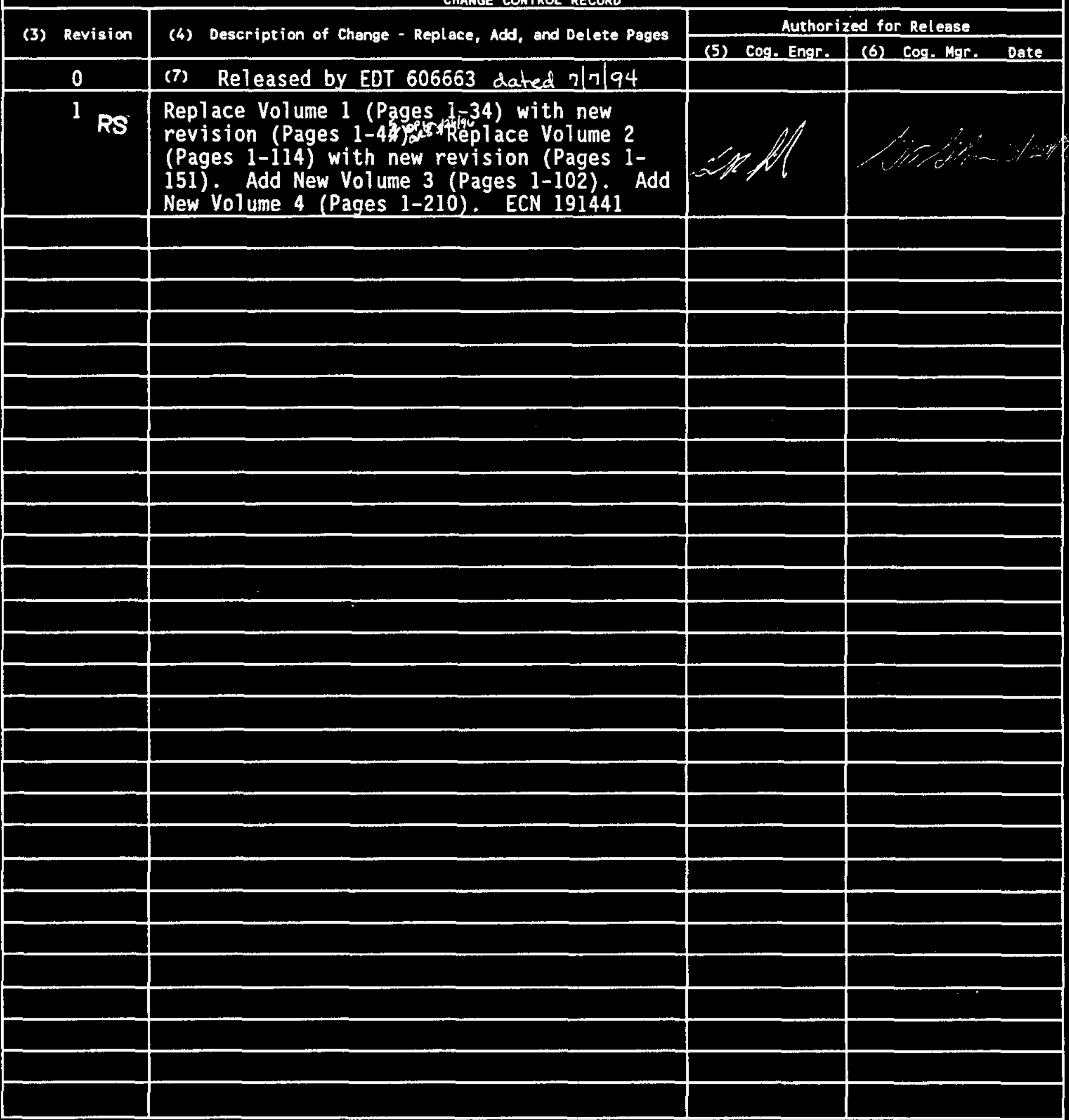

(1) Document Number

WHC-SD-CP-CSWD016 
WHC-SD-CP-CSWD-016

Volume 1

Revision 1

Page 1

TABLE OF CONTENTS

INTRODUCTION ....................... 3

GROUP DISPLAYS ....................... 6

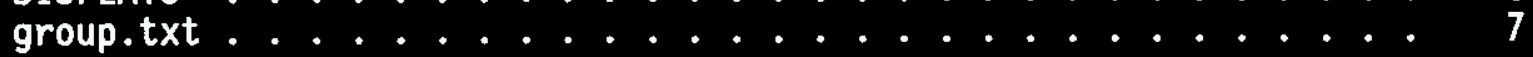

GRAPHIC DISPLAYS ................. 11

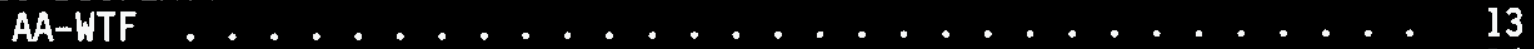

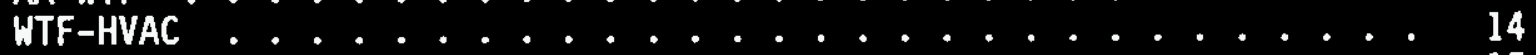

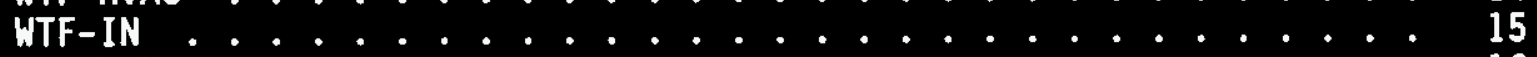

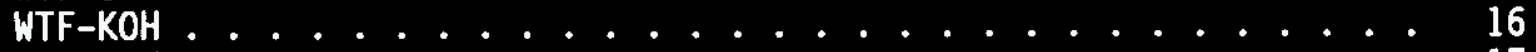

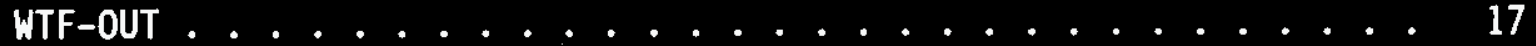

$\mathrm{AA}-\mathrm{CLC} \ldots \ldots \ldots \ldots \ldots \ldots$

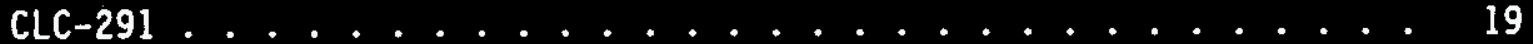

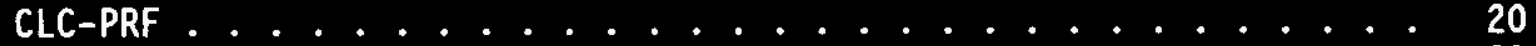

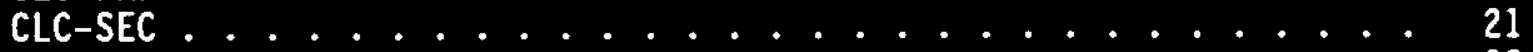

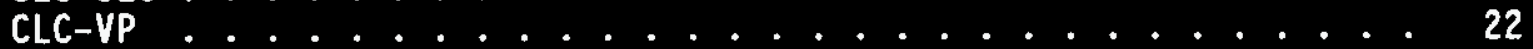

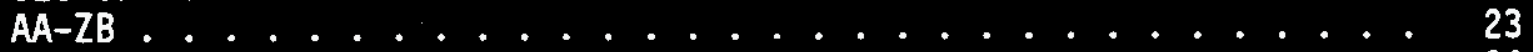

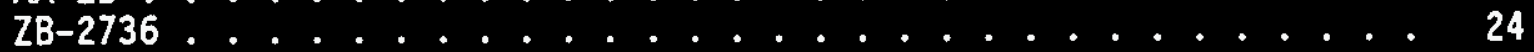

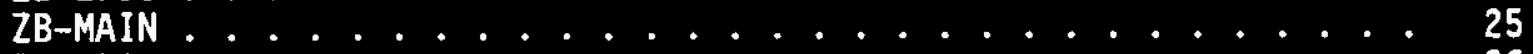

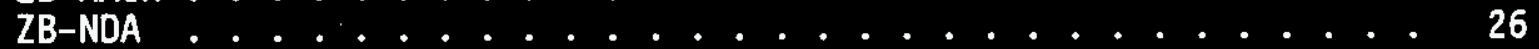

APPENDIX A: SPARC SET-UP FOR MICON VIEW . . . . . . . . . . 27

Disk Partitions ...................... 27

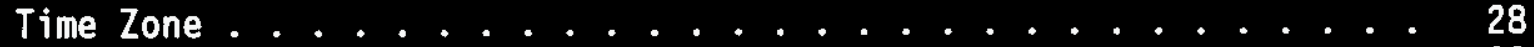

Network Host Information .............. 28

Creating a New Kernel .............. 29

Micon User Password, Directories and Files ......... 30

Extra Swap ......................... 30

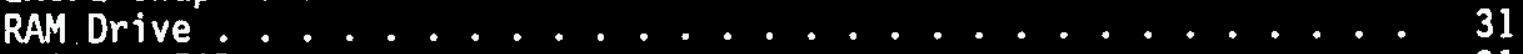

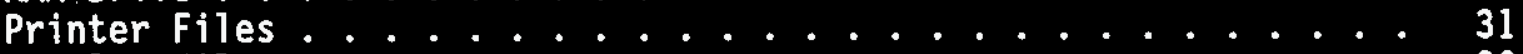

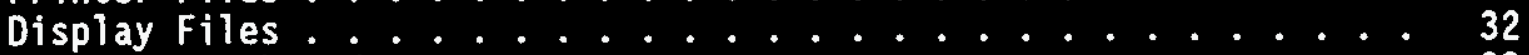

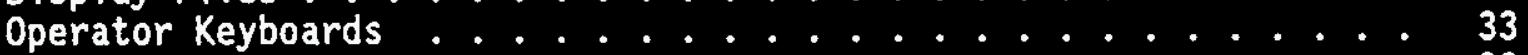

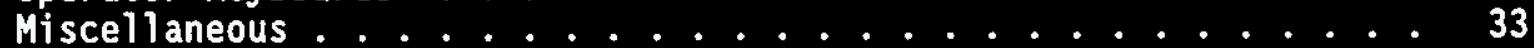

MICON View \& Configuration Files .................. 34 
WHC-SD-CP-CSWD-016

Volume 1

Revision 1

Page 2

APPENDIX B: SYSTEM FILES ................ 35

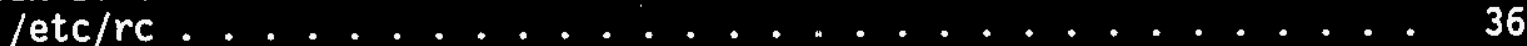

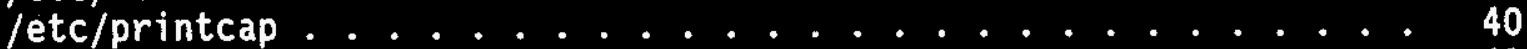

/home/users/micon/.cshrc .............. 41

/home/users/micon/.login ................ 42

/home/users/micon/.openwin-init ........... 43

/home/users/micon/openwin-menu-ops (.openwin-menu) ....... 44

/home/users/micon/openwin-menu-eng ........... 44

/home/users/micon/.openwin-menu-micon .......... 44

/home/users/micon/menu-eng ............. 44

/home/users/micon/menu-ops ............... 44

APPENDIX C ....................... 45 
WHC-SD-CP-CSWD-016

Volume 1

Revision 1

Page 3

\section{INTRODUCTION}

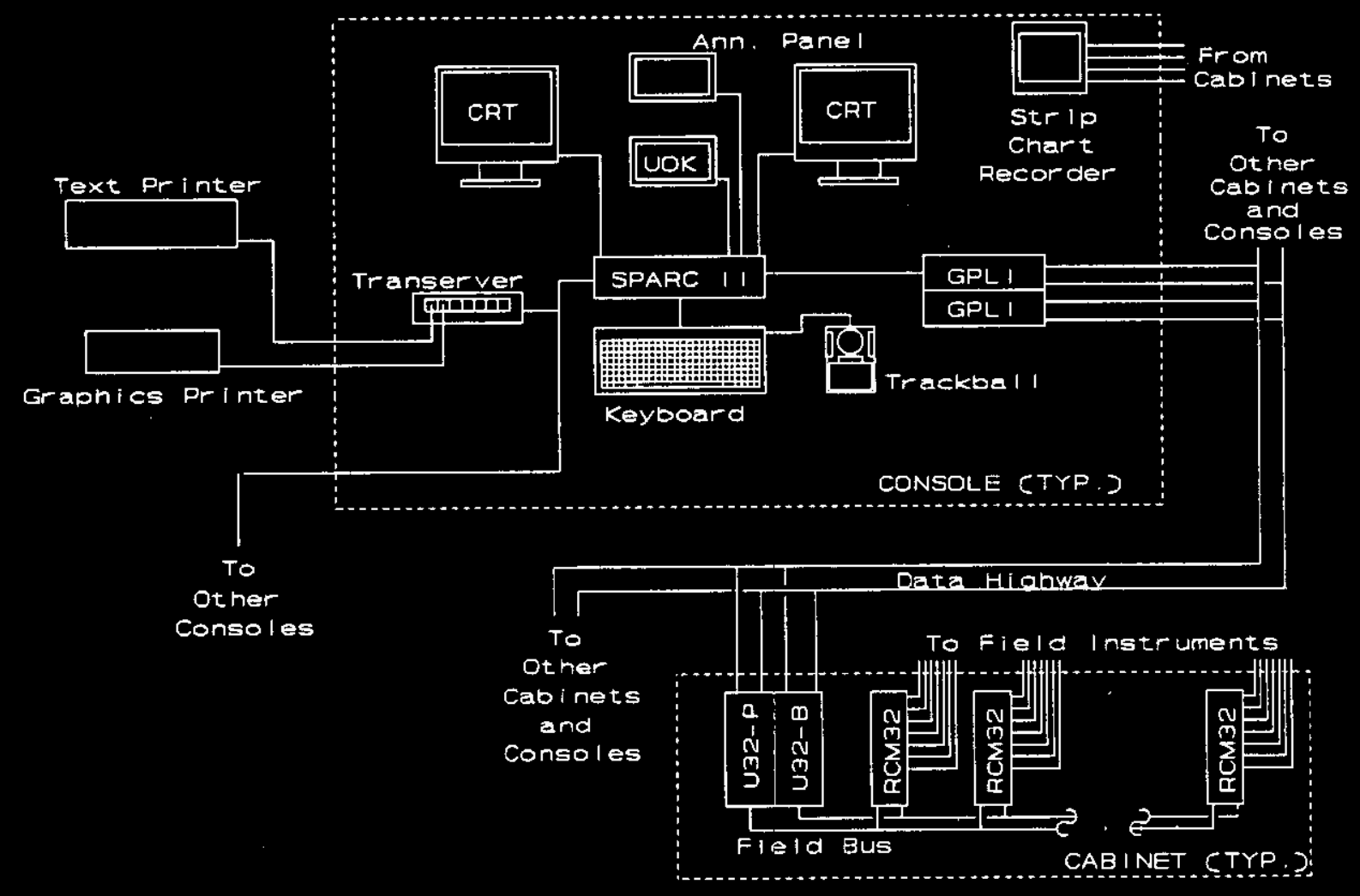

The MICON ${ }^{1}$ A/S distributed control system consists of four consoles or workstations (2 operator, 1 supervisor, and 1 engineer), a data highway, and seven process controller cabinets.

Each console is based on a Sun Microsystem's SPARC 20 workstation. The SPARC 20 workstation is based on a Scalable. Process Architecture (SPARC) micro-processor. Each workstation contains 64 megabytes of memory, a one gigabyte hard drive, a 150 megabyte tape drive, a keyboard, and a trackball. Select SARC statations also contain an additional one gigabyte hard drive, a CD-ROM drive, and a 5 gigabyte tape drive. In addition, each SPARC 20

${ }^{\text {F }}$ For a list of trademarks see APPENDIX C 


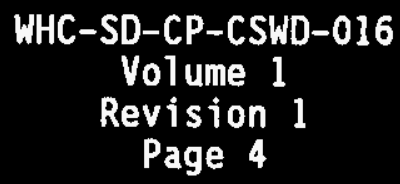

provides two video outputs, two RS-232 serial ports, and two ethernet ports (a thick-net and a thin-net). The video ports are connected to two high resolution color monitors. The RS-232 ports are connected to two Emerald model FPXT single board IBM XT compatible computers with touchscreen. One of the FPXTs is designated as an annunciator panel and is unused. The other is used as a Universal Operator Keyboard (UOK). One of the two ethernet ports -the thin-net -- is connected to the data highway via a MICON Company General Purpose LAN Interface (GPLI). The thicknet provides a Local Area Network (LAN) between the four consoles. It also connects each SPARC 20 with a Rockwe11/CMC TranServer. The TranServer provides eight additional RS-232 serial ports for each workstation. One of these serial ports is connected to an Output Technology Corporation 800 series text printer. Another port is connected to a Hewlett-Packard PaintJet color graphics printer.

The Data Highway is the main data link between the consoles and the process control cabinets. It consists of two redundant coax cables that run between all four consoles and six cabinets. The Data Highway is a local area network using a high speed token ring passing protocol. The Data Highway is also redundant. This means that if one of the two cables fail, communication is automatically passed to the other.

The process control cabinets consist of two redundant main controllers, called $\mathrm{U}-32 \mathrm{~s}$, and several process controllers called RCM-32s. The U-32s and RCM-32s communicate via a small local area network called a Field Bus. The U-32 is a MICON built controller based on the Motorola 68020 processor. Although it can be used to directly control instruments and devices in the field, its primary purpose is to pass information between the consoles and the RCM-32s Each U-32 has a redundant backup. That way if one fails, the other can automatically take over. The RCM-32s are also built by MICON and are based on a Motorola 68030 processor. These controllers provide input and output directly to field instruments and other devices.

There are four types of RCM-32s used in this system: $A, C, D$, and Dr. The $A$ and $C$ cards are used primarily for analog signals. They provide hardware for any standard analog instrument input (4-20 mA, 10-50 mA, and DC voltages) and provide a standard 4-20 $\mathrm{mA}$ output signal. In addition, they provide two 28 Volt DC discrete (on/off) channels. These may be used for input, output, or both. An A card provides 26 analog inputs and 4 analog outputs. Each C card provides 18 analog inputs and 12 analog outputs. The D card is used only for discrete control. It provides 32 on/off type channels that can be used as inputs, outputs, or both. Both inputs and outputs are 28 volt DC. The Dr cards are similar to the $D$ cards except inputs are 120 volt $A C$ or $D C$ and the outputs are 3 amp (120 VAC) dry contact relays.

Since MICON A/S systems are installed by engineers rather than computer programmers, they are designed to minimize the need for programming. All of the software needed to monitor and control a process is already programmed into the system be MICON Company. The engineer need only "configure" the system to a specific application. 


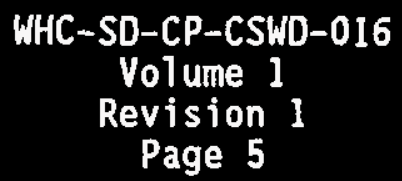

The process is very similar to the method used to install an older analog instrument system. First, a set of Pipe and instrument Diagrams (P\&ID) are created. These drawings show a schematic of the chemical process and the instrumentation to be installed to control it. With an analog system, the engineer would purchase the necessary individual components and wire them together to create the control strategy. With the MICON A/S system, the engineer would call function blocks to invoke preprogrammed modules and create the control strategy. The only difference between the two is that one uses hardware devices wired together, and the other uses software modules connected via a data base.

The MICON configuration is done in two parts. First the RCM-32 and, if needed the U-32 controllers are configured. Next, the workstation displays are created.

The RCM-32 and U-32 configuration consists of both the controller "program" and all display information for each "tag". Tags consists of all inputs and outputs from the controller, PID control loops, and any other displayed value. This information is requested by the MICON View software as the engineer creates the controller configuration.

Since al1 the display information (colors, ranges, etc.) was input during the controller configuration, the workstation configuration consists only of display creation. These are created by calling up a form and filling in the blanks. The only exception to this are the custom created graphics displays. Graphics are created by drawing a picture of the process and overlaying this with tag information. This is done with a special drawing program supplied with MICON View.

To keep size manageable, this document has been divided into several volumes. Volume 1 contains the display configuration for the four workstations. The other volumes each contain the configuration for one process Controller cabinet.

This volume (number 1) contains information on the group and graphic displays. All other type of displays are information only and not controlled. 


\section{WHC-SD-CP-CSWD-016 \\ Volume 1 \\ Revision 1 \\ Page 6}

\section{GROUP DISPLAYS}

Group displays are a group of up to eight "instruments". These instruments can be analog inputs, virtual analogs, discrete (on/off) indicators, switches, or controllers. See the PFP MICON View users document (WHC-SD-CP-CSUD-004) for information on calling up and using these displays.

These groups are created by the configure group option in MICON View under System Maintenance. The data base created by this option, however, isn't in a readable format. To get a readable file a program called grpexp is available in the SPARC II's /home2/Run directory. When run, this program creates a file called group.txt in the /home2/Data directory.

The group.txt file consists of 3,168 lines. One for each of the 3,168 possible groups on the console. Each line is divided into eleven fields. Each field is followed by the "I" character. The first field is the group number. The Second field is the group name. And, the third field a brief description of the group. The last eight fields contain the tag names for the "instruments" displayed in each of the group's eight slots. If a field is blank, then the corresponding slot is empty. Lines with all fields blank except the number indicate an unused group. 

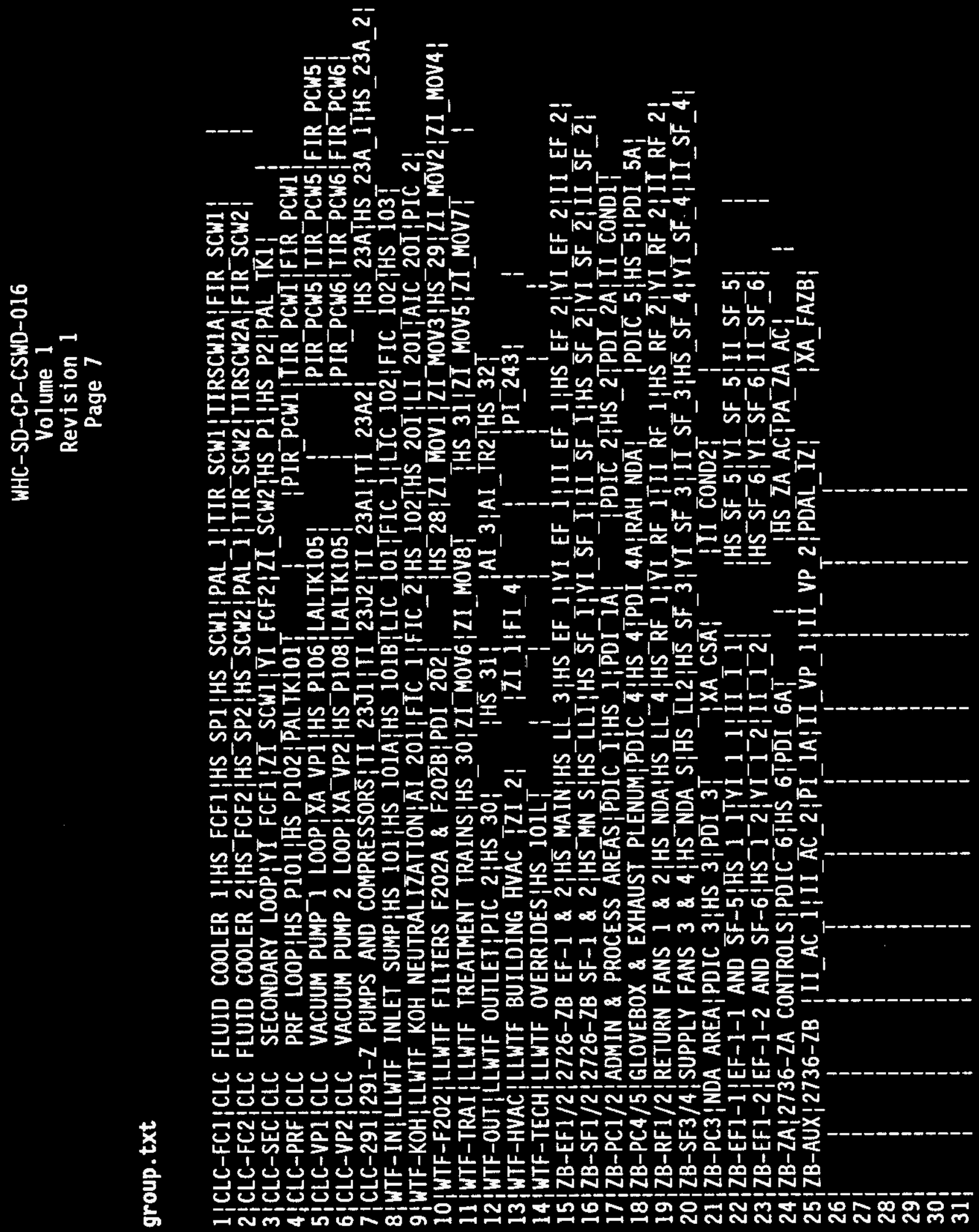


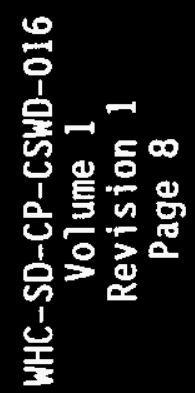

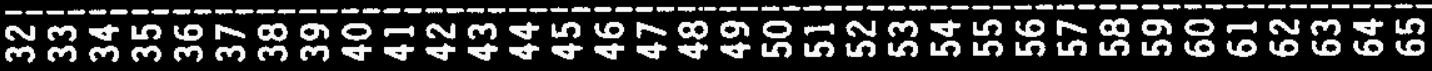




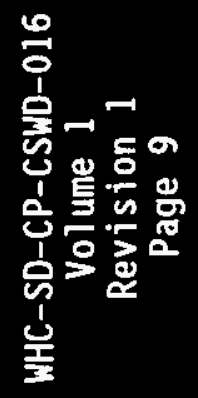

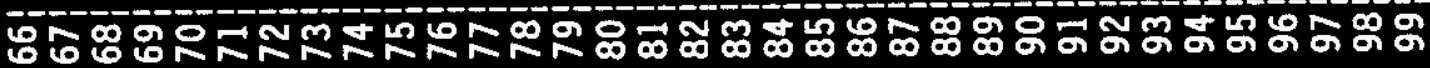




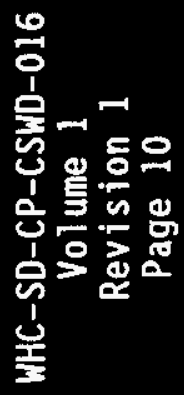


WHC-SD-CP-CSWD-016

Volume 1

Revision 1

Page 11

\section{GRAPHIC DISPLAYS}

Graphics displays are a special "picture" of the process. They consist of a static background picture overlayed with active process information. The picture is usually a drawing of the process being controlled. And, the process information can take the form of a digital text readout, moving bargraphs, or color changing symbols. See the PFP MICON View users document (WHC-SD-CP-CSUD-004) for information on calling up and using these displays.

These displays are created from the Display Builder option available in MICON View through the System Maintenance menu. Display builder creates up to four files for each graphic display. Unfortunately, none of these files are readable. Therefor, we have created drawings of each graphic display used on each console. These displays show the background picture and the instrument tag names for all the process data displayed. All tag names are shown in italics.

Using the Display builder option, these drawing can be compared against the actual display to verify proper configuration. 
WHC-SD-CP-CSWD-016

Volume 1

Revision 1

Page 12

S-201A

AI_201.cur

Standard text indicates background, non-changing, text.

\section{LEGEND}

Italics indicate tagged data displayed as text information. The letters before the period are the tag name, and the letters after are the type of information.

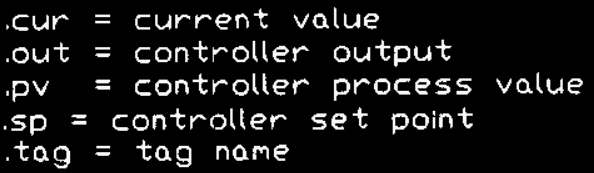

Goto Tag.

Moving vertical bar braph. The driving tag is shown inside the bargraph or pointing to it.

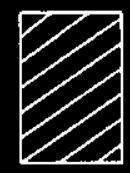<smiles>C1=CC2(CC2)CC12CC2</smiles>
ZI_MOV2.cur

Color changing symbol. The color change is based on the tag pointing to it. 
WHC-SD-CP-CSWD-016

Volume 1

Revision 1

Page 13

\section{AA-NTF}

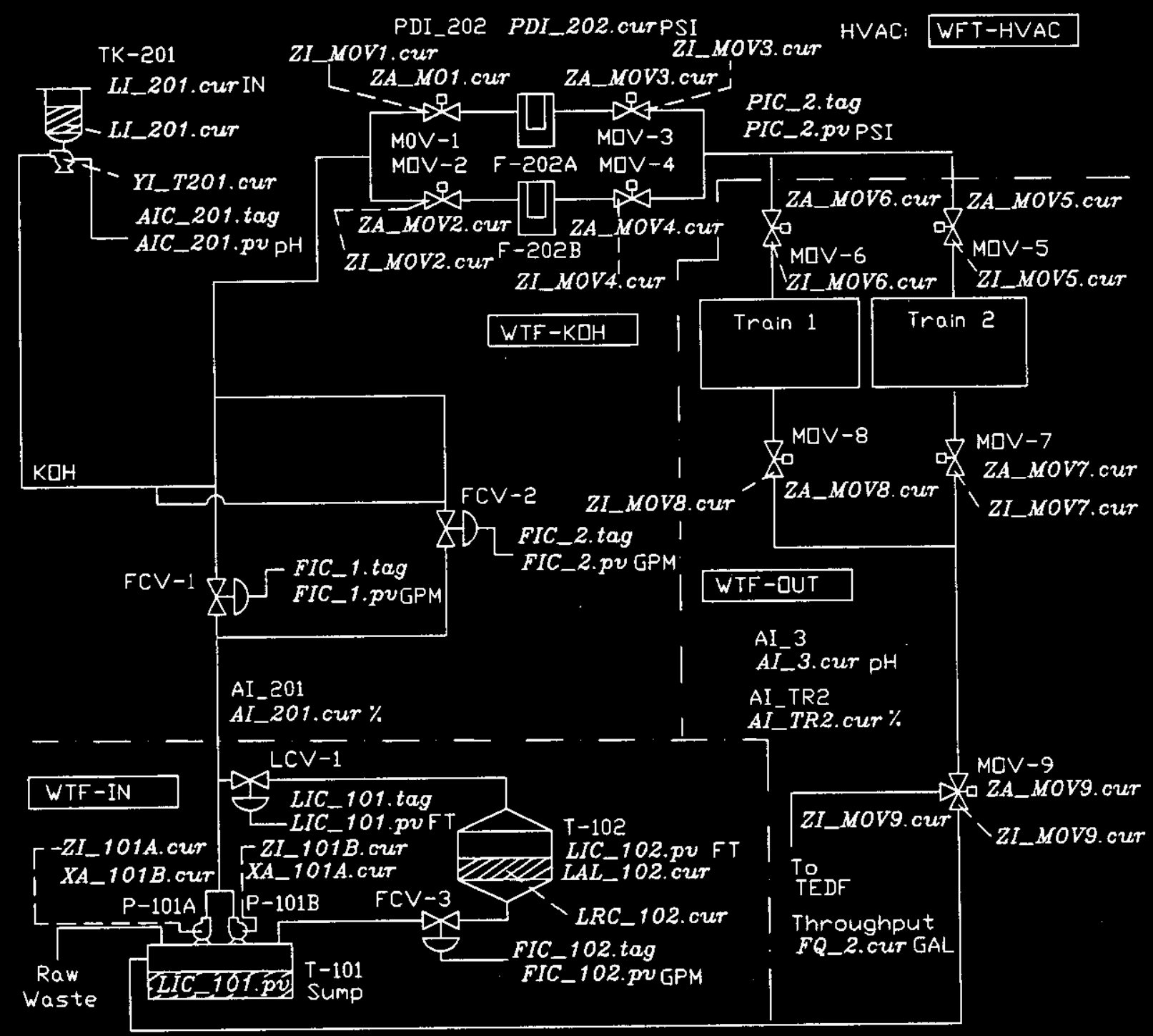


WHC-SD-CP-CSWD-016 Volume 1

Revision 1

Page 14

\section{VTF-HVAC}

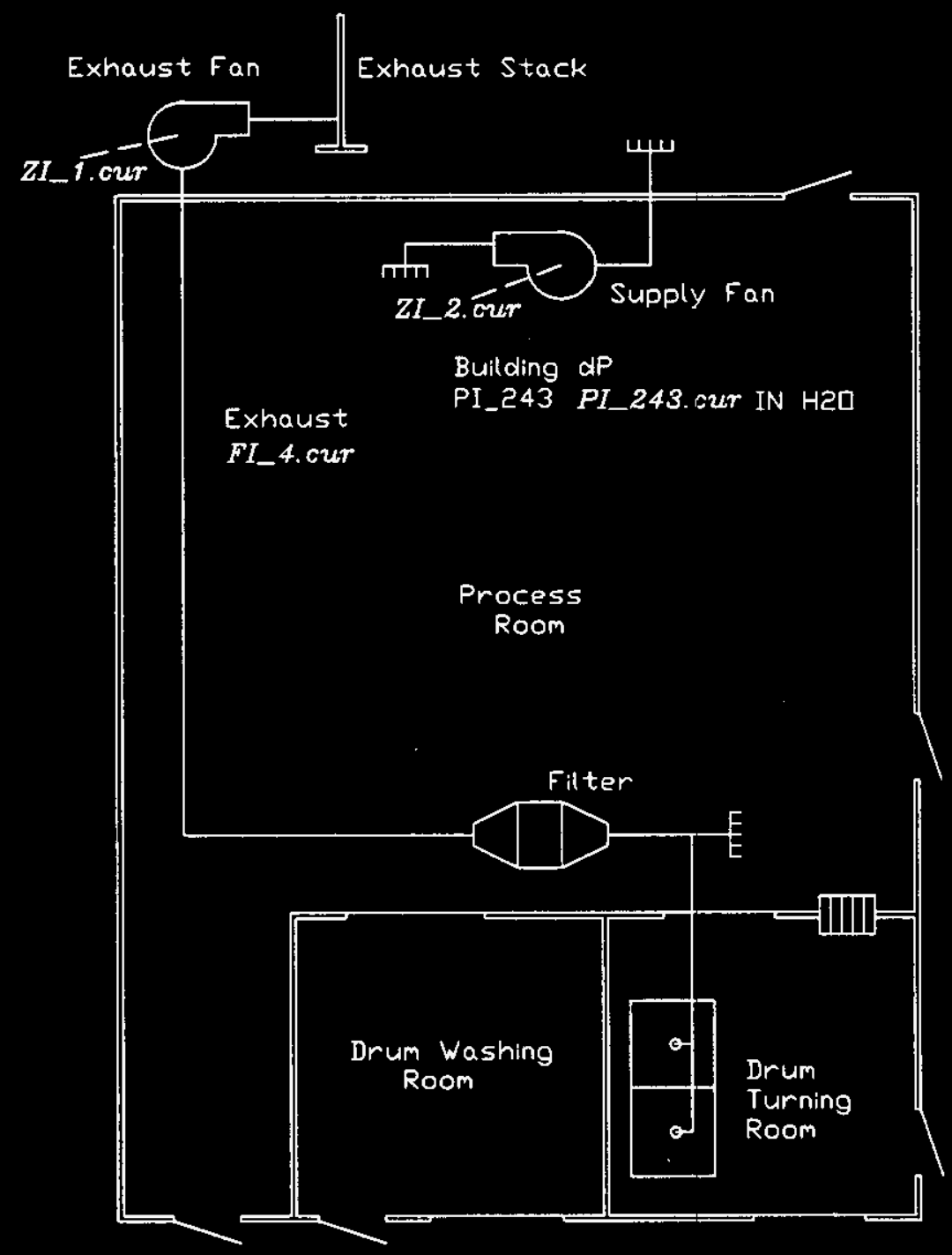

Low Level Waste

Treatment Facility 243-Z

PRDCESS: AA-WTF 
WHC-SD-CP-CSWD-016

Volume 1

Revision 1

Page 15

\section{NTF-IN}

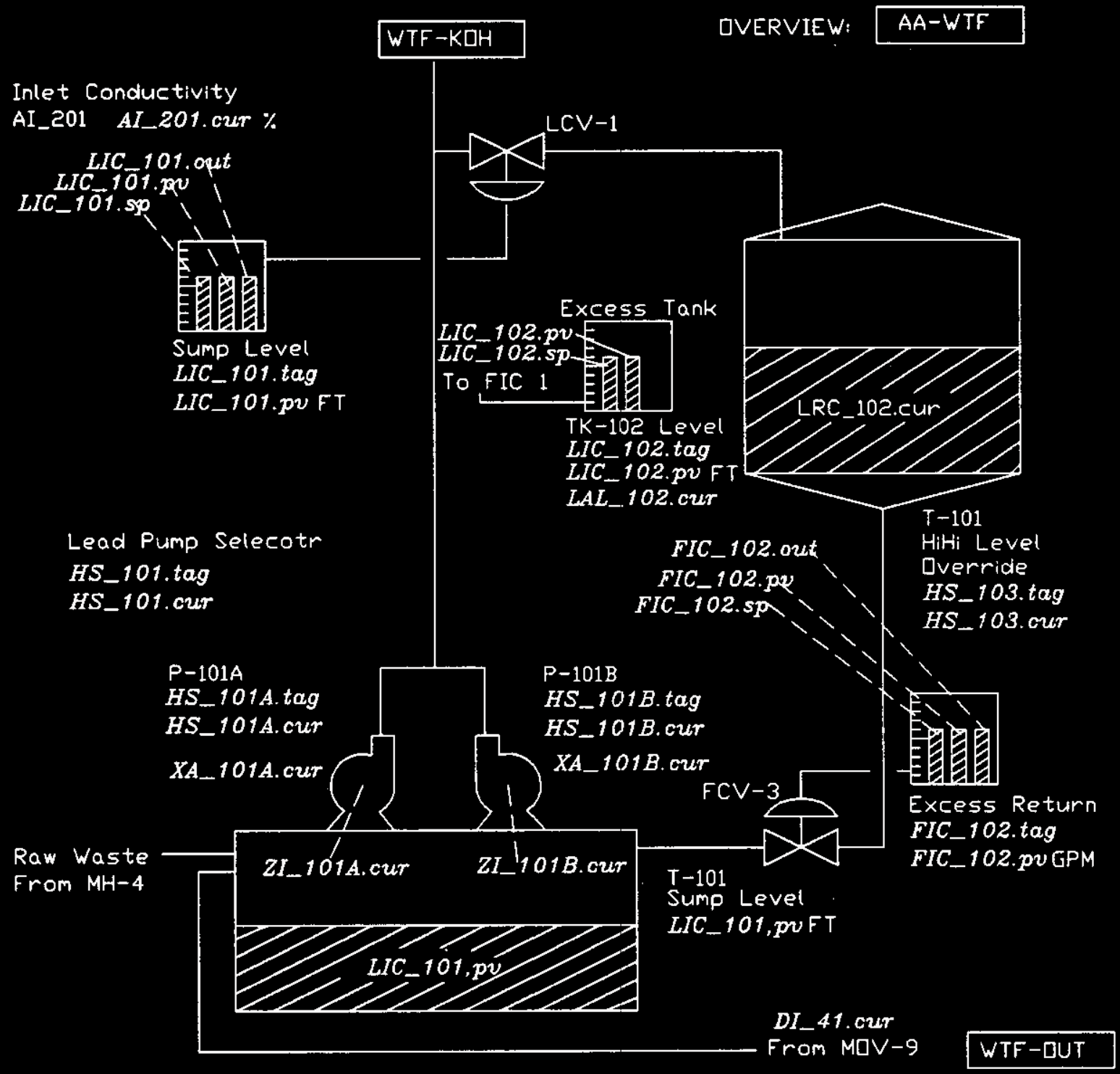


WHC-SD-CP-CSWD-016

Volume 1

Revision 1 .

Page 16

\section{WTF-KOH}

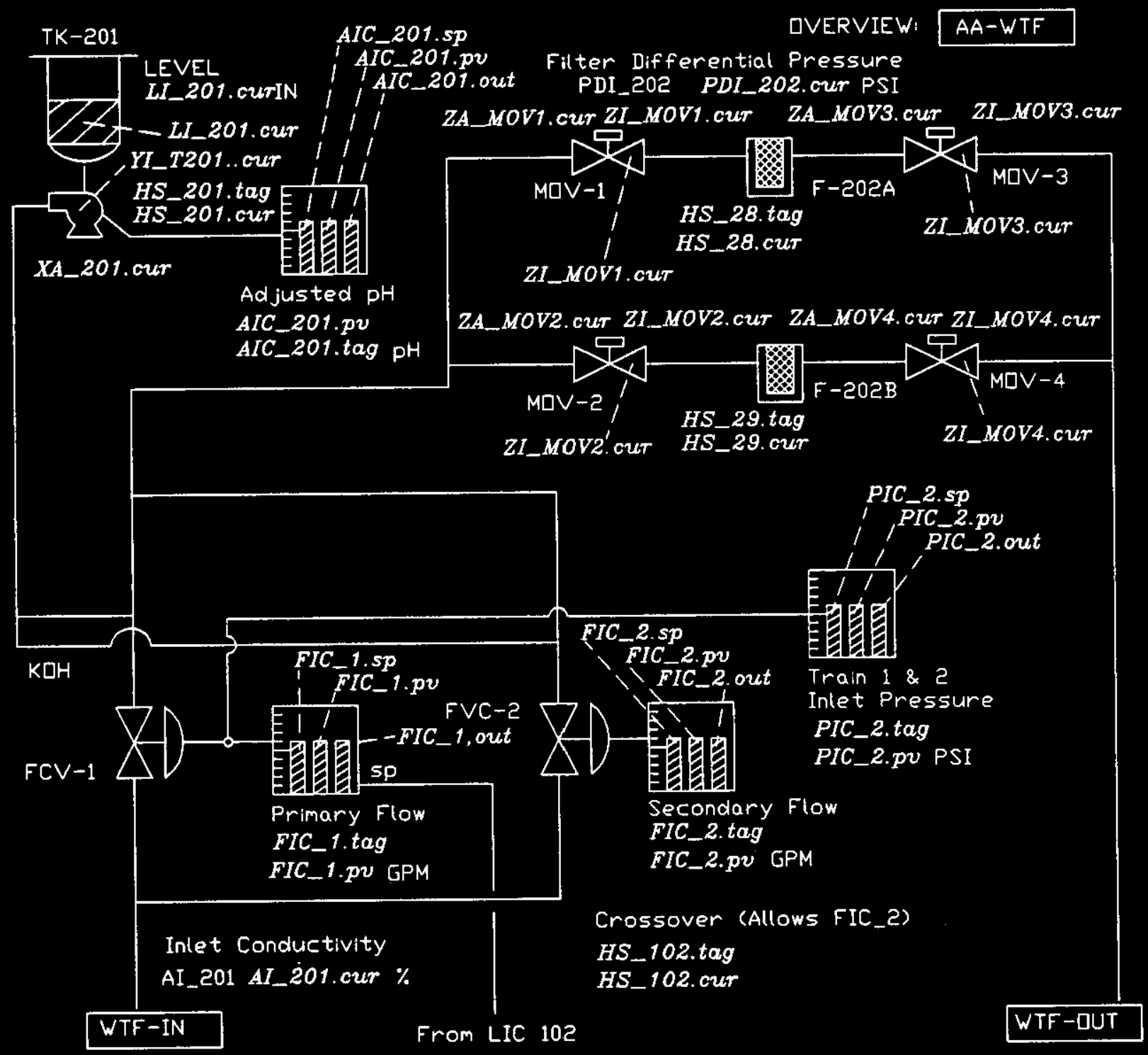


WHC-SD-CP-CSWD-016

Volume 1

Revision 1

Page 17

\section{VTF-OUT}

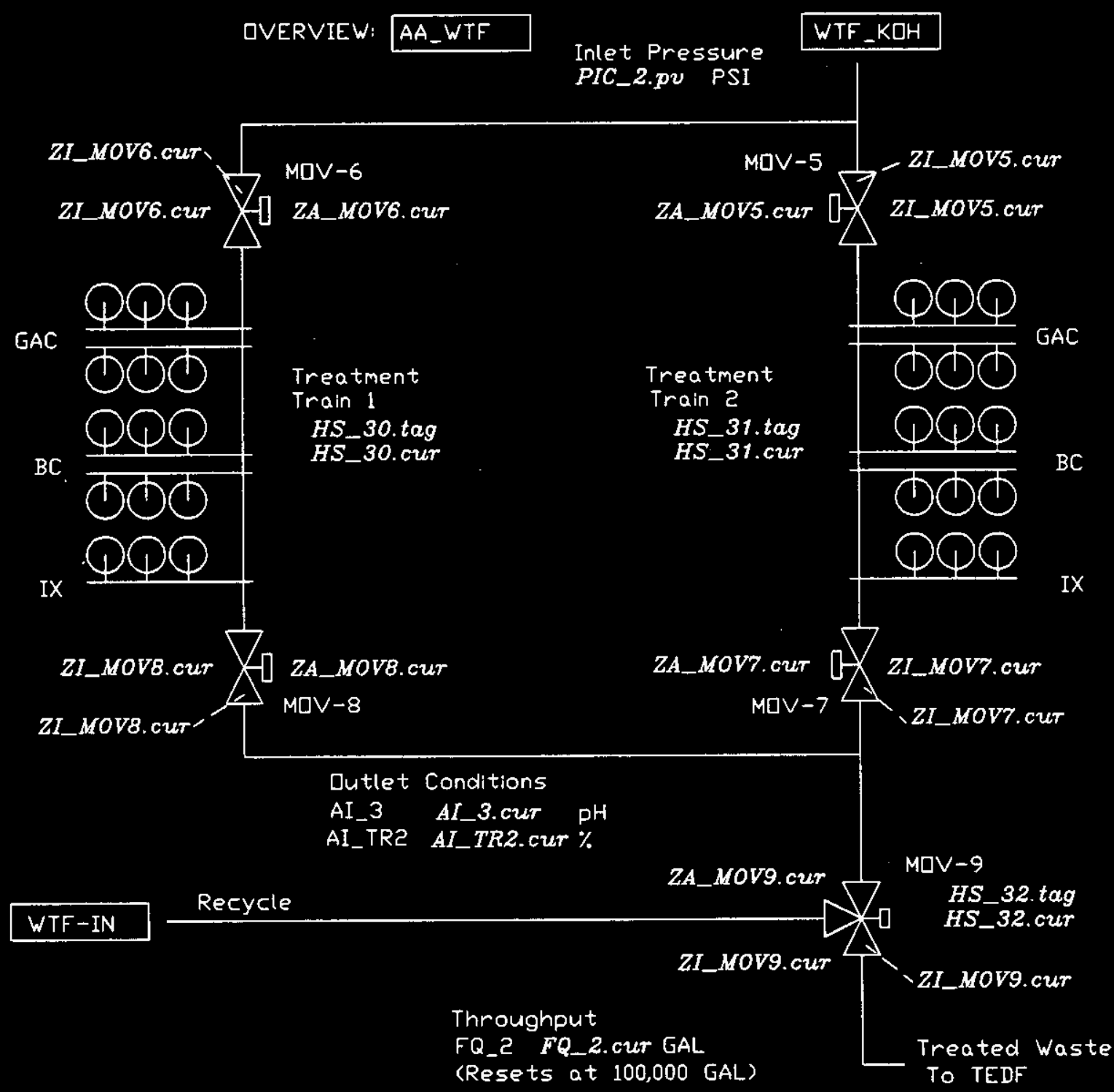


WHC-SD-CP-CSWD-016

Volume 1

Revision 1

Page 18

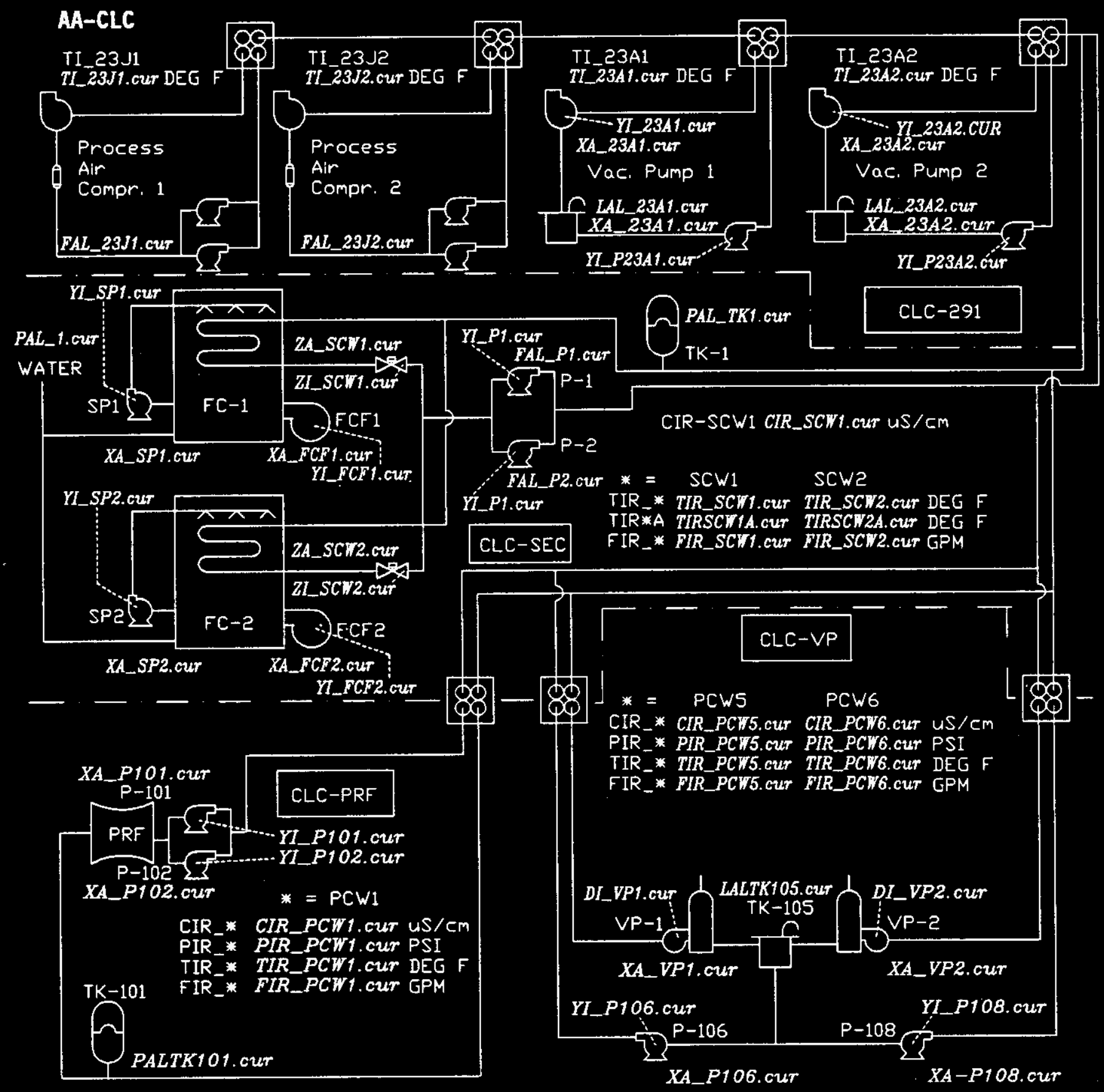


WHC-SD-CP-CSWD-016

Volume 1

Revision 1

Page 19

CLC-291

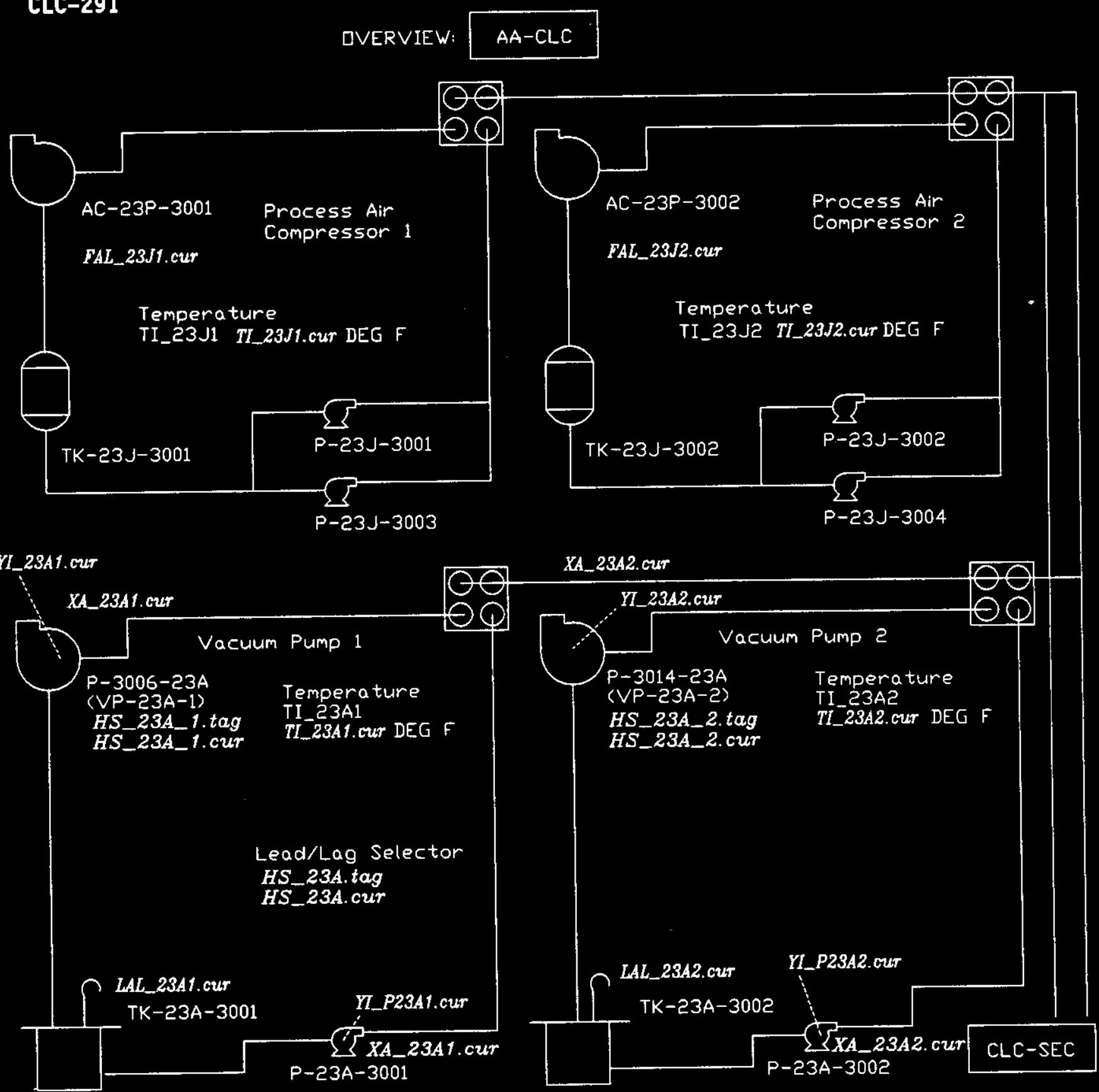


WHC-SD-CP-CSWD-016

Volume 1

Revision 1

Page 20

\section{CLC-PRF}

$$
\text { QVERVIEW: AA-CLC }
$$

$$
\text { CLC-SEC }
$$

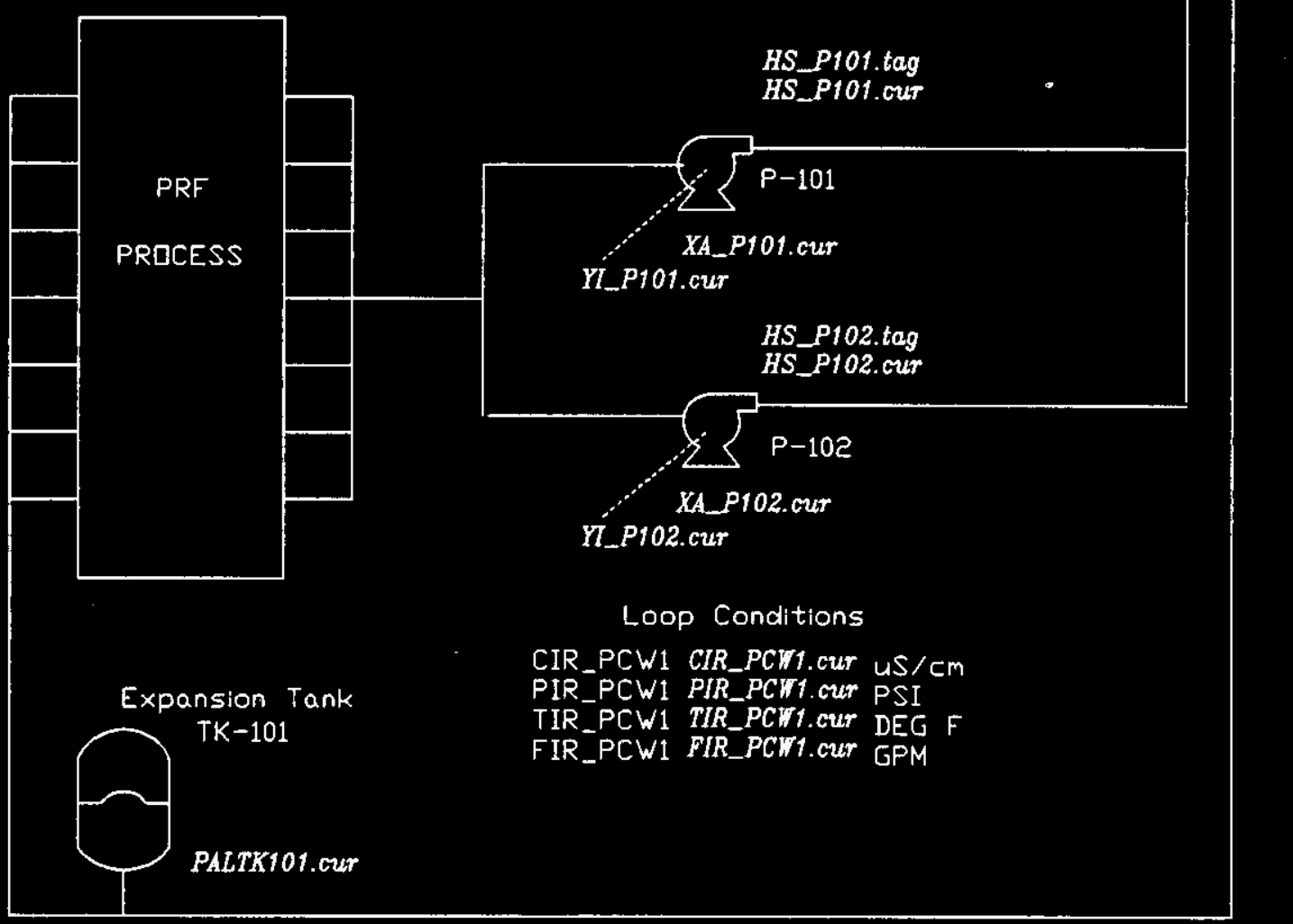


WHC-SD-CP-CSWD-016

Volume 1

Revision 1

Page 21

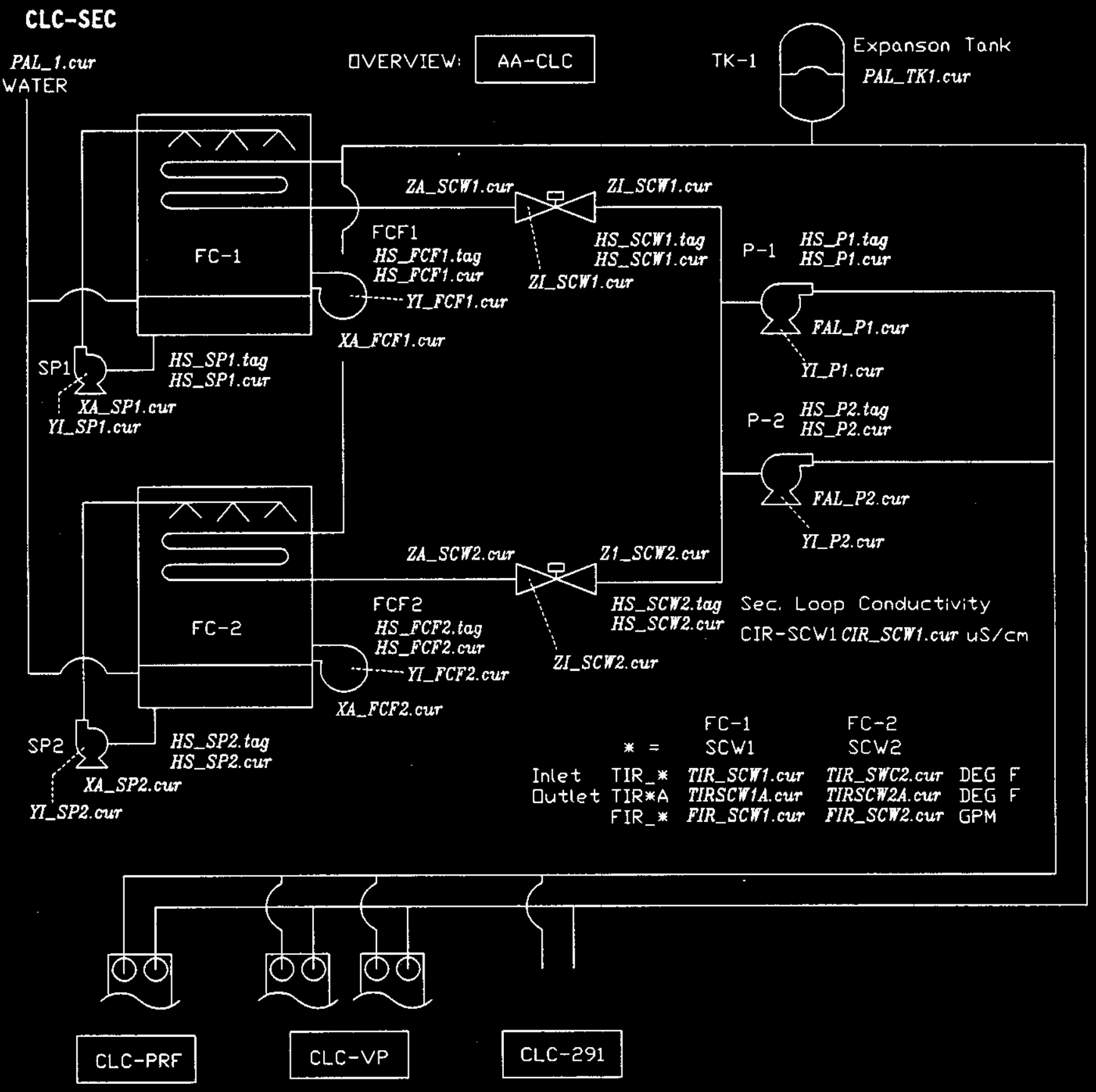


WHC-SD-CP-CSWD-016

Volume 1

Revision 1

Page 22

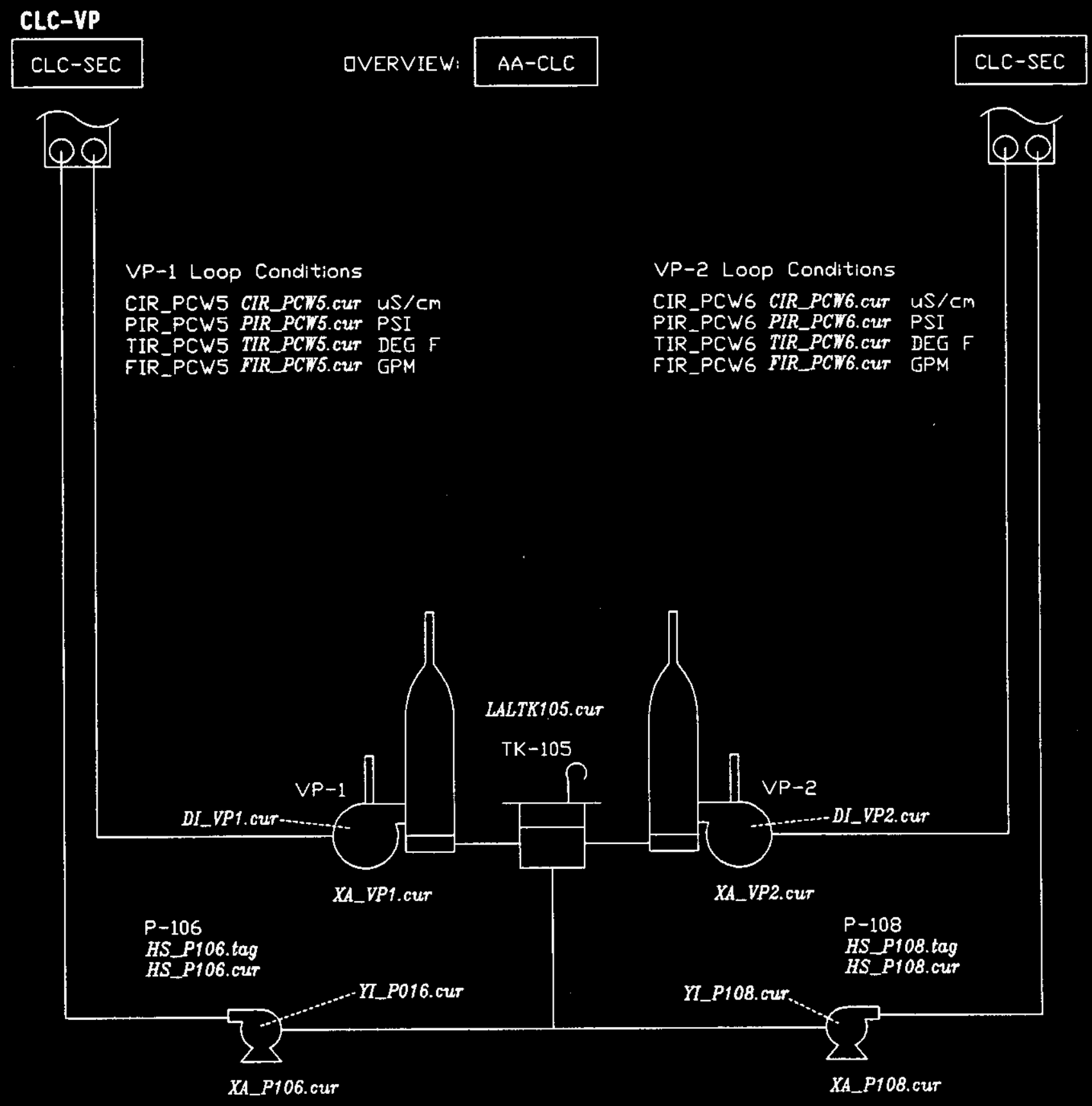


WHC-SD-CP-CSWD-016

Volume 1

Revision 1

Page 23

\section{AA-ZB}

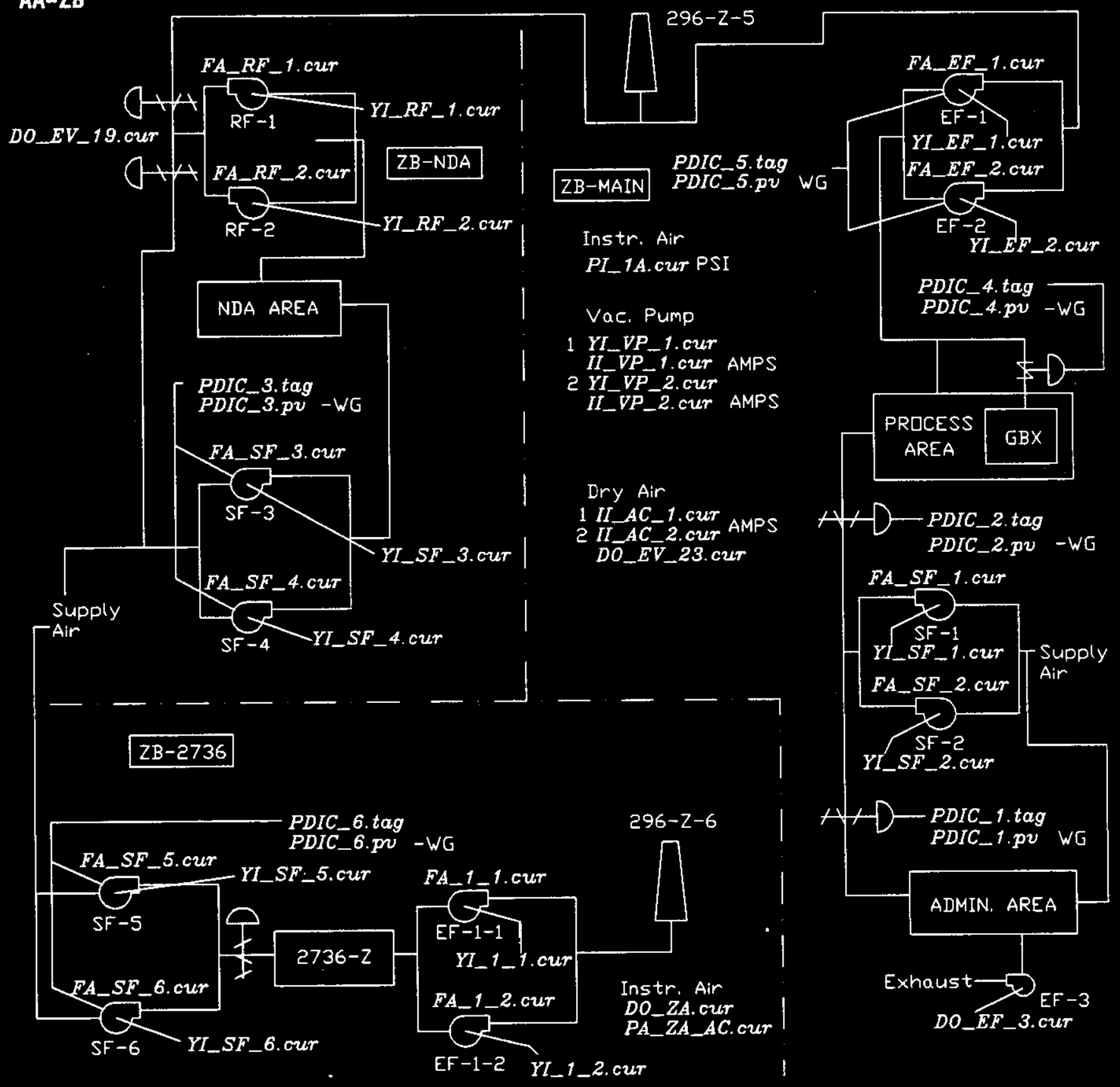


WHC-SD-CP-CSWD-016

Volume 1

Revision 1

Page 24

ZB-2736

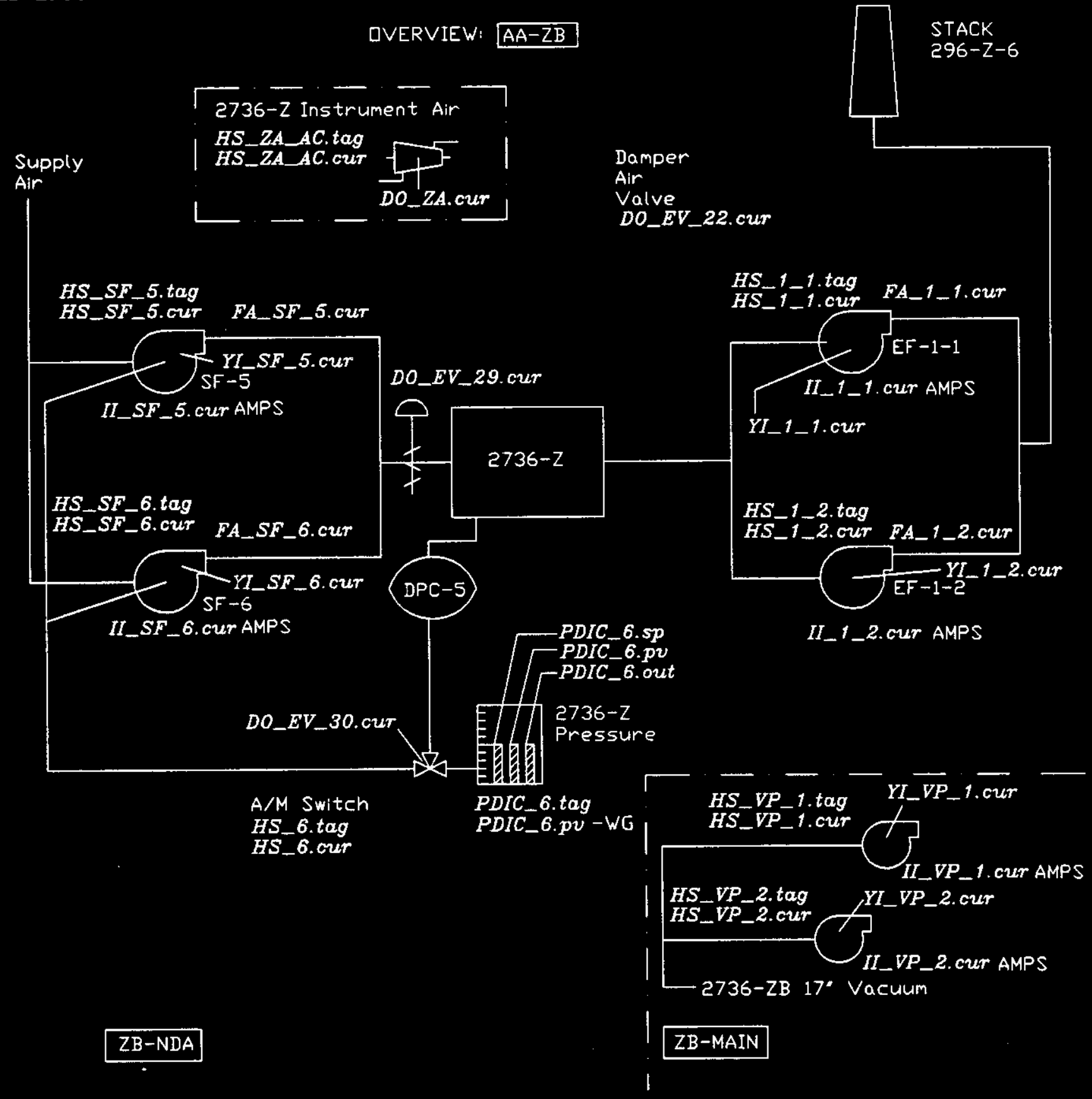


WHC-SD-CP-CSWD-016

Volume 1

Revision 1

Page 25

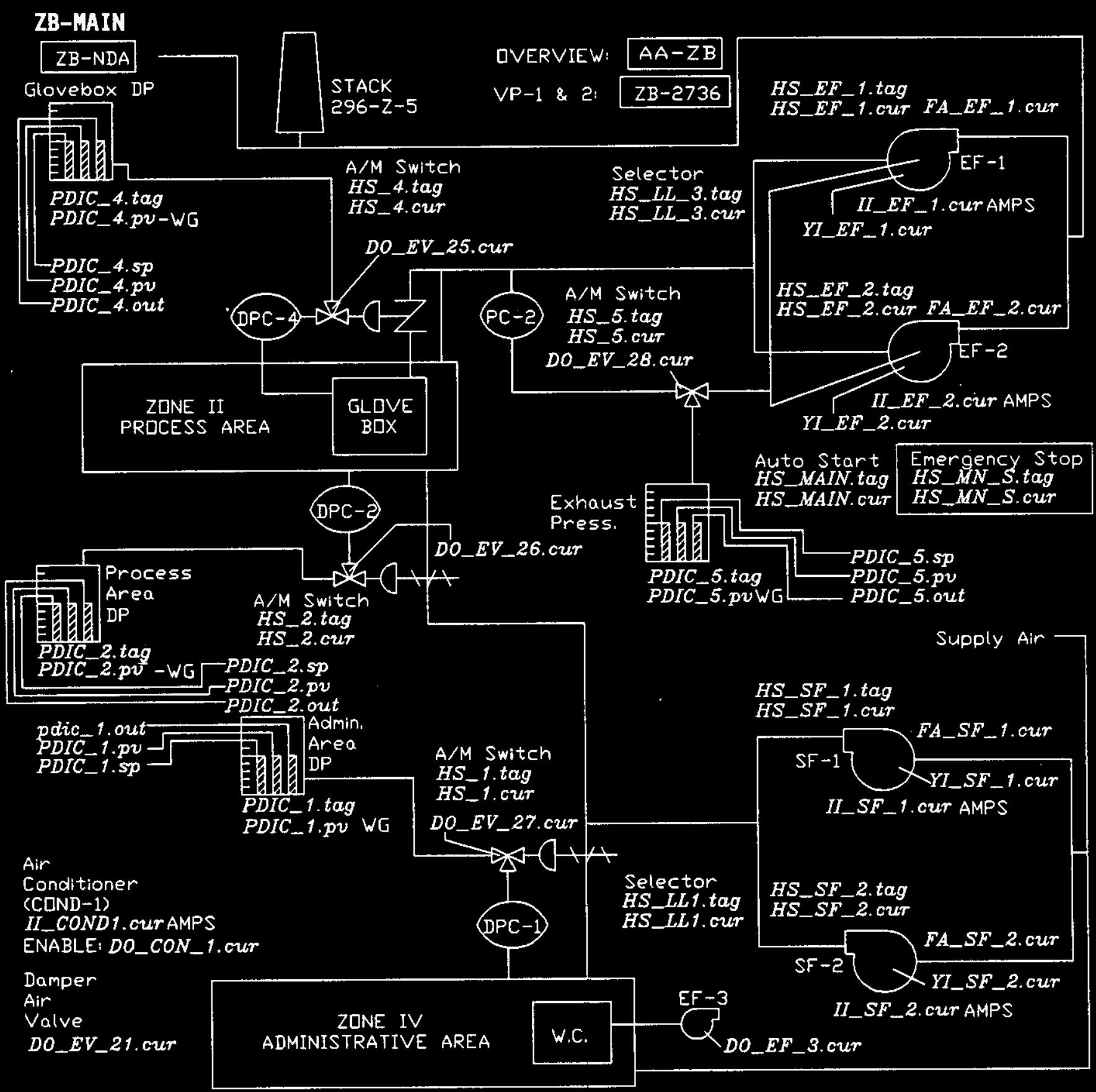


WHC-SD-CP-CSWD-016

Volume 1

Revision 1

Page 26

\section{ZB-NDA}

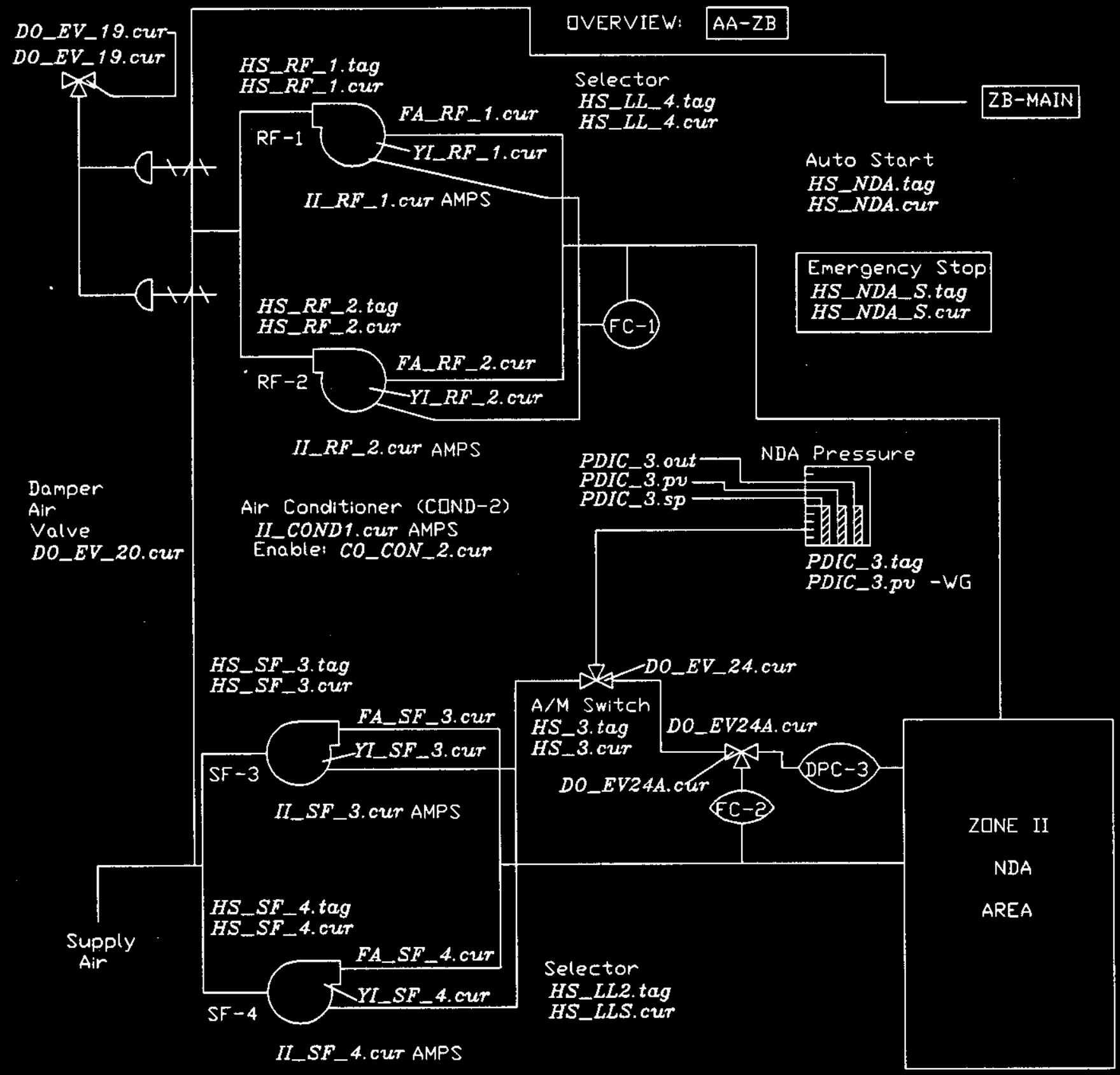




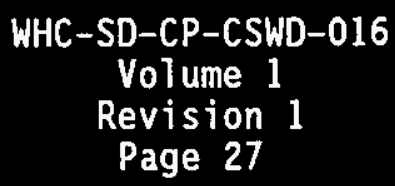

\section{APPENDIX A: SPARC SET-UP FOR MICON VIEN}

(SPARC 20)

This appendix is intended as a reference only. It assumes that the user has a working knowledge of the SUN Unix operating system and has completed the MICON $A / S$ engineer's training course. Refer to the SUN System and Network Administration manual and the MICON $A / S$ View Installation Manual for more information. In addition, help can be obtained from MICON Company $(1-713-947-9470)$.

Unless otherwise told, you must be logged in as root to perform any of these procedures.

\section{Disk Partitions}

The SPARC 20 workstations used at PFP have one or two internal 1 gigabyte hard drives (sdo \& sdl). The partition table for these drives are:

\begin{tabular}{|c|c|c|c|c|}
\hline Partition & Starting Cylinder & \# Blocks & & Mounted On \\
\hline$a$ & 0 & 66528 & $(66 / 0 / 0)$ & $\bar{T}$ \\
\hline b & 66 & 133056 & $(132 / 0 / 0)$ & \\
\hline $\begin{array}{l}c \\
d\end{array}$ & 0 & 2052288 & $(2036 / 0 / 0)$ & \\
\hline d & 0 & 0 & $(0 / 0 / 0)$ & \\
\hline e & 0 & 0 & $(0 / 0 / 0)$ & \\
\hline$f$ & 0 & & $(0 / 0 / 0)$ & \\
\hline$g$ & 198 & 559440 & $(555 / 0 / 0)$ & $\begin{array}{l}\text { /usr } \\
\text { /home }\end{array}$ \\
\hline & 753 & 1293264 & $(1283 / 0 / 0)$ & \\
\hline
\end{tabular}

\begin{tabular}{lllll}
$\begin{array}{llll}\text { sdl } \\
\text { Partition }\end{array}$ & Starting Cylinder & \multicolumn{2}{l}{ \#Blocks } & Mounted On \\
\cline { 2 - 5 } a & 0 & 0 & $(0 / 0 / 0)$ & \\
b & 0 & 0 & $(0 / 0 / 0)$ & \\
c & 0 & $2052288(2036 / 0 / 0)$ & \\
d & 0 & 0 & $(0 / 0 / 0)$ & \\
e & 0 & 0 & $(0 / 0 / 0)$ & \\
f & 0 & 0 & $(0 / 0 / 0)$ & /home2 \\
g & 0 & $2052288(2036 / 0 / 0)$ & \\
h & 0 & 0 & $(0 / 0 / 0)$ &
\end{tabular}

NOTE: For sdl to be recognized, make sure the following line exists in /etc/fstab:

$$
\text { /dev/sdlg /home2 } 4.2 \mathrm{rw} \quad 11
$$

Also, make sure that there is a /home2 directory to mount onto. 
WHC-SD-CP-CSWD-016

Volume 1

Revision 1

Page 28

NOTE: If you reformat sdl, and you don't need to run SunInstall, you must run "newfs" to create a new file system. This must be run from a single user shell.

1. Halt the system if you're running multi-user.

2. From the boot prompt (">"), type "b $-s "$

3. From the single user prompt ("\#"), type "newfs sdlg"

\section{Time Zone}

When installing the operating system, SunInstall will ask for the time zone. This time zone is US/Pacific.

\section{Network Host Information}

Two networks are used on each workstation, leo and 1el. The required information for these networks are:

leo (thicknet)

Workstation

Engineer

Operator \#1

Operator \#2

Supervisor

le1 (thinnet)

Workstation

Engineer

Operator \#1

operator \#2

Supervisor
Ethernet Address

$8: 0: 20: 22: e 9: 2 d$

$8: 0: 20: 77: 42: 93$

$8: 0: 20: 23: 19: 1 d$

$8: 0: 20: 73: d 8: 76$
Internet Address

192.9.200.5

192.9 .200 .11

192.9 .200 .6

92.9.200.4
Workstation Name

ews

ows 1

ows2

SWS

Each workstation must have three files to identify its network names and address. These files are /etc/ethers, /etc/hostname.1e0, and /etc/hostname.1el. Ethers contains that stations ethernet address (eg. 8.0.20.d.e8.3 for the engineer's station). Hostname. leo contains the workstation name for $1 \mathrm{eO}$ (ews for the engineer's station). And, hostname.lel contains the workstation name for lel (engineer for the engineer's station).

Also, each workstation should have a /etc/hosts and a /etc/hosts.equiv file. These files are required for the stations to have access to each other over 1e0. MICON View requires this access.

The /etc/hosts has one line for localhost (required even if you don't have a network) and one line each for all stations you need access to. Each 1 ine contains the station's internet address and its name. The localhost internet address is always 127.0.0.1. In addition, the station should have a 1 ine for 
WHC-SD-CP-CSWD-016

Volume 1

Revision 1

Page 29

itself followed by "loghost", a line for the lel address and a line for the TranServer attached to it. The TranServers address are:

\begin{tabular}{lll} 
Workstation & Internet Address & TranServer Name \\
\cline { 1 - 2 } Engineer & 192.9 .200 .52 & tserv1 \\
Operator $\# 1$ & 192.9 .200 .50 & tserv1 \\
Operator $\# 2$ & 192.9 .200 .53 & tserv1 \\
Supervisor & 192.9 .200 .51 & tserv1
\end{tabular}

For example, the /etc/hosts file for the supervisor's workstation looks like:

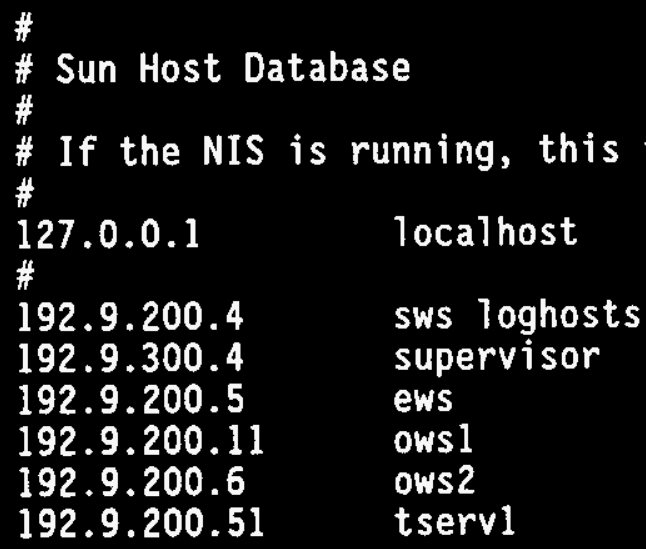

The /etc/hosts.equiv file is a permission file to allow access from other workstations. This file should have two lines:

$$
\begin{aligned}
& + \text { micon } \\
& +
\end{aligned}
$$

\section{Creating a New Kernel}

In order for MICON View to run properly the standard kernel must be modified. See the Sun System and Network Administration manual, section 9.4, method 1 for information on modifying the kernel.

/usr/share/sys/sun 4m/conf/POWELL contains the needed modifications. You can create a new kernel from this file. If it's not on the workstation, copy it over from one of the other stations or create a new POWELL file from the GENERIC file supplied by Sun. 
WHC-SD-CP-CSWD-016

Volume 1

Revision 1

Page 30

To modify the GENERIC file, change maxusers from 8 to 48 , and add the following lines to the end of the file:

$$
\begin{aligned}
& \text { options MSGPOOL }=250 \\
& \text { options SEMMNI }=200 \\
& \text { options SEMMNS }=250 \\
& \text { options FIFOCNT }=250
\end{aligned}
$$

\section{Micon User Password, Directories and Files}

MICON View runs under the Sun user name "micon". Therefore, each station must be set up to recognize this user and provide a home directory.

To set up recognition, add the following 1 ine to /etc/passwd:

$$
\text { micon::101:100:A/S View User:/home/users/micon:/bin/csh }
$$

And, add this line to /etc/group just before the last line ( $+:)$ :

$$
\text { micon:*:100:micon }
$$

To create a home directory for micon, make a new directory called /home/users/micon, and change ownership of this directory to micon ("chown micon /home/users/micon"). NOTE: Change ownership of /home/users/micon only. /home and /home/users should still be owned by root.

Finally, copy the following files from another station or a backup tape into /home/users/micon: .cshrc, .login, .openwin-init, .openwin-menu, .openwin-menu-micon, .openwin-menu-programs, .openwin-menu-utilities, menu-eng, and menu-ops. NOTE: only cshrc, login, and .openwin-init are required by MICON View. The other files are designed to modify the standard menu into either an operator's or an engineer's format. If these are left out, a default menu is used. This menu is similar to the engineer's menu.

\section{Extra Swap}

The standard swap space provided by Sun is not enough for MICON View. To increase it, create /home/extraswap. Change directories to /home, and create extraswap using the following command:

$$
\text { mkfile } 40 \mathrm{~m} \text { extraswap }
$$

Next add this line to /etc/fstab:

$$
\text { /home/extraswap swap swap rw } 00
$$


WHC-SD-CP-CSWD-016

Volume 1

Revision 1

Page 31

\section{RAM Drive}

MICON View requires the use of a RAM drive to speed program operation. To create one, make a new directory, /home/Ram, and set it up for read write list access by everybody ("chmod 777 /home/Ram").

Add this line to /etc/fstab:

$$
\text { swap /home/Ram tmp rw } 00
$$

And, add this line to /etc/rc.local

mount /home/Ram

\section{Printer Files}

Both a video color printer and black and white text printer are connected to each workstation through a TranServer on the thicknet (lea). Several files must be created and/or modified to allow the workstation access to them.

To begin with, copy the following files from a backup tape or another workstation into /usr/lib:

\begin{tabular}{ll} 
File Name & Description \\
\hline hppaintjet & Video Printer Driver \\
ipf & Text Printer Driver \\
tcpcon & TranServer Driver \\
nd & TranServer Driver
\end{tabular}

These files are not part of the Sun operating system. They are supplied by the printer and TranServer manufacturers.

Also, make sure the /etc/hosts file has an entry for tservel. See Network Host Information (above).

Next, create a /usr/spool/hp and a /usr/spool/alarm directory. These will be the printer spool directories. 
WHC-SD-CP-CSWD-016

Volume 1

Revision 1

Page 32

Next, add the following 1 ines to the bottom of /etc/printcap:

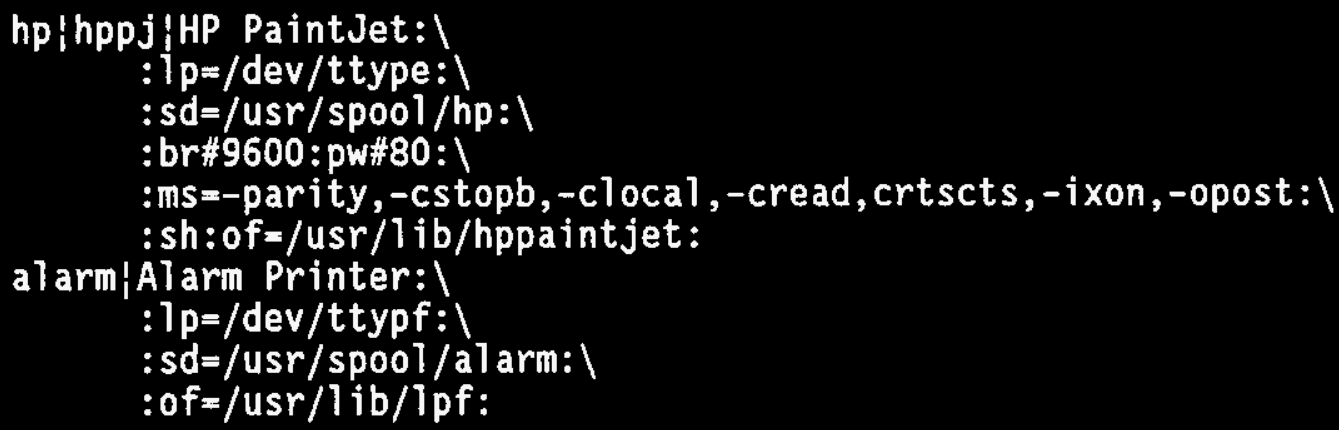

Then, insert the following lines in /etc/rc. Existing lines are shown in bold italics.

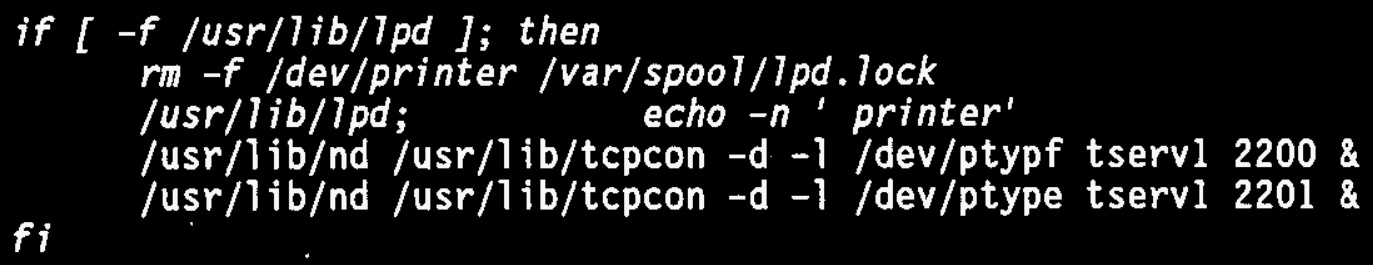

You may find it easier just to copy printcap and rc from another workstation.

Finally, create/dev/lpline and link it to ttypf so MICON View will recognize the text printer as the alarm printer. Change directories to /dev, and type:

link ttypf lpline

\section{Display Files}

Each workstation uses two color monitors. Since the second monitor isn't standard, a driver must be created for it. Also, due to a quirk in the software, some manipulation of the device drivers is needed before Openlindows will recognize both screens.

Change directories to /dev, and create the second screen driver using:

\section{MAKEDEV cgsix 1}

Interchange the screen drivers as follows:

Move cgthreeo to cgthree0.orig

Move cgsixo to cgthree 0

Move cgsixl to cgsixo 
WHC-SD-CP-CSWD-016

Volume 1

Revision 1

Page 33

\section{Operator Keyboards}

The annunciator panel and Universal Operator Keyboard are connected to the two serial ports on the SPARC II. In order for MICON View to recognize them, two link files must be created.

Change to the /dev directory and:

create kbdpl, and link it to ttya using "link ttya kbdpl" create kbdp2, and link it to ttyb using "link ttyb kbdp2"

\section{Miscellaneous}

There are a few other things that must be done for MICON View to work properly.

The /tmp directory must be changed to allow read, write, and 1 ist access for everybody ("chmod 777 /tmp").

The /dev/nit driver must be changed to allow read and write access for everybody ("chmod $666 / \mathrm{dev} / \mathrm{nit}$ ").

All stations, except operator workstation \#1, must be set up to update their time everyday at midnight. Operator workstation \#l is used as the time keeper. To accomplish this, edit the crontab file (using "crontab -e"), and add the following line to the bottom:

$$
00 * \star / \text { usr } / \text { ucb/rdate ows l >/dev/nul1 }
$$

Although this isn't required, inserting these 1 ines at the bottom of /etc/rc before "exit o" will cause a message to print on the screen when the SPARC is done booting.

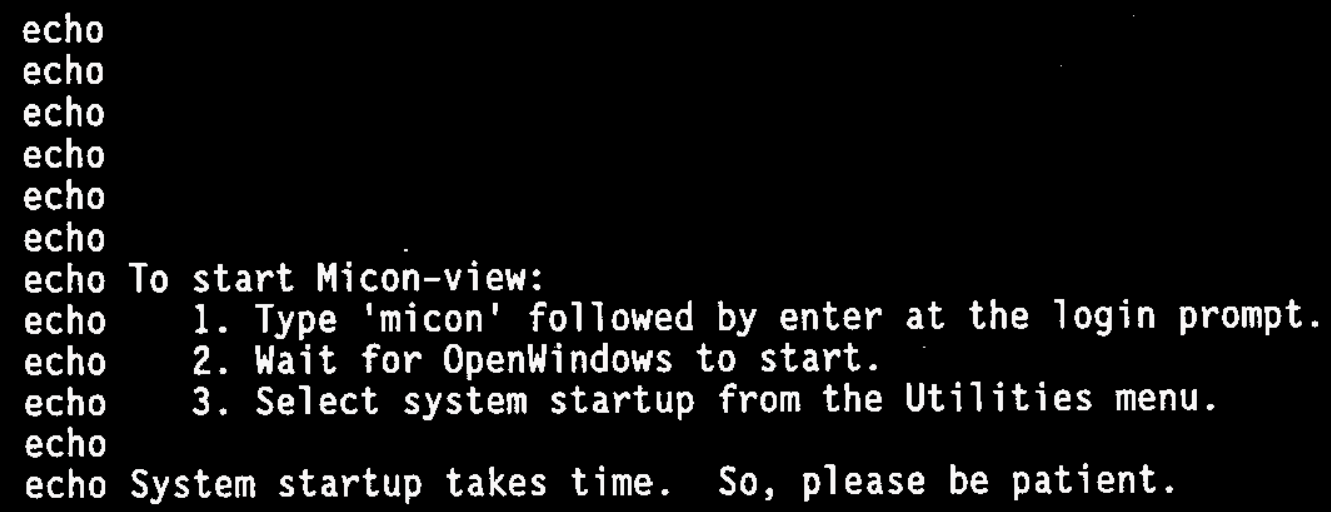


WHC-SD-CP-CSWD-016

Volume 1

Revision 1

Page 34

\section{MICON View \& Configuration Files}

Now that everything's been set-up, MICON View can be loaded.

First, create three new directories: /home/Run, /home/Data, and /home/tmp.

Change ownership of /home/Run and /home/Data to micon ("chown micon /home/Run" and "chown micon /home/Data").

Next, change the /home/tmp directory to read, write, and list access for everybody ("chmod 777 /home/tmp").

After that, load the Run and Data directory contents from a backup tape or another workstation. NOTE: If this is a new or completely cleaned Data directory, copy it from another workstation. The normal Data directory backup tape doesn't include several subdirectories required by MICON View.

Finally, create/home/users/micon/hosts.dat. This file contains the leo names of the other workstations. See Network Host Information for the list of leo network names. When MICON View first starts, it uses the names in this file to poll all workstations to see if any other stations are already running. If no other stations are running, then MICON View starts up in master mode. If MICON View is already running on another station, then this station starts up in a slave mode. The station running as master has a red "master" in the upper portion of its recent alarm display window.

It's very important that only one station be running as master at one time. If more than one station is master, the communications can become confused and crash. For this reason, the /home/users/micon/hosts.dat file must contain the names off all workstations that you expect to be running MICON View. As an example, the hosts.dat file for the supervisor workstation should look like this: 
WHC-SD-CP-CSWD-016

Volume 1

Revision 1

Page 35

\section{APPENDIX B: SYSTEM FILES}

As shown in Appendix A, several Sun operating system files must be modified to allow MICON View to work properly. These files are documented in this appendix. Please note that it's possible for the files in the workstations to differ from the files shown here and still work. For this reason, the files shown here are presented as a reference only. 
WHC-SD-CP-CSWD-016

Volume 1

Revision 1

Page 36

/etc/rc

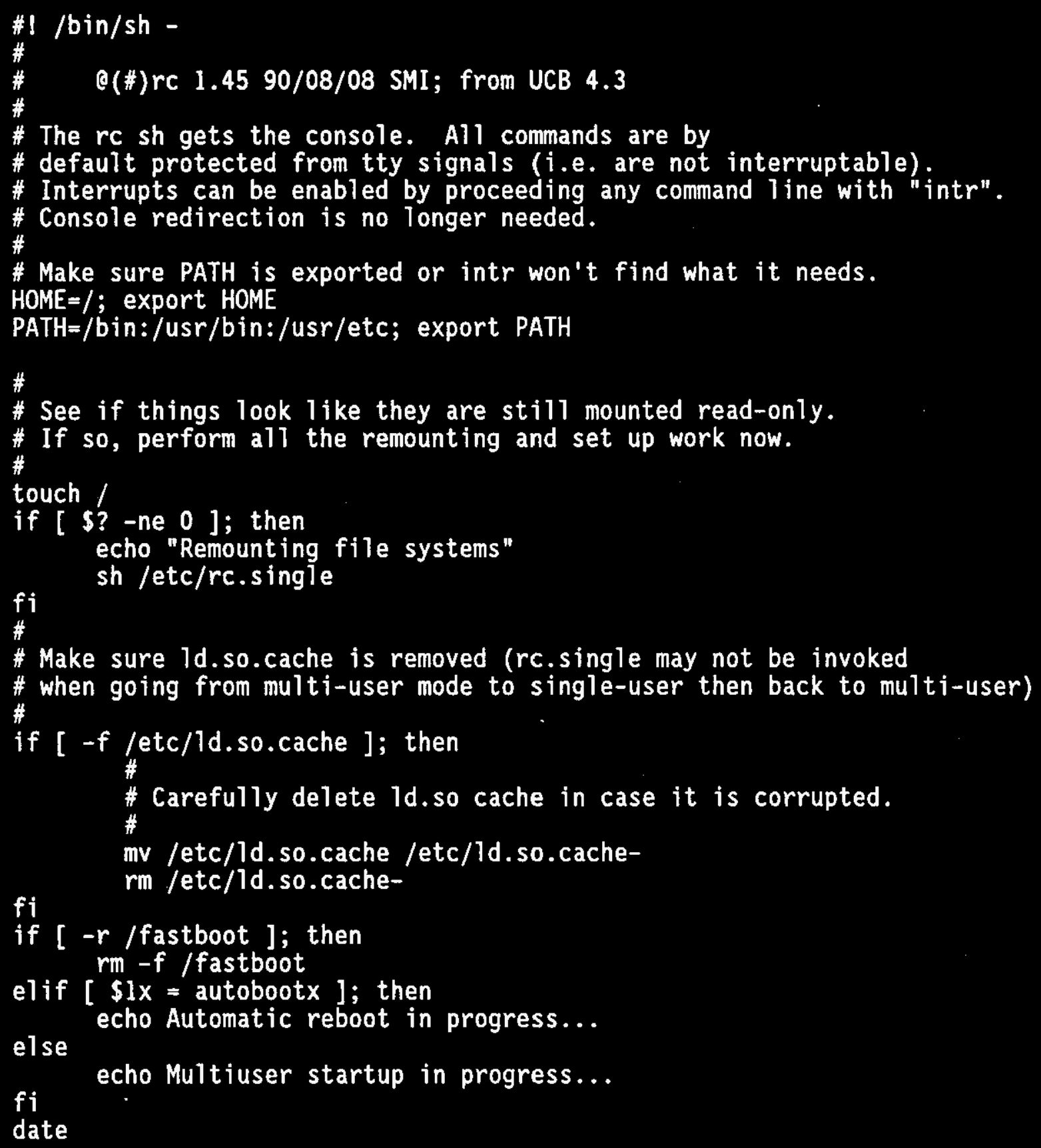


WHC-SD-CP-CSWD-016

Volume 1

Revision 1

Page 37

\# attempt to rationally recover the passwd file if needed

if [ $-\mathrm{s} / \mathrm{etc} / \mathrm{ptmp}]$; then

if [ $-\mathrm{s} / \mathrm{etc} / \mathrm{passwd}]$; then

1s -1 /etc/passwd /etc/ptmp

rm -f /etc/ptmp \# should really remove the shorter

else

echo 'passwd file recovered from ptmp'

fi

$\mathrm{mv} / \mathrm{etc} / \mathrm{ptmp} / \mathrm{etc} / \mathrm{passwd}$

elif [ $-r$ /etc/ptmp ]; then echo 'removing passwd lock file'

fi $r m-f$ /etc/ptmp

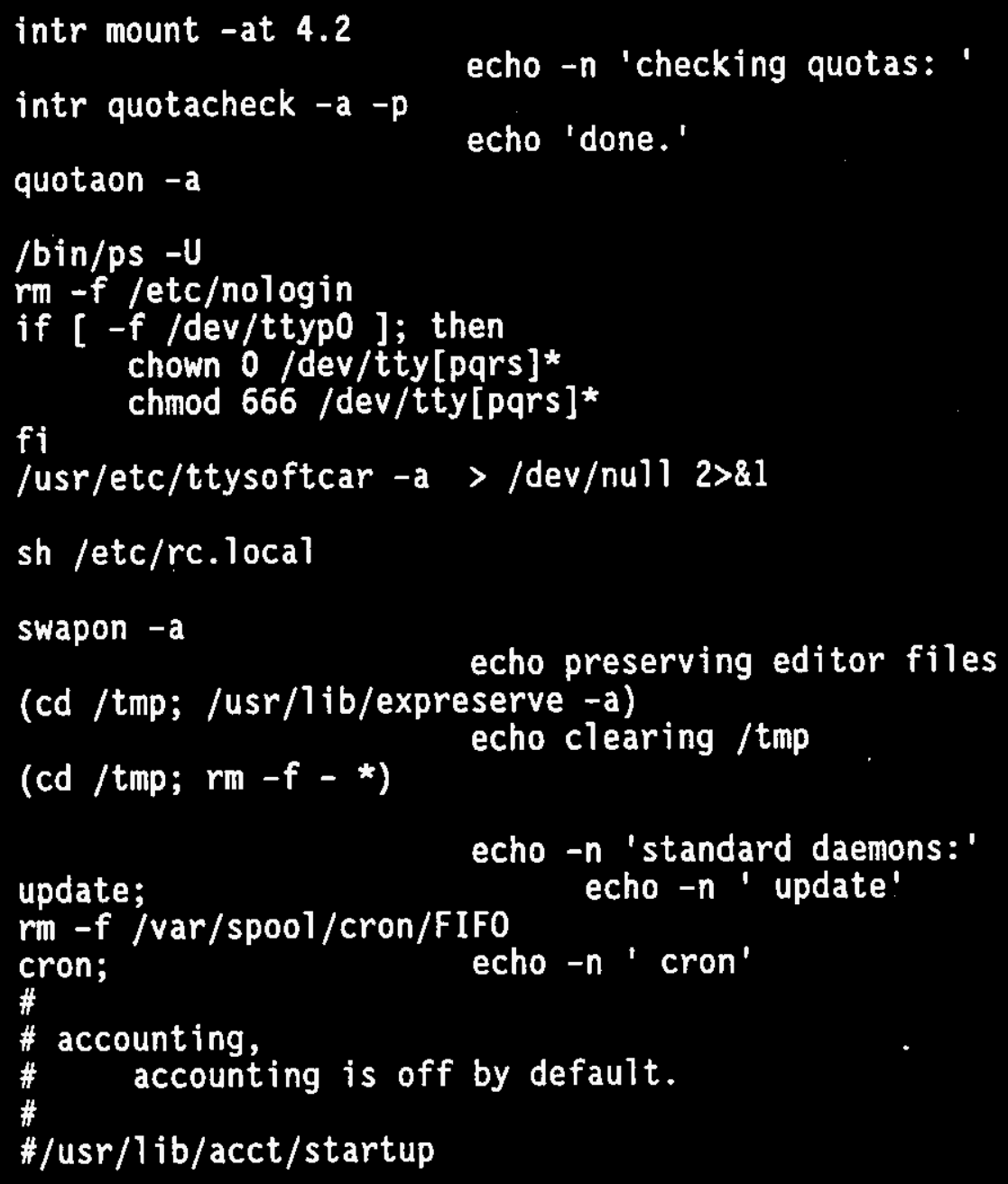


WHC-SD-CP-CSWD-016

Volume 1

Revision 1

Page 38

if [ -d /var/spool/uucp ]; then (cd/var/spool/uucp

$>$ LCK.O

rm -f LCK.*

if [ -d /usr/lib/uucp ]; then

su uucp -c/usr/1 ib/uucp/uusched \& I

fi) echo $-n$ ' uucp'

fi

$$
\text { echo '.' }
$$

echo $-n$ 'starting network daemons:'

\# rwhod (currentiy) is a real performance pig for systems with limited memory. \#if [ -f /usr/etc/in. rwhod ]; then

\# in. rwhod; echo $-n$ ' rwhod'

$\# \mathbf{f i}$

if [ -f / usr/etc/inetd ]; then inetd; echo $-n$ ' inetd'

fi

if [ $-f /$ usr/lib/lpd ]; then $\mathrm{rm}-\mathrm{f} / \mathrm{dev} / \mathrm{printer} / \mathrm{var} / \mathrm{spool} / \mathrm{pd}$. lock /usr/1 ib/1pd; echo $-n$ 'printer' /usr/1ib/nd /usr/1ib/tcpcon -d -1 /dev/ptypf tservl $2200 \&$

fi /usr/1ib/nd /usr/lib/tcpcon -d -1 /dev/ptype tservl $2201 \&$

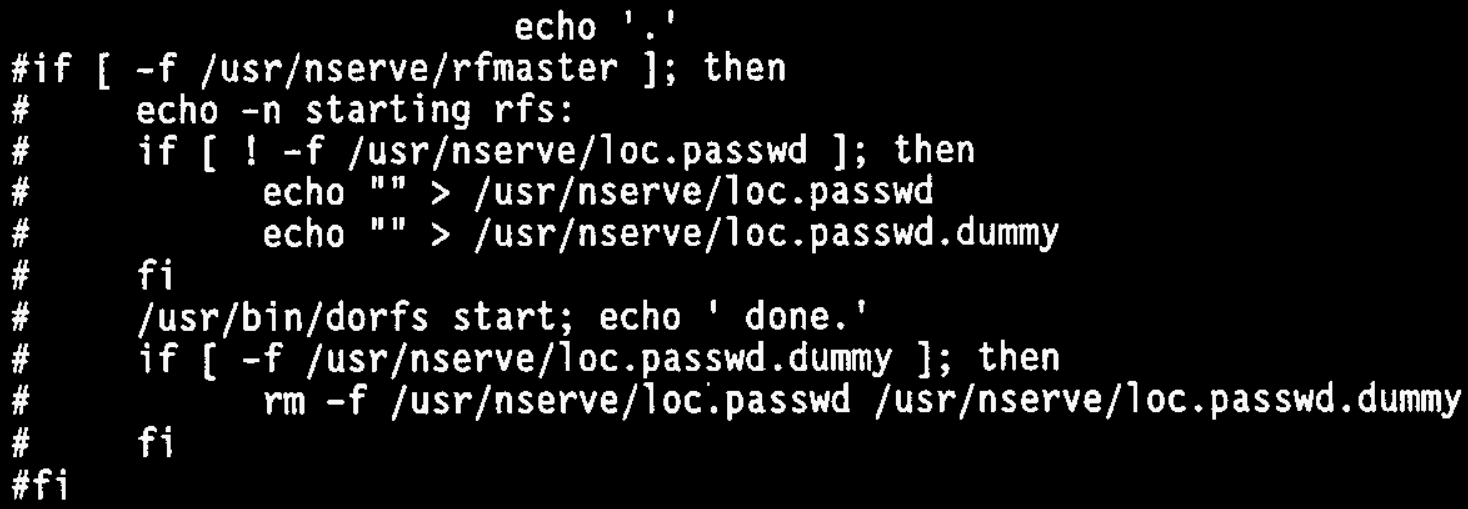


WHC-SD-CP-CSWD-016 Volume 1

Revision 1

Page 39

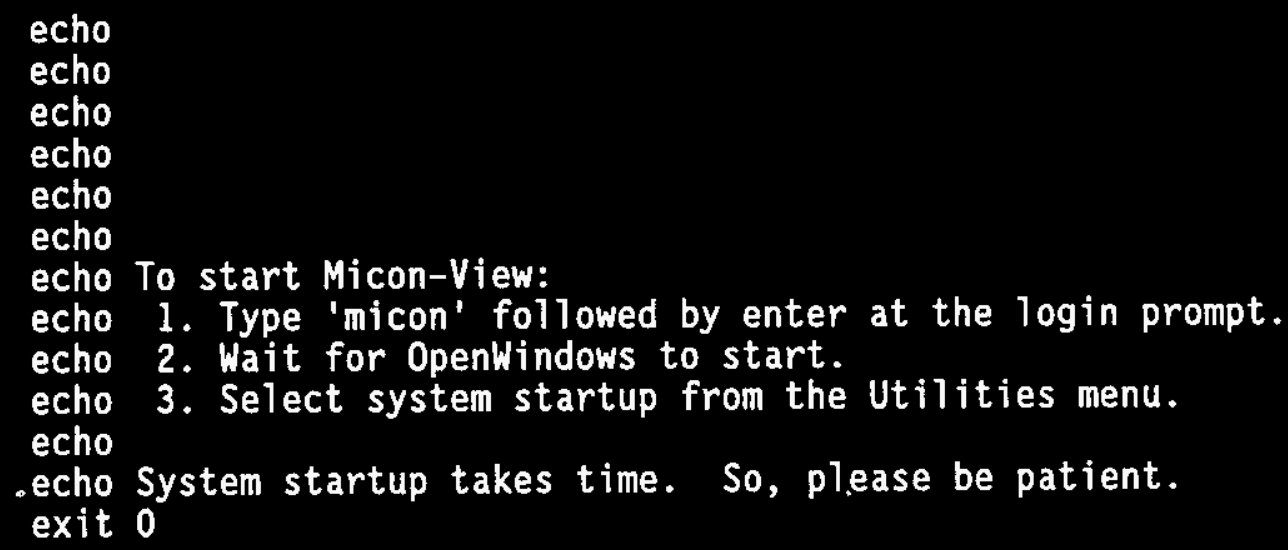


WHC-SD-CP-CSWD-016

Volume 1

Revision 1

Page 40

/etc/printcap

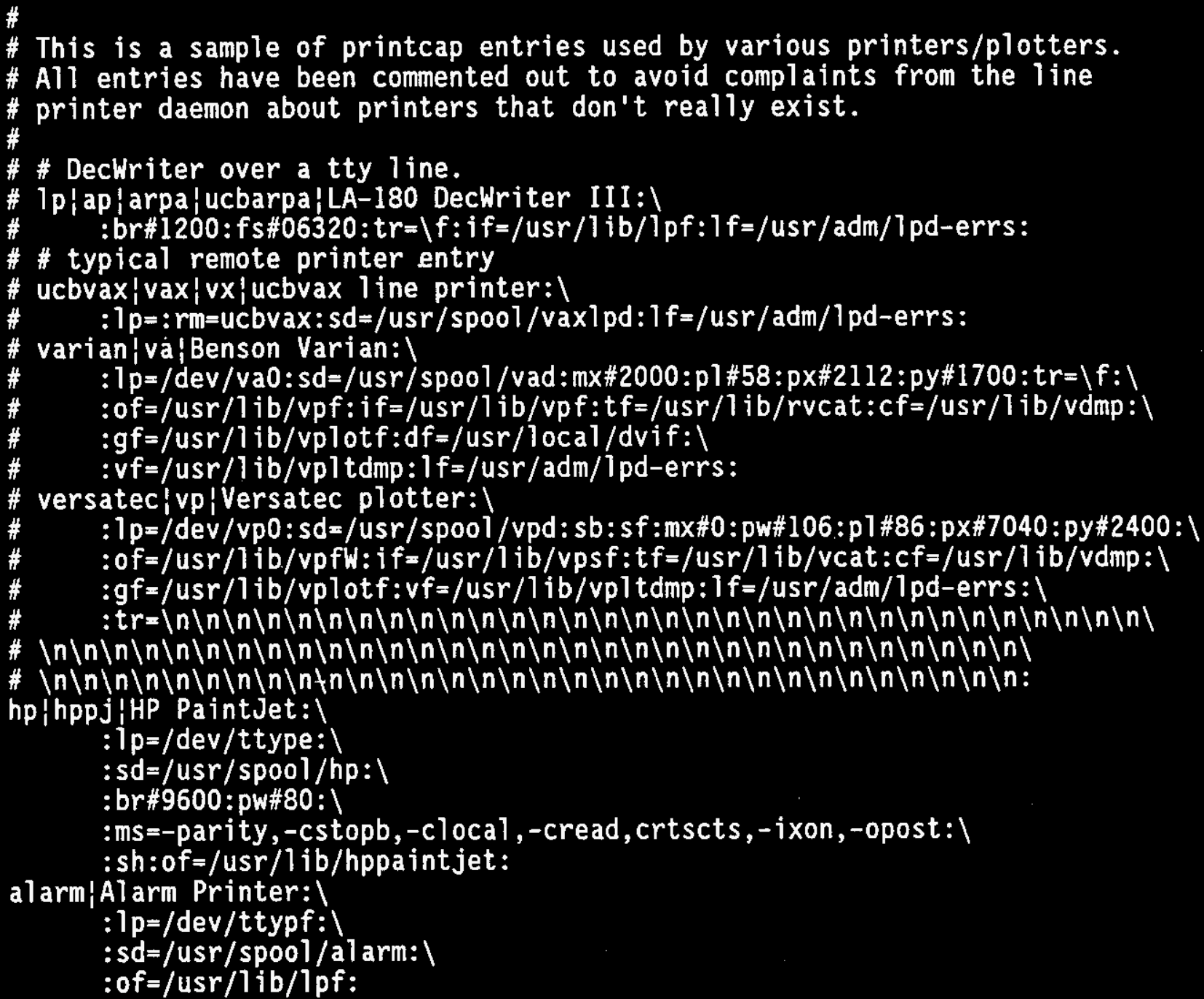


WHC-SD-CP-CSWD-016

Volume 1

Revision 1

Page 41

/home/users/micon/. cshre

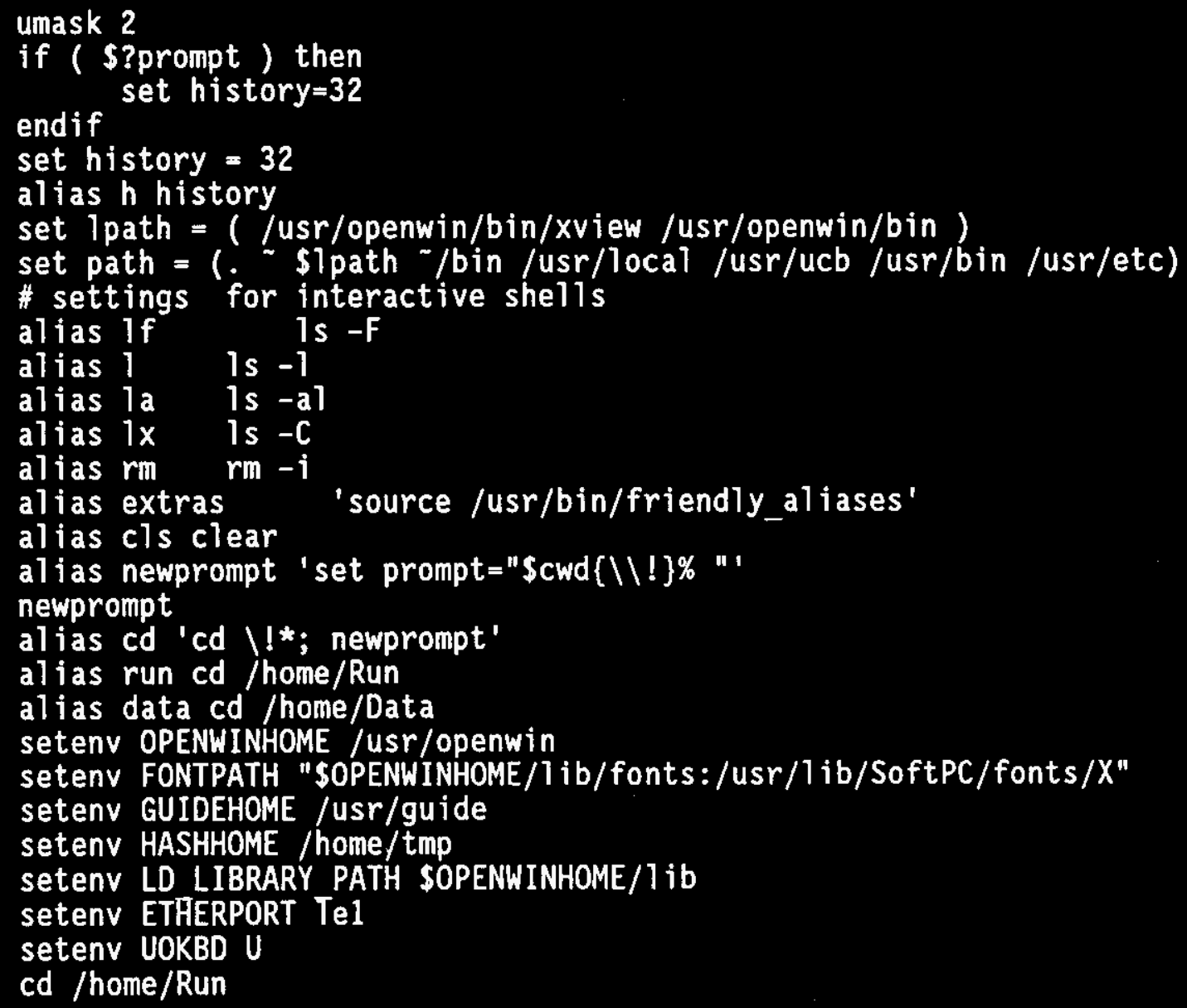


WHC-SD-CP-CSWD-016

Volume 1

Revision 1

Page 42

/home/users/micon/.login

\# If possible, start the windows system. Give user a chance to bail out $\ddot{\#}$

if ( "tty" != "/dev/console" || \$TERM != "sun") then

endif exit \# leave user at regular $C$ shell prompt

setenv OPENWINHOME /usr/openwin

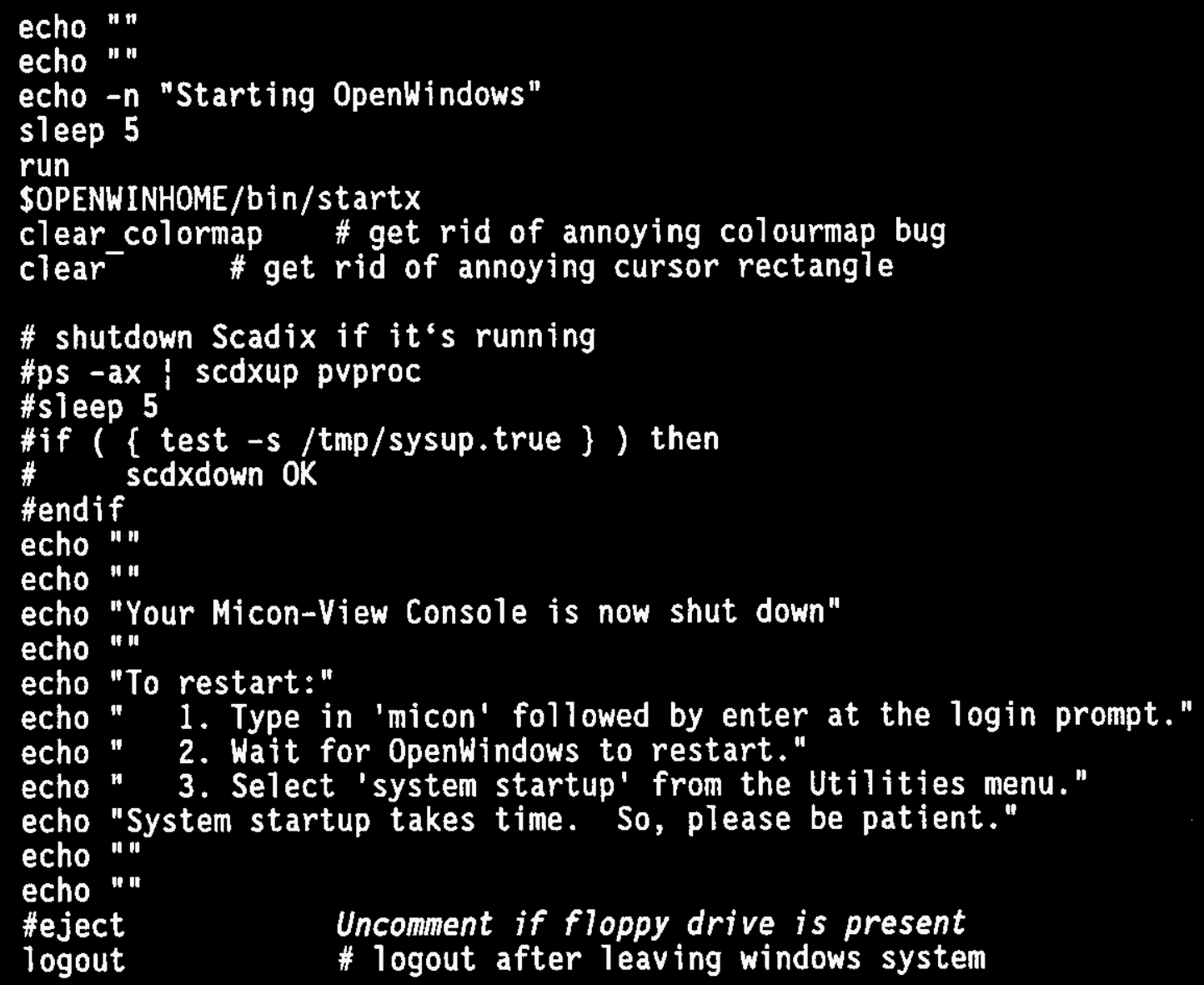


WHC-SD-CP-CSWD-016

Volume 1

Revision 1

Page 43

/home/users/micon/.openwin-init

\# astool replaces the normal console window astool -fg white -bg navy -Wp 10650 -Ws 110550 -C -M 50000 \&

\# this shuts off the screen saver routine $x$ set $s$ off 
WHC-SD-CP-CSWD-016

Volume 1

Revision 1

Page 44

/home/users/micon/openwin-menu-ops (.openwin-menu)

\#

\# $Q(\#)$ openwin-menu $\quad 23.1591 / 09 / 14$ openwin-menu

\# OpenWindows default root menu file - top level menu

\#

"Menu Not Available" TITLE

/home/users/micon/openwin-menu-eng

\#

\# $e(\#)$ openwin-menu $\quad 23.1591 / 09 / 14$ openwin-menu

\# OpenWindows default root menu file - top level menu

$\#$

"Workspace" TITLE

"Programs" MENU SOPENWINHOME/1 ib/openwin-menu-programs

"Utilities" MENU SOPENWINHOME/1ib/openwin-menu-utilities

"Properties..." PROPERTIES

SEPARATOR

"Micon" MENU SHOME/.openwin-menu-micon

SEPARATOR

"Exit..." EXIT

/home/users/micon/. openwin-menu-micon

"Micon View" TITLE PIN

"MCP" exec/home/Run/mcp

"ANNUNCIATOR" exec/home/Run/anunpanel system

/home/users/micon/menu-eng

cp openwin-menu-eng .openwin-menu

/home/users/micon/menu-ops

cp openwin-menu-ops .openwin-menu 
WHC-SD-CP-CSWD-016

Volume 1

Revision 1

Page 45

\section{APPENDIX C}

Trademarks

Trademark
CMC
DecWriter
Emerald
FPXT
Hewlett Packard
IBM
MICON
Motorola
Rockwe11
Sun
TranServer
UNIX
Versatec

Company

CMC Corp.

Digital Equipment Corp.

Emerald Computers Inc.

Emerald Computers Inc.

Hewlett Packard Corp.

International Business Machines Corp.

Powell Process Systems Inc.

Motorola Corp.

Rockwell International Corp.

Sun Microsystems Inc.

CMC Corporation

X/Open Company Ltd.

Versatec Inc. 


\section{WHC-SD-CP-CSWD-016 \\ Volume 2 \\ Revision 1 \\ Page 1}

\section{TABLE OF CONTENTS}

INTRODUCTION . . . . . . . . . . . . . . . . . . . . 2

MICON CONFIGURATION FILES . . . . . . . . . . . . . . . . . . 5

WTF $13 . \mathrm{mic2}$ ro ...................... 7

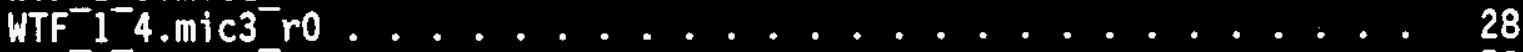

WTF $^{-1 . m i c 4}$ ro ......................... 52

$\mathrm{WTF}^{-2} 2 . \mathrm{mic5}$ ro ....................... 79

WTF_2_3.mic6_ro........................ 106

MICON PROGRAM FILES . . . . . . . . . . . . . . . . . . . . 119

mic2 ro........................... 120

mic3ro........................... 125

mic4ro............................ . 130

mic5 ro . . . . . . . . . . ............. 136

mic6_ro............................. . . 142

APPENDIX A: MICON INPUT/OUTPUT ................... 146

MICON Number: 2......................... 147

MICON Number: 3.......................... 148

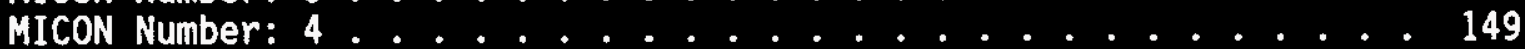

MICON Number: 5......................... 150

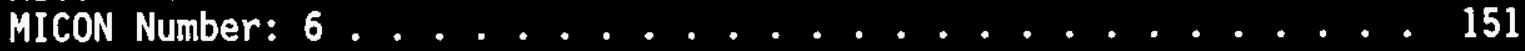


WHC-SD-CP-CSWD-016

Volume 2

Revision 1

Page 2

\section{INTRODUCTION}

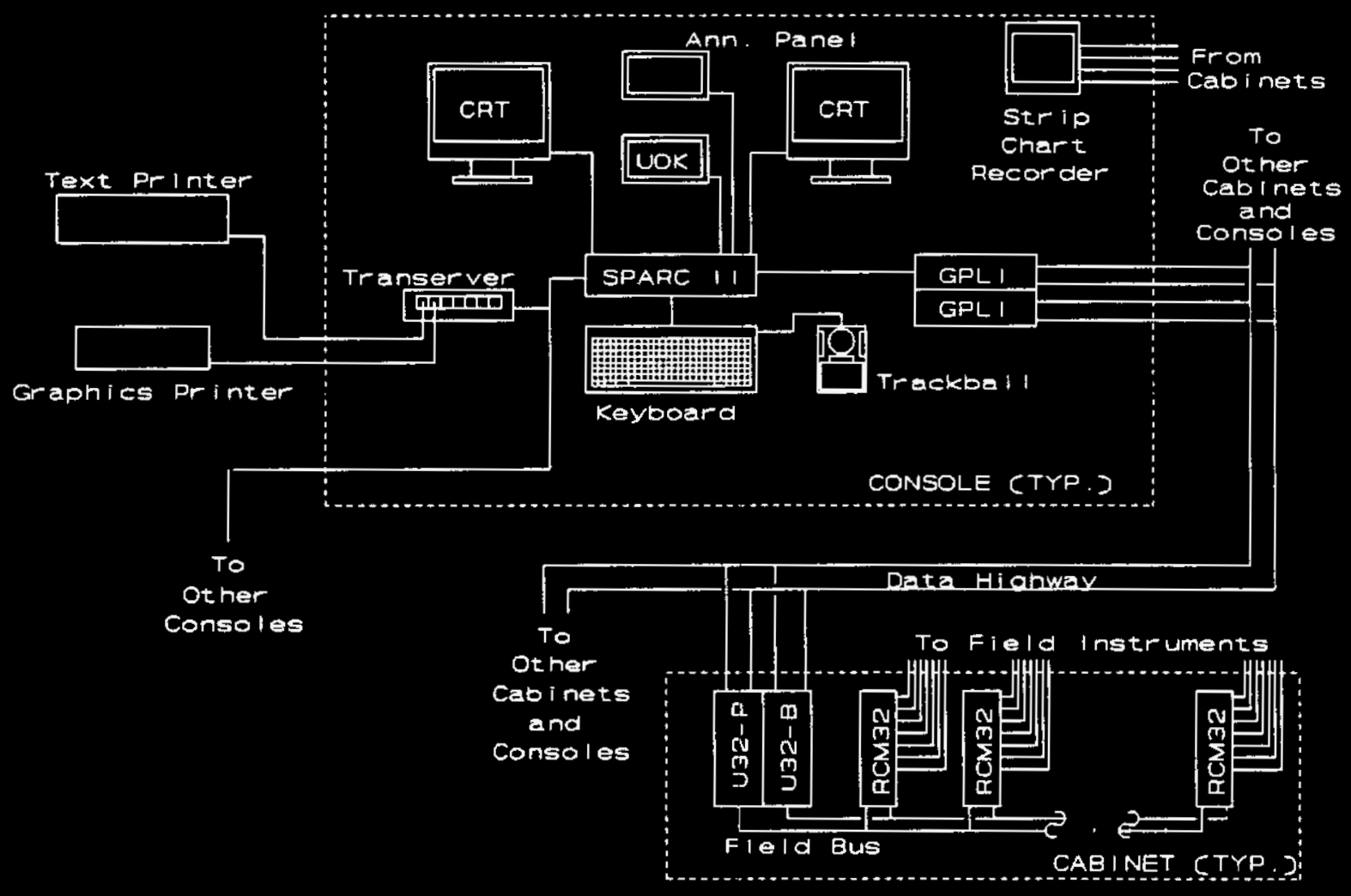

The MICON ${ }^{1}$ A/S distributed control system consists of four consoles or workstations (2 operator, 1 supervisor, and 1 engineer), a data highway, and eight process controller cabinets.

Each console is based on a Sun Microsysten's SPARC 20 workstation. The SPARC 20 workstation is based on a Scalable Process Architecture (SPARC) micro-processor. Each workstation contains 64 megabytes of memory, a one gigabyte hard drive, a 150 megabyte tape drive, a keyboard, and a trackbal1. Select SARC statations also contain an additional one gigabyte hard drive, a CD-ROM drive, and a 5 gigabyte tape drive. In addition, each SPARC 20

${ }^{1}$ For a list of Trademarks, see Volume 1, APPENDIX C 

WHC-SD-CP-CSWD-016
Volume 2
Revision 1
Page 3

provides two video outputs, two RS-232 serial ports, and two ethernet ports (a thick-net and a thin-net). The video ports are connected to two high

resolution color monitors. The RS-232 ports are connected to two Emerald model FPXT single board IBM XT compatible computers with touchscreen. One of the FPXTs is designated as an annunciator panel and is unused. The other is used as a Universal Operator Keyboard (UOK). One of the two ethernet ports -the thin-net -- is connected to the data highway via a MICON Company General Purpose LAN Interface (GPLI). The thicknet provides a Local Area Network (LAN) between the four consoles. It also connects each SPARC 20 with a Rockwe11/CMC TranServer. The TranServer provides eight additional RS-232 serial ports for each workstation. One of these serial ports is connected to an Output Technology Corporation 800 series text printer. Another port is connected to a Hewlett-Packard PaintJet color graphics printer.

The Data Highway is the main data link between the consoles and the process control cabinets. It consists of two redundant coax cables that run between all four consoles and six cabinets. The Data Highway is a local area network using a high speed token ring passing protocol. The Data Highway is also redundant. This means that if one of the two cables fail, communication is automatically passed to the other.

The process control cabinets consist of two redundant main controllers, called $U-32 s$, and several process controllers called RCM-32s. The U-32s and RCM-32s communicate via a small local area network called a Field Bus. The U-32 is a MICON built controller based on the Motorola 68020 processor. Although it can be used to directiy control instruments and devices in the field, its primary purpose is to pass information between the consoles and the RCM-32s Each U-32 has a redundant backup. That way if one fails, the other can automatically take over. The RCM-32s are also built by MICON and are based on a Motorola 68030 processor. These controllers provide input and output directly to field instruments and other devices.

There are four types of RCM-32s used in this system: $A, C, D$, and Dr. The $A$ and $C$ cards are used primarily for analog signals. They provide hardware for any standard analog instrument input (4-20 $\mathrm{mA}, 10-50 \mathrm{~mA}$, and $D C$ voltages) and provide a standard 4-20 mA output signal. In addition, they provide two 28 Volt DC discrete (on/off) channels. These may be used for input, output, or both. An A card provides 26 analog inputs and 4 analog outputs. Each C card provides 18 analog inputs and 12 analog outputs. The $D$ card is used only for discrete control. It provides 32 on/off type channels that can be used as inputs, outputs, or both. Both inputs and outputs are 28 volt DC. The Dr cards are similar to the D cards except inputs are 120 volt $A C$ or $D C$ and the outputs are 3 amp (120 VAC) dry contact relays.

Since MICON A/S systems are installed by engineers rather than computer programmers, they are designed to minimize the need for programming. All of the software needed to monitor and control a process is already programmed into the system be MICON Company. The engineer need only "configure" the system to a specific application. 


\section{WHC-SD-CP-CSWD-016 \\ Volume 2 \\ Revision 1 \\ Page 4}

The process is very similar to the method used to install an older analog instrument system. First, a set of Pipe and instrument Diagrams (P\&ID) are created. These drawings show a schematic of the chemical process and the instrumentation to be installed to control it. With an analog system, the engineer would purchase the necessary individual components and wire them together to create the control strategy. With the MICON A/S system, the engineer would call function blocks to invoke preprogrammed modules and create the control strategy. The only difference between the two is that one uses hardware devices wired together, and the other uses software modules connected via a data base.

The MICON configuration is done in two parts. First the RCM-32 and, if needed the U-32 controllers are configured. Next, the workstation displays are created.

The RCM-32 and U-32 configuration consists of both the controller "program" and all display information for each "tag". Tags consists of all inputs and outputs from the controller, PID control loops, and any other displayed value. This information is requested by the MICON View software as the engineer creates the controller configuration.

Since all the display information (colors, ranges, etc.) was input during the controller configuration, the workstation configuration consists only of display creation. These are created by calling up a form and filling in the blanks. The only exception to this are the custom created graphics displays. Graphics are created by drawing a picture of the process and overlaying this with tag information. This is done with a special drawing program supplied with MICON View.

To keep size manageable, this document has been divided into several volumes. Volume 1 contains the display configuration for the four workstations. The other volumes each contain the configuration for one process Controller cabinet.

This volume (number 2) contains the configuration files for the cabinet located in 243-Z. This cabinet consists of six controllers:

1. U-32 (with backup)

2. RCM-32 C

3. RCM-32 C

4. $\mathrm{RCM}-32 \mathrm{Dr}$

5. RCM-32 Dr

6. $\mathrm{RCM}-32 \mathrm{Dr}$
Controller Number: 1 Controller Number: 2 Controller Number: 3 Controller Number: 4 Controller Number: 5 Controller Number: 6
Tagname: WTF MAIN
Tagname: WTF 1 -3
Tagname: WTF $-1-4$
Tagname: WTF $2-1$
Tagname: WTF $2-2$
Tagname: WTF $^{-}{ }^{-3}$

WTF MAIN is being used for communications only and does not contain a configuration. Therefore, only configurations for the other five controllers are shown. 
WHC-SD-CP-CSWD-016

Volume 2

Revision 1

Page 5

\section{MICON CONFIGURATION FILES}

The MICON configuration file is a text file that the MICON View software creates as the user configures or "programs" each MICON controller. A MICON controller is configured by selecting preprogrammed functions and filling in the required information. As this is done, MICON View places this information into the configuration file. This file contains both display and program information. For a detailed description see U-32 and RCM-32 Controller/RTU Function Description published by MICON Company (Vendor Information file 22068).

This file is named by MICON View based on input from the user. The file name's format is TAG.file where TAG is the controllers tag name and file is the program file name. The first eight lines of the file are the information on the controller itself. These lines are:

MICON TYPE - The type of MICON being programmed (A, $C$, or $D)$. In this case, $D$ and Dr cards are considered the same.

MICON UNIT NO - The controller card's number (user assigned).

MICON TAG - The controller card's tag name (user assigned). For consistency, we have assigned tags in the following format:

\section{CABINET_RACK_SLOT}

CABINET is the MICON controller cabinet location. RACK is the rack number ( 1 to 3 ). Rack 1 is at the top of the cabinet. SLOT is the slot number ( 1 to 6$)$. Slot 1 is at the far left of the rack. Therefor, a controller tagged WTF 13 is the card in rack 1 slot 3 of the cabinet located in the Low Level Waste Treatment Facility (243-Z).

MICON DESCRIPTION - A brief description of the controller card

MICON CONFIGURATION FILE - The name of the file that contains the controller card's program (user assigned). 


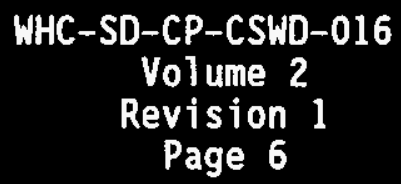

POINT ALLOCATION - The memory allocation for the controller. Memory allocation is based on the types of inputs and outputs (I/0) and the number of storage locations desired. The types of $1 / 0$ and storage locations are:
VAI - Virtual Analog (User assigned). An analog value storage location.
VDI - Virtual Discrete (User assigned). A Discrete (on/off) storage location with alarm capability.
ISW - Internal Switch (User assigned). A Discrete storage location without al arm capability.
LOOPS - Loops (User Assigned). Loops are used to hold a series of program steps. If one of those steps is a PID controller function, The loop tag name becomes the controller tag name.
MSD - Multi-State Discrete (User assigned). This is a special sixteen position indicator.
AI - Analog Inputs (Assigned based on card type used).
AO - Analog Outputs (Assigned based on card type used).
DI - Discrete Inputs (Assigned based on card type used).
DO - Discrete Outputs (Assigned based on card type used).

The remaining information is the actual controller configuration. Right at first, you will notice several blocks of information titled VIRTUAL ANALOG and DISCRETE TAG. These blocks are the configuration information for the virtual analog and internal switch type storage locations. These must always be configured first. After that are the controller loops. Each loop starts with a block of information titled LOOP NO followed by several steps. The last step is always a function 0 (END). Everything except virtual analogs and internal switches are configured inside of a loop.

Loops are a way of organizing the controller configuration. Although most controllers could be programmed using only one loop, using several loops makes the program more understandable. Loops are generaliy invisible to the operator. The exception to this rule is when a PID function (function 20) is used in the loop. When this happens, the loop name becomes the controller name. Otherwise, the loop name is used as an internal reference only

NOTE: Each loop using a PID function has two lines showing the tuning paramaters -- for example:

TUNING PARAMETER :

$P($ GAIN $): 1.00, I$ (REP/MIN) : 5.00, D(RATE) : 0.00 , MANUAL RESET : 0.00

These tuning parameters are not controlled by this document. Therefore, these numbers may be different than the ones that appear in the workstation listings. 
WHC-SD-CP-CSWD-016

Volume 2

Revision 1

Page 7

WTF_1_3.mic2_ro

MICON TYPE :RCM-C

MICON UNIT NO : WTF : 2

MICON DESCRIPTION :-243-Z RACK 1 SLOT 3

MICON CONFIGURATION FILE : mic2_ro

POINT ALLOCATION :

VAI : 32, VDI : 32, ISW : 32 , LOOPS : 12, MSD $: 1$,

AI $: 18, A O: 12$, DI $: 2, D 0: 2$

VIRTUAL ANALOG : AI 3

INPUT \# : 1

INPUT DESC : OUTPUT

INPUT UNIT : PH

INPUT SYNC SCAN : 1 SEC HIST SCAN : 30 SEC

INPUT HIST. DEFINITION : 1

INPUT PLOT LO : 0.00 PLOT HI : 14.00 BAR LO : 0.00 BAR HI $: 14.00$

ACTUATE XMTR ALARM DISCRETES :

XMTR Hi AL DISCRETE : ASH_3

XMTR LO AL DISCRETE :

XMTR HI HI AL DISCRETE :

XMTR LO LO AL DISCRETE :

XMTR MAX AL DISCRETE :

XMTR MIN AL DISCRETE :

XMTR ALARM :

XMTR ALARM Hi 10.00 PRIORITY URGENT

XMTR ALARM LO 6.00 PRIORITY URGENT

XMTR ALARM HI HI 99999.99 PRIORITY EVENT

XMTR ALARM LO LO -9999.99 PRIORITY EVENT

XMTR ALARM MAX 99999.99 PRIORITY EVENT

XMTR ALARM MIN -9999.99 PRIORITY EVENT

XMTR ALARM DESC :243-Z OUTLET PH

XMTR ALARM DEADBAND : 0.10

XMTR ALARM GROUP :0

XMTR ALARM MEMT :0

XMTR ALARM COMMAND DISCR :

XMTR ALARM INDEX :0 
WHC-SD-CP-CSWD-016

Volume 2

Revision 1

Page 8

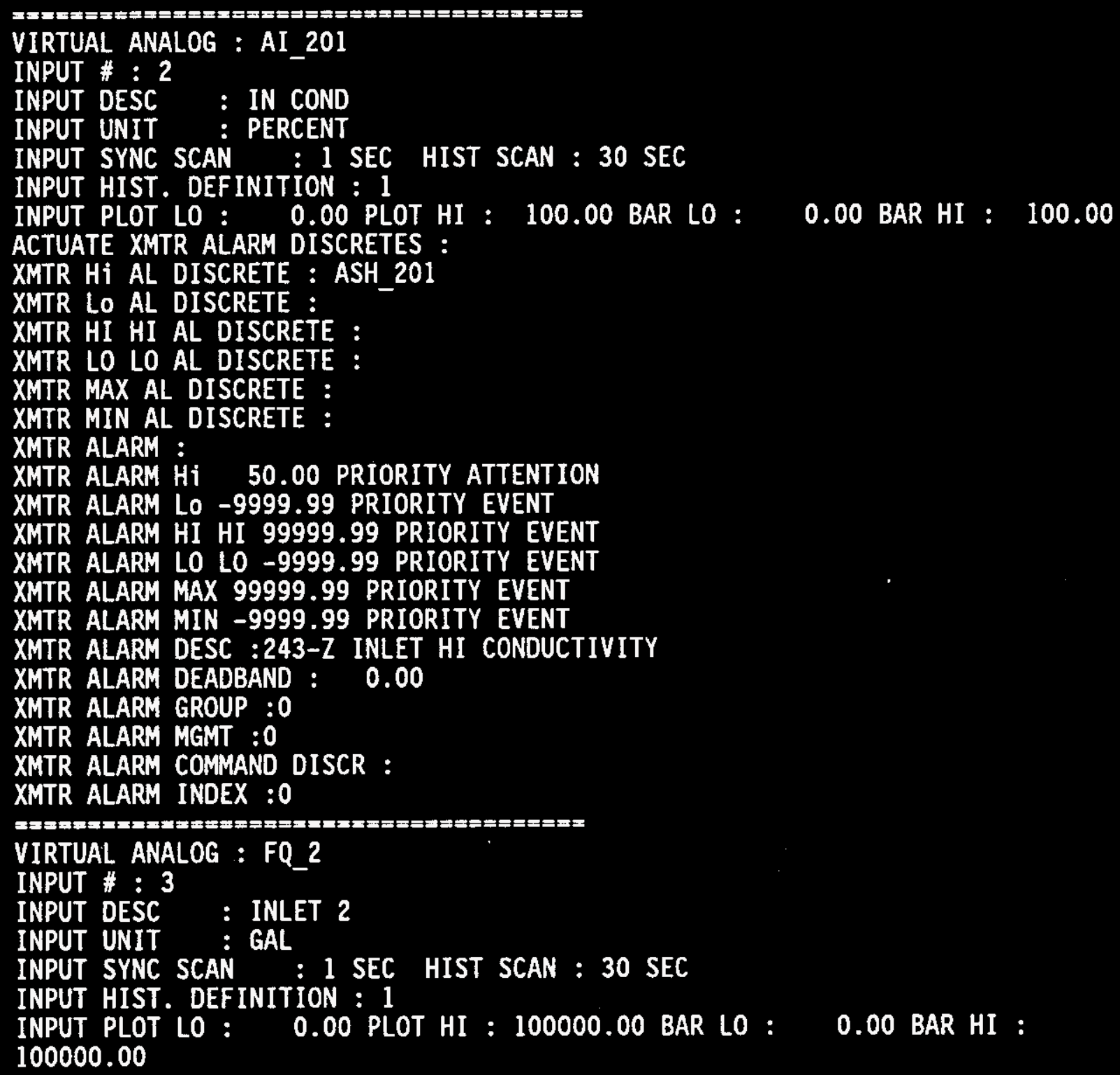


WHC-SD-CP-CSWD-016

Volume 2

Revision 1

Page 9

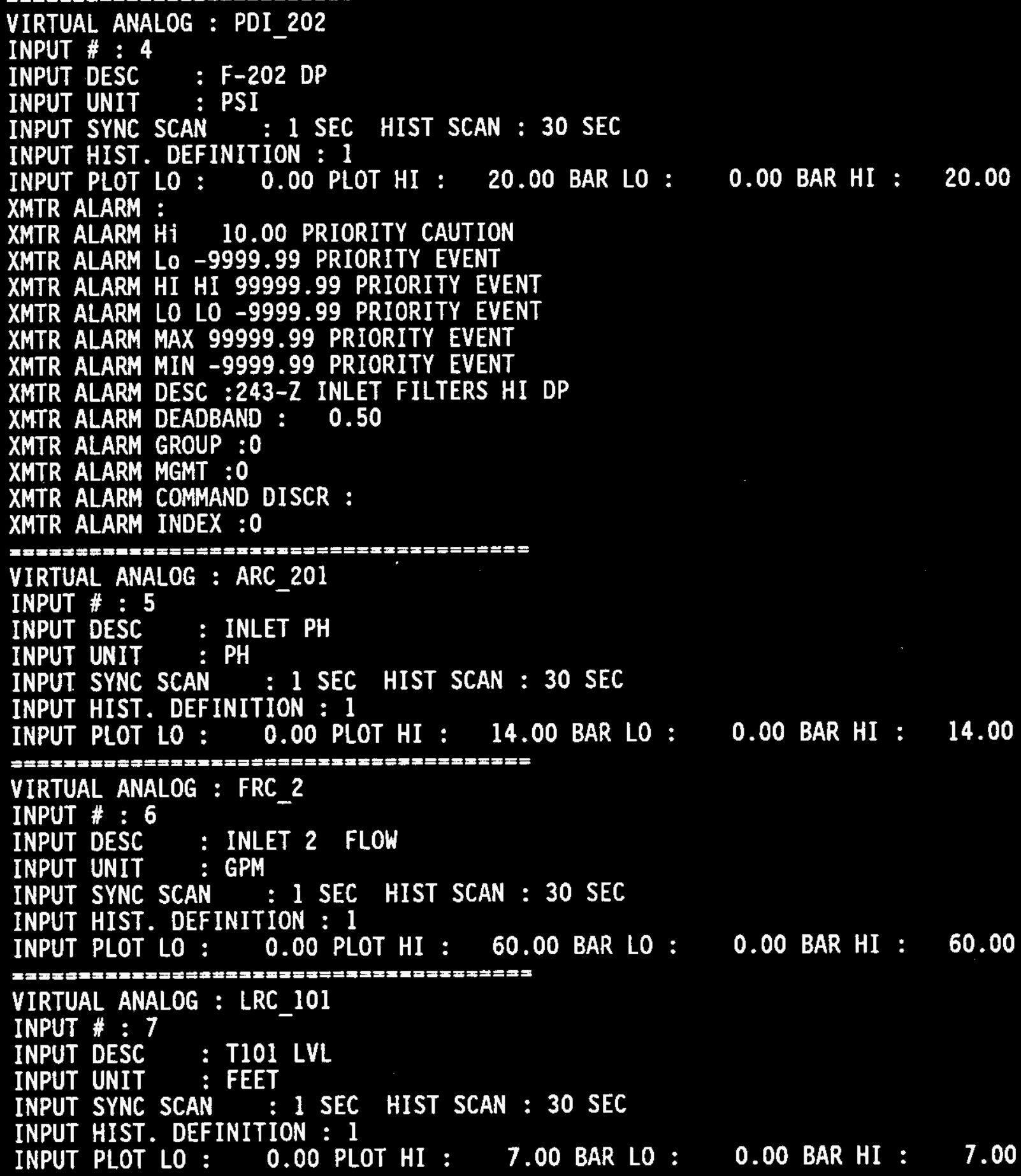


WHC-SD-CP-CSWD-016

Volume 2

Revision 1

Page 10

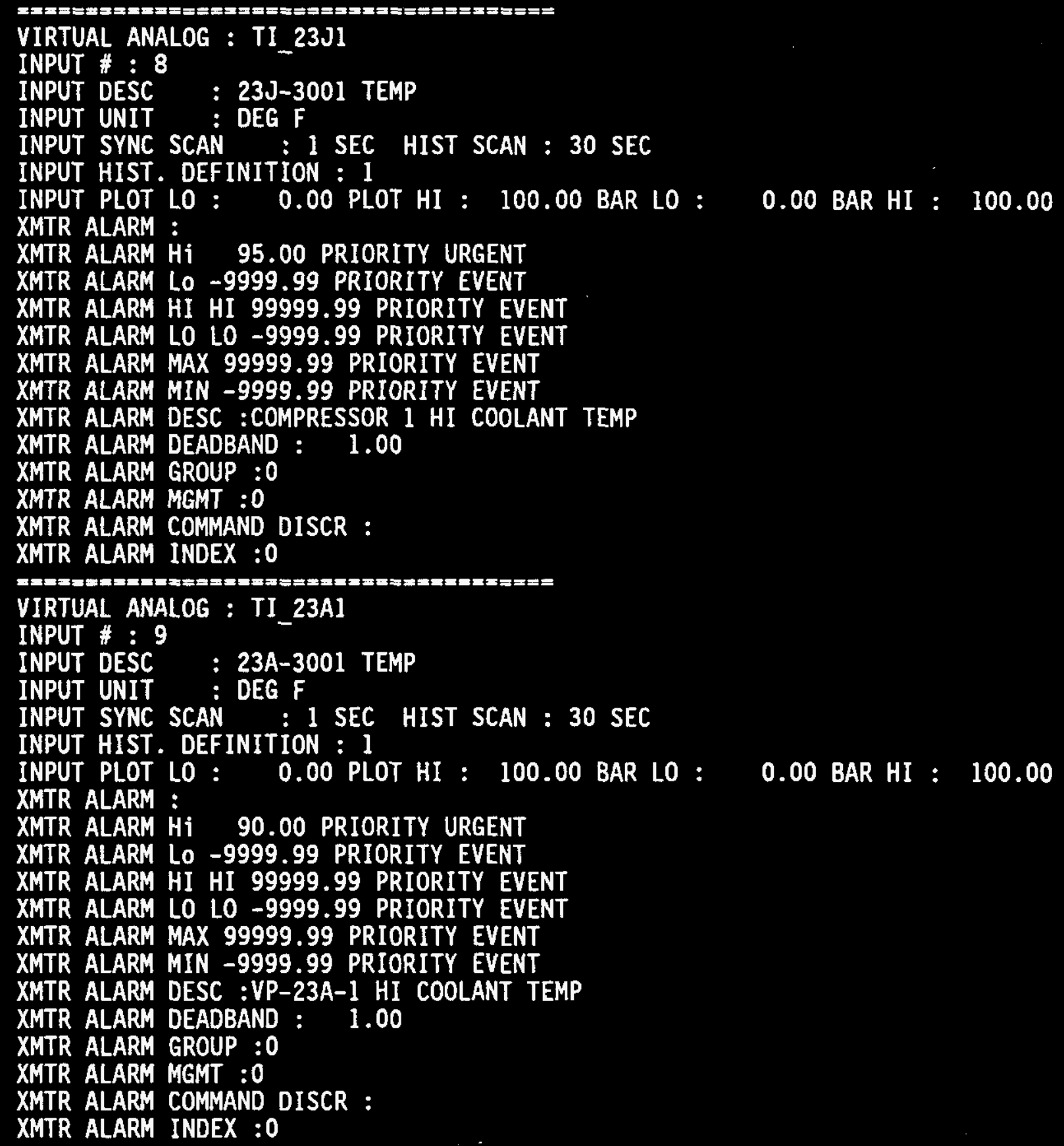


WHC-SD-CP-CSWD-016

Volume 2

Revision 1

Page 11

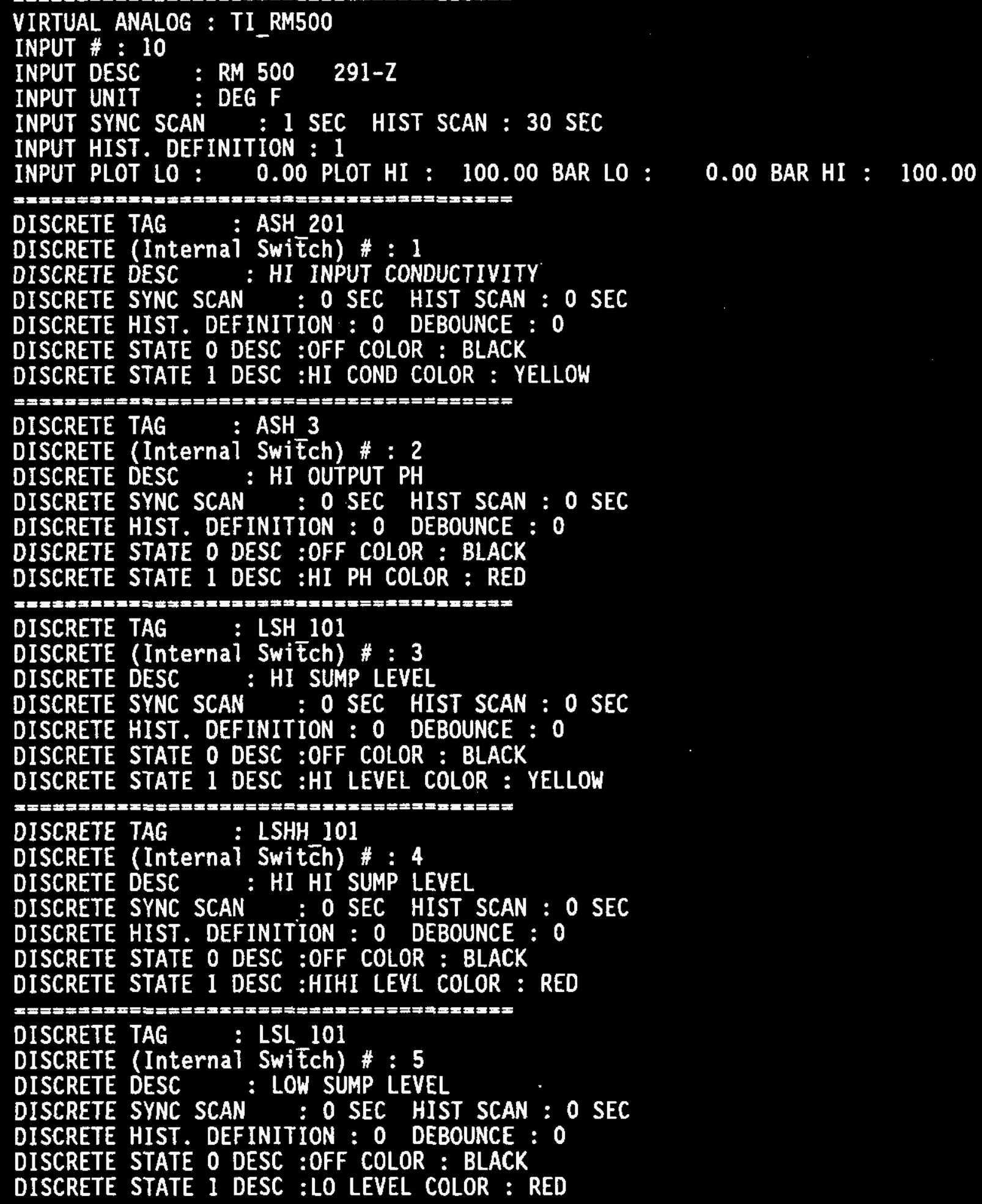


WHC-SD-CP-CSWD-016

Volume 2

Revision 1

Page 12

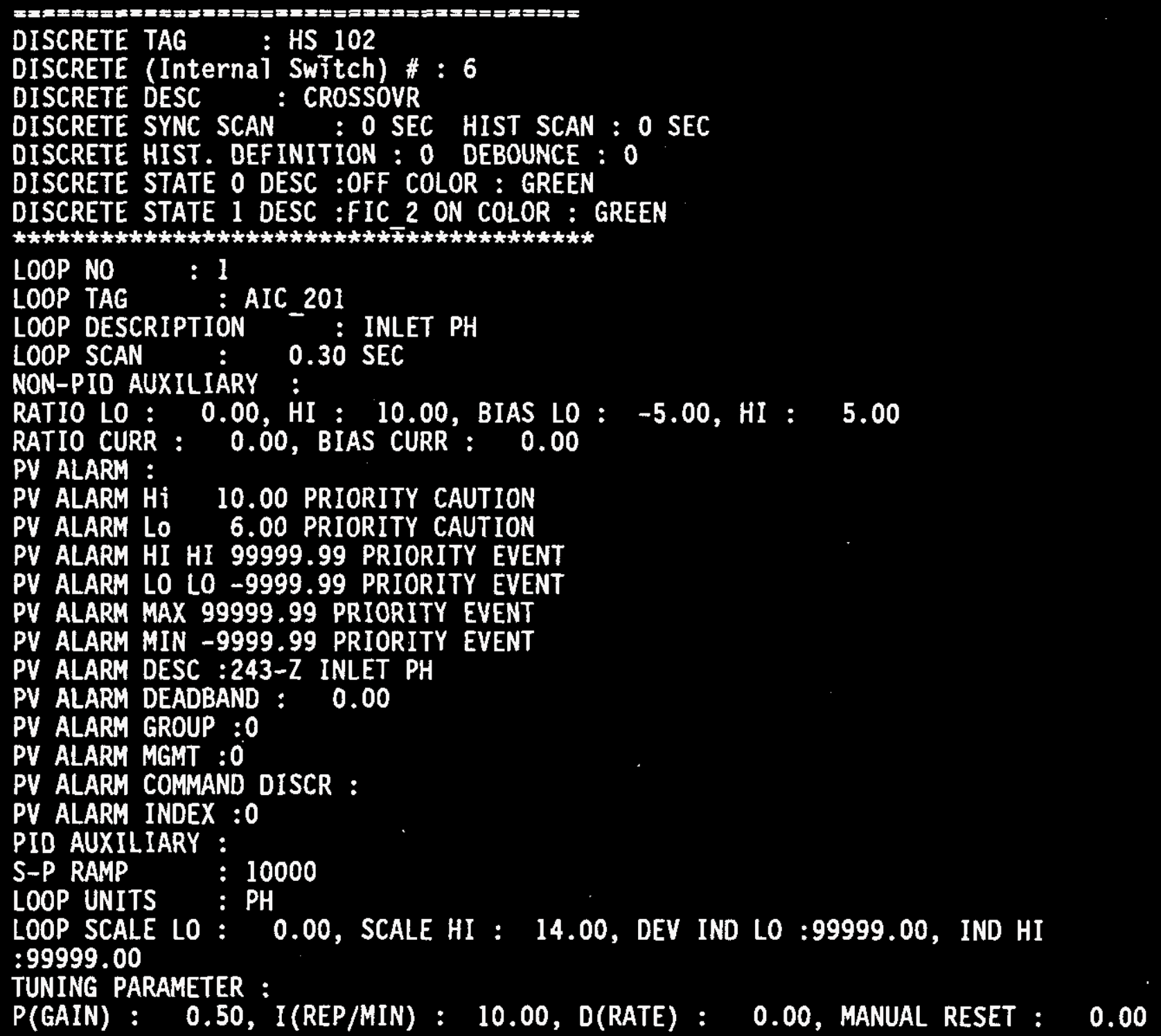


WHC-SD-CP-CSWD-016

Volume 2

Revision 1

Page 13

\#\#\#\#\#\#\#\#\#\#\#\#\#\#\#\#\#\#\#\#\#\#\#\#\#\#\#\#\#\#\#\#\#\#\#\#\#\#\#\#

STEP NO : 1

LABEL :

FUNCTION : 11 (AI)

COMMENT :

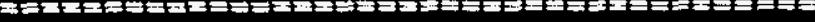

ANALOG INPUT : AI_8

INPUT \# : 4

INPUT DESC : INLET PH (TO AIC_201)

INPUT UNIT : PH

INPUT SYNC SCAN : O SEC HIST SCAN : O SEC

INPUT HIST. DEFINITION : 0

INPUT PLOT LO : 0.00 PLOT HI : 14.00

INPUT TYPE : LINEAR 4-20mA

INPUT RANGE LO : 0.00 RANGE HI : 14.00

DIGITAL FILTER : 0

\#\#\#\#\#\#\#\#\#\#\#\#\#\#\#\#\#\#\#\#\#\#\#\#\#\#\#\#\#\#\#\#\#\#\#\#\#\#\#

STEP NO : 2

LABEL :

FUNCTION : 10 (L )

COMMENT : SAVE INPUT TO ARC 201 FOR HISTORIAN

PARAMETERS :

1) ARC 201 2) AI 8

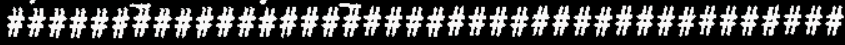

STEP NO : 3

LABEL :

FUNCTION : 20 (PID)

COMMENT : LINEARIZE PH WITH INPUT COMPENSATION

PARAMETERS :

1) $P V=A I 8$ 2) Non-Balancing 3) Reverse 4) Yes 5) LOOP AO

6) Interāctive 7) PV 8) Positional 9) 0

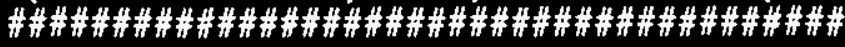

STEP NO

$: 4$

LABEL :

FUNCTION : 55 (CAO )

COMMENT :

OUTPUT TAG : AO 5

OUTPUT \#: 6

OUTPUT DESC : INLET PH

OUTPUT CURRENT : $4-20$

\#\#\#\#\#\#\#\#\#\#\#\#\#\#\#\#\#\#\#\#\#\#\#\#\#\#\#\#\#\#\#\#\#\#\#\#\#\#\#

STEP NO : 5

LABEL :

FUNCTION : 0 (END)

COMMENT : 
WHC-SD-CP-CSWD-016

Volume 2

Revision 1

Page 14

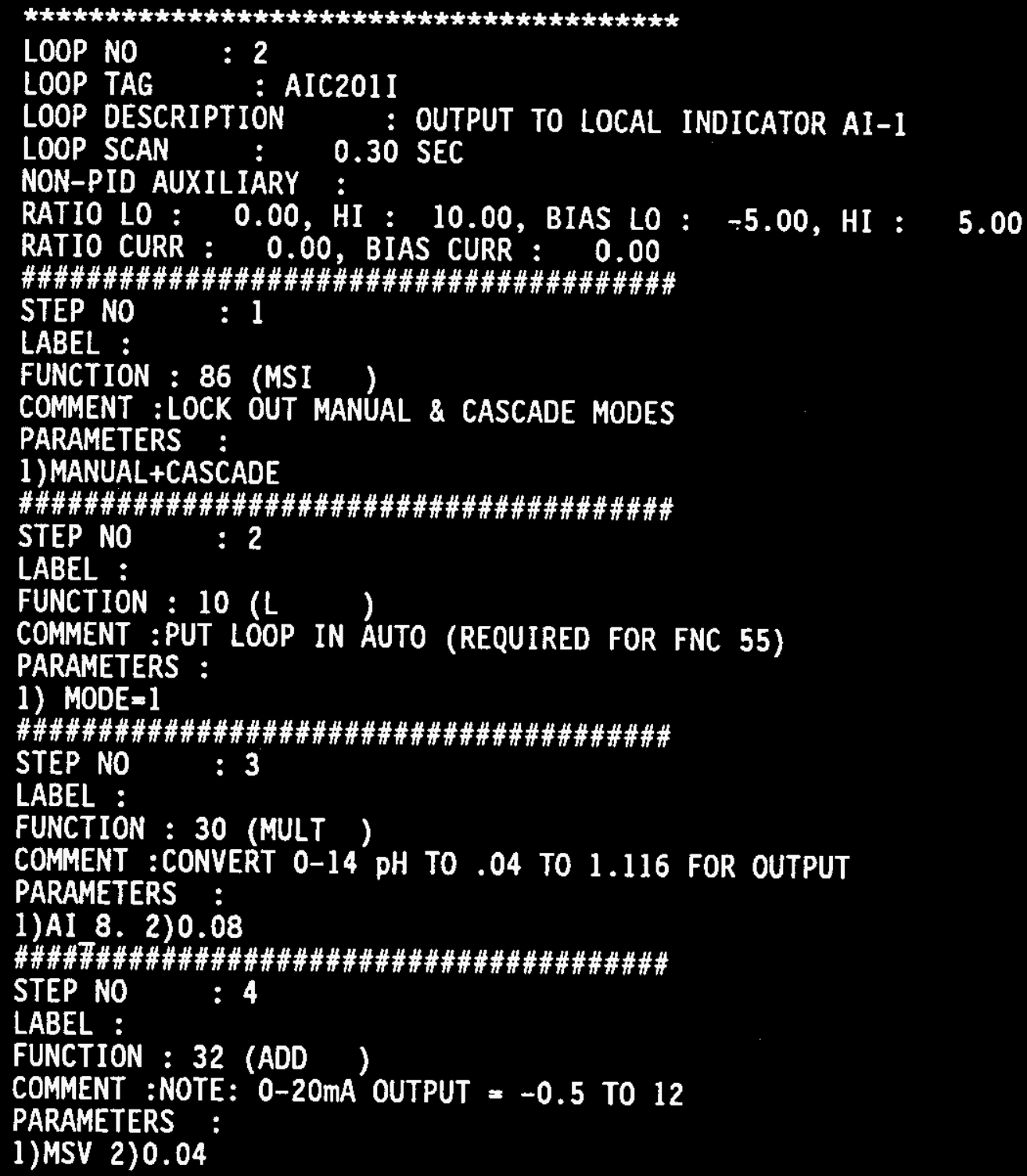


WHC-SD-CP-CSWD-016

Volume 2

Revision 1

Page 15

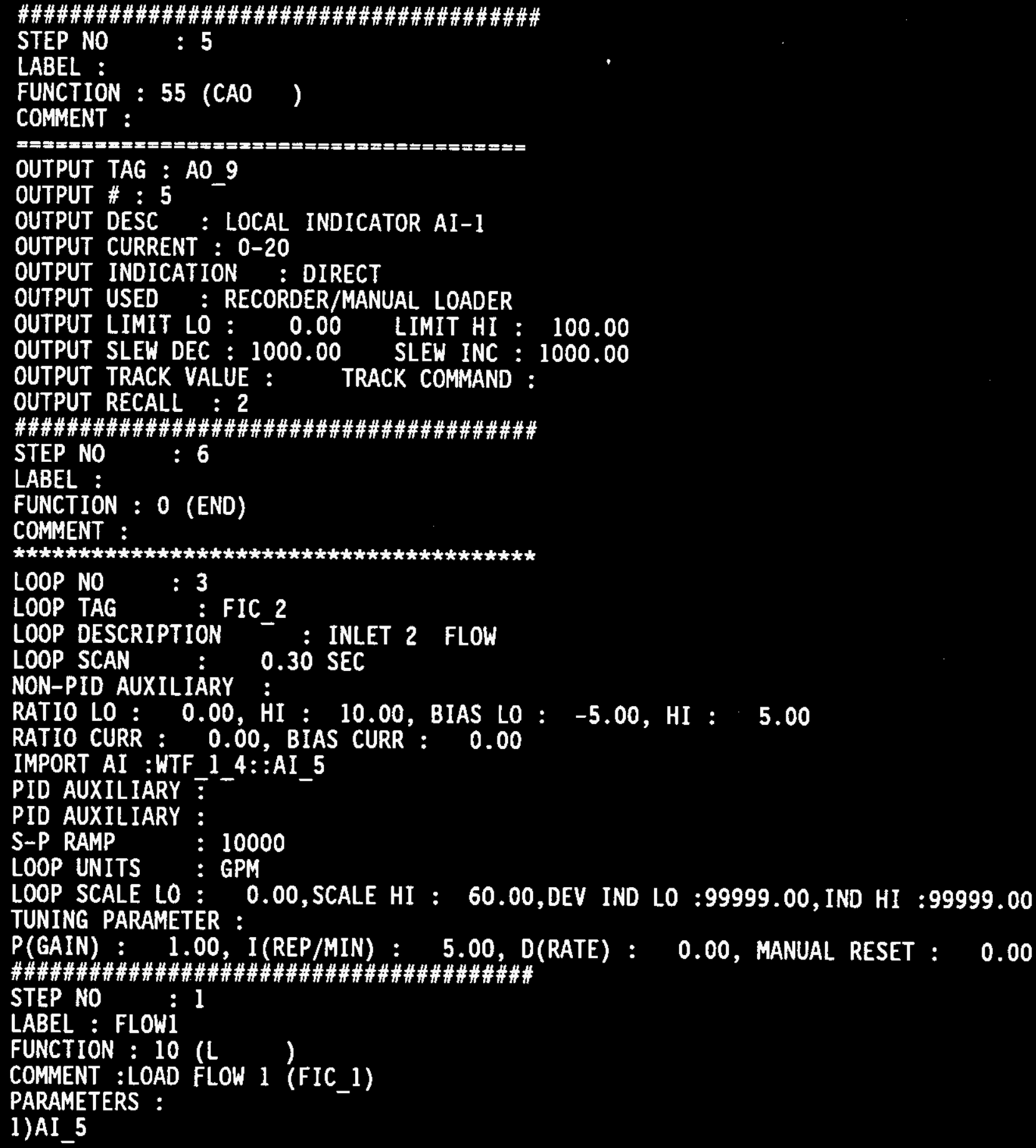


WHC-SD-CP-CSWD-016

Volume 2

Revision 1

Page 16

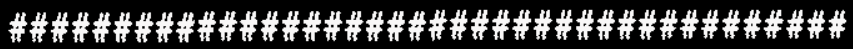

STEP NO

$: 2$

LABEL : TOTFLOW

FUNCTION : 32 (ADD)

COMMENT :ADD INLET 1 AND INLET 2 FLOWS

PARAMETERS :

$\begin{array}{ll}\text { 1)AI } 6 & \text { 2)FLOW1 }\end{array}$

\#\#\#\#界\#\#\#\#\#\#\#\#\#\#\#\#\#\#\#\#\#\#\#\#\#\#\#\#\#\#\#\#\#\#\#\#\#

STEP NO : 3

LABEL : INTERLOCK

FUNCTION : 69 (LC

COMMENT : INTERLOCK, WHEN HS_ 102 IS OFF OR (FIC_1 $=1$ AND FIC_1+FIC_2<30)

PARAMETERS :

1)-HS 102! ( (FLOW1 $>=1) \&($ TOTFLOW $<30)$ )

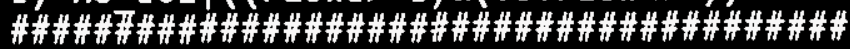

STEP NO : 4

LABEL :

FUNCTION : 11 (AI)

COMMENT :

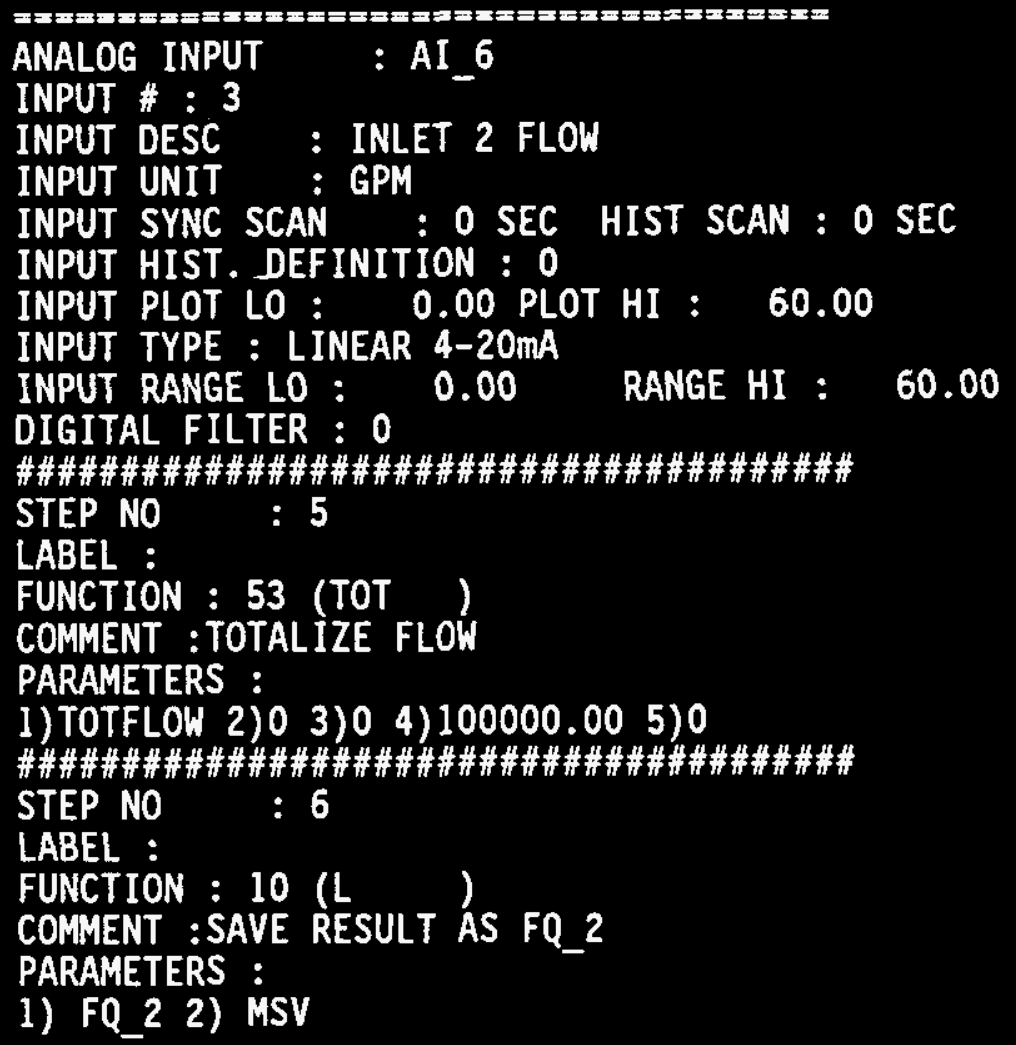


WHC-SO-CP-CSWD-016

Vol ume 2

Revision 1

Page 17

\#\#\#\#\#\#\#\#\#\#\#\#\#\#\#\#\#\#\#\#\#\#\#\#\#\#\#\#\#\#\#\#\#\#\#\#\#\#\#\#\#

STEP NO : 7

LABEL :

FUNCTION : 10 (L

COMMENT :SAVE AI_6 AS INLET 2 FLOW FOR HISTORIAN

PARAMETERS :

1) FRC 2 2) AI 6

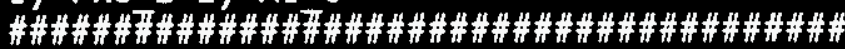

STEP NO : 8

LABEL :

FUNCTION : 20 (PID )

COMMENT :

PARAMETERS :

1) $P V=A I 6$ 2) Non-Balancing 3) Reverse 4) Yes 5) LOOP AO

6) Interāctive 7) PV 8) Positional 9) 0

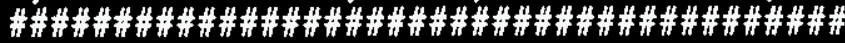

STEP NO : 9

LABEL :

FUNCTION : 55 (CAO)

COMMENT :

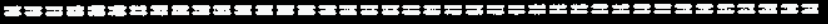

OUTPUT TAG : AO $3^{3}$

OUTPUT \#: 4

OUTPUT DESC : INLET 2 FLOW

OUTPUT CURRENT : 4-20

OUTPUT INDICATION : DIRECT

OUTPUT USED : CONTROL

OUTPUT LIMIT LO : 0.00 LIMIT HI : 100.00

OUTPUT SLEW DEC : 1000.00 SLEW INC : 1000.00

OUTPUT TRACK VALUE : 0 TRACK COMMAND : INTERLOCK

OUTPUT RECALL : 2

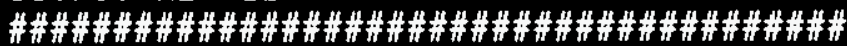

STEP NO : 10

LABEL :

FUNCTION : 0 (END )

COMMENT :

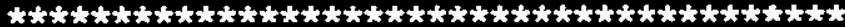

LOOP NO : 4

LOOP TAG : FIC2I

LOOP DESCRIPTION : INLET 2 FLON INDICATOR

LOOP SCAN : 0.30 SEC

NON-PID AUXILIARY :

RATIO LO: 0.00 , HI : 10.00 , BIAS LO: -5.00 , HI : 5.00

RATIO CURR : 0.00 , BIAS CURR : 0.00 
WHC-SD-CP-CSWD-016

Volume 2

Revision 1

Page 18

\#\#\#\#\#\#\#\#\#\#\#\#\#\#\#\#\#\#\#\#\#\#\#\#\#\#\#\#\#\#\#\#\#\#\#\#\#\#\#\#

STEP NO

$: 1$

LABEL :

FUNCTION : 86 (MSI

COMMENT :LOCK OUT MANUAL \& CASCADE MODES

PARAMETERS :

1) MANUAL+CASCADE

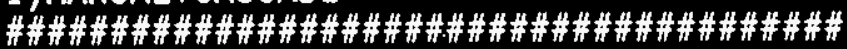

STEP NO : 2

LABEL :

FUNCTION : 10 (L

COMMENT : PUT LOOP IN AUTO

PARAMETERS :

1) $M O D E=1$

\#\#\#\#\#\#\#\#\#\#\#\#\#\#\#\#\#\#\#\#\#\#\#\#\#\#\#\#\#\#\#\#\#\#\#\#\#\#\#\#

STEP NO : 3

LABEL :

FUNCTION : 31 (DIV )

PARAMETERS :

$\begin{array}{ll}\text { 1)AI } 6 & 2) 60\end{array}$

COMMENT :RESCALE 0-60 TO $0-1$ FOR OUTPUT

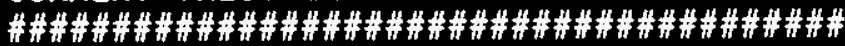

STEP NO : 4

LABEL :

FUNCTION : 55 (CAO)

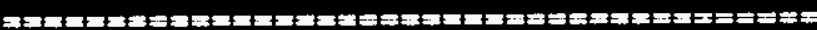

OUTPUT TAG : AO_16

OUTPUT \#: 3

OUTPUT DESC : OUTPUT TO FI-2

OUTPUT CURRENT : 4-20

OUTPUT INDICATION : DIRECT

OUTPUT USED : RECORDER/MANUAL LOADER

OUTPUT LIMIT LO : 0.00 LIMIT HI : 100.00

OUTPUT SLEN DEC : 1000.00 SLEW INC : 1000.00

OUTPUT TRACK VALUE : TRACK COMMAND :

OUTPUT RECALL : 2

COMMENT :

\#\#\#\#\#\#\#\#\#\#\#\#\#\#\#\#\#\#\#\#\#\#\#\#\#\#\#\#\#\#\#\#\#\#\#\#\#\#\#\#

STEP NO : 5

LABEL :

FUNCTION : 0 (END )

COMMENT : 
WHC-SD-CP-CSWD-016

Volume 2

Revision 1

Page 19

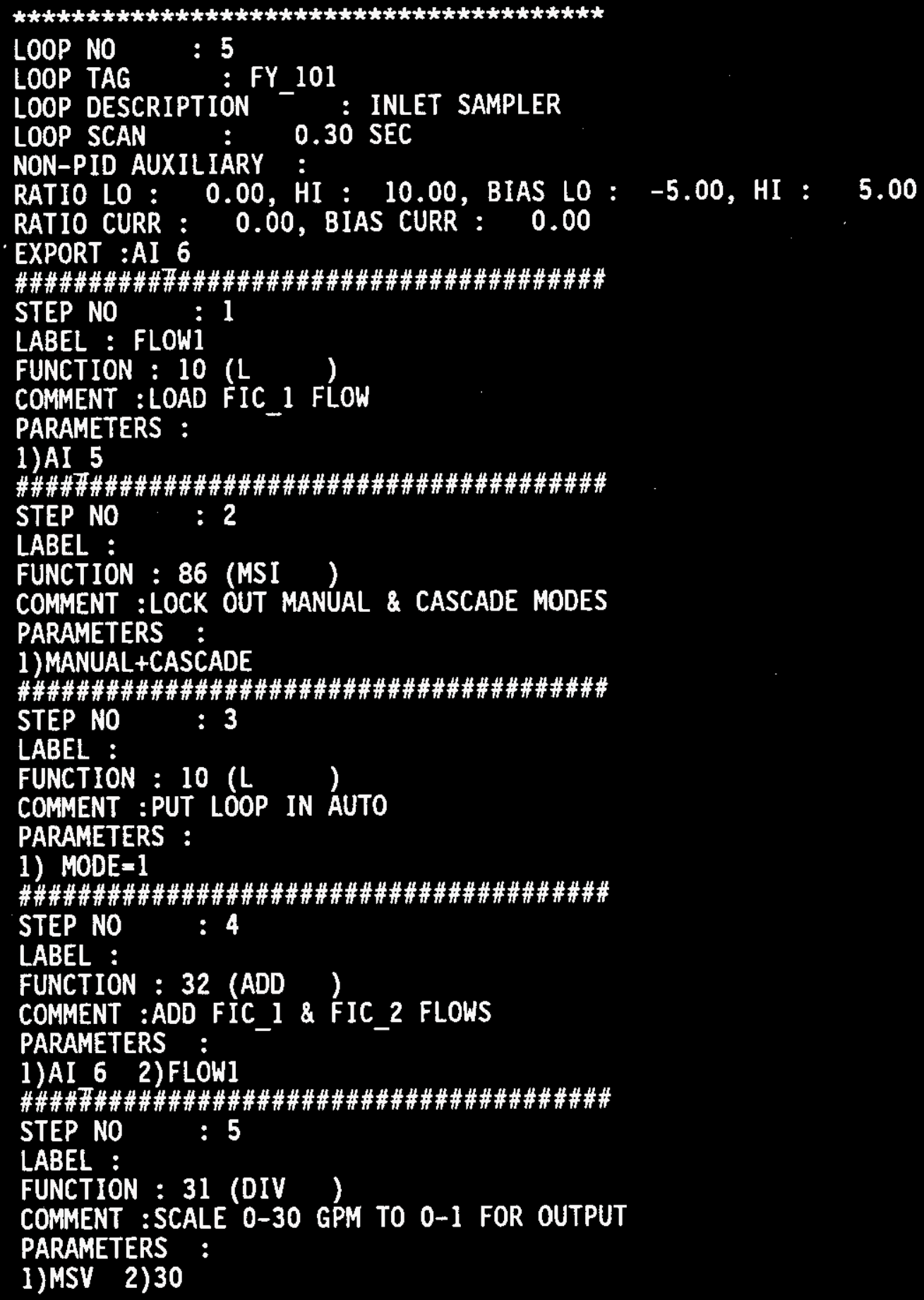


\#\#\#\#\#\#\#\#\#\#\#\#\#\#\#\#\#\#\#\#\#\#\#\#\#\#\#\#\#\#\#\#\#\#\#\#\#\#\#\#

STEP NO : 6

LABEL :

FUNCTION : 47 (CBA)

COMMENT :SET MAX TO OUTPUT AT 1

PARAMETERS :

1)MSV 2)1 3) A>=B 4)1

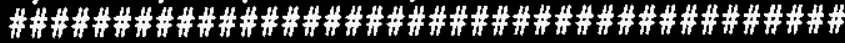

STEP NO : 7

LABEL :

FUNCTION : 55 (CAO)

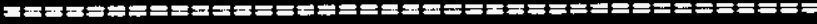

OUTPUT TAG : AO 6

OUTPUT \# : 2

OUTPUT DESC : INLET SAMPLER

OUTPUT CURRENT : 4-20

OUTPUT INDICATION : DIRECT

OUTPUT USED : RECORDER/MANUAL LOADER

OUTPUT LIMIT LO : 0.00 LIMIT HI : 100.00

OUTPUT SLEW DEC : 1000.00 SLEW INC : 1000.00

OUTPUT TRACK VALUE : TRACK COMMAND :

OUTPUT RECALL : 2

COMMENT :

\#\#\#\#\#\#\#\#\#\#\#\#\#\#\#\#\#\#\#\#\#\#\#\#\#\#\#\#\#\#\#\#\#\#\#\#\#\#\#\#

STEP NO $: \mathbf{8}$

LABEL :

FUNCTION : O (END)

COMMENT : 
WHC-SD-CP-CSWD-016

Volume 2

Revision 1

Page 21

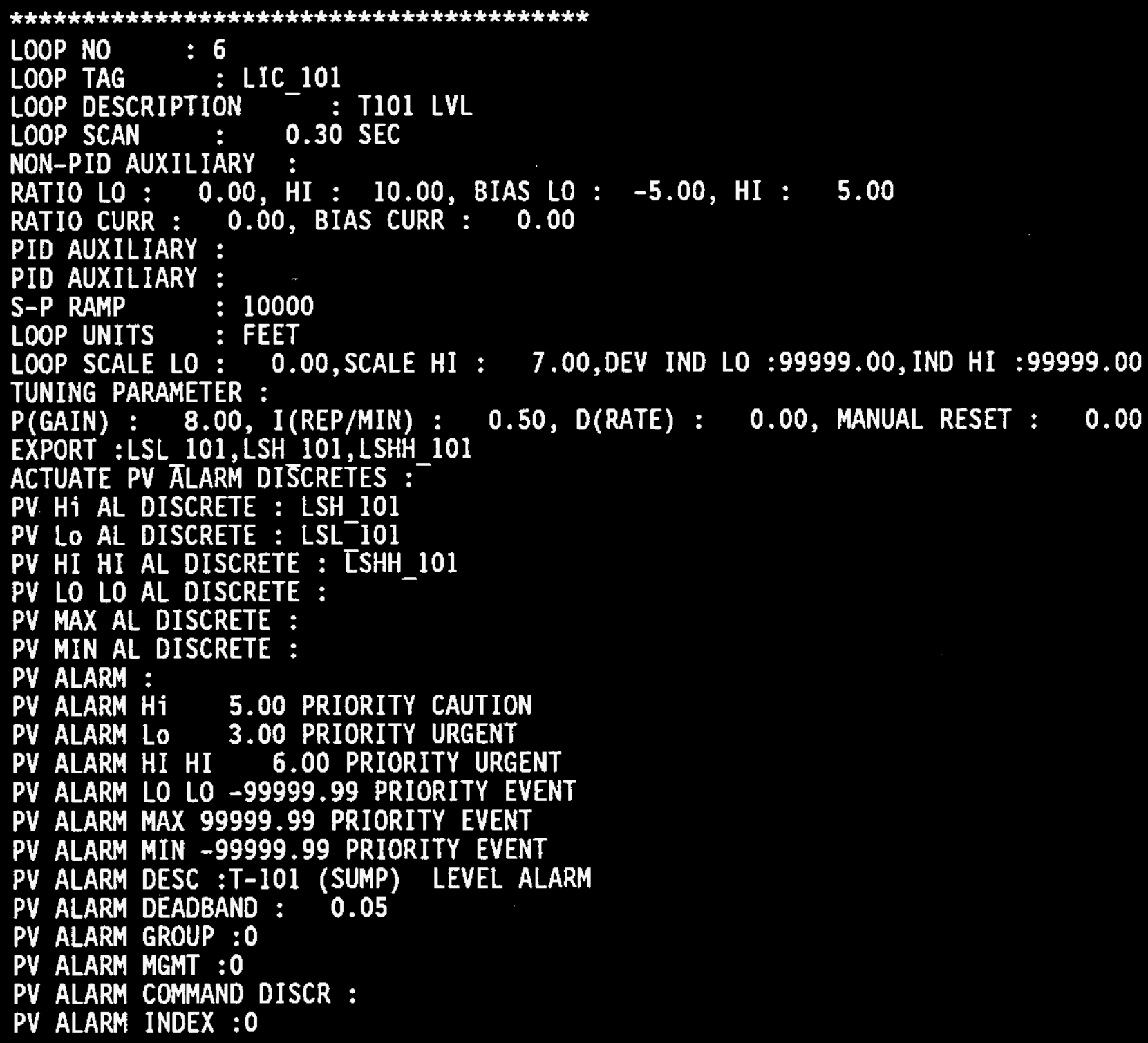


WHC-SD-CP-CSWD-016

Volume 2

Revision 1

Page 22

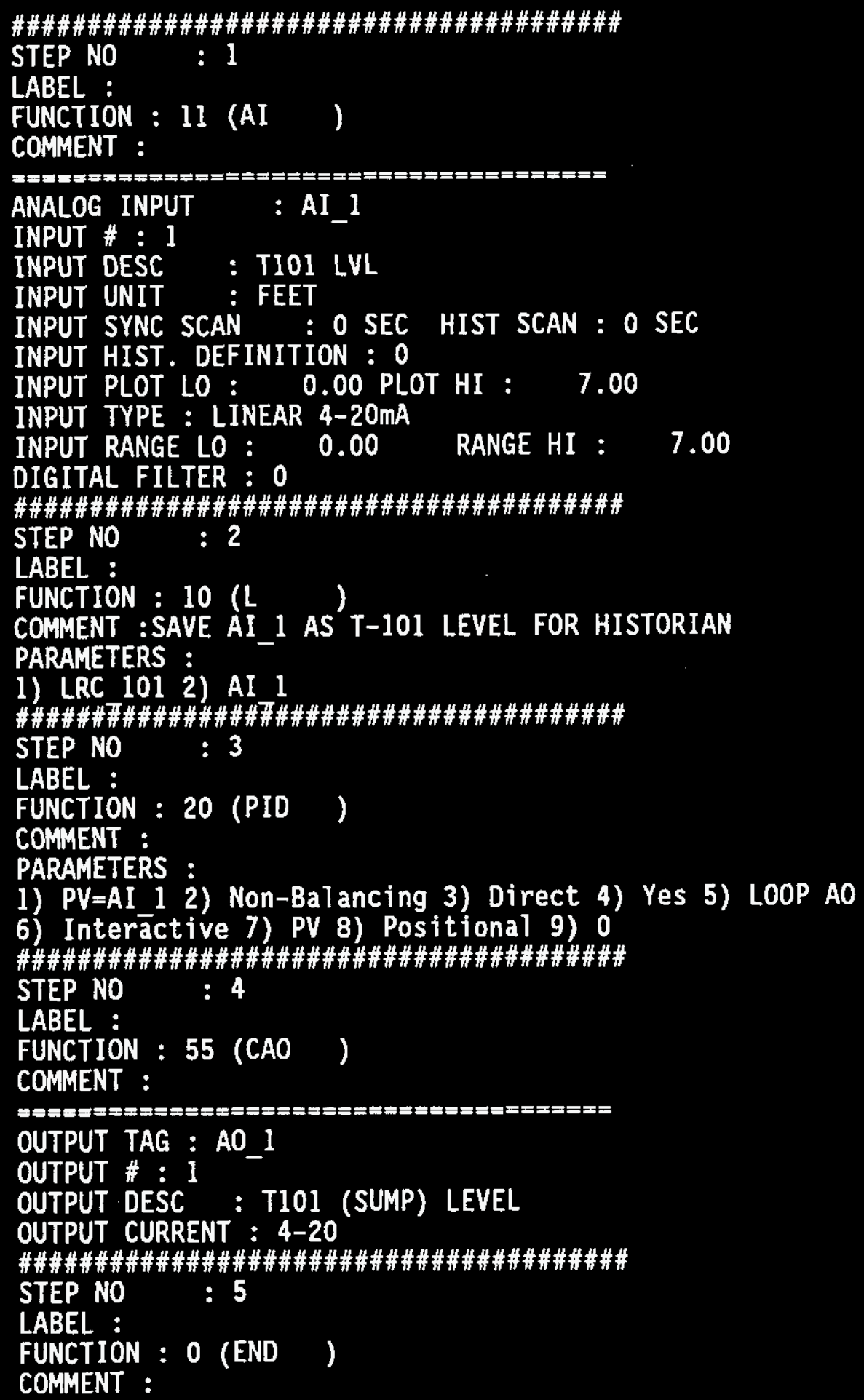




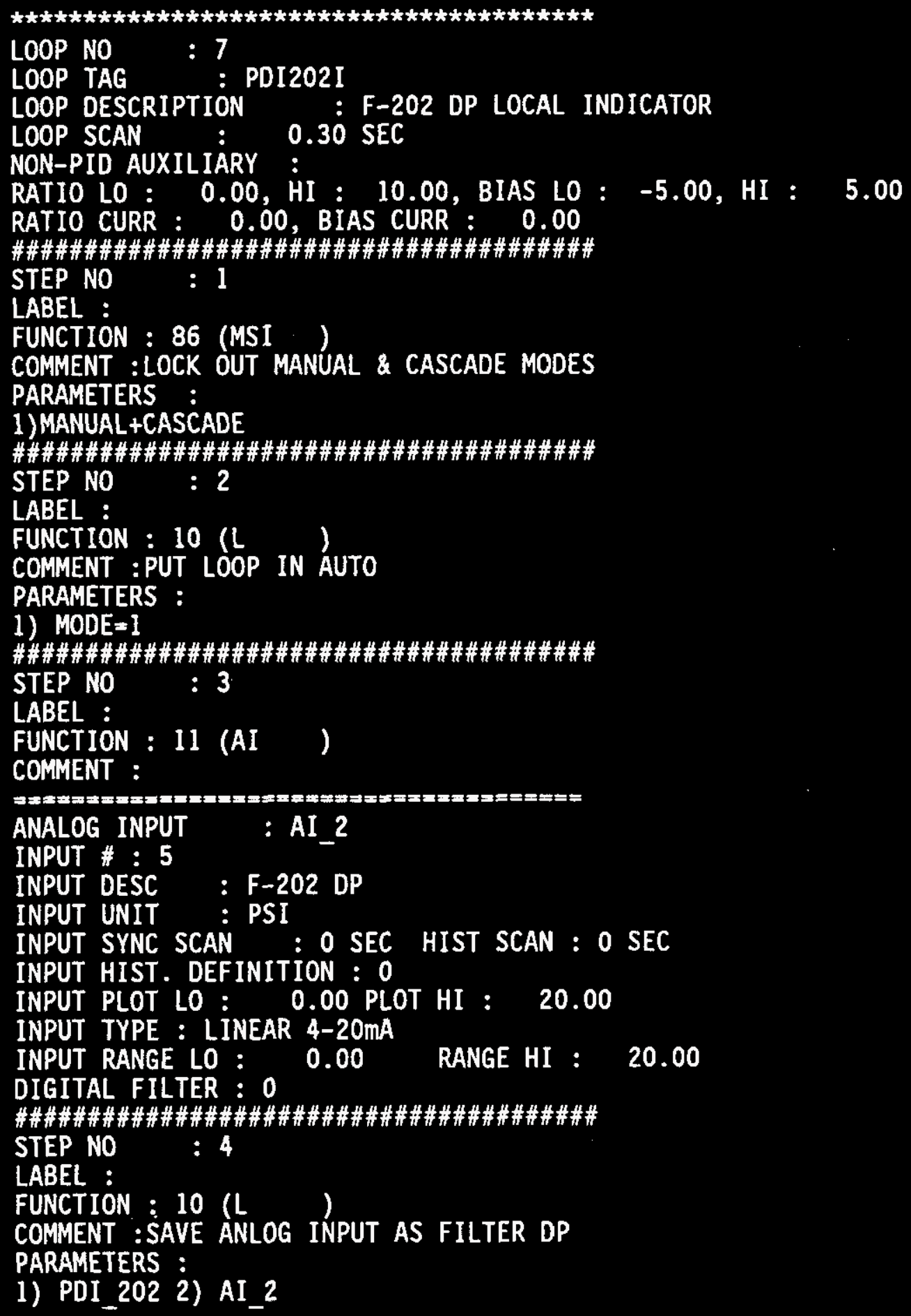




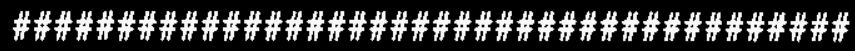

STEP NO : 5

LABEL :

FUNCTION : 31 (DIV)

PARAMETERS :

1)AI 2. 2) 20

COMMENT : RESCALE $0-20$ PSI TO $0-1$ FOR OUTPUT

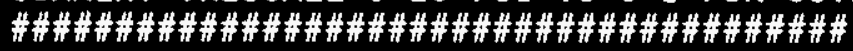

STEP NO : 6

LABEL :

FUNCTION : 55 (CAO)

OUTPUT TAG : A0_13

OUTPUT \#: 7

OUTPUT DESC : LOCAL INDICATOR PDI-1

OUTPUT CURRENT : 4-20

OUTPUT INDICATION : DIRECT

OUTPUT USED : RECORDER/MANUAL LOADER

OUTPUT LIMIT LO : 0.00 LIMIT HI : 100.00

OUTPUT SLEW DEC : 1000.00 SLEW INC : 1000.00

OUTPUT TRACK VALUE : TRACK COMMAND :

OUTPUT RECALL : 2

COMMENT :

\#\#\#\#\#\#\#\#\#\#\#\#\#\#\#\#\#\#\#\#\#\#\#\#\#\#\#\#\#\#\#\#

STEP NO

$: 7$

LABEL :

FUNCTION : 0 (END)

COMMENT :

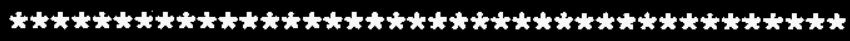

LOOP NO : 8

LOOP TAG : MISC_M2

LOOP DESCRIPTION - : MISC INPUTS

LOOP SCAN : 0.30 SEC

NON-PID AUXILIARY :

RATIO LO : 0.00, HI : 10.00 , BIAS LO : -5.00 , HI : 5.00

RATIO CURR : 0.00 , BIAS CURR : 0.00

EXPORT :ASH_201,ASH_3 


\section{WHC-SD-CP-CSWD-016 \\ Volume 2 \\ Revision 1 \\ Page 25}

\#\#\#\#\#\#\#\#\#\#\#\#\#\#\#\#\#\#\#\#\#\#\#\#\#\#\#\#\#\#\#\#\#\#\#\#\#\#\#\#

STEP NO : 1

LABEL :

FUNCTION : 11 (AI)

COMMENT :

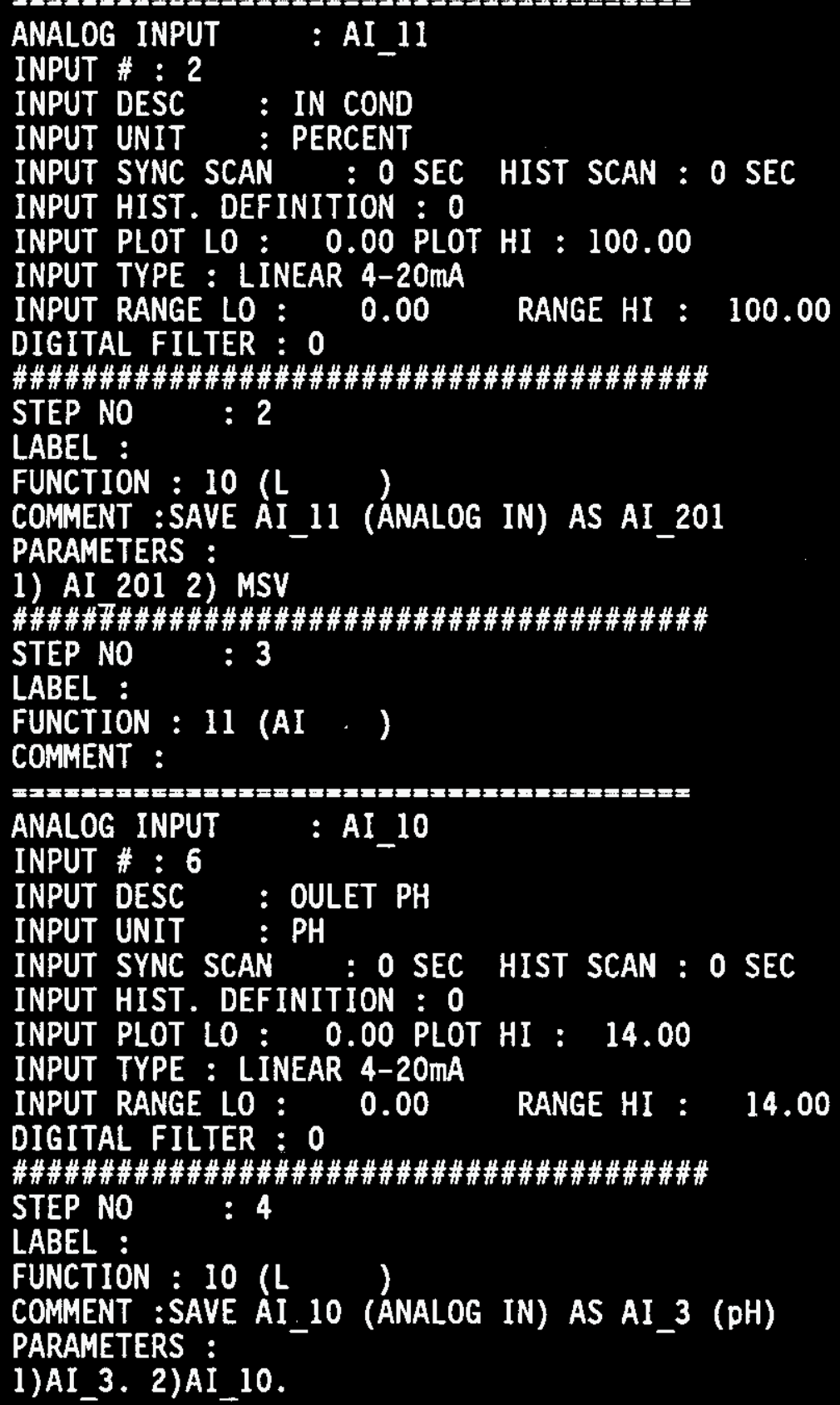


WHC-SD-CP-CSWD-016

Volume 2

Revision 1

Page 26

\#\#\#\#\#\#\#\#\#\#\#\#\#\#\#\#\#\#\#\#\#\#\#\#\#\#\#\#\#\#\#\#\#\#\#

STEP NO : 5

LABEL :

FUNCTION : 11 (AI)

COMMENT :

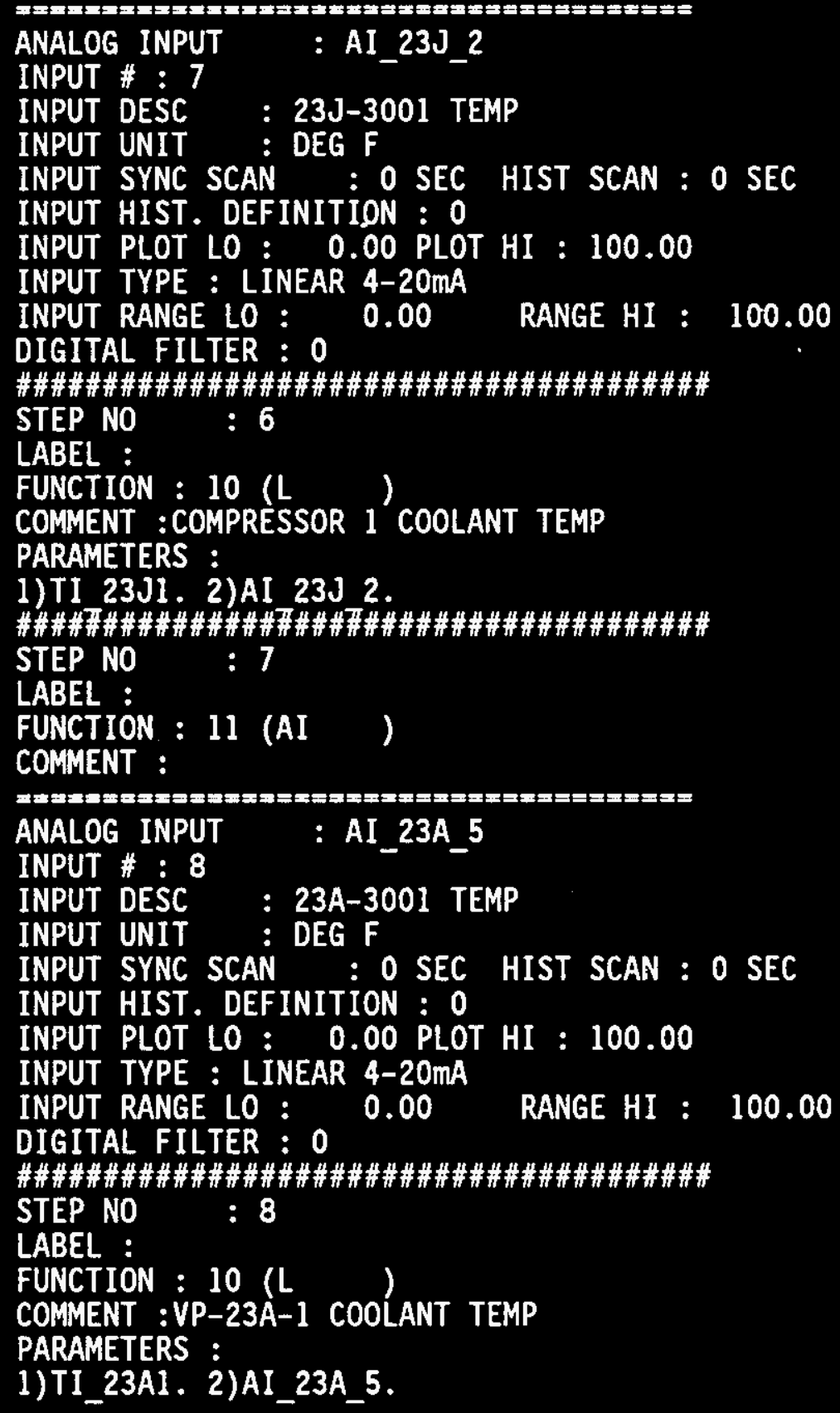




\section{WHC-SD-CP-CSWD-016 \\ Volume 2 \\ Revision 1 \\ Page 27}

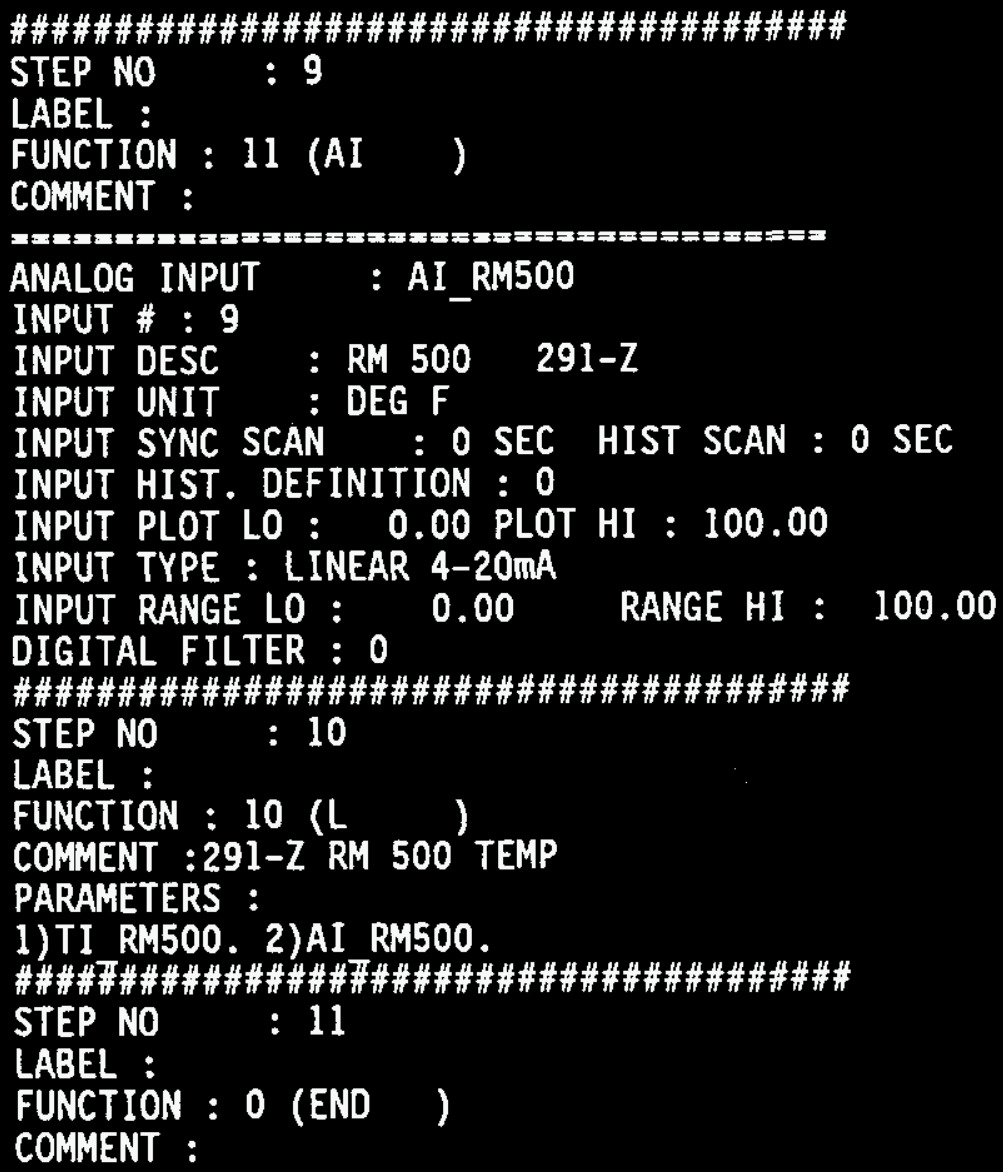


WHC-SD-CP-CSWD-016

Volume 2

Revision 1

Page 28

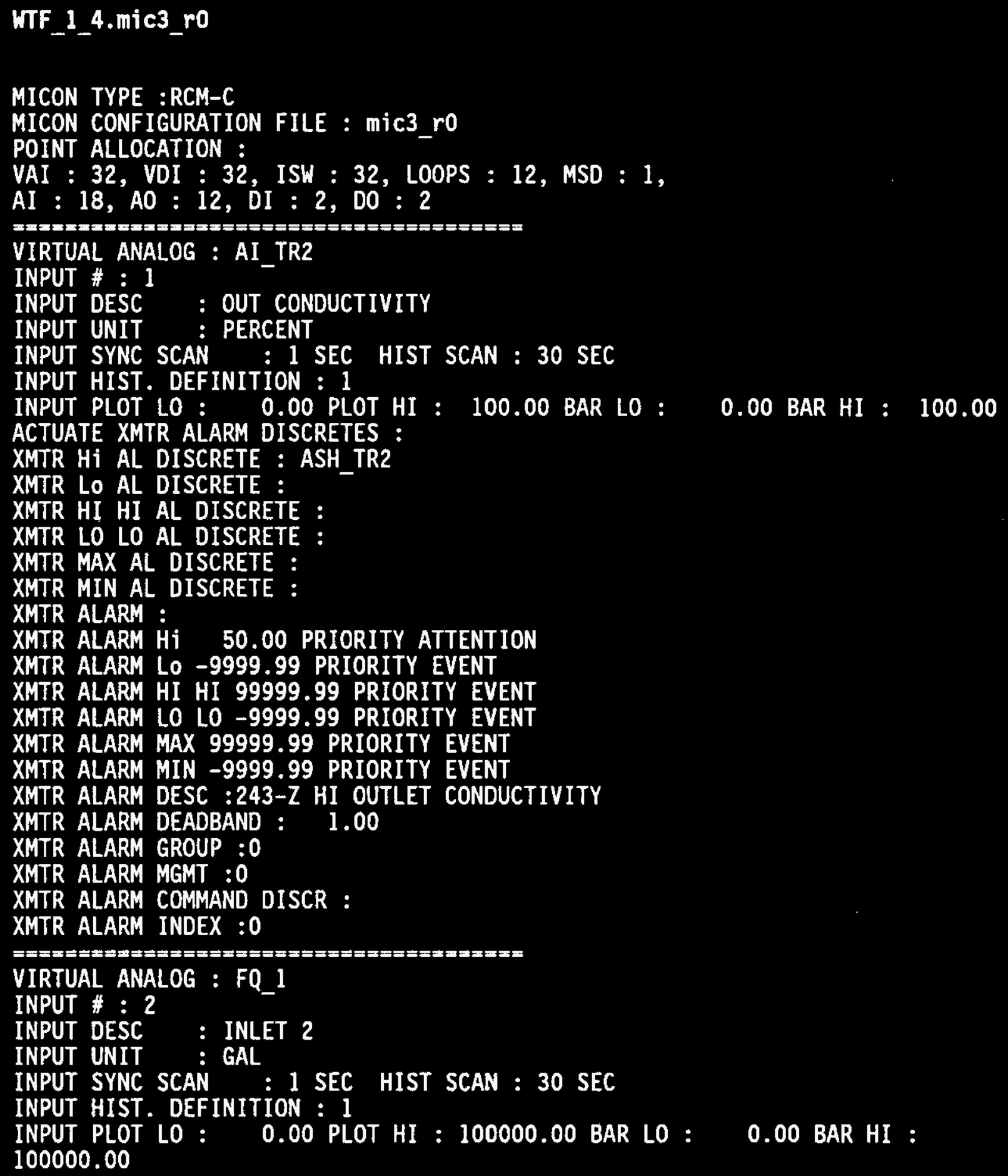




\section{WHC-SD-CP-CSWD-016 \\ Volume 2 \\ Revision 1 \\ Page 29}

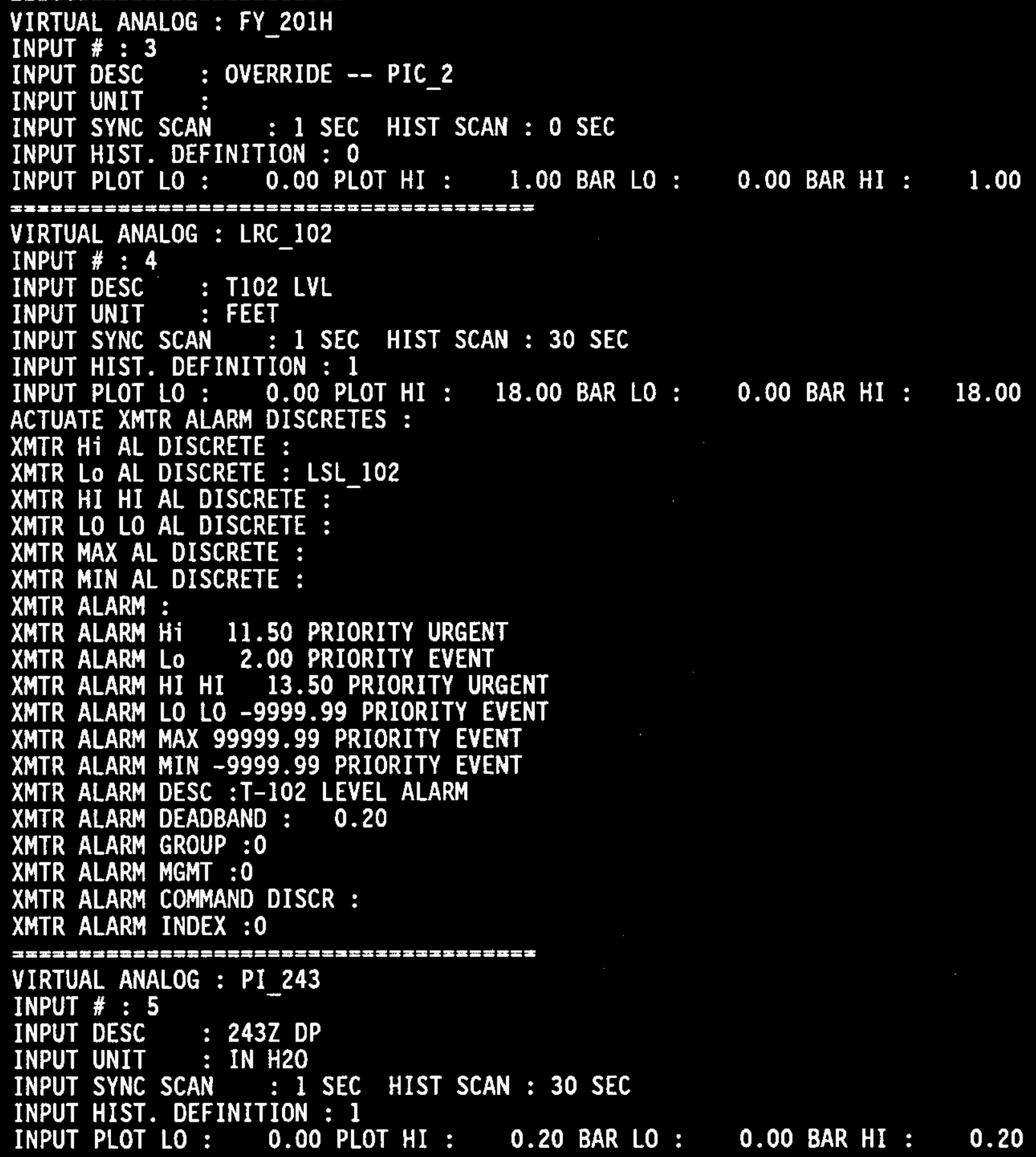


WHC-SD-CP-CSWD-016

Volume 2

Revision 1

Page 30

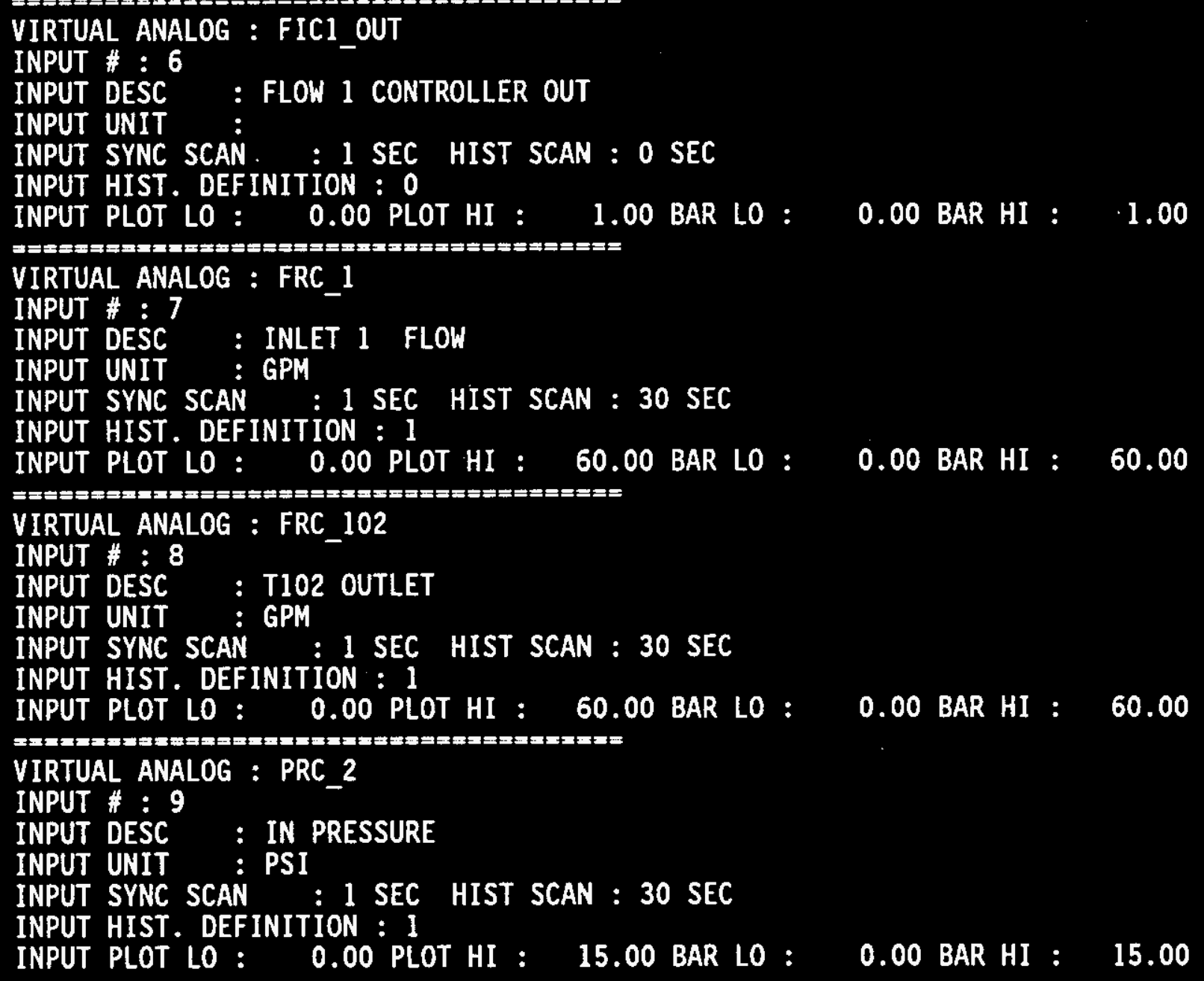




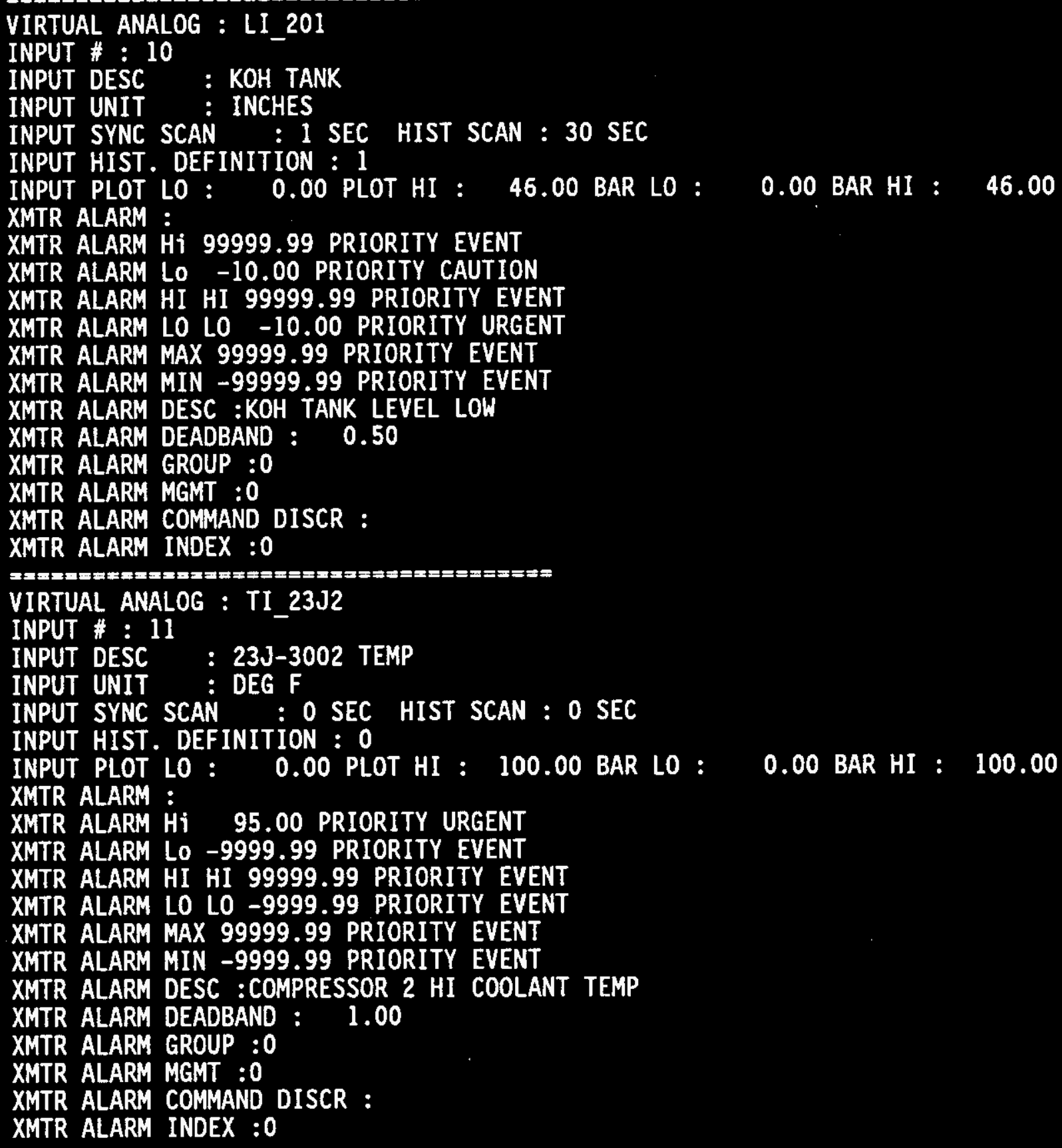


WHC-SD-CP-CSWD-016

Volume 2

Revision 1

Page 32

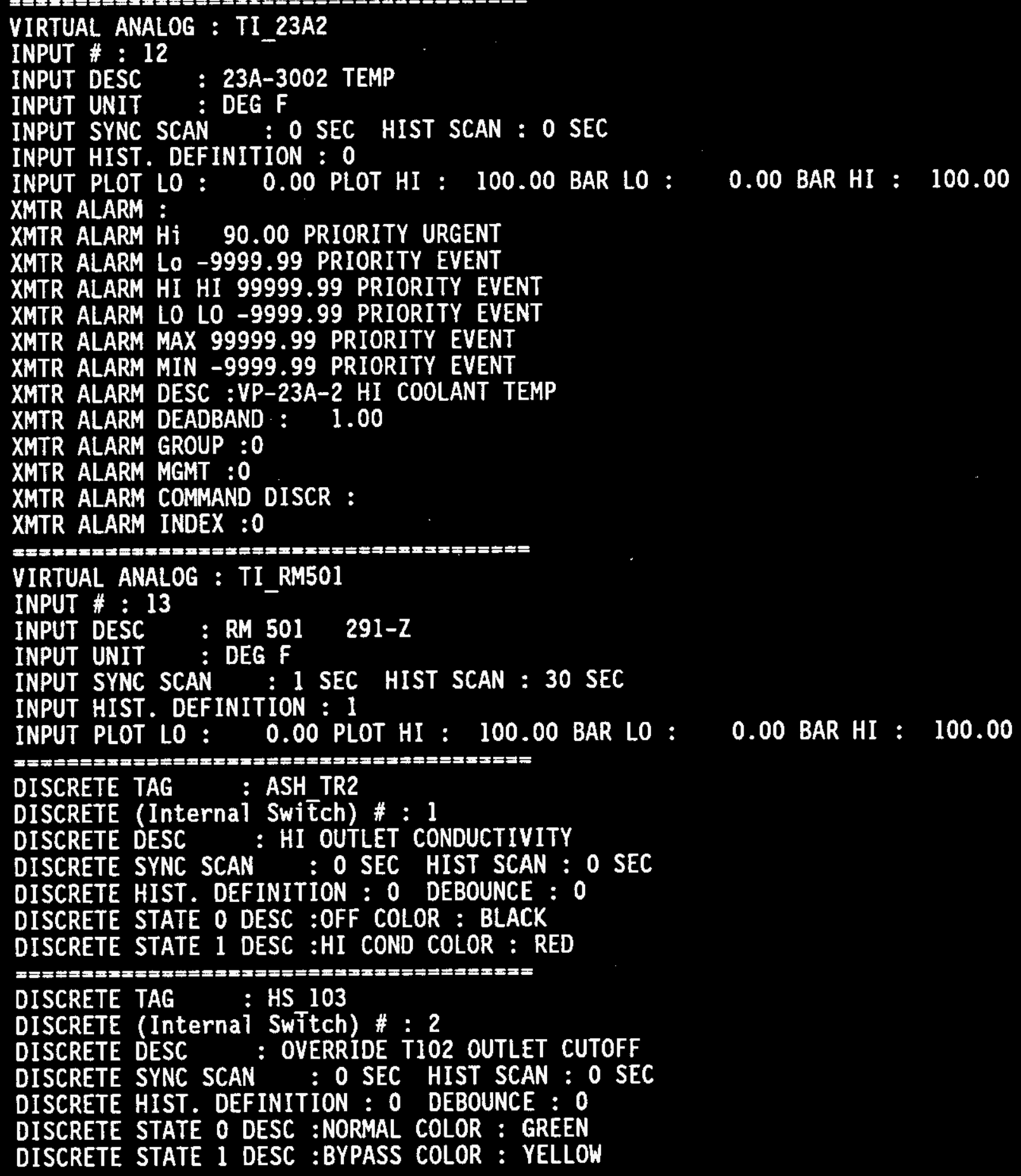


WHC-SD-CP-CSWD-016

Volume 2

Revision 1

Page 33

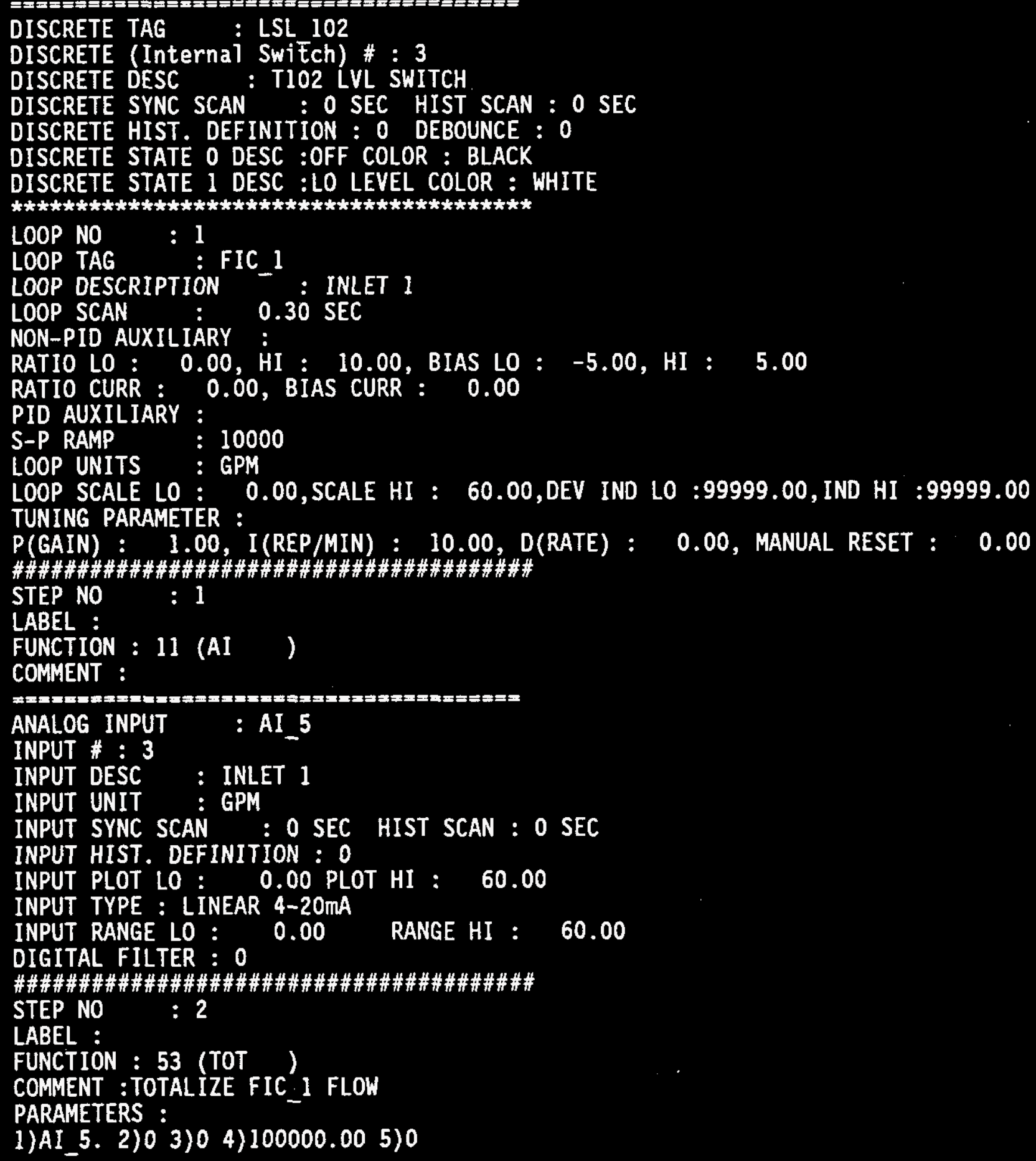


WHC-SD-CP-CSWD-016

Volume 2

Revision 1

Page 34

\#\#\#\#\#\#\#\#\#\#\#\#\#\#\#\#\#\#\#\#\#\#\#\#\#\#\#\#\#\#\#\#\#\#\#\#\#\#\#\#

STEP NO : 3

LABEL :

FUNCTION : 10 (L) )

COMMENT :SAVE RESULT AS FQ_1

PARAMETERS :

1) $\mathrm{FQ} 12$ 2) MSV

\#\#\#\#\#界\#\#\#\#\#\#\#\#\#\#\#\#\#\#\#\#\#\#\#\#\#\#\#\#\#\#\#\#\#\#\#

STEP NO : 4

LABEL :

FUNCTION : $10(\mathrm{~L})$

COMMENT :SAVE AI 5 AS INLET 1 FLOW FOR HISTORIAN

PARAMETERS :

1) FRC 1 2) AI 5

\#\#\#\#\#\#男\#\#\#\#\#\#\#五\#\#\#\#\#\#\#\#\#\#\#\#\#\#\#\#\#\#\#\#\#\#\#\#

STEP NO

$: 5$

LABEL :

FUNCTION : 20 (PID )

COMMENT :

PARAMETERS :

1) PV=AI_5 2) Non-Balancing 3) Reverse 4) Yes 5) LOOP AO

6) Interäctive 7) PV 8) Positional 9) 0

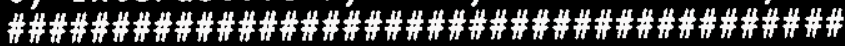

STEP NO

$: 6$

LABEL :

FUNCTION : 10 ( $\mathrm{L}, \mathrm{)})$

COMMENT : SAVE PID FIC_ 1 OUTPUT FOR PIC_ 2 EXTERNAL RESET

PARAMETERS :

1) FIC1 OUT 2) MSV

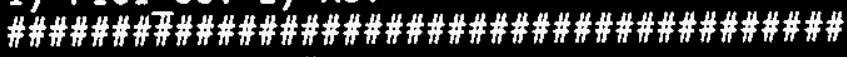

STEP NO : 7

LABEL :

FUNCTION : 47 (CBA

COMMENT :OVERRIDE SIGNAL FROM PIC_2

PARAMETERS :

1) MSV 2)FY 201H 3) A>=B 4)FY 201H

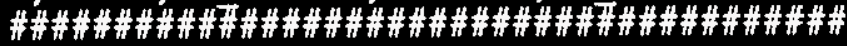

STEP NO : 8

LABEL :

FUNCTION : 55 (CAO )

COMMENT :

OUTPUT TAG : AO_2

OUTPUT \# : 6

OUTPUT DESC : INLET 1 FLOW

OUTPUT CURRENT : $4-20$ 


\section{WHC-SD-CP-CSWD-016 \\ Volume 2 \\ Revision 1 \\ Page 35}

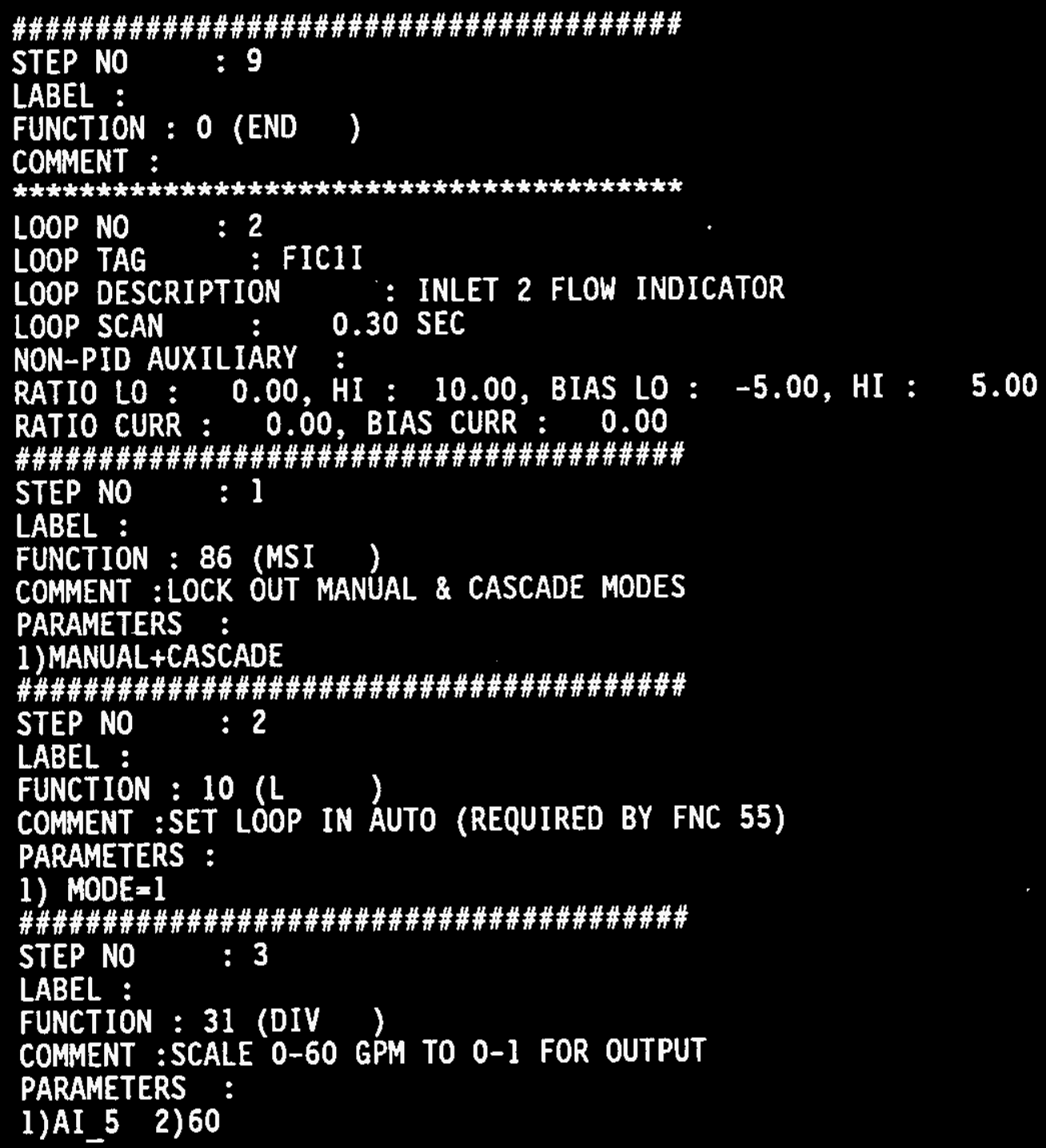


WHC-SD-CP-CSWD-016

Volume 2

Revision 1

Page 36

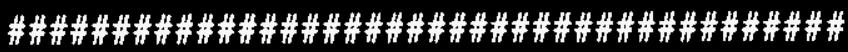

STEP NO : 4

LABEL :

FUNCTION : 55 (CAO )

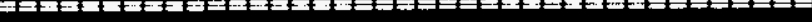

OUTPUT TAG : AO_15

OUTPUT \# : 5

OUTPUT DESC : OUTPUT TO FI 1

OUTPUT CURRENT : 4-20

OUTPUT INDICATION : DIRECT

OUTPUT USED : MSV

OUTPUT LIMIT LO : $\quad 0.00$ LIMIT HI : 100.00

OUTPUT SLEW DEC : 1000.00 SLEW INC : 1000.00

OUTPUT TRACK VALUE : TRACK COMMAND :

OUTPUT RECALL : 2

COMMENT :

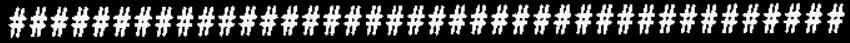

STEP NO : 5

LABEL :

FUNCTION : 0 (END)

COMMENT :

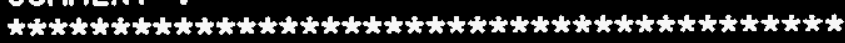

LOOP NO : 3

LOOP TAG : FIC_102

LOOP DESCRIPTION - : T102 OUTLET

LOOP SCAN : 0.30 SEC

NON-PID AUXILIARY :

RATIO LO : 0.00 , HI : 10.00 , BIAS LO : -5.00 , HI : 5.00

RATIO CURR : 0.00 , BIAS CURR : 0.00

IMPORT DI :WTF 1 3::!LSHH_101

PID AUXILIARY $\bar{\vdots}$

S-P RAMP : 10000

LOOP UNITS : GPM

LOOP SCALE LO : 0.00 , SCALE HI : 60.00 , DEV IND LO $: 99999.00$, IND HI $: 99999.00$

TUNING PARAMETER :

P(GAIN) : 0.50 , I (REP/MIN) : 10.00, D(RATE) : 0.00 , MANUAL RESET : 0.00

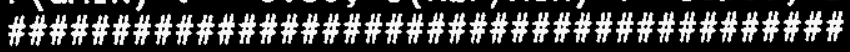

STEP NO : 1

LABEL : LSHH

FUNCTION : $10(\mathrm{~L})$

COMMENT :LOAD T-101 HIHI LEVEL ALARM SWITCH

PARAMETERS :

1)LSHH_101 


\section{WHC-SD-CP-CSWD-016 \\ Volume 2 \\ Revision 1 \\ Page 37}

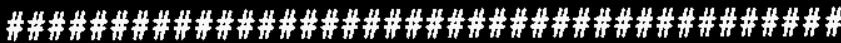

STEP NO : 2

LABEL : INTERLOCK

FUNCTION : 69 (LC

COMMENT : INTERLOCK ON T-101 HIHI LEVEL WHEN HS_103 IS OFF (NORMAL)

PARAMETERS :

1)-HS 103\&LSHH

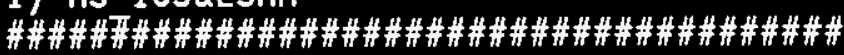

STEP NO : 3

LABEL :

FUNCTION : 11 (AI)

COMMENT :

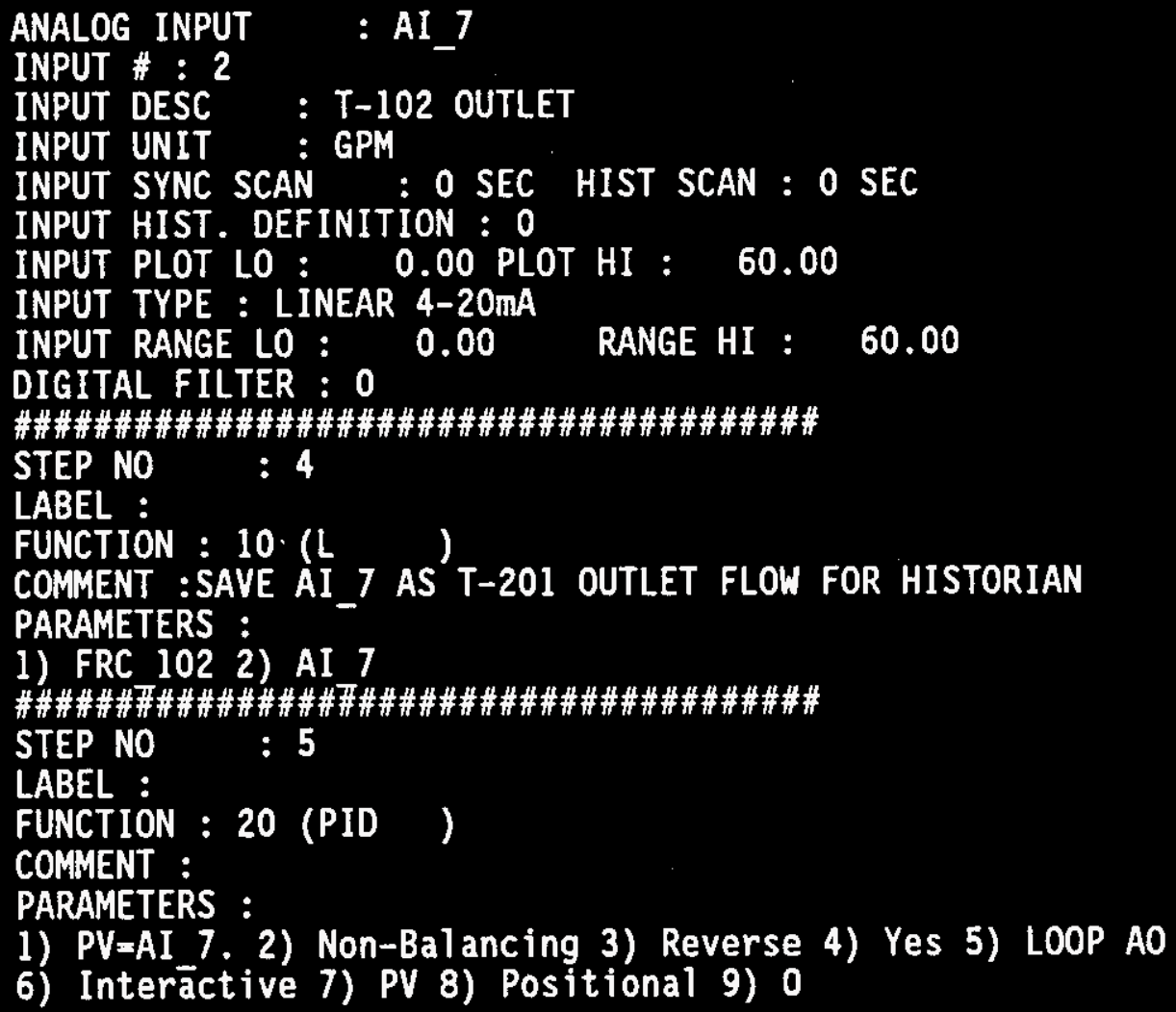


WHC-SD-CP-CSWD-016

Volume 2

Revision 1

Page 38

\#\#\#\#\#\#\#\#\#\#\#\#\#\#\#\#\#\#\#\#\#\#\#\#\#\#\#\#\#\#\#\#\#\#\#\#\#\#\#\#

STEP NO : 6

LABEL :

FUNCTION : 55 (CAO )

COMMENT :

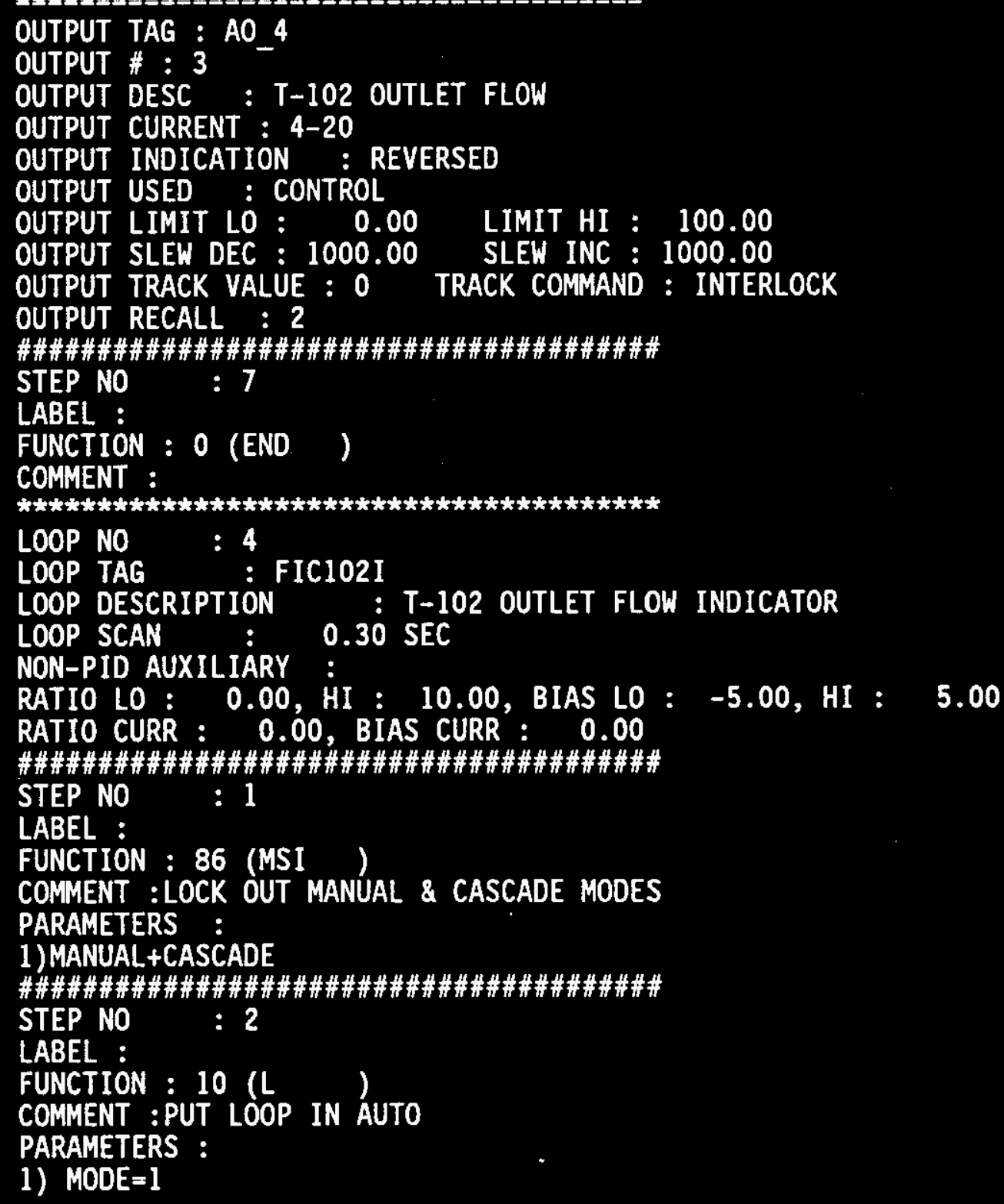


WHC-SD-CP-CSWD-016

Volume 2

Revision 1

Page 39

\#\#\#\#\#\#\#\#\#\#\#\#\#\#\#\#\#\#\#\#\#\#\#\#\#\#\#\#\#\#\#\#\#\#\#\#\#\#\#\#

STEP NO

$: 3$

LABEL :

FUNCTION : 31 (DIV)

COMMENT :SCALE $0-60$ GPM TO $0-1$ FOR OUTPUT

PARAMETERS :

$\begin{array}{ll}\text { 1)AI } 7 & \text { 2) } 60\end{array}$

\#\#\#\#界\#\#\#\#\#\#\#\#\#\#\#\#\#\#\#\#\#\#\#\#\#\#\#\#\#\#\#\#\#\#\#\#\#

STEP NO

$: 4$

LABEL :

FUNCTION : 55 (CAO)

OUTPUT TAG : AO_17

OUTPUT \# : 2

OUTPUT DESC : OUTPUT TO FI-3

OUTPUT CURRENT : 4-20

OUTPUT INDICATION : DIRECT

OUTPUT USED : MSV

OUTPUT LIMIT LO : 0.00 LIMIT HI : 100.00

OUTPUT SLEW DEC : 1000.00 SLEW INC : 1000.00

OUTPUT TRACK VALUE : TRACK COMMAND :

OUTPUT RECALL : 2

COMMENT :

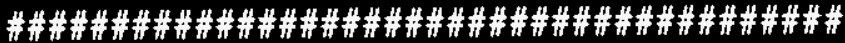

STEP NO : 5

LABEL :

FUNCTION : 0 (END)

COMMENT :

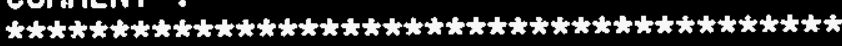

LOOP NO : 5

LOOP TAG : FY 203

LOOP DESCRIPTION - : OUTLET SAMPLER

LOOP SCAN : $0.30 \mathrm{SEC}$

NON-PID AUXILIARY :

RATIO LO : 0.00 , HI : 10.00 , BIAS LO : $\mathbf{- 5 . 0 0 , ~ H I : ~} 5.00$

RATIO CURR : 0.00 , BIAS CURR : 0.00

IMPORT AI :WTF_1_3::AI_6

EXPORT :AI 5

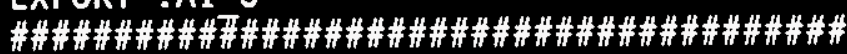

STEP NO

LABEL : FLOW2

FUNCTION : 10 (L )

COMMENT :LOAD INLET 2 FLOW (FIC_2)

PARAMETERS :

1)AI 6 
WHC-SD-CP-CSWD-016

Volume 2

Revision 1

Page 40

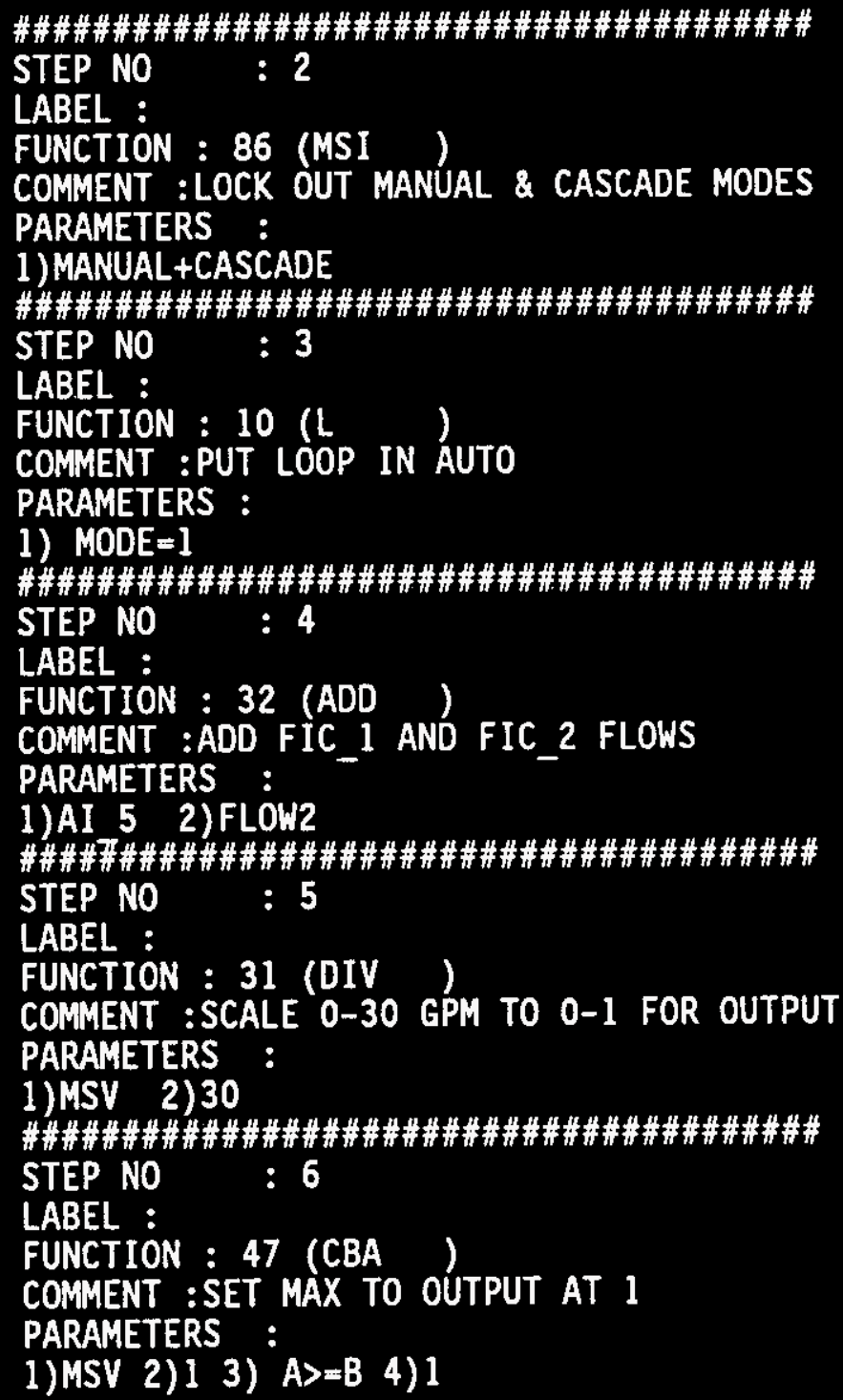




\section{WHC-SD-CP-CSWD-016 \\ Volume 2 \\ Revision 1 \\ Page 41}

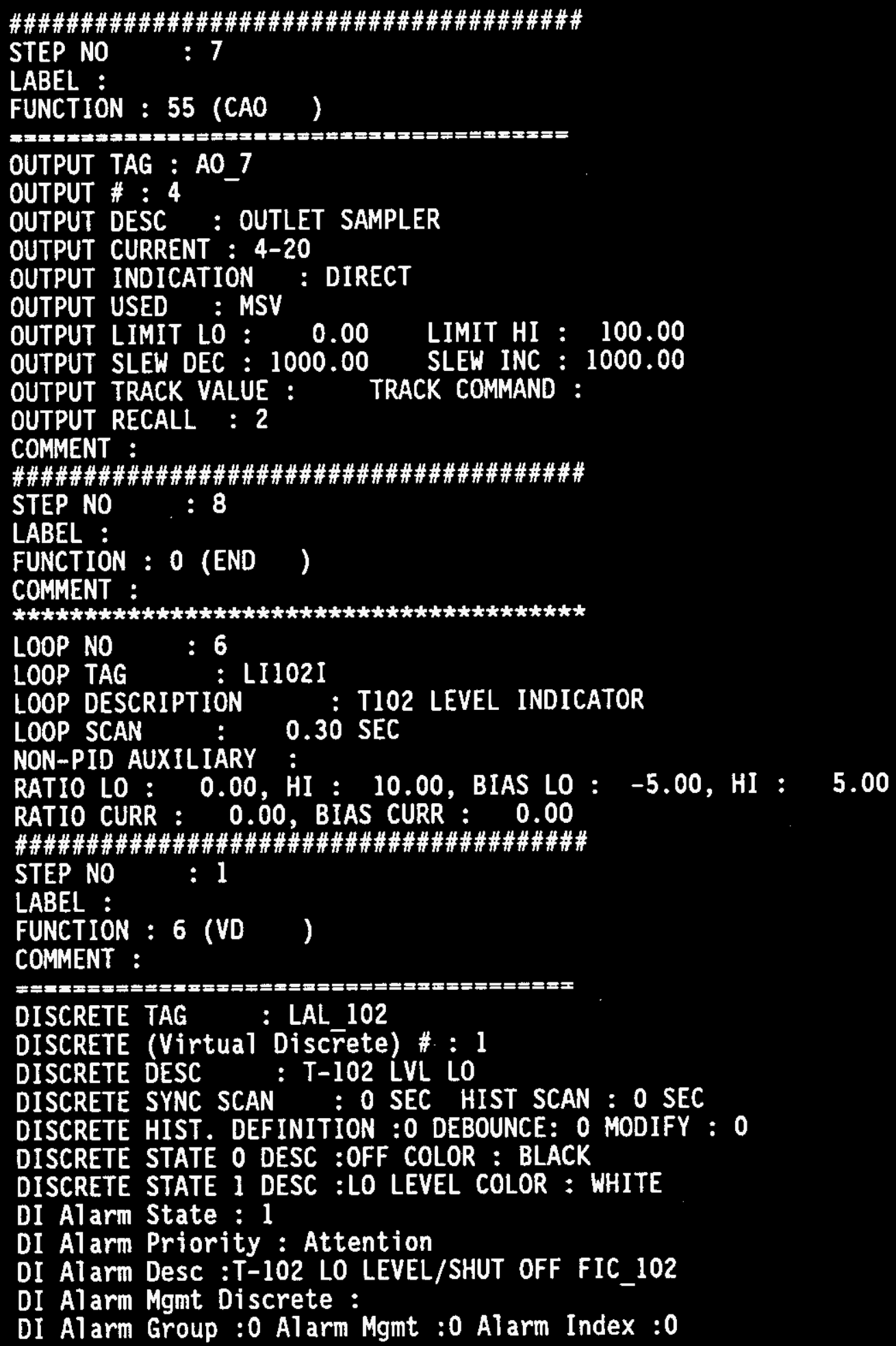




\section{WHC-SD-CP-CSWD-016 \\ Volume 2 \\ Revision 1 \\ Page 42}

\#\#\#\#\#\#\#\#\#\#\#\#\#\#\#\#\#\#\#\#\#\#\#\#\#\#\#\#\#\#\#\#\#\#\#\#\#\#\#\#

STEP NO : 2

LABEL :

FUNCTION : 75 (DST)

COMMENT : ACTIVATE LO LEVEL ALARM FOR 5 MIN ON T-102 LO LEVEL

PARAMETERS :

1) LSL 102 2)0 3) 1 4) 300 5) LAL 102

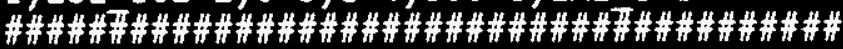

STEP NO : 3

LABEL :

FUNCTION : 11 (AI)

COMMENT :

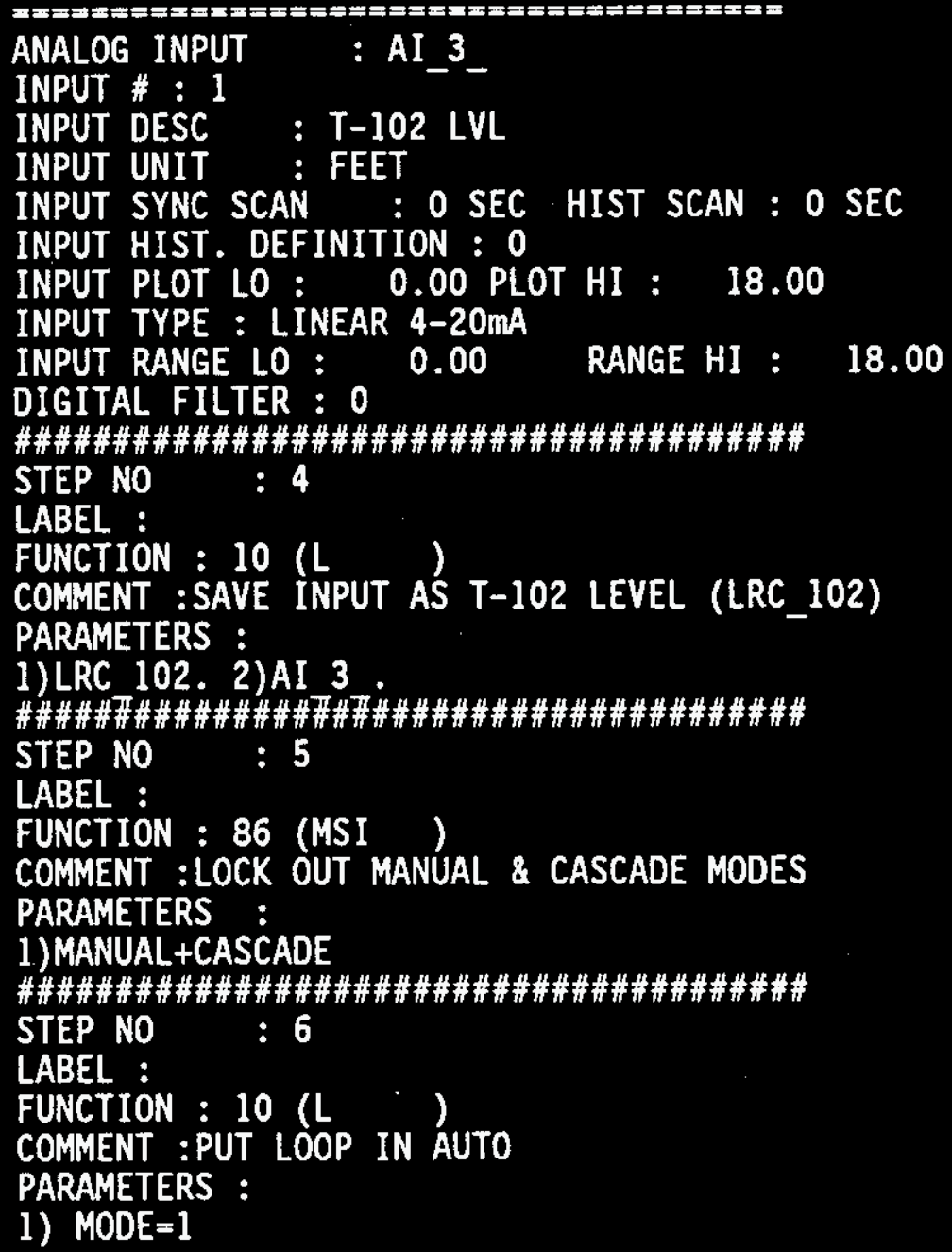




\section{WHC-SD-CP-CSWD-016 \\ Volume 2 \\ Revision 1 \\ Page 43}

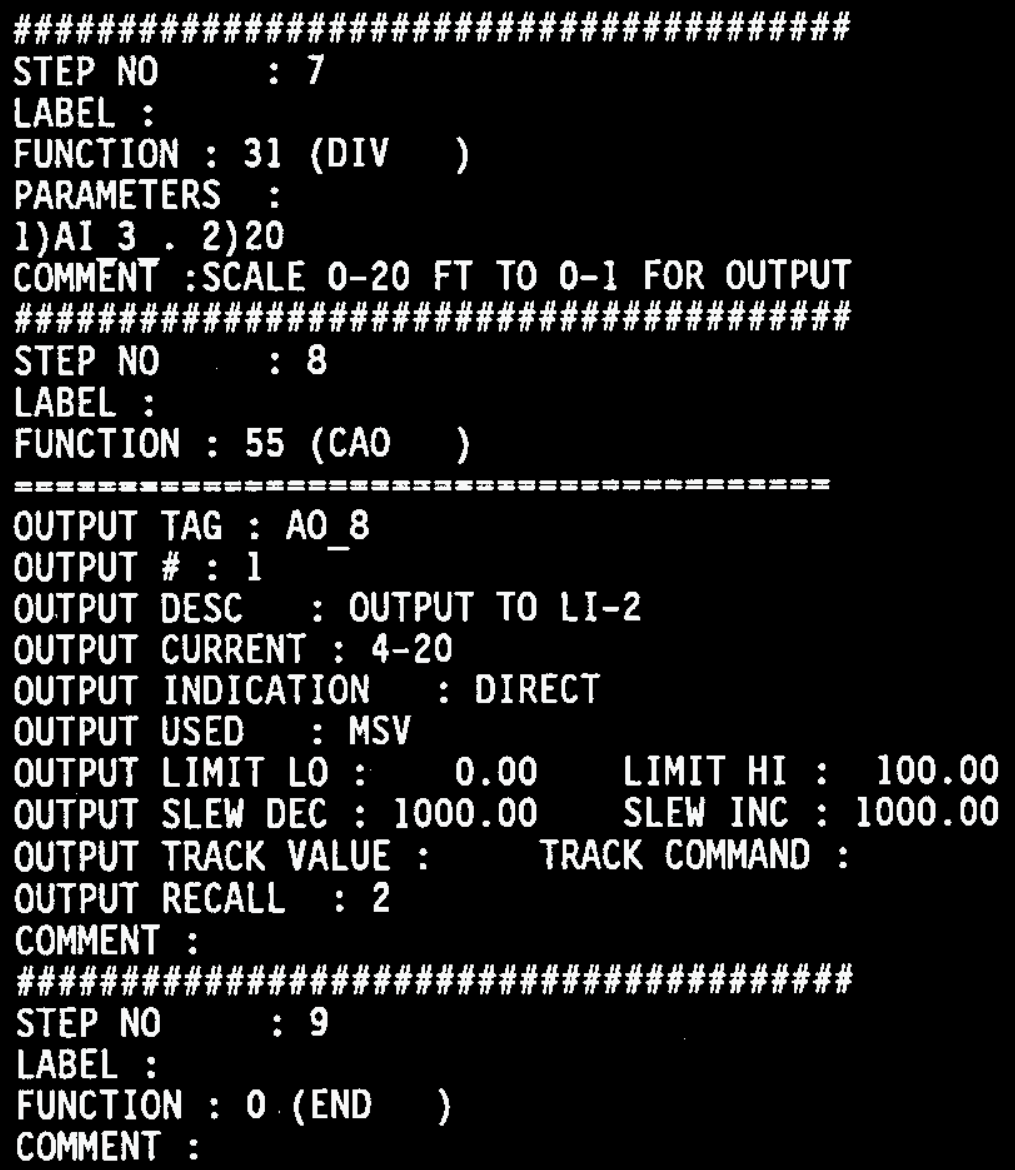


WHC-SO-CP-CSWD-016

Volume 2

Revision 1

Page 44

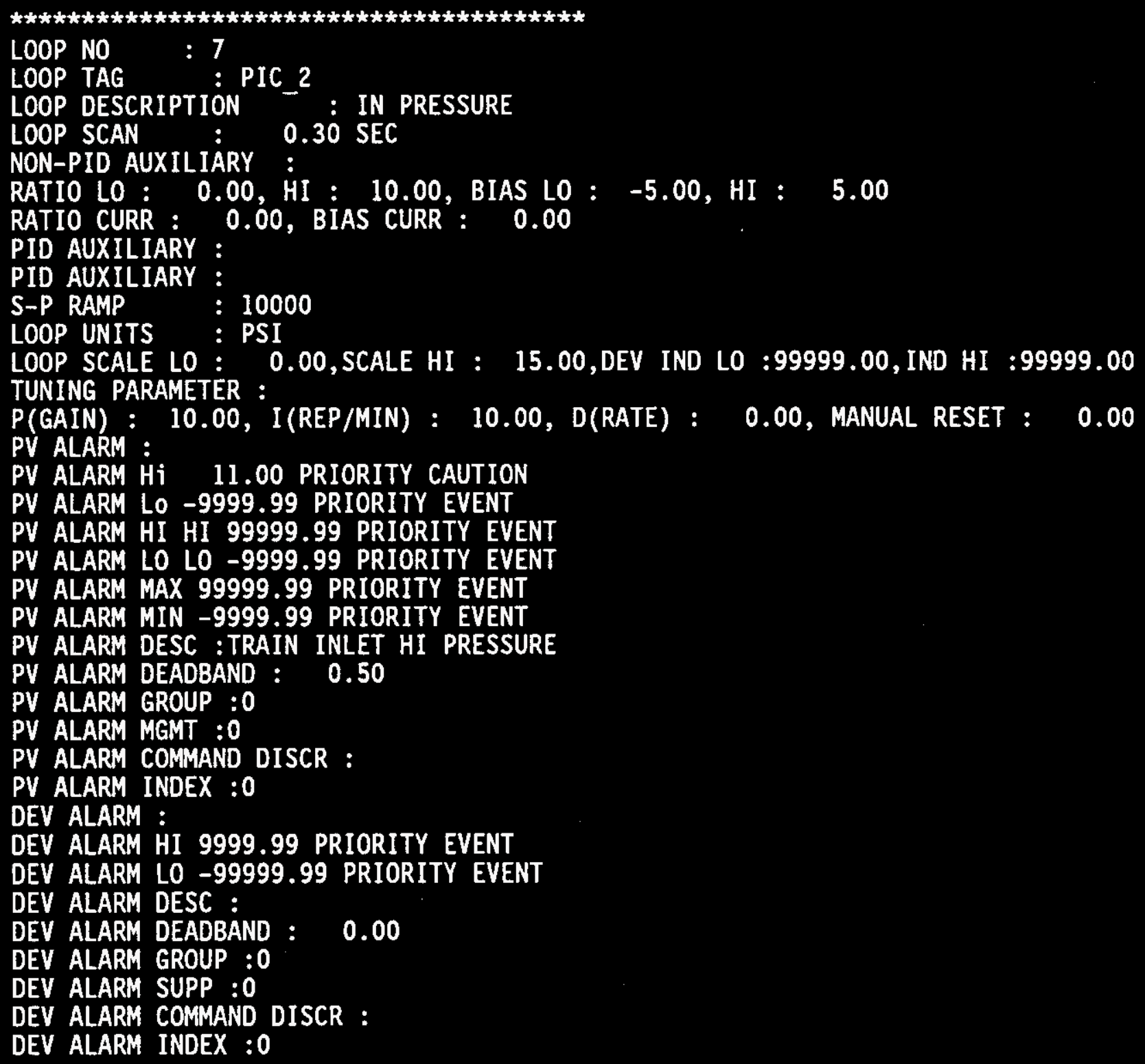




\section{WHC-SD-CP-CSWD-016 \\ Volume 2 \\ Revision 1 \\ Page 45}

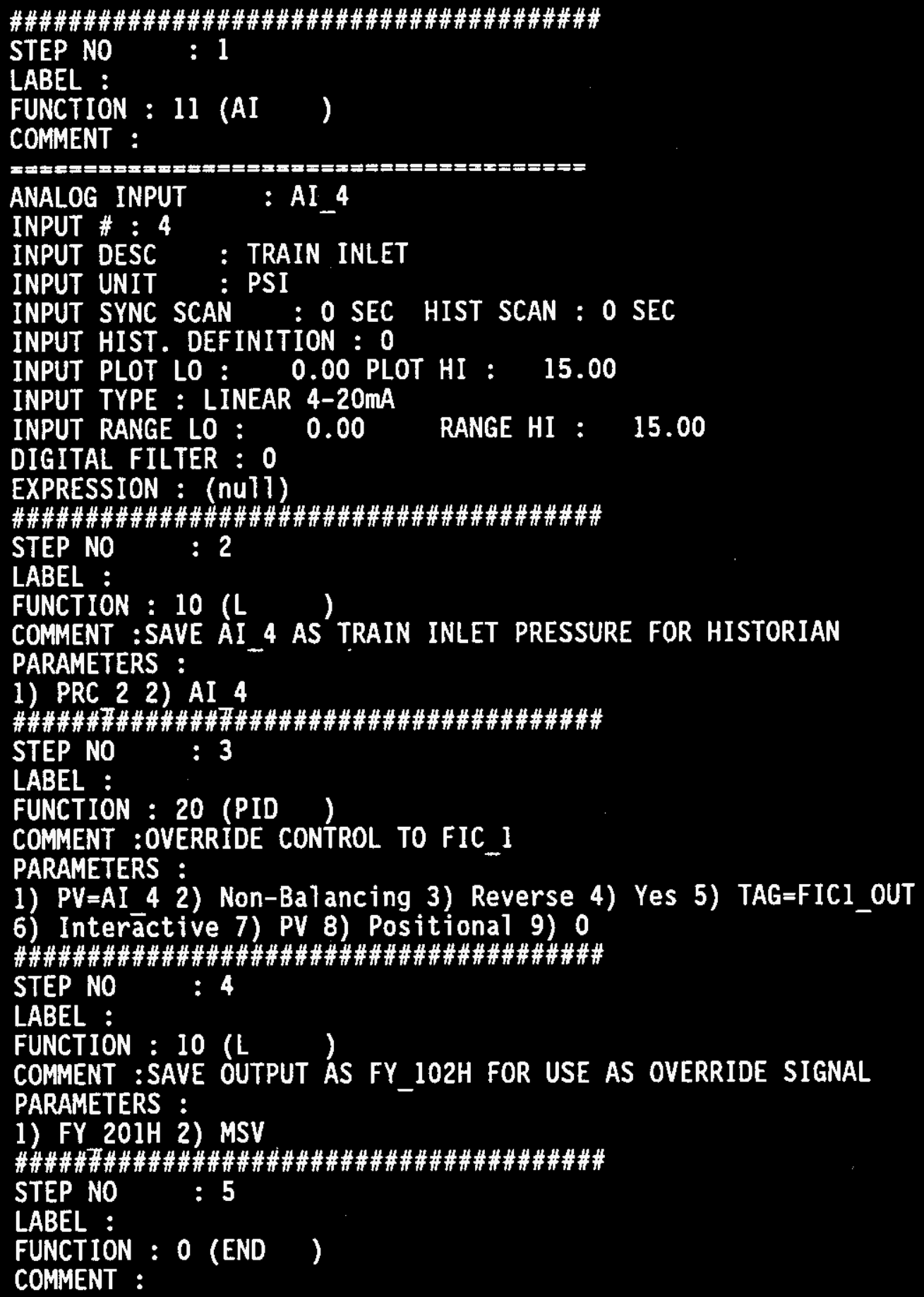


WHC-SD-CP-CSWD-016

Volume 2

Revision 1

Page 46

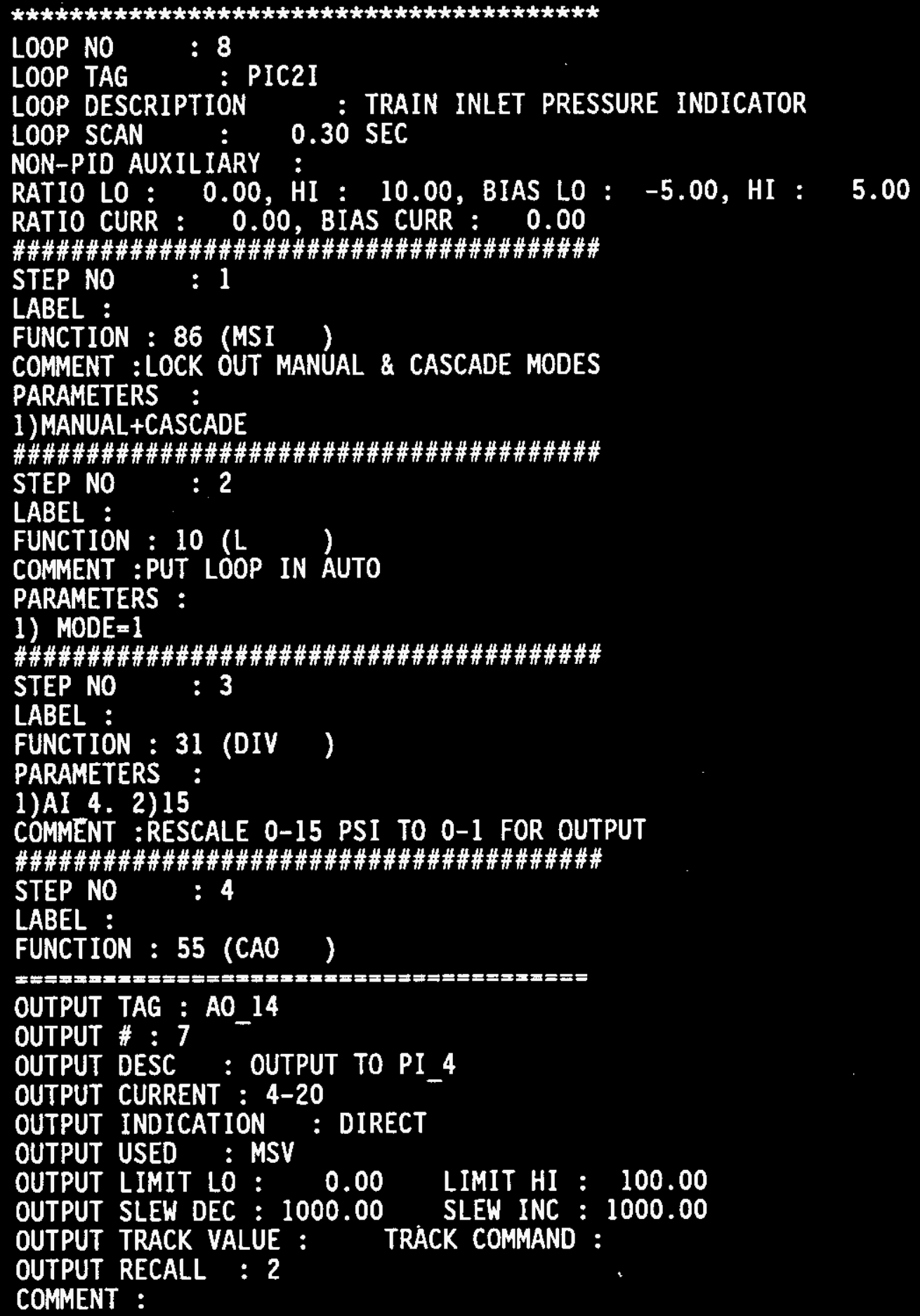




\section{WHC-SD-CP-CSWD-016 \\ Volume 2 \\ Revision 1 \\ Page 47}

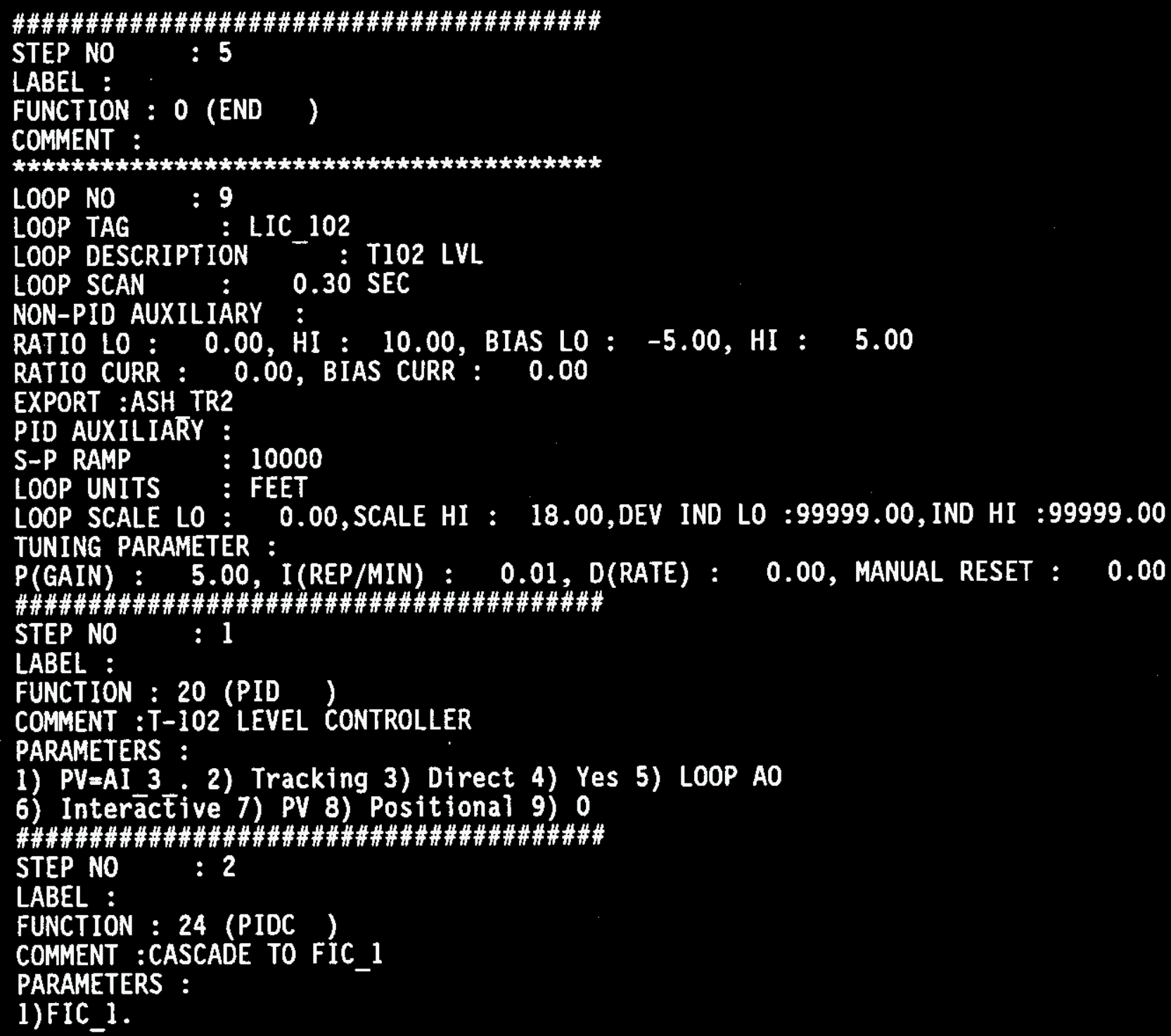




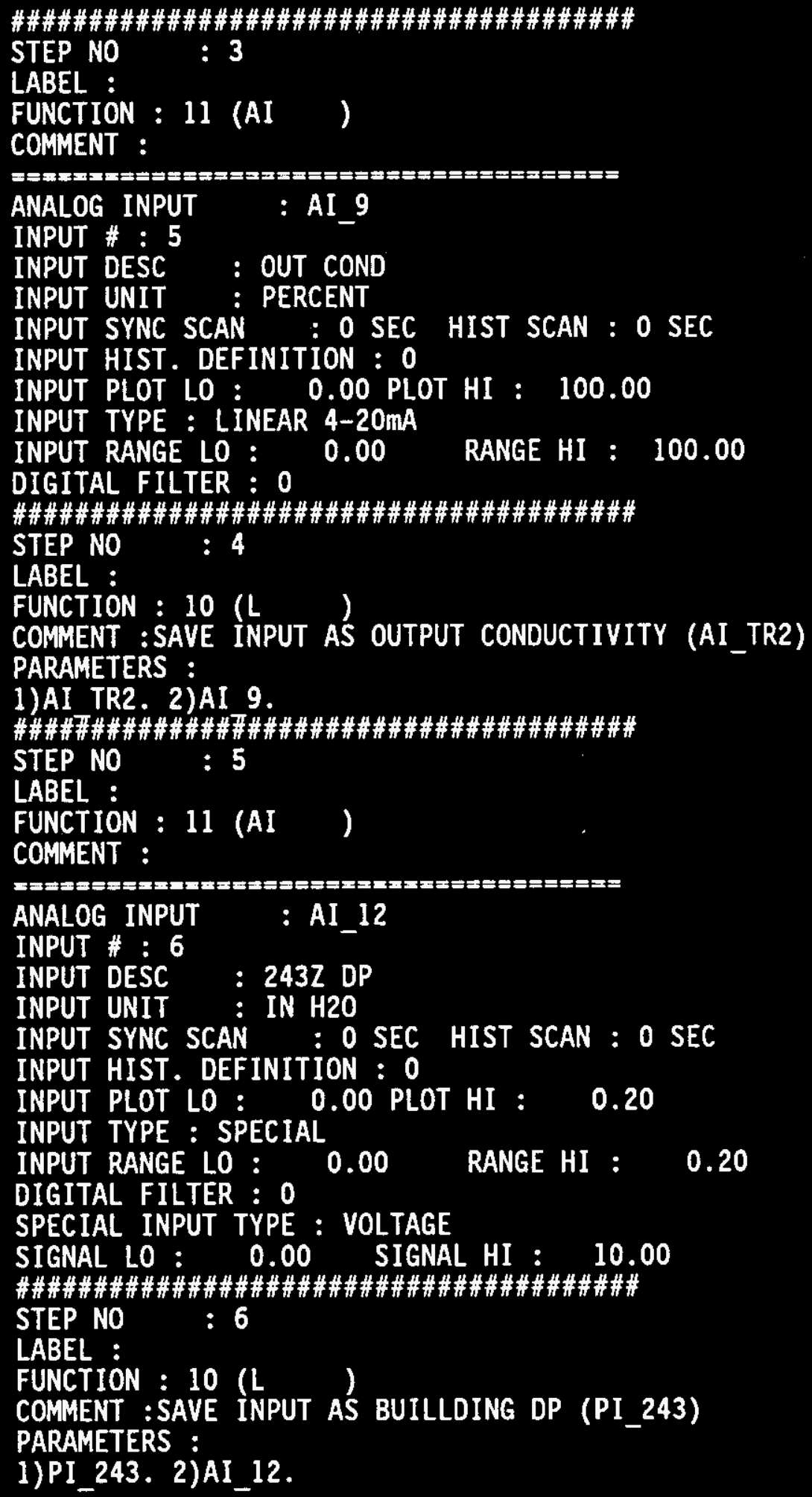


WHC-SD-CP-CSWD-016

Volume 2

Revision 1

Page 49

\#\#\#\#\#\#\#\#\#\#\#\#\#\#\#\#\#\#\#\#\#\#\#\#\#\#\#\#\#\#\#\#\#\#\#\#\#\#\#\#

STEP NO

$: 7$

LABEL :

FUNCTION : 11 (AI )

COMMENT :

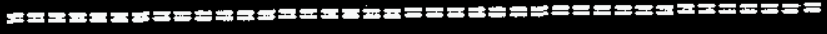

ANALOG INPUT

INPUT \# : 7

: AI_15

INPUT DESC

: KOH TANK LEVEL

INPUT UNIT : INCHES

INPUT SYNC SCAN : O SEC HIST SCAN : O SEC

INPUT HIST. DEFINITION : 0

INPUT PLOT LO: $\quad 0.00$ PLOT HI : 46.00

INPUT TYPE : LINEAR 4-20mA

INPUT RANGE LO : 0.00 RANGE HI : 46.00

DIGITAL FILTER : 0

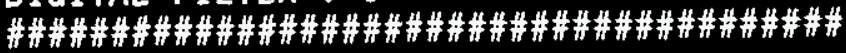

STEP NO : 8

LABEL :

FUNCTION : 10 (L )

COMMENT :SAVE AI_15 AS KOH TANK LEVEL

PARAMETERS :

1)LI 201. 2) AI 15.

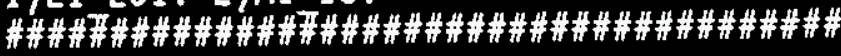

STEP NO $: 9$

LABEL :

FUNCTION : 11 (AI)

COMMENT :

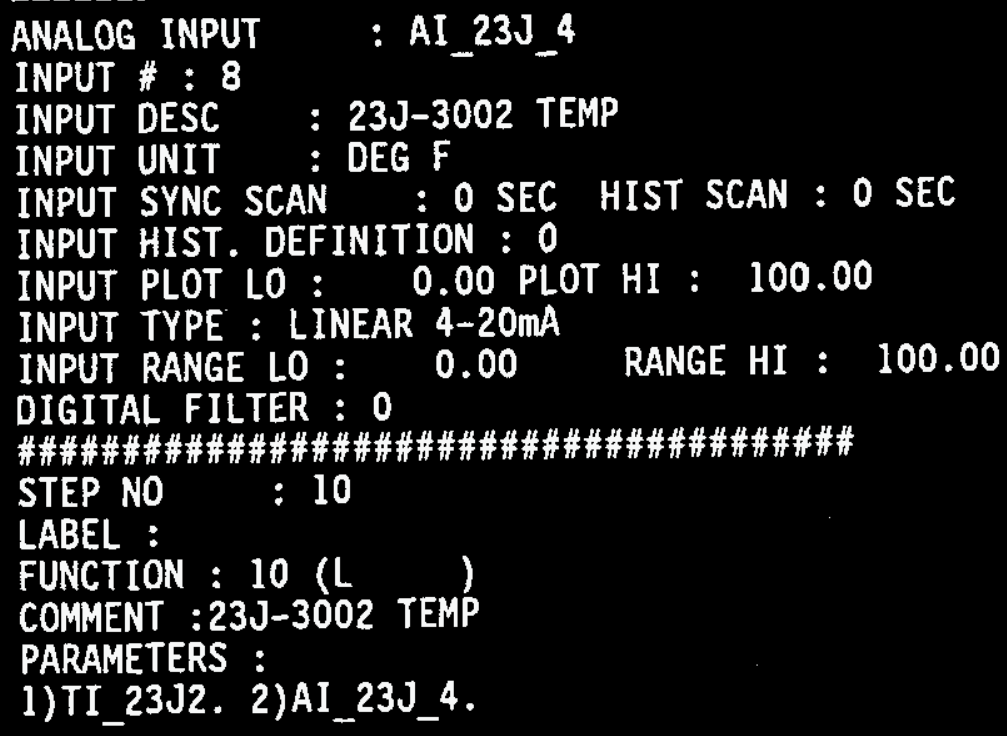




\section{WHC-SD-CP-CSWD-016 \\ Volume 2 \\ Revision 1 \\ Page 50}

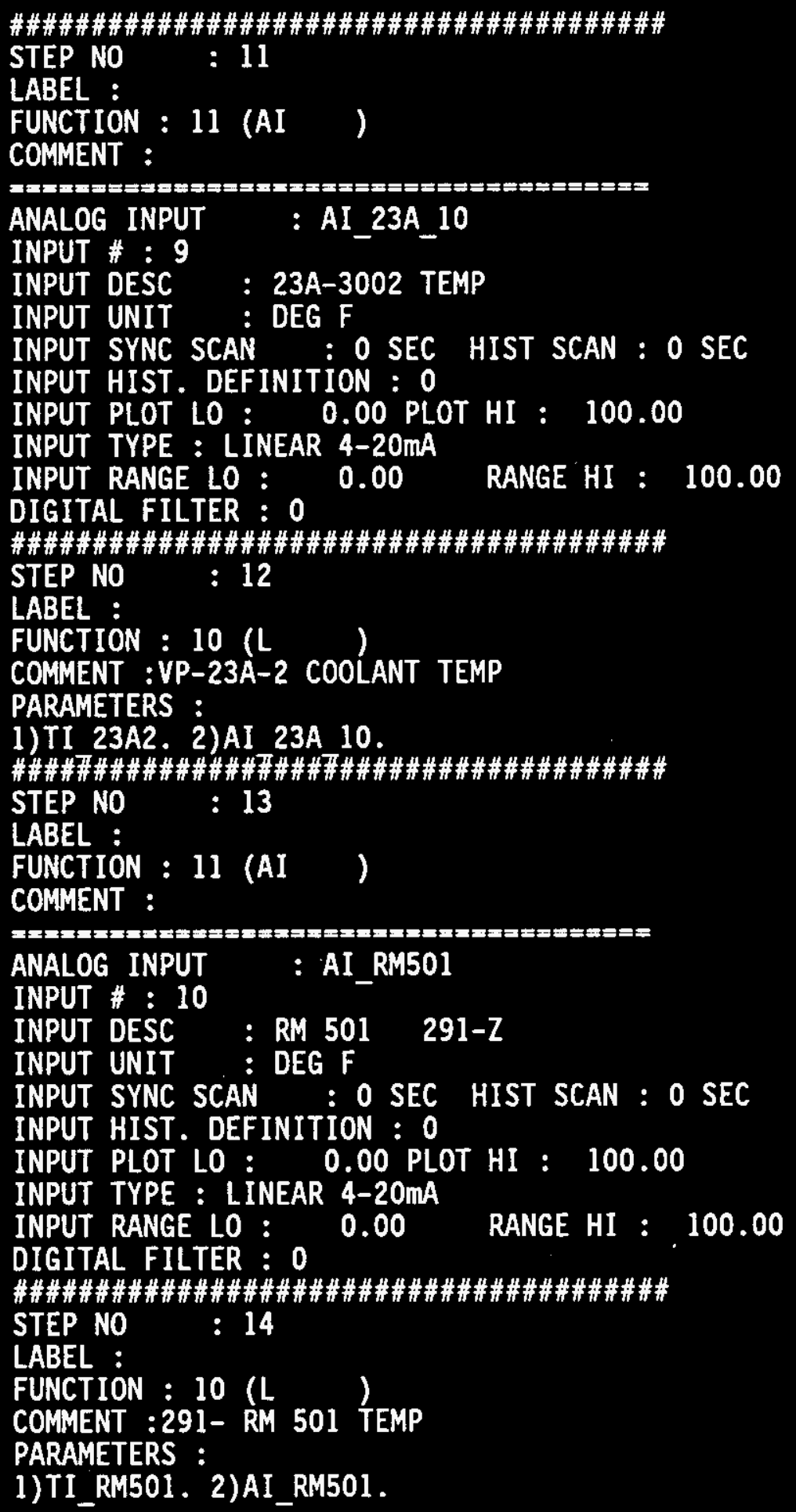


WHC-SD-CP-CSWD-016

Volume 2

Revision 1

Page 51

\#\#\#\#\#\#\#\#\#\#\#\#\#\#\#\#\#\#\#\#\#\#\#\#\#\#\#\#\#\#\#\#\#\#\#\#\#\#\#\#

STEP NO : 15

LABEL :

FUNCTION : 0 (END)

COMMENT : 


\section{WHC-SD-CP-CSWD-016 \\ Volume 2 \\ Revision 1 \\ Page 52}

NTF_2_1.mic4_ro

MICON TYPE :RCM-D

MICON UNIT NO : 4

MICON TAG : WTF 21

MICON DESCRIPTION :- $243-Z$ RACK 2 SLOT 1

MICON CONFIGURATION FILE : mic4_rO

POINT ALLOCATION :

VAI : 32, VDI : 32 , ISW : 32 , LOOPS : 12 , MSD : 16 ,

AI : 0, AO : 0, DI : 32, DO $: 32$

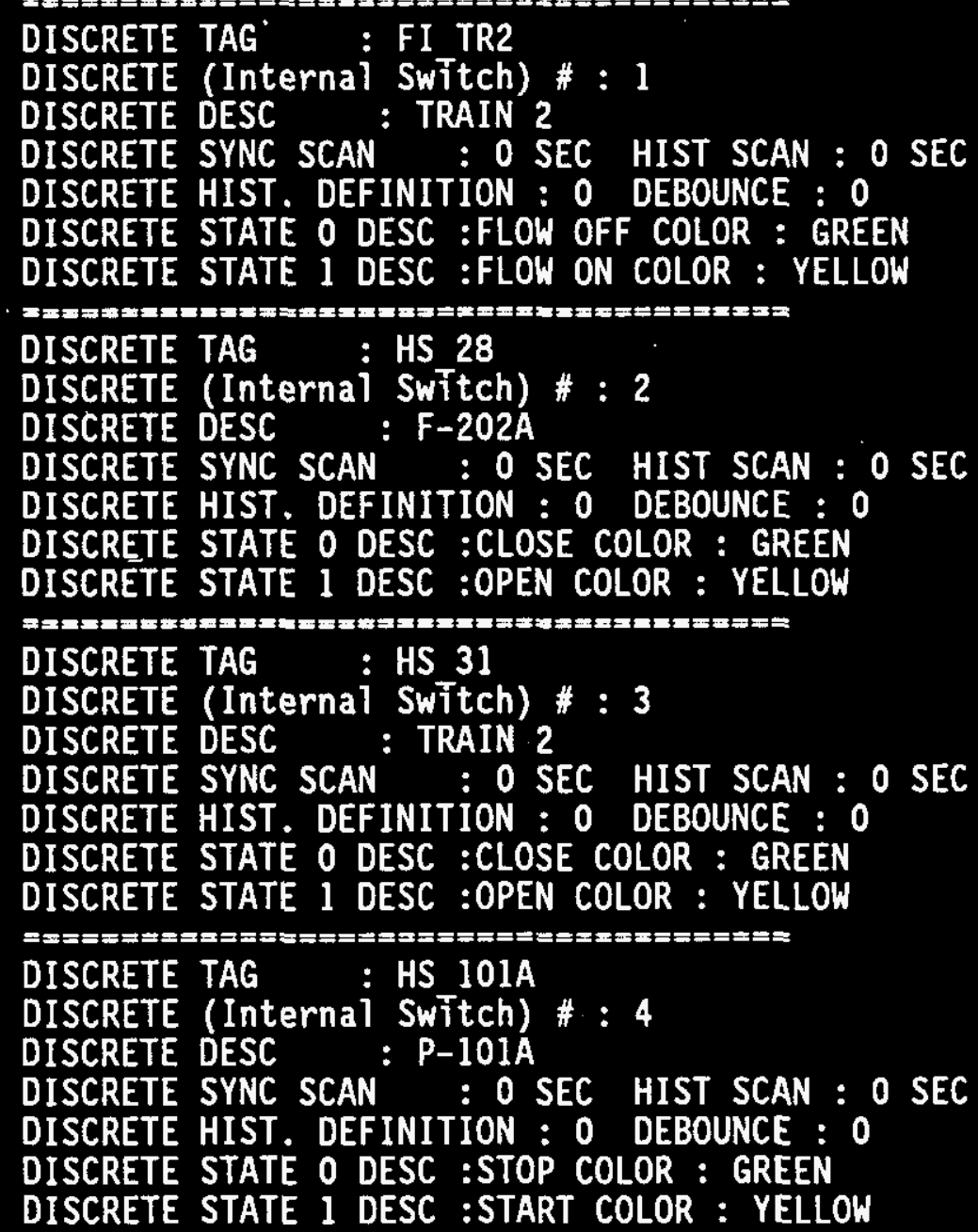


WHC-SO-CP-CSWD-016

Volume 2

Revision 1

Page 53

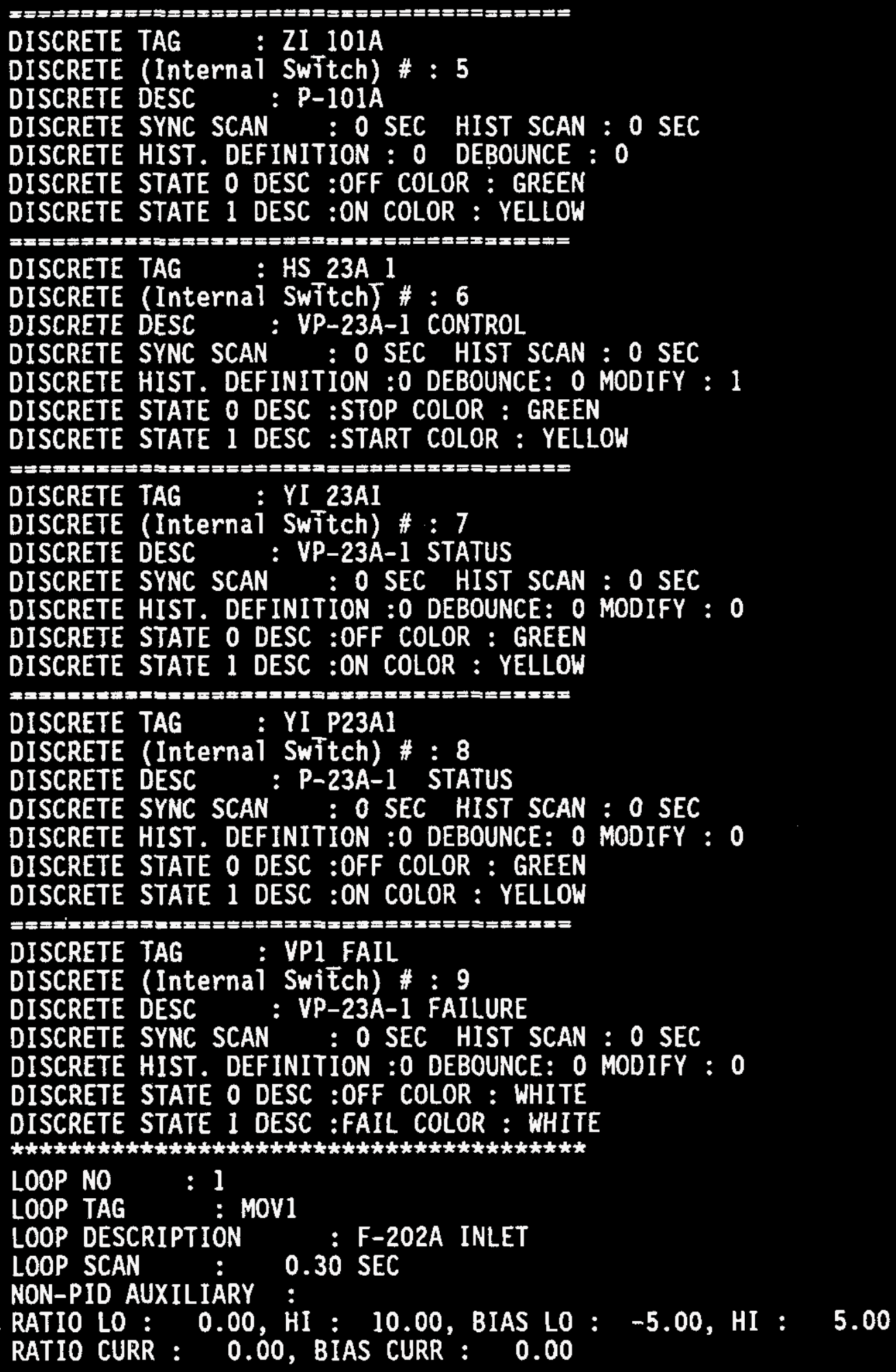




\section{WHC-SD-CP-CSWD-016 \\ Volume 2 \\ Revision 1 \\ Page 54}

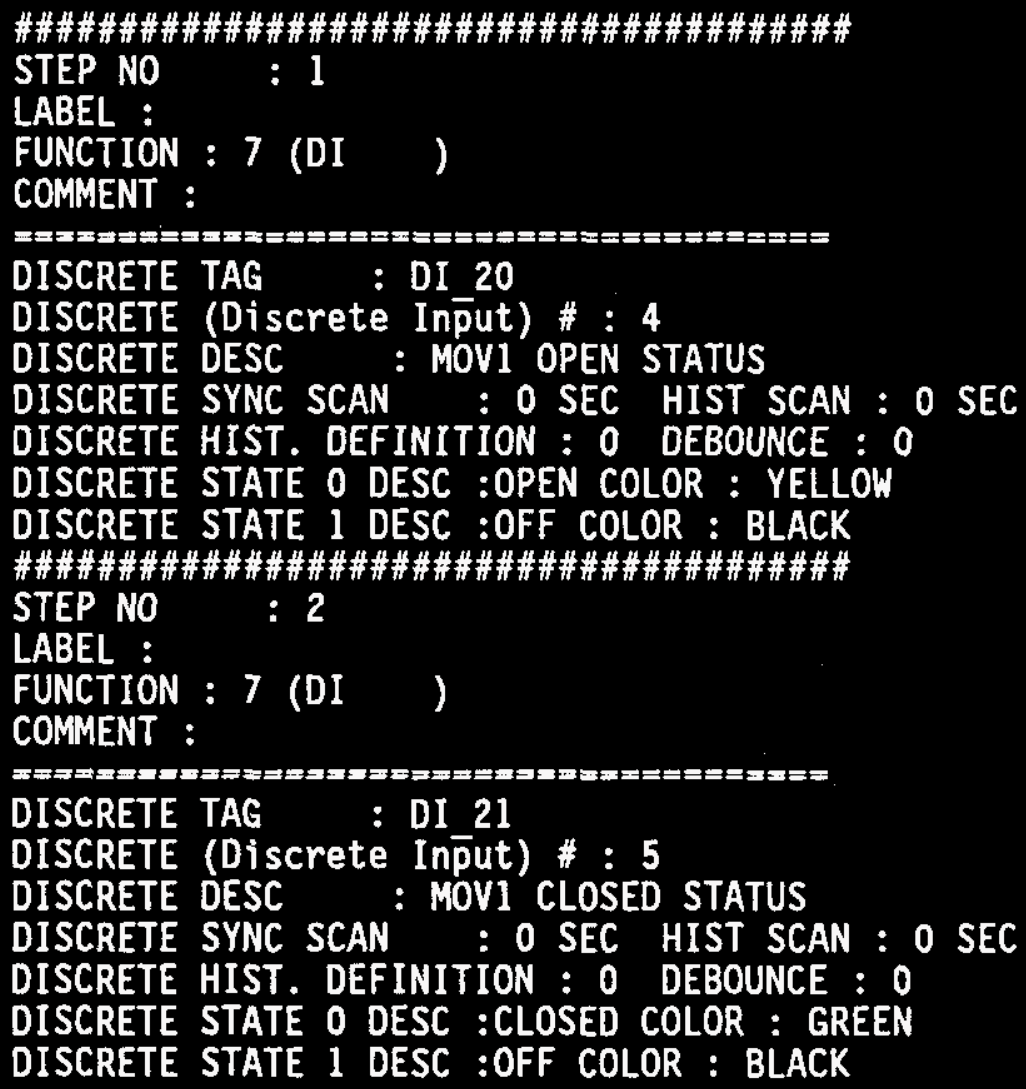


WHC-SD-CP-CSWD-016

Volume 2

Revision 1

Page 55

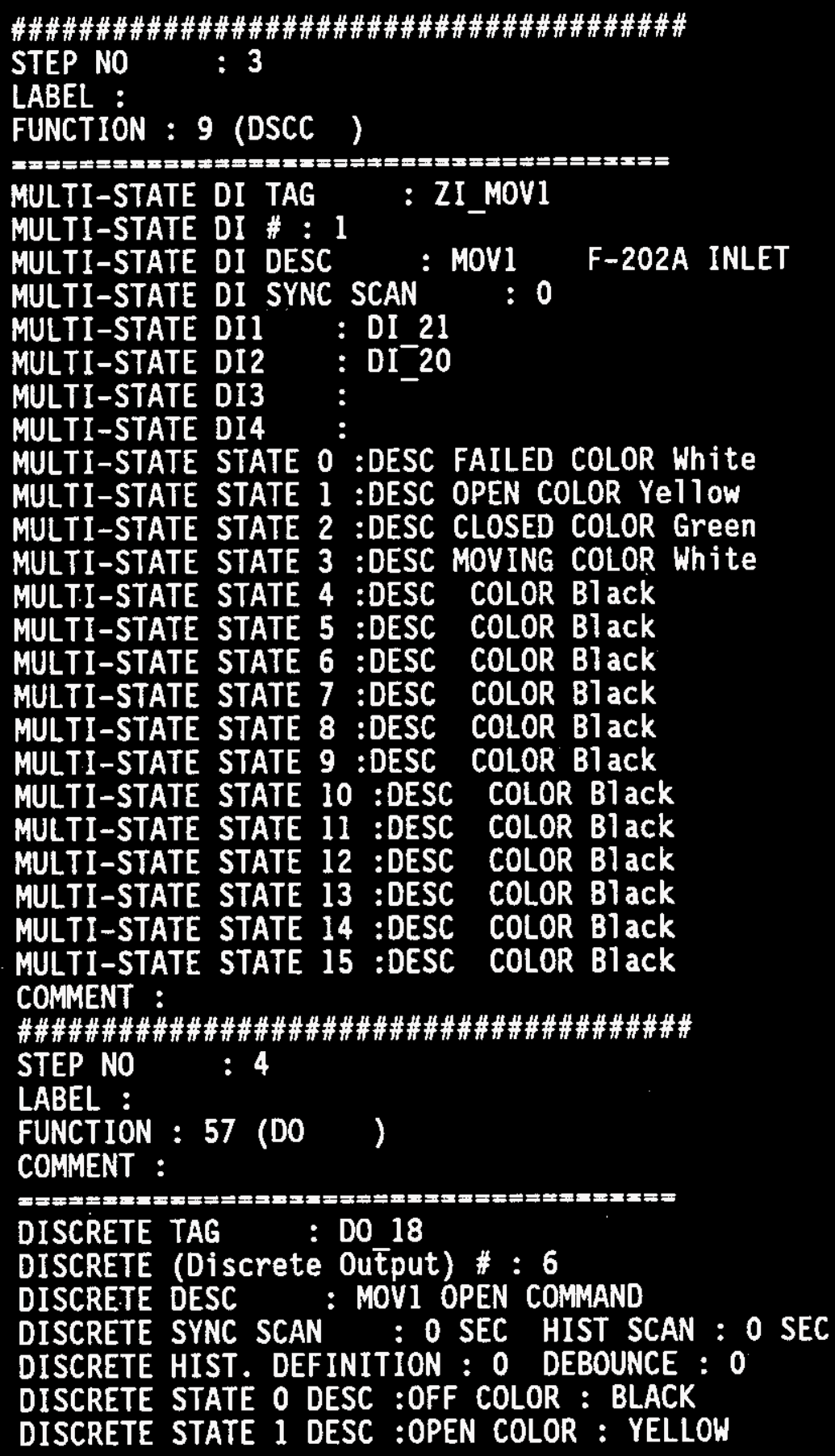




\section{WHC-SD-CP-CSWD-016 \\ Volume 2 \\ Revision 1 \\ Page 56}

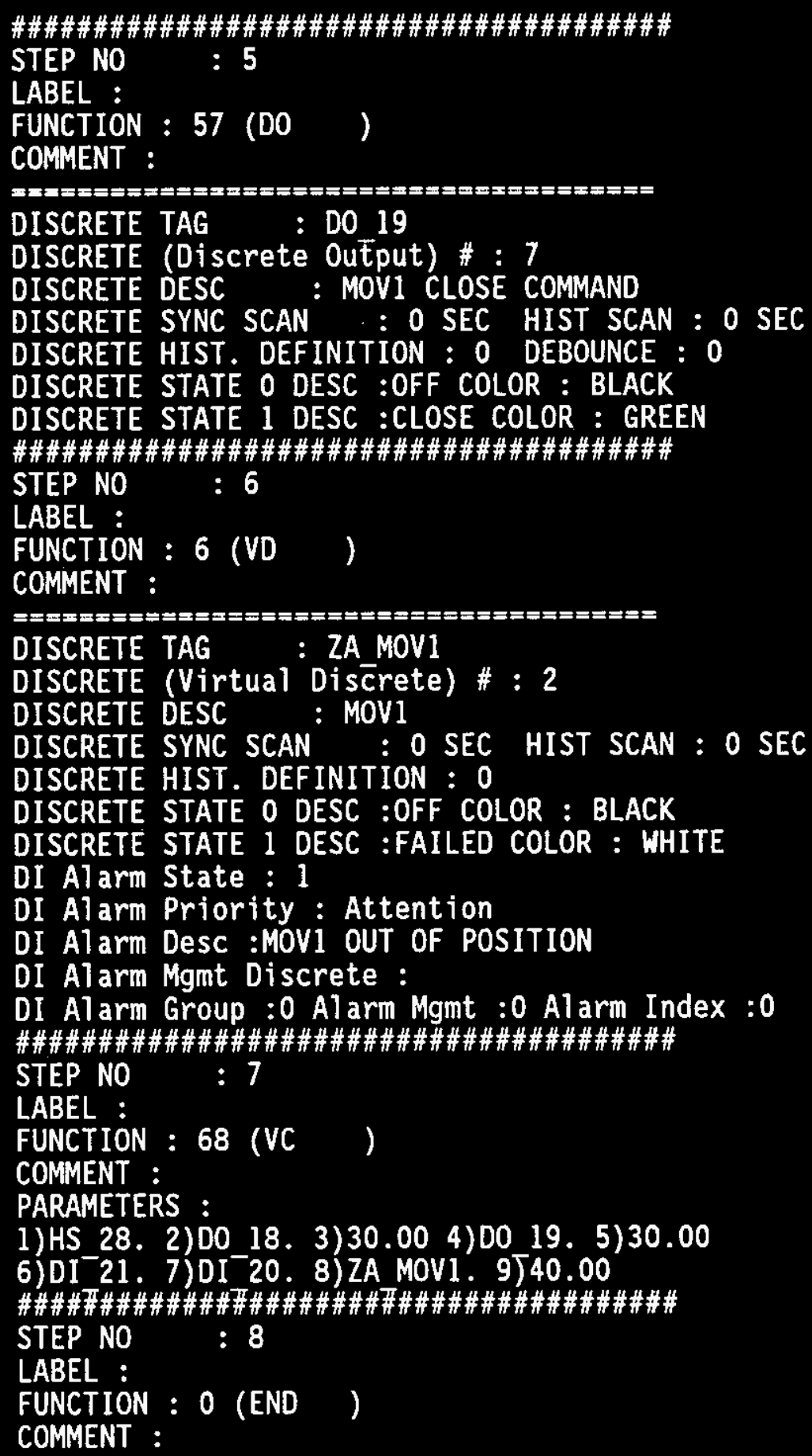




\section{WHC-SD-CP-CSWD-016 \\ Volume 2 \\ Revision 1 \\ Page 57}

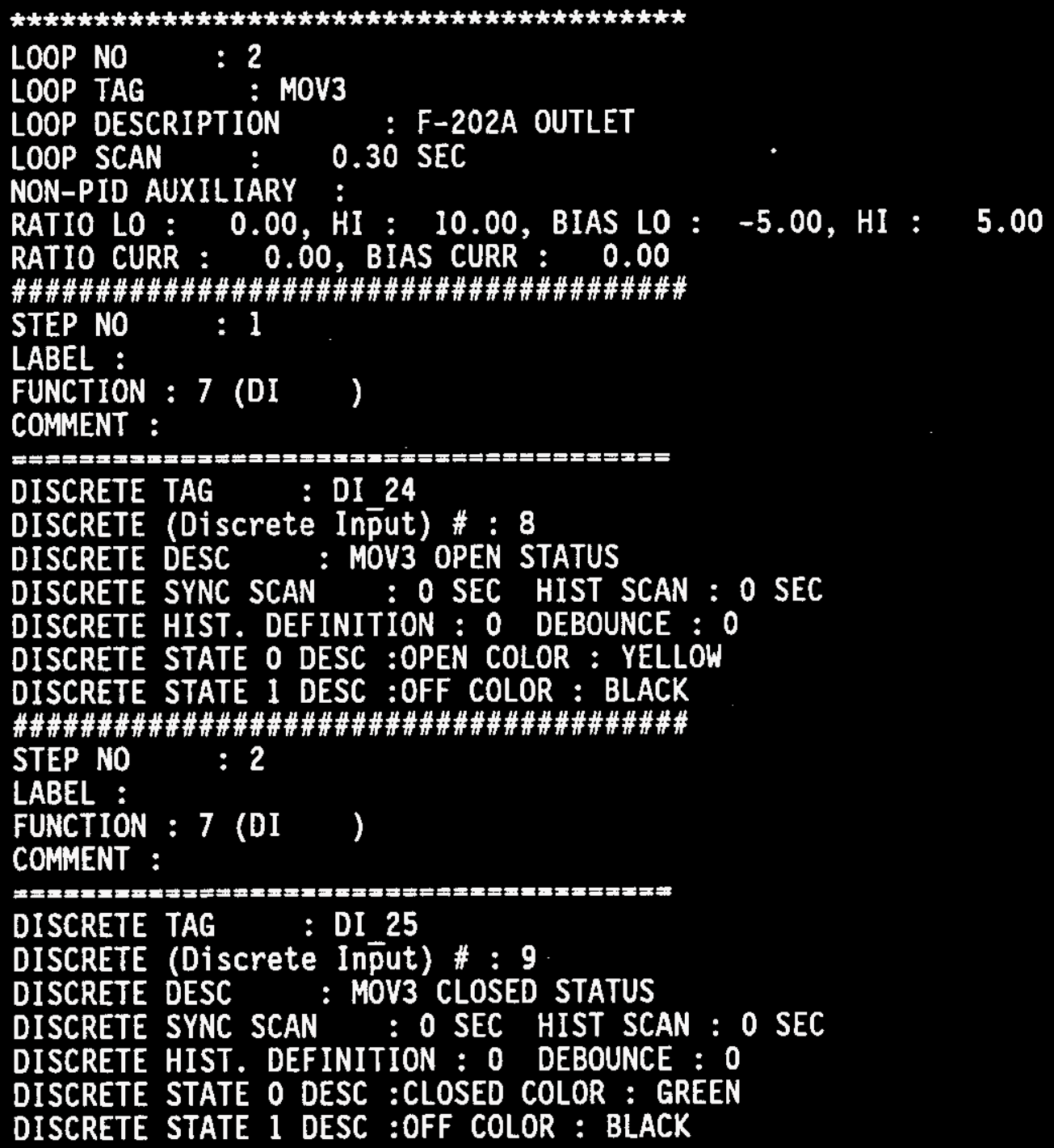


WHC-SD-CP-CSWD-016

Volume 2

Revision 1

Page 58

\#\#\#\#\#\#\#\#\#\#\#\#\#\#\#\#\#\#\#\#\#\#\#\#\#\#\#\#\#\#\#\#\#\#\#\#\#\#\#

STEP NO : 3

LABEL :

FUNCTION : 9 (DSCC )

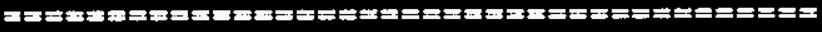

MULTI-STATE DI TAG : ZI_MOV3

MULTI-STATE DI \#: 2

MULTI-STATE DI DESC : MOV3 F-202A INLET

MULTI-STATE DI SYNC SCAN : 0

MULTI-STATE DI1 : DI 25

MULTI-STATE DI2 : $\mathrm{DI}^{-} 24$

MULTI-STATE DI3 :

MULTI-STATE DI4 :

MULTI-STATE STATE 0 :DESC FAILED COLOR White

MULTI-STATE STATE 1 :DESC OPEN COLOR Yellow

MULTI-STATE STATE 2 :DESC CLOSED COLOR Green

MULTI-STATE STATE 3 :DESC MOVING COLOR White

MULTI-STATE STATE 4 :DESC COLOR B1ack

MULTI-STATE STATE 5 :DESC COLOR Black

MULTI-STATE STATE 6 :DESC COLOR B1ack

MULTI-STATE STATE 7 :DESC COLOR B1 ack

MULTI-STATE STATE 8 :DESC COLOR Black

MULTI-STATE STATE 9 :DESC COLOR Black

MULTI-STATE STATE 10 :DESC COLOR Black

MULTI-STATE STATE 11 :DESC COLOR Black

MULTI-STATE STATE 12 :DESC COLOR Black

MULTI-STATE STATE 13 :DESC COLOR Black

MULTI-STATE STATE 14 :DESC COLOR Black

MULTI-STATE STATE 15 :DESC COLOR Black

COMMENT :

\#\#\#\#\#\#\#\#\#\#\#\#\#\#\#\#\#\#\#\#\#\#\#\#\#\#\#\#\#\#\#\#\#\#\#\#\#\#\#\#

STEP NO : 4

LABEL :

FUNCTION : 57 (DO)

COMMENT :

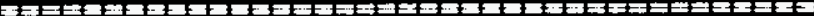

DISCRETE TAG : DO 22

DISCRETE (Discrete Output) \# : 10

DISCRETE DESC : MOV3 OPEN COMMAND

DISCRETE SYNC SCAN : O SEC HIST SCAN : O SEC

DISCRETE HIST. DEFINITION : 0 DEBOUNCE : 0

DISCRETE STATE 0 DESC :OFF COLOR : BLACK

DISCRETE STATE 1 DESC :OPEN COLOR : YELLOW 


\section{WHC-SD-CP-CSWD-016 \\ Volume 2 \\ Revision 1 \\ Page 59}

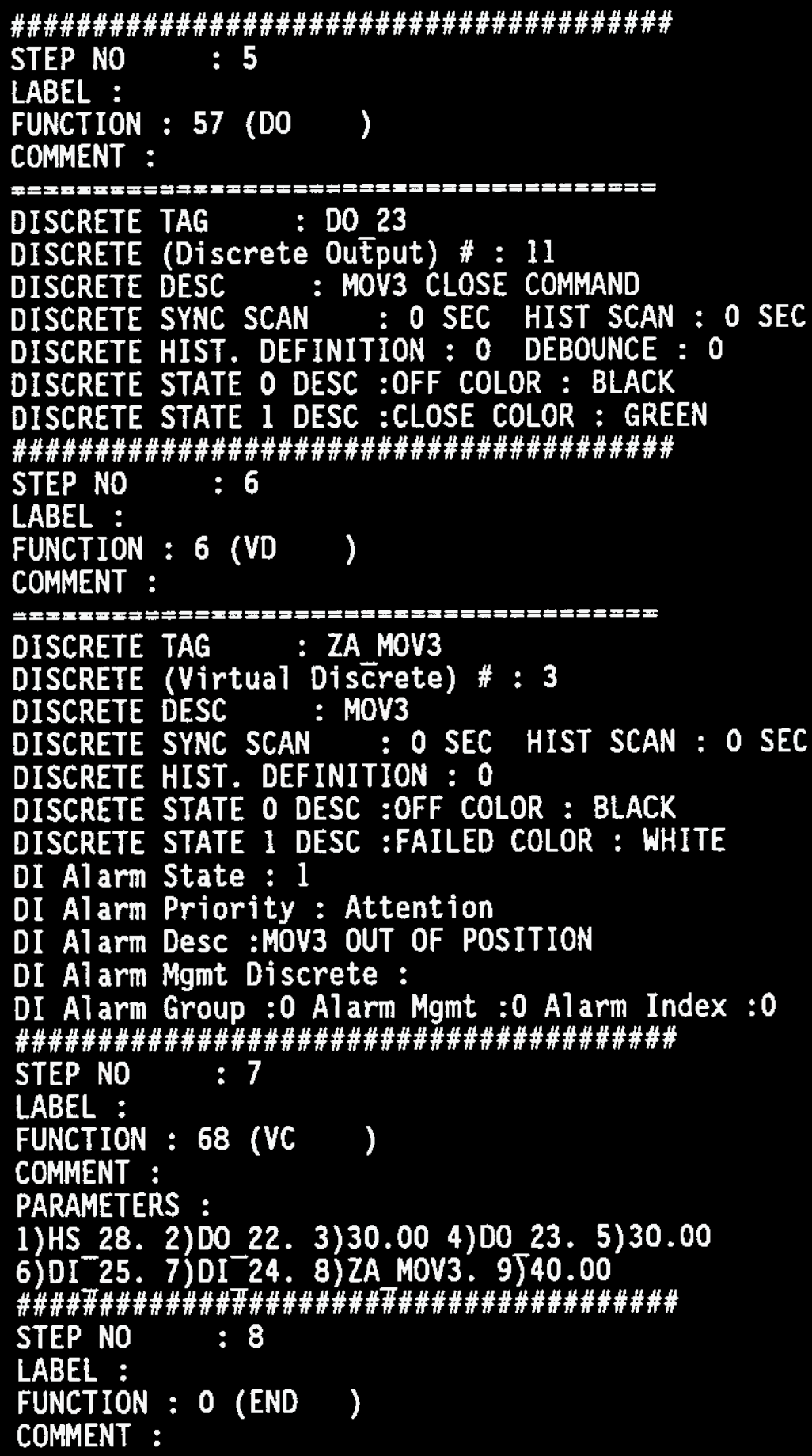


WHC-SD-CP-CSWD-016

Volume 2

Revision 1

Page 60

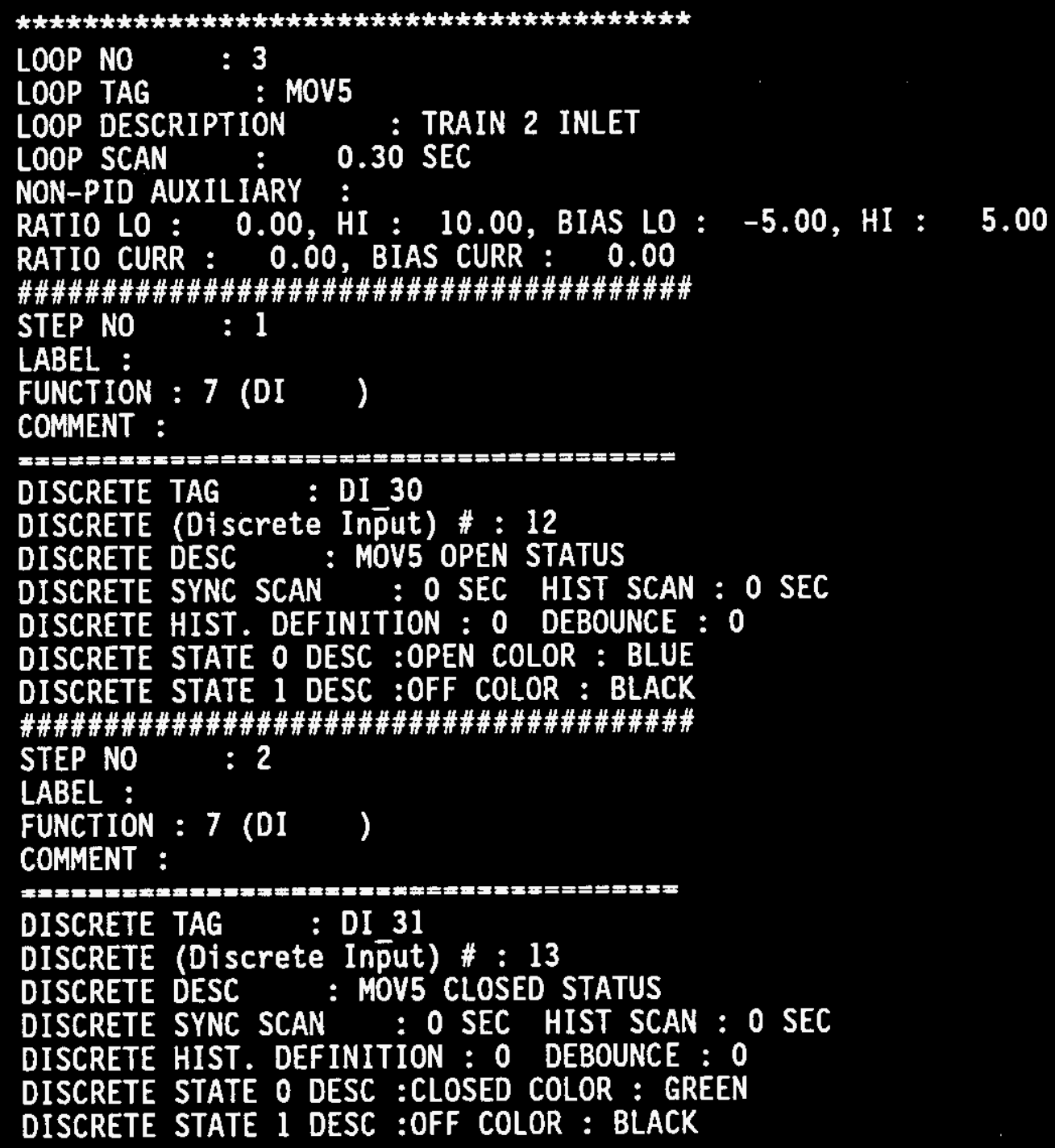


WHC-SD-CP-CSWD-016

Volume 2

Revision 1

Page 61

\#\#\#\#\#\#\#\#\#\#\#\#\#\#\#\#\#\#\#\#\#\#\#\#\#\#\#\#\#\#\#\#\#\#\#\#\#\#\#\#

STEP NO : 3

LABEL :

FUNCTION : 9 (DSCC)

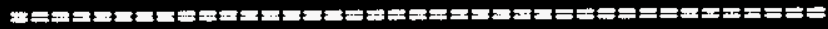

MULTI-STATE DI TAG : ZI MOV5

MULTI-STATE DI \# : 3

MULTI-STATE DI DESC

MULTI-STATE DI SYNC SCAN

: MOV5 TRAIN 2 INLET

MULTI-STATE DI1 : DI 31

MULTI-STATE DI2 : DI 30

MULTI-STATE DI3 :

MULTI-STATE DI4:

MULTI-STATE STATE 0 :DESC FAILED COLOR White

MULTI-STATE STATE 1 :DESC OPEN COLOR Yellow

MULTI-STATE STATE 2 :DESC CLOSED COLOR Green

MULTI-STATE STATE 3 :DESC MOVING COLOR White

MULTI-STATE STATE 4 :DESC COLOR Black

MULTI-STATE STATE 5 :DESC COLOR Black

MULTI-STATE STATE 6 :DESC COLOR Black

MULTI-STATE STATE 7 :DESC COLOR Black

MULTI-STATE STATE 8 :DESC COLOR B1ack

MULTI-STATE STATE 9 :DESC COLOR B1 ack

MULTI-STATE STATE 10 :DESC COLOR Black

MULTI-STATE STATE 11 :DESC COLOR Black

MULTI-STATE STATE 12 :DESC COLOR Black

MULTI-STATE STATE 13 :DESC COLOR Biack

MULTI-STATE STATE 14 :DESC COLOR Black

MULTI-STATE STATE 15 :DESC COLOR B1ack

COMMENT :

\#\#\#\#\#\#\#\#\#\#\#\#\#\#\#\#\#\#\#\#\#\#\#\#\#\#\#\#\#\#\#\#\#\#\#\#\#\#\#\#

STEP NO : 4

LABEL :

FUNCTION : $57(00)$

COMMENT :

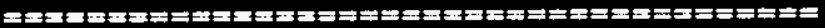

DISCRETE TAG : DO 28

DISCRETE (Discrete Output) \#: 14

DISCRETE DESC : MOVS OPEN COMMAND

DISCRETE SYNC SCAN : O SEC HIST SCAN : O SEC

DISCRETE HIST. DEFINITION : 0 DEBOUNCE : 0

DISCRETE STATE 0 DESC :OFF COLOR : BLACK

DISCRETE STATE 1 DESC :OPEN COLOR : YELLOW 

WHC-SD-CP-CSWD-016
Volume 2
Revision 1
Page 62

\#\#\#\#\#\#\#\#\#\#\#\#\#\#\#\#\#\#\#\#\#\#\#\#\#\#\#\#\#\#\#\#\#\#\#\#\#\#\#\#

STEP NO : 5

LABEL :

FUNCTION : $57(00)$

COMMENT :

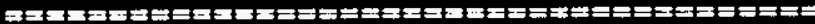

DISCRETE TAG : DO 29

DISCRETE (Discrete Output) \# : 15

DISCRETE DESC : MOV5 CLOSE COMMAND

DISCRETE SYNC SCAN : O SEC HIST SCAN : O SEC

DISCRETE HIST. DEFINITION : 0 DEBOUNCE : 0

DISCRETE STATE 0 DESC :OFF COLOR : BLACK

DISCRETE STATE 1 DESC :CLOSE COLOR : GREEN

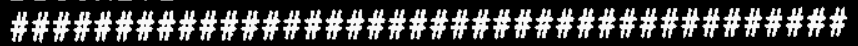

STEP NO : 6

LABEL :

FUNCTION : 6 (VD )

COMMENT :

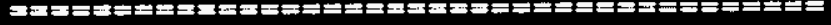

DISCRETE TAG : ZA MOV5

DISCRETE (Virtual Discrete) \# : 4

DISCRETE DESC : MOV5

DISCRETE SYNC SCAN : O SEC HIST SCAN : O SEC

DISCRETE HIST. DEFINITION : 0

DISCRETE STATE 0 DESC :OFF COLOR : BLACK

DISCRETE STATE 1 DESC :FAILED COLOR : WHITE

DI Alarm State : 1

DI Alarm Priority : Attention

DI A1arm Desc :MOV5 OUT: OF POSITION

DI Alarm Mgmt Discrete :

DI Alarm Group :0 Alarm Mgmt :0 Alarm Index :0

\#\#\#\#\#\#\#\#\#\#\#\#\#\#\#\#\#\#\#\#\#\#\#\#\#\#\#\#\#\#\#\#\#\#\#\#\#\#\#\#

STEP NO : 7

LABEL :

FUNCTION : 68 (VC )

COMMENT :

PARAMETERS :

1)HS 31. 2)DO 28. 3) 30.00 4)00 29. 5)30.00

6) $\mathrm{DI}^{-} 31$. 7) $\mathrm{DI}^{-} 30$. 8) ZA MOV5. 9) 40.00

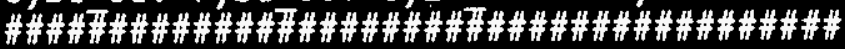

STEP NO : 8

LABEL :

FUNCTION : 0 (END)

COMMENT : 
WHC-SD-CP-CSWD-016

Volume 2

Revision 1

Page 63

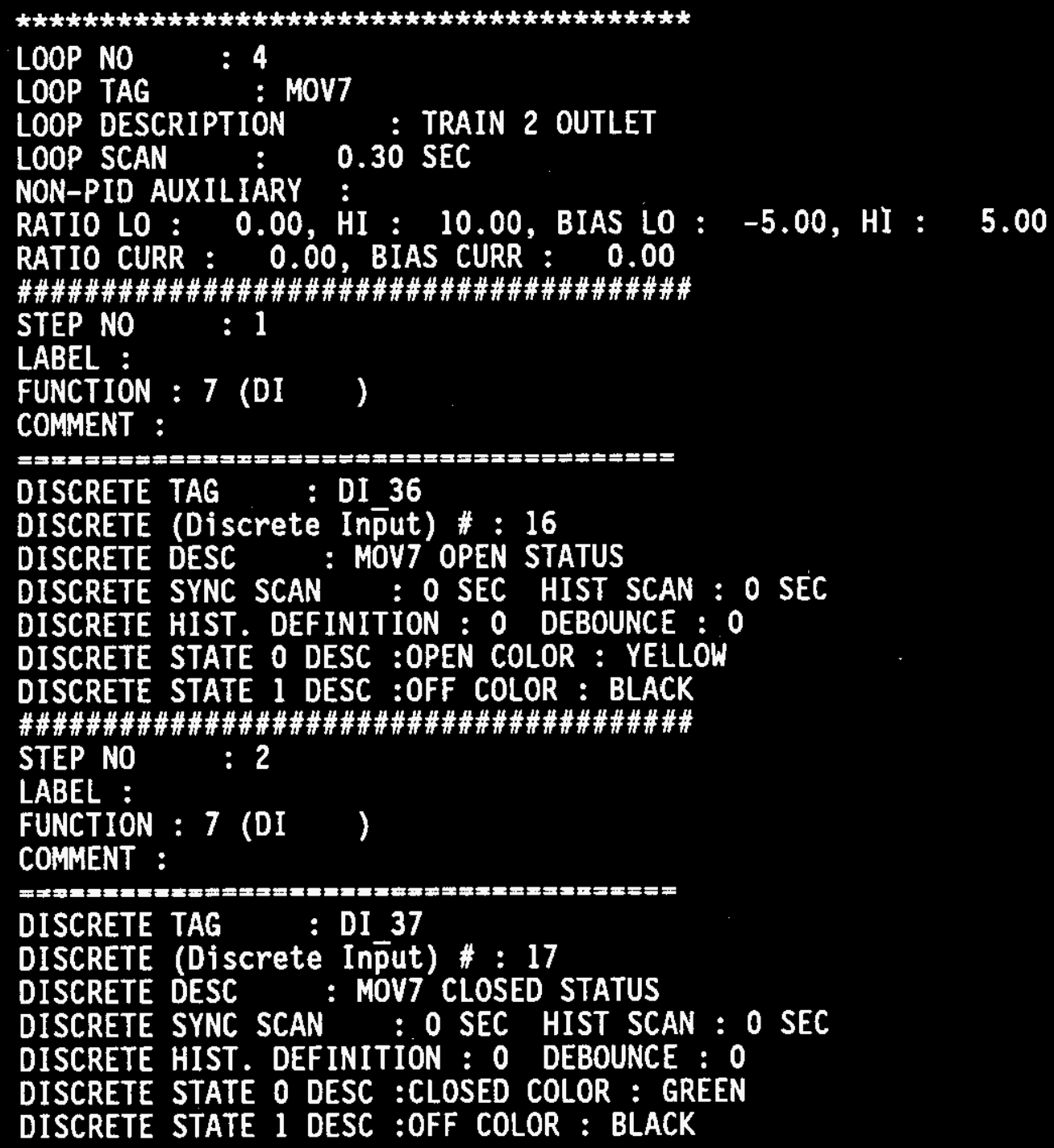


WHC-SD-CP-CSWD-016

Volume 2

Revision 1

Page 64

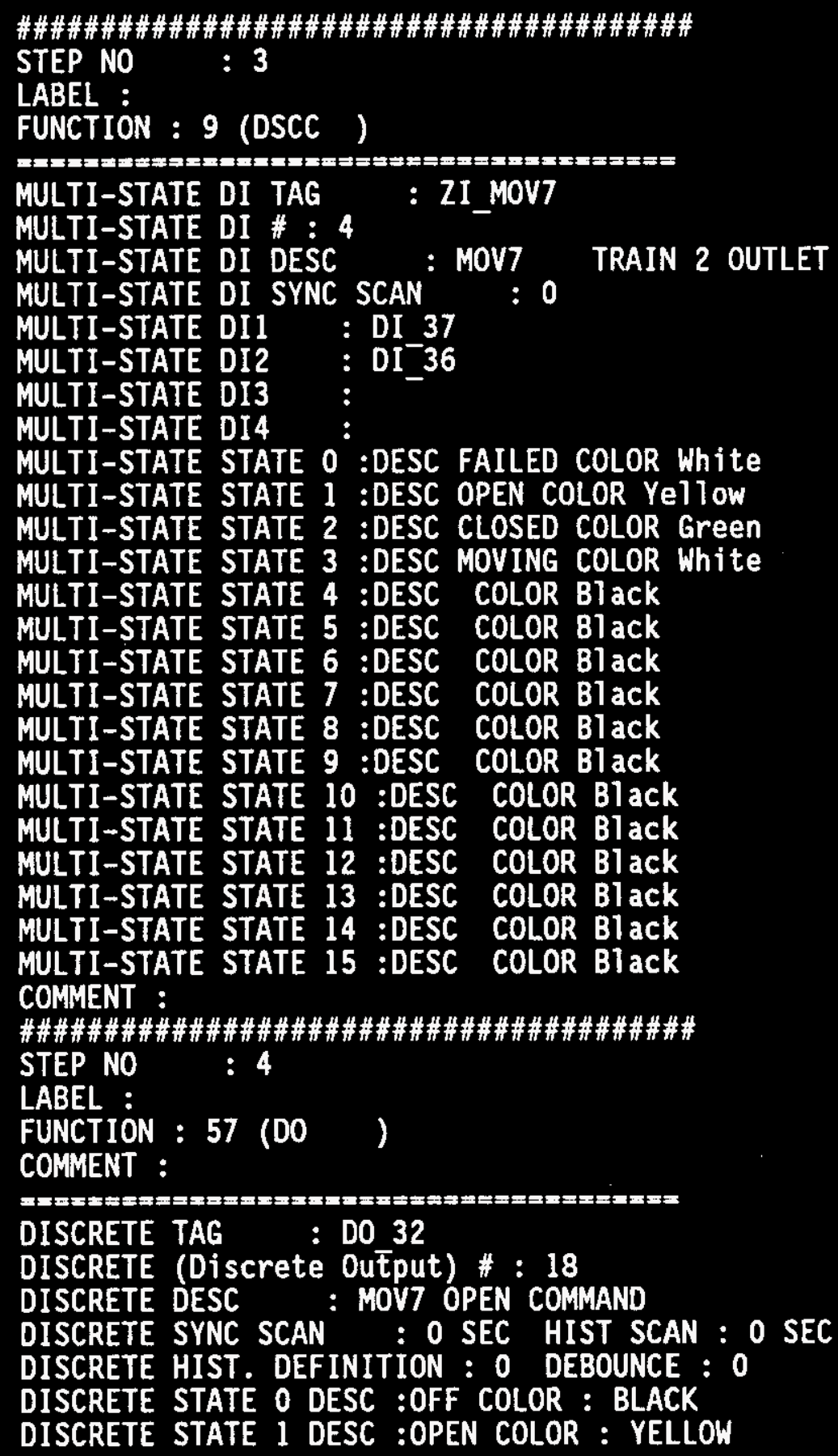


WHC-SD-CP-CSWD-016

Volume 2

Revision 1

Page 65

\#\#\#\#\#\#\#\#\#\#\#\#\#\#\#\#\#\#\#\#\#\#\#\#\#\#\#\#\#\#\#\#\#\#\#\#\#\#\#\#

STEP NO : 5

LABEL :

FUNCTION : $57(\mathrm{DO})$

COMMENT :

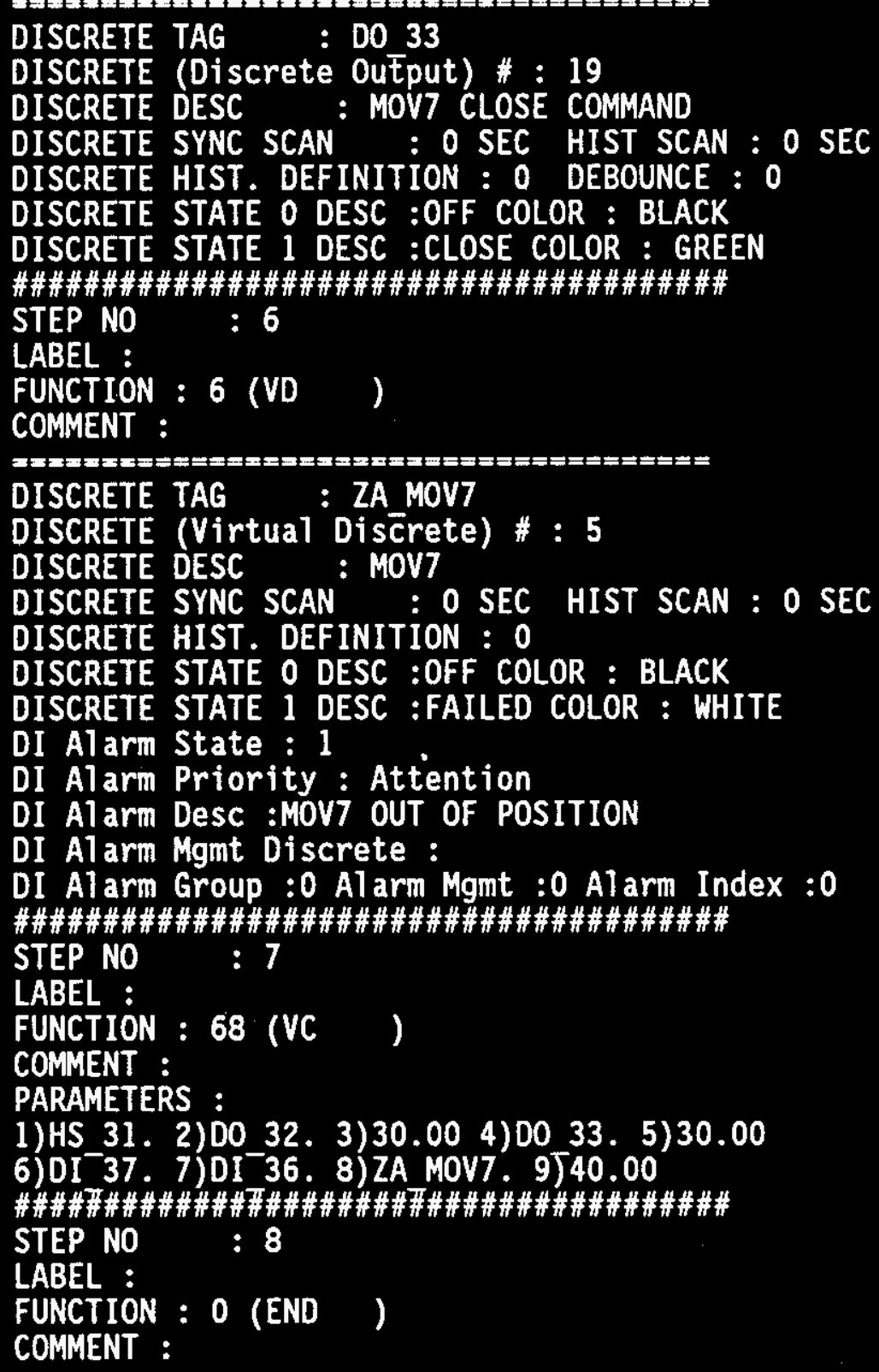




\section{WHC-SD-CP-CSWD-016 \\ Volume 2 \\ Revision 1 \\ Page 66}

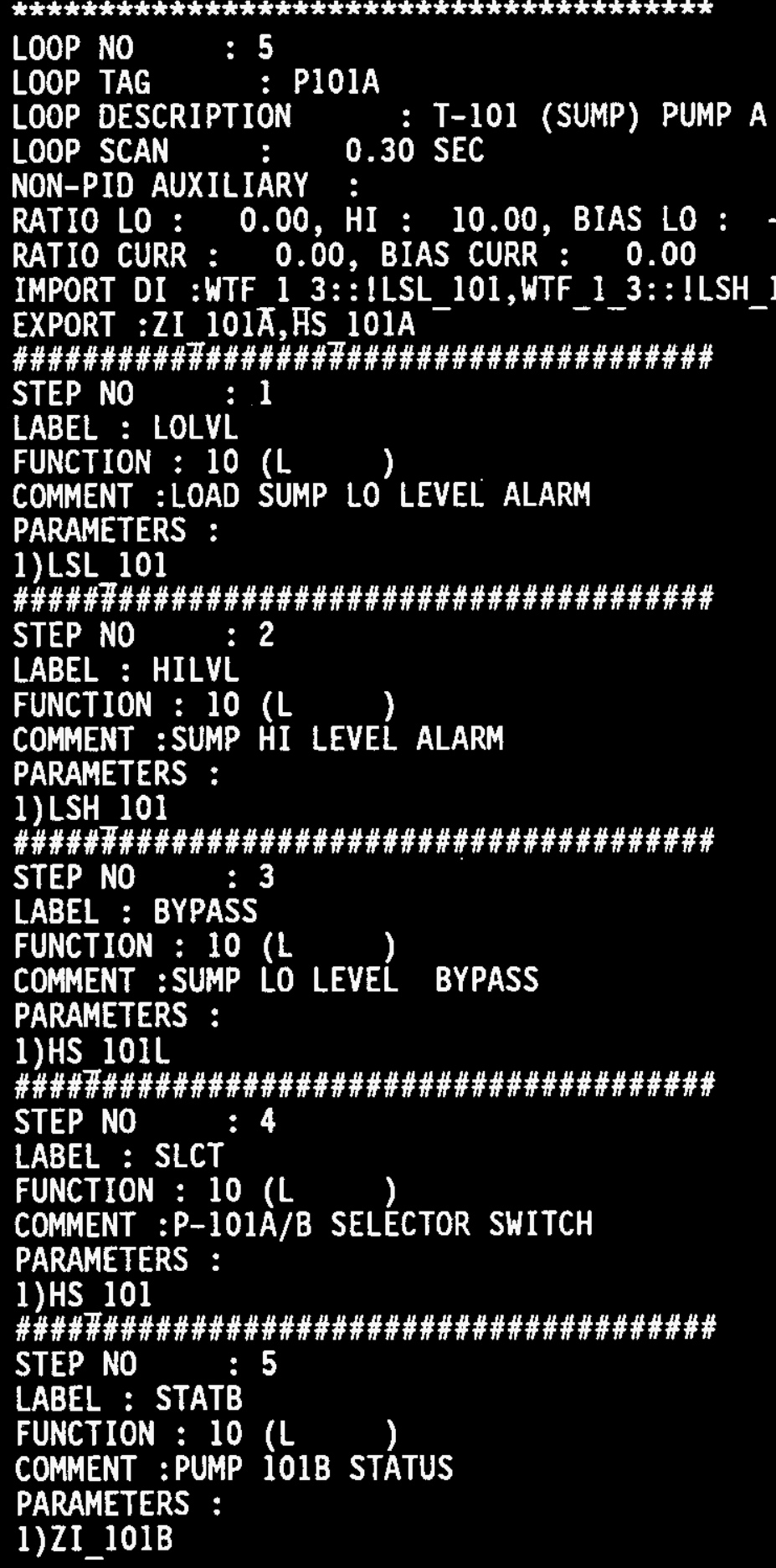




\section{WHC-SD-CP-CSWD-016 \\ Volume 2 \\ Revision 1 \\ Page 67}

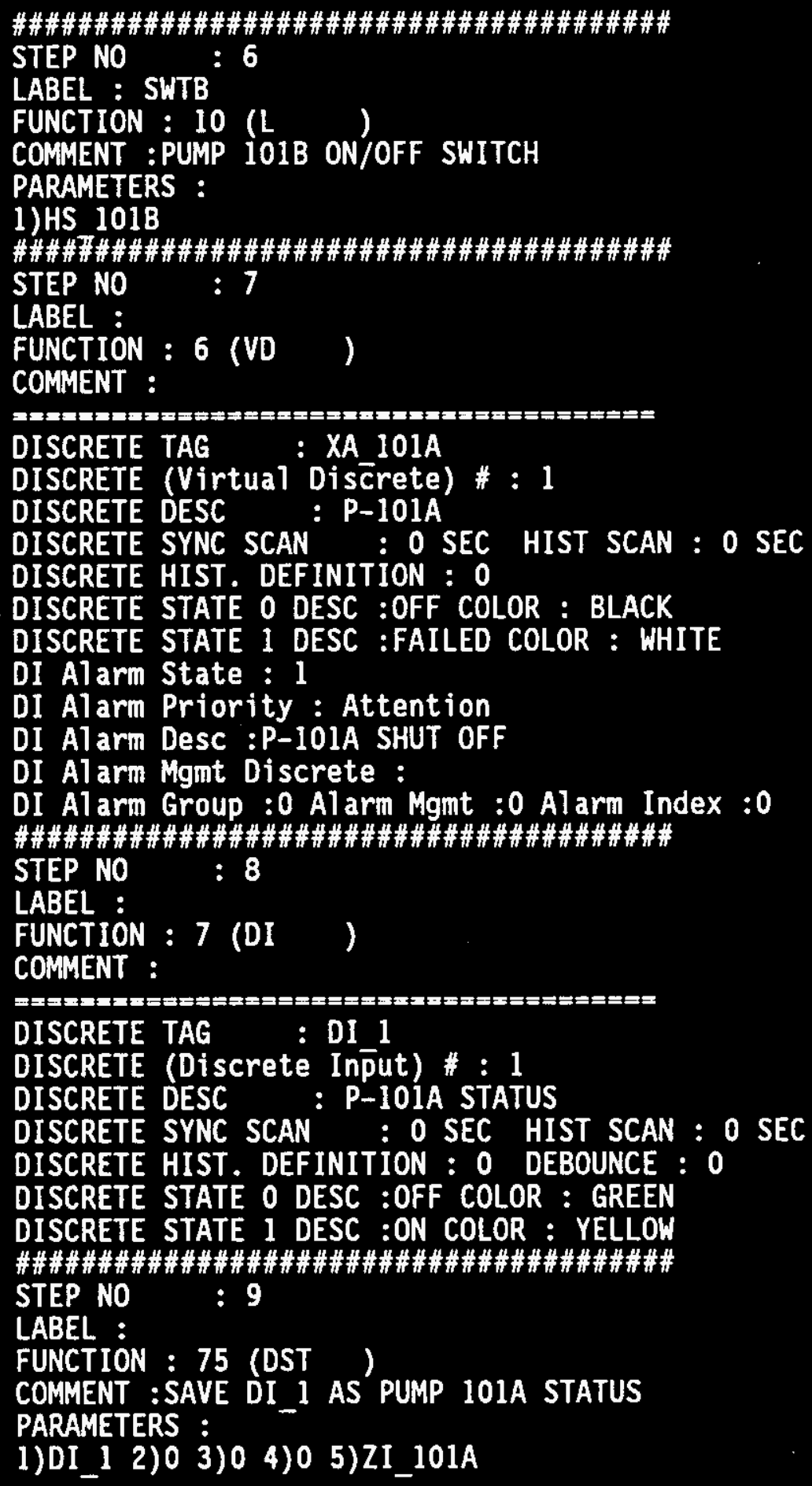


\#\#\#\#\#\#\#\#\#\#\#\#\#\#\#\#\#\#\#\#\#\#\#\#\#\#\#\#\#\#\#\#\#\#\#\#\#\#\#\#

STEP NO : 10

LABEL :

FUNCTION : 57 (DO)

COMMENT :

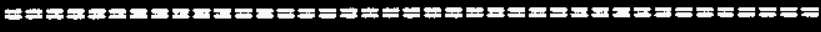

DISCRETE TAG : DO 1

DISCRETE (Discrete Output) \# : 2

DISCRETE DESC : P-101A START COMMAND

DISCRETE SYNC SCAN : O SEC HIST SCAN : O SEC

DISCRETE HIST. DEFINITION : 0 DEBOUNCE : 0

DISCRETE STATE 0 DESC :OFF COLOR : BLACK

DISCRETE STATE 1 DESC :START COLOR : YELLOW

\#\#\#\#\#\#\#\#\#\#\#\#\#\#\#\#\#\#\#\#\#\#\#\#\#\#\#\#\#\#\#\#\#\#\#\#\#\#\#

STEP NO : 11

LABEL :

FUNCTION : $57(\mathrm{DO})$

COMMENT :

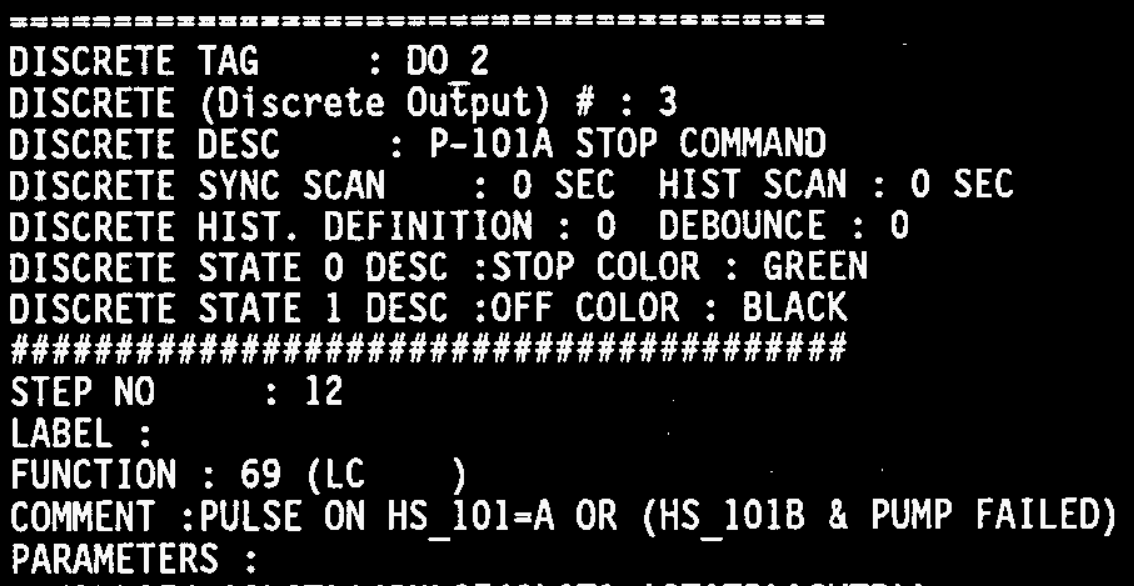

1) (PULSE $(-!$ SLCT) (PULSE(SLCT\&-! STATB)\&SWTB))

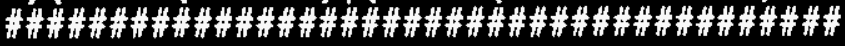

STEP NO : 13

LABEL :

FUNCTION : 62 (GTD )

COMMENT : JUMP NEXT STEP IF NO PULSE

PARAMETERS :

1) IMSV 2) Normal 3) JUMPA

\#\#\#\#\#\#\#\#\#\#\#\#\#\#\#\#\#\#\#\#\#\#\#\#\#\#\#\#\#\#\#\#\#\#\#\#\#\#\#

STEP NO : 14

LABEL : JUMPA

FUNCTION : $10(\mathrm{~L}$

COMMENT :TURN PUMP ON WHEN HS 101 "ON" INTERLOCK ACTIVE

PARAMETERS :

1) HS_101A 2) 1 


\section{WHC-SD-CP-CSWD-016 \\ Volume 2 \\ Revision 1 \\ Page 69}

\#\#\#\#\#\#\#\#\#\#\#\#\#\#\#\#\#\#\#\#\#\#\#\#\#\#\#\#\#\#\#\#\#\#\#\#\#\#\#\#

STEP NO

$: 15$

LABEL :

FUNCTION : 69 (LC )

COMMENT : SEND PULSE WHEN HS_101="P-101B" (ON)

PARAMETERS :

1) PULSE (SLCT)

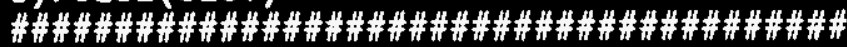

STEP NO : 16

LABEL :

FUNCTION : 62 (GTD )

COMMENT : JUMP NEXT LINE WHEN NO PULSE

PARAMETERS :

1) !MSV 2) Normal 3) JUMPB

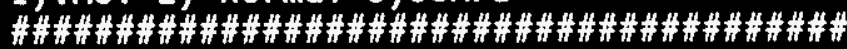

STEP NO : 17

LABEL : JUMPB

FUNCTION : 10 (L) ) WHEN HS 101 "OFF" INTERLOCK ACTIVE
COMMENT :TURN OFF PUMP WHEN

PARAMETERS :

1) HS 101A 2) 0

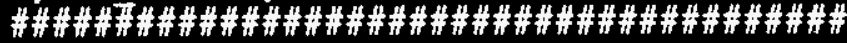

STEP NO : 18

LABEL :

FUNCTION : 69 (LC

COMMENT :LO INTERLOCK (ONLY WHEN BYPASS IS OFF)

PARAMETERS :

1)-1BYPASS\&LOLVL

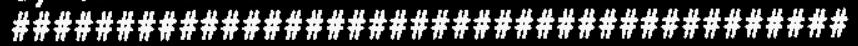

STEP NO : 19

LABEL :

FUNCTION : 62 (GTD )

COMMENT :JUMP NEXT STEP IF NO LOW LEVEL ALARM

PARAMETERS :

1) IMSV 2) Normal 3) JMPLO

\#\#\#\#\#\#\#\#\#\#\#\#\#\#\#\#\#\#\#\#\#\#\#\#\#\#\#\#\#\#\#\#\#\#

STEP NO

LABEL : JMPLO

FUNCTION : 10 (L

COMMENT : PUMP OFF ON LO LEVEL INTERLOCK

PARAMETERS :

1) HS 101A 2) 0

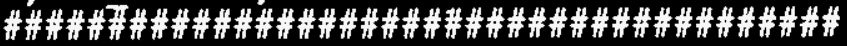

STEP NO : 21

LABEL :

FUNCTION : 69 (LC )

COMMENT : PULSE ON HI LEVEL ALARM WHEN HS 101='A'

PARAMETERS :

1) PULSE(HILVL\&-!SLCT) 


\section{WHC-SD-CP-CSWD-016 \\ Volume 2 \\ Revision 1 \\ Page 70}

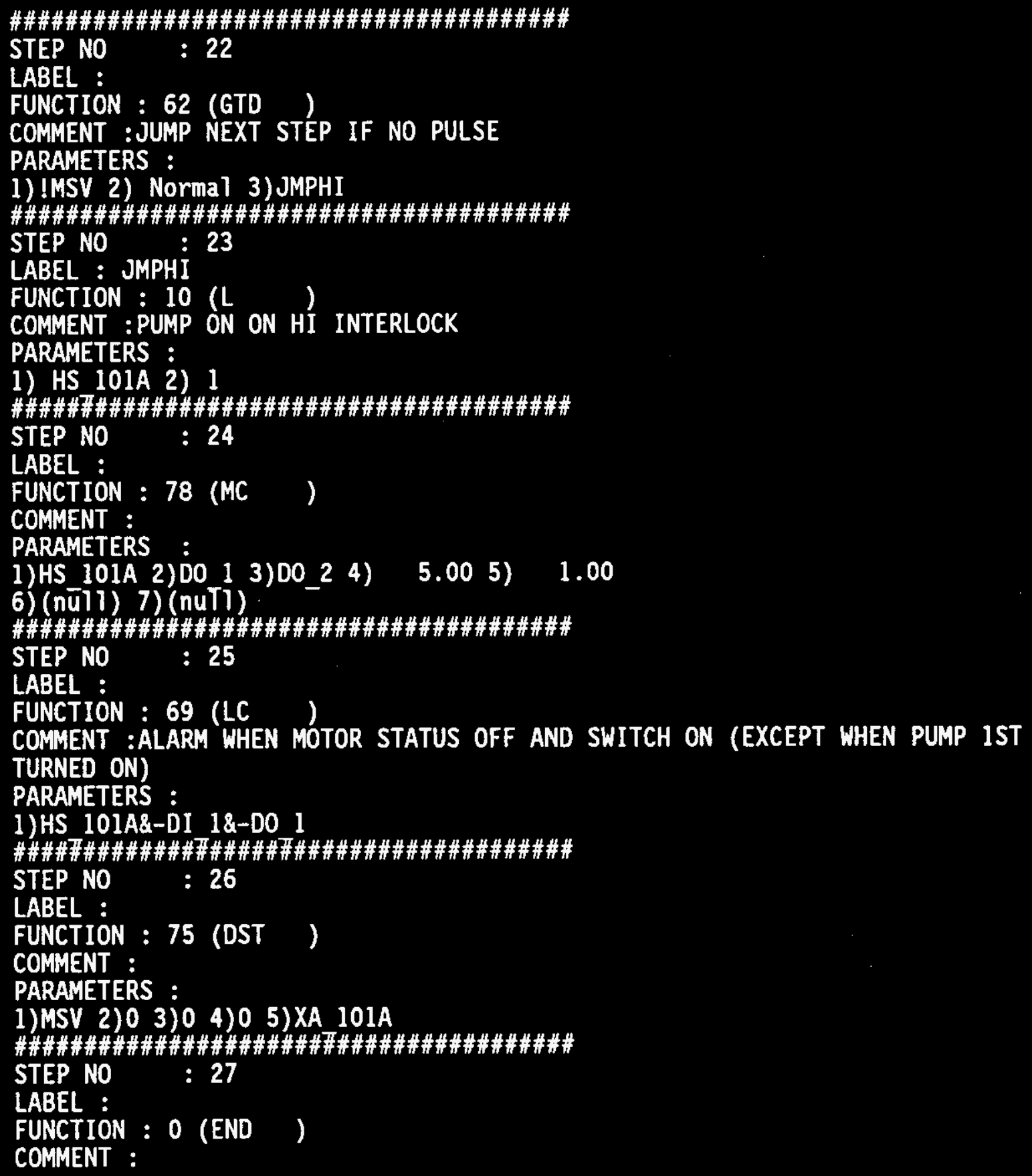




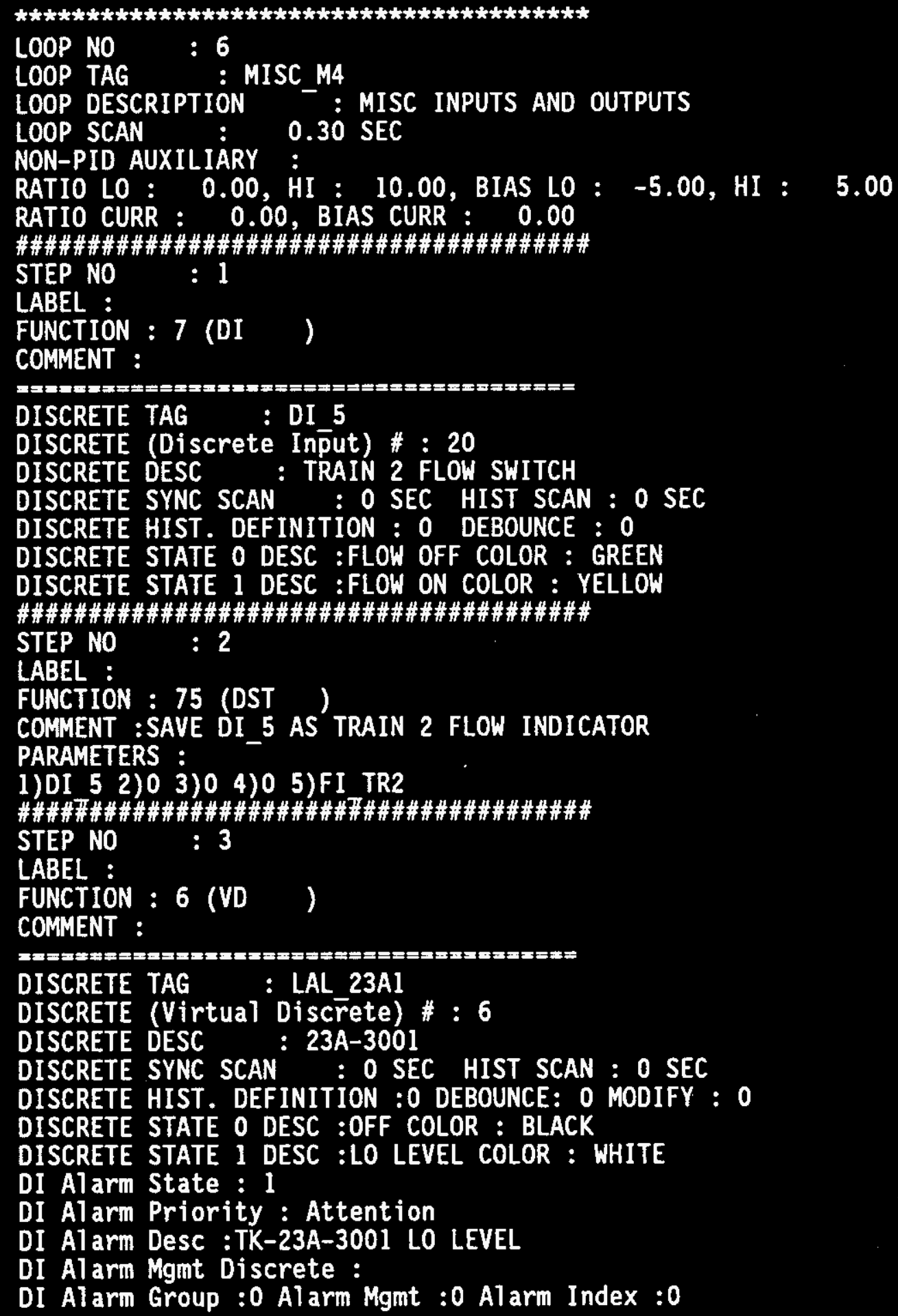


WHC-SD-CP-CSWD-016

Volume 2

Revision 1

Page 72

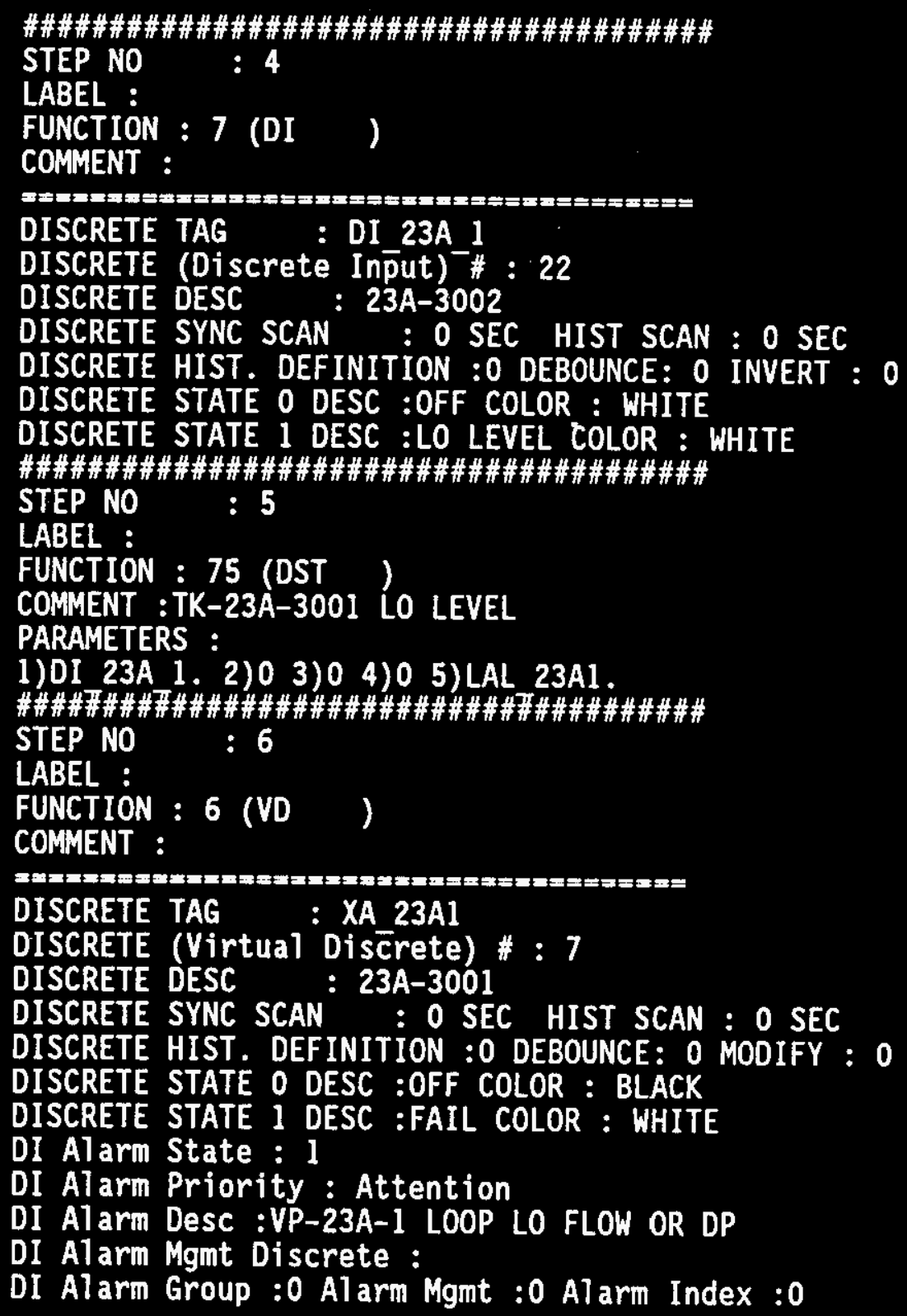


WHC-SD-CP-CSWD-016

Volume 2

Revision 1

Page 73

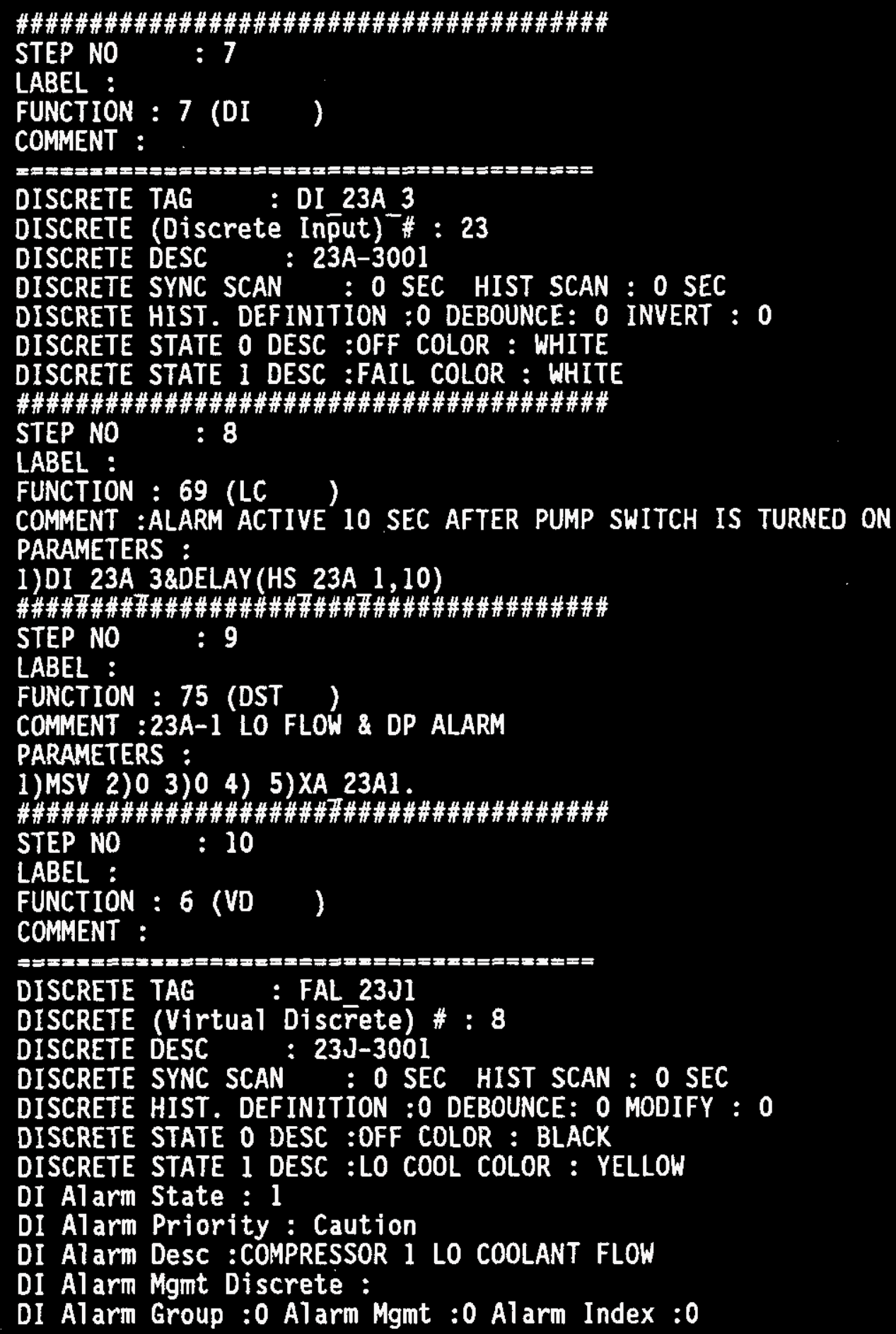


WHC-SD-CP-CSWD-016

Volume 2

Revision 1

Page 74

\#\#\#\#\#\#\#\#\#\#\#\#\#\#\#\#\#\#\#\#\#\#\#\#\#\#\#\#\#\#\#\#\#

STEP NO : 11

LABEL :

FUNCTION : 7 (DI )

COMMENT :

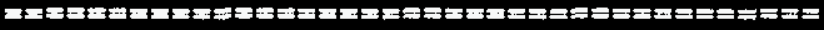

DISCRETE TAG : DI 23J 1

DISCRETE (Discrete Input) ${ }^{-\#}: 24$

DISCRETE DESC : COMP 1 LO FLOW

DISCRETE SYNC SCAN : O SEC HIST SCAN : O SEC

DISCRETE HIST. DEFINITION :0 DEBOUNCE: 0 INVERT : 0

DISCRETE STATE O DESC :OFF COLOR : WHITE

DISCRETE STATE 1 DESC :LO COOL COLOR : WHITE

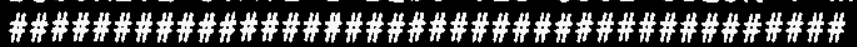

STEP NO

$: 12$

LABEL :

FUNCTION : 75 (DST

COMMENT :COMPRESSOR 1 LO FLOW

PARAMETERS :

1)DI 23J 1. 2)0 3)0 4)0 5)FAL 23J1.

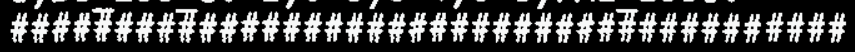

STEP NO : 13

LABEL :

FUNCTION : 0 (END)

COMMENT :

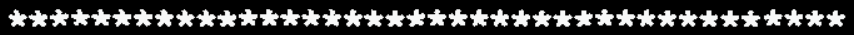

LOOP NO : 7

LOOP TAG : VP_23A_1

LOOP DESCRIPTION - $\quad$ P-2006-23A

LOOP SCAN : 0.30 SEC

NON-PID AUXILIARY :

RATIO LO: $0.00, \mathrm{HI}: 10.00$, BIAS LO : $-5.00, \mathrm{HI}: 5.00$

RATIO CURR : 0.00 , BIAS CURR : 0.00

EXPORT :VPI FAIL

IMPORT DI :WTF_2_2::!VP2_FAIL,WTF_2_3::!HS_23A 
WHC-SD-CP-CSWD-016

Volume 2

Revision 1

Page 75

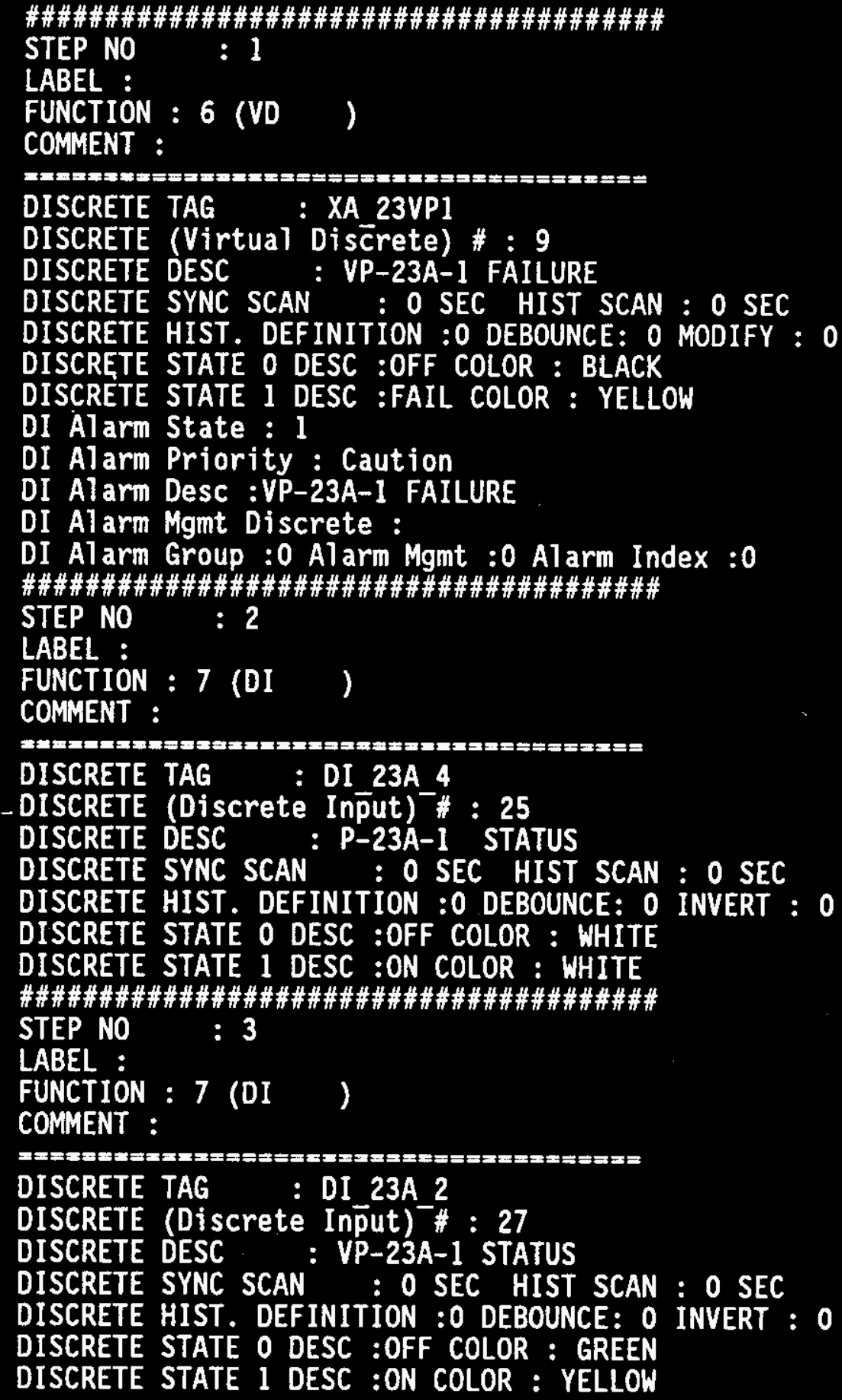


WHC-SD-CP-CSWD-016

Volume 2

Revision 1

Page 76

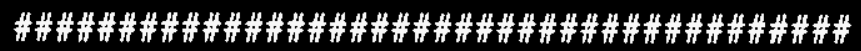

STEP NO : 4

LABEL :

FUNCTION : 57 (DO )

COMMENT :

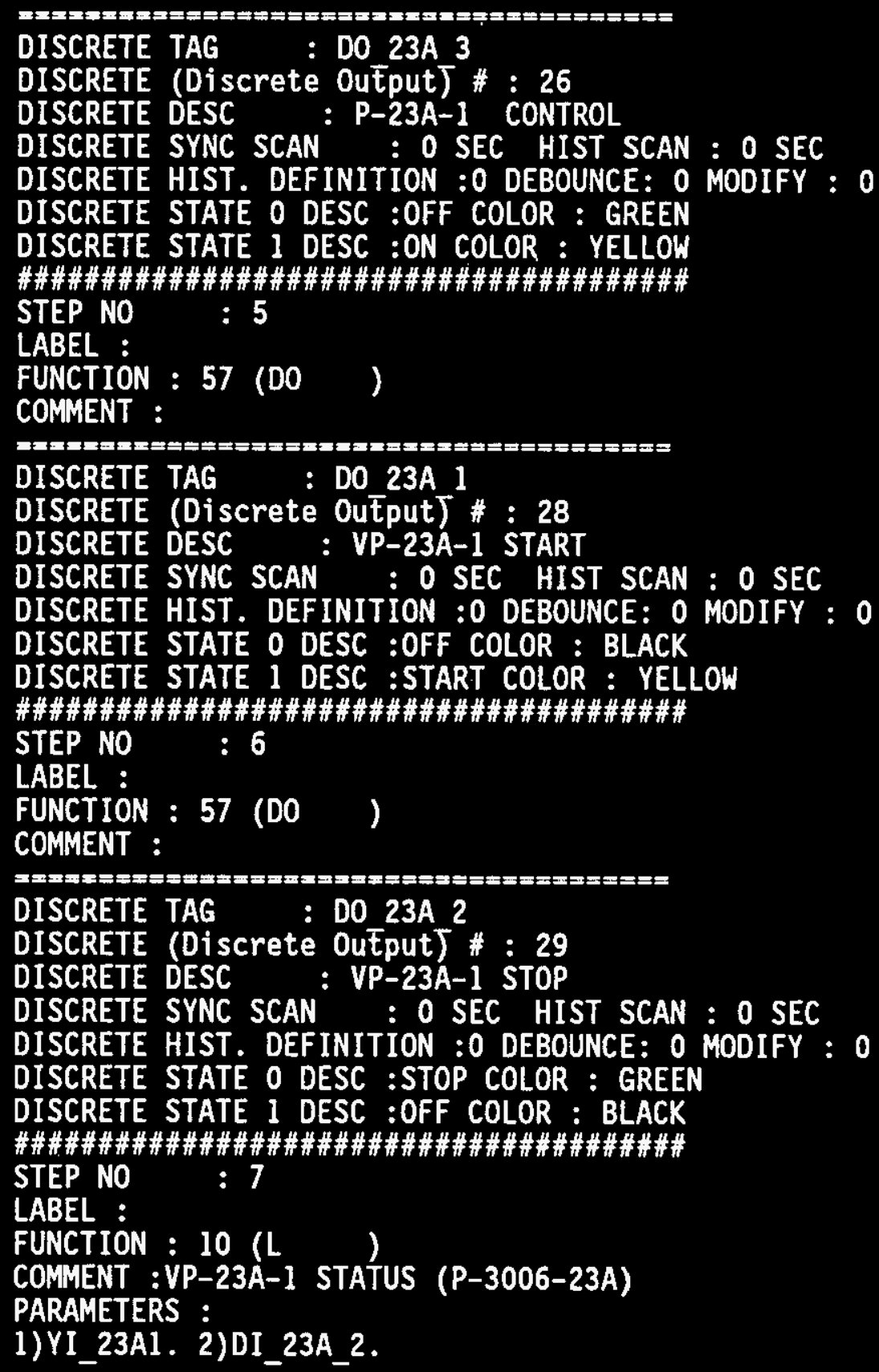


\#\#\#\#\#\#\#\#\#\#\#\#\#\#\#\#\#\#\#\#\#\#\#\#\#\#\#\#\#\#\#\#\#\#\#\#\#\#\#\#

STEP NO : 8

LABEL :

FUNCTION : $10(\mathrm{~L}$

COMMENT : $\mathrm{P}-23 A-3001$ STATUS

PARAMETERS :

1)YI P23A1. 2)DI $23 A 4$.

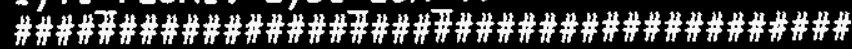

STEP NO : 9

LABEL :

FUNCTION : 69 (LC) )

COMMENT : " 0 " PULSE ON HS 23A=VP2 AND FAILURE ALARM

PARAMETERS :

1)-! PULSE(HS 23A\&VP2 FAIL)

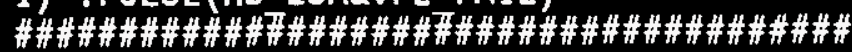

STEP NO : 10

LABEL :

FUNCTION : 62 (GTD)

COMMENT : JUMP IF NO PULSE

PARAMETERS :

1)MSV 2) off-Nomal 3) JMPON

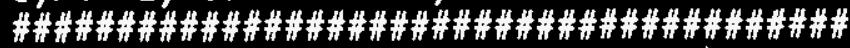

STEP NO

LABEL : JMPON

FUNCTION : 10 (L

COMMENT : TURN VP1 ON IF VP2 HAS FAILED

PARAMETERS :

1)HS 23A 1.2) 1

\#\#\#\#罗\#\#\#罗\#\#\#\#\#\#\#\#\#\#\#\#\#\#\#\#\#\#\#\#\#\#\#\#\#\#\#\#\#\#\#

STEP NO

: 12

LABEL :

FUNCTION : 73 (LAT)

COMMENT : UNLATCH PUMP OUTPUT IF NO STATUS AFTER 5 SEC

PARAMETERS :

1) LATCH $=$ HS 23A 1.2) UNLATCH $=(-Y I$ P23A18DELAY (HS_3)0 4)D0_23A_3. 5)0

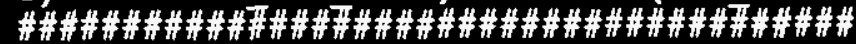

STEP NO : 13

LABEL :

FUNCTION : 69 (LC )

COMMENT :FAILURE ON LO FLOW \& DP ALARM OR VP1 FAIL

PARAMETERS :

1) XA 23A1 \XA 23VP1

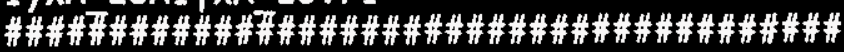

STEP NO : 14

LABEL :

FUNCTION : 75 (DST)

COMMENT :

PARAMETERS :
1)MSV 2)0 3)0
4) 5)VP1_FAIL. 


\section{WHC-SD-CP-CSWD-016 \\ Volume 2 \\ Revision 1 \\ Page 78}

\#\#\#\#\#\#\#\#\#\#\#\#\#\#\#\#\#\#\#\#\#\#\#\#\#\#\#\#\#\#\#\#\#\#\#\#\#\#\#\#

STEP NO : 15

LABEL : VPON

FUNCTION : 69 (LC )

COMMENT :START VAC PUMP 15 SEC AFTER CIRC PUMP IF NO ALARM

PARAMETERS :

1)DELAY(YI P23A1, 15)\&-DI 23A 3\&HS 23A 1

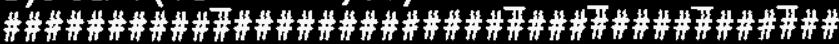

STEP NO : 16

LABEL :

FUNCTION : 78 (MC )

COMMENT :

PARAMETERS :

1)MSV 2)DO 23A 1. 3)DO 23A_2. 4) 10.00 5) 1.00

6) (nu11) 7J(nuT1) 8) (nū11) -9)(nu11) 10)(nu11)

11) (nu11)

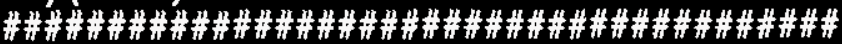

STEP NO

$: 17$

LABEL :

FUNCTION : 69 (LC

COMMENT :ALARM ON VP STATUS OFF AND SHITCH ON (EXCEPT STARTUP)

PARAMETERS :

1) VPON\&-YI 23A1\&-DO 23A 1

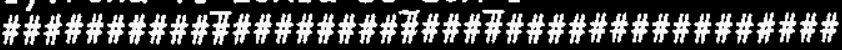

STEP NO : 18

LABEL :

FUNCTION : $10(\mathrm{~L} \quad)$

COMMENT :

PARAMETERS :

1)XA 23VP1. 2)MSV

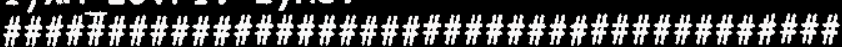

STEP NO : 19

LABEL :

FUNCTION : 0 (END)

COMMENT : 
WHC-SD-CP-CSWD-016

Volume 2

Revision 1

Page 79

VTF_2_2.mic5_ro

MICON TYPE :RCM-D

MICON UNIT NO : 5

MICON TAG : WTF_2 2

MICON DESCRIPTION :- $243-Z$ RACK 2 SLOT 2

MICON CONFIGURATION FILE : mic5_rO

POINT ALLOCATION :

VAI : 32, VDI : 32, ISW : 32, LOOPS : 12 , MSD : 16 ,

AI : $0, A 0: 0, D I: 32,00: 32$

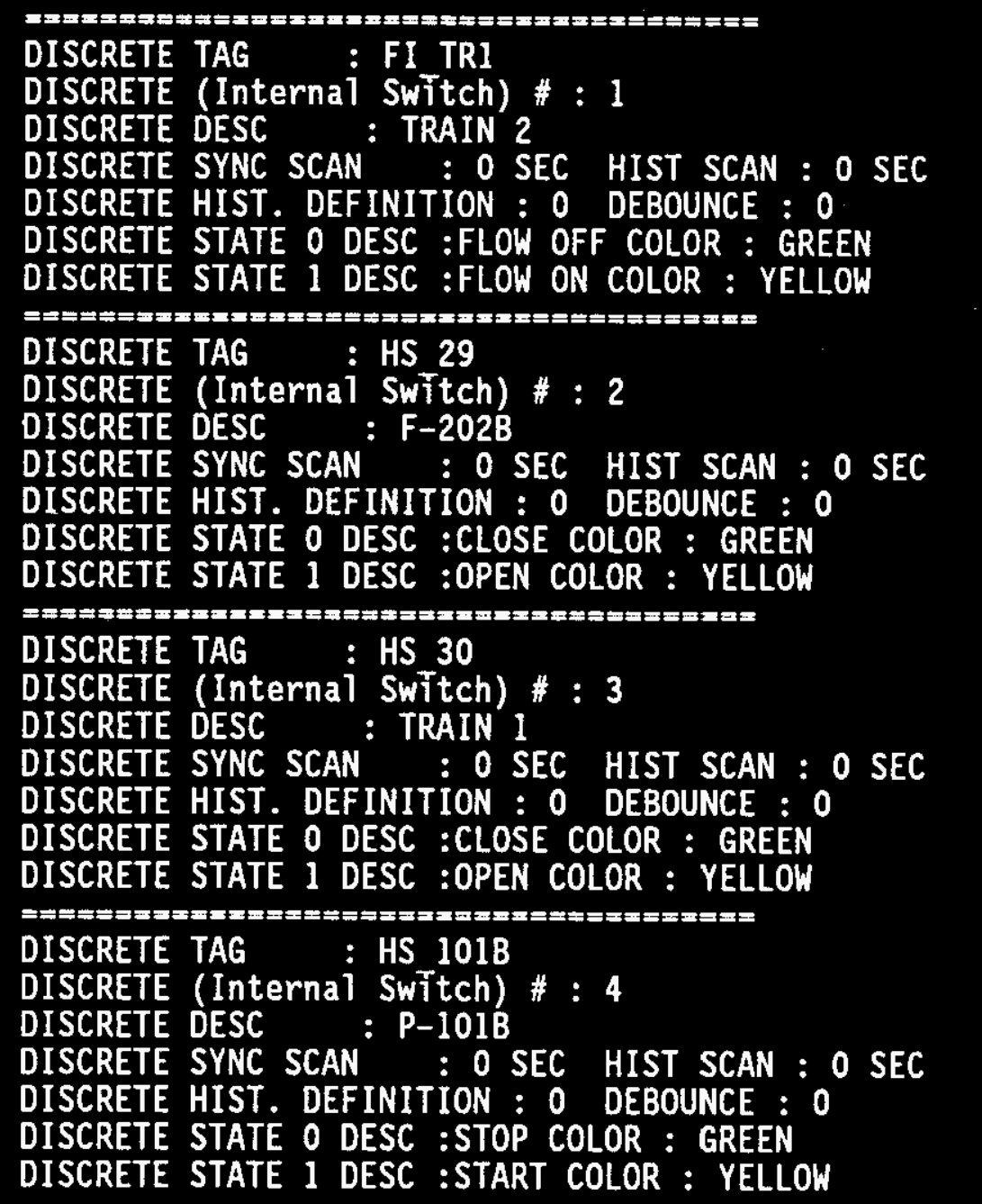


WHC-SD-CP-CSWD-016

Volume 2

Revision 1

Page 80

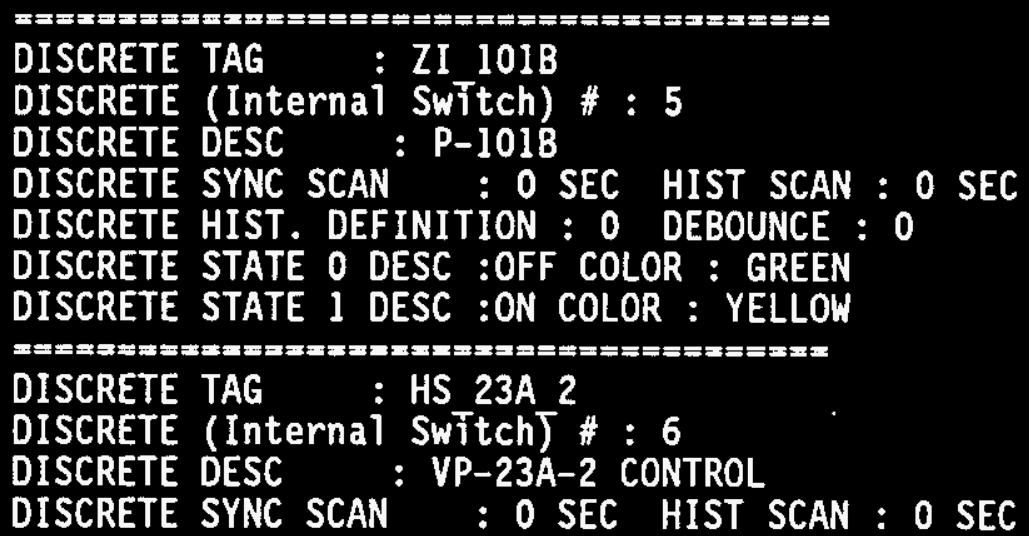

DISCRETE HIST. DEFINITION :0 DEBOUNCE: 0 MODIFY : 1

DISCRETE STATE O DESC :STOP COLOR : GREEN

DISCRETE STATE 1 DESC :START COLOR : YELLOW

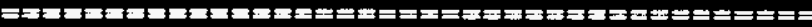

DISCRETE TAG : YI 23A2

DISCRETE (Internal Switch) \#: 7

DISCRETE DESC : VP-23A-2 STATUS

DISCRETE SYNC SCAN : O SEC HIST SCAN : O SEC

DISCRETE HIST. DEFINITION :0 DEBOUNCE: 0 MODIFY : 0

DISCRETE STATE O DESC :OFF COLOR : GREEN

DISCRETE STATE 1 DESC :ON COLOR : YELLOW

;

DISCRETE TAG : YI P23A2

DISCRETE (Internal Switch) \#: 8

DISCRETE DESC : P-23A-2 STATUS

DISCRETE SYNC SCAN : O SEC HIST SCAN : O SEC

DISCRETE HIST. DEFINITION :0 DEBOUNCE: 0 MODIFY : 0

DISCRETE STATE O DESC :OFF COLOR : GREEN

DISCRETE STATE 1 DESC :ON COLOR : YELLOW

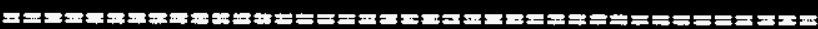

DISCRETE TAG : VP2 FAIL

DISCRETE (Internal Switch) \#: 9

DISCRETE DESC : VP-23A-2 FAILURE

DISCRETE SYNC SCAN : O SEC HIST SCAN : O SEC

DISCRETE HIST. DEFINITION :0 DEBOUNCE: 0 MODIFY : 0

DISCRETE STATE O DESC :OFF COLOR : WHITE

DISCRETE STATE 1 DESC :FAIL COLOR : WHITE

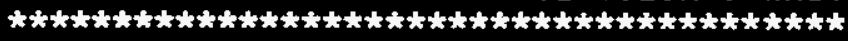

LOOP NO : 1

LOOP TAG : MOV2

LOOP DESCRIPTION : F-202B INLET

LOOP SCAN : 0.30 SEC

NON-PID AUXILIARY :

RATIO LO : 0.00 , HI : 10.00 , BIAS LO : -5.00 , HI : 5.00

RATIO CURR : 0.00 , BIAS CURR : 0.00 
WHC-SD-CP-CSWD-016

Volume 2

Revision 1

Page 81

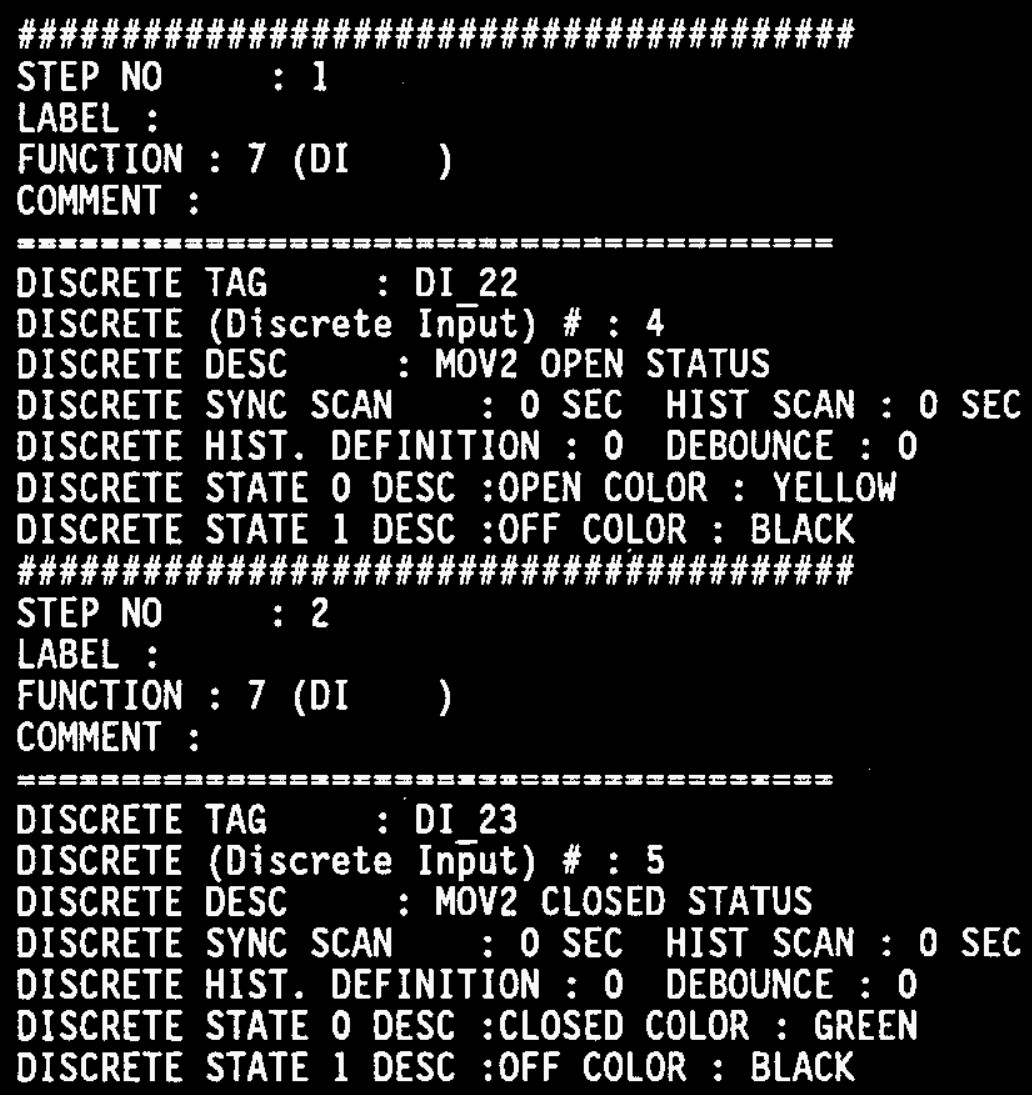




\title{
WHC-SD-CP-CSWD-016 \\ Volume 2 \\ Revision 1 \\ Page 82
}

\author{
\#\#\#\#\#\#\#\#\#\#\#\#\#\#\#\#\#\#\#\#\#\#\#\#\#\#\#\#\#\#\#\#\#\#\#\#\#\#\# \\ STEP NO : 3 \\ LABEL : \\ FUNCTION : 9 (DSCC) \\ MULTI-STATE DI TAG : ZI_MOV2 \\ MULTI-STATE DI \#: 1 \\ MULTI-STATE DI DESC : MOV2 F-202B INLET \\ MULTI-STATE DI SYNC SCAN : 0 \\ MULTI-STATE DI1 : DI 23 \\ MULTI-STATE DI2 : DI_22 \\ MULTI-STATE DI3 : \\ MULTI-STATE DI4: \\ MULTI-STATE STATE 0 :DESC FAILED COLOR White \\ MULTI-STATE STATE 1 :DESC OPEN COLOR Yellow \\ MULTI-STATE STATE 2 :DESC CLOSED COLOR Green \\ MULTI-STATE STATE 3 :DESC MOVING COLOR white \\ MULTI-STATE STATE 4 :DESC COLOR BTack \\ MULTI-STATE STATE 5 :DESC COLOR Black \\ MULTI-STATE STATE 6 :DESC COLOR B1ack \\ MULTI-STATE STATE 7 :DESC COLOR Black \\ MULTI-STATE STATE 8 :DESC COLOR Black \\ MULTI-STATE STATE 9 :DESC COLOR Black \\ MULTI-STATE STATE 10 :DESC COLOR Black \\ MULTI-STATE STATE 11 :DESC COLOR B1ack \\ MULTI-STATE STATE 12 :DESC COLOR Black \\ MULTI-STATE STATE 13 :OESC COLOR B1ack \\ MULTI-STATE STATE 14 :DESC COLOR B1ack \\ MULTI-STATE STATE 15 :OESC COLOR Black \\ COMMENT :
}


WHC-SD-CP-CSWD-016

Volume 2

Revision 1

Page 83

\#\#\#\#\#\#\#\#\#\#\#\#\#\#\#\#\#\#\#\#\#\#\#\#\#\#\#\#\#\#\#\#\#\#\#\#\#\#\#\#

STEP NO : 4

LABEL :

FUNCTION : $57(00)$

COMMENT :

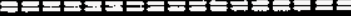

DISCRETE TAG : DO 20

DISCRETE (Discrete Output) \#: 6

DISCRETE DESC : MOV2 OPEN COMMAND

DISCRETE SYNC SCAN : O SEC HIST SCAN : O SEC

DISCRETE HIST. DEFINITION : 0 DEBOUNCE : 0

DISCRETE STATE $O$ DESC :OFF COLOR : BLACK

DISCRETE STATE 1 DESC :OPEN COLOR : YELLOW

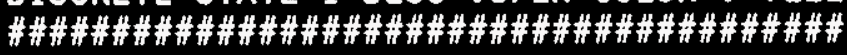

STEP NO : 5

LABEL :

FUNCTION : $57(00)$

COMMENT :

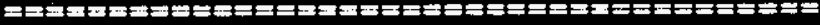

DISCRETE TAG : DO 21

DISCRETE (Discrete Output) \#: 7

DISCRETE DESC : MOV2 CLOSE COMMAND

DISCRETE SYNC SCAN : O SEC HIST SCAN : O SEC

DISCRETE HIST. DEFINITION : 0 DEBOUNCE : 0

DISCRETE STATE O DESC :OFF COLOR : BLACK

DISCRETE STATE 1 DESC :CLOSE COLOR : GREEN

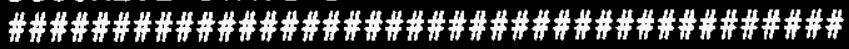

STEP NO : 6

LABEL :

FUNCTION : 6 (VD)

COMMENT :

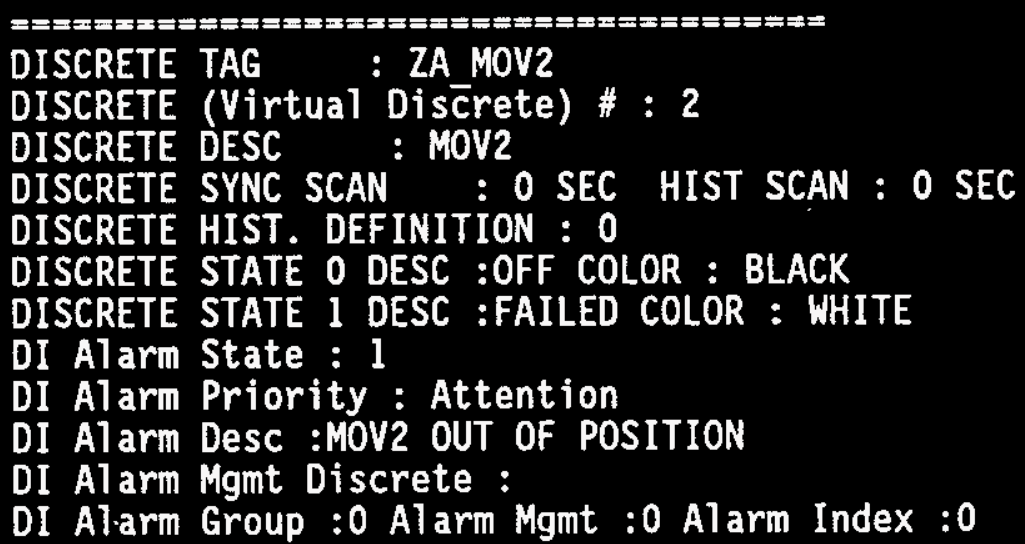



WHC-SD-CP-CSWD-016
Volume 2
Revision 1
Page 84

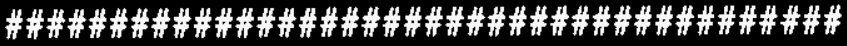

STEP NO : 7

LABEL :

FUNCTION : 68 (VC )

COMMENT :

PARAMETERS :

1)HS_29. 2)DO 20. 3)30.00 4)00 21. 5) 30.00

6) DI 23. 7) DI 22. 8) ZA MOV2. 9) 40.00

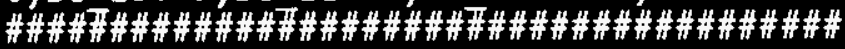

STEP NO : 8

LABEL :

FUNCTION : 0 (END)

COMMENT :

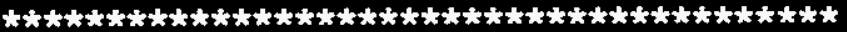

LOOP NO : 2
LOOP TAG : MOV4
LOOP DESCRIPTION $\quad:$ F-202B OUTLET
LOOP SCAN : 0.30 SEC

NON-PID AUXILIARY :

RATIO LO: 0.00 , HI : 10.00 , BIAS LO: -5.00 , HI : 5.00

RATIO CURR : 0.00 , BIAS CURR : 0.00

\#\#\#\#\#\#\#\#\#\#\#\#\#\#\#\#\#\#\#\#\#\#\#\#\#\#\#\#\#\#\#\#\#\#\#\#\#\#\#\#

STEP NO : 1

LABEL :

FUNCTION : 7 (DI )

COMMENT :

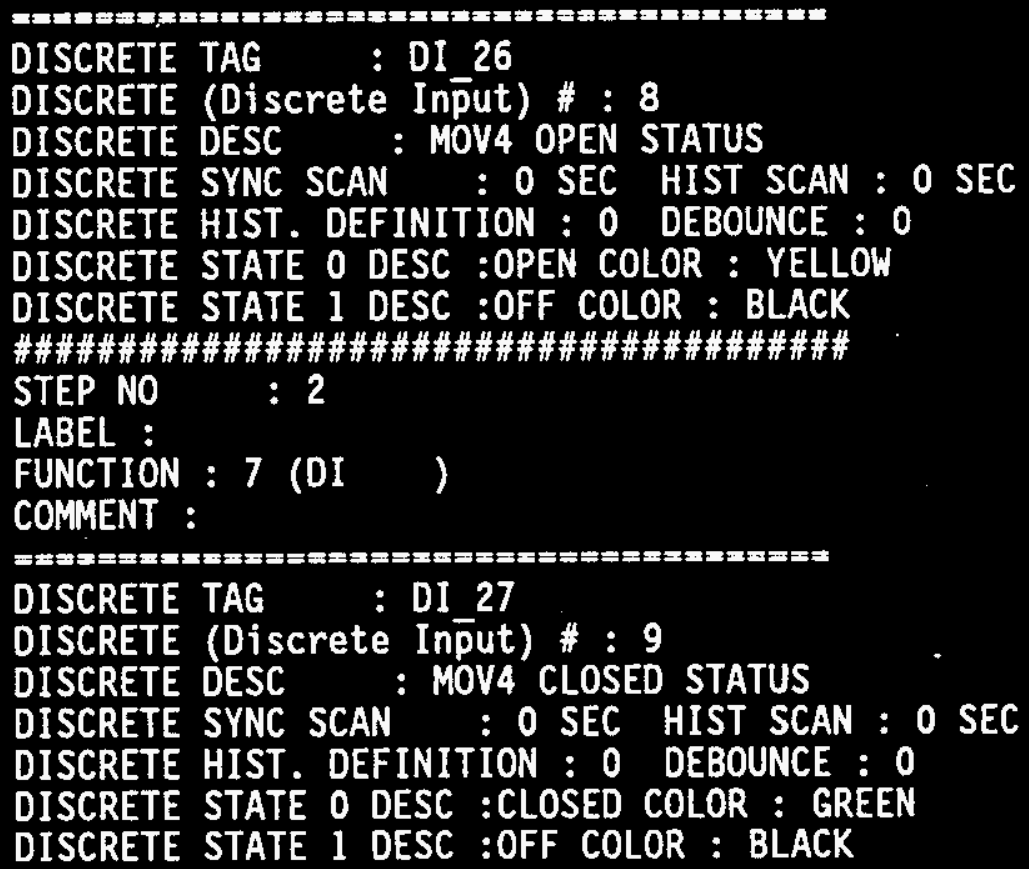




\title{
WHC-SD-CP-CSWD-016 \\ Volume 2 \\ Revision 1 \\ Page 85
}

\author{
\#\#\#\#\#\#\#\#\#\#\#\#\#\#\#\#\#\#\#\#\#\#\#\#\#\#\#\#\#\#\#\#\#\#\#\#\#\#\#\# \\ STEP NO : 3 \\ LABEL : \\ FUNCTION : 9 (DSCC )

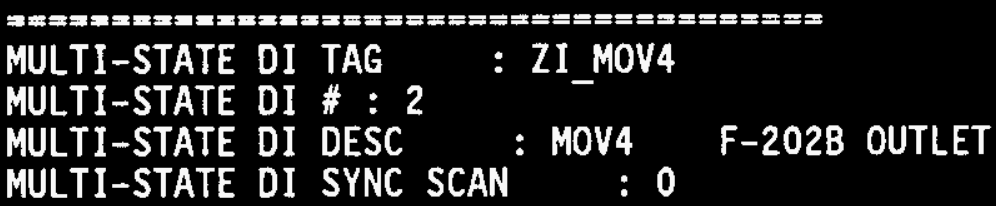 \\ MULTI-STATE DII : DI 27 \\ MULTI-STATE DI2 : DI_26 \\ MULTI-STATE DI3 : \\ MULTI-STATE DI4 : \\ MULTI-STATE STATE 0 :DESC FAILED COLOR White \\ MULTI-STATE STATE 1 :DESC OPEN COLOR Yellow \\ MULTI-STATE STATE 2 :DESC CLOSED COLOR Green \\ MULTI-STATE STATE 3 :DESC MOVING COLOR White \\ MULTI-STATE STATE 4 :DESC COLOR Black \\ MULTI-STATE STATE 5 :DESC COLOR Black \\ MULTI-STATE STATE 6 :DESC COLOR Black \\ MULTI-STATE STATE 7 :DESC COLOR Black \\ MULTI-STATE STATE 8 :DESC COLOR Black \\ MULTI-STATE STATE 9 :DESC COLOR B1ack \\ MULTI-STATE STATE 10 :DESC COLOR Black \\ MULTI-STATE STATE 11 :DESC COLOR Black \\ MULTI-STATE STATE 12 :DESC COLOR Black \\ MULTI-STATE STATE 13 :DESC COLOR Black \\ MULTI-STATE STATE 14 :DESC COLOR Black \\ MULTI-STATE STATE 15 :DESC COLOR Black \\ COMMENT :

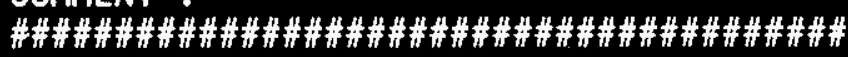 \\ STEP NO : 4 \\ LABEL : \\ FUNCTION : $57(\mathrm{DO})$ \\ COMMENT :

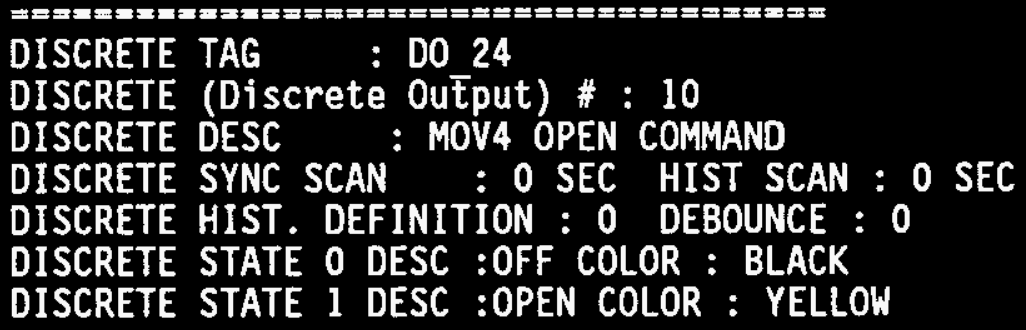




\section{WHC-SD-CP-CSWD-016 \\ Volume 2 \\ Revision 1 \\ Page 86}

\#\#\#\#\#\#\#\#\#\#\#\#\#\#\#\#\#\#\#\#\#\#\#\#\#\#\#\#\#\#\#\#\#\#\#\#\#\#\#\#

STEP NO : 5

LABEL :

FUNCTION : $57(\mathrm{DO})$

CONMENT :

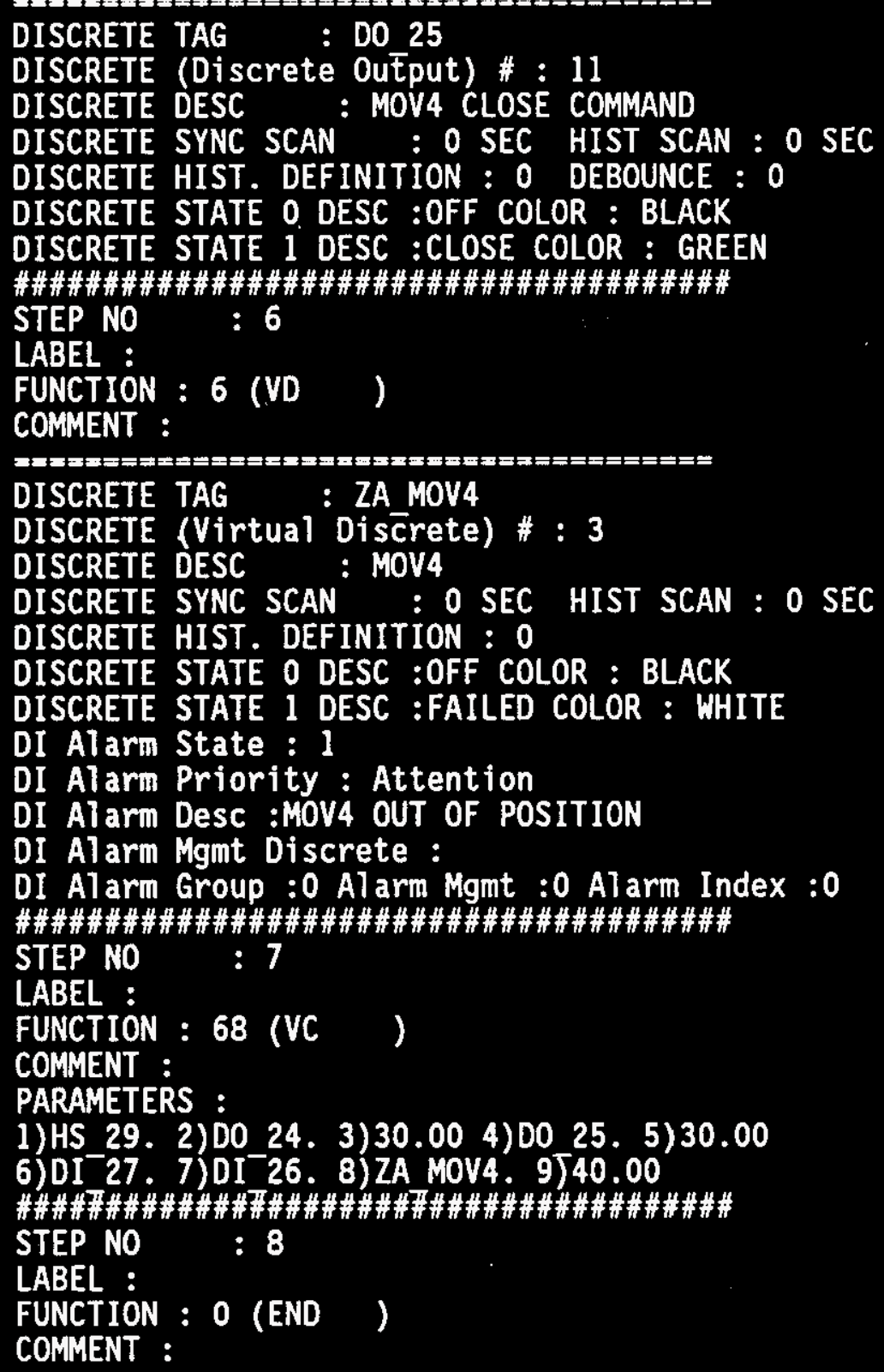


WHC-SD-CP-CSWD-016

Volume 2

Revision 1

Page 87

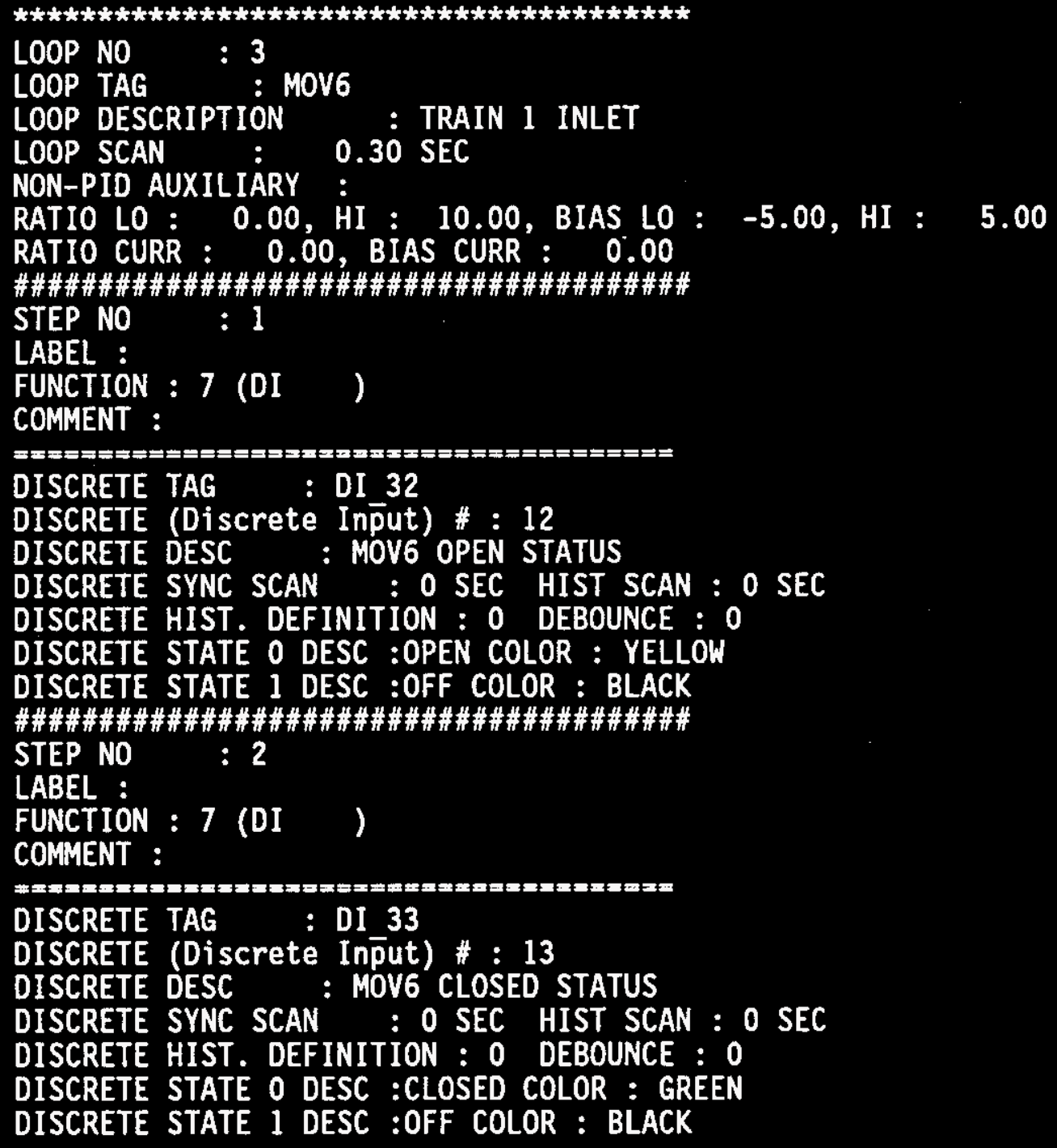


\#\#\#\#\#\#\#\#\#\#\#\#\#\#\#\#\#\#\#\#\#\#\#\#\#\#\#\#\#\#\#\#\#\#

STEP NO : 3

LABEL :

FUNCTION : 9 (DSCC)

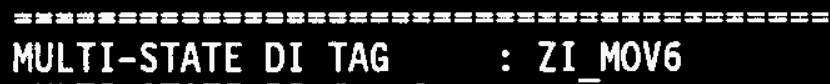

MULTI-STATE DI \#: 3

MULTI-STATE DI DESC : MOV6 TRAIN INLET

MULTI-STATE DI SYNC SCAN : 0

MULTI-STATE DI1 : DI 33

MULTI-STATE DI2 : DI 32

MULTI-STATE DI3 :

MULTI-STATE DI4 :

MULTI-STATE STATE 0 :DESC FAILED COLOR White

MULTI-STATE STATE 1 :DESC OPEN COLOR Yellow

MULTI-STATE STATE 2 :DESC CLOSED COLOR Green

MULTI-STATE STATE 3 :DESC MOVING COLOR White

MULTI-STATE STATE 4 :DESC COLOR Black

MULTI-STATE STATE 5 :DESC COLOR Black

MULTI-STATE STATE 6 :DESC COLOR Black

MULTI-STATE STATE 7 :DESC COLOR Black

MULTI-STATE STATE 8 :DESC COLOR Black

MULTI-STATE STATE 9 :DESC COLOR Black

MULTI-STATE STATE 10 :DESC COLOR Black

MULTI-STATE STATE 11 :DESC COLOR Black

MULTI-STATE STATE 12 :DESC COLOR Black

MULTI-STATE STATE 13 :DESC COLOR Black

MULTI-STATE STATE 14 :DESC COLOR Black

MULTI-STATE STATE 15 :DESC COLOR Black

COMMENT :

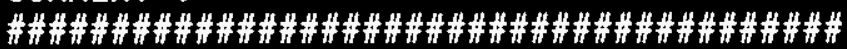

STEP NO : 4

LABEL :

FUNCTION : 57 (DO)

COMMENT :

-

DISCRETE TAG : DO 30

DISCRETE (Discrete Output) \#: 14

DISCRETE DESC : MOV6 OPEN COMMAND

DISCRETE SYNC SCAN : O SEC HIST SCAN : O SEC

DISCRETE HIST. DEFINITION : 0 DEBOUNCE : 0

DISCRETE STATE O DESC :OFF COLOR : BLACK

DISCRETE STATE 1 DESC :OPEN COLOR : YELLON 
WHC-SD-CP-CSWD-016

Volume 2

Revision 1

Page 89

\#\#\#\#\#\#\#\#\#\#\#\#\#\#\#\#\#\#\#\#\#\#\#\#\#\#\#\#\#\#\#\#\#\#\#\#\#\#\#\#

STEP NO : 5

LABEL :

FUNCTION : $57(00)$

COMMENT :

DISCRETE TAG : 0031

DISCRETE (Discrete Output) \#: 15

DISCRETE DESC : MOVG CLOSE COMMAND

DISCRETE SYNC SCAN : O SEC HIST SCAN : O SEC

DISCRETE HIST. DEFINITION : 0 DEBOUNCE : 0

DISCRETE STATE 0 DESC :OFF COLOR : BLACK

DISCRETE STATE 1 DESC :CLOSE COLOR : GREEN

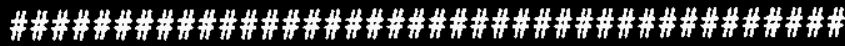

STEP NO : 6

LABEL :

FUNCTION : 6 (VD)

COMMENT :

DISCRETE TAG : ZA MOV6

DISCRETE (Virtual Disçrete) \# : 4

DISCRETE DESC : MOV6

DISCRETE SYNC SCAN : O SEC HIST SCAN : O SEC

DISCRETE HIST. DEFINITION : 0

DISCRETE STATE 0 DESC :OFF COLOR : BLACK

DISCRETE STATE 1 DESC :FAILED COLOR : WHITE

DI Alarm State : 1

DI Alarm Priority : Attention

DI Alarm Desc :MOV6 OUT OF POSITION

DI Alarm Mgmt Discrete :

DI A1 arm Group :0 Alarm Mgmt :0 Alarm Index :0

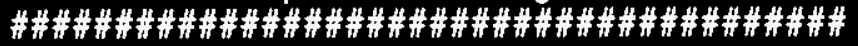

STEP NO : 7

LABEL :

FUNCTION : 68 (VC )

COMMENT :

PARAMETERS :

1)HS 30. 2) DO 30 . 3) 30.00 4)DO 31. 5) 30.00

6) $\mathrm{DI}^{-33}$. 7) DI 32. 8) ZA MOV6. 9) 40.00

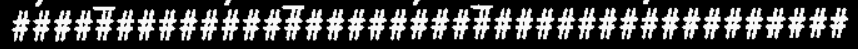

STEP NO : 8

LABEL :

FUNCTION : 0 (END)

COMMENT : 
WHC-SD-CP-CSWD-016

Volume 2

Revision 1

Page 90

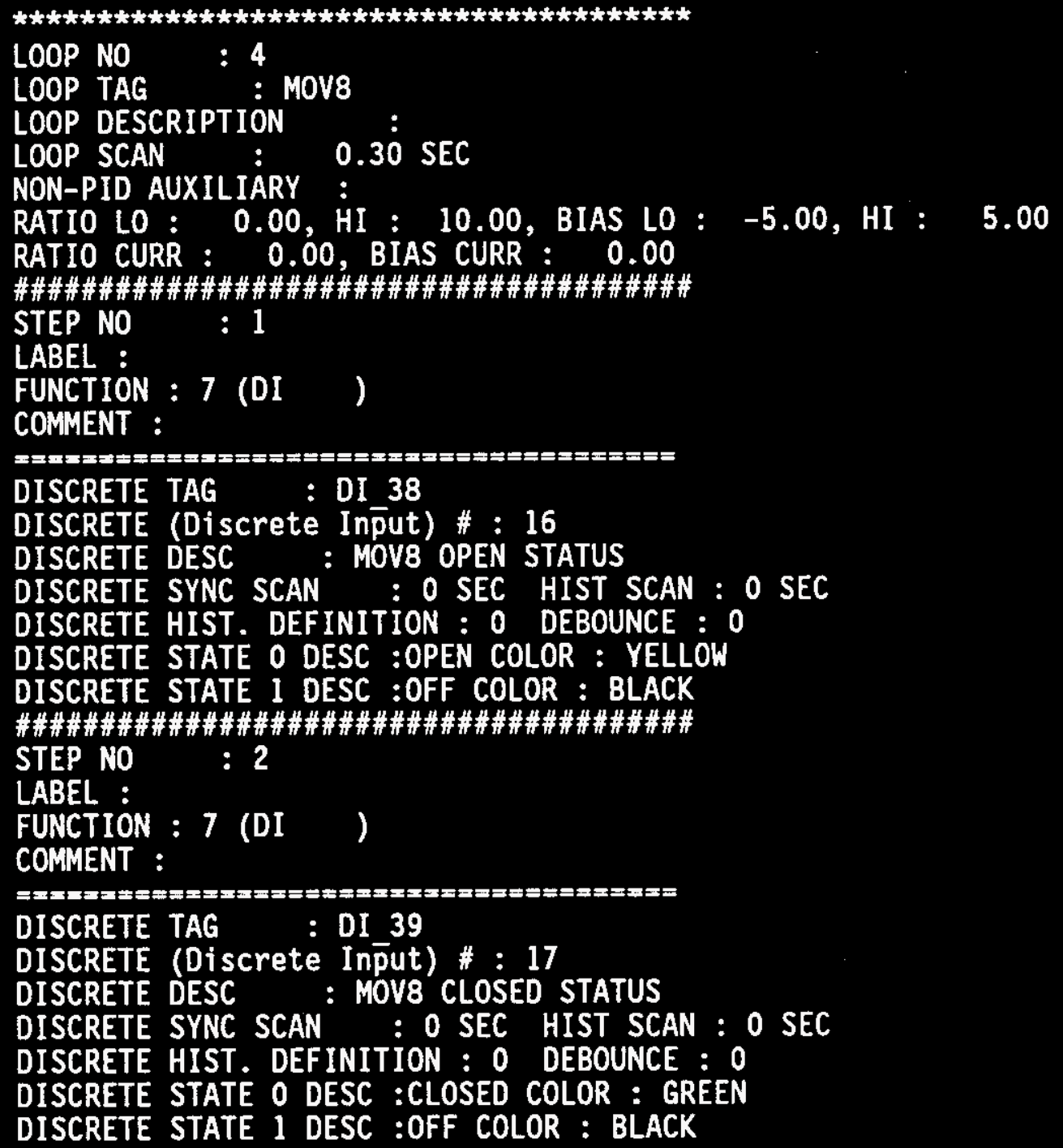




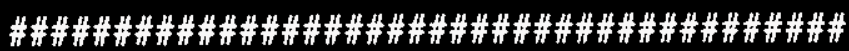

STEP NO : 3

LABEL :

FUNCTION : 9 (DSCC)

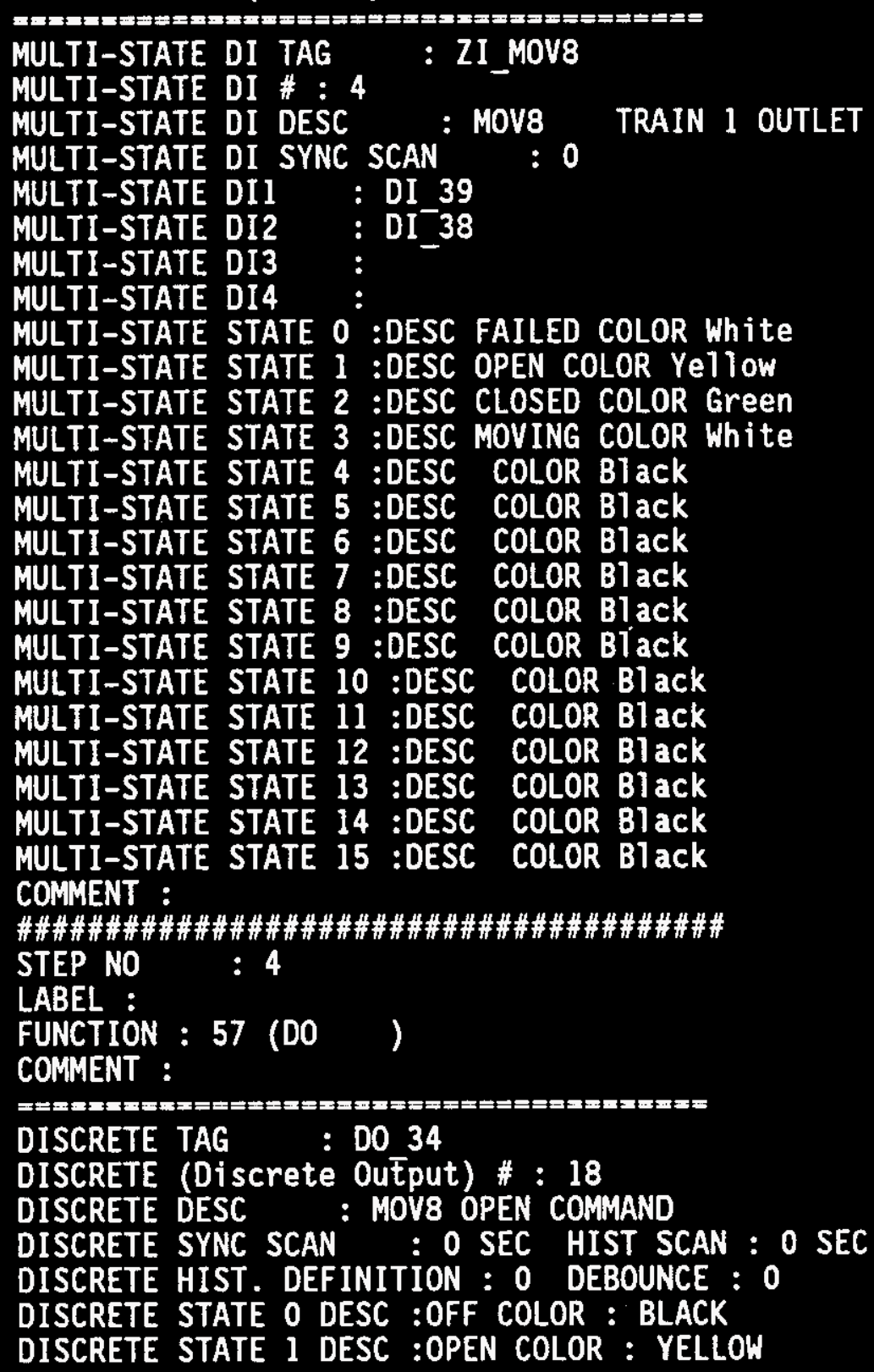


WHC-SD-CP-CSWD-016

Volume 2

Revision 1

Page 92

\#\#\#\#\#\#\#\#\#\#\#\#\#\#\#\#\#\#\#\#\#\#\#\#\#\#\#\#\#\#\#\#\#\#\#\#\#\#\#\#

STEP NO : 5

LABEL :

FUNCTION : $57(\mathrm{DO})$

COMMENT :

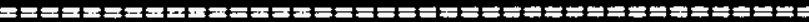

DISCRETE TAG : D0 35

DISCRETE (Discrete Output) \# : 19

DISCRETE DESC : MOVB CLOSE STATUS

DISCRETE SYNC SCAN : O SEC HIST SCAN : O SEC

DISCRETE HIST. DEFINITION : 0 DEBOUNCE : 0

DISCRETE STATE O DESC :OFF COLOR : BLACK

DISCRETE STATE 1 DESC :CLOSE COLOR : GREEN

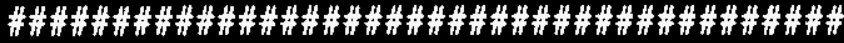

STEP NO : 6

LABEL :

FUNCTION : 6 (VD)

COMMENT :

DISCRETE TAG : ZA MOV8

DISCRETE (Virtual Disçrete) \#: 5

DISCRETE DESC : MOV8

DISCRETE SYNC SCAN : O SEC HIST SCAN : O SEC

DISCRETE HIST. DEFINITION : 0

DISCRETE STATE O DESC :OFF COLOR : BLACK

DISCRETE STATE 1 DESC :FAILED COLOR : WHITE

DI Alarm State : 1

DI Alarm Priority : Attention

DI Alarm Desc :MOV8 OUT OF POSITION

DI Alarm Mgmt Discrete :

DI Alarm Group :0 Alarm Mgmt :0 Alarm Index :0

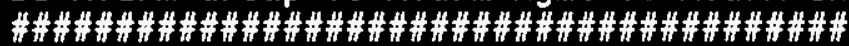

STEP NO : 7

LABEL :

FUNCTION : 68 (VC )

COMMENT :

PARAMETERS :

1)HS_30. 2) DO 34. 3) 30.00 4)DO 35. 5) 30.00

6) $\left.\mathrm{DI}^{-} 39.7\right) \mathrm{DI}^{-} 38$. 8) ZA MOV8. 9广 40.00

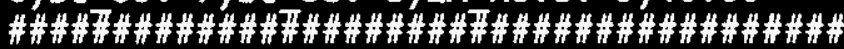

STEP NO : 8

LABEL :

FUNCTION : 0 (END)

COMMENT : 


\section{WHC-SD-CP-CSWD-016 \\ Volume 2 \\ Revision 1 \\ Page 93}

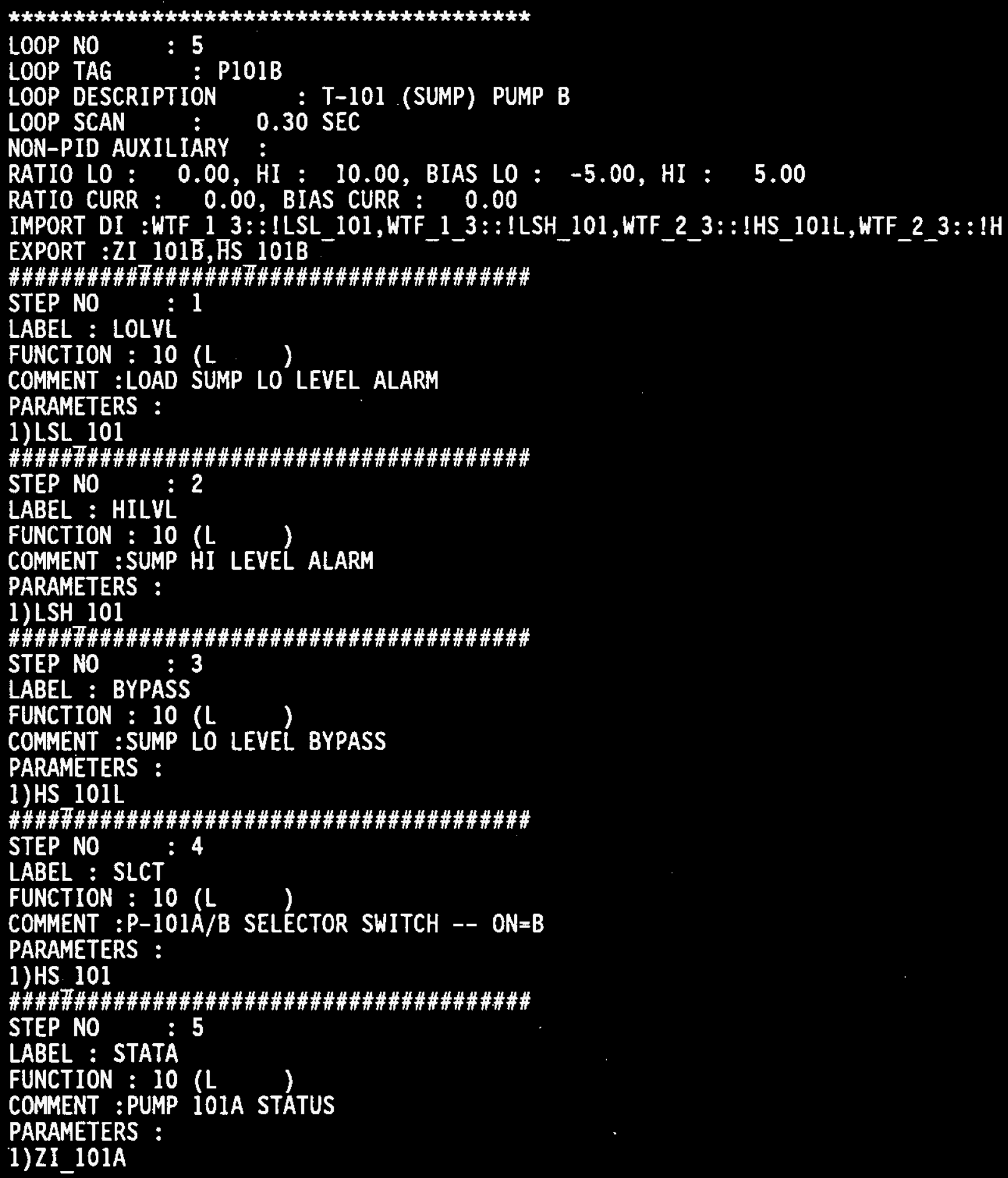




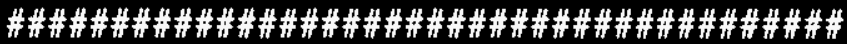

STEP NO : 6

LABEL : SWTA

FUNCTION : 10 (L

COMMENT : PUMP 101A START/STOP SWITCH

PARAMETERS :

1)HS 101A

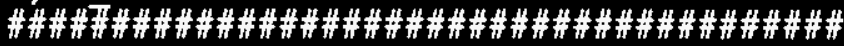

STEP NO $: 7$

LABEL :

FUNCTION : 6 (VD )

COMMENT :

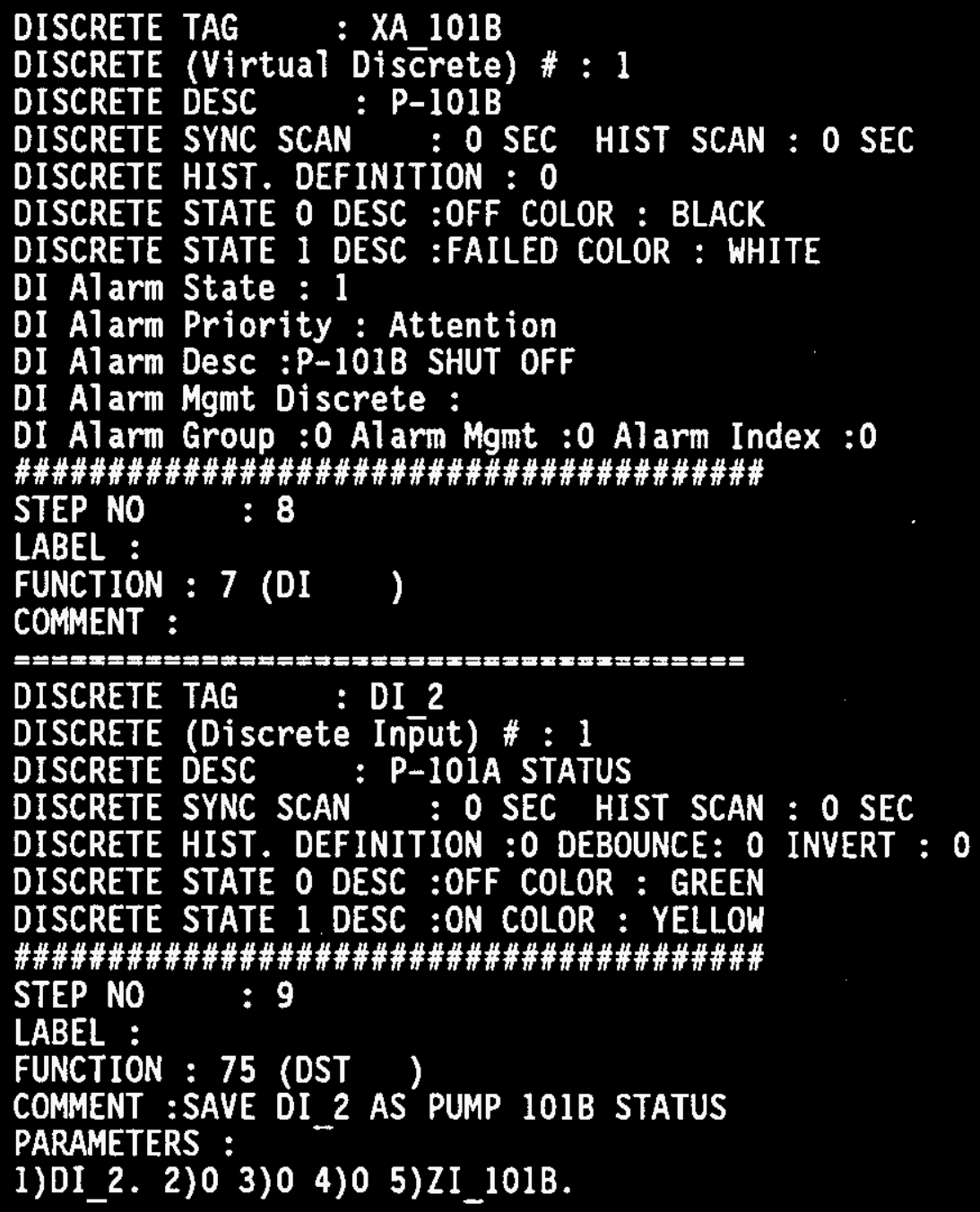




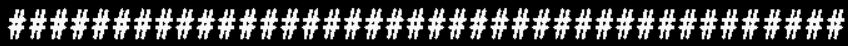

STEP NO : 10

LABEL :

FUNCTION : 57 (DO) )

COMMENT :

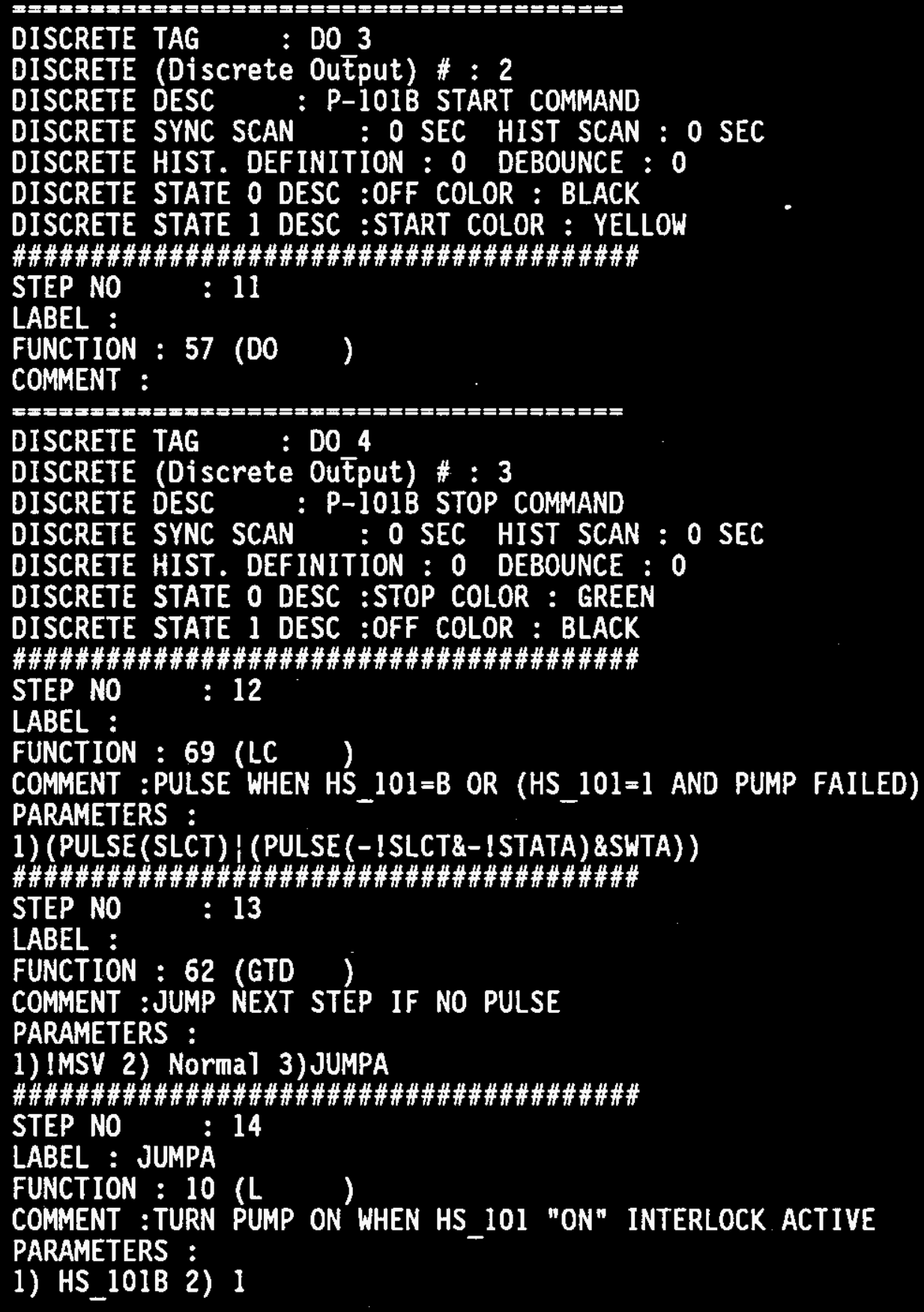
1) HS_101B 2) I 
WHC-SD-CP-CSWD-016

Volume 2

Revision 1

Page 96

\#\#\#\#\#\#\#\#\#\#\#\#\#\#\#\#\#\#\#\#\#\#\#\#\#\#\#\#\#\#\#\#\#\#\#\#\#\#\#

STEP NO : 15

LABEL :

FUNCTION : 69 (LC

COMMENT :SEND PULSE WHEN HS_101="P-101A" (OFF)

PARAMETERS :

1) PULSE (-1SLCT)

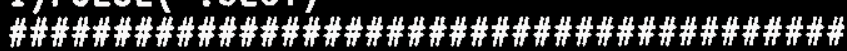

STEP NO : 16

LABEL :

FUNCTION : 62 (GTD )

COMMENT :SKIP NEXT STEP WHEN NO PULSE

PARAMETERS :

1) IMSV 2) Normal 3) JUMPB

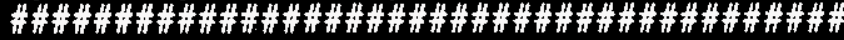

STEP NO : 17

LABEL : JUMPB

FUNCTION : 10 (L

COMMENT :TURN OFF PUMP WHEN HS 101 "OFF" INTERLOCK ACTIVE

PARAMETERS :

1) HS 1018 2) 0

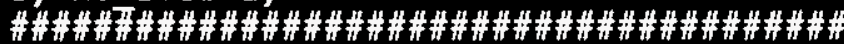

STEP NO : 18

LABEL :

FUNCTION : 69 (LC

COMMENT :LO INTERLOCK (ONLY WHEN BYPASS IS OFF)

PARAMETERS :

1)-IBYPASS\&LOLVL

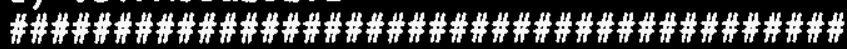

STEP NO : 19

LABEL :

FUNCTION : 62 (GTD )

COMMENT : JUMP NEXT STEP IF NO LOW LEVEL ALARM

PARAMETERS :

1) !MSV 2) Normal 3) JMPLO

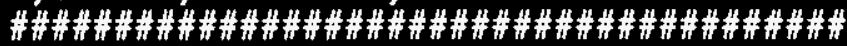

STEP NO

$: 20$

LABEL : JMPLO

FUNCTION : 10 (L

COMMENT : PUMP OFF ON LO LEVEL INTERLOCK

PARAMETERS :

1) HS 101B 2) 0

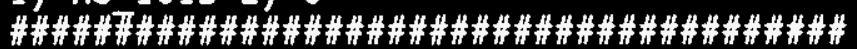

STEP NO : 21

LABEL :

FUNCTION : 69 (LC)

COMMENT : PULSE ON HI LEVEL ALARM \& P 101='B'

PARAMETERS :

1) PULSE(HILVL\&SLCT) 


\section{WHC-SD-CP-CSWD-016 \\ Volume 2 \\ Revision 1 \\ Page 97}

\#\#\#\#\#\#\#\#\#\#\#\#\#\#\#\#\#\#\#\#\#\#\#\#\#\#\#\#\#\#\#\#\#\#\#\#\#\#\#\#

STEP NO : 22

LABEL :

FUNCTION : 62 (GTD )

COMMENT : JUMP NEXT STEP IF NO PULSE

PARAMETERS :

1) !MSV 2) Norma1 3) JMPHI

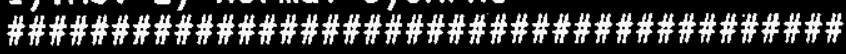

STEP NO : 23

LABEL : JMPHI

FUNCTION : 10 (L

COMMENT : PUMP ON ON HI LEVEL INTERLOCK

PARAMETERS :

1) HS 101B 2) 1

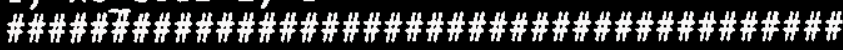

STEP NO : 24

LABEL :

FUNCTION : 78 (MC )

COMMENT :

PARAMETERS :

1)HS_101B 2)DO 3 3)DO_4 4) 5.00 5) 1.00

6) (nü11) 7) (nuT1)

\#\#\#\#\#\#\#\#\#\#\#\#\#\#\#\#\#\#\#\#\#\#\#\#\#\#\#\#\#\#\#\#\#\#\#\#\#\#\#\#

STEP NO : 25

LABEL :

FUNCTION : 69 (LC)

COMMENT : ALARM WHEN MOTOR STAUS OFF AND SHITCH ON (EXCEPT WHEN PUMP 1ST TURNED ON)

PARAMETERS :

1)HS 101B\&-DI 2\&-DO 3

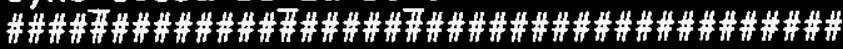

STEP NO : 26

LABEL :

FUNCTION : 75 (DST )

COMMENT :

PARAMETERS :

1)MSV 2) 0 3) 0 4)0 5) XA 101B

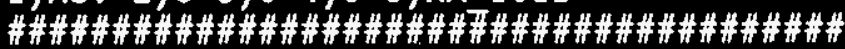

STEP NO : 27

LABEL :

FUNCTION : O (END )

COMMENT : 
WHC-SD-CP-CSWD-016

Volume 2

Revision 1

Page 98

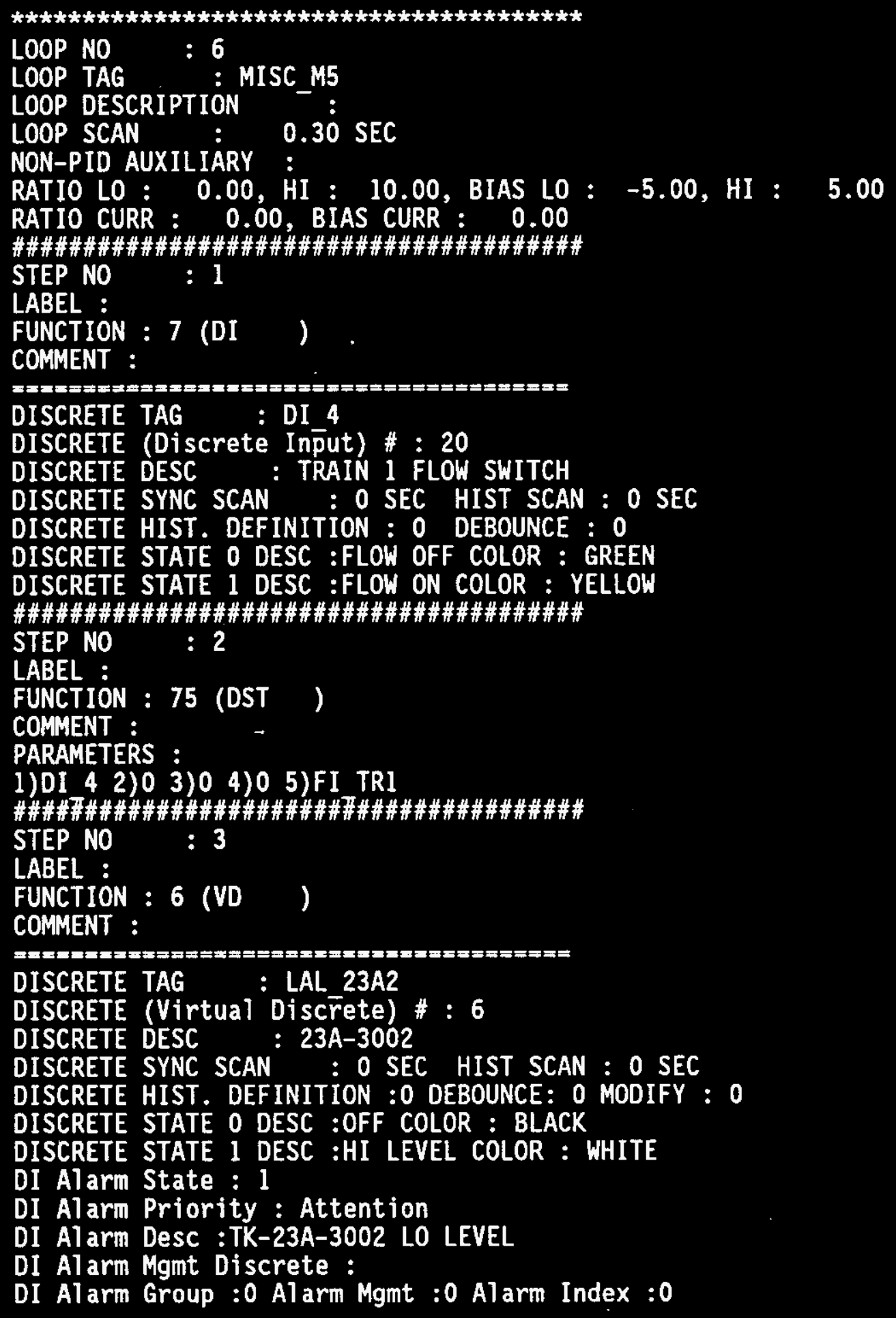




\section{WHC-SD-CP-CSWD-016 \\ Volume 2 \\ Revision 1 \\ Page 99}

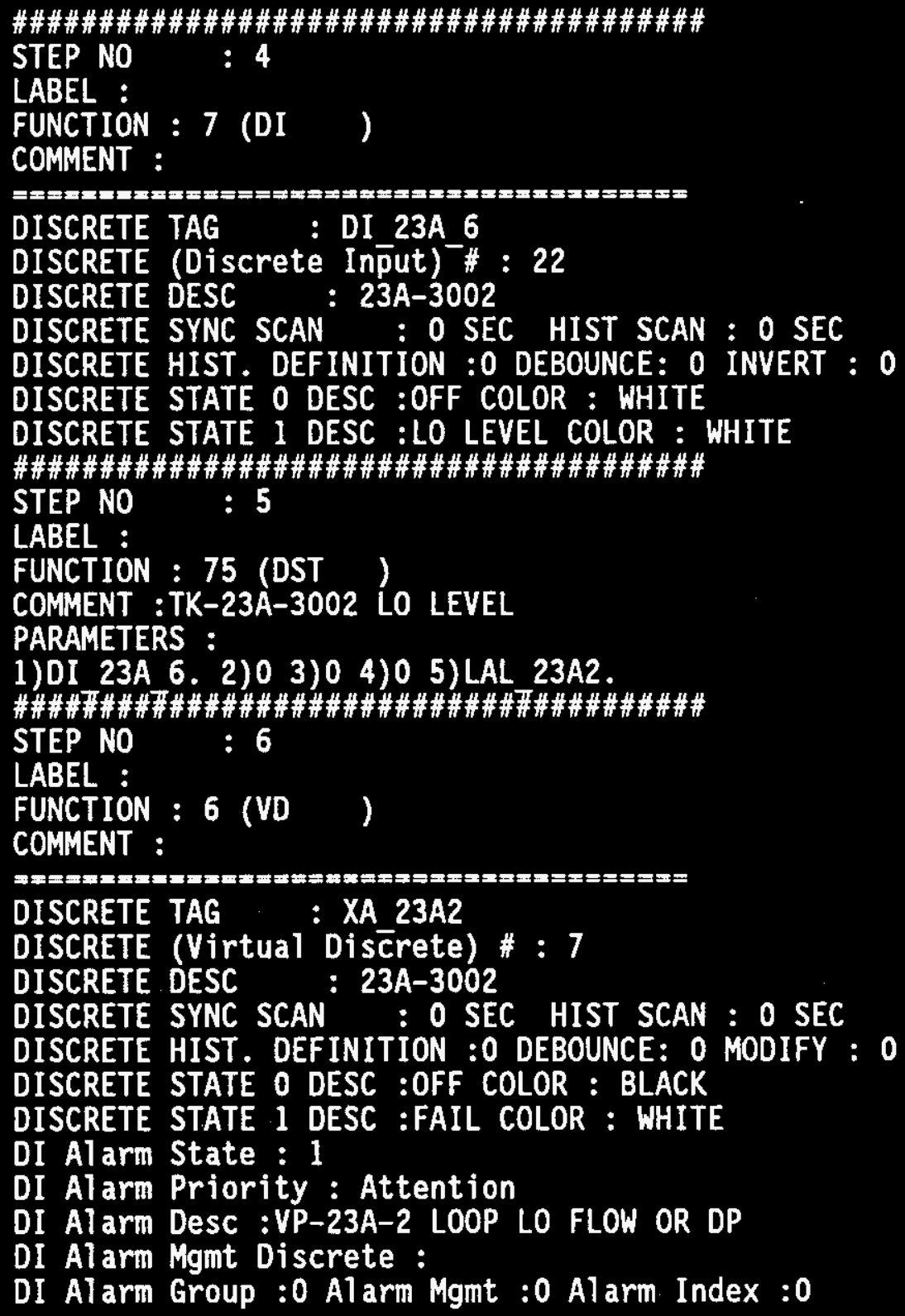


\#\#\#\#\#\#\#\#\#\#\#\#\#\#\#\#\#\#\#\#\#\#\#\#\#\#\#\#\#\#\#\#\#\#\#

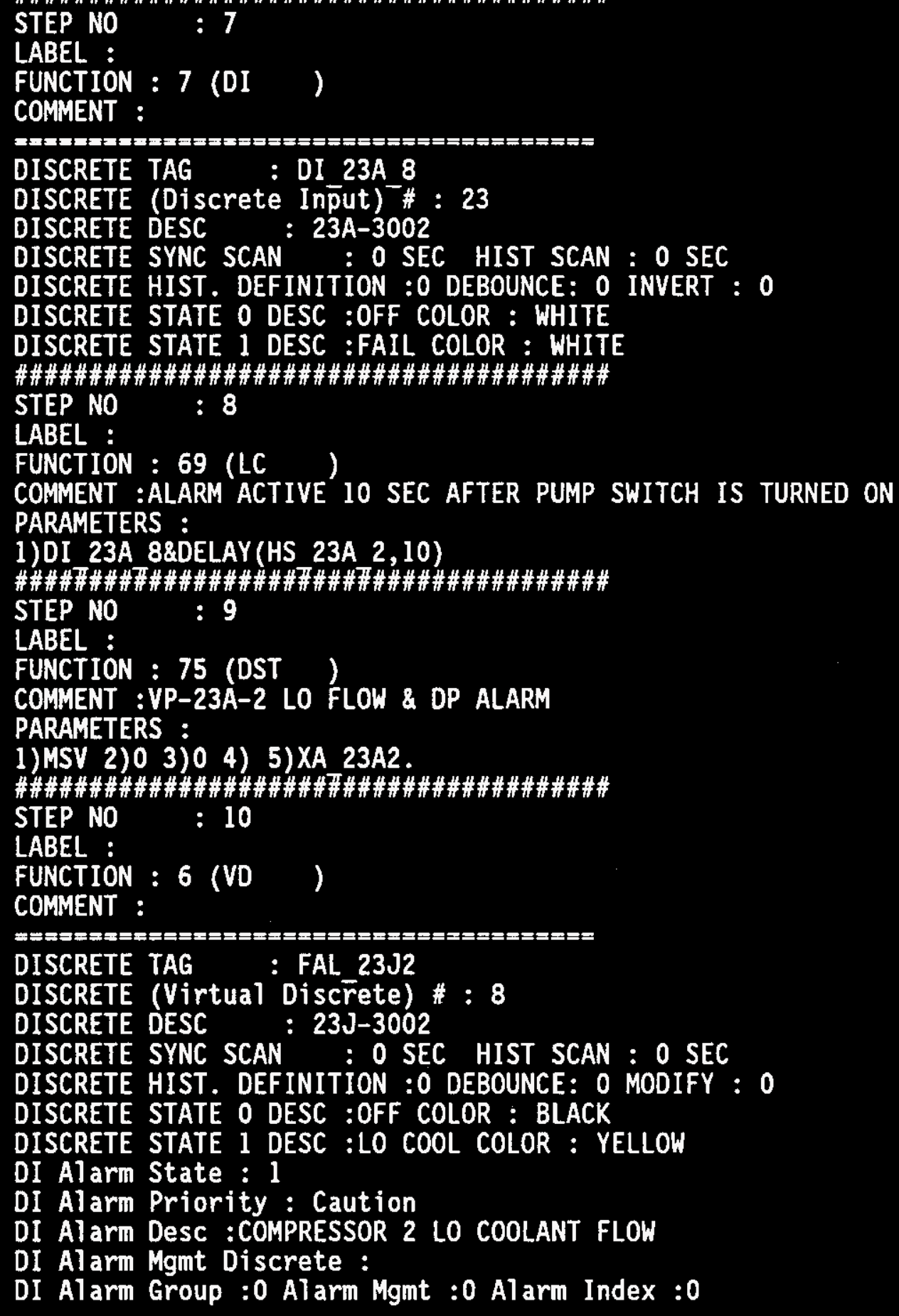


WHC-SD-CP-CSWD-016

Volume 2

Revision 1

Page 101

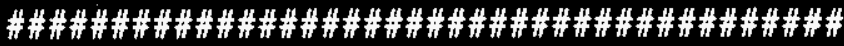

STEP NO : 11

LABEL :

FUNCTION : 7 (DI)

COMMENT :

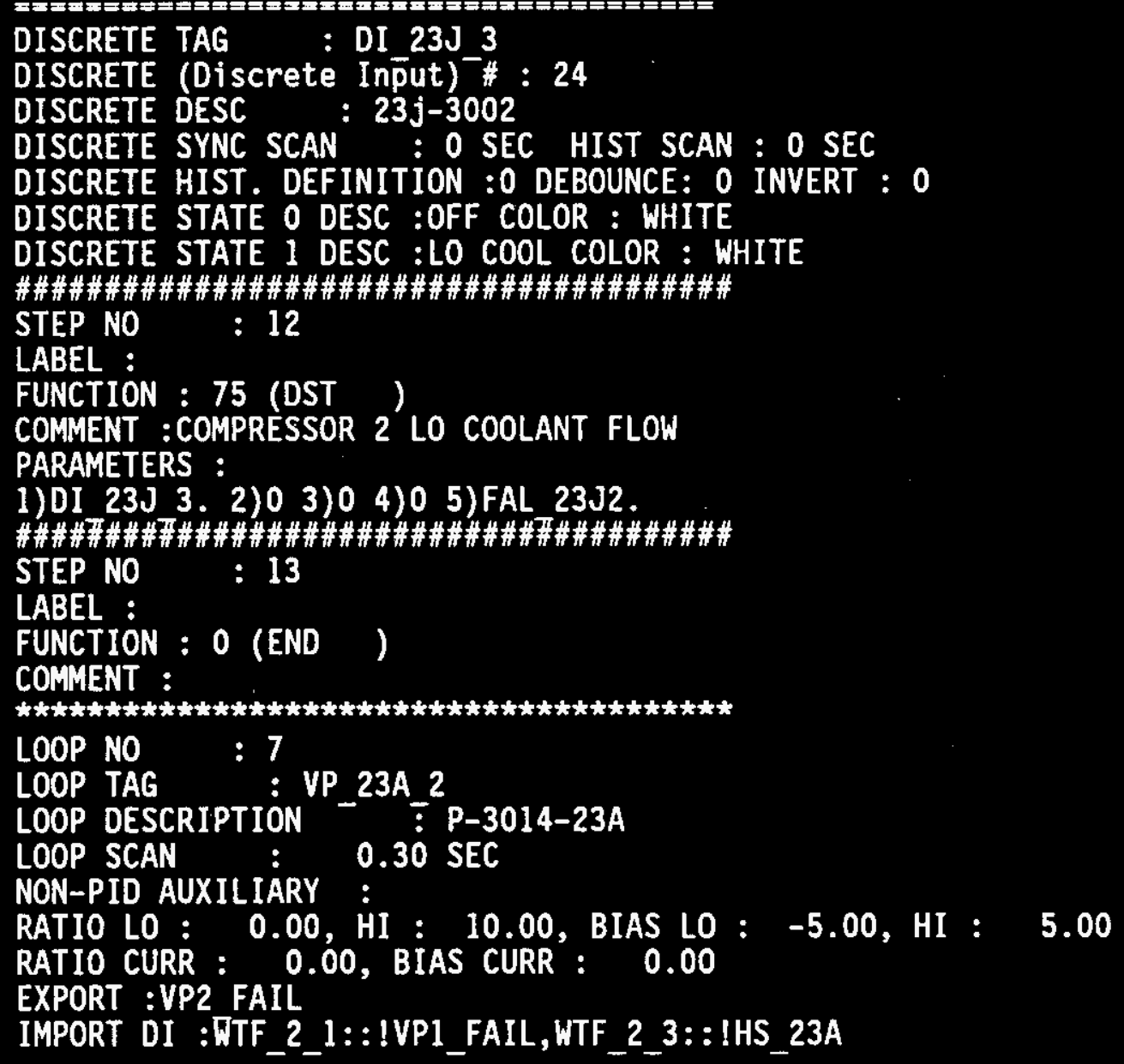


WHC-SD-CP-CSWD-016

Volume 2

Revision 1

Page 102

\#\#\#\#\#\#\#\#\#\#\#\#\#\#\#\#\#\#\#\#\#\#\#\#\#\#\#\#\#\#\#\#\#\#\#\#\#\#\#\#

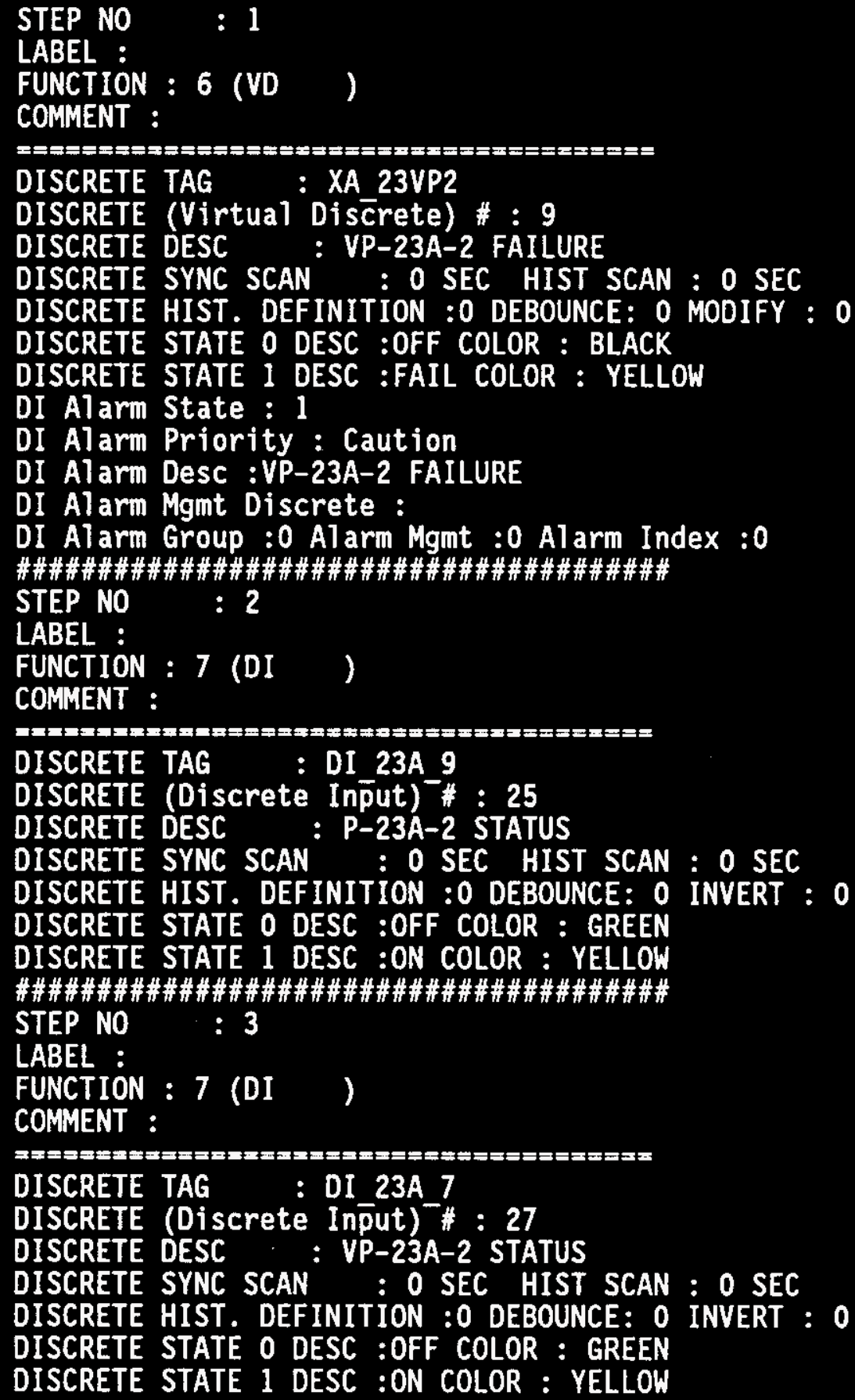


WHC-SD-CP-CSWD-016

Volume 2

Revision 1

Page 103

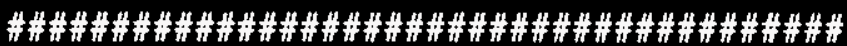

STEP NO : 4

LABEL :

FUNCTION : $57(00)$

COMMENT :

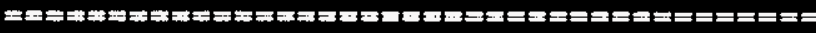

DISCRETE TAG : DO $23 A 6$

DISCRETE (Discrete Output) \# : 26

DISCRETE DESC : P-23A-2 CONTROL

DISCRETE SYNC SCAN : O SEC HIST SCAN : O SEC

DISCRETE HIST. DEFINITION :0 DEBOUNCE: 0 MODIFY : 0

DISCRETE STATE O DESC :OFF COLOR : GREEN

DISCRETE STATE 1 DESC :ON COLOR : YELLOW

\#\#\#\#\#\#\#\#\#\#\#\#\#\#\#\#\#\#\#\#\#\#\#\#\#\#\#\#\#\#\#\#\#\#\#\#\#\#\#\#

STEP NO : 5

LABEL :

FUNCTION : $57(00)$

COMMENT :

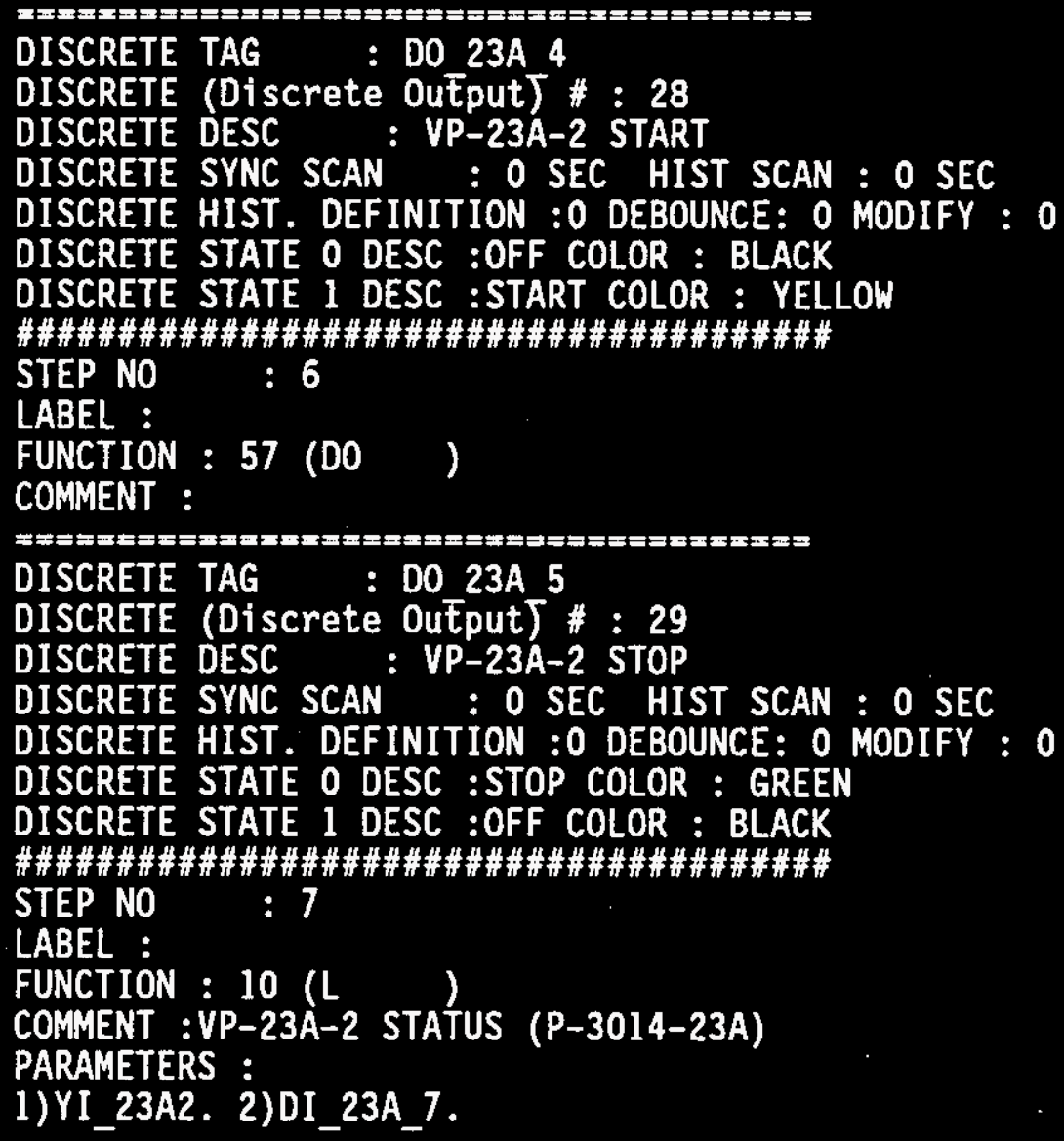


WHC-SD-CP-CSWD-016

Volume 2

Revision 1

Page 104

\#\#\#\#\#\#\#\#\#\#\#\#\#\#\#\#\#\#\#\#\#\#\#\#\#\#\#\#\#\#\#\#\#\#\#\#\#\#\#\#

STEP NO : 8

LABEL :

FUNCTION : $10(\mathrm{~L}$
COMMENT : $\mathrm{P}-23 \mathrm{~A}-3002$ STATUS

PARAMETERS :

1)YI P23A2. 2)DI $23 A 9$.

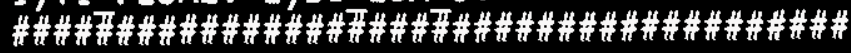

STEP NO : 9

LABEL :

FUNCTION : 69 (LC)

COMMENT : "O" PULSE ON HS 23A=VP1 AND FAILURE ALARM

PARAMETERS :

1)-! PULSE(-HS 23A\&VP1 FAIL)

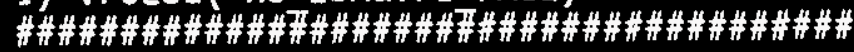

STEP NO : 10

LABEL :

FUNCTION : 62 (GTD )

COMMENT : JUMP IF NO PULSE

PARAMETERS :

1)MSV 2) Off-Nomal 3) JMPON

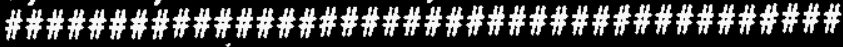

STEP NO

LABEL : JMPON

FUNCTION : 10 (L

COMMENT : TURN VP2 ON IF VP1 HAS FAILED

PARAMETERS :

1)HS 23A 2. 2)1

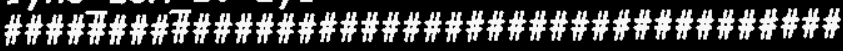

STEP NO : 12

LABEL :

FUNCTION : 73 (LAT

COMMENT :UNLATCH PUMP OUTPUT IF NO STATUS AFTER 5 SEC

PARAMETERS :

1) $L A T C H=H S$ 23A 2. 2) UNLATCH $=(-Y I$ P23A28DELAY (HS_ 3)0 4)DO_23A_6. 5)0

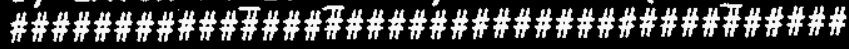

STEP NO : 13

LABEL :

FUNCTION : 69 (LC )

COMMENT :FAILURE ON LO FLOW \& DP OR VP2 FAIL

PARAMETERS :

1) XA 23A2 XA 23VP2

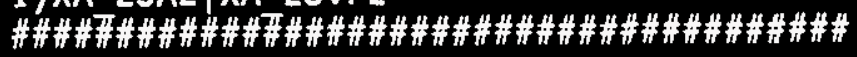

STEP NO : 14

LABEL :

FUNCTION : 75 (DST)

COMMENT :

PARAMETERS :

1)MSV 2)0 3)0 4) 5)VP2_FAIL. 
WHC-SD-CP-CSWD-016

Volume 2

Revision 1

Page 105

\#\#\#\#\#\#\#\#\#\#\#\#\#\#\#\#\#\#\#\#\#\#\#\#\#\#\#\#\#\#\#\#\#\#\#\#\#\#\#\#

STEP NO : 15

LABEL : VPON

FUNCTION : 69 (LC

COMMENT :START VAC PUMP 15 SEC AFTER CIRC PUMP IF NO ALARM

PARAMETERS :

1)DELAY(YI P23A2, 15)\&-DI 23A 8\&HS 23A 2

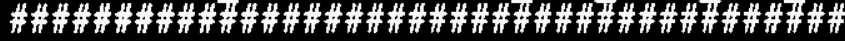

STEP NO : 16

LABEL :

FUNCTION : 78 (MC )

COMMENT :

PARAMETERS :

1)MSV 2)DO 23A 4. 3)DO 23A 5. 4) 10.00 5) 1.00

6) (nu11) $7 \zeta\left(\right.$ nuTl) 8) (nū11) ${ }^{-9}$ (nul1) 10)(nu11)

11) (nul1)

\#\#\#\#\#\#\#\#\#\#\#\#\#\#\#\#\#\#\#\#\#\#\#\#\#\#\#\#\#\#\#\#\#\#\#\#\#\#\#\#

STEP NO : 17

LABEL :

FUNCTION : 69 (LC

COMMENT :ALARM ON VP STATUS OFF AND SWITCH ON (EXCEPT STARTUP)

PARAMETERS :

1) VPON\&-YI 23A28-DO 23A 4

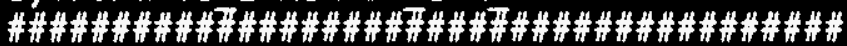

STEP NO : 18

LABEL :

FUNCTION : $10(\mathrm{~L})$

COMMENT :

PARAMETERS :

1)XA 23VP2. 2) MSV

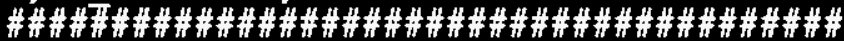

STEP NO : 19

LABEL :

FUNCTION : 0 (END)

COMMENT : 


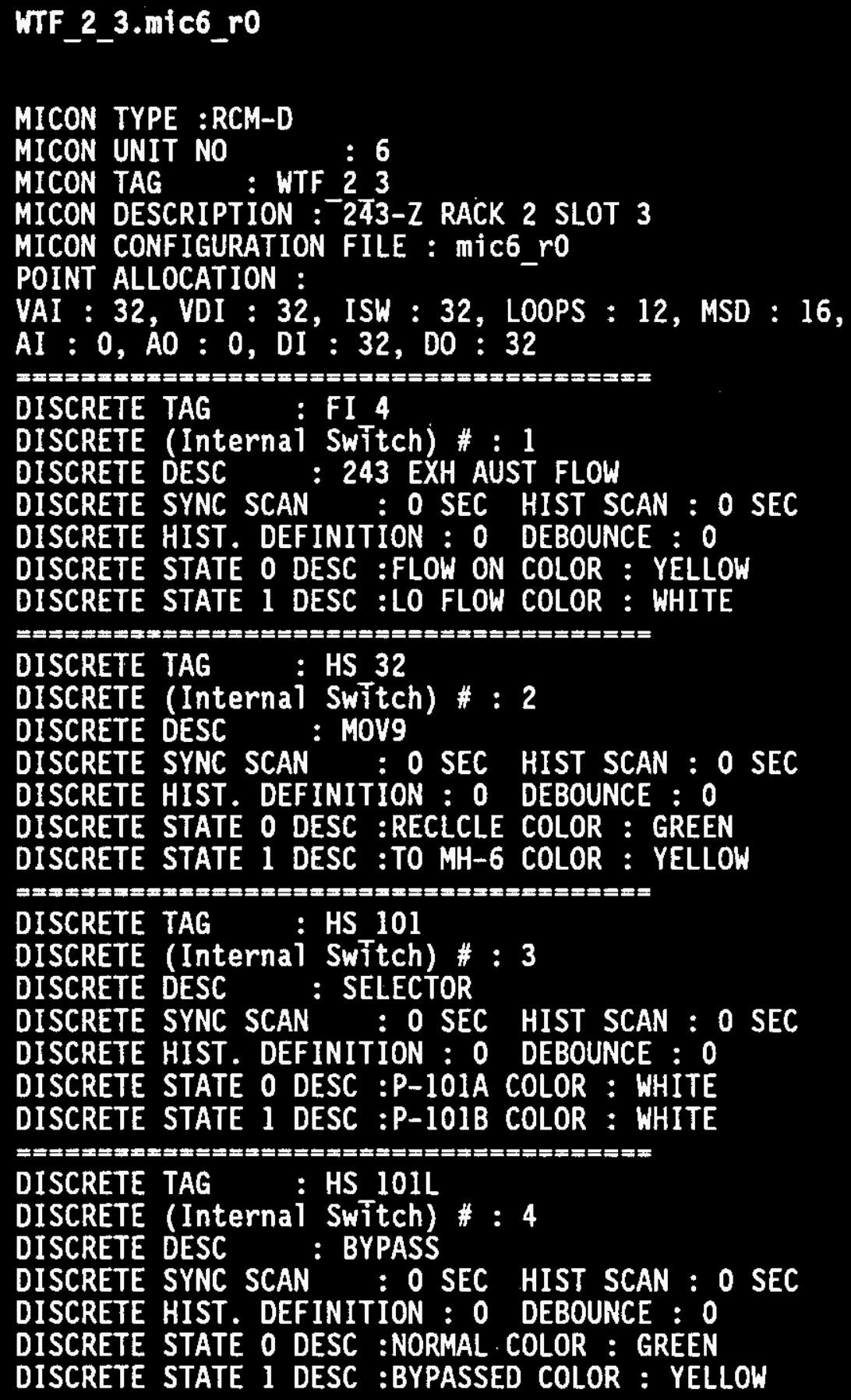


WHC-SD-CP-CSWD-016

Volume 2

Revision 1

Page 107

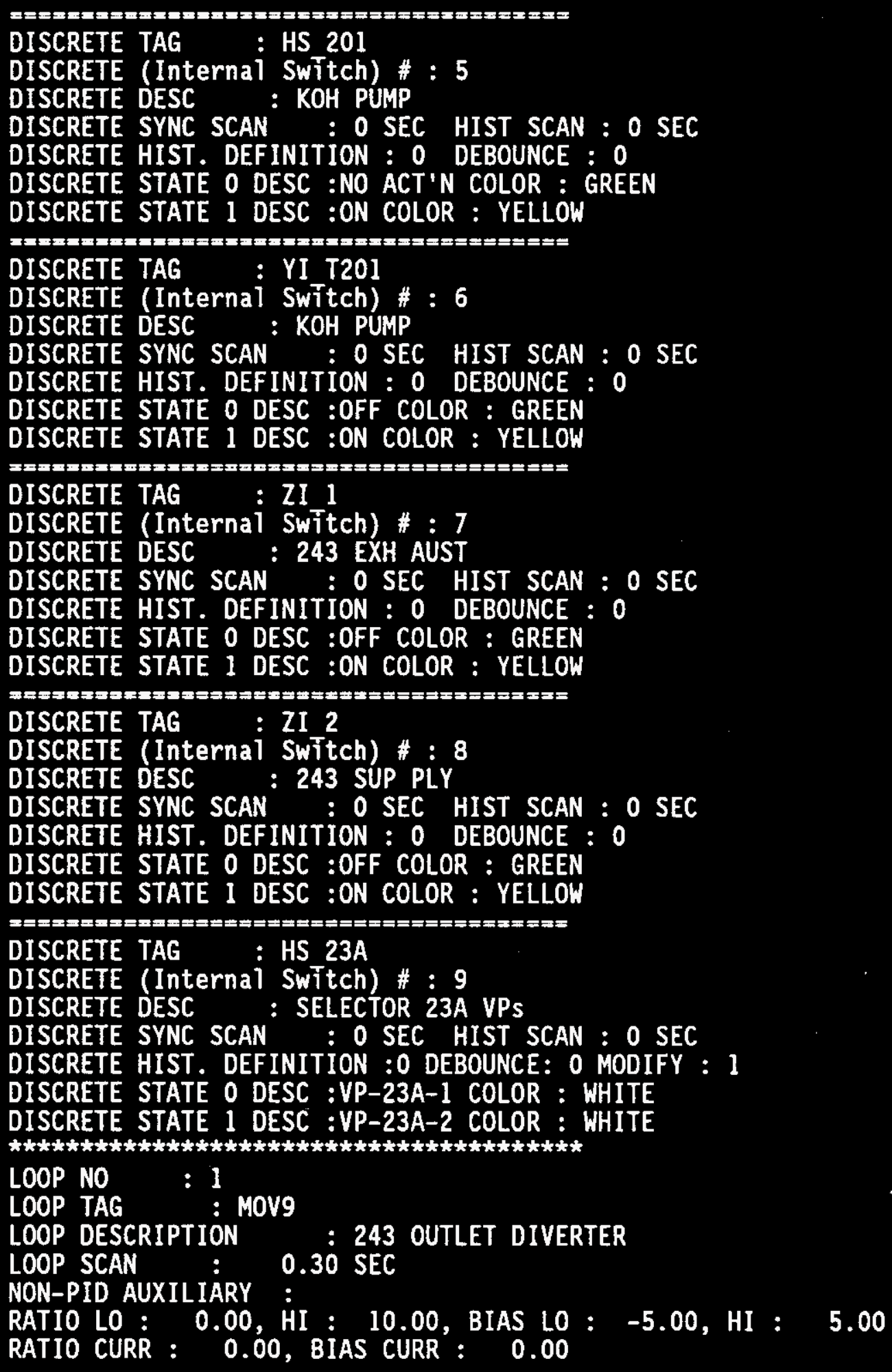


WHC-SD-CP-CSWD-016

Volume 2

Revision 1

Page 108

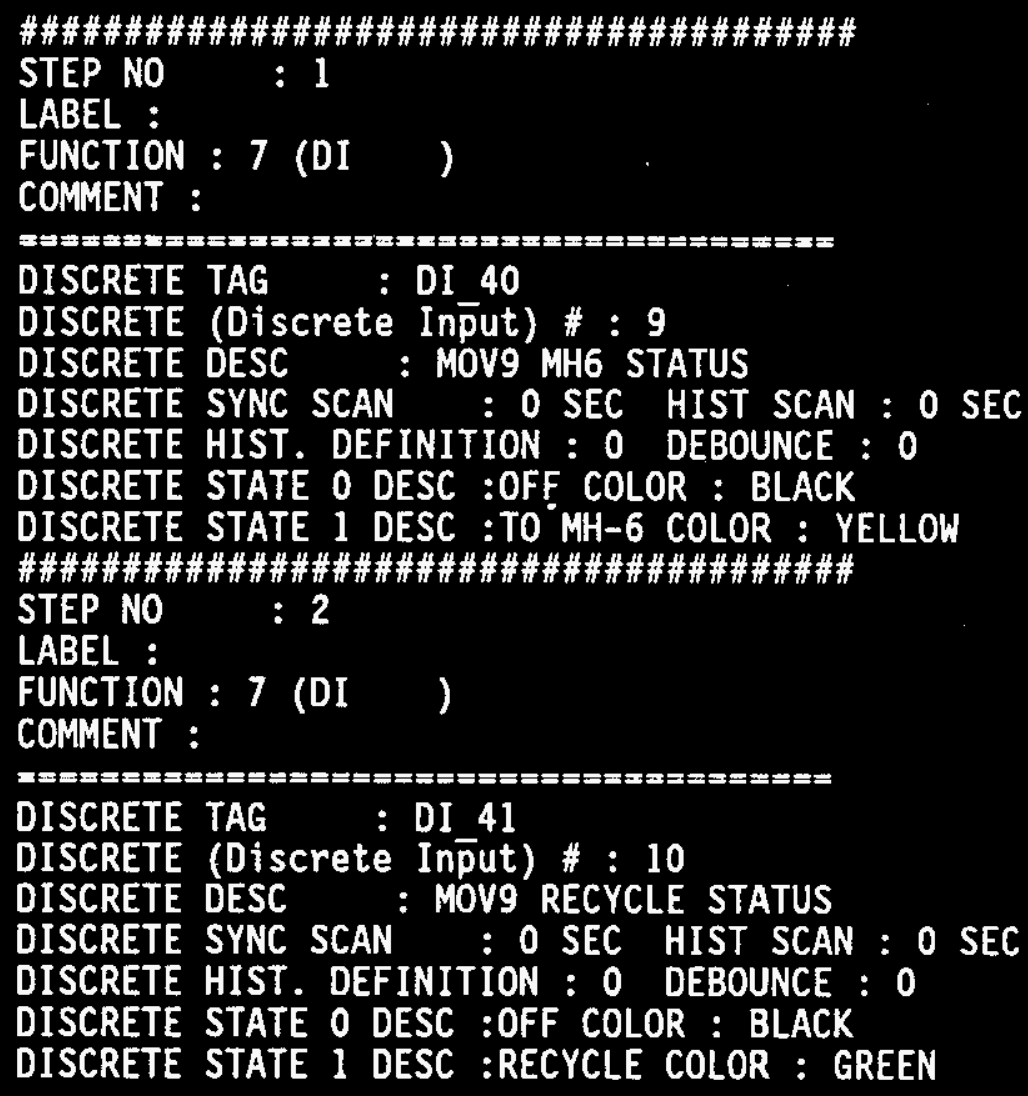


WHC-SD-CP-CSWD-016

Volume 2

Revision 1

Page 109

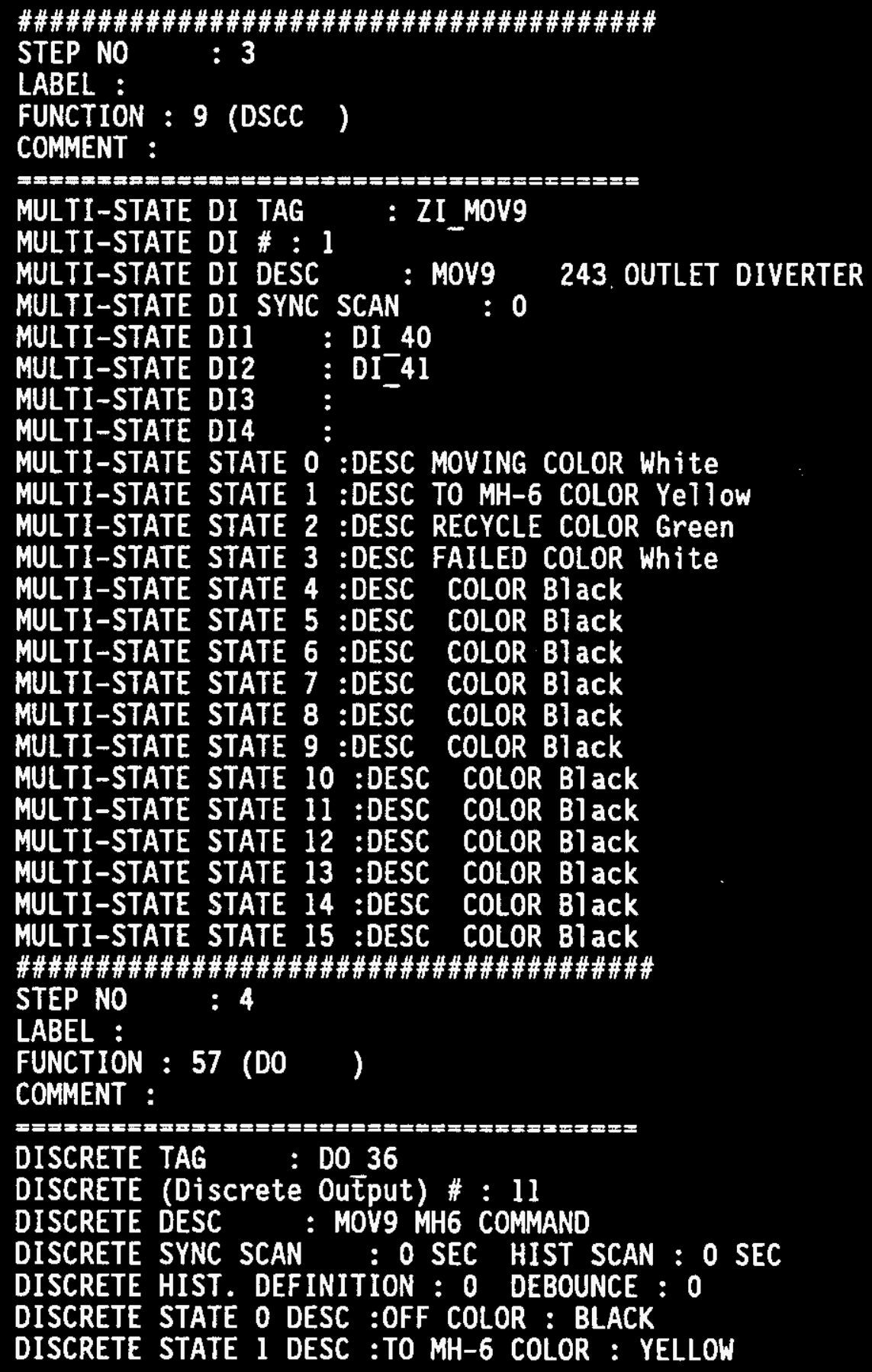


WHC-SD-CP-CSWD-016

Volume 2

Revision 1

Page 110

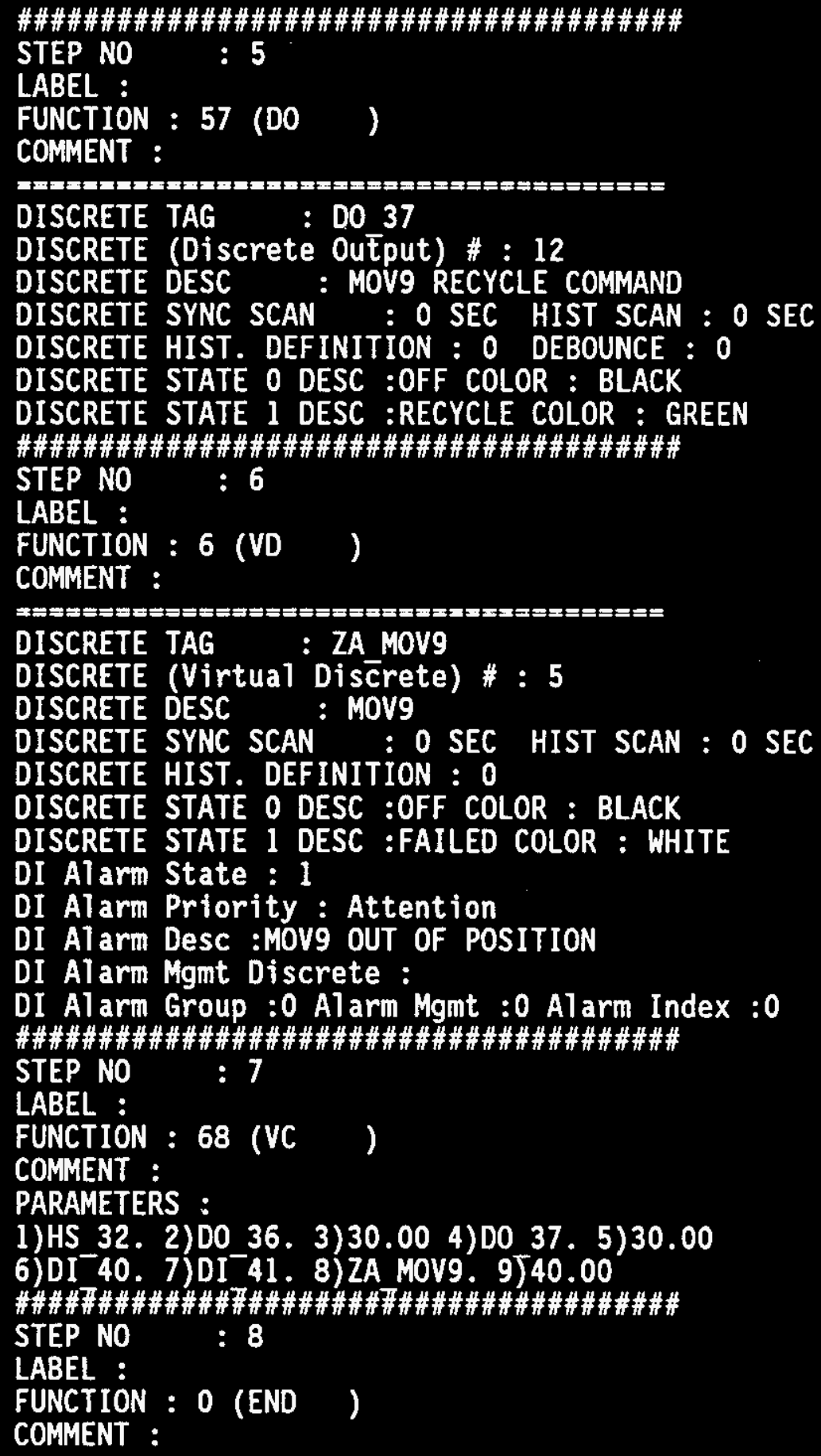


WHC-SD-CP-CSWD-016

Volume 2

Revision 1

Page 111

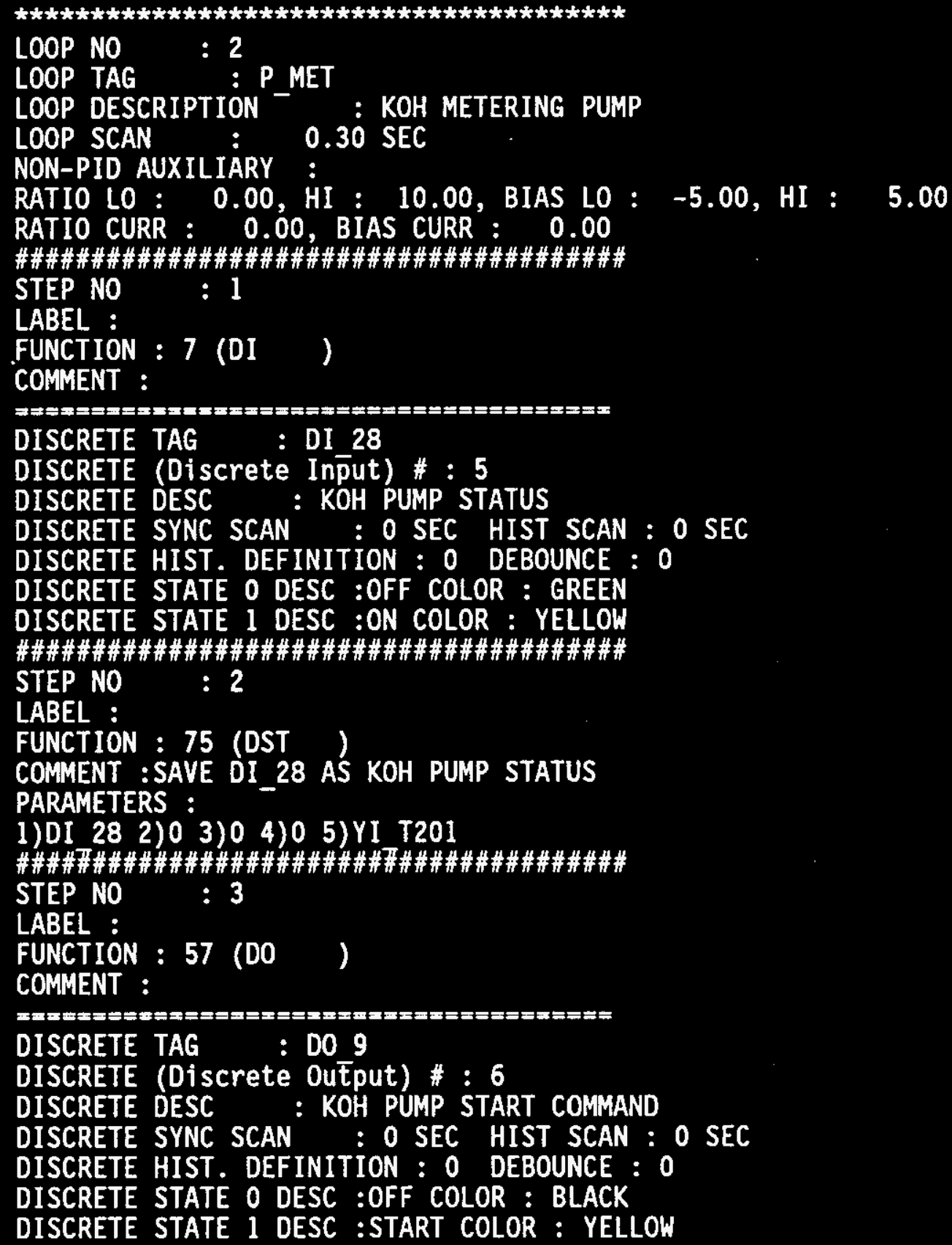


WHC-SD-CP-CSWD-016

Volume 2

Revision 1

Page 112

\#\#\#\#\#\#\#\#\#\#\#\#\#\#\#\#\#\#\#\#\#\#\#\#\#\#\#\#\#\#\#\#\#

STEP NO : 4

LABEL :

FUNCTION : 6 (VD )

COMMENT :

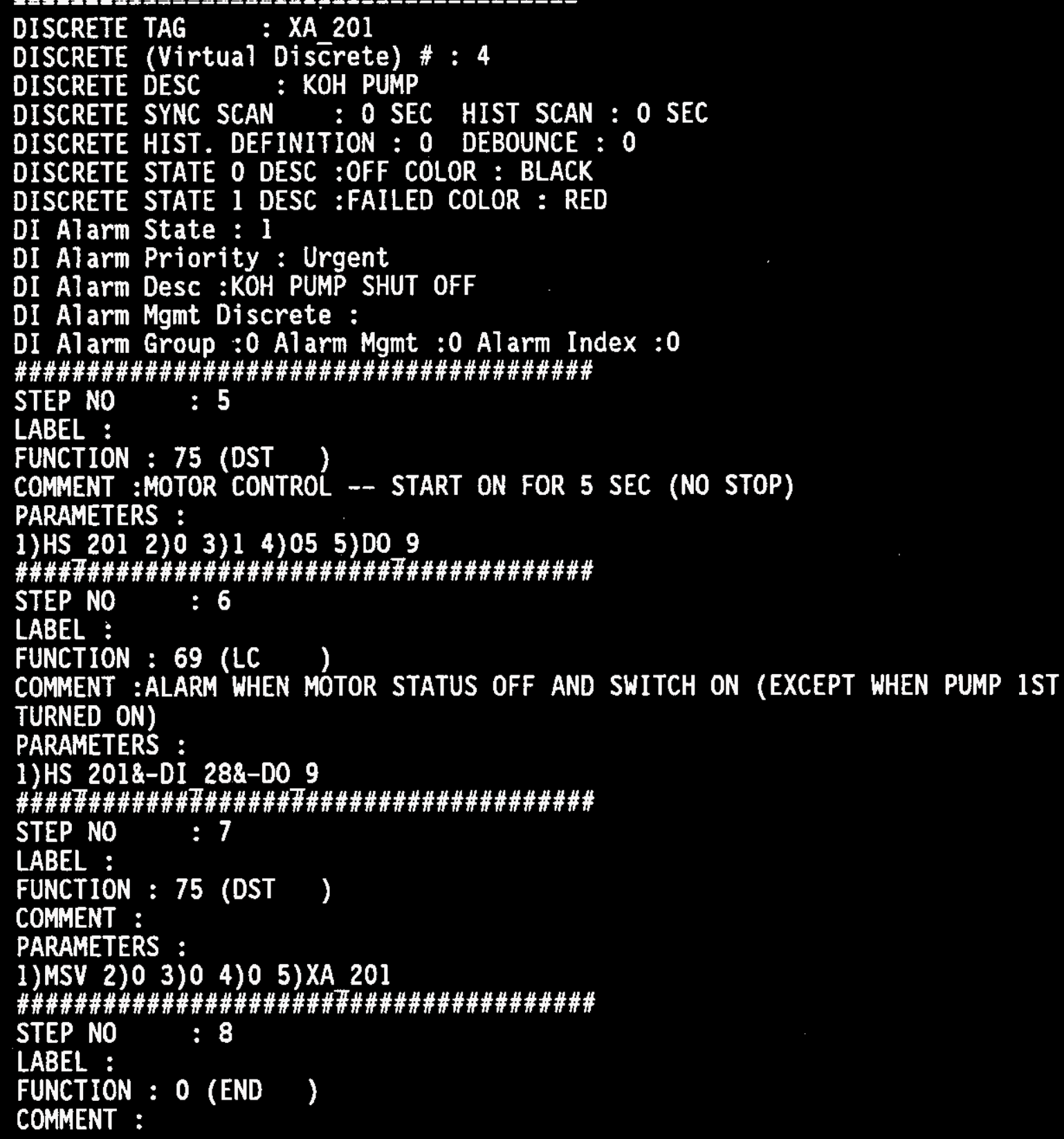




\section{WHC-SD-CP-CSWD-016 \\ Volume 2 \\ Revision 1 \\ Page 113}

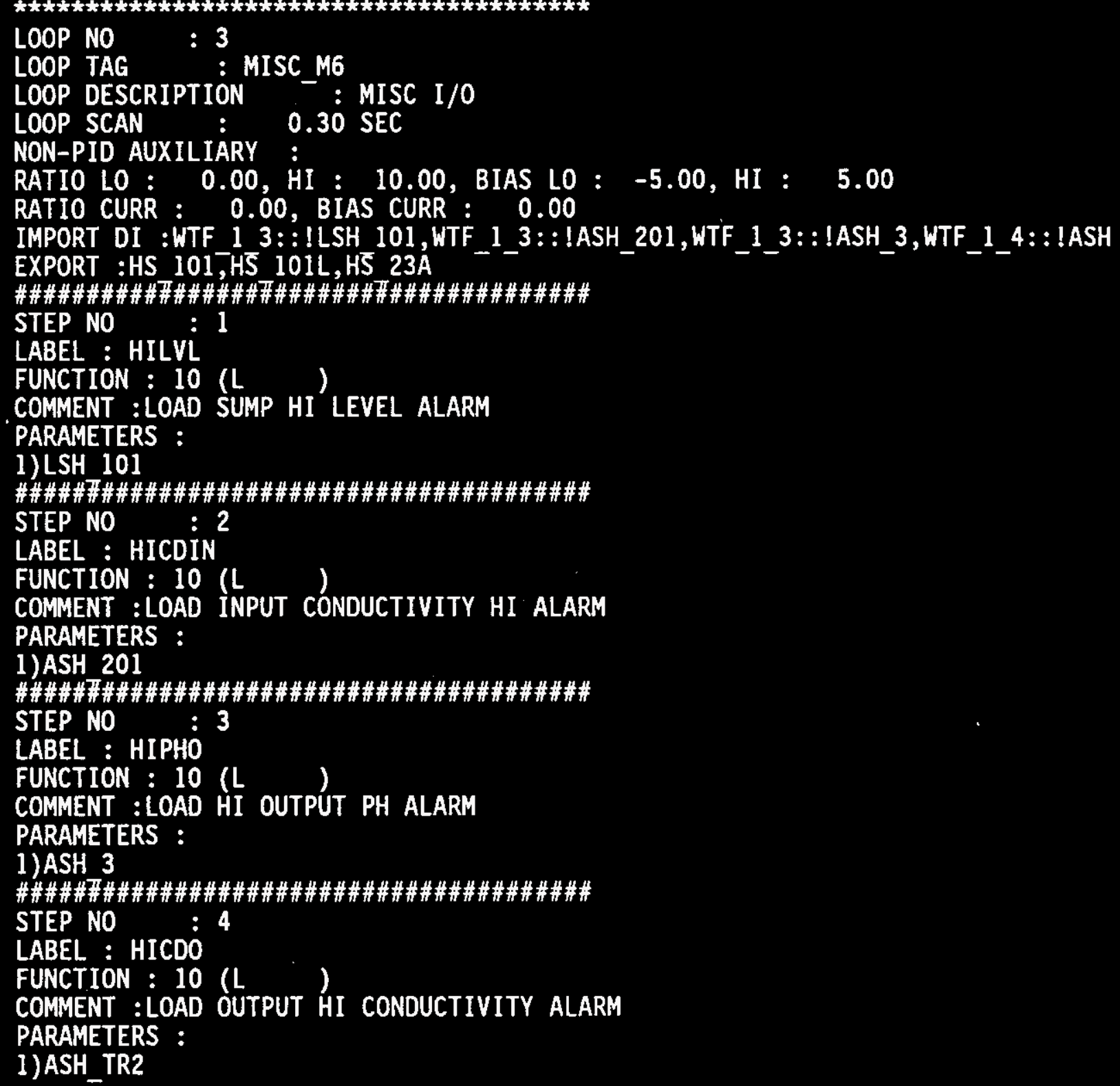


WHC-SD-CP-CSWD-016

Volume 2

Revision 1

Page 114

\#\#\#\#\#\#\#\#\#\#\#\#\#\#\#\#\#\#\#\#\#\#\#\#\#\#\#\#\#\#\#\#\#\#\#\#\#\#\#\#

STEP NO : 5

LABEL :

FUNCTION : 6 (VD )

COMMENT :

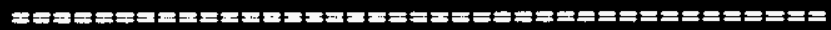

DISCRETE TAG

: FI 102

DISCRETE (Virtual Discrete) \# : 1

DISCRETE DESC : T-102 INLET

DISCRETE SYNC SCAN : 0 SEC HIST SCAN : O SEC

DISCRETE HIST. DEFINITION :0 DEBOUNCE: 0 MODIFY : 0

DISCRETE STATE O DESC :FLOW OFF COLOR : GREEN

DISCRETE STATE 1 DESC :FLON ON COLOR : YELLON

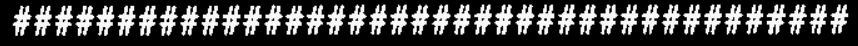

STEP NO : 6

LABEL :

FUNCTION : 7 (DI )

COMMENT :

DISCRETE TAG : DI 3

DISCRETE (Discrete Input) \#: 1

DISCRETE DESC : T-102 INLET LO FLOW SWITCH

DISCRETE SYNC SCAN : O SEC HIST SCAN : O SEC

DISCRETE HIST. DEFINITION : 0 DEBOUNCE : 0

DISCRETE STATE O DESC :OFF COLOR : BLACK

DISCRETE STATE 1 DESC :LO FLOW COLOR : WHITE

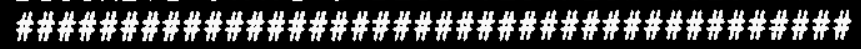

STEP NO : 7

LABEL :

FUNCTION : 7 (DI)

COMMENT :

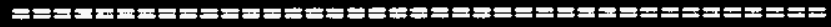

DISCRETE TAG : DI 6

DISCRETE (Discrete Input) \#: 13

DISCRETE DESC : 243-Z EXHAUST FLOW SHITCH

DISCRETE SYNC SCAN : O SEC HIST SCAN : O SEC

DISCRETE HIST. DEFINITION : 0 DEBOUNCE : 0

DISCRETE STATE O DESC :OFF COLOR : BLACK

DISCRETE STATE 1 DESC :LO FLOW COLOR : YELLOW 
WHC-SD-CP-CSWD-016

Volume 2

Revision 1

Page 115

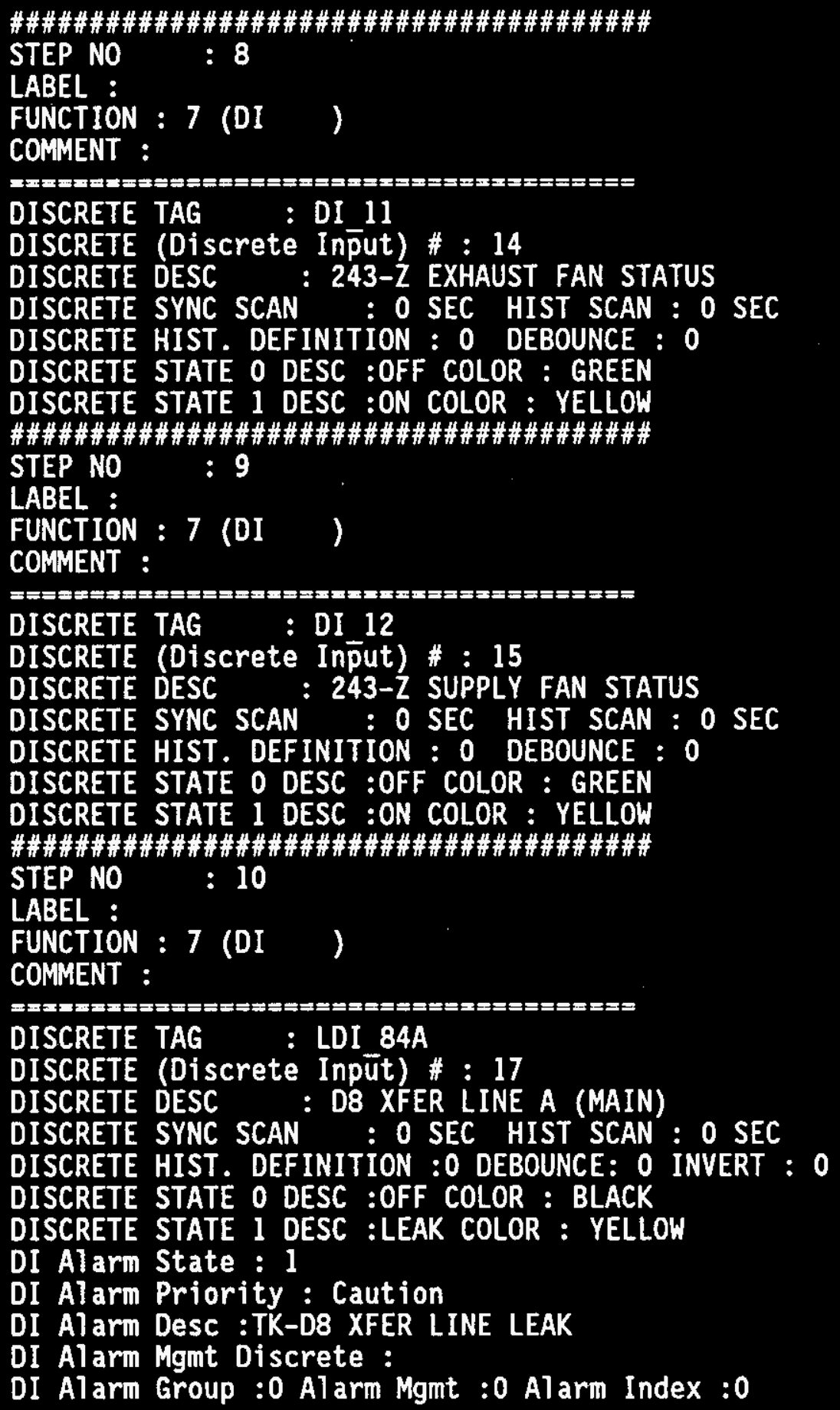




\section{WHC-SD-CP-CSWD-016 \\ Volume 2 \\ Revision 1 \\ Page 116}

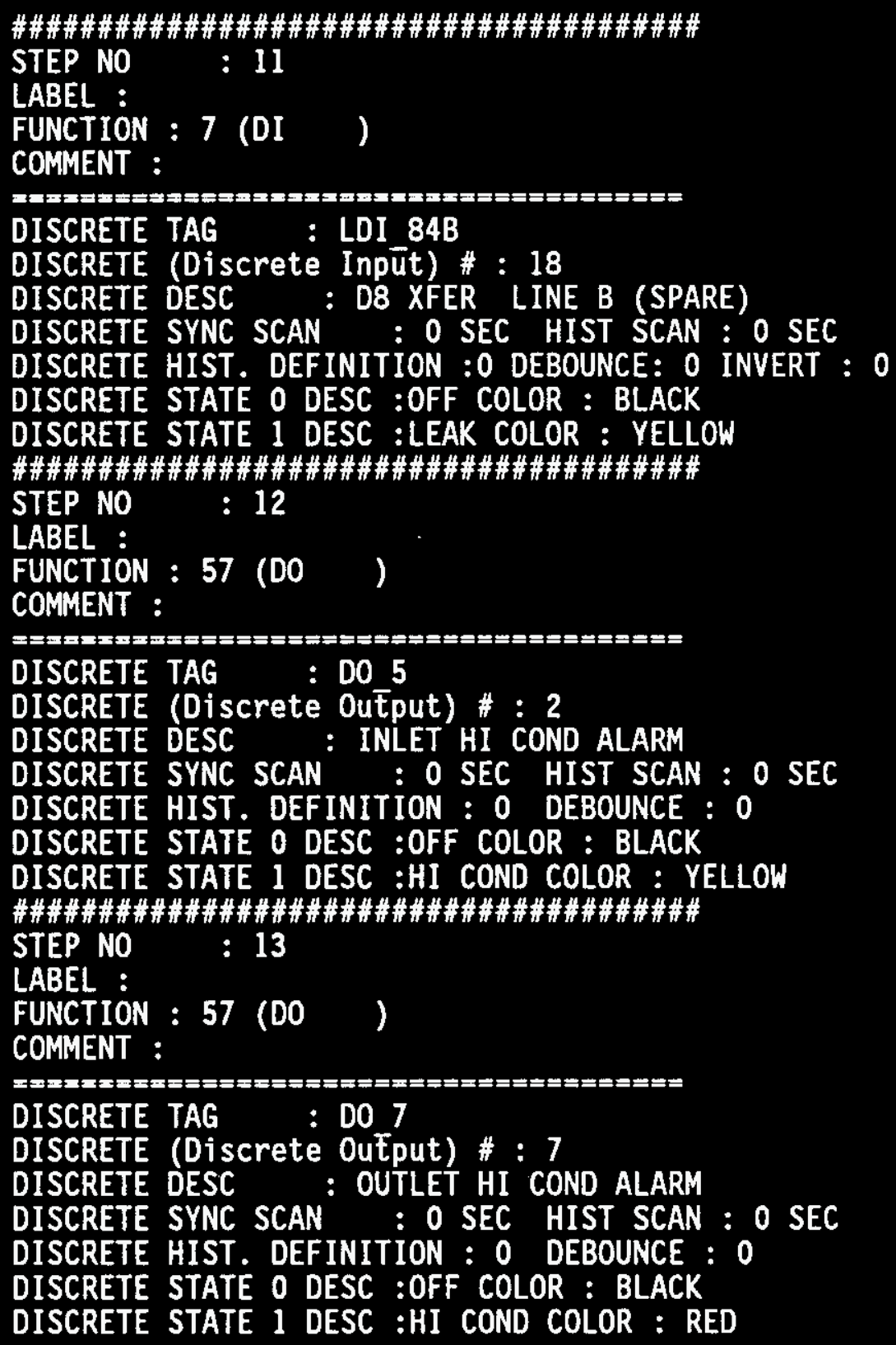


WHC-SD-CP-CSWD-016

Volume 2

Revision 1

Page 117

\#\#\#\#\#\#\#\#\#\#\#\#\#\#\#\#\#\#\#\#\#\#\#\#\#\#\#\#\#\#\#\#\#\#

STEP NO : 14

LABEL :

FUNCTION : 57 (DO )

COMMENT :

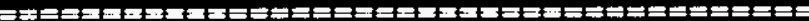

DISCRETE TAG : D0 8

DISCRETE (Discrete Output) \#:8

DISCRETE DESC : OUTLET HI PH ALARM

DISCRETE SYNC SCAN :O SEC HIST SCAN : O SEC

DISCRETE HIST. DEFINITION : 0 DEBOUNCE : 0

DISCRETE STATE 0 DESC :OFF COLOR : BLACK

DISCRETE STATE 1 DESC :HI PH COLOR : RED

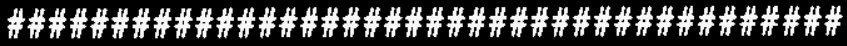

STEP NO : 15

LABEL :

FUNCTION : 57 (DO )

COMMENT :

DISCRETE TAG : DO 6

DISCRETE (Discrete Output) \#: 16

DISCRETE DESC : T-101 (SUMP) HI LEVEL ALARM

DISCRETE SYNC SCAN :O SEC HIST SCAN : O SEC

DISCRETE HIST. DEFINITION : 0 DEBOUNCE : 0

DISCRETE STATE O DESC :OFF COLOR : BLACK

DISCRETE STATE 1 DESC :HI LEVEL COLOR : YELLOW

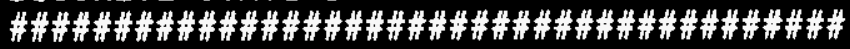

STEP NO : 16

LABEL :

FUNCTION : 75 (DST)

COMMENT :OUTPUT T-101 HI LEVEL ALARM TO LIGHT

PARAMETERS :

1)HILVL 2)0 3)0 4)0 5)00 6

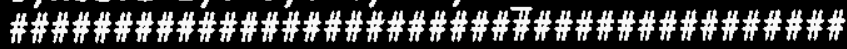

STEP NO : 17

LABEL :

FUNCTION : 75 (DST

COMMENT :OUTPUT HI INPUT COND ALARM TO LIGHT

PARAMETERS :

1) HICDIN 2)O 3)O 4)0 5)DO 5

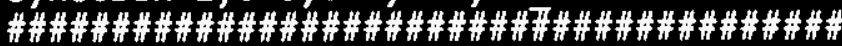

STEP NO : 18

LABEL :

FUNCTION : 75 (DST

COMMENT :OUTPUT HI OUTPUT PH ALARM TO LIGHT

PARAMETERS :

1)HIPHO 2)0 3)0 4)0 5)DO 8 
\#\#\#\#\#\#\#\#\#\#\#\#\#\#\#\#\#\#\#\#\#\#\#\#\#\#\#\#\#\#\#\#\#\#\#\#\#\#\#\#

STEP NO : 19

LABEL :

FUNCTION : 75 (DST)

COMMENT :OUTPUT HI OUTPUT COND ALARM TO LIGHT

PARAMETERS :

1)HICDO 2)0 3)0 4)0 5)D0 7

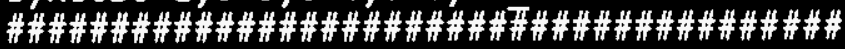

STEP NO : 20

LABEL :

FUNCTION : 75 (DST)

COMMENT :SAVE DI_6 AS 253-Z EXHAUST FLOW INDICATOR

PARAMETERS :

1)DI 6 2)0 3)0 4)0 5)FI 4

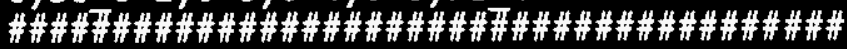

STEP NO : 21

LABEL :

FUNCTION : 75 (DST )

COMMENT :SAVE DI_11 AS EXHAUST FAN INDICATOR

PARAMETERS :

1)DI 11. 2)0 3)0 4)0 5)ZI 1 .

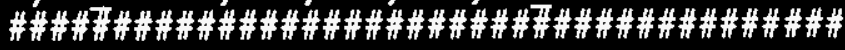

STEP NO : 22

LABEL :

FUNCTION : 75 (DST)

COMMENT : SAVE DI_12 AS SUPPLY FAN INDICATOR

PARAMETERS :

1)DI 12. 2) 0 3) 0 4)0 5)ZI 2.

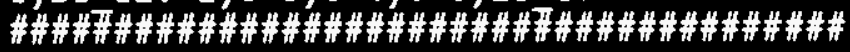

STEP NO : 23

LABEL :

FUNCTION : 75 (DST)

COMMENT :SAVE DI_3 AS T-102 INLET FLOW INDICATION

PARAMETERS :

1)DI 3. 2)0 3)0 4) 5)FI 102.

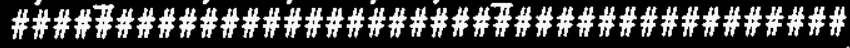

STEP NO : 24

LABEL :

FUNCTION : 0 (END)

COMMENT : 
WHC-SD-CP-CSWD-016

Volume 2

Revision 1

Page 119

\section{MICON PROGRAM FILES}

MICON program files are created by MICON View from the configuration file listings (above). This file is the actual program that is run by the controlier card. When you request a program upload from a controller card, these are the files you'll get.

These files should not be directly modified by the user. All modifications should be through the configuration file using the configure controller option on MICON View.

The programming language is a custom language developed by MICON Company. A description of this language can be found in the U-32/RCM Function Compiler Documentation available from MICON Company (Houston, TX 1-713-947-9470).

You will notice that, like the configuration file, the program file is organized into loops. With the exception of two loops, these are the same ones created by the user in the controller's configuration file. The two exceptions are loop "ZERO" at the top and loop "NINETY NINE" at the bottom. These are house-keeping loops created by FICON Viēw.

The following file listings have been edited to make them easier to read. Spaces have been put between the loops and extra long lines have been indented Therefor, the actual file's format may look a little different.

NOTE: The DEF line under each loop name contains fields showing the tuning paramaters -- for example:

$$
\text { CGAIN }=0.50, \text { CRESET }=10.00, \text { CRATE }=0.00, M \_ \text {RESET }=0.00
$$

These tuning parameters are not controlled by this document. Therefore, these numbers may be different than the ones that appear in the workstation listings. This will also be true of program files uploaded from the controllers. 
WHC-SD-CP-CSWD-016

Volume 2

Revision 1

Page 120

mic2_ro

LOOP ZERO;

DEF :TAG=AI 1.,AI_11.,AI 6.,AI 8.,AI 2.,AI 10.,AI 23J 2.,AI 23A 5.,AI RM500.;

DEF :TAG=AI 3.,AI_201.,F̄ 2.,PDI_202.,ARC_201.,FRC_2.,LRC_101.,TI_23JI.,TI_23A1.,TI_RM500.;

DEF : $A C C E S S=1$ : TAG $=$ !HS 102.;

DEF :TAG=!ASH 201., IASH_3.,!LSH_101., ILSHH_101., ILSL_101.;

DEF : TAG $=$ PERIOD $=1$;

END;

LOOP AIC 201.;

DEF :TAG $=$ PERIOD $=0.30, \mathrm{SP}$ RATE $=10000.00, \mathrm{SPLOLM}=0.00, \mathrm{SPHILM}=14.00, \mathrm{SPANL}=0.00, \mathrm{SPANHI}=14.00, \mathrm{RATI}=0.00$, BIAS $=0.00$, CGAIN $=0.50$, CRESET $=10.00$, CRATE $=0.00, M$ RESET $=0.00$;

AI :TAG=AI 8.: CHAN $=4: S P A N L O=0.00: S P A N H I=14.00$;

LET :TAG=ARTC 201=AI 8;/*SAVE INPUT TO ARC_201 FOR HISTORIAN*/

PID :PV=AI 8:RECALL $=1 ; /$ - INEARIZE PH WITH INPUT COMPENSATION*/

$A 0: T A G=A 0^{-} 5 .: C H A N=24: S T E P=M S V: 0 P L O L M=0.00: 0 P H I L M=100.00: 0 P N R L M=1000.00: 0 P P R L M=1000.00 ;$

ALARM :TAG=AIC_201.PV:TYPE $=3:$ PRI $=149778:$ LIMITS $=\{?-, ?-, 6.00,10.00, ?+, ?+\}:$ DEADBAND=0.00;

END;

LOOP AIC201I.;

DEF :TAG $=$ PERIOOD=0.30, SP RATE $=10000.00$, SPLOLM $=0.00, S P H I M=100.00$, SPANLO $=0.00, S P A N H I=100.00$, RATI0=0.00, BIAS $=0.00$, CGAIN $=0.20$, CRESET $=10.00$, CRATE $=0.00, M$ RESET $=0.00 ;$

MODE INTERLOCK :REF MODE $=5 ; / *$ LOCK OUT MANUAL \& CASCADE MODES*/

LET :TAG $=$ MODE $=1 ; / *$ PUT LOOP IN AUTO (REQUIRED FOR FNC 55)*/

LET :TAG $=$ AI $8 . * 0.08 ; / *$ CONVERT $0-14$ pH TO .04 TO 1.116 FOR OUTPUT*/

LET :TAG $=M S V+0.04 ; / *$ NOTE: $0-20 \mathrm{~mA}$ OUTPUT $=-0.5$ TO $12 * /$

$A 0: T A G=A 0$ 9. $:$ CHAN=23: TYPE=0:POSITION=HSV:OPLOLM=0.00:0PHILH=100.00:0PNRLM=1000.00:0PPRLM=1000.00;

END; 
WHC-SD-CP-CSWD-016

Volume 2

Revision 1

Page 121

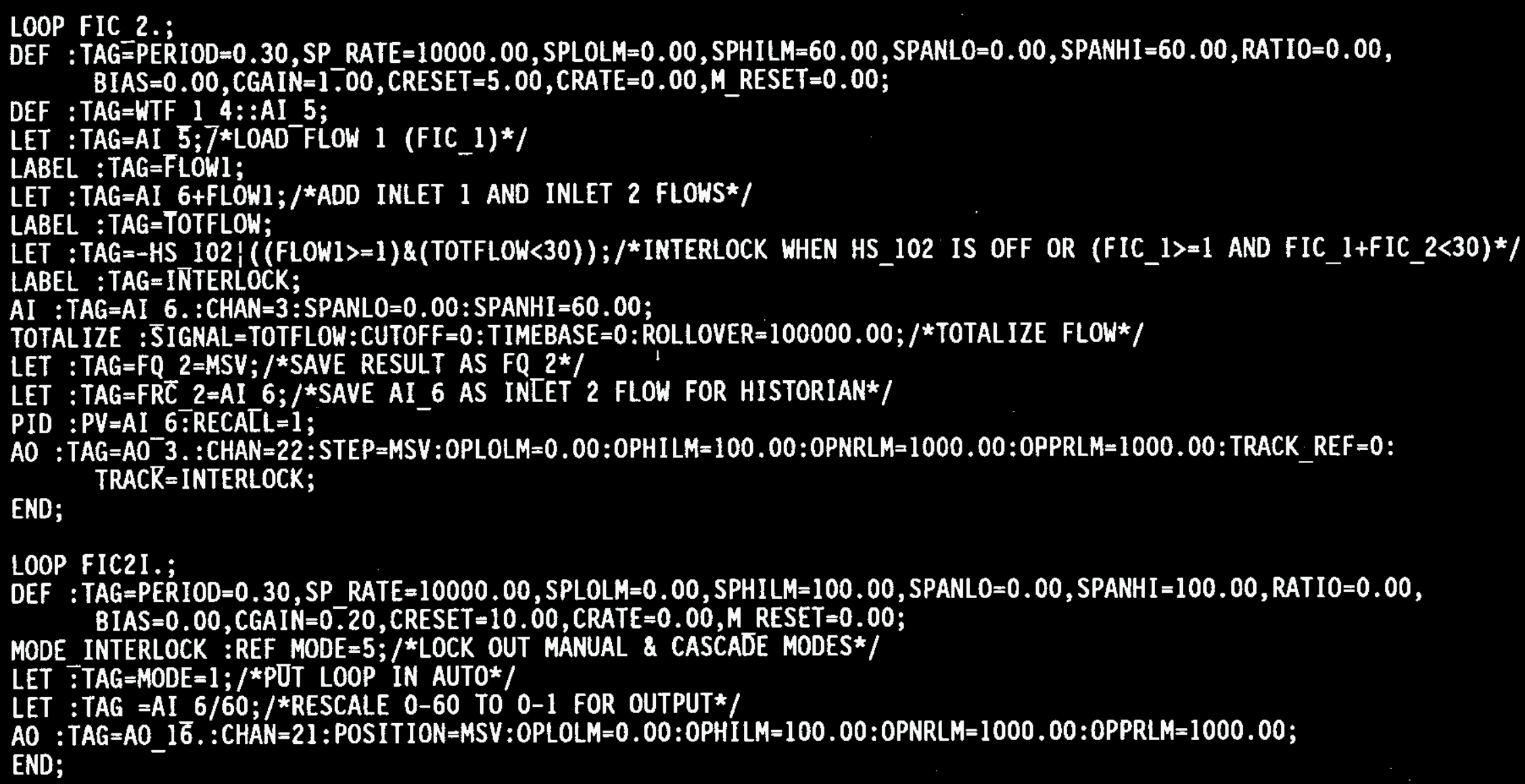


WHC-SD-CP-CSHD-016

Volume 2

Revision 1

Page 122

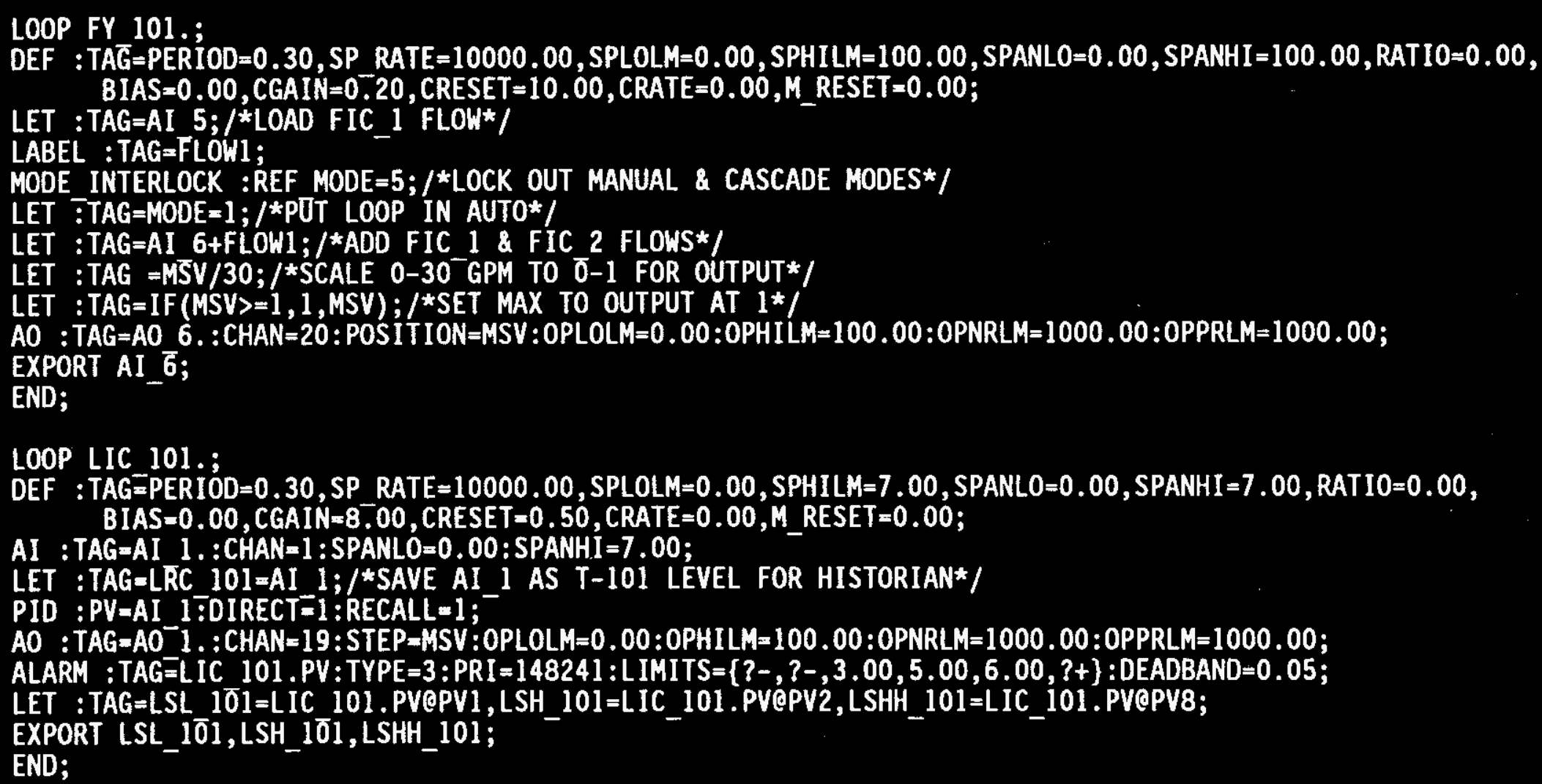



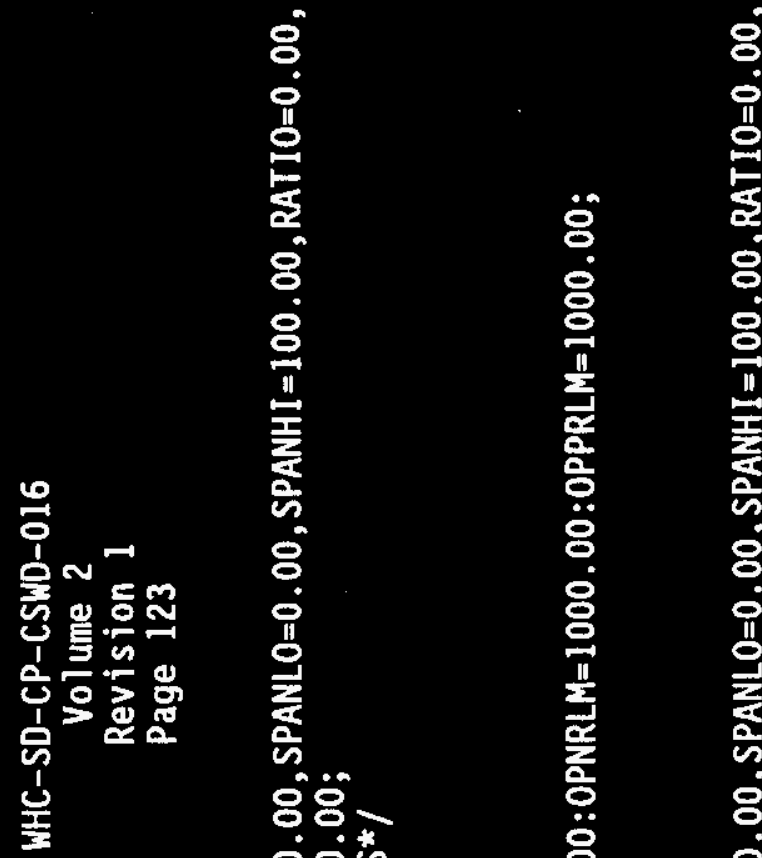

sọ.

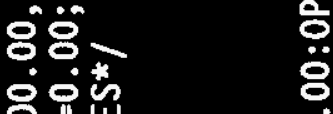

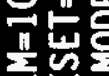

부을

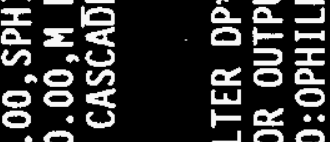

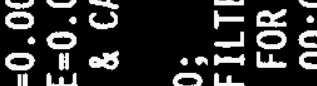

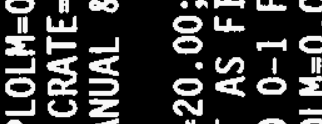

응 는응

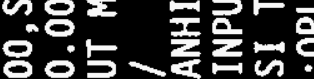

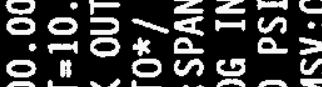

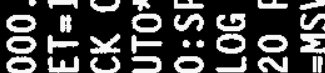
응

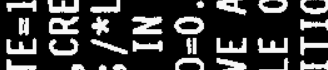
웅의

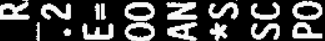

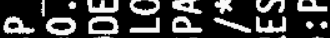

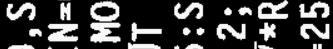

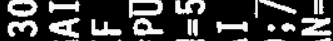

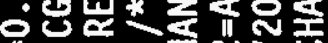

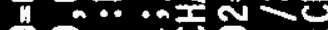
. 象茨

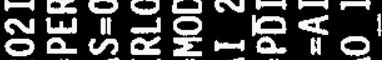

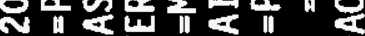

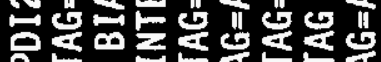

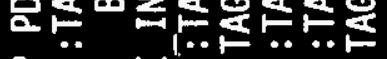

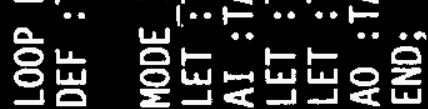

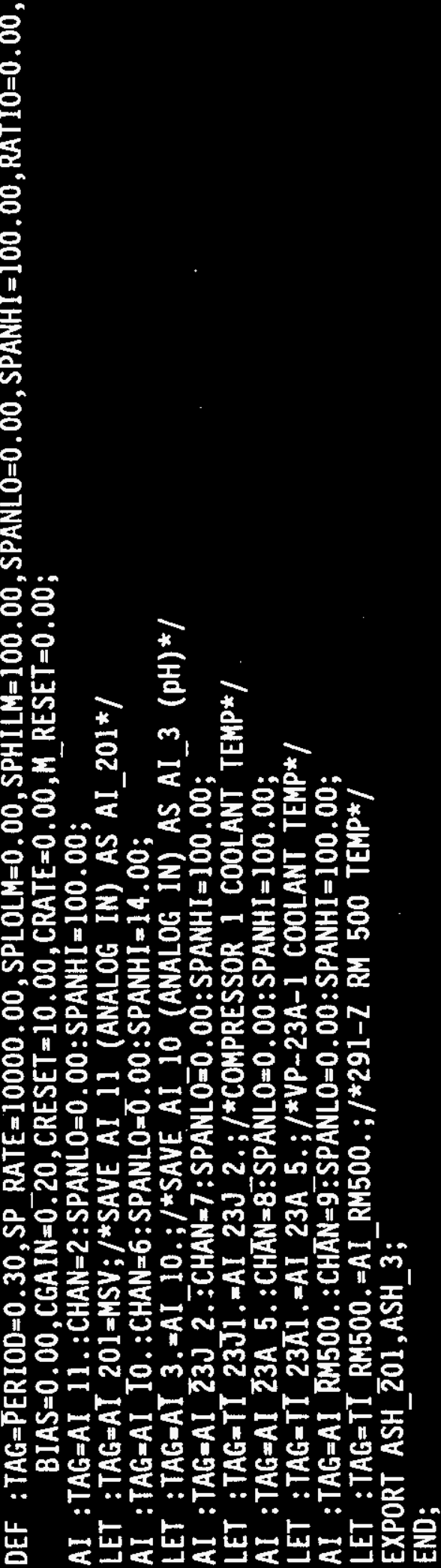




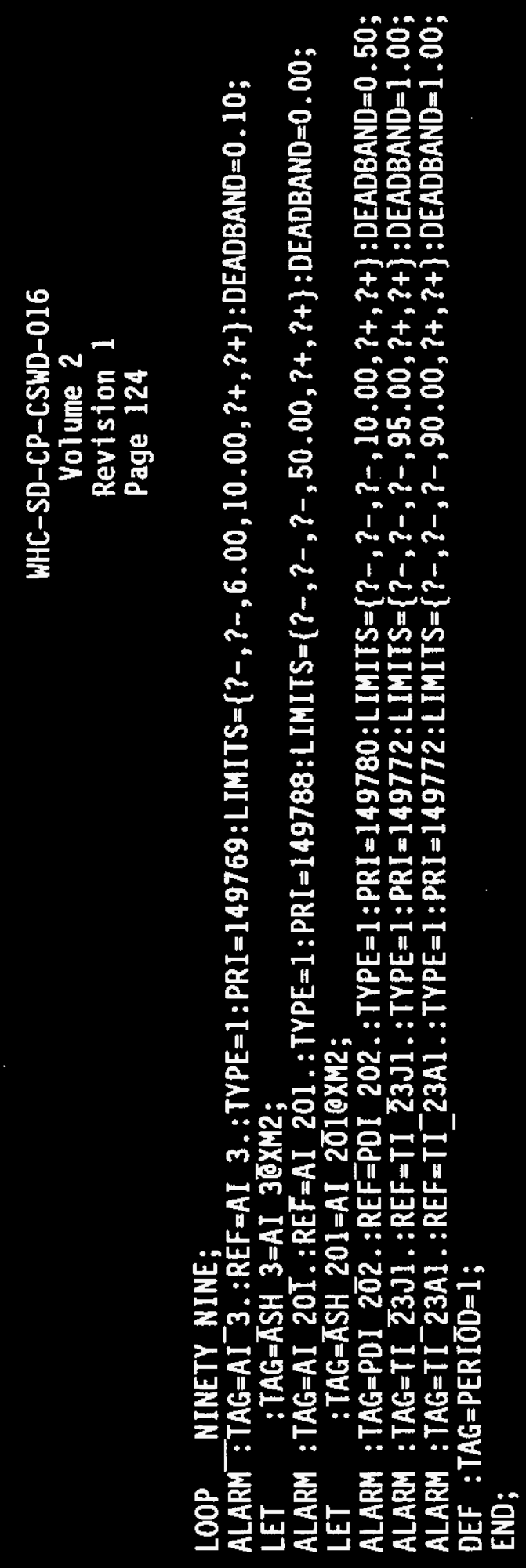



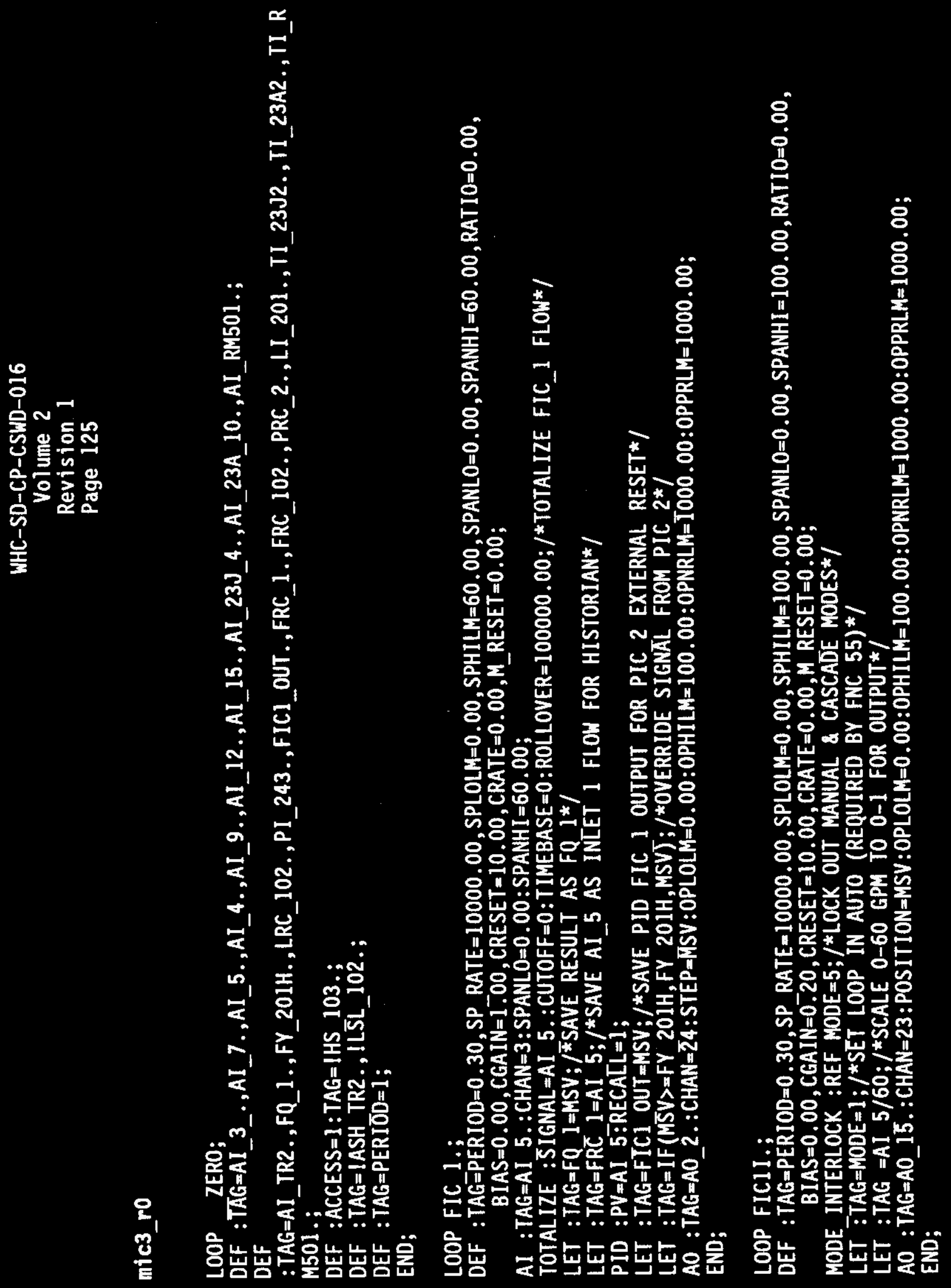

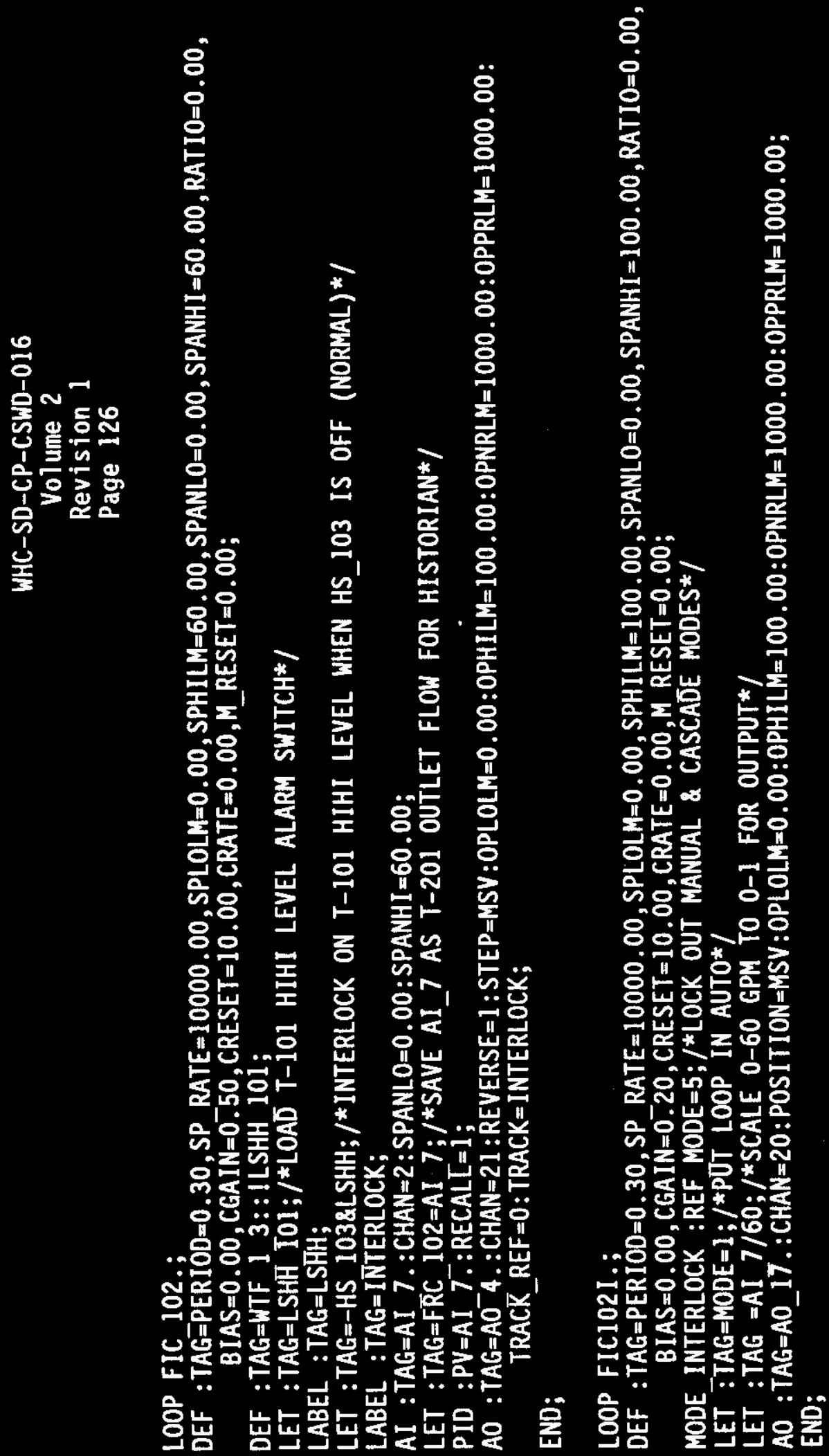
WHC-SD-CP-CSWD-016

Volume 2

Revision 1

Page 127

LOOP FY 203.

DEF :TAG $=P E R I O D=0.30, S P$ RATE $=10000.00, S P L O L M=0.00, S P H I L M=100.00$, SPANLO $=0.00, S P A N H I=100.00$, RATIO=0.00, $B I A S=0.00$, CGAIN $=0.20$, CRESET $=10.00$, CRATE $=0.00, M \_$RESET $=0.00$;

DEF :TAG=NTF 1 3: :AI 6;

LET :TAG $=A I-\overline{6} ; 7^{*}$ LOAD INLET 2 FLON $($ FIC_ 2$) * /$

LABEL :TAG=FLOW2;

MODE INTERLOCK :REF MODE $=5 ; / *$ LOCK OUT MANUAL \& CASCADE MODES*/

LET :TAG $=$ MODE $=1 ; / *$ PUT LOOP IN AUTO*/

LET :TAG $=$ AI $5+$ FLOW2; $/ * A D D$ FIC 1 AND FIC 2 FLOWS*/

LET :TAG =MSV $/ 30 ; / * S C A L E$ 0-30- GPH TO 0 -T FOR OUTPUT*/

LET :TAG $=$ IF (MSV $>=1,1$, MSV) $; /$ SET MAX TO OUTPUT AT $1 * /$

$A 0: T A G=A 07 .: C H A N=22: P O S I T I O N=M S V: O P L O L M=0.00: 0 P H I L M=100.00: 0 P N R L M=1000.00: 0 P P R L M=1000.00$;

EXPORT AI 5;

END;

LOOP LI102I.;

DEF :TAG $=P E R I O D=0.30, S P$ RATE $=10000.00, S P L O L M=0.00, S P H I L M=100.00, S P A N L O=0.00, S P A N H I=100.00, R A T I 0=0.00$, BIAS $=0.00$, CGAIN $=0.20$, CRESET $=10.00$, CRATE $=0.00$, M RESET $=0.00$;

DEF : TAG $=$ + +3 LAL 102.;

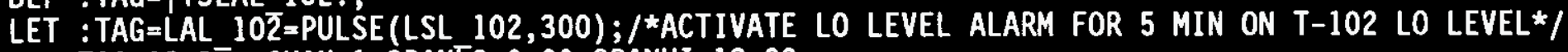

AI :TAG=AI 3-: :CHAN $=1:$ SPAN $[0=0.00:$ SPANHI $=18.00$

LET :TAG=LRC $102 .=A I 3 ; / *$ SAVE INPUT AS T-102 LEVEL (LRC 102)*/

MODE INTERLOCK :REF FODE $=5 ; / *$ LOCK OUT MANUAL \& CASCADE MODES*/

LET $:$ TAG $=$ MODE $=1 ; / *$ PUT LOOP IN AUTO*/

LET : TAG $=$ AI $3.120 ; / *$ SCALE $0-20$ FT TO 0 - 1 FOR OUTPUT* $/$

$A 0: T A G=A 0$ 8. $:$ CHAN $=19:$ POSITION=MSV:OPLOLM=0.00:0PHILM=100.00:0PNRLM $=1000.00: 0 P P R L M=1000.00$;

END; 

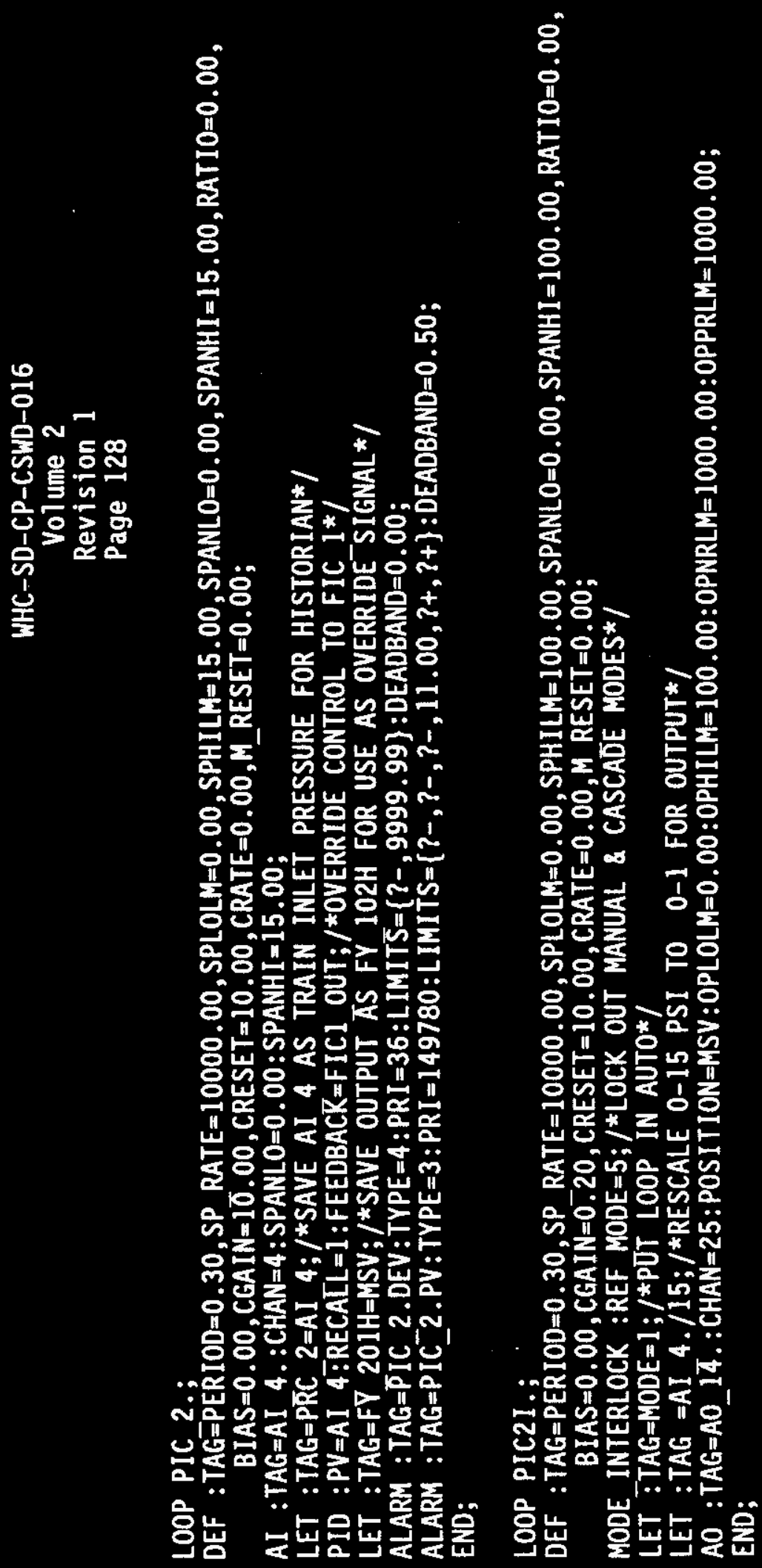
LOOP LIC 102;

DEF :TAG $=$ PERIÓD $=0.30$, SP RATE $=10000.00$, SPLOLM $=0.00$, SPHILM $=18.00$, SPANLO $=0.00$, SPANHI $=18.00$, RATI0 $=0.00$, $B I A S=0.00, C G A I N=5.00$, CRESET $=0.01, C R A T E=0.00, M$ RESET $=0.00$;

PID : PV $=A I 3 \ldots$ : BALANCING $=2:$ DIRECT $=1:$ RECALL $=1 ; / * T-10 \overline{2}$ LEVEL CONTROLLER* $/$

PID CASCADE :TAG $=$ FIC $1 . ; /$ CASCADE TO FIC $1 * /$

AI :TAG=AI 9.: CHAN=5: SPANLO=0.00:SPANHI $=100.00$;

LET :TAG=AI TR2.=AI 9.;/*SAVE INPUT AS OUTPUT CONDUCTIVITY (AI TR2)*/

AI : TAG $=A I$ I2.: $C H A N=6:$ TYPE $=10: L 0=0.00: H I=10.00: S P A N L O=0.00: S P A N H I=0.20$;

LET :TAG $=$ PI 243.=AI 12.; *SAVE INPUT AS BUILLDING DP (PI 243)*/

AI : TAG=AI I5.: CHAN=7:SPANL0=0.00:SPANHI $=46.00$;

LET :TAG=LI 201.=AI 15.;/*SAVE AI 15 AS KOH TANK LEVEL*/

AI :TAG $=A I$ 23J 4.: CHAN $=8:$ SPANLO=0.00:SPANHI $=100.00$

LET :TAG $=$ TI 23J2.=AI 23J 4.;/*23J-3002 TEMP*/

AI $: T A G=A I$ 23A 10.: CHAN $=\overline{9}:$ SPANLO $=0.00:$ SPANHI $=100.00$;

LET :TAG=TI 23A2. =AI 23A 10.;/*VP-23A-2 COOLANT TEMP*/

AI : TAG $=A I$ RM501.: CHAN $=10$ : $S P A N L O=0.00: S P A N H I=100.00$;

LET :TAG=TI RM501.=AI RM501.;/*291- RM 501 TEMP*/

EXPORT ASH TR2;

END;

LOOP NINETY NINE;

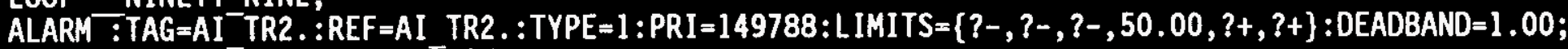

LET :TAG=ASH TR2=AI TR2OXM2:

ALARM :TAG=LRC 102.: REF=LRC 102.: TYPE=1:PRI =148236:LIMITS=\{?-,?-,2.00,11.50,13.50, ?+\}:DEADBAND=0.20;

LET :TAG=LSL 102=LRC 102OXM1;

ALARM :TAG=LI 20T.:REF $=$ LI 201.:TYPE $=1:$ PRI $=149602:$ LIMITS $=\{?-,-10.00,-10.00, ?+, ?+, ?+\}:$ DEADBAND=0.50;

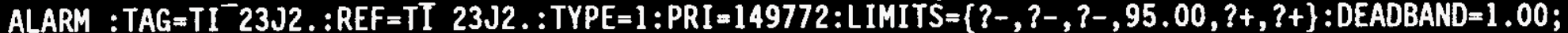

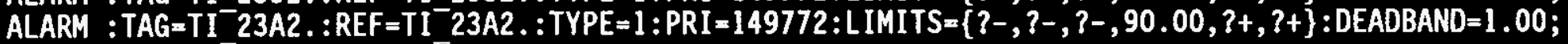

DEF :TAG=PERIOD $=1$;

END; 


\section{WHC-SD-CP-CSWD-016}

Volume 2

Revision 1

Page 130

\section{mic4_ro}

LOOP ZERO;

DEF :TAG=!DI $1 .$, IDI 20.,IDI 21.,IDI_24.,IDI 25.,IDI_30.,IDI_31.,IDI_36.,IDI_37., IDI_5., IDI_23A_1., IDI 23A 3., IOT 23J 1., IDI 23A 4., IDI $23 A$ 2.;

DEF : ACCESS $=1$ : TAG $=$ !HS 28 ., IHS 3T., IHS 101A., IHS $23 \mathrm{~A} 1 . ;$

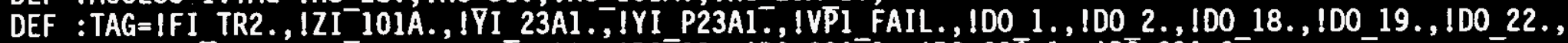

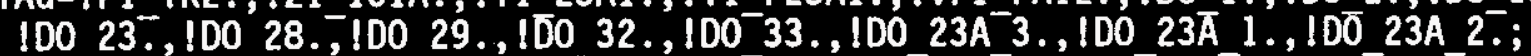

DEF :TAG=\#ZI MOV1., \#ZI_MOV3.,\#ZI_MOV5., \#ZI_MOV7.;

DEF : TAG $=$ PERTOD $=1$;

END;

LOOP MOV1.;

DEF : TAG $=$ PERIOD $=0.30$, SP RATE $=10000.00$, SPLOLM $=0.00$, SPHIL $H=100.00$, SPANLO $=0.00$, SPANHI $=100.00$, RATI $10=0.00$, BIAS $=0.00$, CGAIN $=0.20$, CRESET $=10.00$, CRATE $=0.00, M$ RESET $=0.00 ;$

DI : $T A G=D I$ 20. : $C H A N=-4$;

DI :TAG=DI 21 : : $C H A N=-5$;

MULTI STATE DI :TAG=ZI MOV1.:DI 1=DI_21.:DI2=DI 20.;

$D O: T A G=D O$ I8. : $C H A N=6: D A D=0$;

DO : $T A G=D 0^{-} 19 .:$ CHAN $=7: D A D=0$;

DEF : TAG $=1+3 Z$ A $M O V 1 . ;$

VALVE CONTROL $:$ SHITCH=HS 28.:OPEN=DO 18.:OPEN_TIME $=30.00: C L O S E=D 0 \_19 .:$ CLOSE_TIME $=30.00: S E N S E$ OPEN=DI_21.: END; 
WHC-SD-CP-CSWD-016

Volume 2

Revision 1

Page 131

LOOP MOV3.;

DEF :TAG $=$ PERIOD $=0.30$, SP_RATE $=10000.00, S P L O L M=0.00, S P H I L M=100.00, S P A N L 0=0.00, S P A N H I=100.00$, RATI0 $=0.00$, BIAS $=0.00$, CGAIN $=0.20$, CRESET $=10.00$, CRATE $=0.00, M$ RESET $=0.00$;

DI :TAG=DI 24.: CHAN=-8;

DI :TAG $=0 \mathrm{I}-25$. $\mathrm{CHAN}=-9$;

MULTI STATE DI :TAG=ZI MOV3.:DI1=DI 25.:DI2=DI 24.;

$D O: T \bar{A} G=D O$ 22. :CHAN $=10 \overline{0} D A D=0$;

$D 0: T A G=D 0^{-} 23 .: C H A N=11: D A D=0$;

DEF :TAG $=1 \overline{+}+32$ A MOV3.;

VALVE CONTROL :SWITCH=HS 28.:OPEN=DO 22.:OPEN TIME $=30.00: C L O S E=D 0$ 23.:CLOSE TIME=30.00:SENSE OPEN=DI 25.: END;

SENSE CLOSE $=$ DI 24. : ALARM=ZA MOV3.:ALARM TIME=40.00;

LOOP MOV5.:

DEF :TAG $=$ PERIOD $=0.30$, SP RATE $=10000.00, S P L O L M=0.00, S P H I L M=100.00, S P A N L 0=0.00, S P A N H I=100.00, R A T I 0=0.00$, BIAS $=0.00$, CGAIN $=0.20$, CRESET $=10.00$, CRATE $=0.00, M$ RESET $=0.00$;

DI : $T A G=01$ 30. $\mathrm{CHAN}=-12$;

DI :TAG=DI 31. : CHAN $=-13$;

MULTI STATE DI :TAG=ZI_MOV5.:DI1=DI_31.:DI2=DI_30.;

$D 0: T A G=D 0$ 28. $:$ CHAN $=14: D A D=0$;

DO :TAG $=\mathrm{DO}^{-} 29 .: \mathrm{CHAN}=15: \mathrm{DAD}=0$;

DEF :TAG $=1+3 Z$ A HOV5.;

VALVE CONTROL :SWITCH=HS 31.:0PEN=DO 28.:0PEN TIME=30.00:CLOSE=DO 29.:CLOSE TIME=30.00:SENSE OPEN=DI 31.: END; SENSE_CLOSE $=$ DI_30.:ALARM =ZA MOVI5.:ALARM_TIME $=40.00$; 
WHC-SD-CP-CSHD-016

Volume 2

Revision 1

Page 132

LOOP MOV7.:

DEF :TAG $=$ PERIOD=0.30,SP_RATE $=10000.00$, SPLOLM $=0.00$, SPHILM $=100.00$, SPANLO $=0.00, S P A N H I=100.00$, RATI $10=0.00$, $B I A S=0.00$, CGAIN $=0.20$, CRESET $=10.00$, CRATE $=0.00, M$ RESET $=0.00 ;$

DI :TAG $=$ DI 36. : $\mathrm{CHAN}=-16$;

DI :TAG $=0 I^{-} 37 .:$ CHAN $=-17$;

MULTI STATE DI :TAG=ZI MOV7.:DI1=DI_37.:DI2=DI_36.;

$D O: T A G=D O \quad \overline{32}$ : $C H A N=18: D A D=0$;

DO : $\mathrm{TAG}=\mathrm{DO} \mathrm{O}^{-} 33 . \mathrm{CHAN}=19: \mathrm{DAD}=0$;

DEF : TAG $=1 \overline{+} 32$ A MOV7.;

VALVE_CONTROL $:$ SWITCH=HS 31.:OPEN=D0 32.:OPEN_TIME=30.00:CLOSE=D0_33.:CLOSE_TIME=30.00:SENSE_OPEN=DI_37.: END; SENSE CLOSE $=$ DI 36. :ALARM=ZA MOV7.:ALARH TIME $=40.00$;

LOOP P101A.;

DEF : TAG $=P E R I O D=0.30, S P$ RATE $=10000.00, S P L O L M=0.00, S P H I L M=100.00$, SPANL $0=0.00$, SPANHI $=100.00$, RATI0 $=0.00$, $B I A S=0.00, C G A I N=0.20$, CRESET $=10.00$, CRATE $=0.00, M$ RESET $=0.00$

DEF :TAG=WTF 1 3::!lSL 101,WTF_1_3:: ILSH_101,WTF_2_3::IHS_101L,WTF_2_3::!HS_101,WTF_2_2::121_1018,

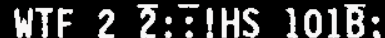

LET :TAG $=\left[S\left[101 ; /^{\mp}\right.\right.$ LOAD SUMP LO LEVEL ALARM*/

LABEL :TAG=LOLVL;

LET :TAG=LSH $101 ; / \star S U M P$ HI LEVEL ALARM*/

LABEL :TAG=HILVL;

LET :TAG=HS 101L;/*SUMP LO LEVEL BYPASS*/

LABEL :TAG $=\bar{B} Y P A S S ;$

LET :TAG=HS $101 ; / * P-101 A / B$ SELECTOR SWITCH*/

LABEL :TAG=SLCT;

LET :TAG $=2$ I 101B; /*PUMP 101B STATUS*/

LABEL :TAG=STATB;

LET :TAG $=$ HS 101B;/*PUMP 1018 ON/OFF SWITCH*/

LABEL : TAG $=$ SWTB;

DEF :TAG $=1+3 \times A$ 101A,;

DI : TAG $=0$ I $1 .: \bar{C} H A N=1$;

LET :TAG=ZI 101A=DI 1;/*SAVE DI_1 AS PUMP 101A STATUS*/

DO :TAG=DO_I.:CHAN= $\overline{2}: D A D=0$; 


\section{WHC-SD-CP-CSWD-016 \\ Volume 2}

Revision 1

Page 133

DO : TAG $=00$ 2.: $\mathrm{CHAN}=3: \mathrm{DAD}=0$;

LET :TAG $=($ PULSE $(-1$ SLCT) (PULSE(SLCT\&-ISTATB)\&SWTB)); $/ *$ PULSE ON HS 101=A OR (HS 101B \& PUMP FAILED)*/

GOTO : COND $=-$ !MSV:TAG=JUMPA; $/$ JUMP NEXT STEP IF NO PULSE*/

LET : TAG $=$ HS 101A=1;/*TURN PUMP ON WHEN HS 101 "ON" INTERLOCK ACTIVE*/

LABEL :TAG= JUMPA;

LET :TAG=PULSE(SLCT); /*SEND PULSE WHEN HS 101="P-1018" (ON)*/

GOTO :COND=-1MSV:TAG=JUMPB; $/ * J U M P$ NEXT LINE WHEN NO PULSE*/

LET : TAG $=$ HS $101 \mathrm{~A}=0 ; /$ *TURN OFF PUMP WHEN HS 101 "OFF" INTERLOCK ACTIVE*/

LABEL :TAG=JUMPB;

LET :TAG $=-$ IBYPASS\&LOLVL; $/ *$ LO INTERLOCK (ONLY WHEN BYPASS IS OFF) $*$ I

GOTO :COND $=-1$ MSV:TAG $=$ JMPLO; $/ *$ JUMP NEXT STEP IF NO LOW LEVEL ALARM*

LET :TAG=HS 101A=0;/*PUMP OFF ON LO LEVEL INTERLOCK*/

LABEL :TAG=JMPLO;

LET :TAG=PULSE(HILVL\&-1SLCT); $/ *$ PULSE ON HI LEVEL ALARM WHEN HS_101 $={ }^{\prime} A^{\prime} * /$

GOTO :COND =-!MSV:TAG=JMPHI; $/$ JUMP NEXT STEP IF NO PULSE* $/$

LET :TAG=HS $101 \mathrm{~A}=1 ; /$ *PUMP ON ON HI INTERLOCK $\star / /$

LABEL :TAG=JMPHI;

MOTOR CONTROL :SWITCH=HS 101A:START=DO 1:STOP=DO 2:START TIME=5.00:STOP TIME=1.00;

LET :TAG=HS 101A\&-DI_18-DŌ_1;/*ALARM WHEN MOTOR STATUS OFF AND SHITCH ON' (EXCEPT WHEN PUMP IST TURNED ON) $/$

LET :TAG =XA 101A=MSV;

EXPORT 2I_101A,HS_101A;

END; 


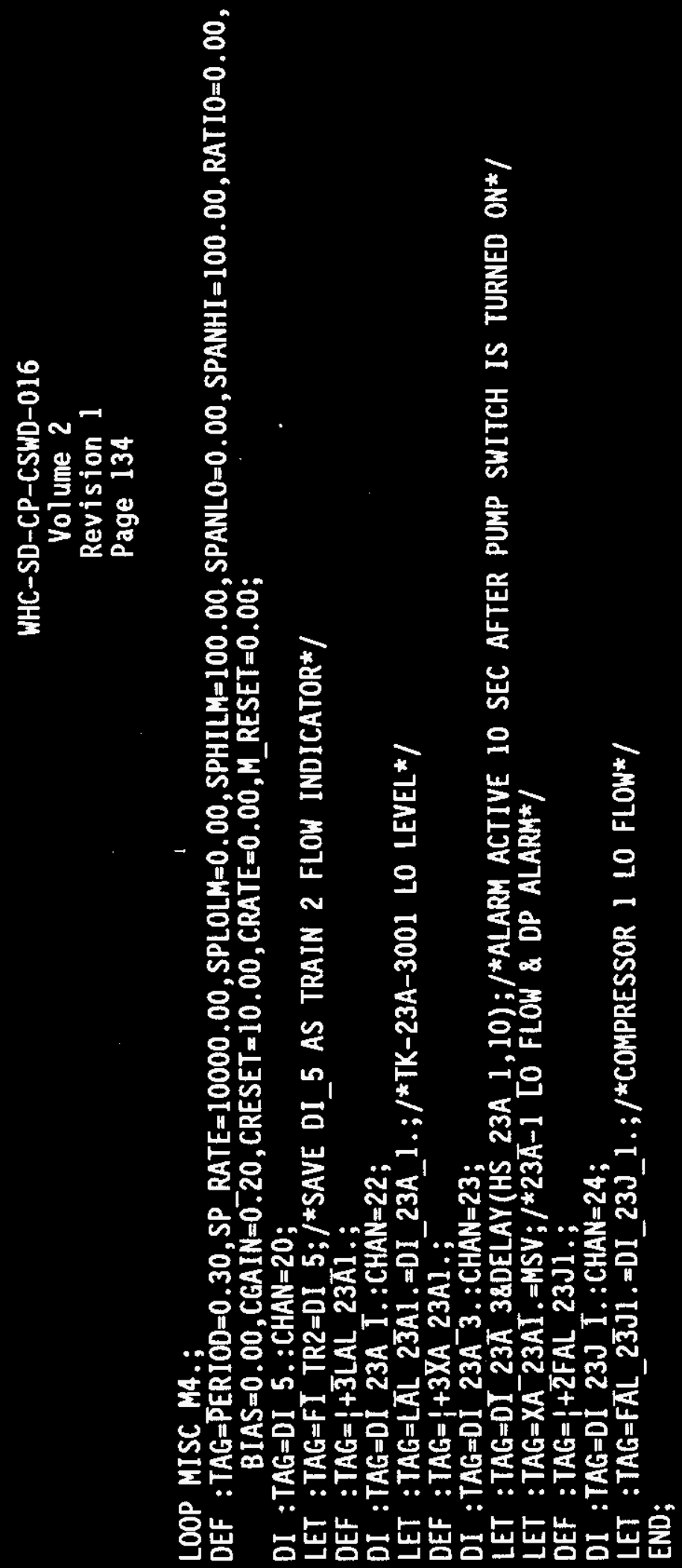




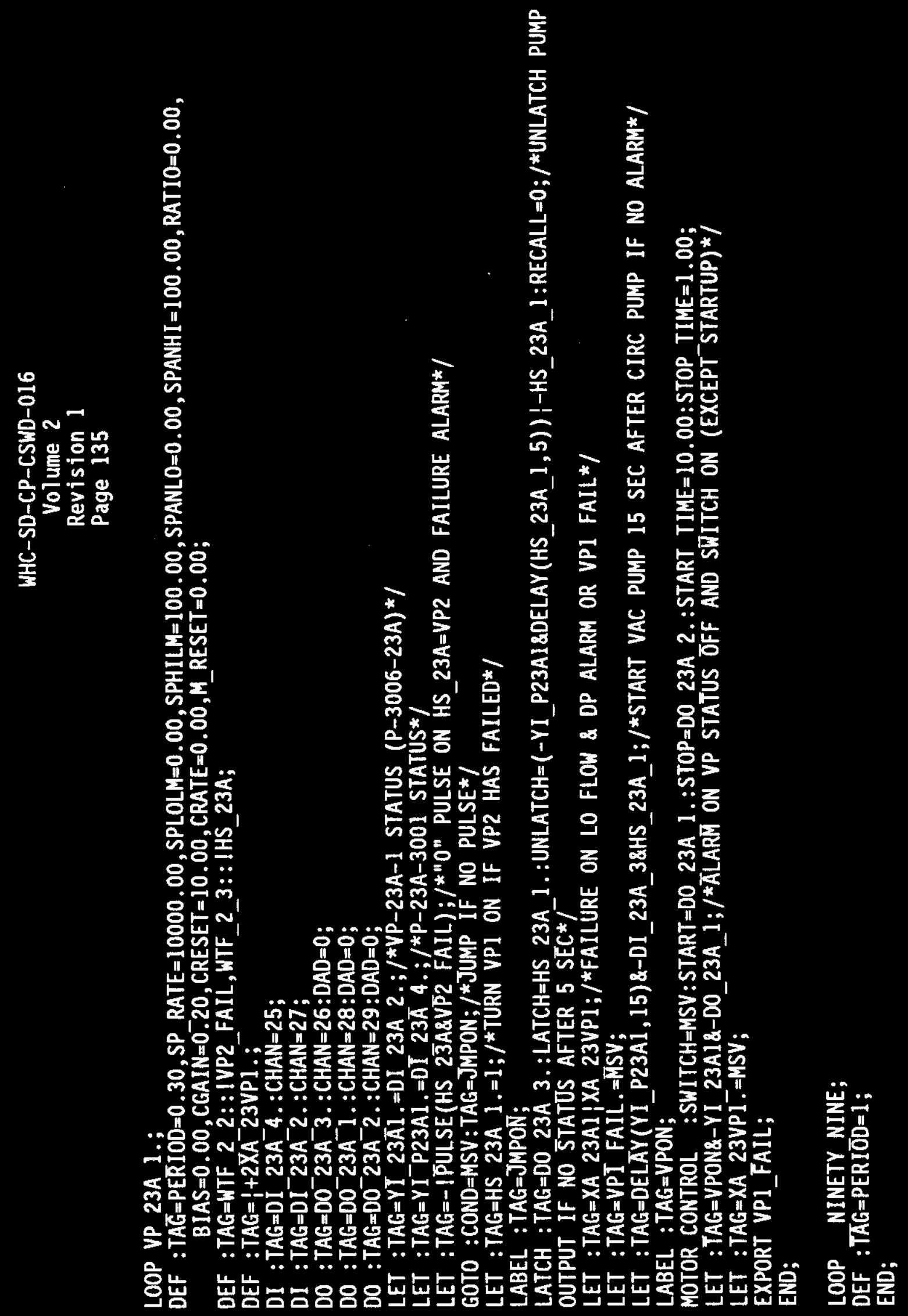



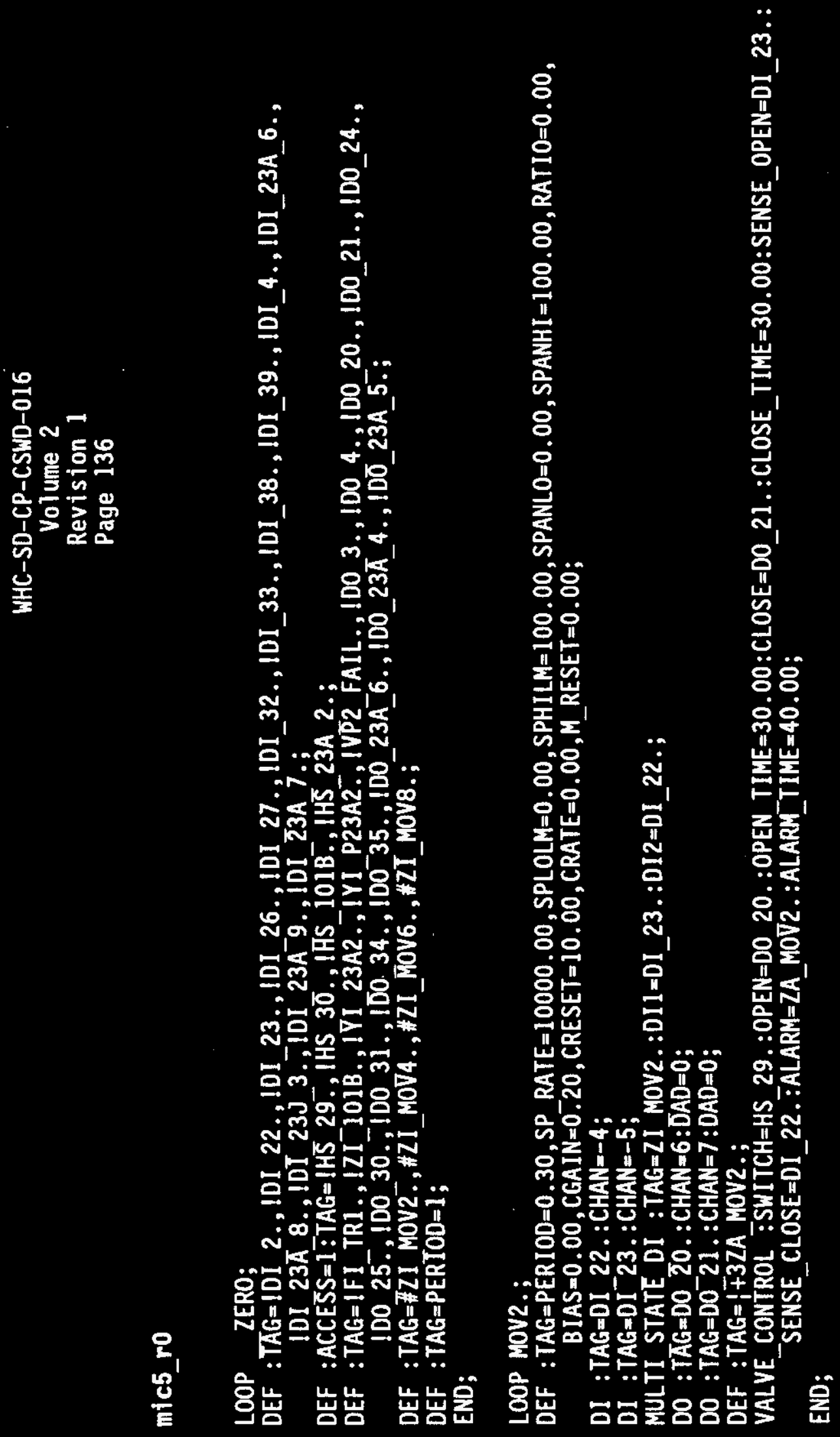


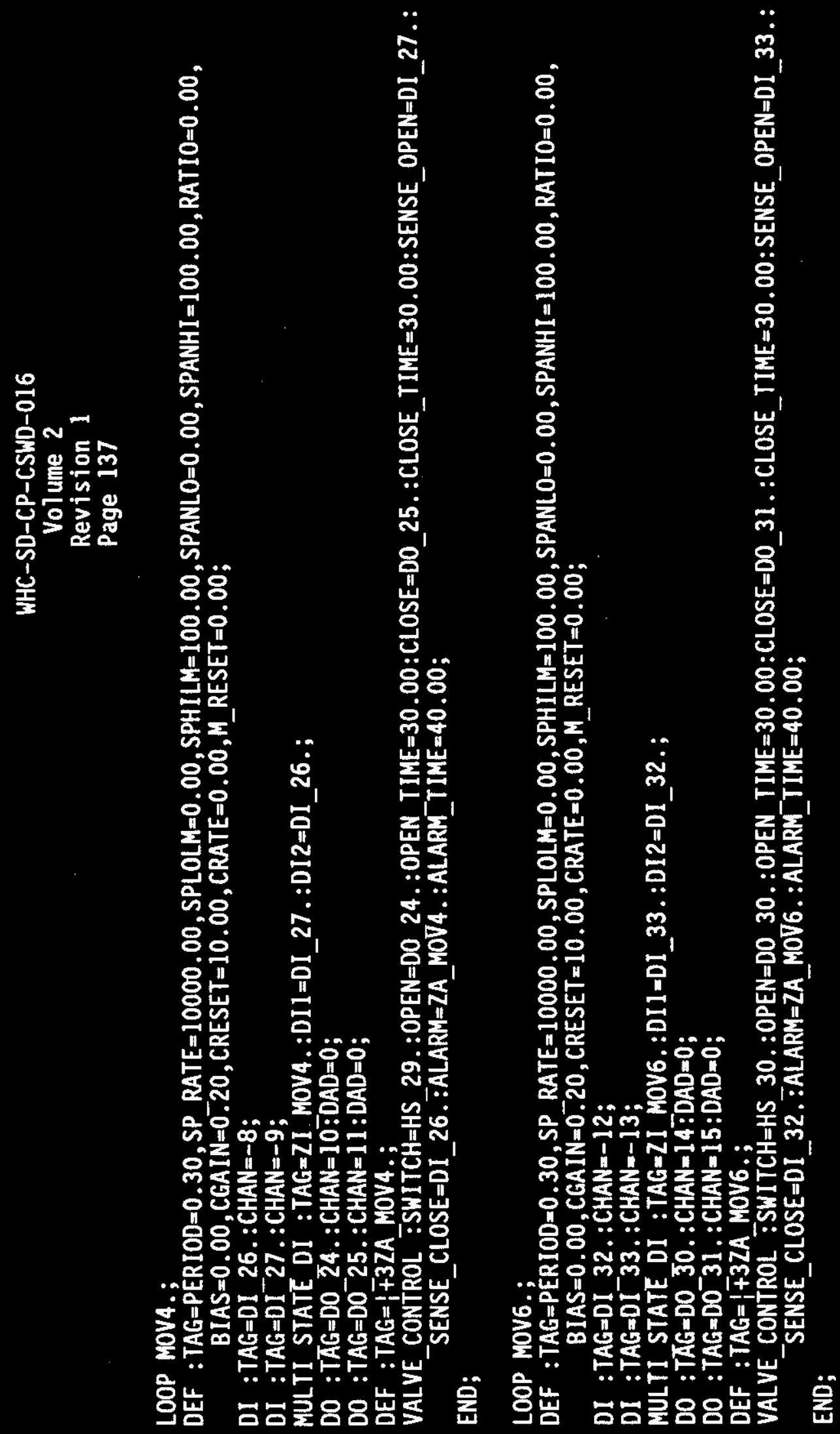




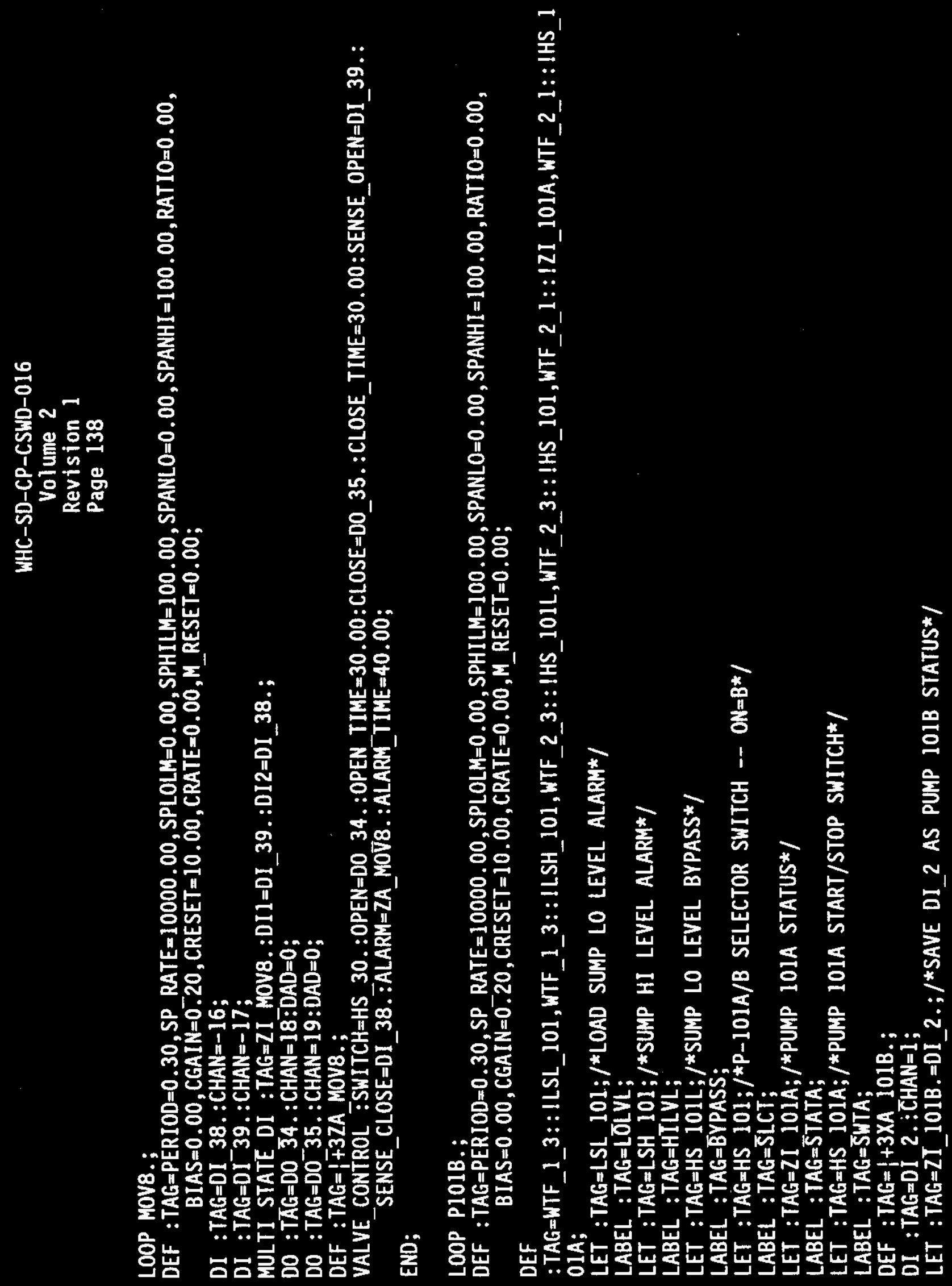




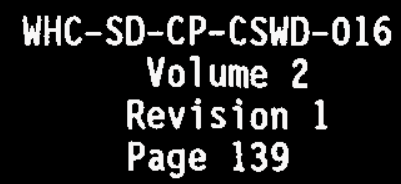

DO :TAG $=00$ 3.:CHAN $=2: D A D=0$;

DO : $T A G=D O-4 .: C H A N=3: D A D=0$;

LET :TAG=(PULSE(SLCT) $\{$ PULSE(-!SLCT\&-ISTATA)\&SWTA));/*PULSE WHEN HS_101=B OR (HS_101=1 AND PUMP FAILED) $/$ GOTO :COND=-!MSV:TAG=JUMPA; *JUMP NEXT STEP IF NO PULSE*/

LET :TAG=HS 101B $=1 ; /$ *TURN PUMP ON WHEN HS_101 "ON" INTERLOCK ACTIVE*/

LABEL :TAG=JUMPA:

LET :TAG=PULSE $(-I S L C T) ; / * S E N D$ PULSE WHEN HS $101=" P-101 A^{n}($ OFF)*/

GOTO : COND $=-1$ MSV:TAG=JUMPB; $/$ *SKIP NEXT STEP WHEN NO PULSE*/

LET :TAG=HS 101B=0;/*TURN OFF PUMP WHEN HS_101 "OFF" INTERLOCK ACTIVE*/

$\angle A B E L$ : TAG=JUMPB;

LET :TAG $=-$ !BYPASS\&LOLVL; $/$ LO INTERLOCK (ONLY WHEN BYPASS IS OFF) $/$

GOTO :COND=-IMSV:TAG=JMPLO; / JUMP NEXT STEP IF NO LOW LEVEL ALARM*/

LET :TAG $=$ HS $1018=0 ; / *$ PUMP OFF ON LO LEVEL INTERLOCK */

LABEL :TAG=JMPLO;

LET :TAG=PULSE(HILVL\&SLCT); $/ *$ PULSE ON HI LEVEL ALARM \& $P$ 101 $=^{\prime} B^{\prime *}$ /

GOTO : COND $=-$ IMSV:TAG=JMPHI; $/$ JUMP NEXT STEP IF NO PULSE $* \overline{7}$

LET :TAG $=$ HS $101 \mathrm{~B}=1 ; /$ *PUMP ON ON HI LEVEL INTERLOCK* $/$

LABEL :TAG=JMPHI;

MOTOR CONTROL :SWITCH=HS 101B:START=DO 3:STOP=DO 4:START_TIME=5.00:STOP TIME=1.00;

LET :TAG=HS 101B\&-DI 2\&-DO 3;/*ALARM WHEN MOTOR STAUS OFF AND SWITCH ON (EXCEPT WHEN PUMP 1ST TURNED ON)*/ LET :TAG $=X A^{-} 101 B=M S V ;$

EXPORT ZI_101B,HS_101B;

END; 
WHC-SD-CP-CSWD-016

Volume 2

Revision 1

Page 140

LOOP MISC M5.;

DEF :TAG $=\bar{P} E R I O D=0.30, S P$ RATE $=10000.00, S P L O L M=0.00, S P H I L M=100.00, S P A N L 0=0.00, S P A N H I=100.00, R A T I 0=0.00$, $B I A S=0.00, C G A I N=0.20$, CRESET $=10.00$, CRATE $=0.00, M$ RESET $=0.00$;

DI : TAG=DI 4.:CHAN=20;

LET : TAG $=F I$ TRI $=D I$ 4;

DEF : TAG $=\{+\overline{3} L A L ~ 23 A 2$;

DI :TAG $=$ DI $23 A \overline{6}$.: CHAN $=22$;

LET :TAG=LAL 23̈A2.=DI_23A_6.; /*TK-23A-3002 LO LEVEL*/

DEF : $T A G=1+3 X A$ 23A2.;

DI :TAG $=0 \mathrm{I}$ 23A-8. $\mathrm{CHAN}=23$;

LET :TAG=DI 23A 88DELAY(HS 23A 2,10); $/$ ALARM ACTIVE 10 SEC AFTER PUMP SWITCH IS TURNED ON*/

LET :TAG $=X A^{-} 23 A \overline{2}$. =MSV; $/ * V P=23 A-2$ LO FLOW \& DP ALARM $*$

DEF : TAG $=1+\overline{2} F A L$ 23J2.;

DI :TAG=DI 23J 3. :CHAN $=24$;

LET :TAG=FAL_23J2.=DI_23J_3.; $/$ COMPRESSOR 2 LO COOLANT FLOW*/

END; 
WHC-SD-CP-CSWD-016

Volume 2

Revision 1

Page 141

LOOP VP 23A 2.;

DEF :TAG $=P E \bar{R} I O D=0.30, S P \_R A T E=10000.00, S P L O L M=0.00, S P H I L M=100.00, S P A N L 0=0.00, S P A N H I=100.00, R A T 10=0.00$, $B I A S=0.00$, CGAIN $=0.20$, CRESET $=10.00$, CRATE $=0.00, M$ RESET $=0.00$;

DEF :TAG=WTF 2 1::IVP1 FAIL, WTF 2 3::IHS 23A;

DEF :TAG $=1+2 X A-23 \cup P 2$;

DI :TAG $=$ DI 23A-9.:CHAN $=25$;

DI :TAG $=\mathrm{DI}^{-23 A^{-}}$7.: $\mathrm{CHAN}=27$;

DO :TAG $=00^{-23 A}$-6.:CHAN $=26: D A D=0$;

$D 0: T A G=D 0^{-23 A} 4 .: C H A N=28: D A D=0$;

DO : $T A G=D 0^{-} 23 A^{-} 5 .: C H A N=29: D A D=0$;

LET :TAG $=Y I$ 23A2. $=0$ I $23 A 7 . ; / * V P-23 A-2$ STATUS $(P-3014-23 A) * /$

LET : TAG $=Y I-P 23 A 2 .=0 T 23 \bar{A} 9 ; / * P-23 A-3002$ STATUS $* 1$

LET :TAG $=-1$ PULSE $(-H S$ 23A\&VP1 FAIL); $/ * 0 "$ PULSE ON HS_23A=VP1 AND FAILURE ALARM*

GOTO :COND=MSV:TAG=JMPON: $/$ JUMP If NO PULSE*/

LET :TAG $=$ HS $23 A 2 .=1 ; / *$ TURN VP2 ON IF VP1 HAS FAILED*/

LABEL :TAG=JMPON;

LATCH :TAG=D0 23A 6.:LATCH=HS 23A 2.:UNLATCH=(-YI_P23A28DELAY(HS_23A_2,5))!-HS_23A_2:RECALL=0;/ *UNLATCH PUMP OUTPUT IF NO-STATUS AFTER 5 SEC*/

LET :TAG $=X A$ 23A2;XA 23VP2;/*FAILURE ON LO FLOW \& DP OR VP2 FAIL*/

LET :TAG=VPZ̃ FAIL. $=$ MSV;

LET :TAG=DELAY(YI_P23A2,15)\&-DI_23A_8\&HS_23A_2;/*START VAC PUMP 15 SEC AFTER CIRC PUMP IF NO ALARM*/

LABEL :TAG=VPON;

MOTOR CONTROL :SHITCH=MSV:START=DO 23A 4.:STOP=DO 23A 5.:START TIME=10.00:STOP TIME=1.00;

LET :TAG=VPON\&-YI $23 A 2 \&-D O 23 A 4 ; / *$ ALARM ON VP STATUS OFF AND SNITCH ON (EXCEPT STARTUP) $\star$;

LET :TAG=XA 23VP2, = HSV;

EXPORT VP2 FAIL;

END;

LOOP NINETY NINE;

DEF : TAG $=P E R I \bar{O} D=1$;

END; 


\section{WHC-SD-CP-CSWD-016}

Volume 2

Revision 1

Page 142

mic6_ro

LOOP ZERO;

DEF $: T A G=! D I 3 .$, IDI 28., IDI 40,, IDI 41.,IDI_6., IDI 11.,!DI 12., !LDI_84A., !LDI_84B.;

DEF : ACCESS $=\mathrm{T}$ : TAG $=$ !HS 32 ., !HS 101., THS 101L., IHS 201., IHS $\overline{2} 3 \mathrm{~A}$.;

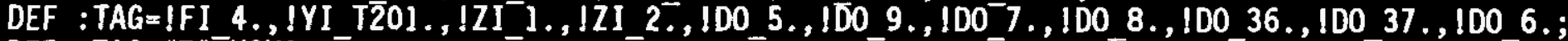

DEF :TAG =\#ZI MOV9.;

DEF : TAG $=P E R T O D=1$;

END;

LOOP MOV9.;

DEF :TAG $=$ PERIOD $=0.30, S P$ RATE $=10000.00, S P L O L M=0.00, S P H I L M=100.00, S P A N L O=0.00, S P A N H I=100.00, R A T 10=0.00$, BIAS $=0.00$, CGAIN $=0.20$, CRESET $=10.00$, CRATE $=0.00, M$ RESET $=0.00$;

DI : $T A G=D I$ 40.: CHAN=9;

DI :TAG $=D I^{-1} 41$ : $C H A N=10$;

MULTI STATE DI :TAG=2I MOV9.:DI1=DI_40:DI2=DI_41;

DO :TAG $=00$ 36.: $\mathrm{CHAN}=11: \mathrm{DAD}=0$;

DO : $T A G=D 0^{-} 37 .:$ CHAN $=12: D A D=0$;

DEF : TAG $=1 \mp 3 Z$ A MOV9.;

VALVE_CONTROL :SWITCH=HS 32.:OPEN=DO 36.:OPEN_TIME $=30.00:$ CLOSE=D0_37.:CLOSE_TIME=30.00:SENSE_OPEN=DI 40.: END; SENSE_CLOSE=DI_41.:ALARM=ZA_MOV9.:ALARM_TIME=40.00; 


\section{WHC-SD-CP-CSWD-016 \\ Volume 2 \\ Revision 1}

Page 143

LOOP P MET.;

DEF :TAG $=P E R I O D=0.30, S P$ RATE $=10000.00, S P L O L M=0.00, S P H I L M=100.00$, SPANLO $=0.00$, SPANHI $=100.00$, RATI0=0.00, BIAS $=0.00$, CGAIN $=0.20$, CRESET $=10.00$, CRATE $=0.00$, M_RESET $=0.00$;

DI :TAG $=$ DI 28.: CHAN $=5$;

LET :TAG=YI T201=DI 28;/*SAVE DI_28 AS KOH PUMP STATUS*/

DO : TAG $=$ DO $\overline{9} .:$ CHAN $=\overline{6}: D A D=0$;

DEF :TAG $=$ + XA_201.;

LET :TAG $=00$ 9=PULSE(HS 201,05);/*MOTOR CONTROL -- START ON FOR 5 SEC (NO STOP) $/$

LET :TAG=HS 2018-DI 28\%-DO 9; $/$ *ALARM WHEN MOTOR STATUS OFF AND SWITCH ON (EXCEPT WHEN PUMP 1ST TURNED ON)*/

LET :TAG $=X A^{-} 201=M S V$;

END; 


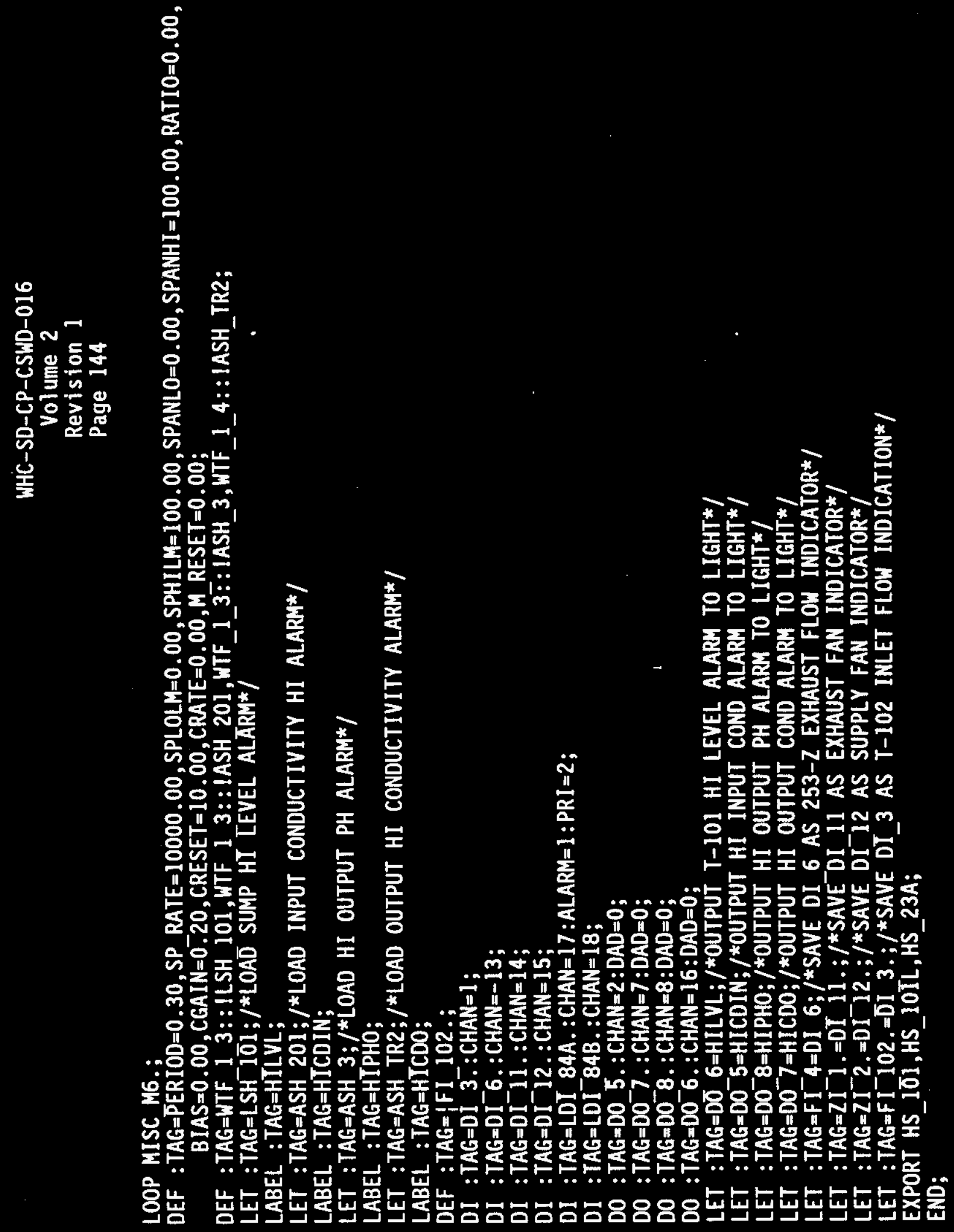



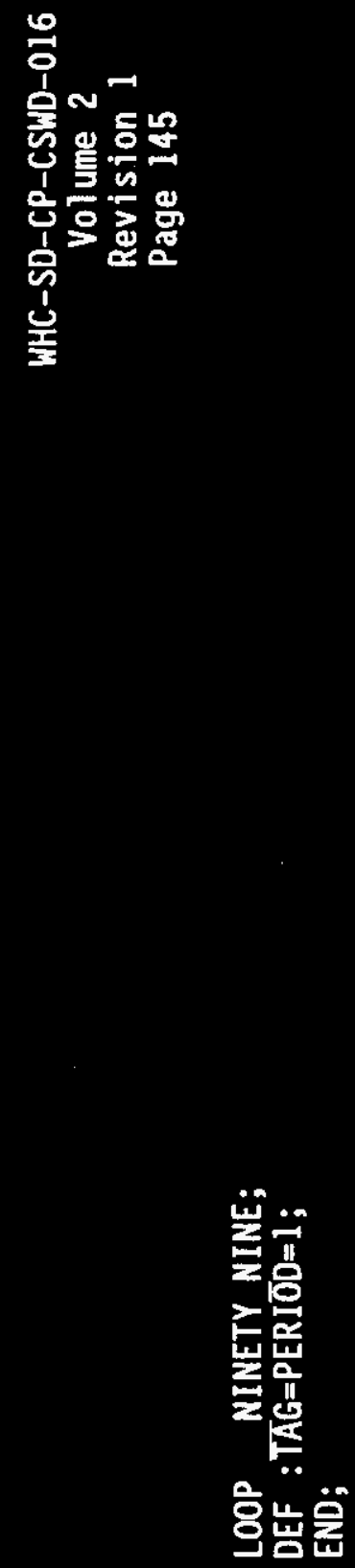
WHC-SD-CP-CSWD-016

Volume 2

Revision 1

Page 146

\section{APPENDIX A: MICON INPUT/OUTPUT}

Analog inputs and discrete Input/Outputs can be set up in different ways. Analog inputs can be set to accept one of three types of signals: a 4-20 miliamp input with the MICON powering the loop, a 4-20 miliamp input with an external power supply, or a voltage input. Twentyfour volt discrete input/outputs can be set up as an input only, an output only, or as both an input and output. Since the hardware requirements are different for each of these setups, the controller cards have on-board jumpers for each analog input or discrete. These jumpers must be set to the desired type of analog input or discrete for the card to work properly.

Analog outputs are 4-20 miliamp internally powered only. Since they can't be changed they don't have any jumpers.

Before replacing an existing card with a new one or installing a backup card, you must make sure the jumpers in the new card are set the same as the jumpers in the old card.

The following tables show the Input/Output types for each controller card in the 243-Z cabinet. See the RCM-32 Remote Control Module User Manual (Vendor Information file 22068) for a description of the I/0 jumper placement. 
WHC-SD-CP-CSWD-016

Volume 2

Revision 1

Page 147

MICON Number: 2 Tag Name:WTF_1_3

Location: 243-Z Rack Number: 1

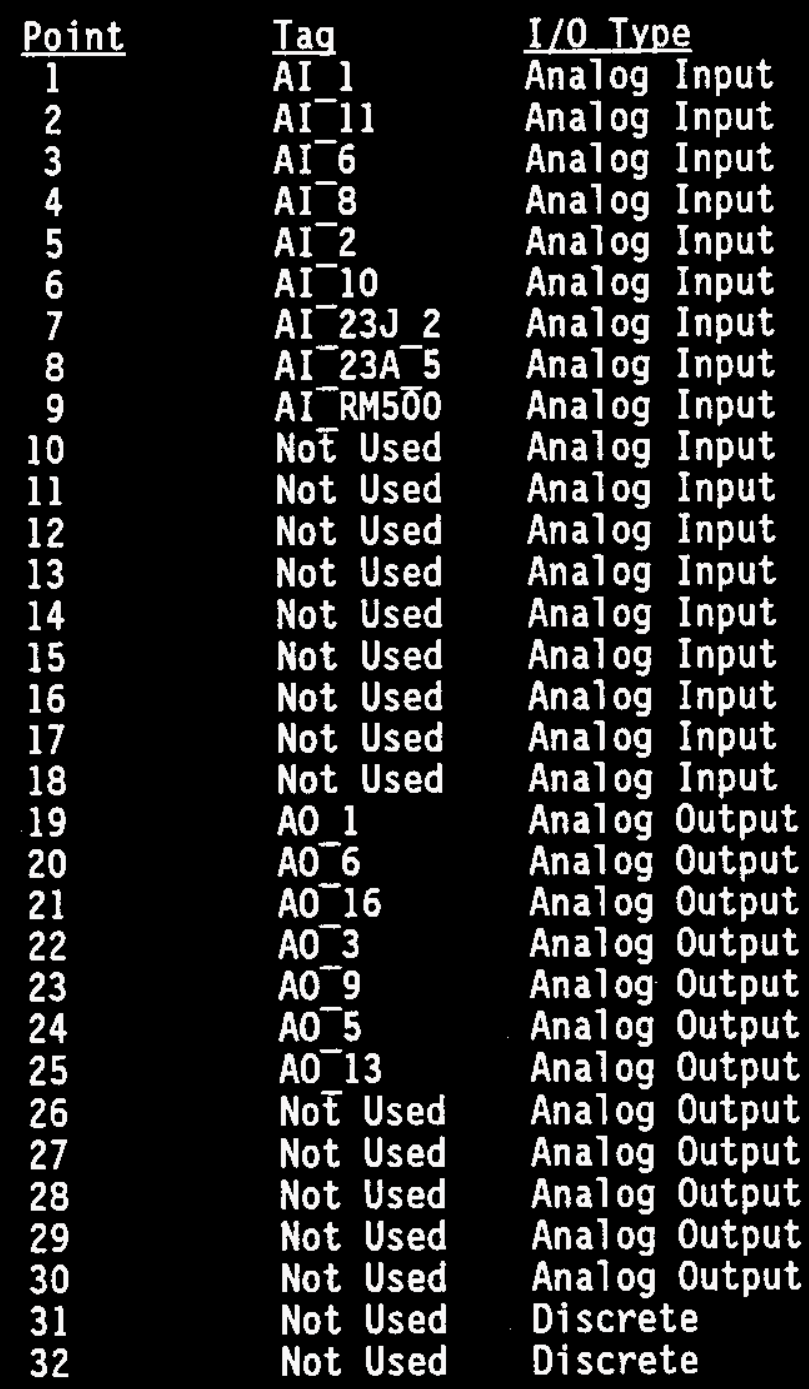

Type: $\mathrm{RCH}-32 \mathrm{C}$

S1ot Number: 3

Jumper Assignment
4-20 mA - External Power
$4-20 \mathrm{~mA}$ - External Power
$4-20 \mathrm{~mA}$ - External Power
4-20 mA - External Power
4-20 mA - MICON Powered
4-20 mA - External Power
4-20 mA - External Power
4-20 mA - External Power
4-20 mA - External Power
Voltage
Voltage
Voltage
Voltage
Voltage
Voltage
Voltage
Voltage
Voltage
N/A
N/A
N/A
N/A
N/A
N/A
N/A
N/A
N/A
N/A
N/A
N/A
Input \& Output
Input \& Output

${ }^{1}$ See RCM-32 Remote Control Module User Manual for description of I/0 jumper placement. 

WHC-SD-CP-CSWD-016
Volume 2
Revision 1
Page 148

MICON Number: 3 Tag Name:WTF_1_4

Location: 243-Z Rack Number: 1

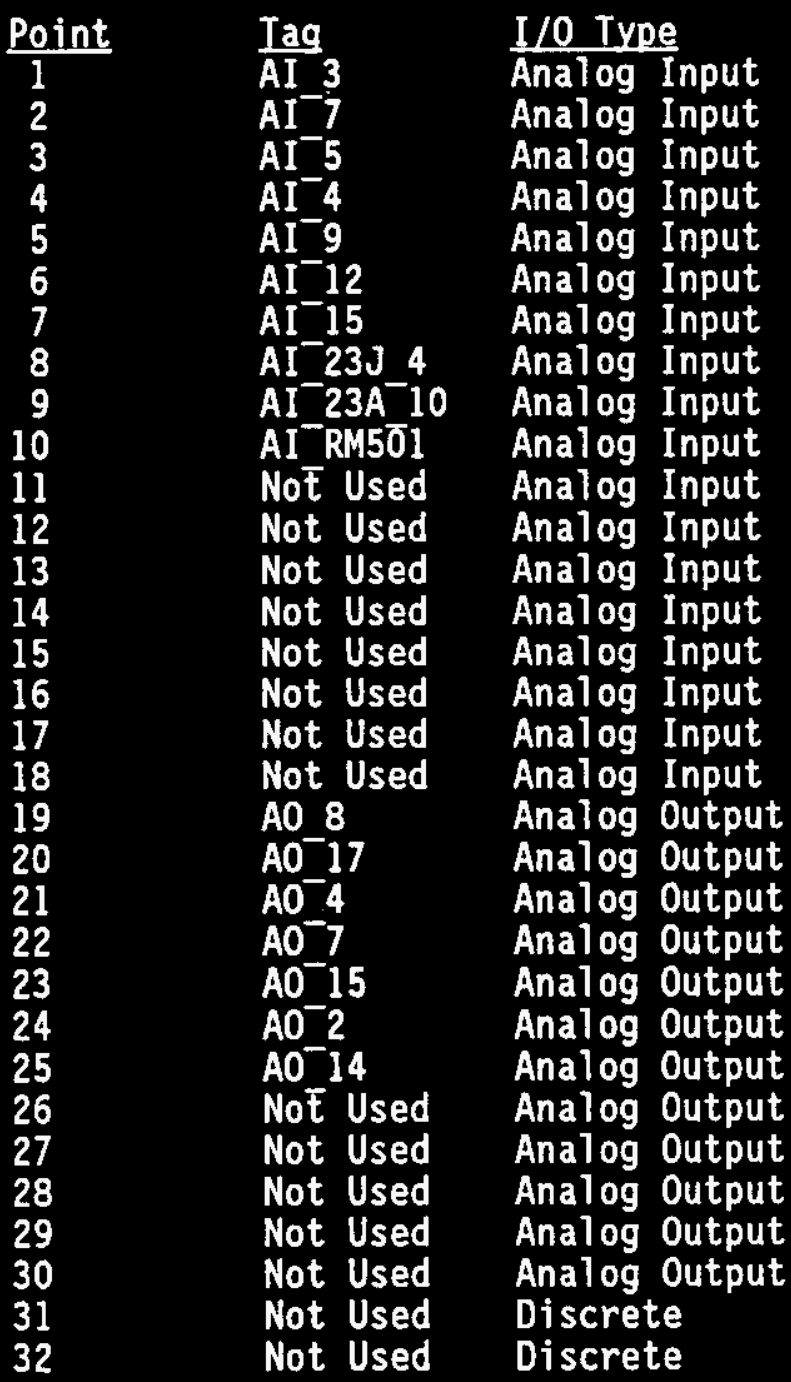

Type: $\mathrm{RCM}-32 \mathrm{C}$

Slot Number: 4

Jumper Assignment
4-20 mA - External Power
4-20 mA - External Power
4-20 mA - External Power
4-20 mA - MICON Powered
4-20 mA - External Power
Votage
4-20 mA - External Power
4-20 mA - External Power
4-20 mA - External Power
4-20 mA - External Power
Voltage
Voltage
Voltage
Voltage
Voltage
Voltage
Voltage
Voltage
N/A
N/A
N/A
N/A
N/A
N/A
N/A
N/A
N/A
N/A
N/A
N/A
Input \& Output
Input \& Output

${ }^{1}$ See RCM-32 Remote Control Module User Manual for description of I/0 jumper placement. 
WHC-SD-CP-CSWD-016

Volume 2

Revision 1

Page 149

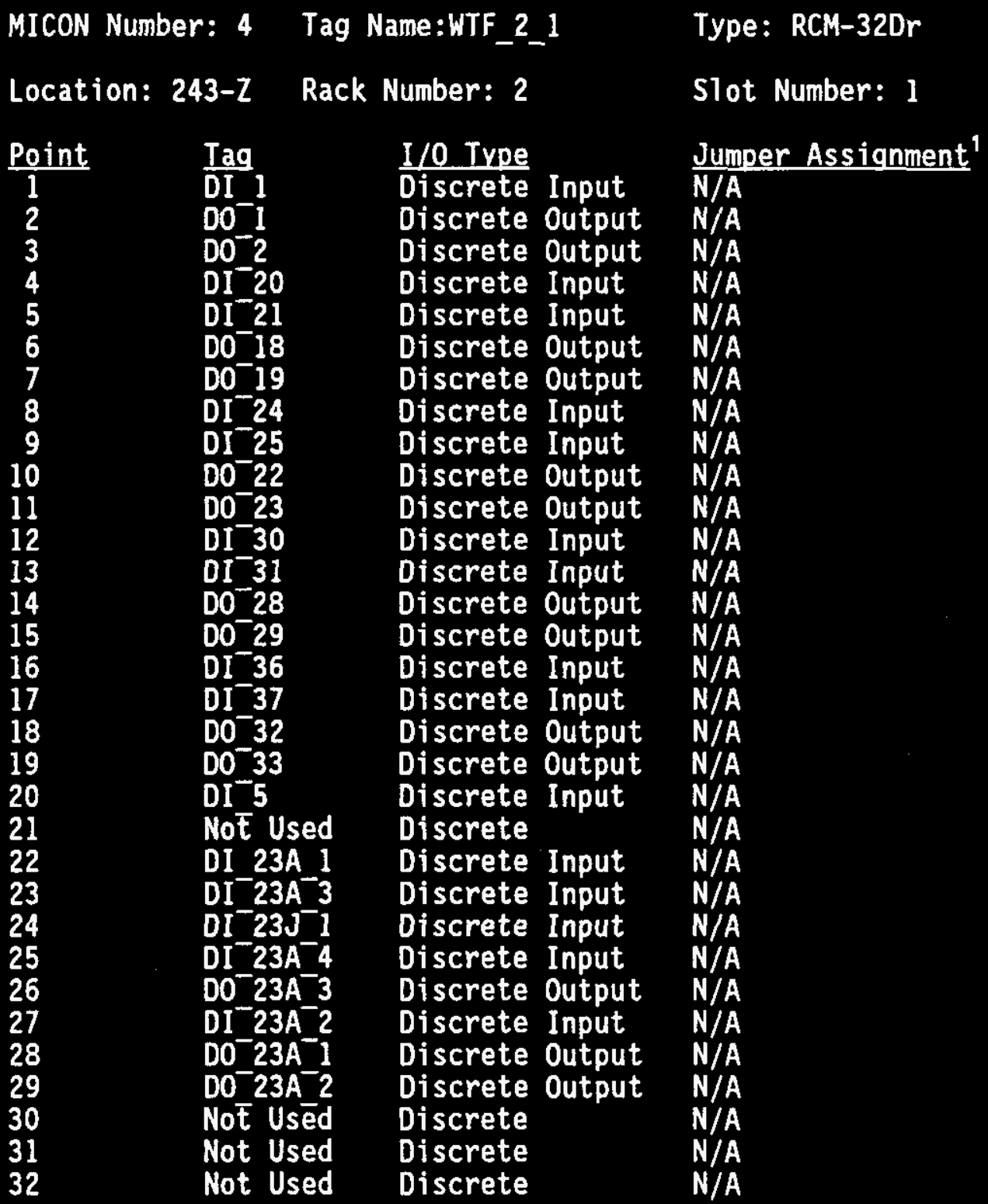

${ }^{1}$ See RCM-32 Remote Control Module User Manual for description of $1 / 0$ jumper placement. 
WHC-SD-CP-CSWD-016

Volume 2

Revision 1

Page 150

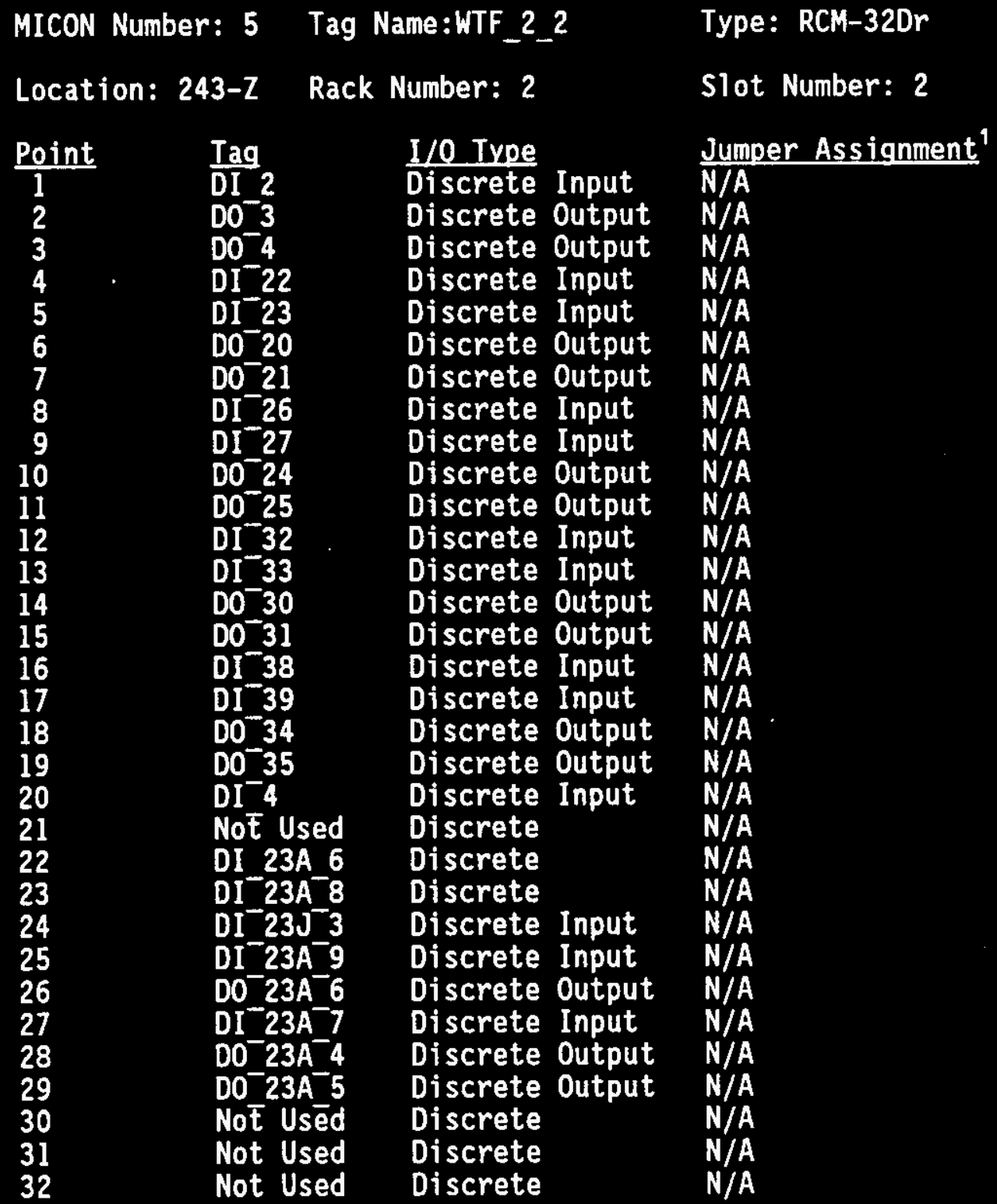

${ }^{1}$ See RCM-32 Remote Control Module User Manual for description of I/0 jumper placement. 


\section{WHC-SD-CP-CSWD-016 \\ Volume 2 Revision 1 \\ Page 151}

MICON Number: 6 Tag Name:WTF_2_3 Type: RCM-32Dr

Location: 243-Z Rack Number: 2 Slot Number: 3

\begin{tabular}{|c|c|c|c|c|}
\hline Point & Tag & I/0 Type & & Jumper Assignment \\
\hline 1 & $\mathrm{DI} 3$ & Discrete & Input & $\mathrm{N} / \mathrm{A}$ \\
\hline 2 & $00^{-5}$ & Discrete & Output & $N / A$ \\
\hline 3 & Not Used & Discrete & & $N / A$ \\
\hline $\begin{array}{l}4 \\
5\end{array}$ & Not Used & Discrete & & $N / A$ \\
\hline $\begin{array}{l}5 \\
6\end{array}$ & DI_28 & Discrete & Input & $N / A$ \\
\hline $\begin{array}{l}6 \\
7\end{array}$ & DO 9 & Discrete & Output & $N / A$ \\
\hline $\begin{array}{l}7 \\
8\end{array}$ & DO 7 & Discrete & Output & $N / A$ \\
\hline $\begin{array}{l}8 \\
9\end{array}$ & $\mathrm{DO}^{-8} 8$ & Discrete & Output & $N / A$ \\
\hline 9 & $\mathrm{DI}^{-} 40$ & Discrete & Input & $N / A$ \\
\hline 10 & $\mathrm{DI}^{-} 41$ & Discrete & Input & $N / A$ \\
\hline 11 & $\mathrm{DO}^{-} 36$ & Discrete & Output & $N / A$ \\
\hline 12 & $\mathrm{DO}^{-} 37$ & Discrete & Output & $N / A$ \\
\hline 13 & $0 I^{-6} 6$ & Discrete & Input & $N / A$ \\
\hline 14 & DI 11 & Discrete & Input & $N / A$ \\
\hline 15 & $\mathrm{DI} 12$ & Discrete & Input & $N / A$ \\
\hline 16 & DO 6 & Discrete & Output & $N / A$ \\
\hline 17 & LDE $84 A$ & Discrete & Input & $N / A$ \\
\hline 18 & LDE 848 & Discrete & Input & $N / A$ \\
\hline 19 & Not ${ }^{-}$Used & Discrete & & $N / A$ \\
\hline 20 & Not Used & Discrete & & $N / A$ \\
\hline 21 & Not Used & Discrete & & $N / A$ \\
\hline 22 & Not Used & Discrete & & $N / A$ \\
\hline 23 & Not Used & Discrete & & N/A \\
\hline 24 & Not Used & Discrete & & $N / A$ \\
\hline 2 & Not Used & Discrete & & $N / A$ \\
\hline 2 & Not Used & Discrete & & $N / A$ \\
\hline 2 & Not Used & Discrete & & $N / A$ \\
\hline 2 & Not Used & Discrete & & $N / A$ \\
\hline 2 & Not Used & Discrete & & $N / A$ \\
\hline 3 & Not Used & Discrete & & $N / A$ \\
\hline 3 & Not Used & Discrete & & $N / A$ \\
\hline 3 & Not Us & Discrete & & $N / A$ \\
\hline
\end{tabular}

${ }^{1}$ See RCM-32 Remote Control Module User Manual for description of I/0 jumper placement. 
WHC-SD-CP-CSWD-016 Volume 3 Revision 1

Page 1

\section{TABLE OF CONTENTS}

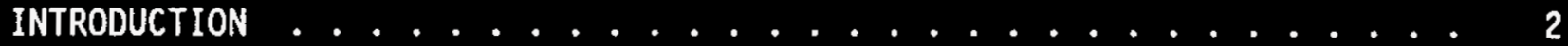

MICON CONFIGURATION FILES . . . . . . . . . . . . . 6

RM45_1 3.mic8_ro ...................... 8

RM451 1 micgro ........................ 17

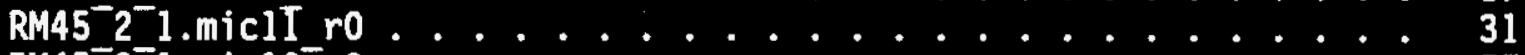

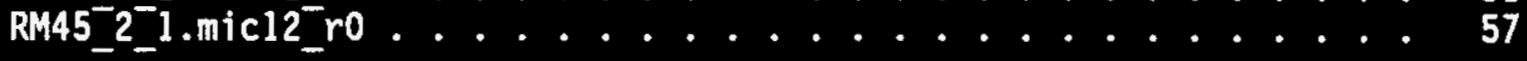

MICON PROGRAM FILES ................... 80

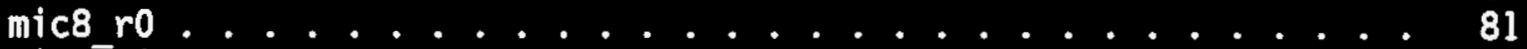

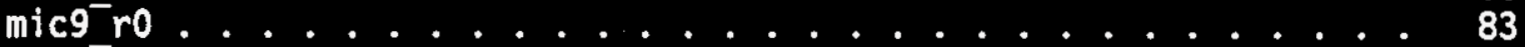

$\operatorname{mic1I} r 0 \ldots \ldots . \ldots . \ldots . . . \ldots 86$

mic12_ro $\ldots \ldots \ldots . \ldots \ldots 2$

APPENDIX A: MICON INPUT/OUTPUT . . . . . . . . . . 99

MICON Number: 8................. 100

MICON Number: $9 . \ldots . . . . . . . . . . . .101$

MICON Number: 11 ................... 102

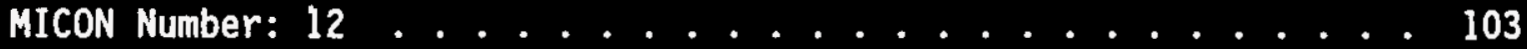


WHC-SD-CP-CSWD-016

Volume 3

Revision 1

Page 2

\section{INTRODUCTION}

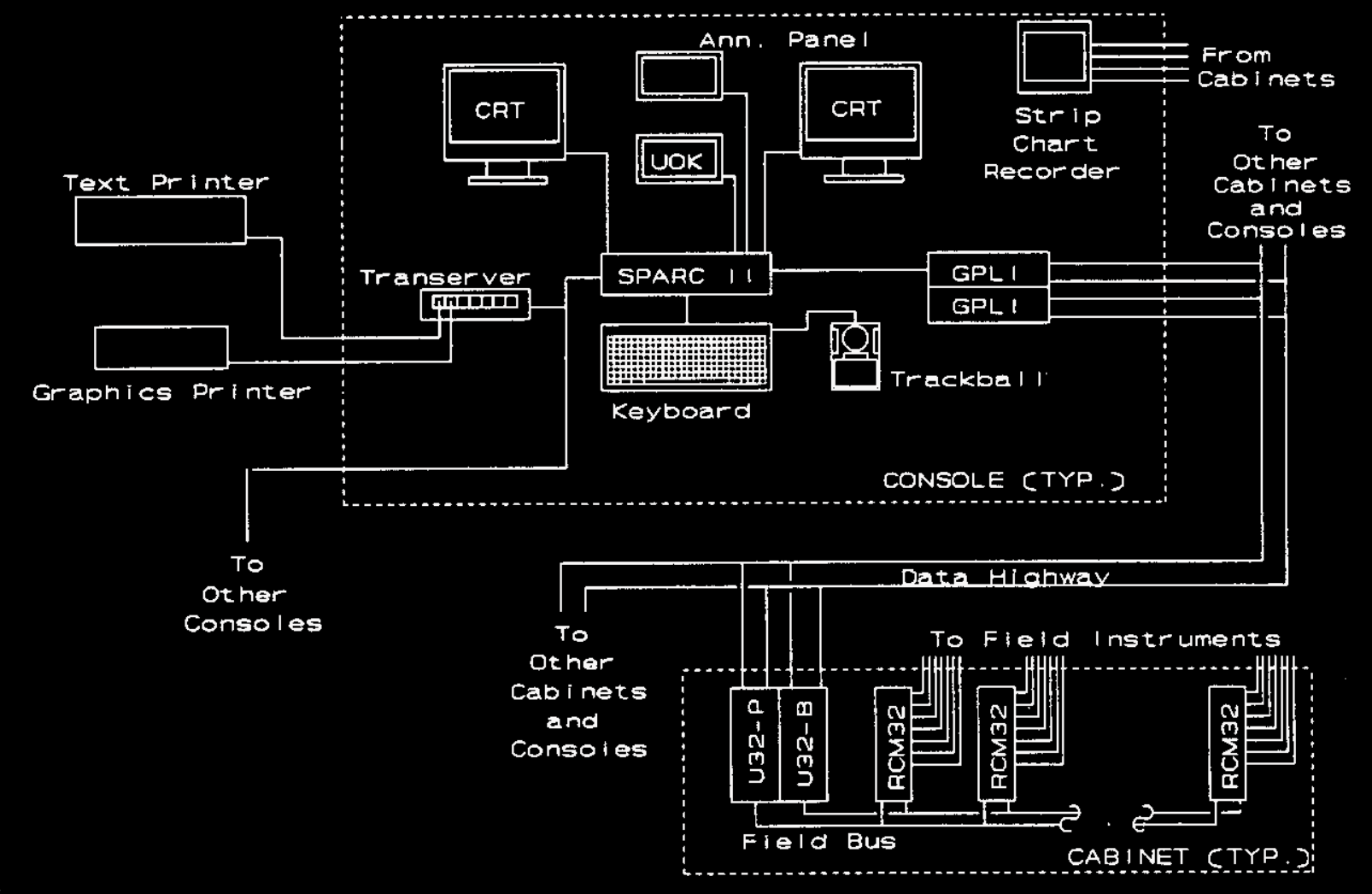

The MICON A/S distributed control system consists of four consoles or workstations ( 2 operator, 1 supervisor, and 1 engineer), a data highway, and eight process controller cabinets.

Each console is based on a Sun Microsystem's SPARC 20 workstation. The SPARC 20 workstation is based on a Scalable Process Architecture (SPARC) micro-processor. Each workstation contains 64 megabytes of memory, a one gigabyte hard drive, a 150 megabyte tape drive, a keyboard, and a trackball. Select SARC statations also contain an additional one gigabyte hard drive, a CD-ROM drive, and a 5 gigabyte tape drive. In addition, each SPARC 20

${ }^{1}$ For a list of Trademarks, see Volume 1, APPENDIX C 


\author{
WHC-SD-CP-CSWD-016 \\ Volume 3 \\ Revision 1 \\ Page 3
}

provides two video outputs, two RS-232 serial ports, and two ethernet ports (a thick-net and a thin-net). The video ports are connected to two high resolution color monitors. The RS-232 ports are connected to two Emerald model FPXT single board IBM XT compatible computers with touchscreen. One of the FPXTs is designated as an annunciator panel and is unused. The other is used as a Universal Operator Keyboard (UOK). One of the two ethernet ports -the thin-net -- is connected to the data highway via a MICON Company General Purpose LAN Interface (GPLI). The thicknet provides a Local Area Network (LAN) between the four consoles. It also connects each SPARC 20 with a Rockwe11/CMC TranServer. The TranServer provides eight additional RS-232 serial ports for each workstation. One of these serial ports is connected to an Output Technology Corporation 800 series text printer. Another port is. connected to a Hewlett-Packard PaintJet color graphics printer.

The Data Highway is the main data link between the consoles and the process control cabinets. It consists of two redundant coax cables that run between all four consoles and six cabinets. The Data Highway is a local area network using a high speed token ring passing protocol. The Data Highway is also redundant. This means that if one of the two cables fail, communication is automatically passed to the other.

The process control cabinets consist of two redundant main controllers, called $\mathrm{U}-32 \mathrm{~s}$, and several process controllers called RCM-32s. The U-32s and RCM-32s communicate via a small local area network called a Field Bus. The U-32 is a MICON built controller based on the Motorola 68020 processor. Although it can be used to directly control instruments and devices in the field, its primary purpose is to pass information between the consoles and the RCM-32s Each U-32 has a redundant backup. That way if one fails, the other can automatically take over. The RCM-32s are also built by MICON and are based on a Motorola 68030 processor. These controllers provide input and output directly to field instruments and other devices.

There are four types of RCM-32s used in this system: A, C, D, and Dr. The A and $C$ cards are used primarily for analog signals. They provide hardware for any standard analog instrument input (4-20 $\mathrm{mA}, 10-50 \mathrm{~mA}$, and $D C$ voltages) and provide a standard 4-20 $\mathrm{mA}$ output signal. In addition, they provide two 28 Volt DC discrete (on/off) channels. These may be used for input, output, or both. An A card provides 26 analog inputs and 4 analog outputs. Each C card provides 18 analog inputs and 12 analog outputs. The D card is used only for discrete control. It provides 32 on/off type channels that can be used as inputs, outputs, or both. Both inputs and outputs are 28 volt DC. The Dr cards are similar to the D cards except inputs are 120 volt $A C$ or DC and the outputs are 3 amp (120 VAC) dry contact relays.

Since MICON A/S systems are installed by engineers rather than computer programmers, they are designed to minimize the need for programming. A11 of the software needed to monitor and control a process is already programmed into the system be MICON Company. The engineer need only "configure" the system to a specific application. 


$$
\begin{gathered}
\text { WHC-SD-CP-CSWD-016 } \\
\text { Volume } 3 \\
\text { Revision } 1 \\
\text { Page } 4
\end{gathered}
$$

The process is very similar to the method used to install an older analog instrument system. First, a set of Pipe and instrument Diagrams (P\&ID) are created. These drawings show a schematic of the chemical process and the instrumentation to be installed to control it. With an analog system, the engineer would purchase the necessary individual components and wire them together to create the control strategy. With the MICON A/S system, the engineer would call function blocks to invoke preprogrammed modules and create the control strategy. The only difference between the two is that one uses hardware devices wired together, and the other uses software modules connected via a data base.

The MICON configuration is done in two parts. First the RCM-32 and, if needed the U-32 controllers are configured. Next, the workstation displays are created.

The RCM-32 and U-32 configuration consists of both the controller "program" and all display information for each "tag". Tags consists of all inputs and outputs from the controller, PID control loops, and any other displayed value. This information is requested by the MICON View software as the engineer creates the controller configuration.

Since all the display information (colors, ranges, etc.) was input during the controller configuration, the workstation configuration consists only of display creation. These are created by calling up a form and filling in the blanks. The only exception to this are the custom created graphics displays. Graphics are created by drawing a picture of the process and overlaying this with tag information. This is done with a special drawing program supplied with MICON View.

To keep size manageable, this document has been divided into several volumes. Volume 1 contains the display configuration for the four workstations. The other volumes each contain the configuration for one process Controller cabinet.

This volume (number 3 ) contains the configuration files for the cabinet located in 236-Z Room 45. This cabinet consists of six controllers:

1. U-32 (with backup)

2. RCM-32 A

3. RCM-32 A

4. $\mathrm{RCM}-32$ A

5. RCM-32 Dr

6. RCM-32 Dr

7. $\mathrm{RCM}-32 \mathrm{Dr}$

8. $\mathrm{RCM}-32 \mathrm{Dr}$
Controller Number: 7

Controller Number: 8

Controller Number: 9

Controller Number: 10

Controller Number: 11

Controller Number: 12

Controller Number: 13

Controller Number: 14
Tagname: RM45 MAIN

Tagname: RM45 13

Tagname: RM45 ${ }^{-}{ }^{-4}$

Tagname: RM45 ${ }^{-} 1^{-5}$

Tagname: RM45 2-1

Tagname: RM45 $2-2$

Tagname: RM45 ${ }^{-} 3$

Tagname: RM45_2-4 
WHC-SD-CP-CSWD-016

Volume 3

Revision 1

Page 5

RM45 MAIN is being used for communications only and does not contain a configuration. In addition, controllers RM45 1 5, RM45_2 3, and RM45_2 4 are not used and, therefore, do not cotain a configüration. - Only configurations for the other four controllers are shown. 
WHC-SD-CP-CSWD-016

Volume 3

Revision 1

Page 6

\section{MICON CONFIGURATION FILES}

The MICON configuration file is a text file that the MICON View software creates as the user configures or "programs" each MICON controller. A MICON controller is configured by selecting preprogrammed functions and filling in the required information. As this is done, MICON View places this information into the configuration file. This file contains both display and program information. For a detailed description see $U-32$ and $R C N-32$ Controller/RTU Function Description published by MICON Company (Vendor Information file 22068).

This file is named by MICON View based on input from the user. The file name's format is TAG.file where TAG is the controllers tag name and file is the program file name. The first eight lines of the file are the information on the controller itself. These lines are:

MICON TYPE - The type of MICON being programmed (A, C, or D). In this case, $D$ and Dr cards are considered the same.

MICON UNIT NO - The controller card's number (user assigned).

MICON TAG - The controller card's tag name (user assigned). For consistency, we have assigned tags in the following format:

$$
\text { CABINET_RACK_SLOT }
$$

CABINET is the MICON controller cabinet location. RACK is the rack number (1 to 3 ). Rack 1 is at the top of the cabinet. SLOT is the slot number ( 1 to 6 ). Slot 1 is at the far left of the rack. Therefor, a controller tagged WTF_1 3 is the card in rack 1 slot 3 of the cabinet located in the Low Level Waste Treatment Facility (243-Z).

MICON DESCRIPTION - A brief description of the controller card

MICON CONFIGURATION FILE - The name of the file that contains the controller card's program (user assigned). 

WHC-SD-CP-CSWD-016
Volume 3
Revision 1
Page 7

POINT ALLOCATION - The memory allocation for the controller. Memory allocation is based on the types of inputs and outputs (I/O) and the number of storage locations desired. The types of $I / 0$ and storage locations are:

VAI - Virtual Analog (User assigned). An analog value storage location.

VDI - Virtual Discrete (User assigned). A Discrete (on/off) storage location with alarm capability.

ISW - Internal Switch (User assigned). A Discrete storage location without alarm capability.

LOOPS - Loops (User Assigned). Loops are used to hold a series of program steps. If one of those steps is a PID controller function, The loop tag name becomes the controller tag name.

MSD - Multi-State Discrete (User assigned). This is a special sixteen position indicator.

AI - Analog Inputs (Assigned based on card type used).

AO - Analog Outputs (Assigned based on card type used).

DI - Discrete Inputs (Assigned based on card type used).

DO - Discrete Outputs (Assigned based on card type used).

The remaining information is the actual controller configuration. Right at first, you will notice several blocks of information titied VIRTUAL ANALOG and DISCRETE TAG. These blocks are the configuration information for the virtual analog and internal switch type storage locations. These must always be configured first. After that are the controller loops. Each loop starts with a block of information titled LOOP NO followed by several steps. The last step is always a function 0 (END). Everything except virtual analogs and internal switches are configured inside of a loop.

Loops are a way of organizing the controller configuration. Although most controllers could be programmed using only one loop, using several loops makes the program more understandable. Loops are generally invisible to the operator. The exception to this rule is when a PID function (function 20) is used in the 100p. When this happens, the loop name becomes the controller name. Otherwise, the loop name is used as an internal reference only

NOTE: Each loop using a.PID function has two lines showing the tuning paramaters -- for example:

TUNING PARAMETER :

P(GAIN): $1.00, \mathrm{I}$ (REP/MIN) : 5.00, D(RATE) : 0.00, MANUAL RESET : 0.00

These tuning parameters are not controlled by this document. Therefore, these numbers may be different than the ones that appear in the workstation listings. 
WHC-SD-CP-CSWD-016

Volume 3

Revision 1

Page 8

RM45_1_3.mic8_ro

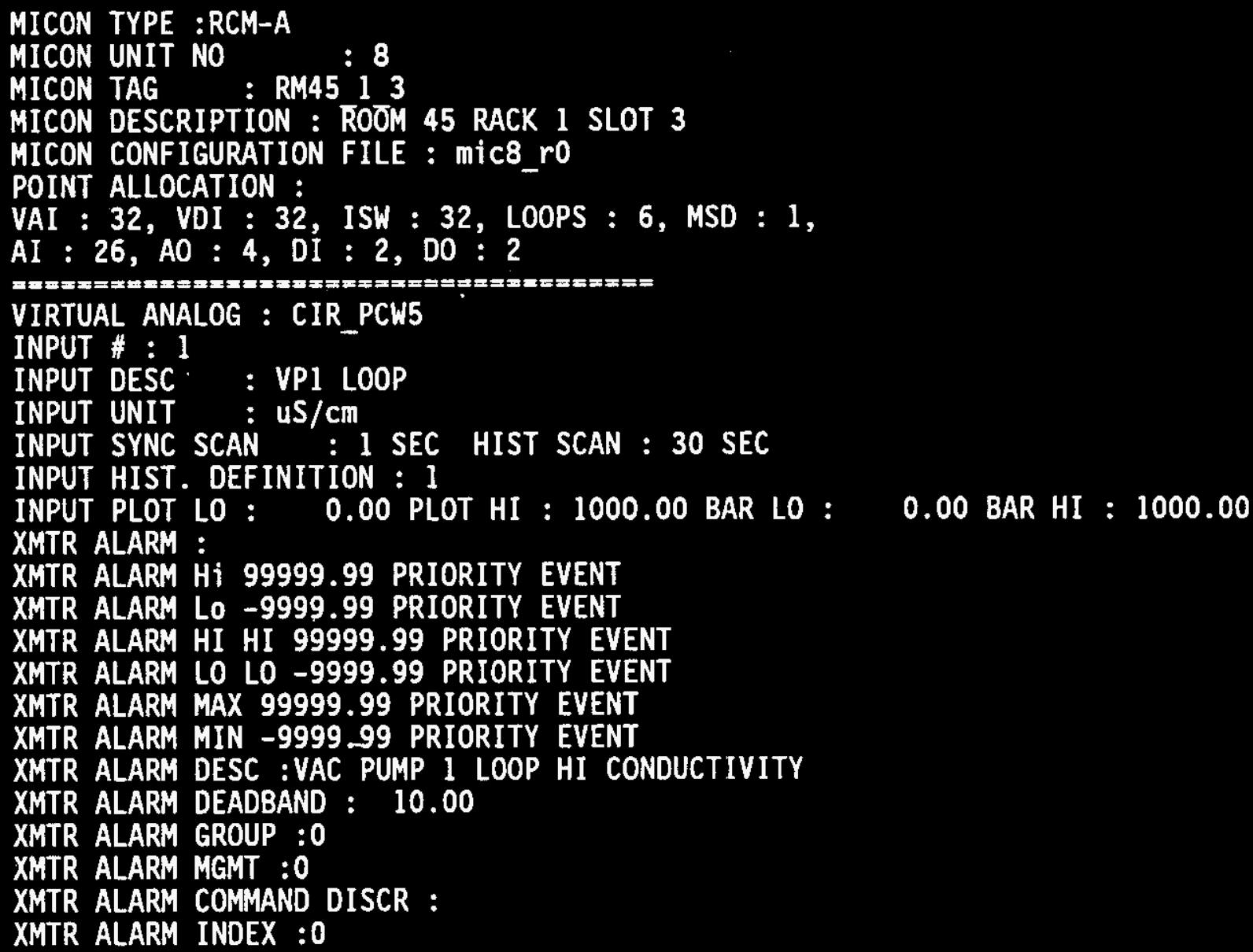


WHC-SD-CP-CSWD-016

Volume 3

Revision 1

Page 9

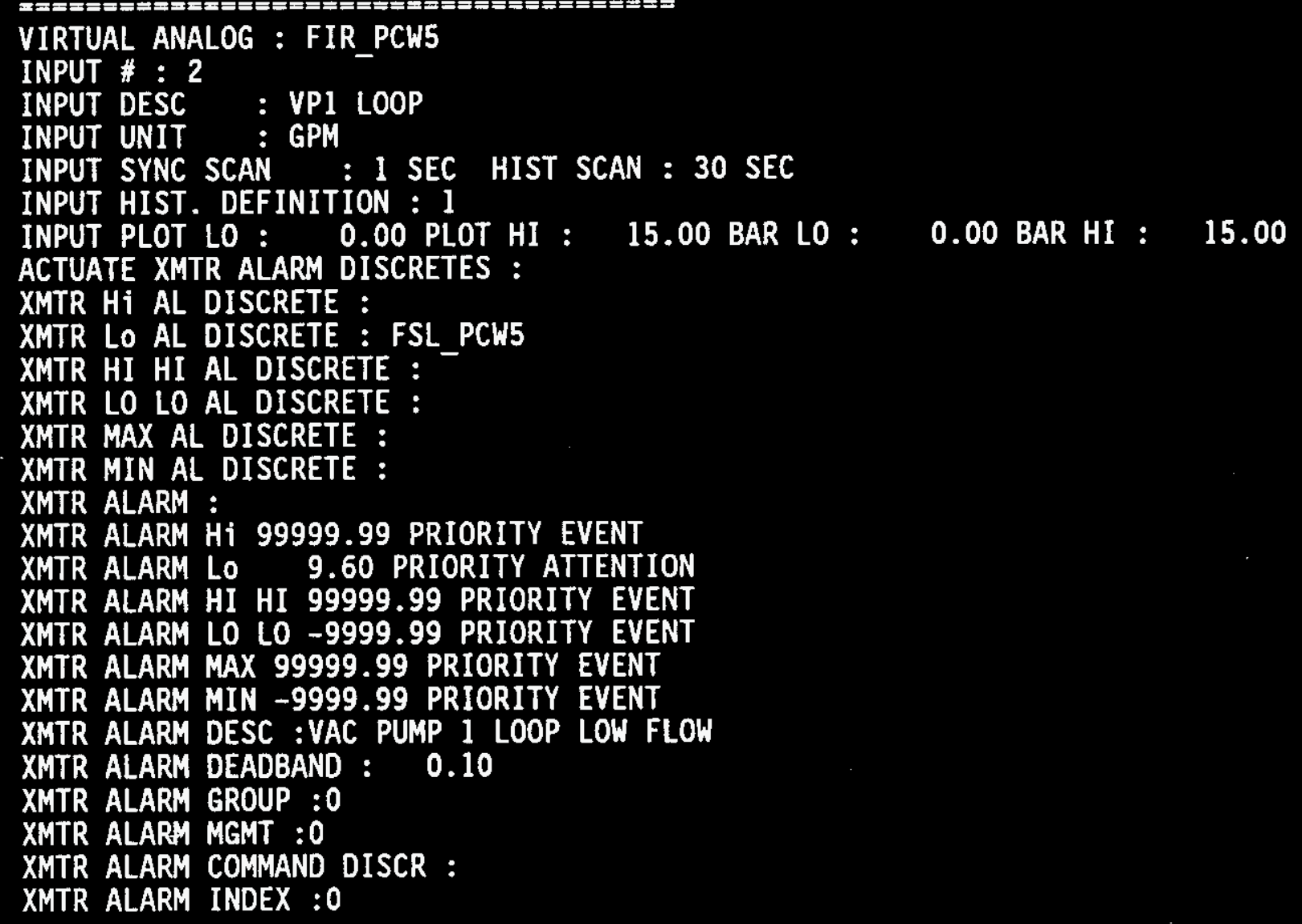


WHC-SD-CP-CSWD-016

Volume 3

Revision 1

Page 10

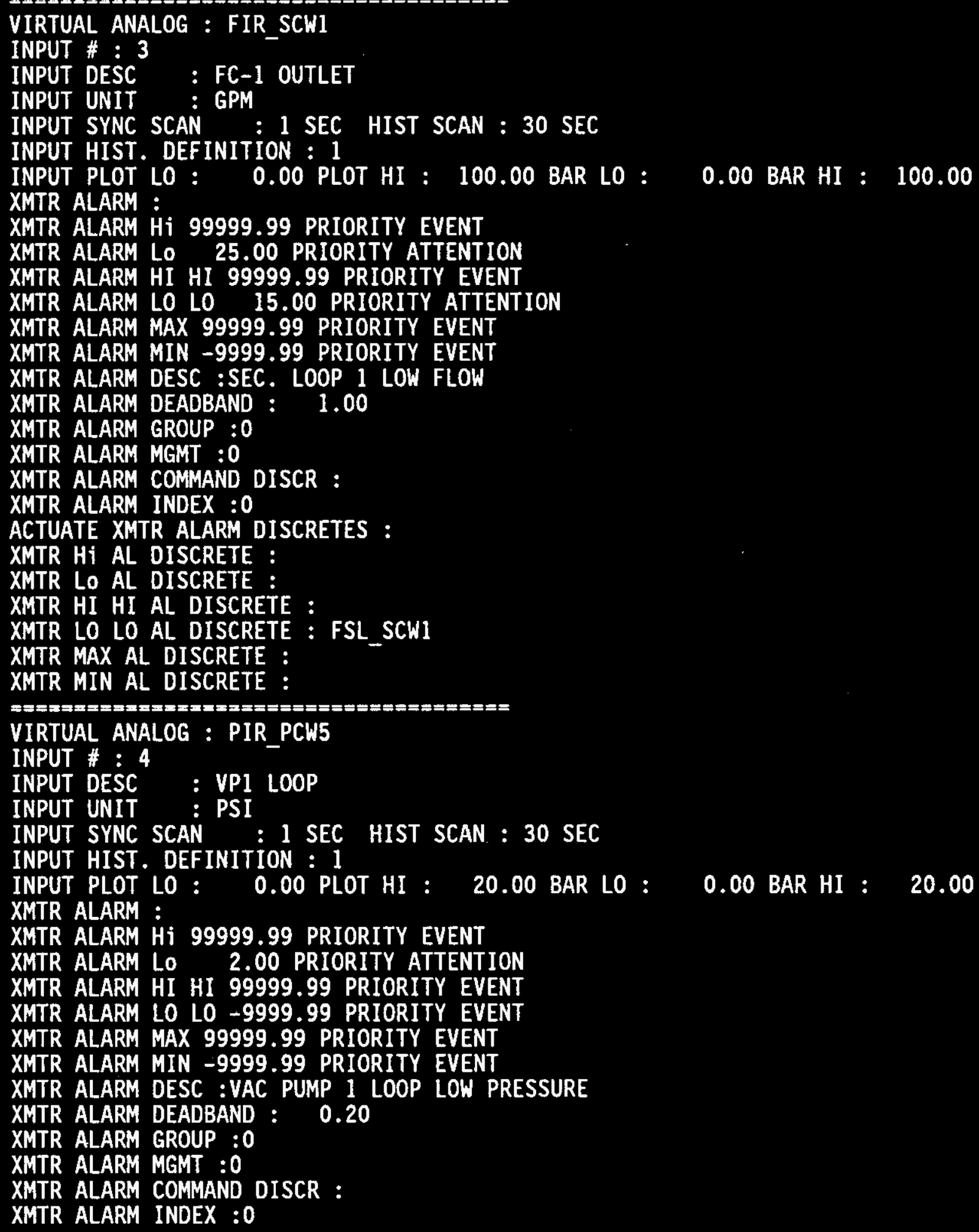


WHC-SD-CP-CSWD-016

Volume 3

Revision 1

Page 11

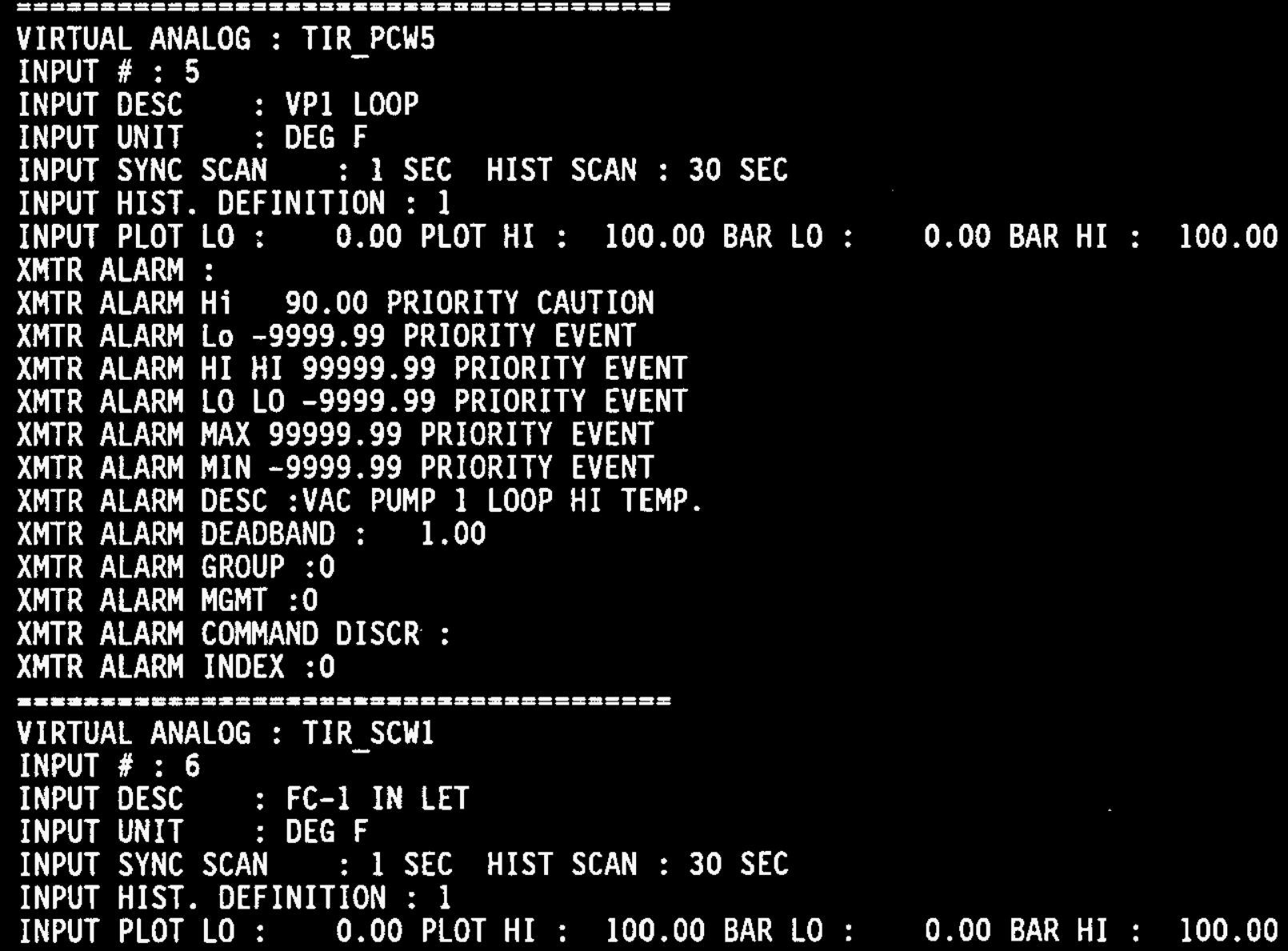


WHC-SD-CP-CSWD-016

Volume 3

Revision 1

Page 12

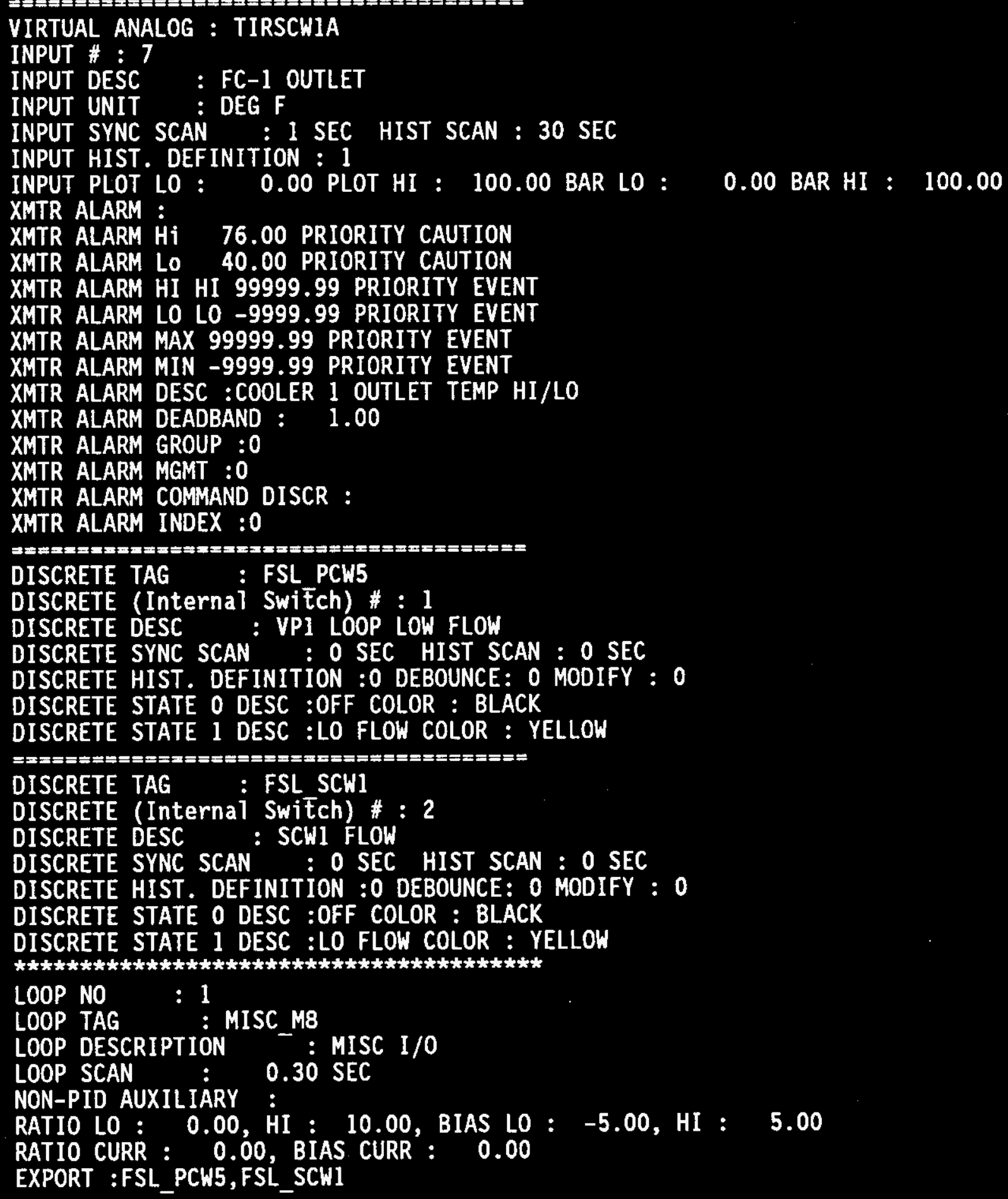


WHC-SD-CP-CSWD-016

Volume 3

Revision 1

Page 13

\#\#\#\#\#\#\#\#\#\#\#\#\#\#\#\#\#\#\#\#\#\#\#\#\#\#\#\#\#\#\#\#\#\#\#\#\#\#\#\#

STEP NO : 1

LABEL :

FUNCTION : 11 (AI)

COMMENT :

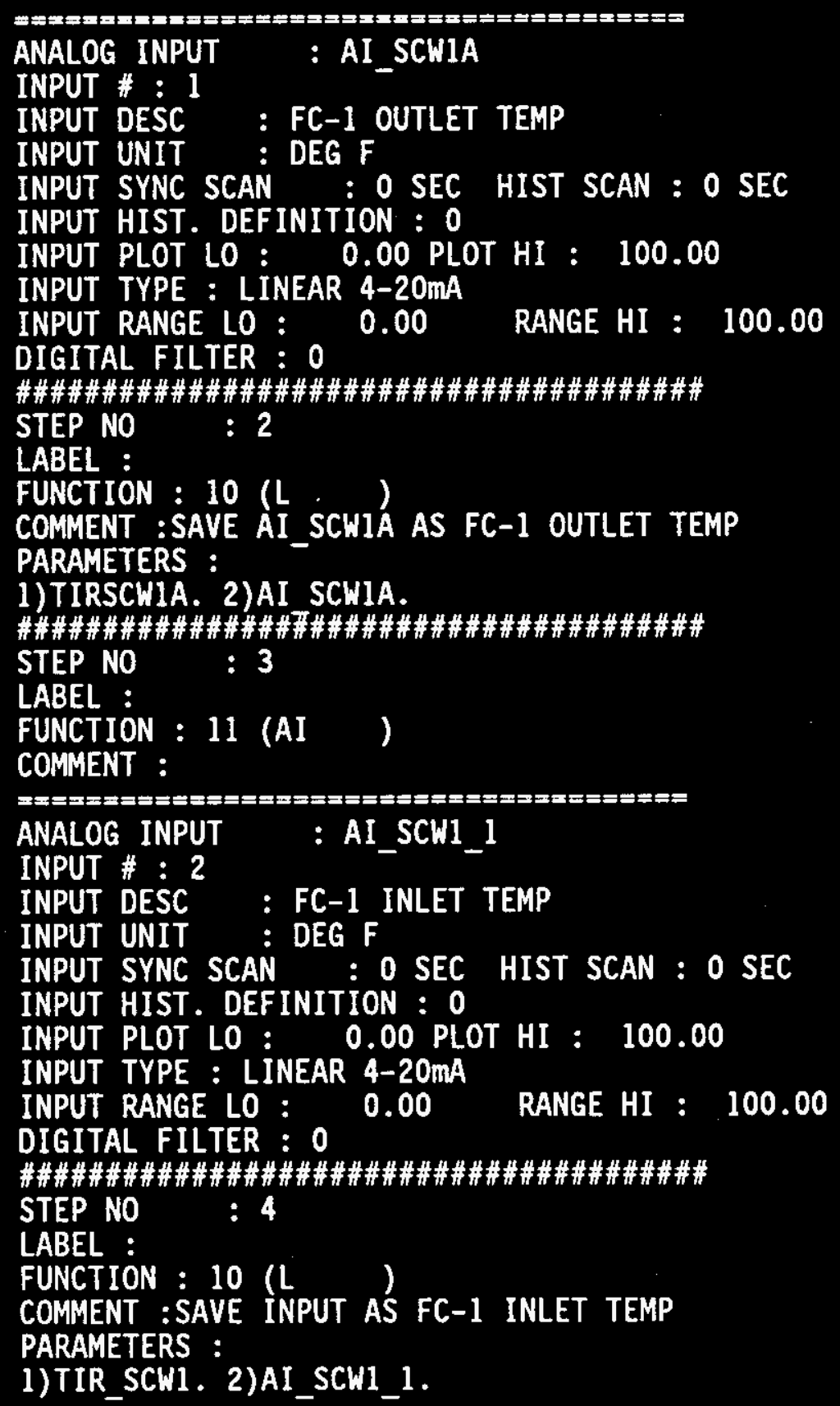


WHC-SD-CP-CSWD-016

Volume 3

Revision 1

Page 14

\#\#\#\#\#\#\#\#\#\#\#\#\#\#\#\#\#\#\#\#\#\#\#\#\#\#\#\#\#\#\#\#\#\#\#\#\#\#\#\#

STEP NO : 5

LABEL :

FUNCTION : 11 (AI)

COMMENT :

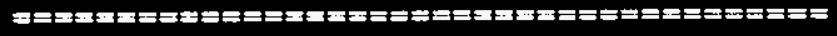

ANALOG INPUT : AI_SCW1_2

INPUT \#: 3

INPUT DESC : FC-1 OUTLET FLOW

INPUT UNIT : GPM

INPUT SYNC SCAN : 0 SEC HIST SCAN : 0 SEC

INPUT HIST. DEFINITION : 0

INPUT PLOT LO : 0.00 PLOT HI : 100.00

INPUT TYPE : LINEAR 4-20mA

INPUT RANGE LO: 0.00 RANGE HI : 100.00

DIGITAL FILTER : 0

\#\#\#\#\#\#\#\#\#\#\#\#\#\#\#\#\#\#\#\#\#\#\#\#\#\#\#\#\#\#\#\#\#\#\#\#\#\#\#\#

STEP NO : 6

LABEL :

FUNCTION : 10 (L )

COMMENT : SAVE AI_SCW1_2 AS FC-1 OUTLET FLOW

PARAMETERS :

1)FIR SCW1. 2)AI SCWI 2.

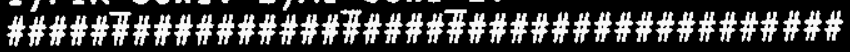

STEP NO : 7

LABEL :

FUNCTION : 11 (AI)

COMMENT :

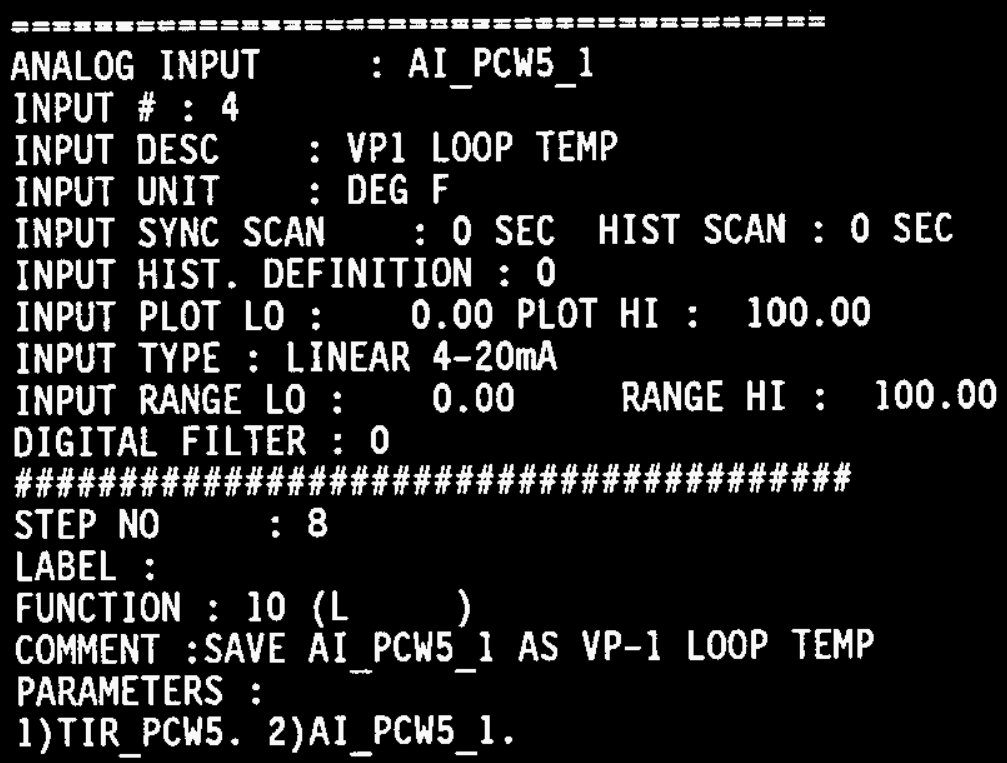




\section{WHC-SD-CP-CSWD-016 \\ Volume 3 \\ Revision 1 \\ Page 15}

\#\#\#\#\#\#\#\#\#\#\#\#\#\#\#\#\#\#\#\#\#\#\#\#\#\#\#\#\#\#\#\#\#\#\#\#\#\#\#\#

STEP NO : 9

LABEL :

FUNCTION : 11 (AI)

COMMENT :

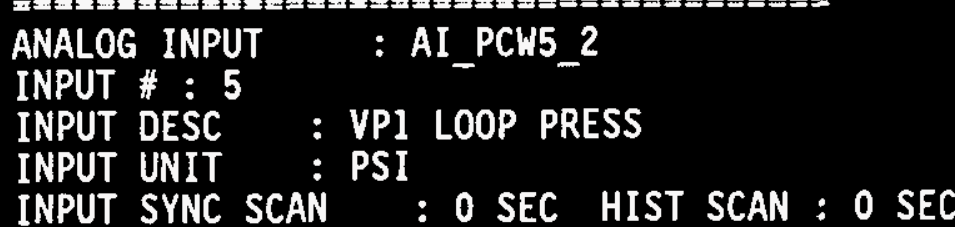

INPUT HIST. DEFINITION : 0

INPUT PLOT LO : 0.00 PLOT HI : 20.00

INPUT TYPE : LINEAR 4-20mA

INPUT RANGE LO: 0.00 RANGE HI : 20.00

DIGITAL FILTER : 0

\#\#\#\#\#\#\#\#\#\#\#\#\#\#\#\#\#\#\#\#\#\#\#\#\#\#\#\#\#\#\#\#\#\#\#\#\#\#

STEP NO : 10

LABEL :

FUNCTION : $10(\mathrm{~L}$

COMMENT : SAVE AI PCH5 2 AS VP-1 LOOP PRESS

PARAMETERS :

1)PIR PCW5. 2)AI PCW5 2.

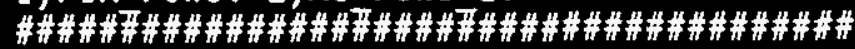

STEP NO : 11

LABEL :

FUNCTION : 11 (AI)

COMMENT :

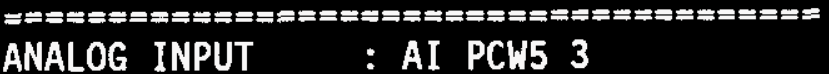

ANALOG INPUT
INPUT \#: 6

INPUT DESC : VP1 LOOP CONDUCTIVITY

INPUT UNIT : uS/cm

INPUT SYNC SCAN : O SEC HIST SCAN : O SEC

INPUT HIST. DEFINITION : 0

INPUT PLOT LO : 0.00 PLOT HI : 1000.00

INPUT TYPE : LINEAR 4-20mA

INPUT RANGE LO : 0.00 RANGE HI : 1000.00

DIGITAL FILTER : 0

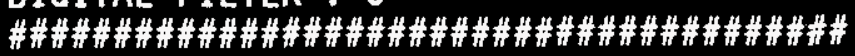

STEP NO : 12

LABEL :

FUNCTION : 10 (L

COMMENT : SAVE AI PCW5 3 AS VP-1 LOOP CONDUCTIVITY

PARAMETERS :

1)CIR_PCW5. 2)AI_PCW5_3. 
WHC-SD-CP-CSWD-016

Volume 3

Revision 1

Page 16

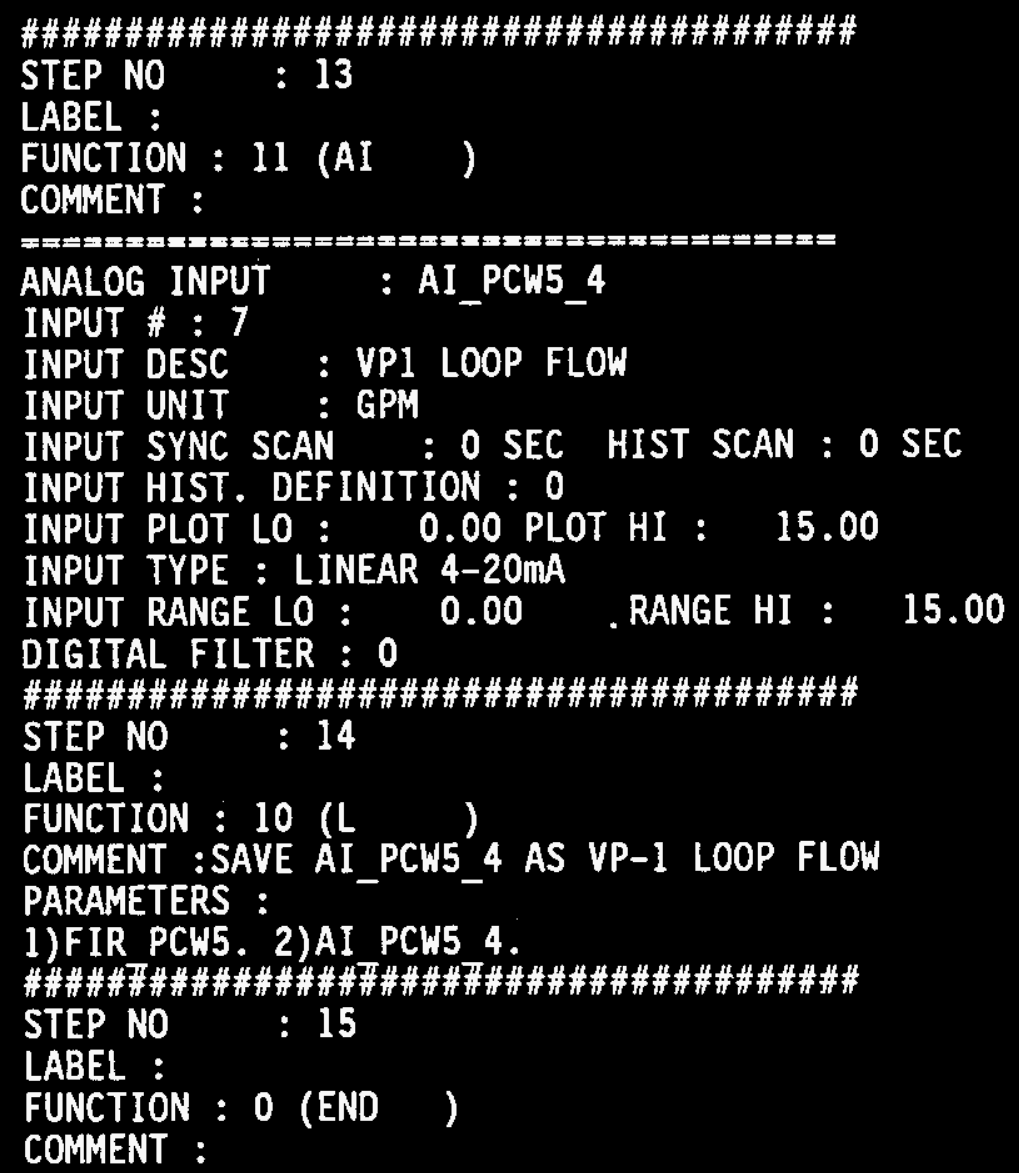


WHC-SD-CP-CSWD-016

Volume 3

Revision 1

Page 17

RM45_1_4.mic9_ro

MICON TYPE :RCM-A

MICON CONFIGURATION FILE : mic9_rO

POINT ALLOCATION :

VAI : 32, VDI : 32, ISW : 32, LOOPS : 6 , MSD : 1 ,

AI $: 26$, AO $: 4$, DI $: 2$, DO : 2

$=x=26$,

VIRTUAL ANALOG : CIR_PCW1

INPUT \# : 1

INPUT DESC : PRF LOOP

INPUT UNIT : US $/ \mathrm{cm}$

INPUT SYNC SCAN :O SEC HIST SCAN : 30 SEC

INPUT HIST. DEFINITION : 1

INPUT PLOT LO : 0.00 PLOT HI : 1000.00 BAR LO : 0.00 BAR HI $: 1000.00$

XMTR ALARM :

XMTR ALARM Hi 99999.99 PRIORITY EVENT

XMTR ALARM Lo -9999.99 PRIORITY EVENT

XMTR ALARM HI HI 99999.99 PRIORITY EVENT

XMTR ALARM LO LO -9999.99 PRIORITY EVENT

XMTR ALARM MAX 99999.99 PRIORITY EVENT

XMTR ALARM MIN -9999.99 PRIORITY EVENT

XMTR ALARM DESC :PRF LOOP HI CONDUCTIVITY

XMTR ALARM DEADBAND : 10.00

XMTR ALARM GROUP :0

XMTR ALARM MGMT :0

XMTR ALARM COMMAND DISCR :

XMTR ALARM INDEX :0 
WHC-SD-CP-CSWD-016

Volume 3

Revision 1

Page 18

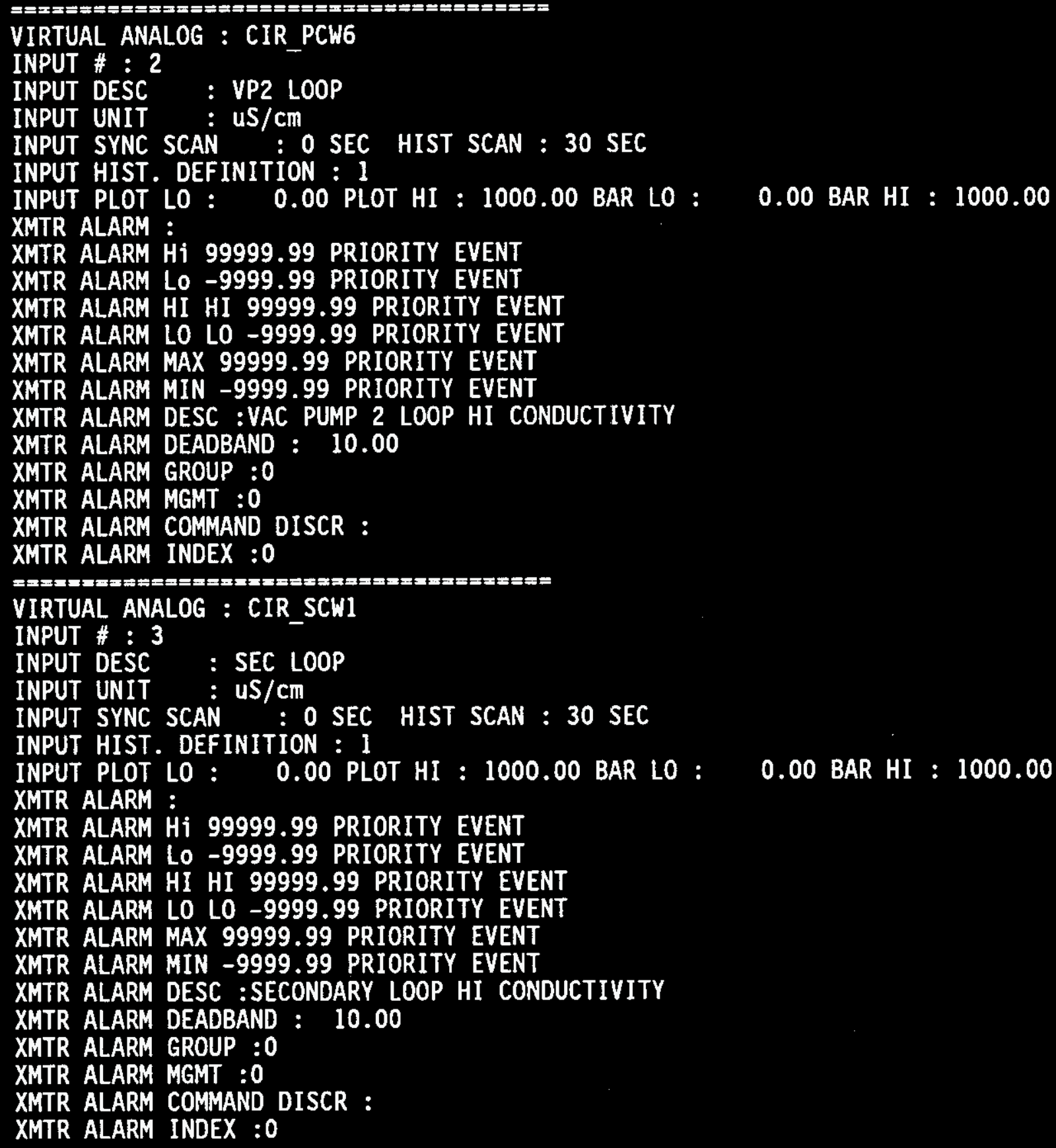


WHC-SD-CP-CSWD-016

Volume 3

Revision 1

Page 19

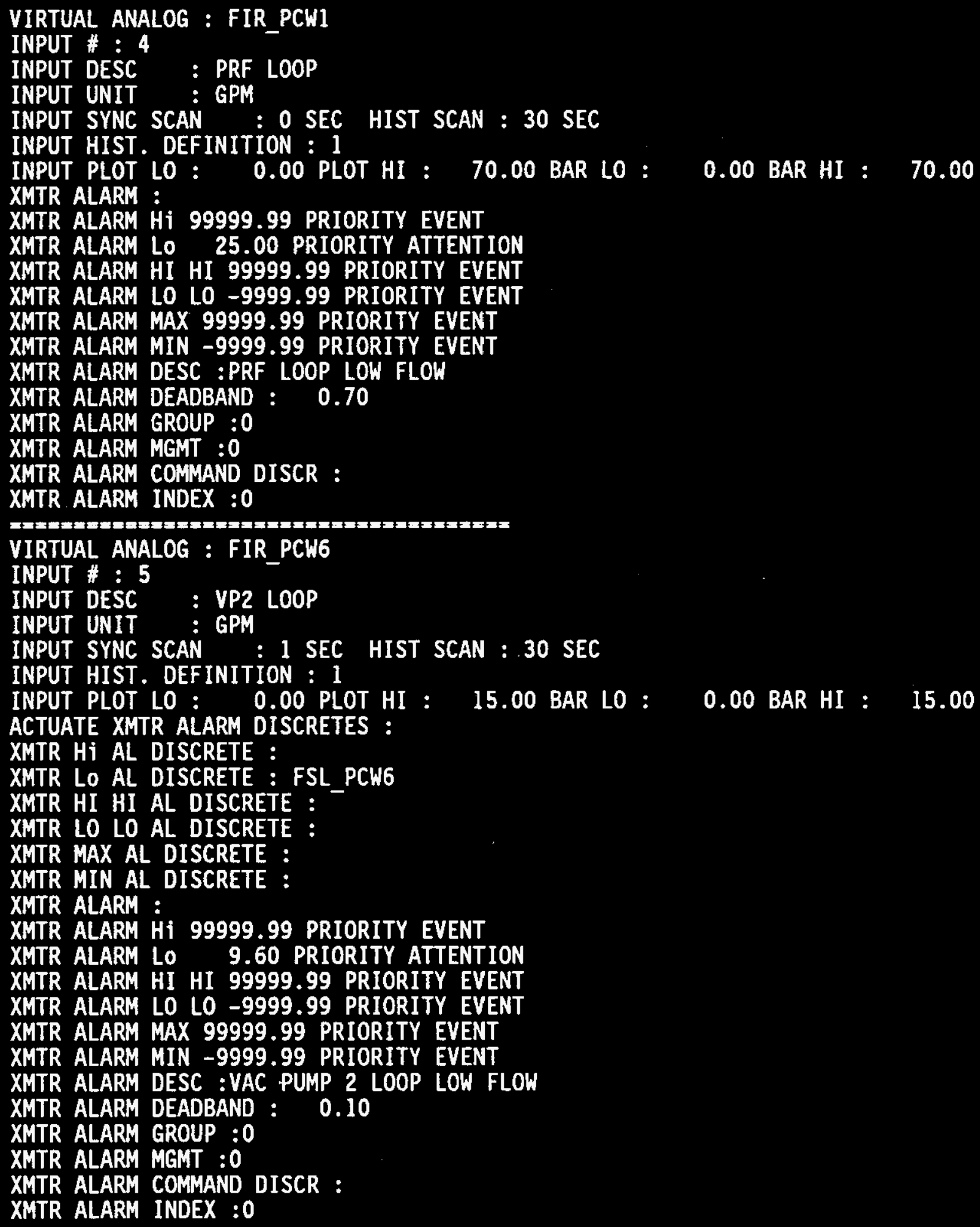


WHC-SD-CP-CSWD-016

Volume 3

Revision 1

Page 20

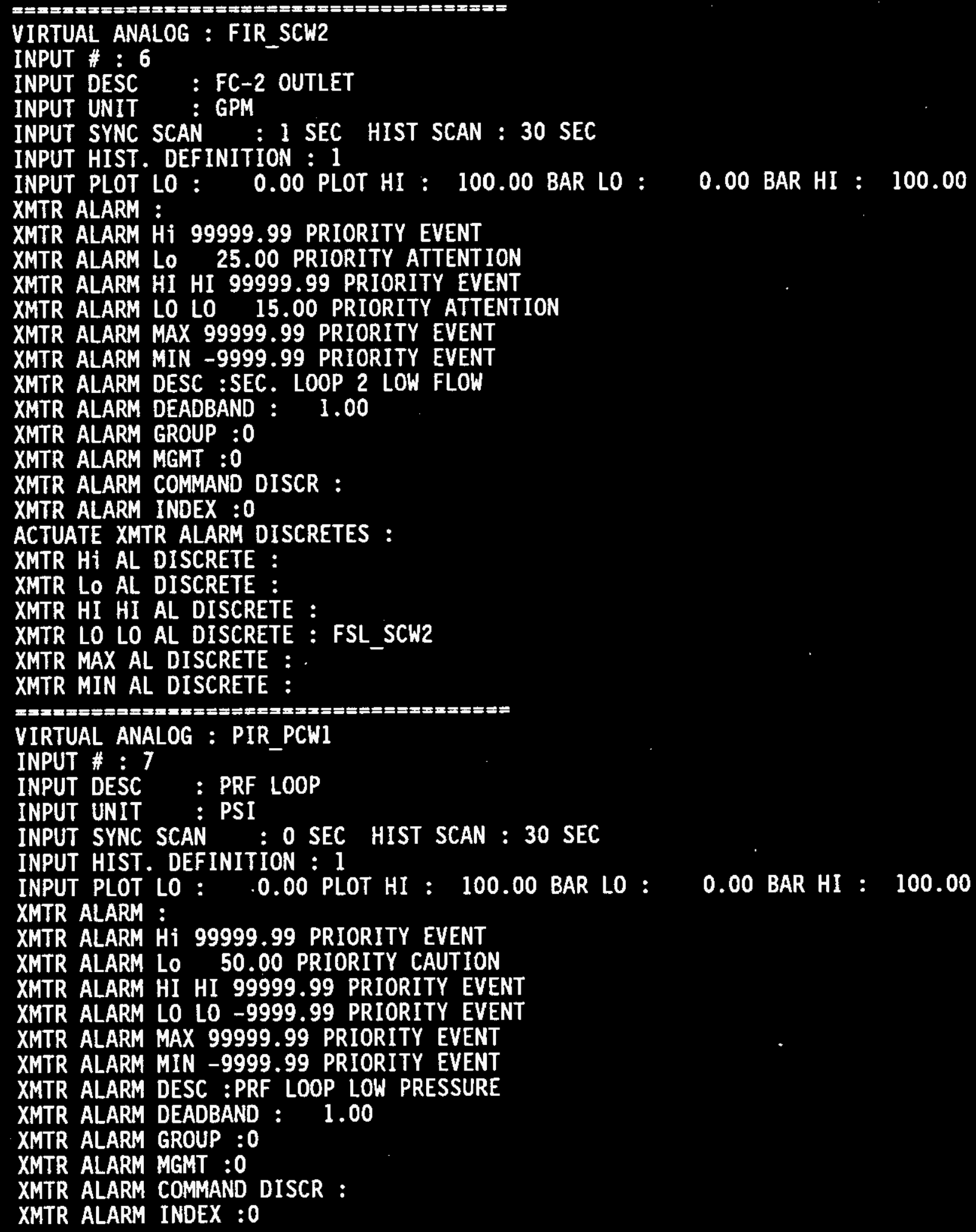


WHC-SD-CP-CSWD-016

Volume 3

Revision 1

Page 21

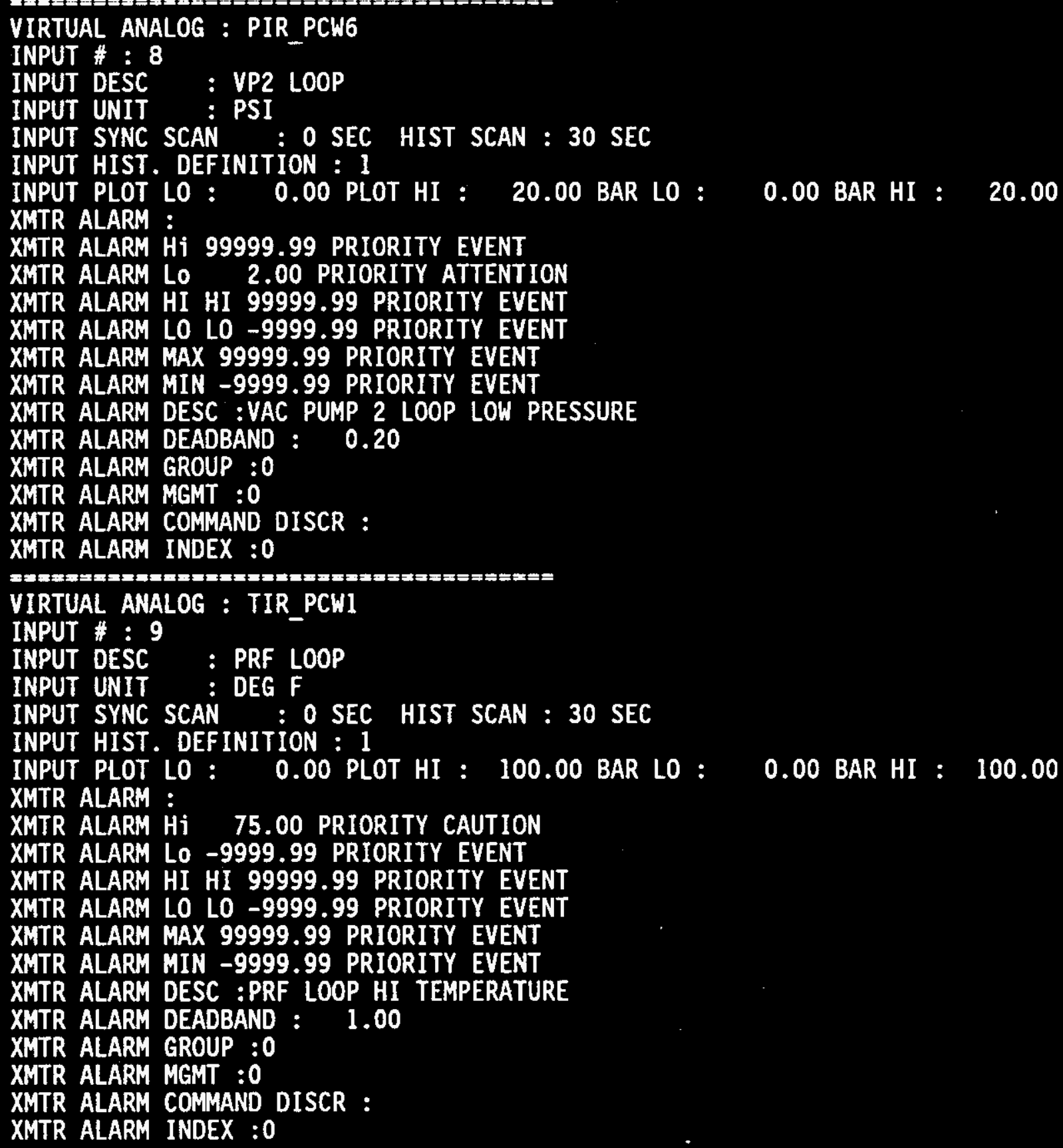


WHC-SD-CP-CSWD-016

Volume 3

Revision 1

Page 22

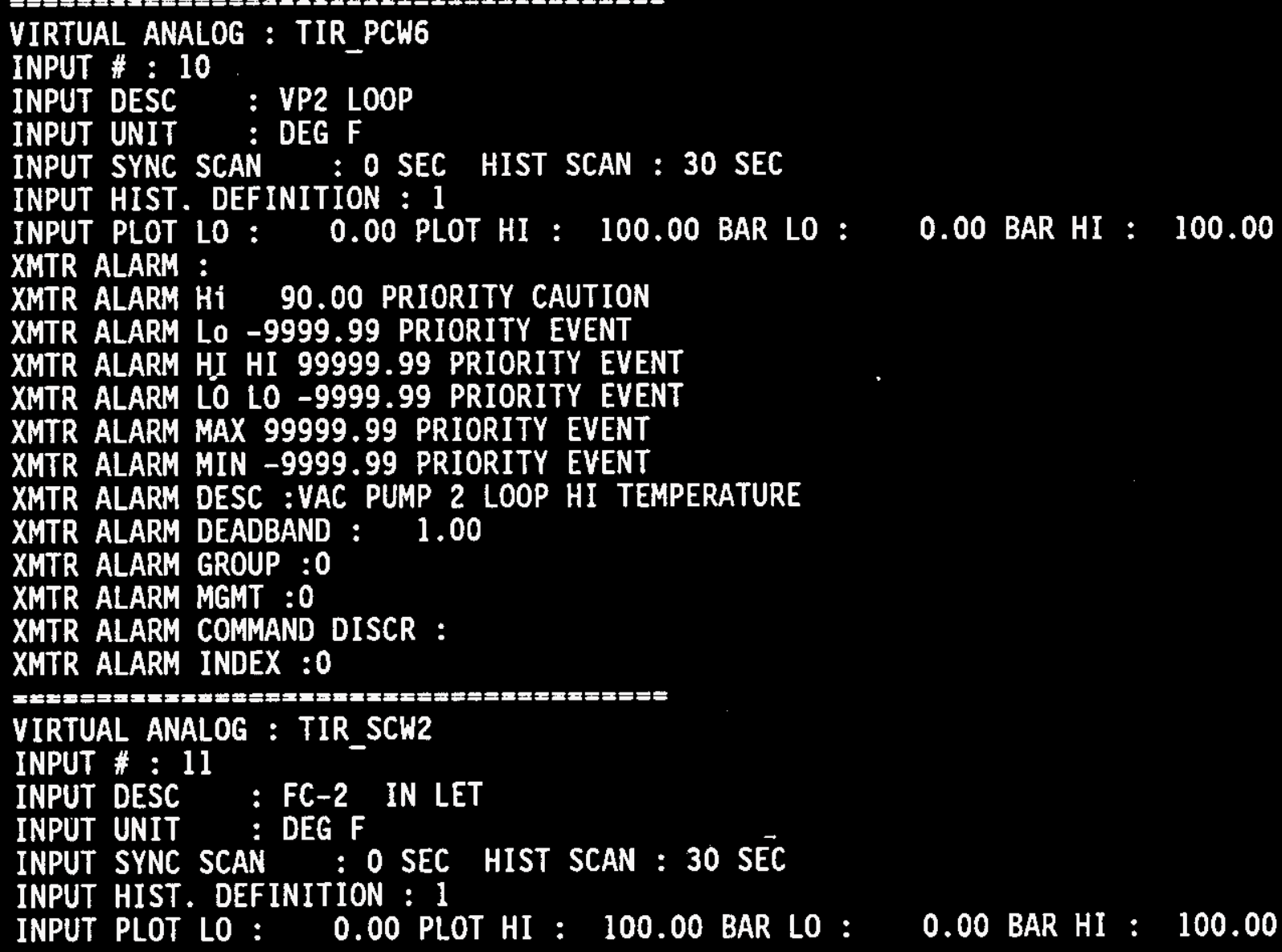


WHC-SD-CP-CSWD-016

Volume 3

Revision 1

Page 23

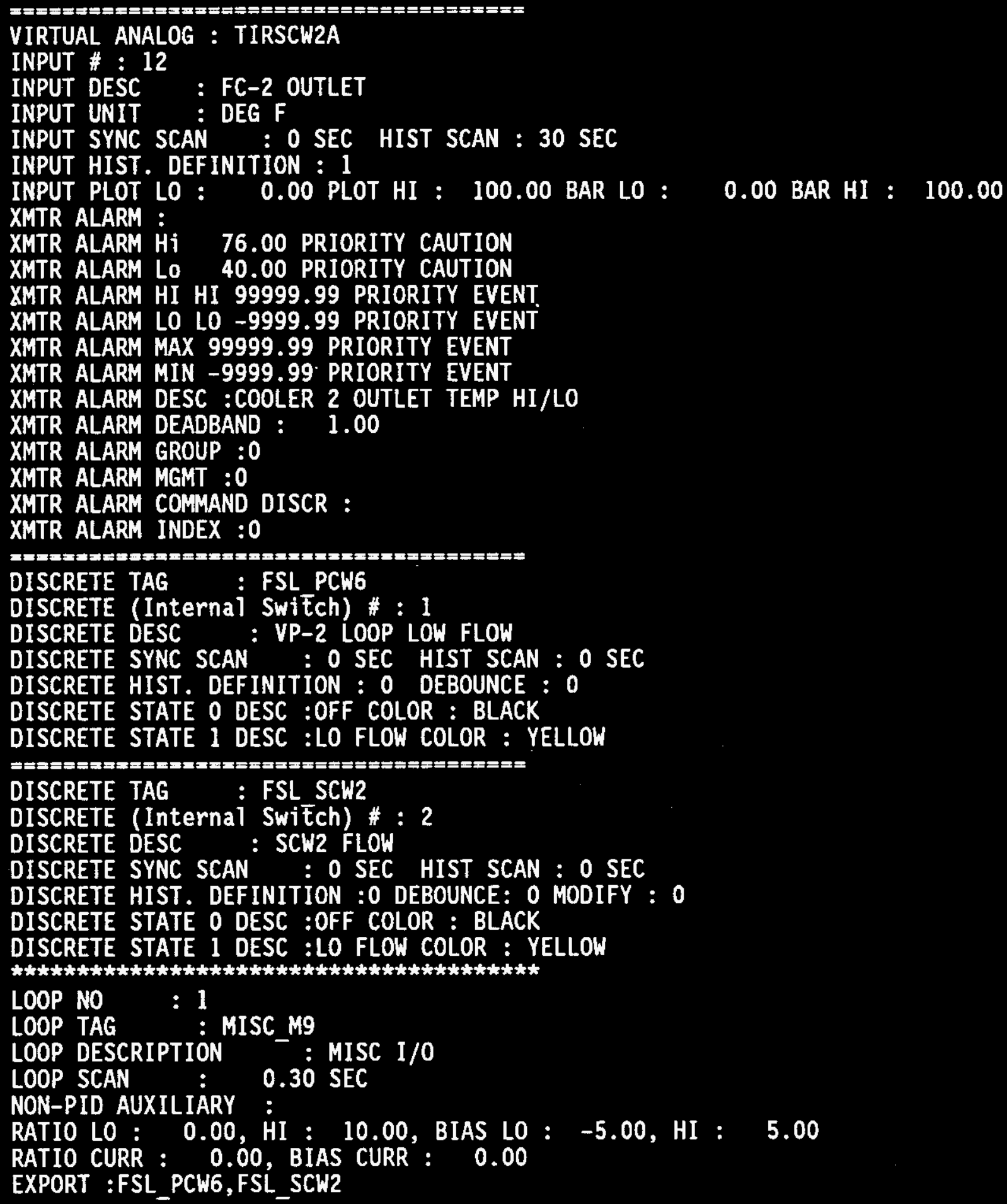


WHC-SD-CP-CSWD-016

Volume 3

Revision 1

Page 24

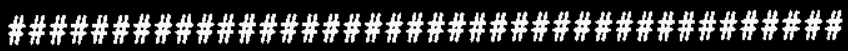

STEP NO : 1

LABEL :

FUNCTION : 11 (AI)

COMMENT :

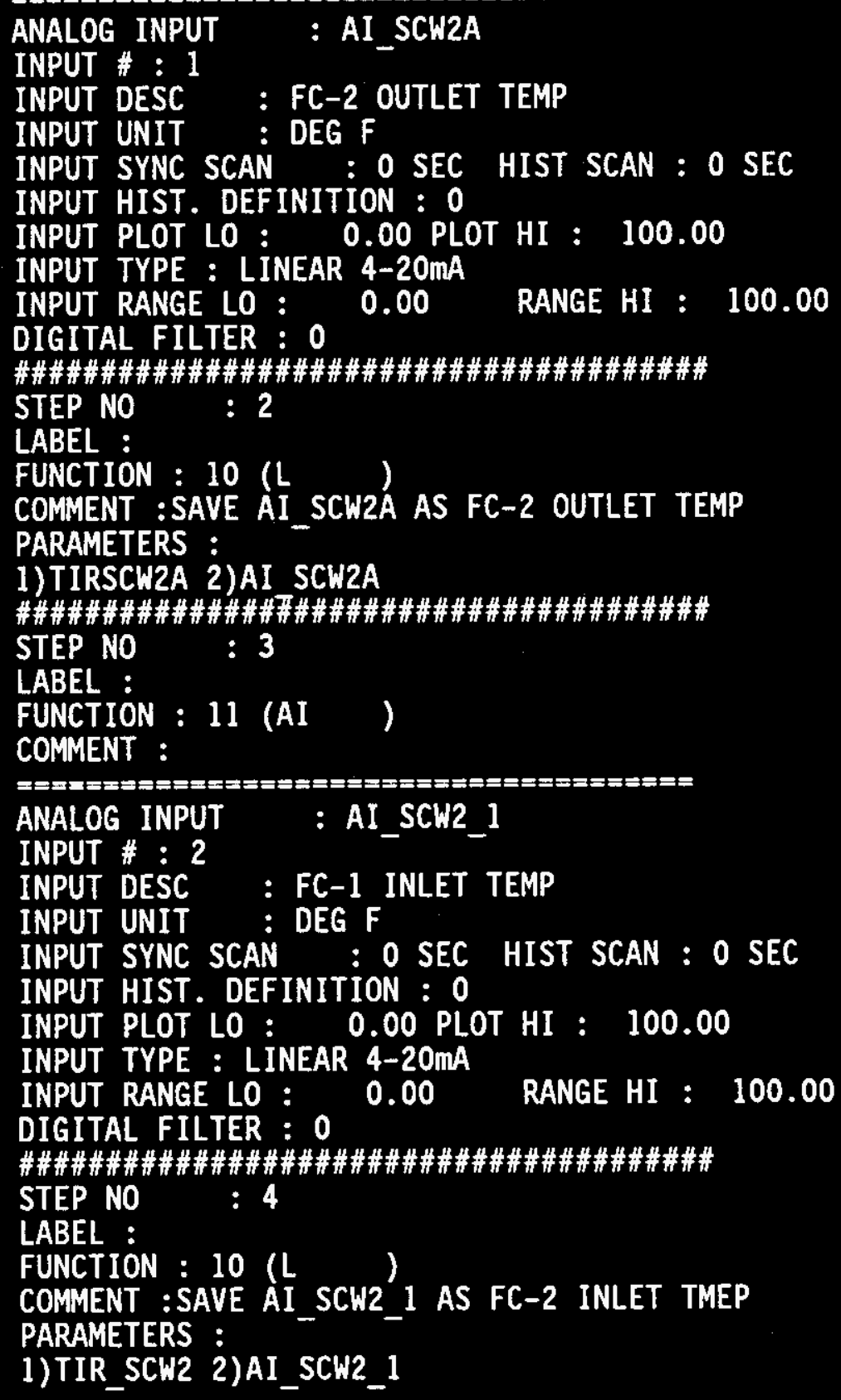


WHC-SD-CP-CSWD-016

Volume 3

Revision 1

Page 25

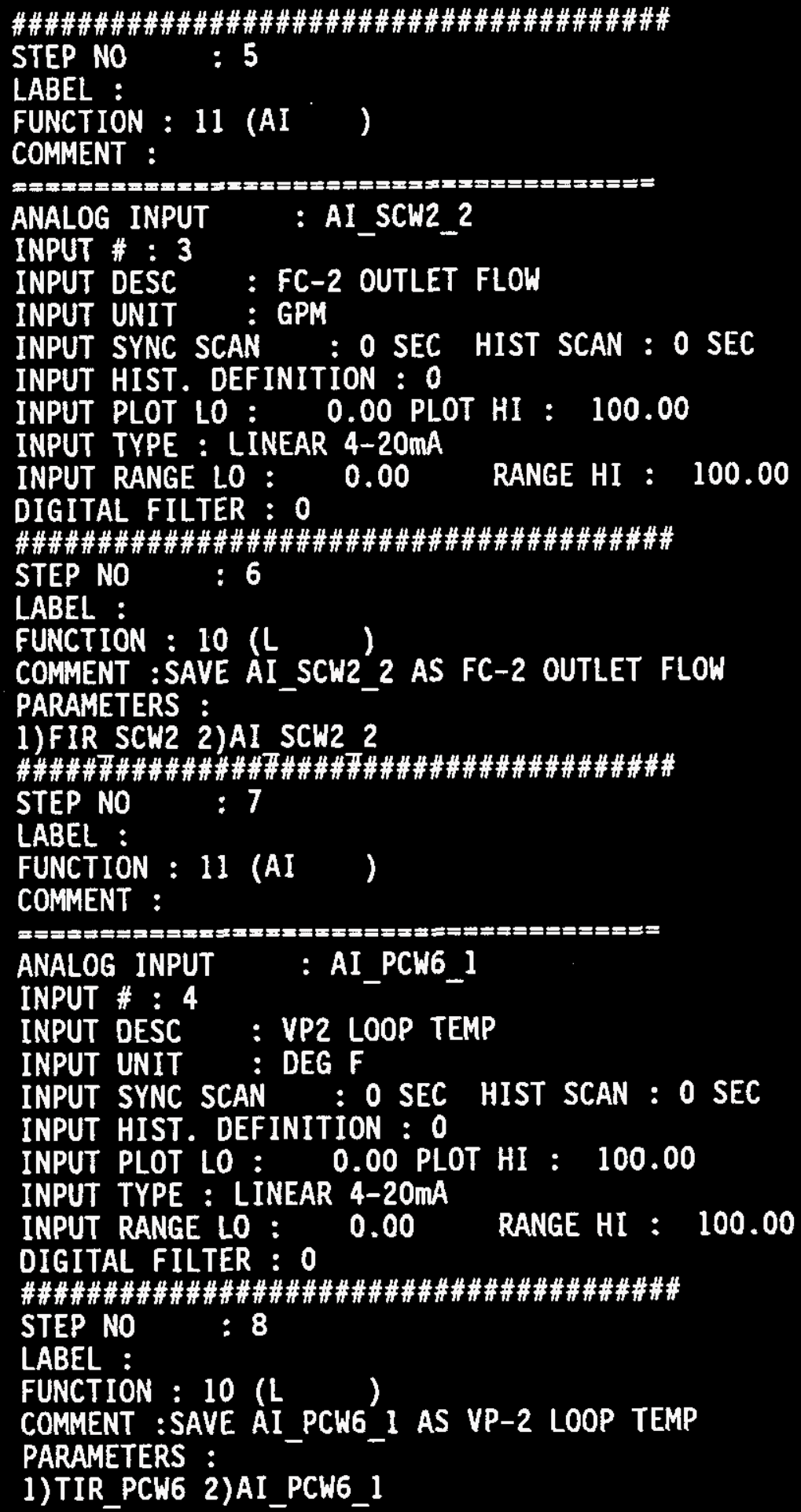


WHC-SD-CP-CSWD-016

Volume 3

Revision 1

Page 26

\#\#\#\#\#\#\#\#\#\#\#\#\#\#\#\#\#\#\#\#\#\#\#\#\#\#\#\#\#\#\#\#\#\#\#\#\#\#\#\#

STEP NO : 9

LABEL :

FUNCTION : 11 (AI)

COMMENT :

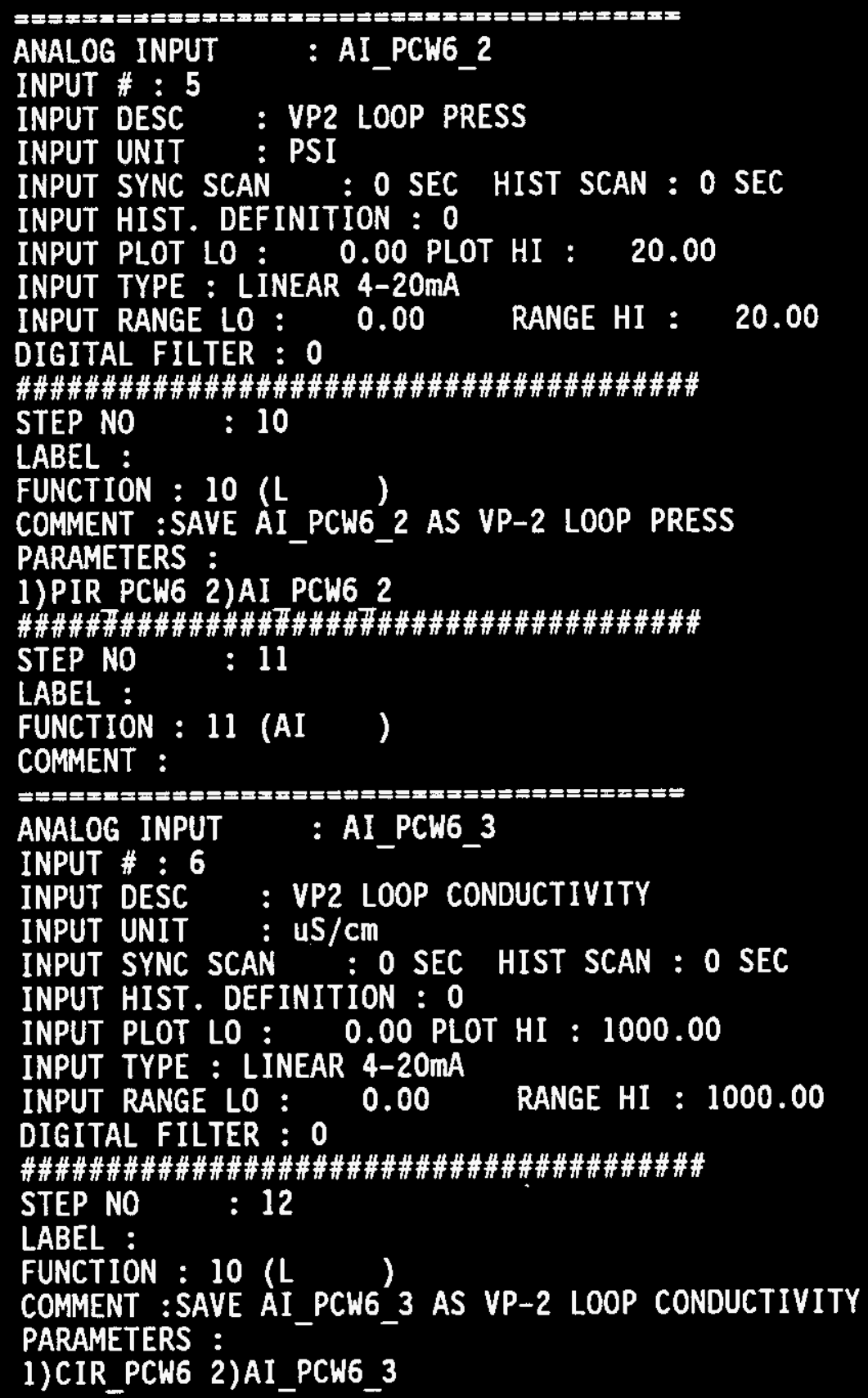


WHC-SD-CP-CSWD-016

Volume 3

Revision 1

Page 27

\#\#\#\#\#\#\#\#\#\#\#\#\#\#\#\#\#\#\#\#\#\#\#\#\#\#\#\#\#\#\#\#\#\#\#\#\#\#\#\#

STEP NO : 13

LABEL :

FUNCTION : 11 (AI)

COMMENT :

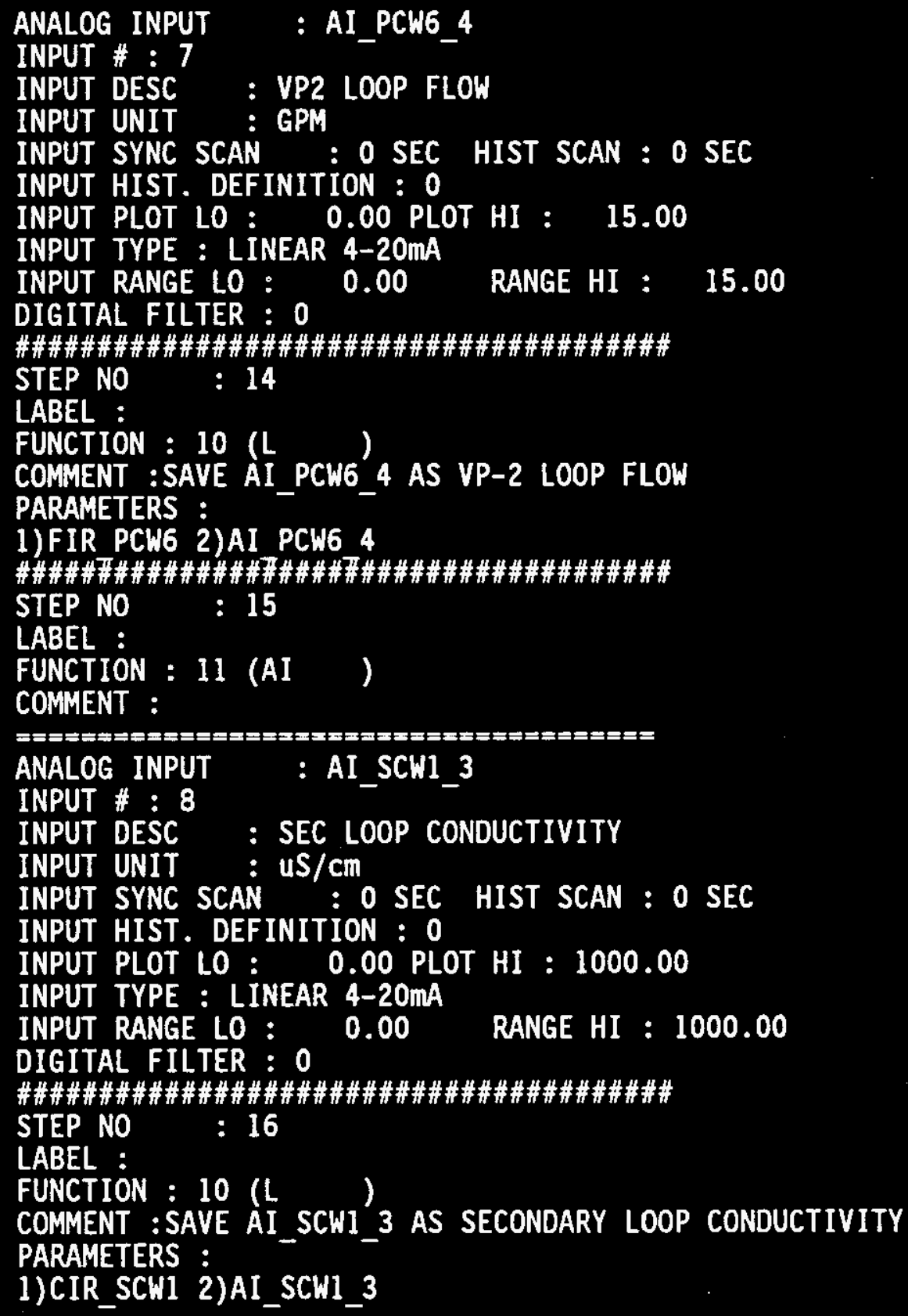


\#\#\#\#\#\#\#\#\#\#\#\#\#\#\#\#\#\#\#\#\#\#\#\#\#\#\#\#\#\#\#\#\#\#\#\#\#\#\#

STEP NO : 17

LABEL :

FUNCTION : 11 (AI)

COMMENT :

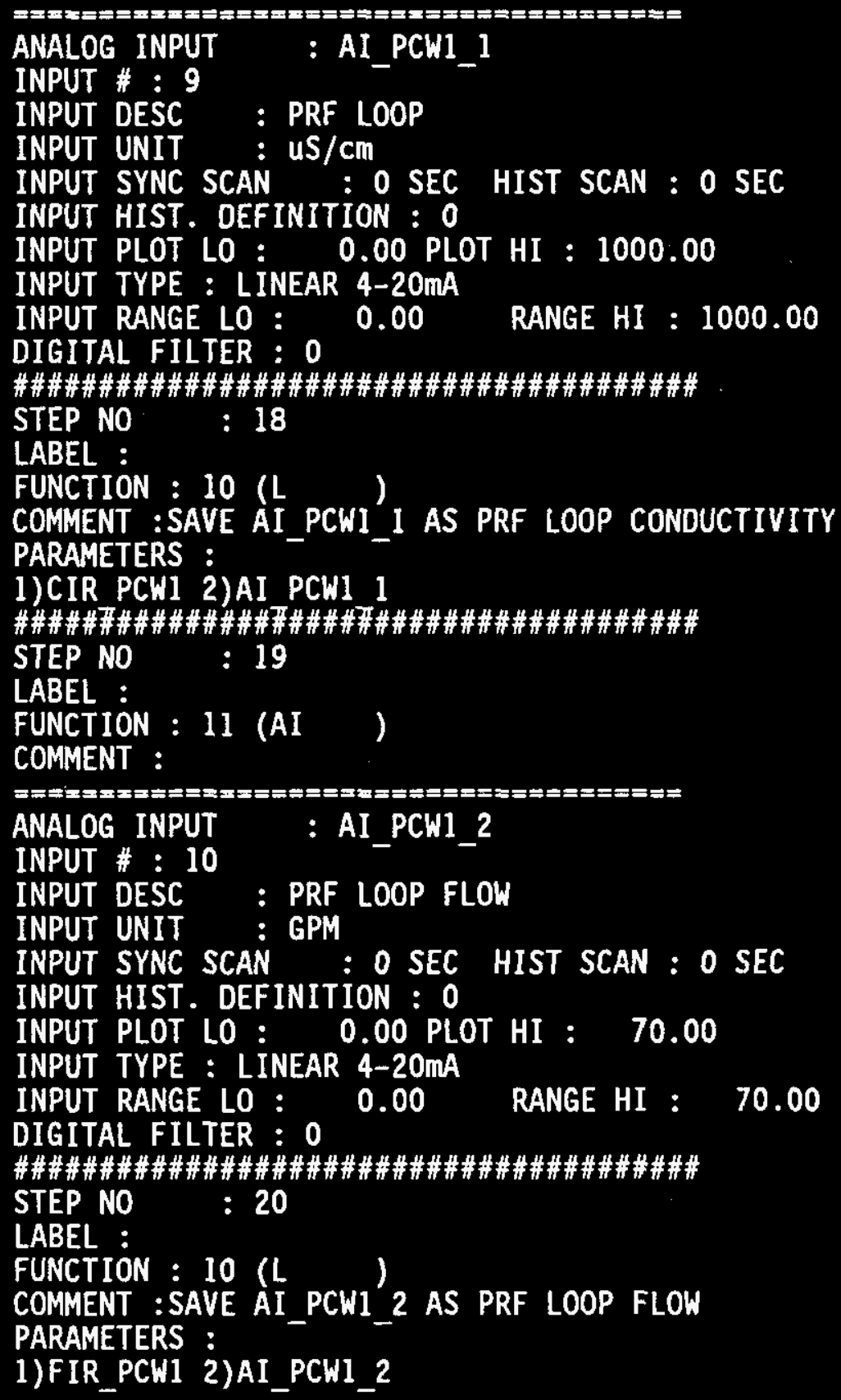


WHC-SD-CP-CSWD-016

Volume 3

Revision 1

Page 29

\#\#\#\#\#\#\#\#\#\#\#\#\#\#\#\#\#\#\#\#\#\#\#\#\#\#\#\#\#\#\#\#\#\#\#\#\#\#\#\#

STEP NO : 21

LABEL :

FUNCTION : 11 (AI )

COMMENT :

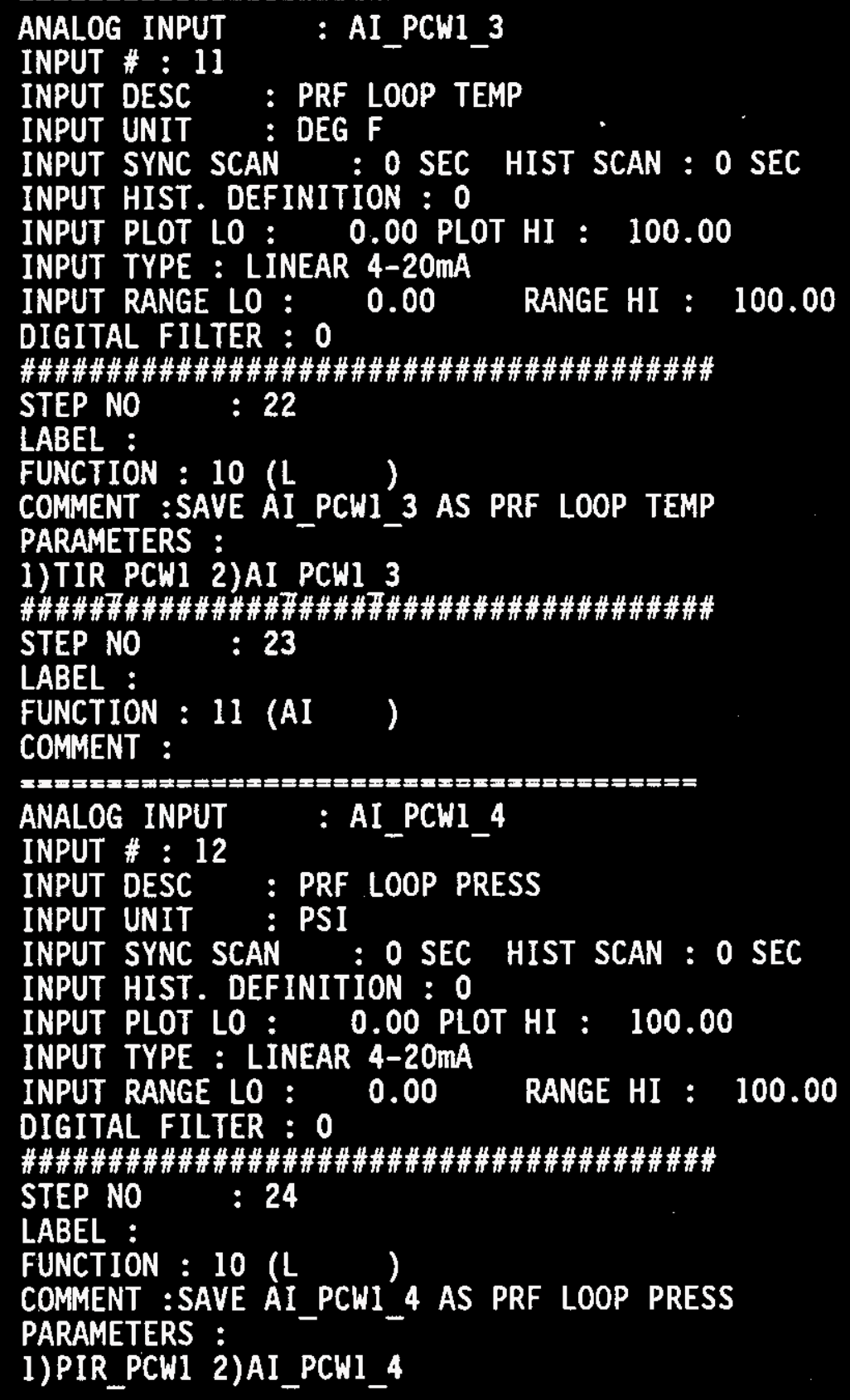


WHC-SD-CP-CSWD-016

Volume 3

Revision 1

Page 30

\#\#\#\#\#\#\#\#\#\#\#\#\#\#\#\#\#\#\#\#\#\#\#\#\#\#\#\#\#\#\#\#\#\#\#\#\#\#\#\#

STEP NO : 25

LABEL :

FUNCTION : 0 (END )

COMMENT : 
WHC-SD-CP-CSWD-016

Volume 3

Revision 1

Page 31

RN45_2_1.mic11_ro

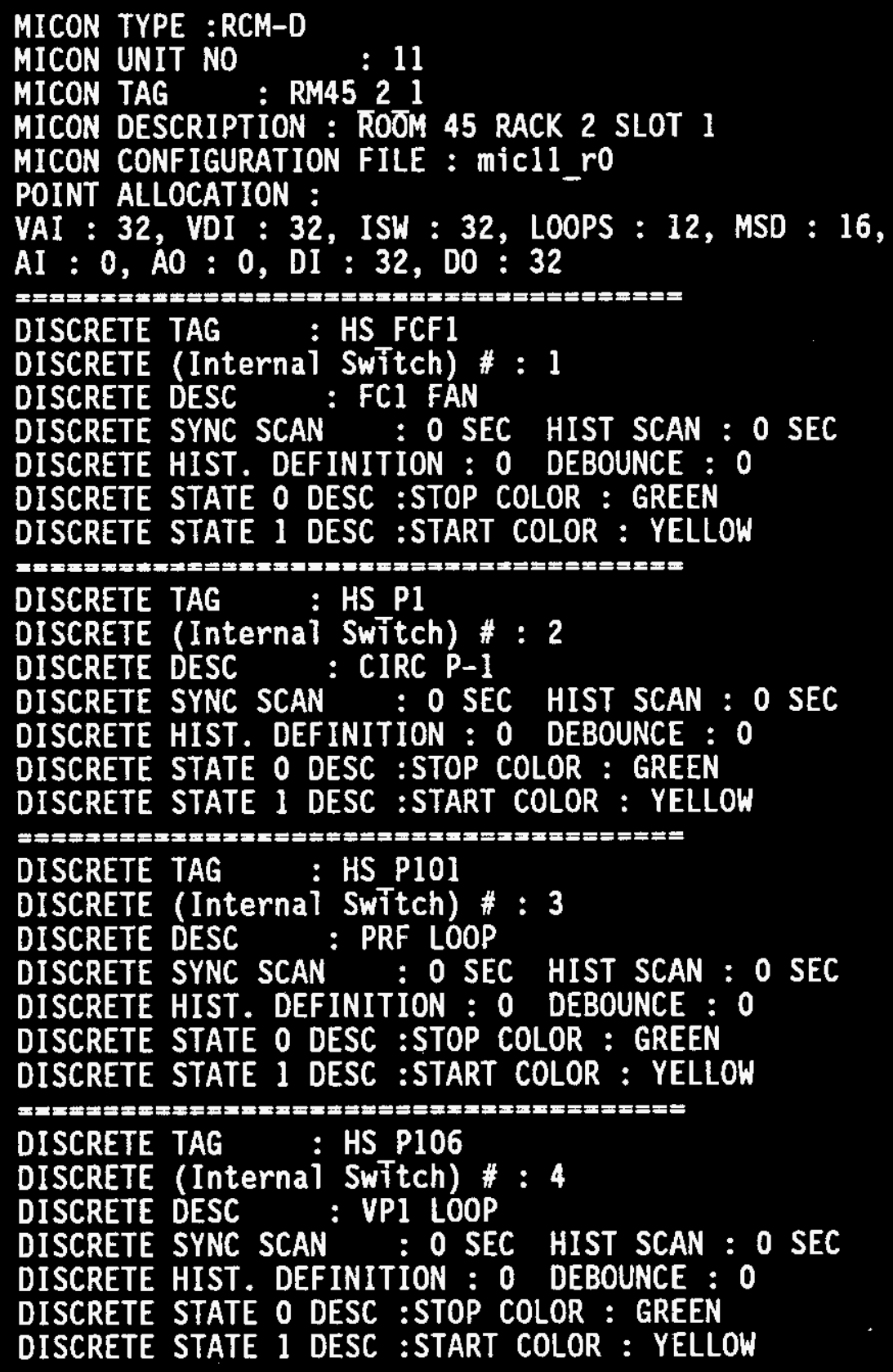


WHC-SD-CP-CSWD-016

Volume 3

Revision 1

Page 32

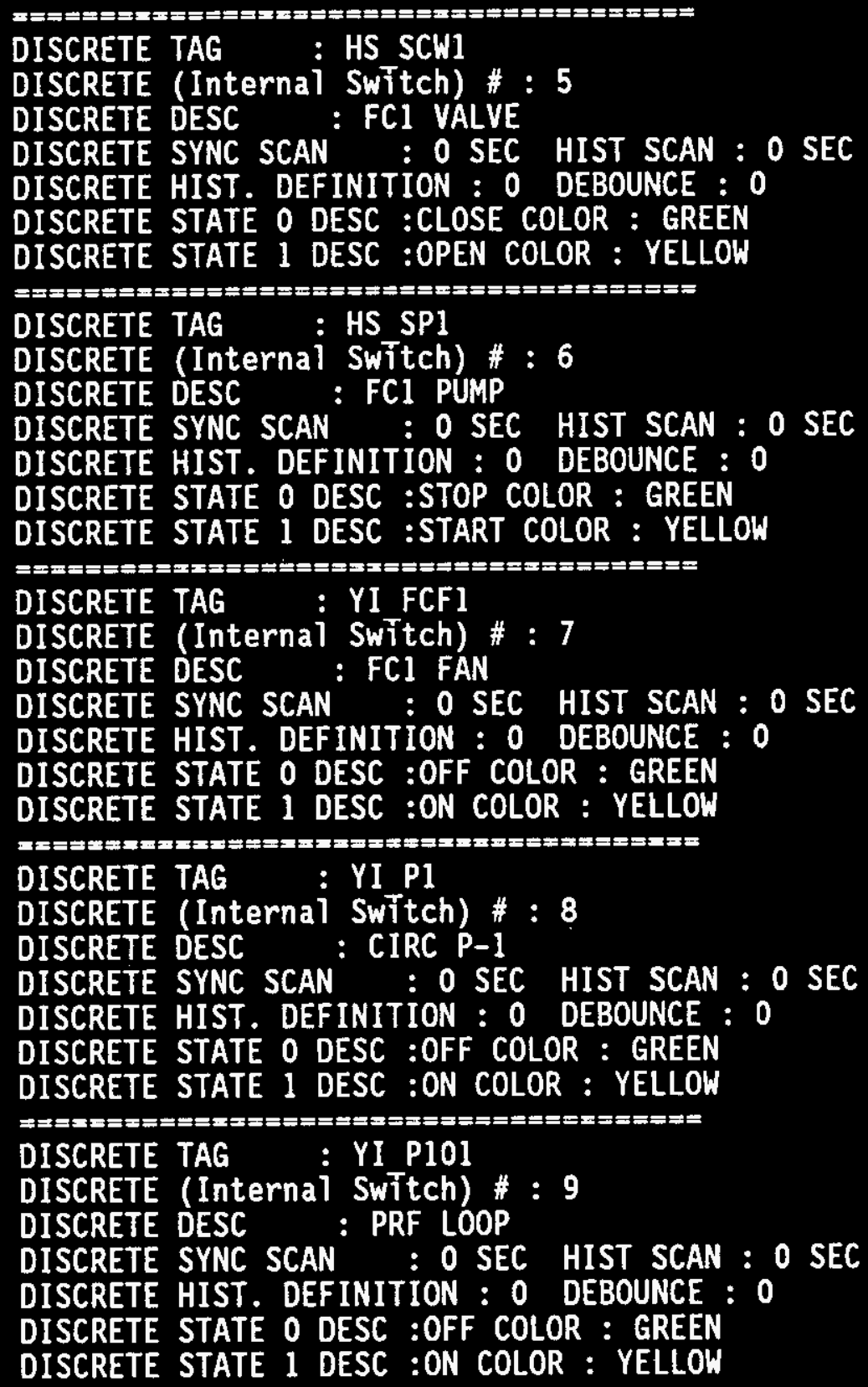


WHC-SD-CP-CSWD-016

Volume 3

Revision 1

Page 33

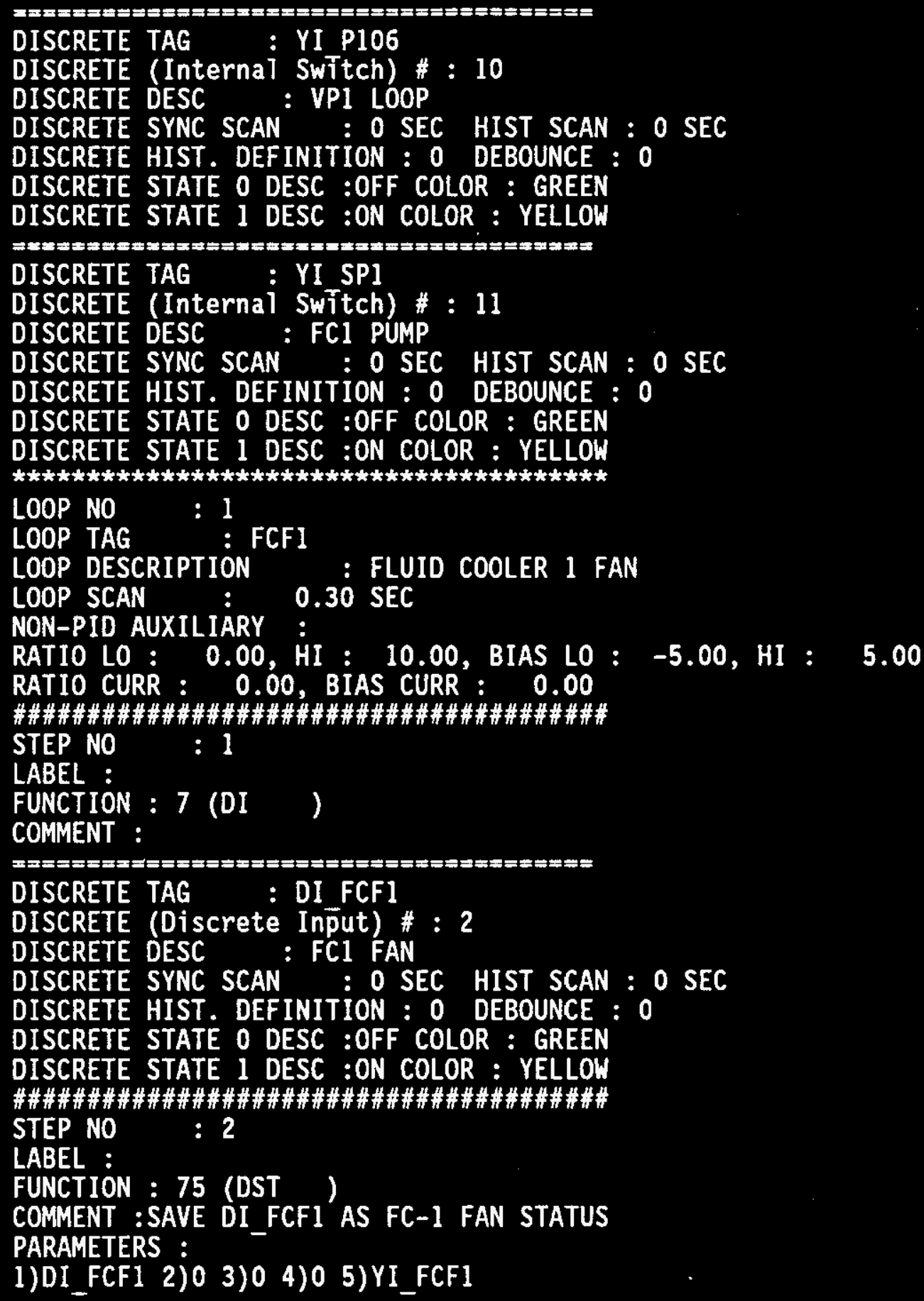




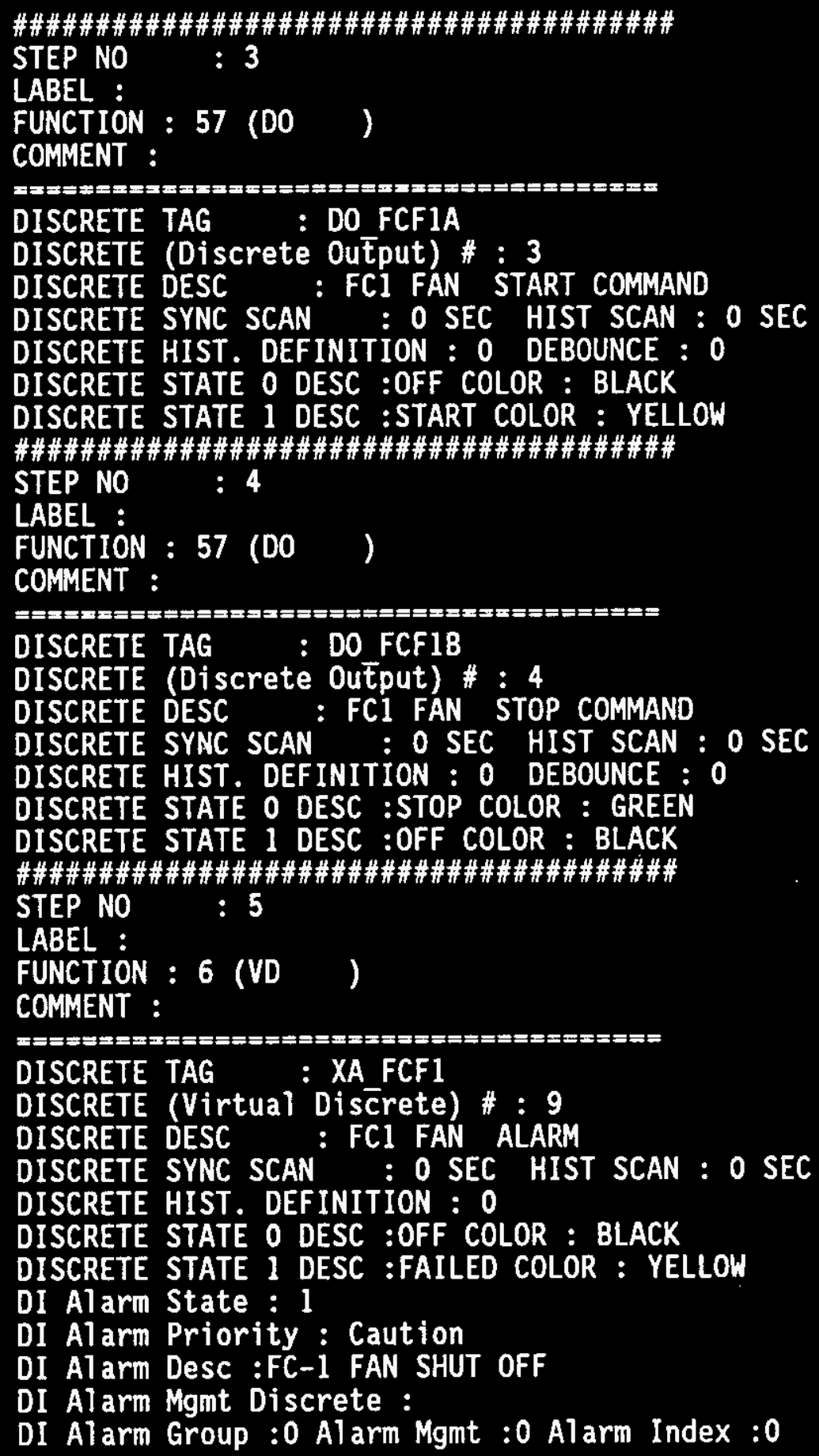



WHC-SD-CP-CSWD-016
Volume 3
Revision 1
Page 35

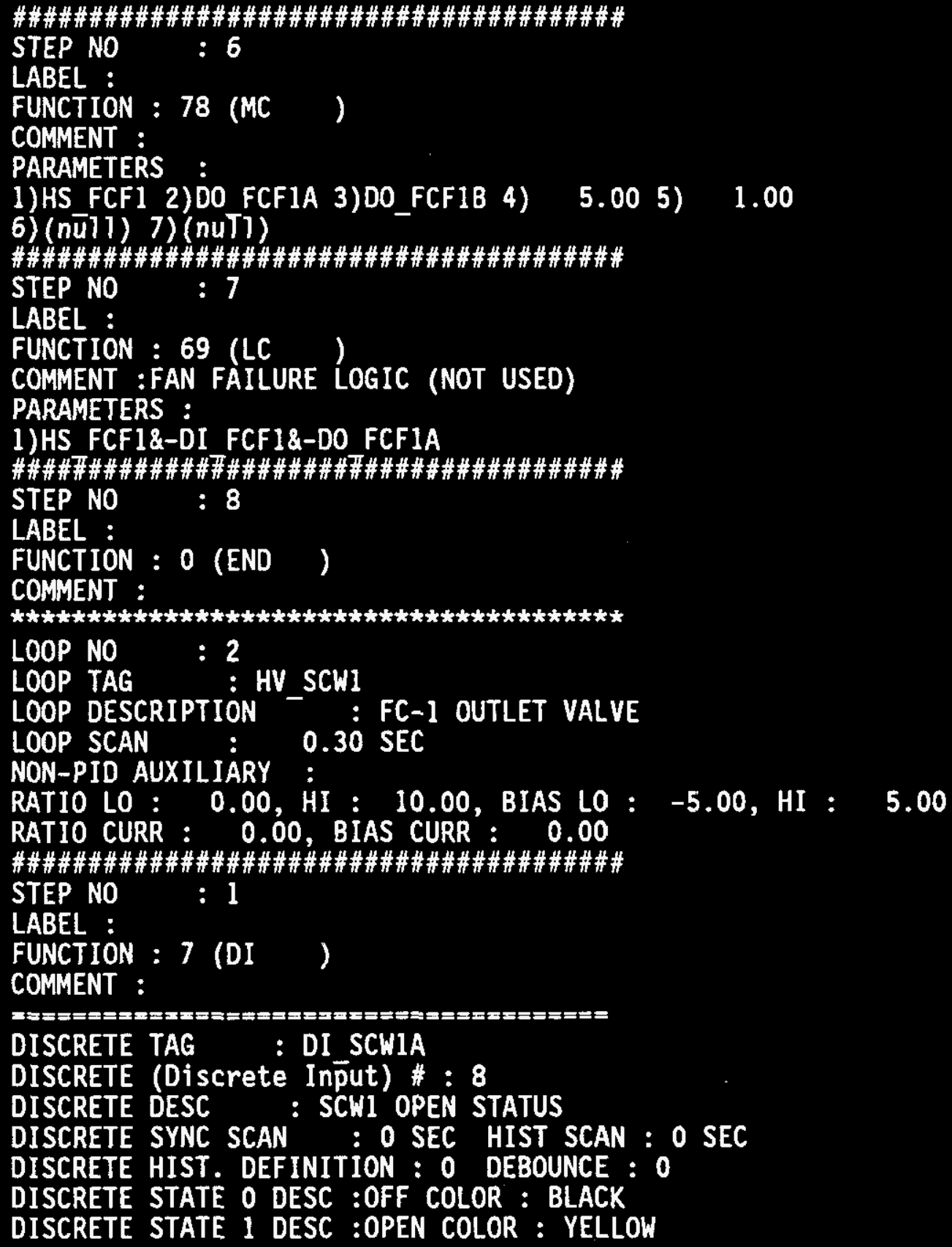


WHC-SD-CP-CSWD-016

Volume 3

Revision 1

Page 36

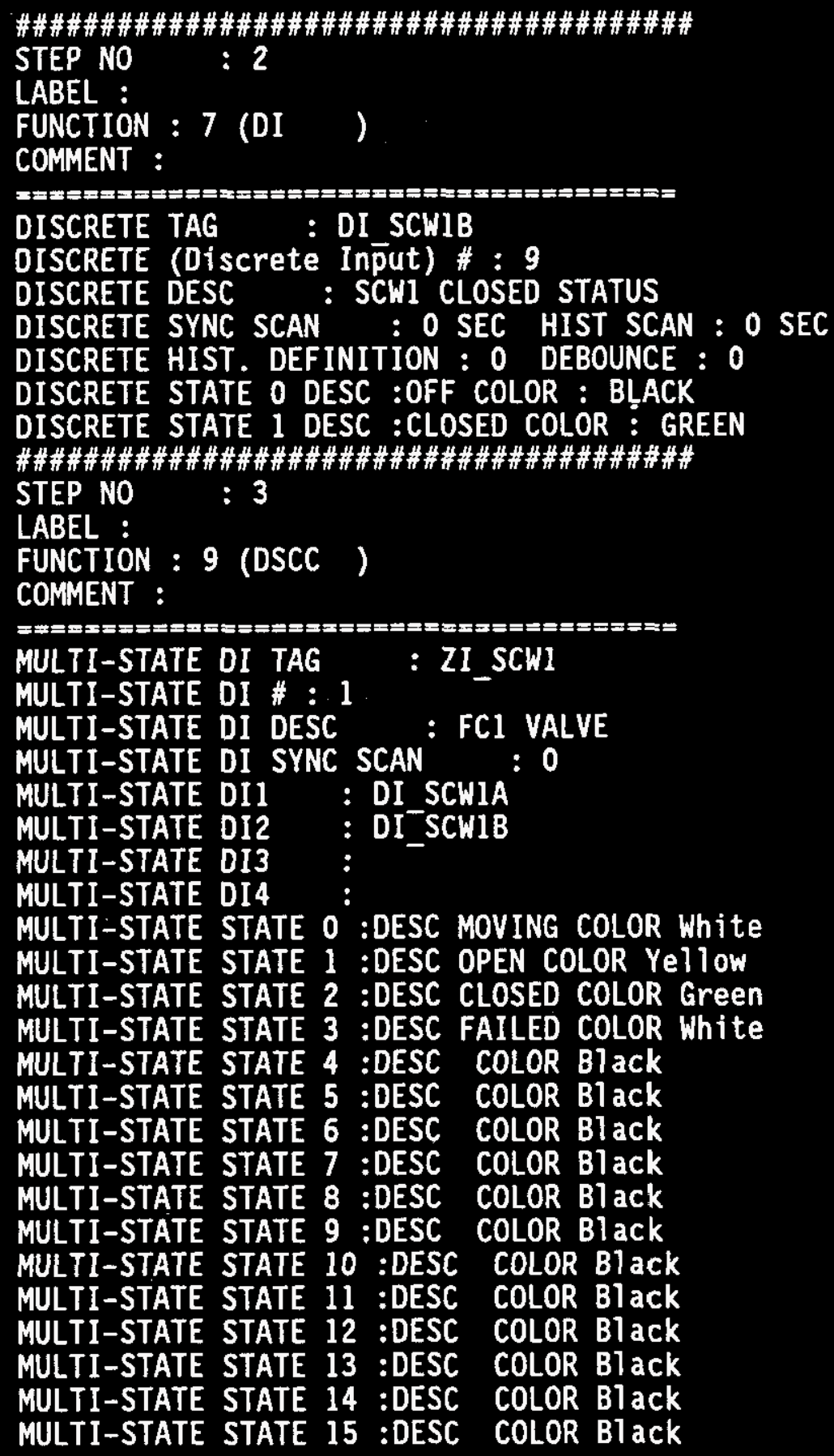


WHC-SD-CP-CSWD-016

Volume 3

Revision 1

Page 37

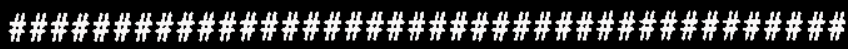

STEP NO : 4

LABEL :

FUNCTION : 57 (DO )

COMMENT :

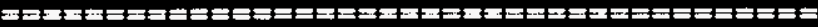

DISCRETE TAG : DO SCWIA

DISCRETE (Discrete Output) \# : 10

DISCRETE DESC : SCWI OPEN COMMAND

DISCRETE SYNC SCAN : O SEC HIST SCAN : O SEC

DISCRETE HIST. DEFINITION : 0 DEBOUNCE : 0

DISCRETE STATE 0 DESC :OFF COLOR : BLACK

DISCRETE STATE 1 DESC :OPEN COLOR : YELLOW

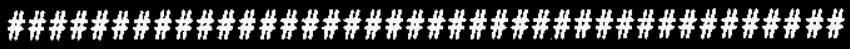

STEP NO

: 5

LABEL :

FUNCTION : 57 (DO)

COMMENT :

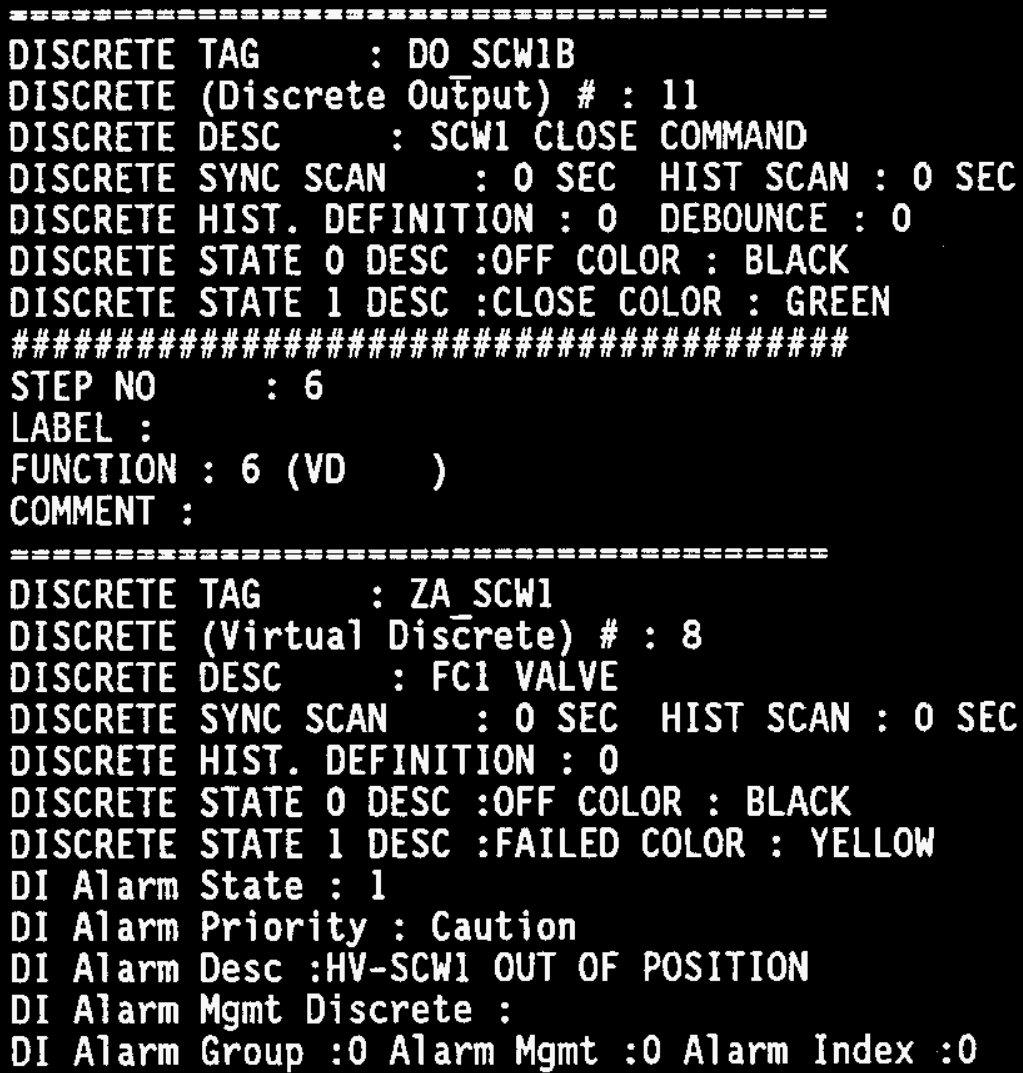


WHC-SD-CP-CSWD-016

Volume 3

Revision 1

Page 38

\#\#\#\#\#\#\#\#\#\#\#\#\#\#\#\#\#\#\#\#\#\#\#\#\#\#\#\#\#\#\#\#\#\#\#\#\#\#\#\#

STEP NO : 7

LABEL :

FUNCTION : 75 (DST)

COMMENT : VALVE OPEN COMMAND

PARAMETERS :

1)HS SCW1. 2)0 3)0 4)0 5)DO SCW1A.

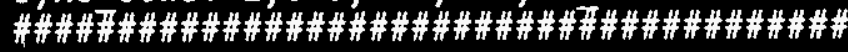

STEP NO : 8

LABEL :

FUNCTION : 75 (DST)

COMMENT :CLOSE COMMAND

PARAMETERS :

1) HS SCW1. 2) 1 3) 0 4)0 5)DO SCW18.

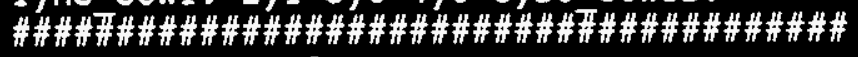

STEP NO : 9

LABEL :

FUNCTION : 69 (LC )

COMMENT : ALARM IF VALVE OUT OF POSITION (AFTER 30 SEC DELAY)

PARAMETERS :

1) (DELAY (HS SCW1, 30)\&-DI SCW1A) ! (DELAY(-HS_SCW1, 30)\&-DI_SCW1B)

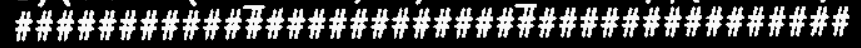

STEP NO : 10

LABEL :

FUNCTION : 75 (DST )

COMMENT :

PARAMETERS :

1)MSV 2)0 3)0 4)0 5)ZA SCW1.

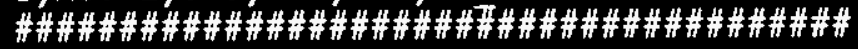

STEP NO : 11

LABEL :

FUNCTION : 0 (END)

COMMENT :

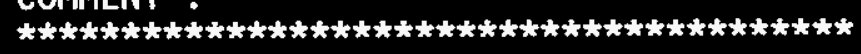

LOOP NO : 3

LOOP TAG : P1

LOOP DESCRIPTION : CIRCULATION PUMP 1

LOOP SCAN : $0.30 \mathrm{SEC}$

NON-PID AUXILIARY :

RATIO LO : 0.00 , HI : 10.00 , BIAS LO : -5.00 , HI : 5.00

RATIO CURR : 0.00 , BIAS CURR : 0.00

IMPORT DI :RM45_1_3::!FSL_SCW1, RM45_1_4::!FSL_SCW2 
WHC-SD-CP-CSWD-016

Volume 3

Revision 1

Page 39

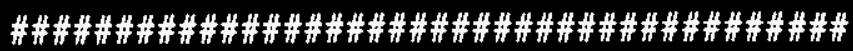

STEP NO : 1

LABEL :

FUNCTION : 7 (DI )

COMMENT :

DISCRETE TAG : DI P1 1

DISCRETE (Discrete Input) \# : 12

DISCRETE DESC : CIRC P-1 STATUS

DISCRETE SYNC SCAN : O SEC HIST SCAN : O SEC

DISCRETE HIST. DEFINITION : 0 DEBOUNCE : 0

DISCRETE STATE O DESC :OFF COLOR : GREEN

DISCRETE STATE 1 DESC :ON COLOR : YELLOW

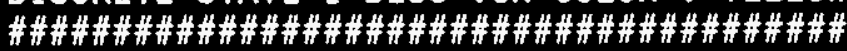

STEP NO

$: 2$

LABEL :

FUNCTION : 75 (DST)

COMMENT :SAVE DI_P1_1 AS P-1 STATUS

PARAMETERS :

1)DI P1 1 2)0 3) 0 4)0 5)YI P1

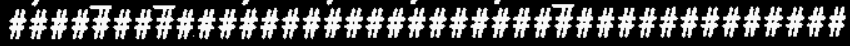

STEP NO : 3

LABEL :

FUNCTION : 7 (DI )

COMMENT :

DISCRETE TAG : DI P1 2

DISCRETE (Discrete Input) \# : 15

DISCRETE DESC : P1 LO FLOW SWITCH

DISCRETE SYNC SCAN : O SEC HIST SCAN : O SEC

DISCRETE HIST. DEFINITION : 0 DEBOUNCE : 0

DISCRETE STATE O DESC :OFF COLOR : BLACK

DISCRETE STATE 1 DESC :LO FLOW COLOR : YELLOW 
WHC-SD-CP-CSWD-016

Volume 3

Revision 1

Page 40

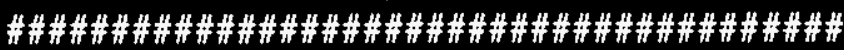

STEP NO

: 4

LABEL :

FUNCTION : 6 (VD )

COMMENT :

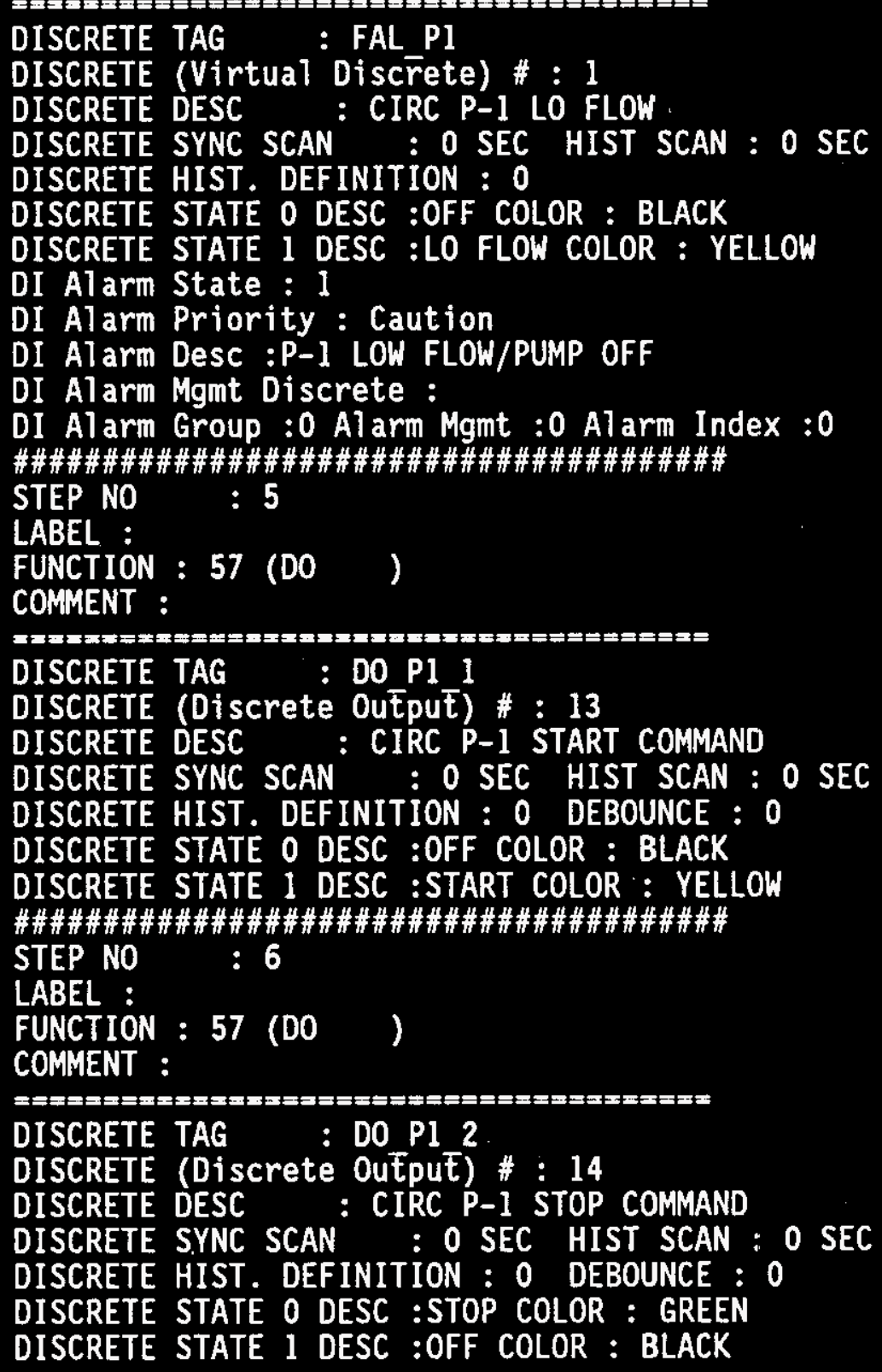


WHC-SD-CP-CSWD-016

Volume 3

Revision 1

Page 41

\#\#\#\#\#\#\#\#\#\#\#\#\#\#\#\#\#\#\#\#\#\#\#\#\#\#\#\#\#\#\#\#\#\#\#\#\#\#\#\#

STEP NO : 7

LABEL :

FUNCTION : 78 (MC )

COMMENT :

PARAMETERS :

1)HS P1 2)DO P1 1 3)DO_P1_2 4) 5.00 5) 1.00

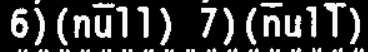

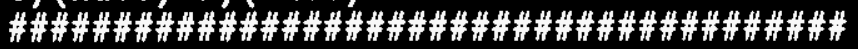

STEP NO : 8

LABEL :

FUNCTION : 69 (LC

COMMENT : ALARM ON LO FLOW \& P1 OFF (EXCEPT STARTUP)

PARAMETERS :

1)-DO P1 18((FSL SCW1\&FSL SCW2) |(HS P1\&-DI_P1_1))

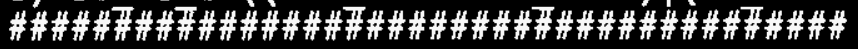

STEP NO : 9

LABEL :

FUNCTION : 75 (DST )

COMMENT :

PARAMETERS :

1)MSV 2)0 3)0 4)0 5)FAL P1

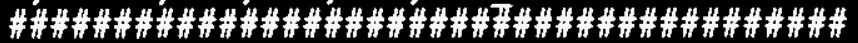

STEP NO : 10

LABEL :

FUNCTION : 69 (LC

COMMENT : SEND PULSE ON LO FLON (RESTART RESETS)

PARAMETERS :

1) PULSE (FSL SCW18FSL SCW2\&-DO P1 1)

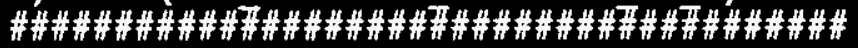

STEP NO : 11

LABEL :

FUNCTION : 62 (GTD

COMMENT :SKIP NEXT STEP IF NO PULSE

PARAMETERS :

1)!MSV 2) Normal 3) JMP

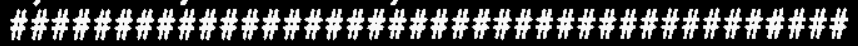

STEP NO : 12

LABEL : JMP

FUNCTION : 10 (L

COMMENT : TURN PUMP OFF IF PULSE (LOW FLOW)

PARAMETERS :

1) HS P1 2) 0

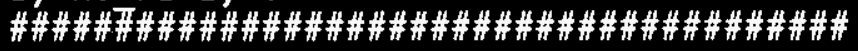

STEP NO : 13

LABEL :

FUNCTION : 0 (END )

COMMENT : 
WHC-SD-CP-CSWD-016

Volume 3

Revision 1

Page 42

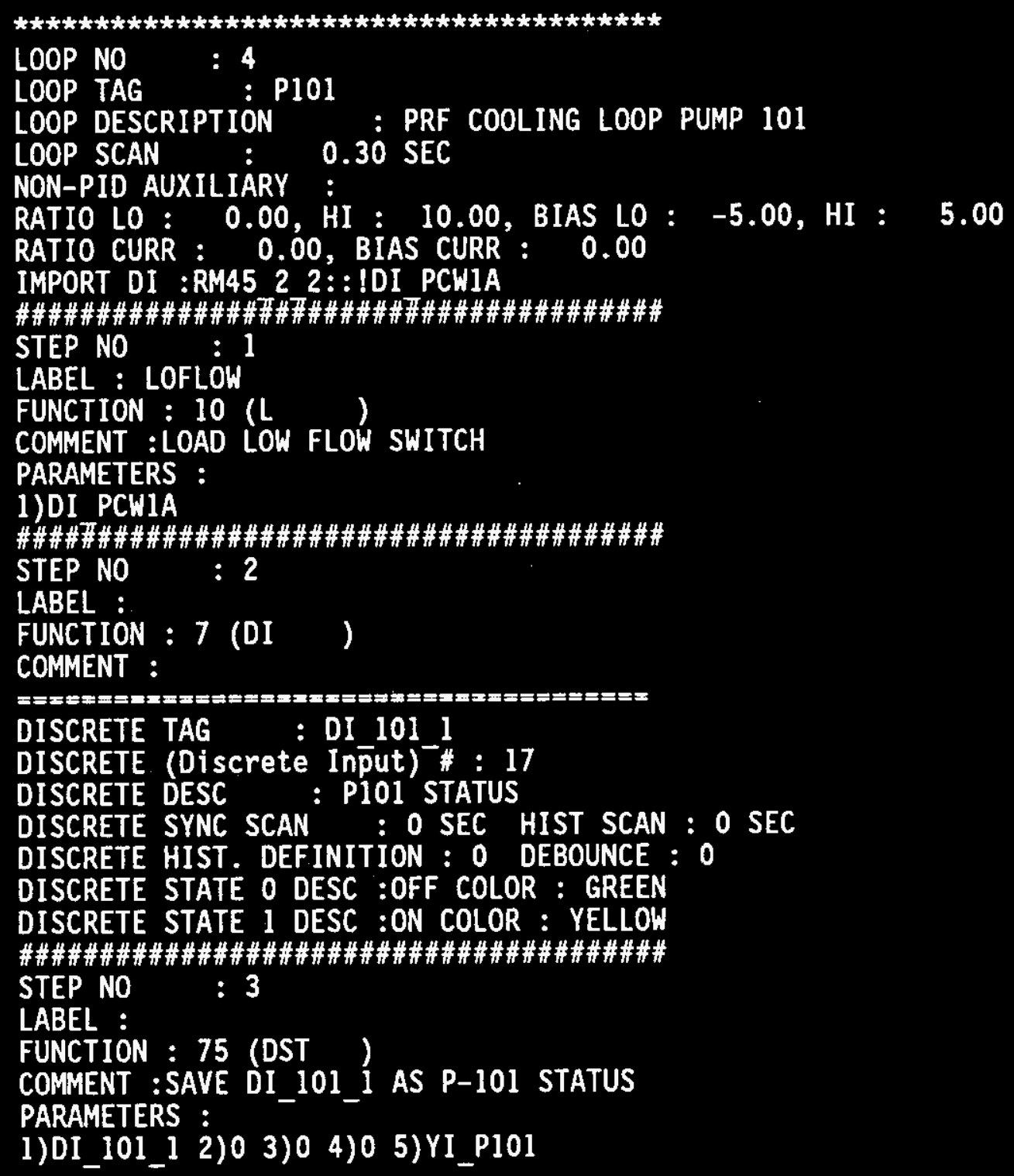


WHC-SD-CP-CSWD-016

Volume 3

Revision 1

Page 43

\#\#\#\#\#\#\#\#\#\#\#\#\#\#\#\#\#\#\#\#\#\#\#\#\#\#\#\#\#\#\#\#\#\#\#\#\#\#\#\#

STEP NO : 4

LABEL :

FUNCTION : 6 (VD )

COMMENT :

DISCRETE TAG : XA P101

DISCRETE (Virtual Discrete) \#: 5

DISCRETE DESC : P101 ALARM

DISCRETE SYNC SCAN : 0 SEC HIST SCAN : O SEC

DISCRETE HIST. DEFINITION : 0

DISCRETE STATE 0 DESC :OFF COLOR : BLACK

DISCRETE STATE 1 DESC :LO FLOW COLOR : YELLOW

DI Alarm State : 1

DI Alarm Priority : Caution

DI Alarm Desc :PRF LOOP LO FLOW/P-101 OFF

DI Alarm Mgmt Discrete :

DI A1arm Group :0 Alarm Mgmt :0 Alarm Index :0

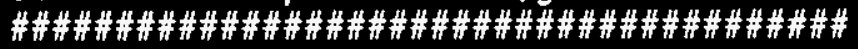

STEP NO : 5

LABEL :

FUNCTION : $57($ DO )

COMMENT :

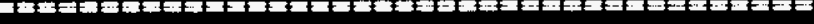

DISCRETE TAG : DO 1011

DISCRETE (Discrete Output) \# : 18

DISCRETE DESC : P101 START COMMAND

DISCRETE SYNC SCAN : O SEC HIST SCAN : O SEC

DISCRETE HIST. DEFINITION : 0 DEBOUNCE : 0

DISCRETE STATE 0 DESC :OFF COLOR : BLACK

DISCRETE STATE 1 DESC :START COLOR : YELLOW

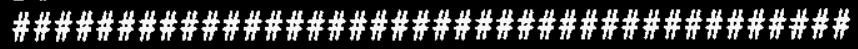

STEP NO : 6

LABEL :

FUNCTION : $57(00)$

COMMENT :

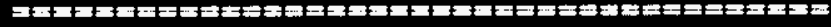

DISCRETE TAG : DO 1012

DISCRETE (Discrete Output) \# : 19

DISCRETE DESC : P101 STOP COMMAND

DISCRETE SYNC SCAN : O SEC HIST SCAN : O SEC

DISCRETE HIST. DEFINITION : 0 DEBOUNCE : 0

DISCRETE STATE O DESC :STOP COLOR : GREEN

DISCRETE STATE 1 DESC :OFF COLOR : BLACK 
WHC-SD-CP-CSWD-016

Volume 3

Revision 1

Page 44

\#\#\#\#\#\#\#\#\#\#\#\#\#\#\#\#\#\#\#\#\#\#\#\#\#\#\#\#\#\#\#\#\#\#\#\#\#\#\#\#

STEP NO : 7

LABEL :

FUNCTION : 78 (MC )

COMMENT :

PARAMETERS :

1)HS P101 2)DO 101_1 3)D0_101_2 4) 5.00 5) 1.00

6) (nū11) 7) (nuT1)

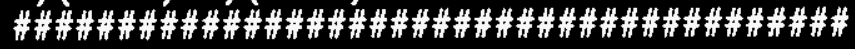

STEP NO : 8

LABEL :

FUNCTION : 69 (LC

COMMENT : ALARM ON LO FLOW \& P101 OFF (EXCEPT STARTUP)

PARAMETERS :

1)-DO 101 18(LOFLOW (HS P101\&-DI 101 1))

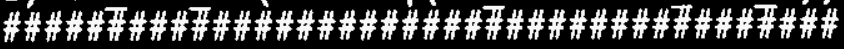

STEP NO : 9

LABEL :

FUNCTION : 75 (DST)

COMMENT :SAVE ALARM

PARAMETERS :

1)MSV 2) 0 3) 0 4) 0 5) XA P101

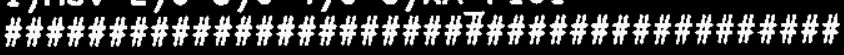

STEP NO : 10

LABEL :

FUNCTION : 69 (LC )

COMMENT :SEND PULSE ON LOW FLOW (RESTART RESETS)

PARAMETERS :

1) PULSE(LOFLOW\&-D0 101 1)

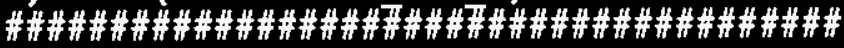

STEP NO : 11

LABEL :

FUNCTION : 62 (GTD )

COMMENT : SKIP NEXT STEP IF NO PULSE

PARAMETERS :

1) IMSV 2) Normal 3) JMP

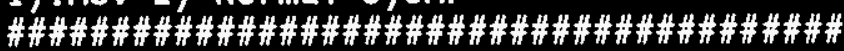

STEP NO : 12

LABEL : JMP

FUNCTION : 10 (L

COMMENT : TURN OFF PUMP ON PULSE (LOW FLOW)

PARAMETERS :

1) HS P101 2) 0

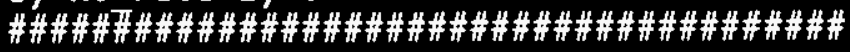

STEP NO : 13

LABEL :

FUNCTION : 0 (END)

COMMENT : 


\section{WHC-SD-CP-CSWO-016 \\ Volume 3 \\ Revision 1 \\ Page 45}

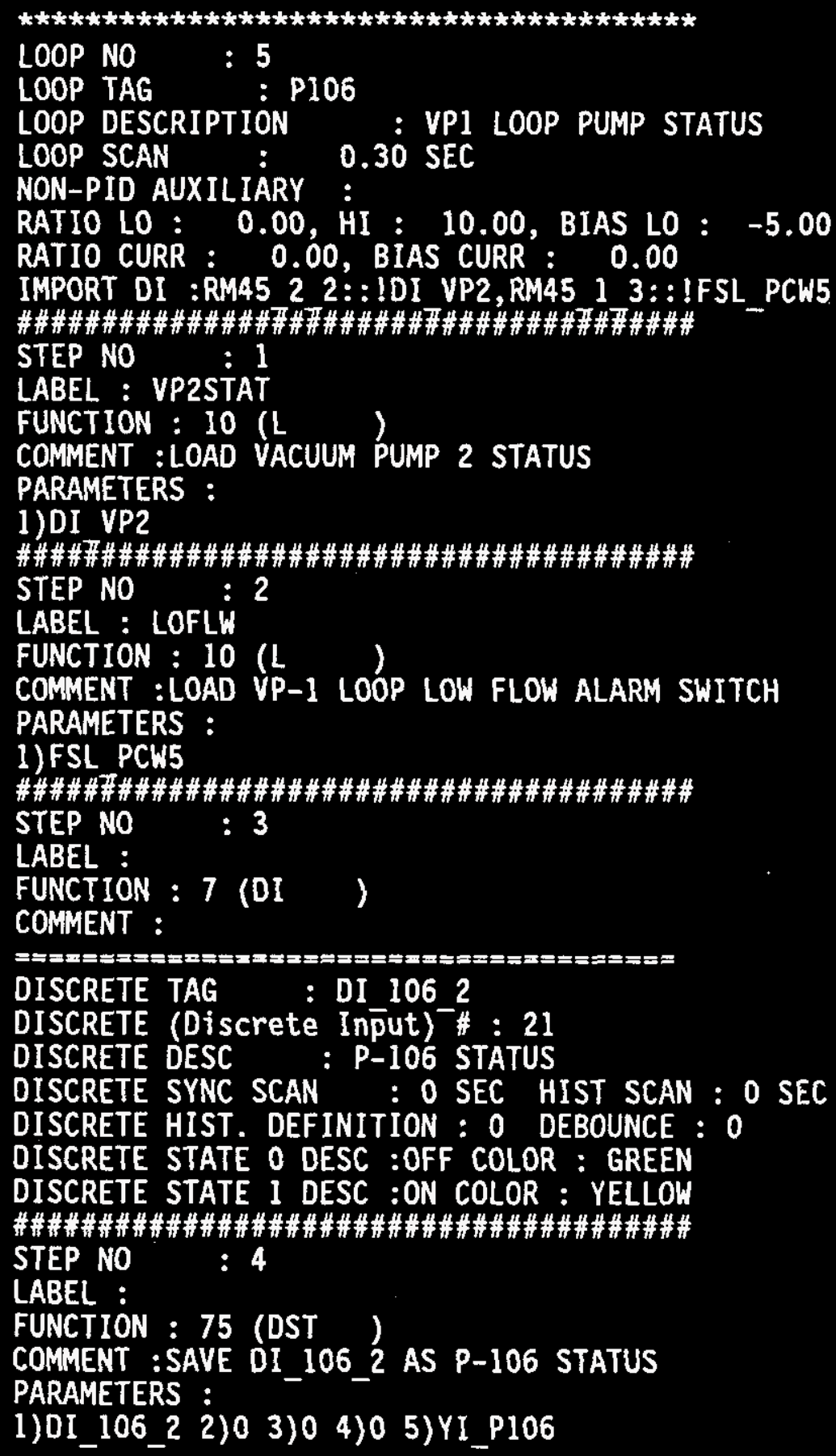




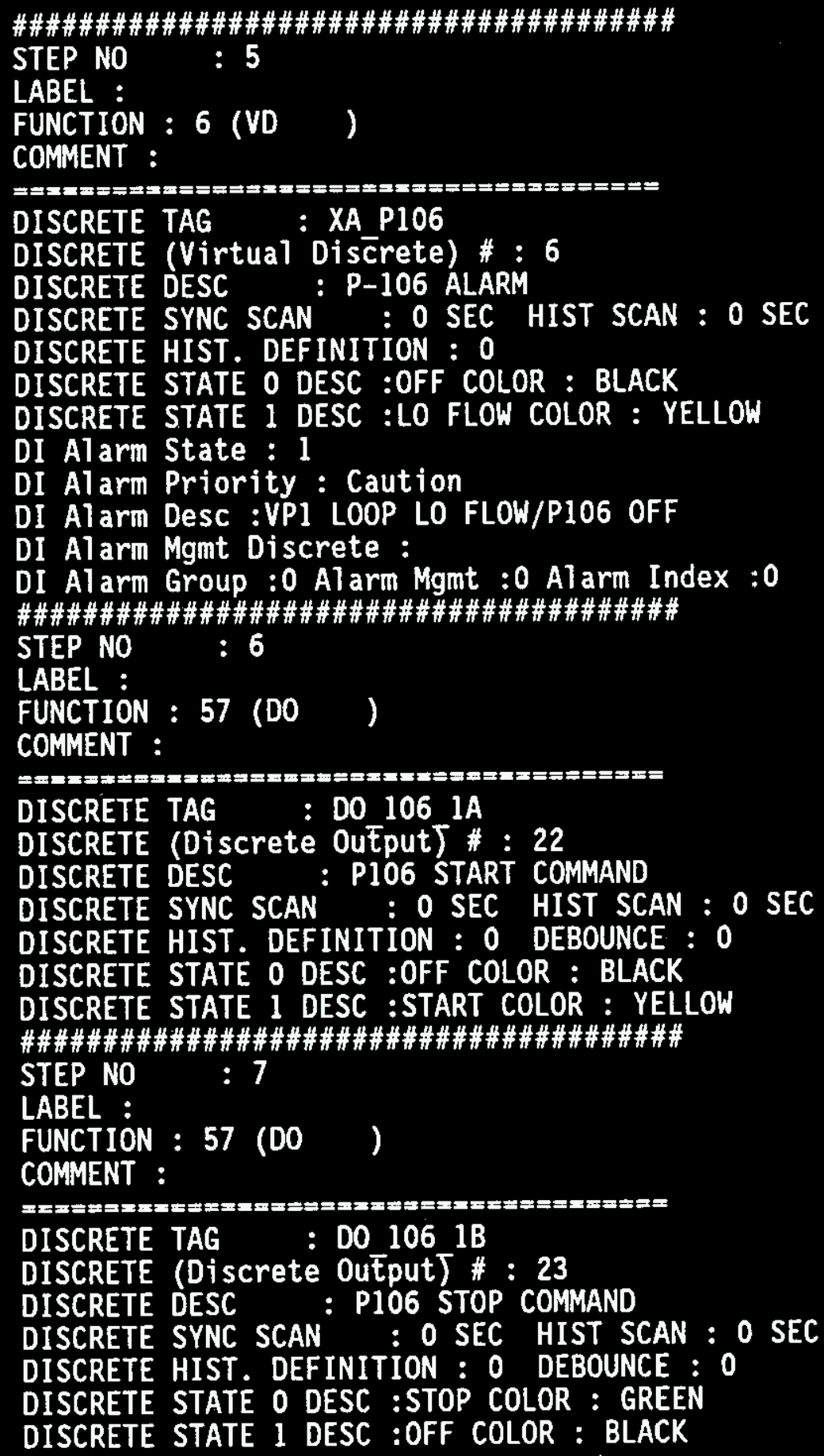




\section{WHC-SD-CP-CSWD-016 \\ Volume 3 \\ Revision 1 \\ Page 47}

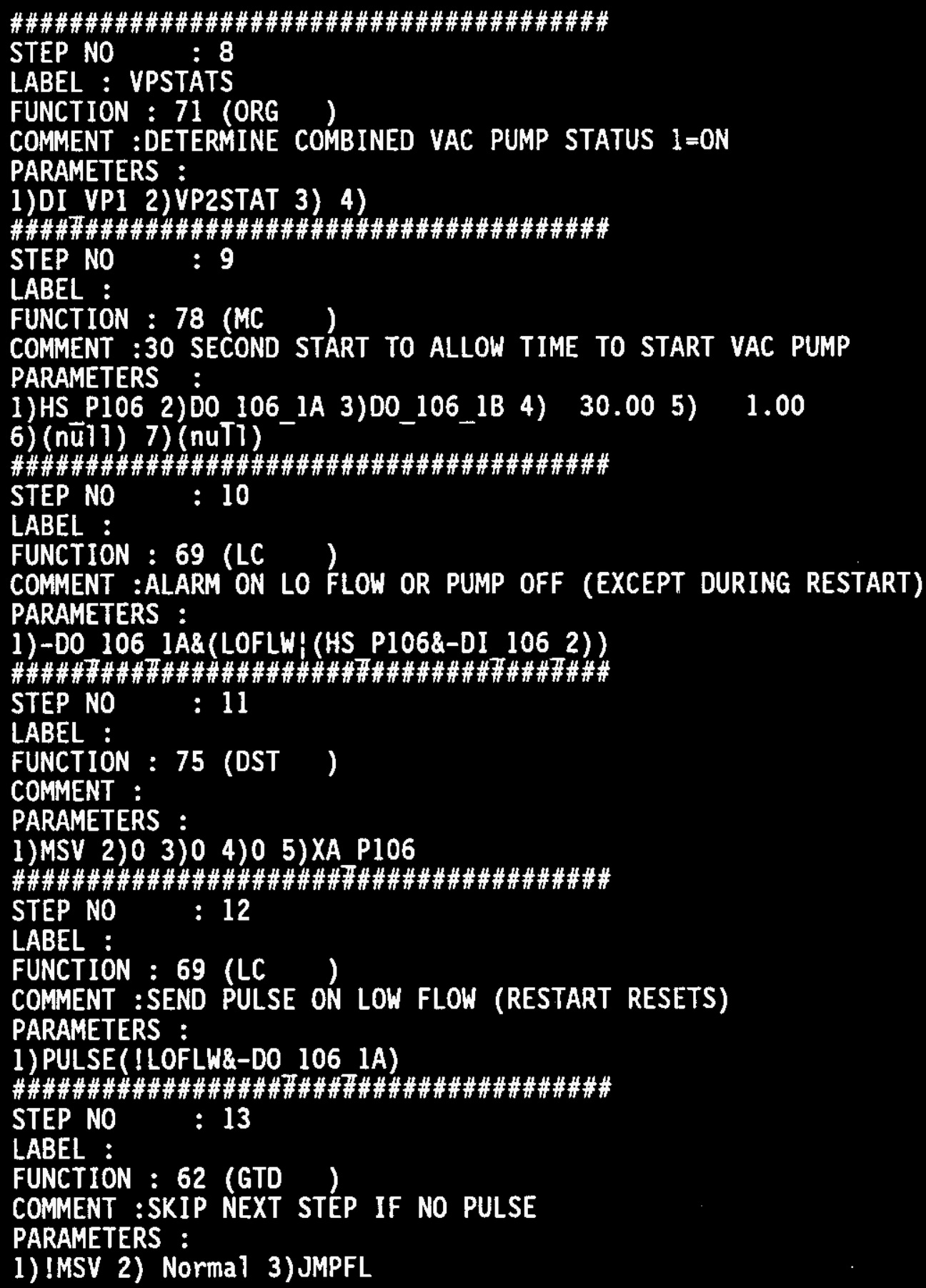




\section{WHC-SD-CP-CSWD-016 \\ Volume 3 \\ Revision 1 \\ Page 48}

\#\#\#\#\#\#\#\#\#\#\#\#\#\#\#\#\#\#\#\#\#\#\#\#\#\#\#\#\#\#\#\#\#\#\#\#\#\#\#

STEP NO : 14

LABEL : JMPFL

FUNCTION : 10 (L

COMMENT : TURN OFF PUMP ON PULSE (LON FLOW)

PARAMETERS :

1) HS P106 2) 0

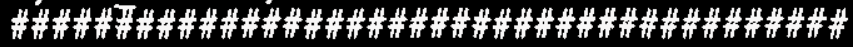

STEP NO : 15

LABEL :

FUNCTION : 71 (ORG

COMMENT :OUTPUT=1 IF A VAC PUMP IS RUNNING OR P106 IS RESTARTING

PARAMETERS :

1) VPSTATS 2)DO 106 IA 3) 4)

\#\#\#\#\#\#\#\#\#\#\#\#\#\#\#\#\#开\#\#\#\#\#\#\#\#\#\#\#\#\#\#\#\#\#\#\#

STEP NO : 16

LABEL :

FUNCTION : 62 (GTD)

COMMENT : SKIP NEXT STEP IF VP's RUNING OR P106 IN RESTART

PARAMETERS :

1) MSV 2) Off-Noma] 3) JMPVP

\#\#\#\#\#\#\#\#\#\#\#\#\#\#\#\#\#\#\#\#\#\#\#\#\#\#\#\#\#\#\#\#\#\#\#\#\#\#\#

STEP NO : 17

LABEL : JMPVP

FUNCTION : 10 (L

COMMENT : TURN OFF PUMP IF BOTH VAC PUMPS OFF (EXCEPT OURING RESTART)

PARAMETERS :

1) HS P106 2) 0

\#\#\#\#\#男\#\#\#\#\#\#\#\#\#\#\#\#\#\#\#\#\#\#\#\#\#\#\#\#\#\#\#\#\#\#

STEP NO : 18

LABEL :

FUNCTION : 0 (END)

COMMENT :

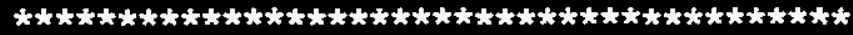

LOOP NO : 6

LOOP TAG : SP1

LOOP DESCRIPTION : FLUID COOLER 1 SPRAY PUMP

LOOP SCAN : $0.30 \mathrm{SEC}$

NON-PID AUXILIARY :

RATIO LO : 0.00 , HI : 10.00 , BIAS LO : -5.00 , HI : 5.00

RATIO CURR : 0.00 , BIAS CURR : 0.00 


\section{WHC-SD-CP-CSWD-016 \\ Volume 3 \\ Revision 1 \\ Page 49}

\#\#\#\#\#\#\#\#\#\#\#\#\#\#\#\#\#\#\#\#\#\#\#\#\#\#\#\#\#\#\#\#\#\#\#\#\#\#\#\#

STEP NO : 1

LABEL :

FUNCTION : 7 (DI )

COMMENT :

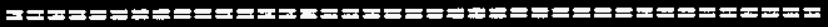

DISCRETE TAG : DI_SPI

DISCRETE (Discrete Input) \# : 5

DISCRETE DESC : SP-1 STATUS

DISCRETE SYNC SCAN : O SEC HIST SCAN : O SEC

DISCRETE HIST. DEFINITION : 0 DEBOUNCE : 0

DISCRETE STATE 0 DESC :OFF COLOR : GREEN

DISCRETE STATE 1 DESC :ON COLOR : YELLOW

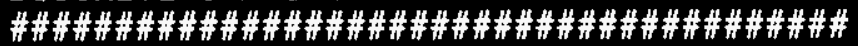

STEP NO $\quad: 2$

LABEL :

FUNCTION : 75 (DST)

COMMENT : SAVE DI_SP1 AS SP-1 STATUS

PARAMETERS :

1)DI SP1 2)0 3)0 4)0 5)YI SP1

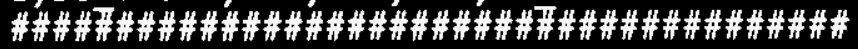

STEP NO : 3

LABEL :

FUNCTION : 57 (DO)

COMMENT :

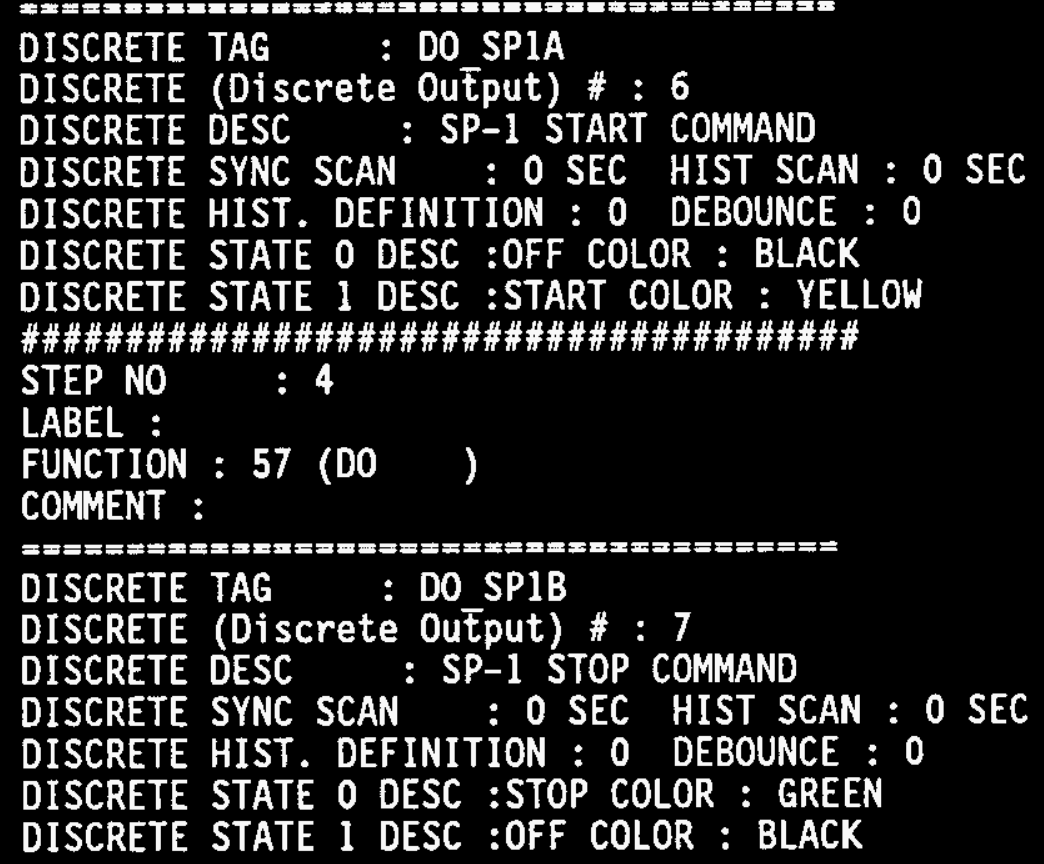



WHC-SD-CP-CSWD-016
Volume 3
Revision 1
Page 50

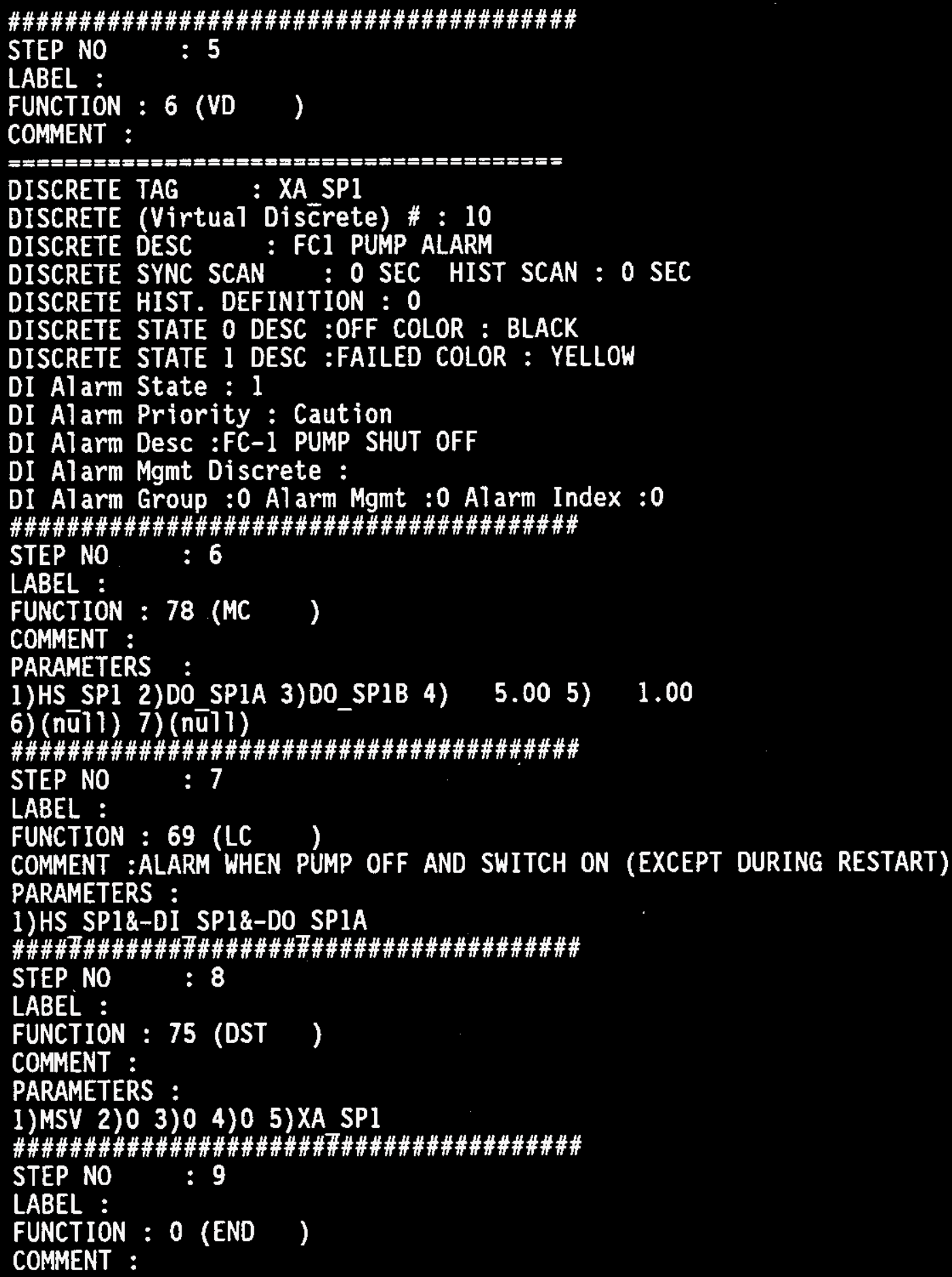


WHC-SD-CP-CSWD-016

Volume 3

Revision 1

Page 51

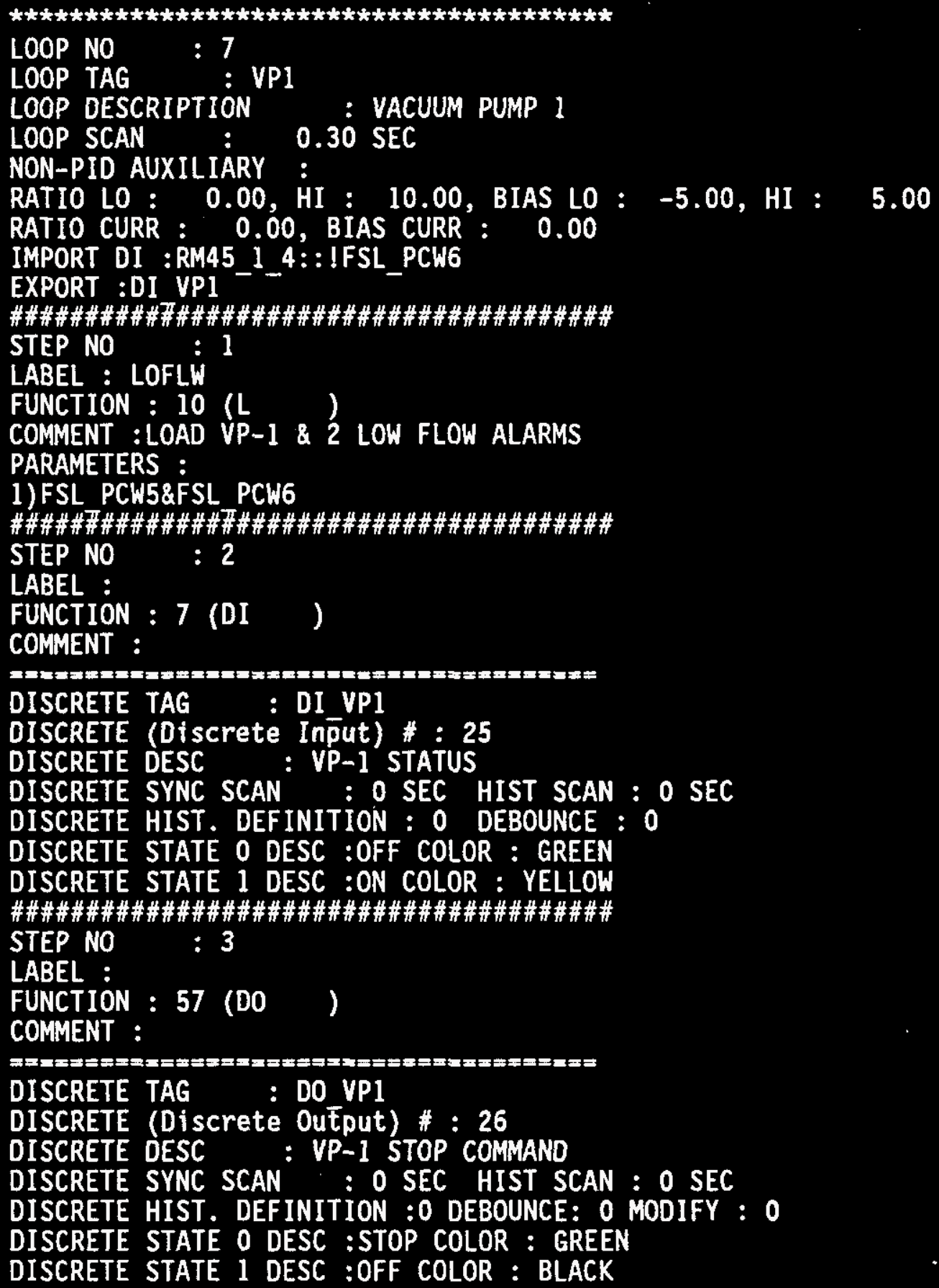


WHC-SD-CP-CSWD-016

Vol ume 3

Revision 1

Page 52

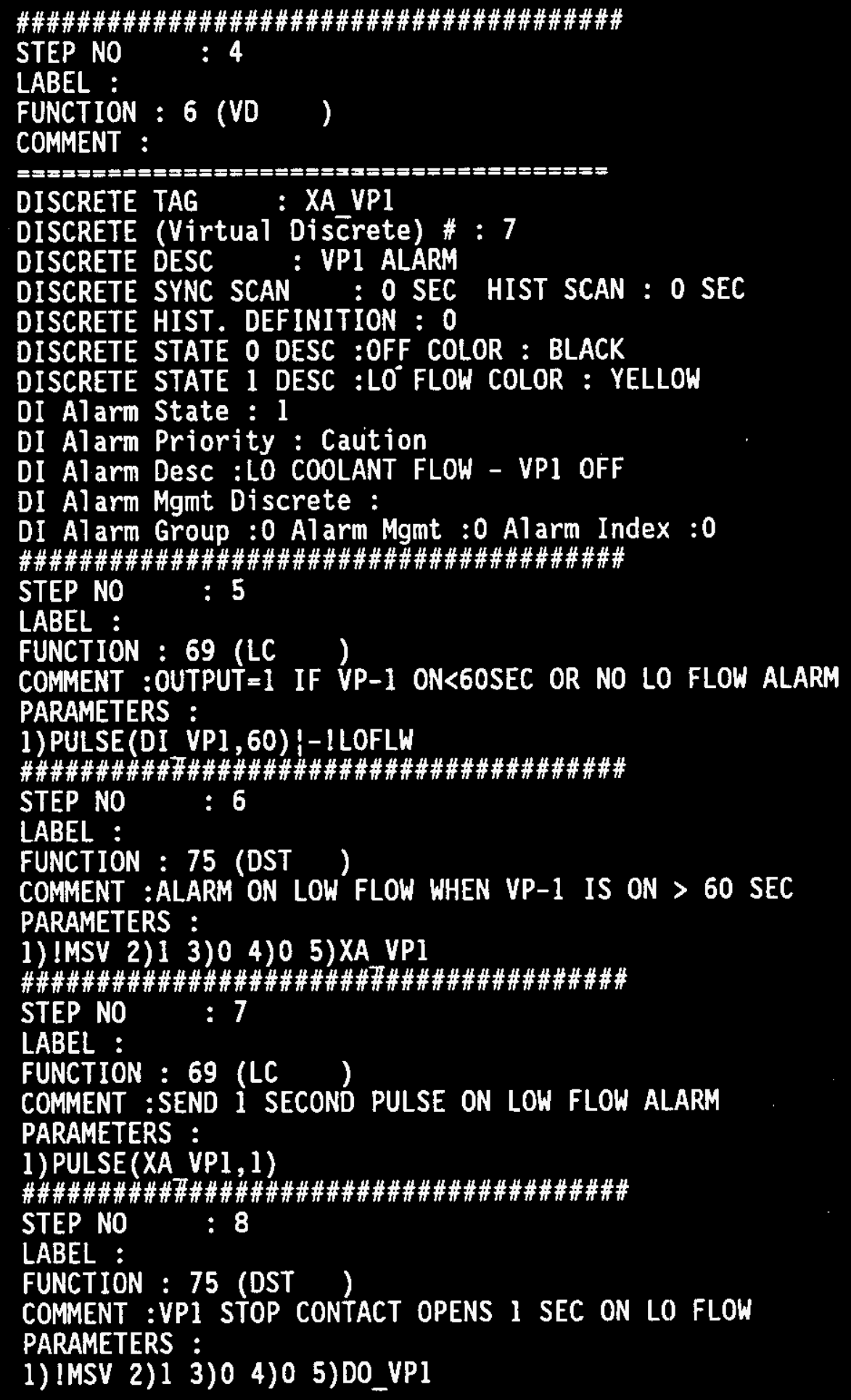


WHC-SD-CP-CSWD-016

Volume 3

Revision 1

Page 53

\#\#\#\#\#\#\#\#\#\#\#\#\#\#\#\#\#\#\#\#\#\#\#\#\#\#\#\#\#\#\#\#\#\#\#\#\#\#\#\#

STEP NO $: 9$

LABEL :

FUNCTION : 0 (END )

COMMENT :

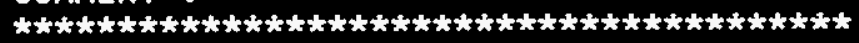

LOOP NO

: 8

LOOP TAG

: MISC M11

LOOP DESCRIPTION

LOOP SCAN :

: MISC I/0

NON-PID AUXILIARY :

RATIO LO : 0.00 , HI : 10.00 , BIAS LO : -5.00, HI : 5.00

RATIO CURR : 0.00 , BIAS CURR : 0.00

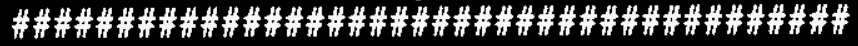

STEP NO : 1

LABEL :

FUNCTION : 6 (VD )

COMMENT :

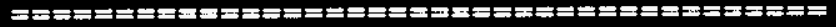

DISCRETE TAG : LALTK105

DISCRETE (Virtual Discrete) \# : 2

DISCRETE DESC : TK-105 ALARM,

DISCRETE SYNC SCAN : O SEC HIST SCAN : O SEC

DISCRETE HIST. DEFINITION : 0

DISCRETE STATE O DESC :OFF COLOR : BLACK

DISCRETE STATE 1 DESC :LO LEVEL COLOR : YELLOW.

DI Alarm State : 1

DI Alarm Priority : Caution

DI Alarm Desc :VAC PUMP SEAL WATER TANK LO LEVEL

DI Alarm Mgmt Discrete :

DI Alarm Group :0 Alarm Mgmt :0 Alarm Index :0

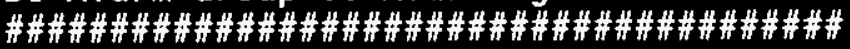

STEP NO : 2

LABEL :

FUNCTION : 7 (DI )

COMMENT :

DISCRETE TAG : DI 1052
DISCRETE (Discrete Input) $\#: 27$
DISCRETE DESC : TK-105 LO LEVEL SWITCH
DISCRETE SYNC SCAN : 0 SEC HIST SCAN : O SEC
DISCRETE HIST. DEFINITION : O DEBOUNCE : 0
DISCRETE STATE O DESC :OFF COLOR : BLACK
DISCRETE STATE 1 DESC :LO LEVEL COLOR : YELLOW


\#\#\#\#\#\#\#\#\#\#\#\#\#\#\#\#\#\#\#\#\#\#\#\#\#\#\#\#\#\#\#\#\#\#\#\#\#\#\#\#

STEP NO : 3

LABEL :

FUNCTION : 75 (DST)

COMMENT : SAVE DI_105_2 AS TK-105 LO LEVEL ALARM

PARAMETERS :

1)DI 1052 2)0 3)0 4)0 5)LALTK105

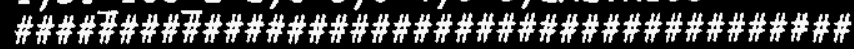

STEP NO : 4

LABEL :

FUNCTION : 6 (VD )

COMMENT :

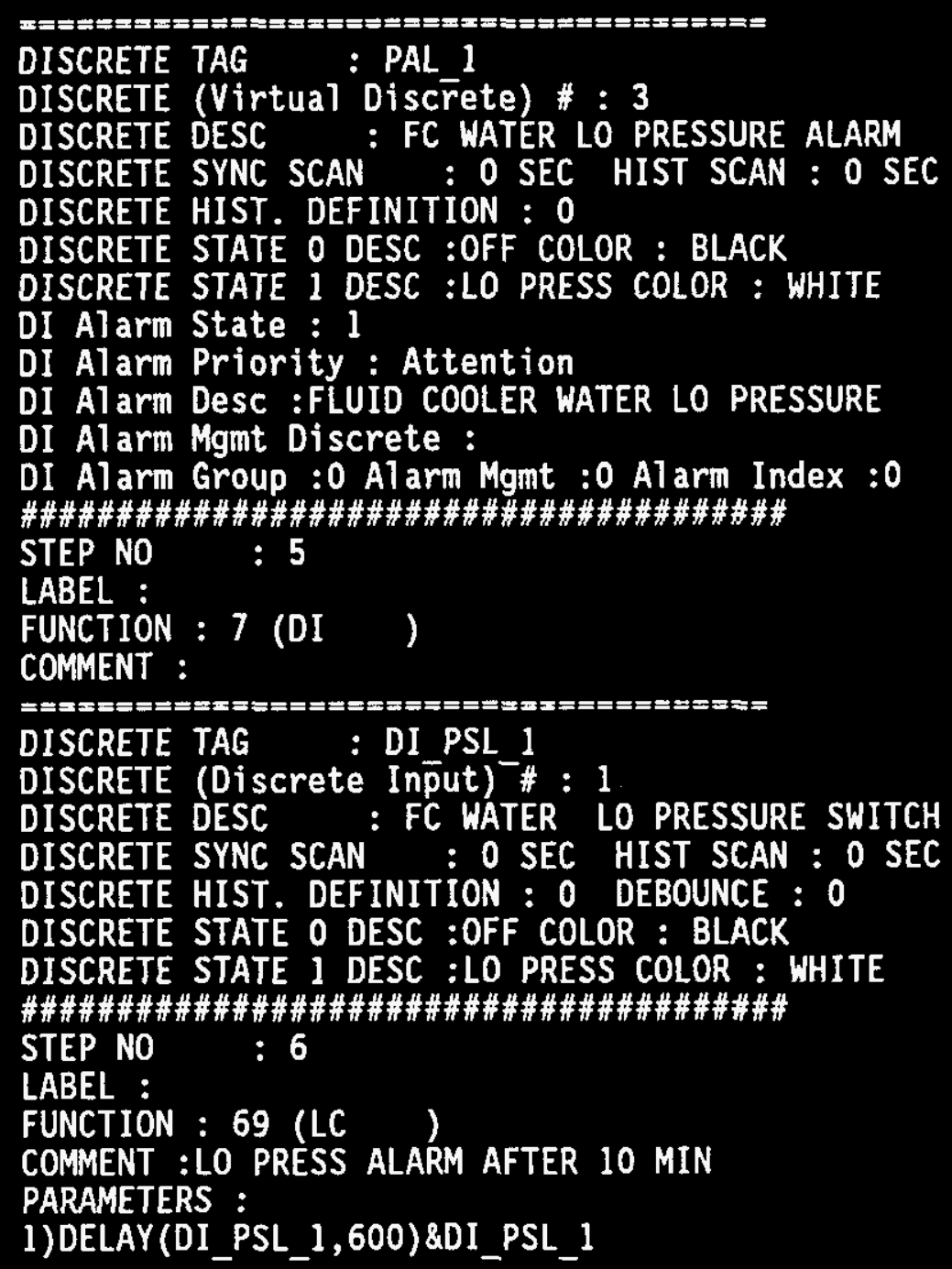




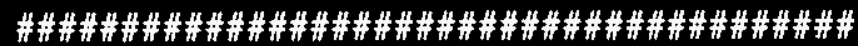

STEP NO $: 7$

LABEL :

FUNCTION : 75 (DST)

COMMENT : SAVE AS FC WATER LO PRESSURE ALARM

PARAMETERS :

1)MSV 2)0 3)0 4)0 5) PAL 1.

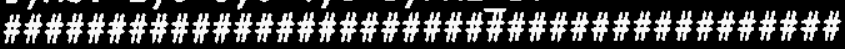

STEP NO : 8

LABEL :

FUNCTION : 6 (VD )

COMMENT :

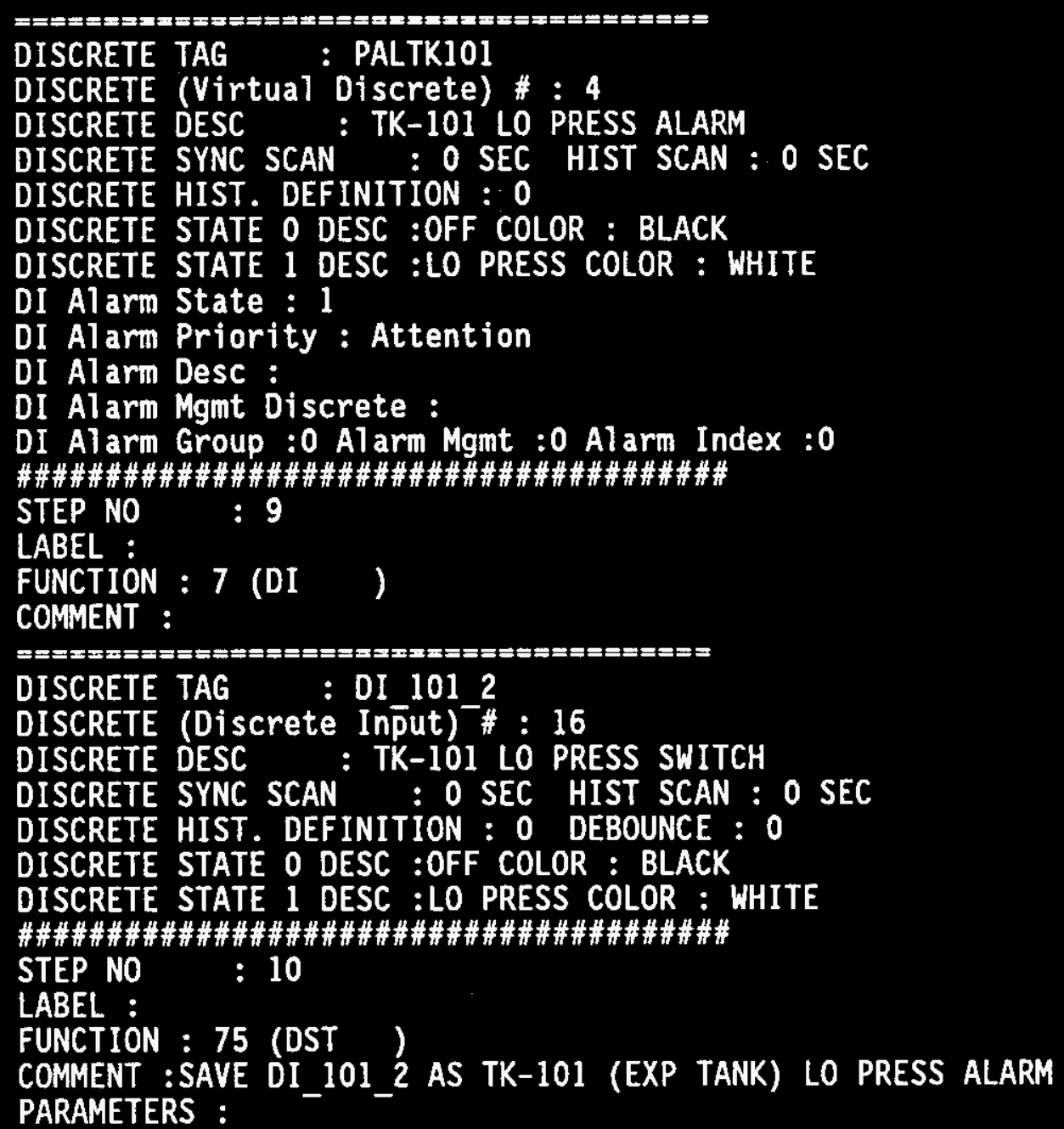
1)DI_101_2
2) 0
$0 4 \longdiv { 0 }$
5) PALTK101 
WHC-SD-CP-CSWD-016

Volume 3

Revision 1

Page 56

\#\#\#\#\#\#\#\#\#\#\#\#\#\#\#\#\#\#\#\#\#\#\#\#\#\#\#\#\#\#\#\#\#\#\#\#\#\#\#\#

STEP NO : 11

LABEL :

FUNCTION : 0 (END )

COMMENT : 
WHC-SD-CP-CSWD-016

Volume 3

Revision 1

Page 57

RH45_2_1.mic12_ro

MICON TYPE :RCM-D

MICON CONFIGURATION FILE : mic12 ro

POINT ALLOCATION :

$== \pm= \pm= \pm= \pm= \pm= \pm=x= \pm= \pm x= \pm= \pm= \pm= \pm= \pm= \pm= \pm= \pm==$

DISCRETE TAG : DI_SP2

DISCRETE (Discrete Input) \#: 5

DISCRETE DESC : SP-2 STATUS

DISCRETE SYNC SCAN : O SEC HIST SCAN : O SEC

DISCRETE HIST. DEFINITION : 0 DEBOUNCE : 0

DISCRETE STATE O DESC :OFF COLOR : GREEN

DISCRETE STATE 1 DESC :ON COLOR : YELLOW

VAI : 32 , VDI : 32, ISW : 32, LOOPS : 12, MSD : 16,

AI : $0, A O: 0, D I: 32, D O: 32$

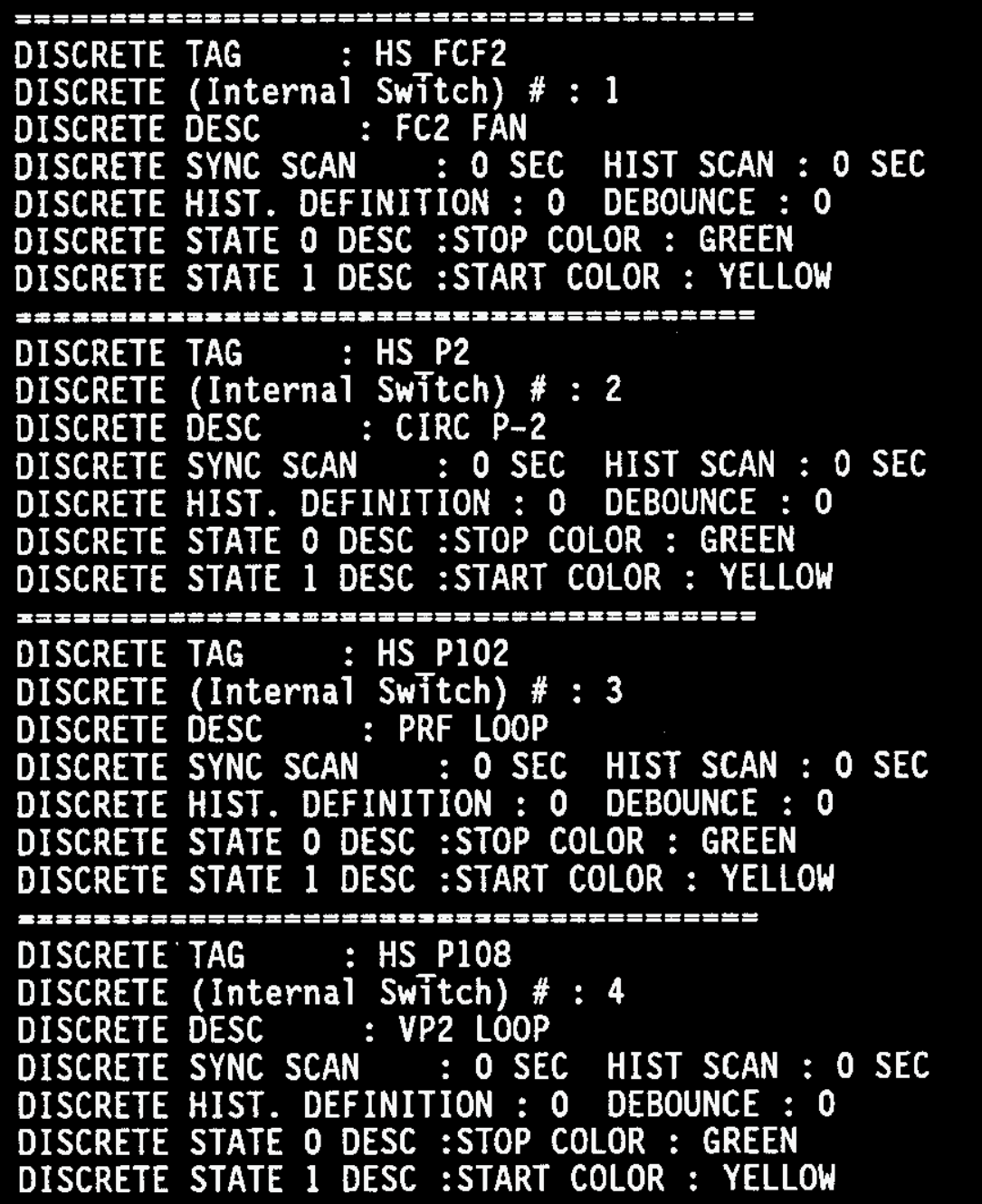


WHC-SD-CP-CSWD-016

Volume 3

Revision 1

Page 58

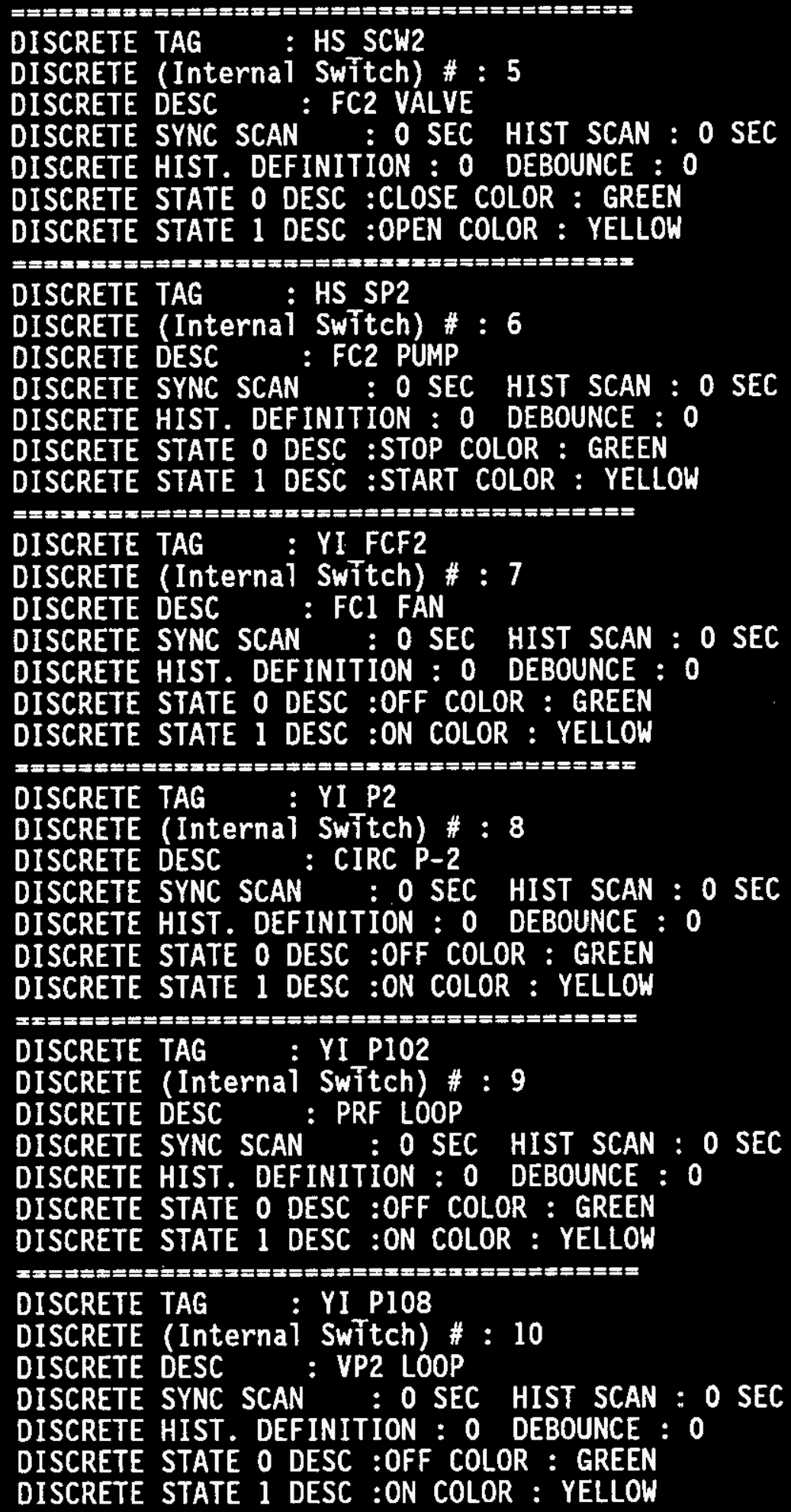


WHC-SD-CP-CSWD-016

Volume 3

Revision 1

Page 59

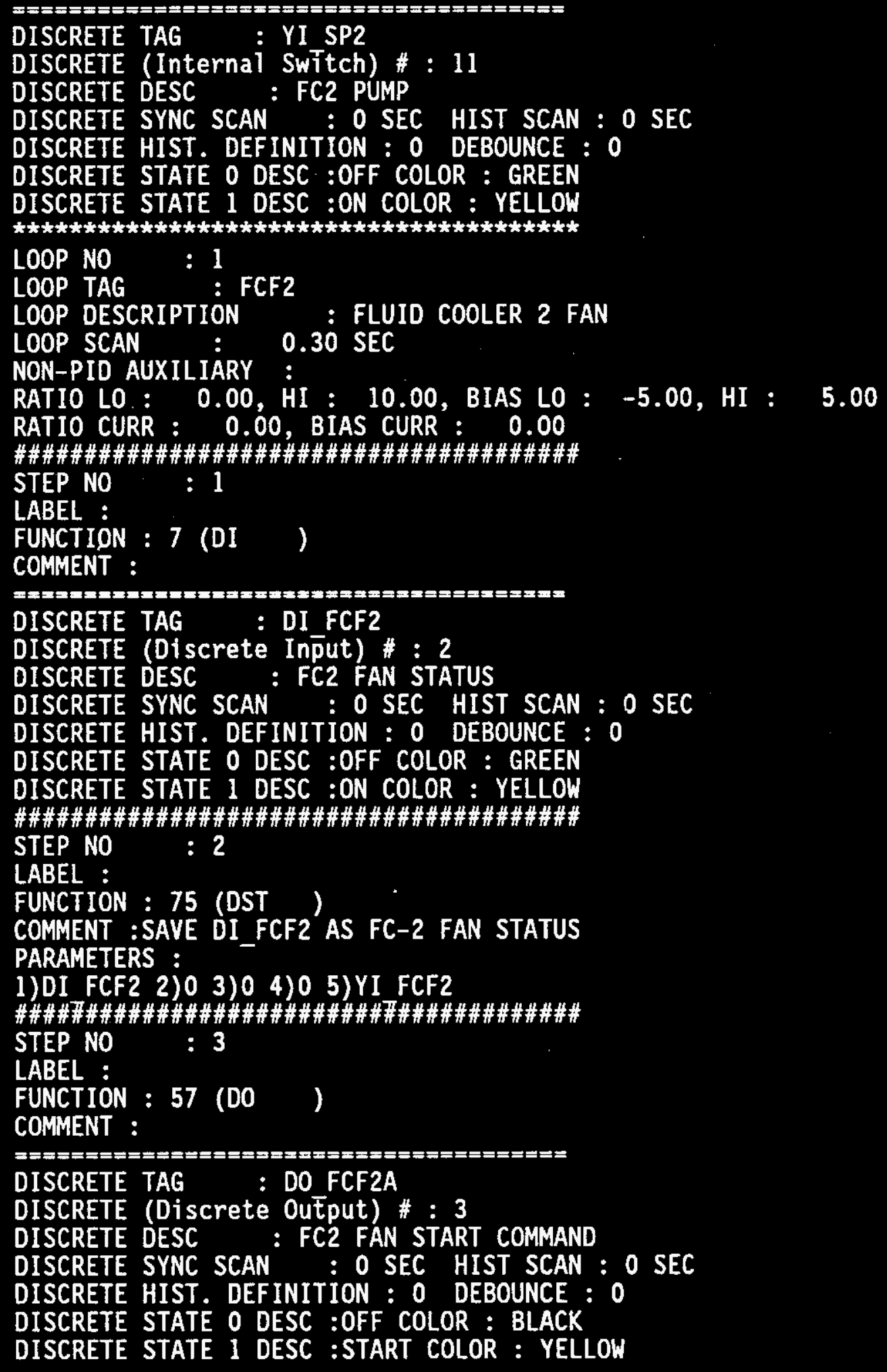


WHC-SD-CP-CSWD-016

Volume 3

Revision 1

Page 60

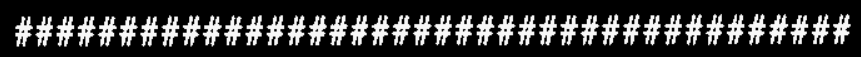

STEP NO : 4

LABEL :

FUNCTION : $57(00)$

COMMENT :

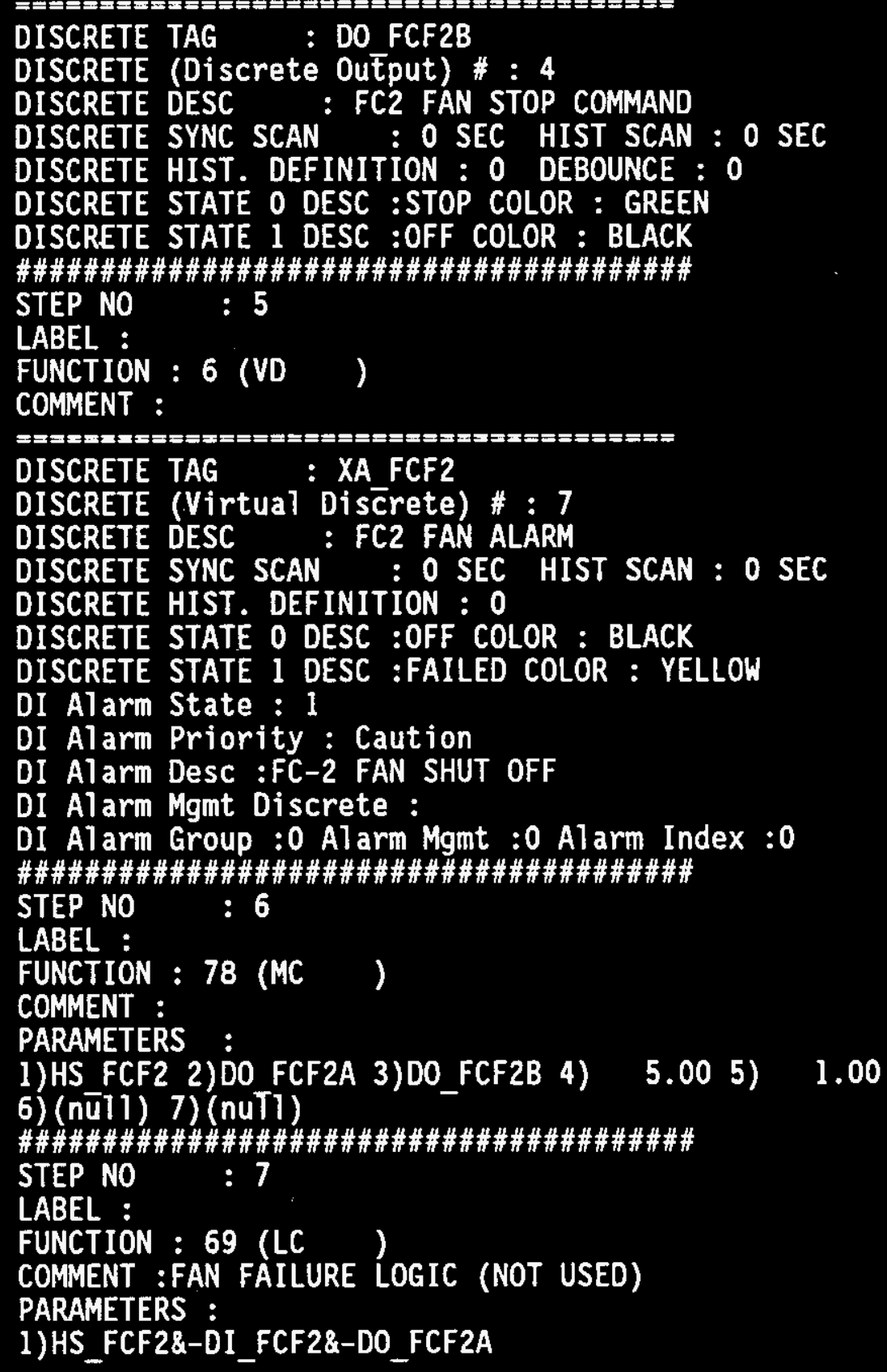



WHC-SD-CP-CSWD-016
Volume 3
Revision 1
Page 61

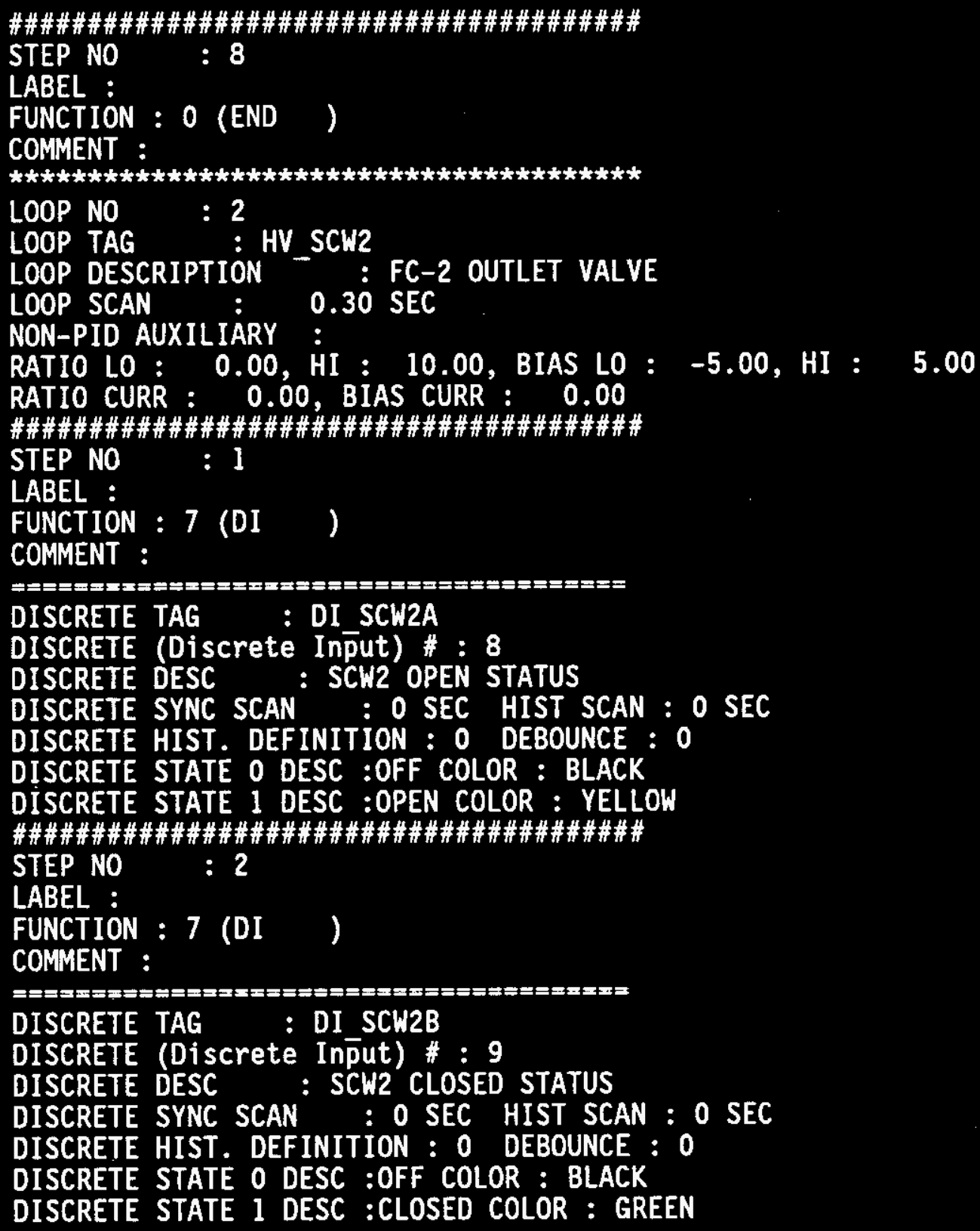


WHC-SD-CP-CSWD-016

Volume 3

Revision 1

Page 62

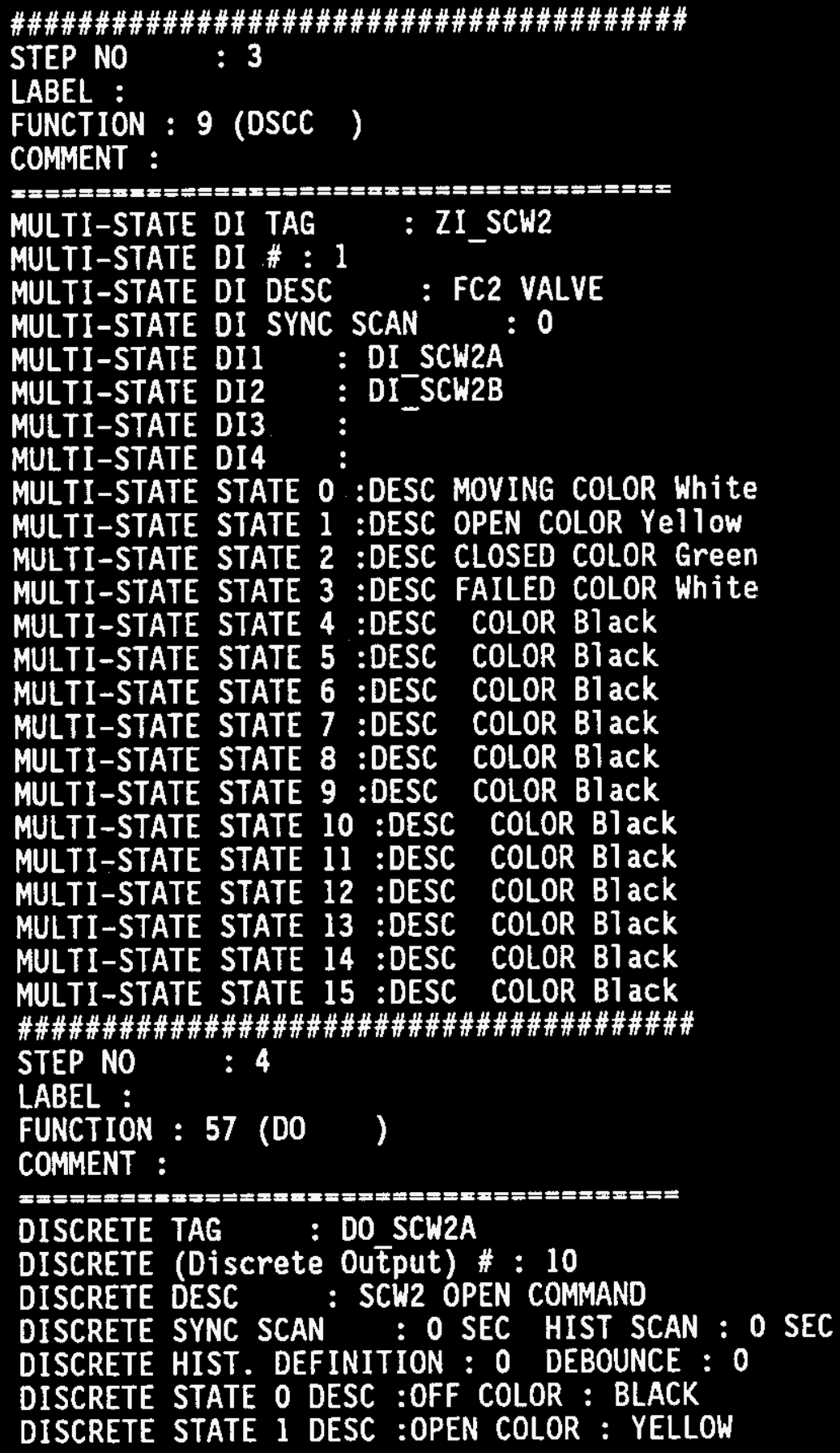


WHC-SD-CP-CSWD-016

Volume 3

Revision 1

Page 63

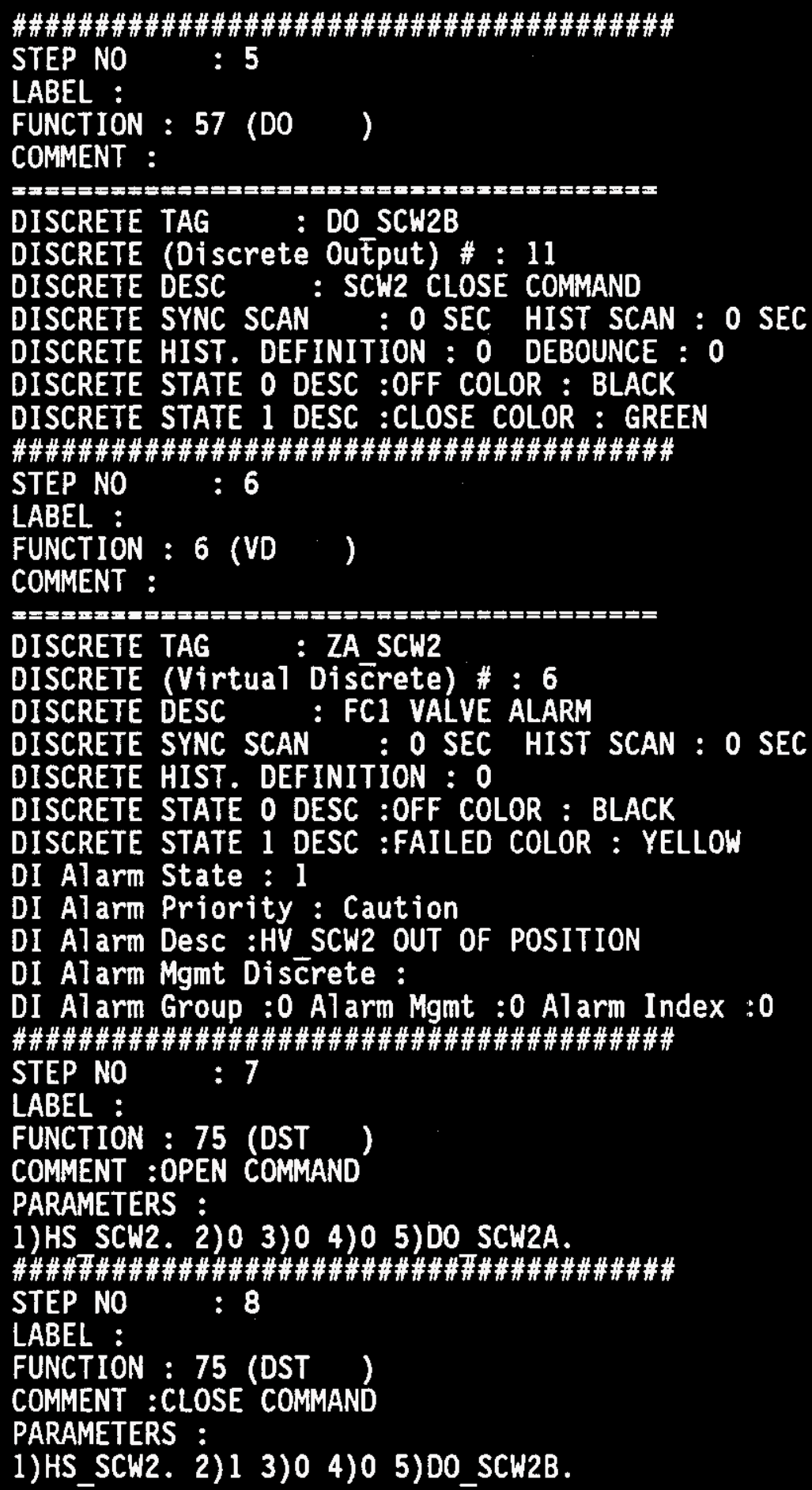


WHC-SD-CP-CSWD-016

Volume 3

Revision 1

Page 64

\#\#\#\#\#\#\#\#\#\#\#\#\#\#\#\#\#\#\#\#\#\#\#\#\#\#\#\#\#\#\#\#\#\#\#\#\#\#\#\#

STEP NO : 9

LABEL :

FUNCTION : 69 (LC )

COMMENT :ALARM IF VALVE OUT OF POSITION (AFTER 30 SEC)

PARAMETERS :

1) (DELAY (HS SCW2,30)\&-DI SCW2A) ! (DELAY (-HS_SCW2, 30)\&-DI_SCW2B)

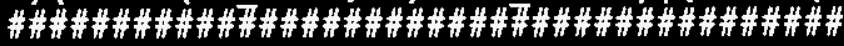

STEP NO : 10

LABEL :

FUNCTION : 75 (DST)

COMMENT :

PARAMETERS :

1)MSV 2)0 3)0 4)0 5)ZA SCW2.

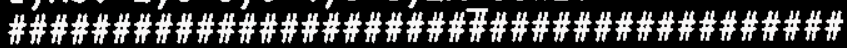

STEP NO : 11

LABEL :

FUNCTION : 0 (END )

COMMENT :

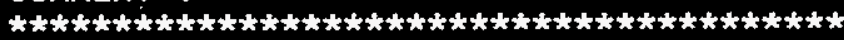

LOOP NO : 3

LOOP TAG : P2

LOOP DESCRIPTION : CIRCULATION PUMP 2

LOOP SCAN : 0.30 SEC

NON-PID AUXILIARY :

RATIO LO: 0.00 , HI : 10.00 , BIAS LO : -5.00 , HI : 5.00

RAT10 CURR : 0.00 , BIAS CURR : 0.00

IMPORT DI :RM45 1 4::!FSL SCW2, RM45 1 3::! FSL_SCW1

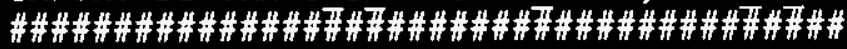

STEP NO : 1

LABEL :

FUNCTION : 7 (DI )

COMMENT :

DISCRETE TAG : DI P21

DISCRETE (Discrete Input) \# : 12

DISCRETE DESC : CIRC P-2 STATUS

DISCRETE SYNC SCAN : O SEC HIST SCAN : O SEC

DISCRETE HIST. DEFINITION : 0 DEBOUNCE : 0

DISCRETE STATE O DESC :OFF COLOR : GREEN

DISCRETE STATE 1 DESC :ON COLOR : YELLOW

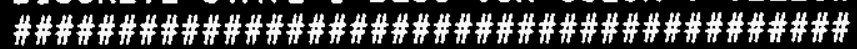

STEP NO : 2

LABEL :

FUNCTION : 75 (DST)

COMMENT : SAVE DI P2 1 AS P-2 STATUS

PARAMETERS :

1)DI_P2_1 2)0 3)0 4)0 5)YI_P2 
WHC-SD-CP-CSWD-016

Volume 3

Revision 1

Page 65

\#\#\#\#\#\#\#\#\#\#\#\#\#\#\#\#\#\#\#\#\#\#\#\#\#\#\#\#\#\#\#\#\#\#\#\#\#\#\#\#

STEP NO : 3

LABEL :

FUNCTION : 7 (DI )

COMMENT :

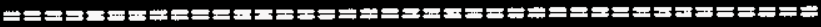

DISCRETE TAG : DI P2 2

DISCRETE (Discrete Inp̄ut) \# : 15

DISCRETE DESC : P2 LO FLOW SWITCH

DISCRETE SYNC SCAN : O SEC HIST SCAN : O SEC

DISCRETE HIST. DEFINITION : 0 DEBOUNCE : 0

DISCRETE STATE $O$ DESC :OFF COLOR : BLACK

DISCRETE STATE 1 DESC :LO FLOW COLOR : YELLOW

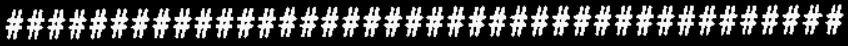

STEP NO : 4

LABEL :

FUNCTION : 6 (VD )

COMMENT :

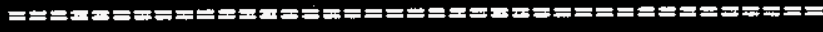

DISCRETE TAG : FAL P2

DISCRETE (Virtual Discrete) \#: I

DISCRETE DESC : CIRC P-2 LOW FLOW ALARM

DISCRETE SYNC SCAN : O SEC HIST SCAN : O SEC

DISCRETE HIST. DEFINITION : 0

DISCRETE STATE O DESC :OFF COLOR : BLACK

DISCRETE STATE 1 DESC :LO FLOW COLOR : YELLOW

DI Alarm State : 1

DI Alarm Priority : Caution

DI Alarm Desc :P-2 LOW FLOW/PUMP OFF

DI Alarm Mgmt Discrete :

DI Alarm Group :0 Alarm Mgmt :0 Alarm Index :0

\#\#\#\#\#\#\#\#\#\#\#\#\#\#\#\#\#\#\#\#\#\#\#\#\#\#\#\#\#\#\#\#\#\#\#\#

STEP NO : 5

LABEL :

FUNCTION : 57 (DO )

COMMENT :

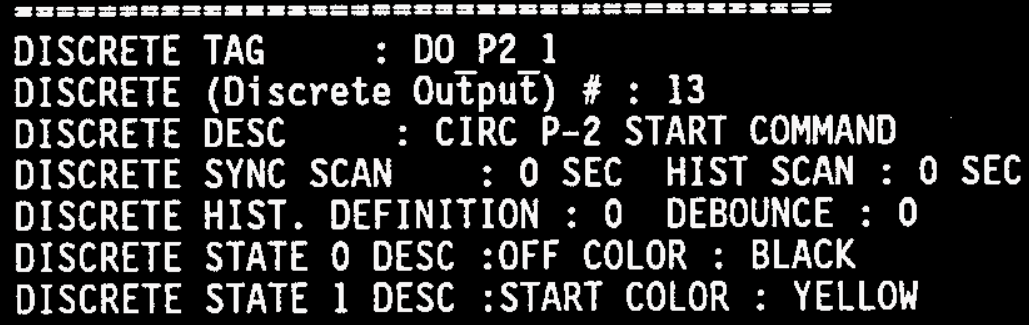


WHC-SD-CP-CSWD-016

Volume 3

Revision 1

Page 66

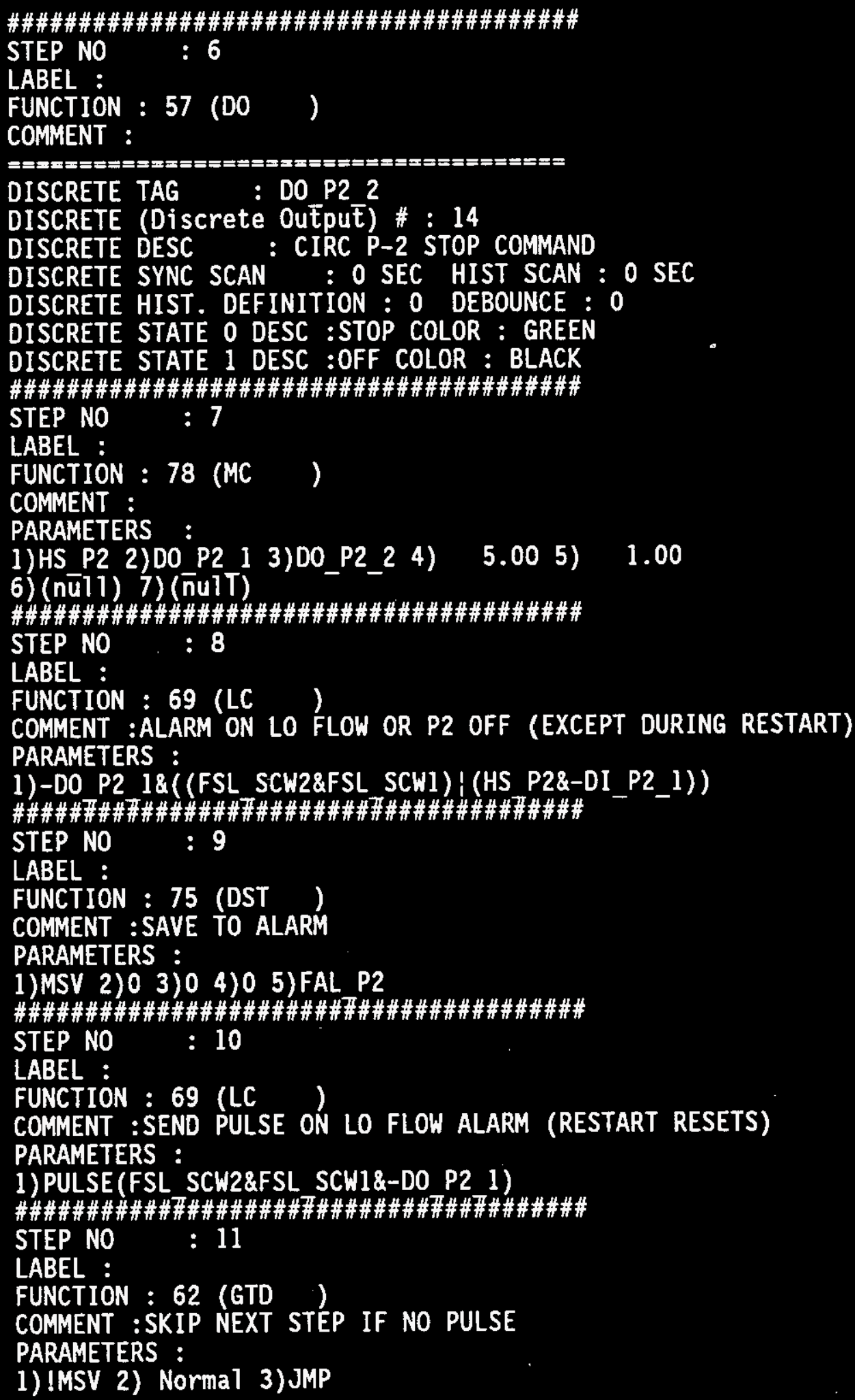



WHC-SD-CP-CSWD-016
Volume 3
Revision 1
Page 67

\#\#\#\#\#\#\#\#\#\#\#\#\#\#\#\#\#\#\#\#\#\#\#\#\#\#\#\#\#\#\#\#\#\#\#\#\#\#\#\#

STEP NO : 12

LABEL : JMP

FUNCTION : 10 (L $)$ )
COMMENT :TURN OFF PUMP IF PULSE (LOW FLOW)

PARAMETERS :

1) HS P2 2) 0

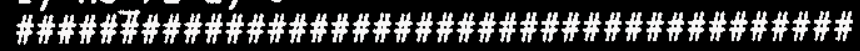

STEP NO : 13

LABEL :

FUNCTION : 0 (END )

COMMENT :

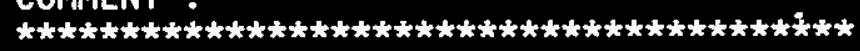

LOOP NO : 4

LOOP TAG: : P102

LOOP DESCRIPTION : PRF COOLING LOOP PUMP 102

LOOP SCAN : 0.30 SEC

NON-PID AUXILIARY :

RATIO LO: 0.00 , HI : 10.00 , BIAS LO : -5.00 , HI : 5.00

RATIO CURR : 0.00 , BIAS CURR : 0.00

EXPORT :DI PCH1A

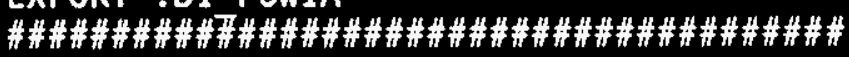

STEP NO : 1

LABEL :

FUNCTION : 7 (DI )

COMMENT :

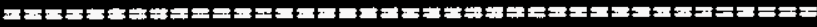

DISCRETE TAG : DI PCWIA

DISCRETE (Discrete Input) \# : 16

DISCRETE DESC : PRF LOOP LO FLOW SWITCH

DISCRETE SYNC SCAN : O SEC HIST SCAN : O SEC

DISCRETE HIST. DEFINITION : 0 DEBOUNCE : 0

DISCRETE STATE O DESC :OFF COLOR : BLACK

DISCRETE STATE 1 DESC :LO FLOW COLOR : YELLOW

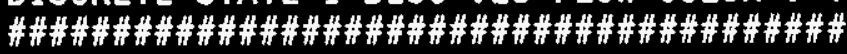

STEP NO

$: 2$

LABEL :

FUNCTION : 7 (DI)

COMMENT :

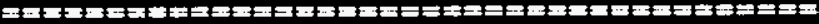

DISCRETE TAG : DI 1021

DISCRETE (Discrete Input) $\#: 17$

DISCRETE DESC : P-102 STATUS

DISCRETE SYNC SCAN : O SEC HIST SCAN : O SEC

DISCRETE HIST. DEFINITION : 0 DEBOUNCE : 0

DISCRETE STATE 0 DESC :OFF COLOR : GREEN

DISCRETE STATE 1 DESC :ON COLOR : YELLOW 
WHC-SD-CP-CSWD-016

Volume 3

Revision 1

Page 68

\#\#\#\#\#\#\#\#\#\#\#\#\#\#\#\#\#\#\#\#\#\#\#\#\#\#\#\#\#\#\#\#\#\#\#\#\#

STEP NO : 3

LABEL :

FUNCTION : 75 (DST)

COMMENT : SAVE DI 102_1 AS PUMP STATUS

PARAMETERS :

1)DI 102 1 2)0 3)0 4)0 5) YI P102

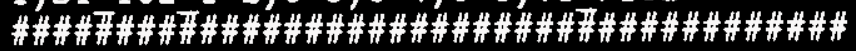

STEP NO : 4

LABEL :

FUNCTION : 6 (VD )

COMMENT :

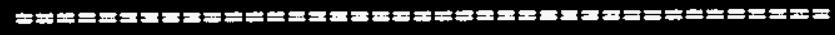

DISCRETE TAG : XA_P102

DISCRETE (Virtual Disçrete) \#: 3

DISCRETE DESC : P102 ALARM

DISCRETE SYNC SCAN : O SEC HIST SCAN : O SEC

DISCRETE HIST. DEFINITION : 0

DISCRETE STATE O DESC :OFF COLOR : BLACK

DISCRETE STATE 1 DESC :LO FLOW COLOR : YELLOW

DI Al arm State : 1

DI Alarm Priority : Caution

DI Alarm Desc :PRF LOOP LO FLOW/P-102 OFF

DI Alarm Mgmt Discrete :

DI Alarm Group :0 Alarm Mgmt :0 Alarm Index :0

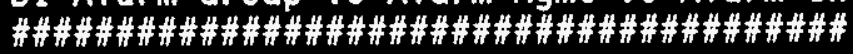

STEP NO : 5

LABEL :

FUNCTION : 57 (DO )

COMMENT :

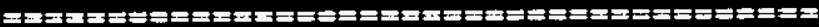

DISCRETE TAG

: D0 1021

DISCRETE (Discrete Output) \# : 18

DISCRETE DESC : P102 START COMMAND

DISCRETE SYNC SCAN : O SEC HIST SCAN : O SEC

DISCRETE HIST. DEFINITION : 0 DEBOUNCE : 0

DISCRETE STATE O DESC :OFF COLOR : BLACK

DISCRETE STATE 1 DESC :START COLOR : YELLOW 
WHC-SD-CP-CSWD-016

Volume 3

Revision 1

Page 69

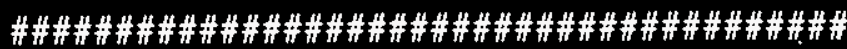

STEP NO : 6

LABEL :

FUNCTION : 57 (DO) )

COMMENT :

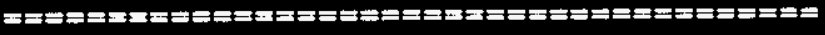

DISCRETE TAG : DO 1022

DISCRETE (Discrete Output) \# : 19

DISCRETE DESC : P102 STOP COMMAND

DISCRETE SYNC SCAN :O SEC HIST SCAN : O SEC

DISCRETE HIST. DEFINITION : 0 DEBOUNCE : 0

DISCRETE STATE O DESC :STOP COLOR : GREEN

DISCRETE STATE 1 DESC :OFF COLOR : BLACK

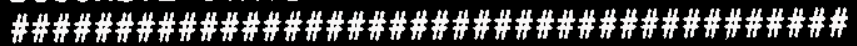

STEP NO : 7

LABEL :

FUNCTION : 78 (MC )

COMMENT :

PARAMETERS :

1)HSP102 2)DO 102_1 3)DO_102_2 4) 5.00 5) 1.00

6) (nū11) 7) (nuTi)

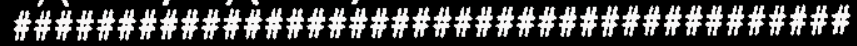

STEP NO : 8

LABEL :

FUNCTION : 69 (LC)

COMMENT :ALARM ON LO FLOW \& P102 OFF (EXCEPT STARTUP)

PARAMETERS :

1) -DO 102 18(DI PCW1A! (HS P102\&-DI 102 1))

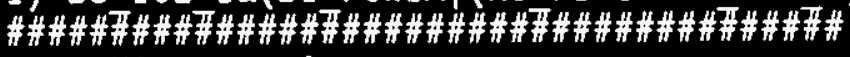

STEP NO : 9

LABEL :

FUNCTION : 75 (DST)

COMMENT :SAVE ALARM

PARAMETERS :

1)MSV 2) 0 3) 0 4)0 5)XA P102

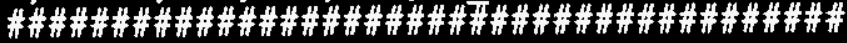

STEP NO : 10

LABEL :

FUNCTION : 69 (LC )

COMMENT : SEND PULSE ON LO FLOW (RESTART RESETS)

PARAMETERS :

1) PULSE (DI PCWIA\&-BO 102 1)

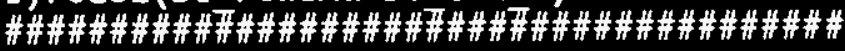

STEP NO : 11

LABEL :

FUNCTION : 62 (GTD )

COMMENT : SKIP NEXT STEP IF NO PULSE

PARAMETERS :

1) !MSV 2) Normal 3) JMP 


$$
\begin{gathered}
\text { WHC-SD-CP-CSWD-016 } \\
\text { Volume } 3 \\
\text { Revision } 1 \\
\text { Page } 70
\end{gathered}
$$

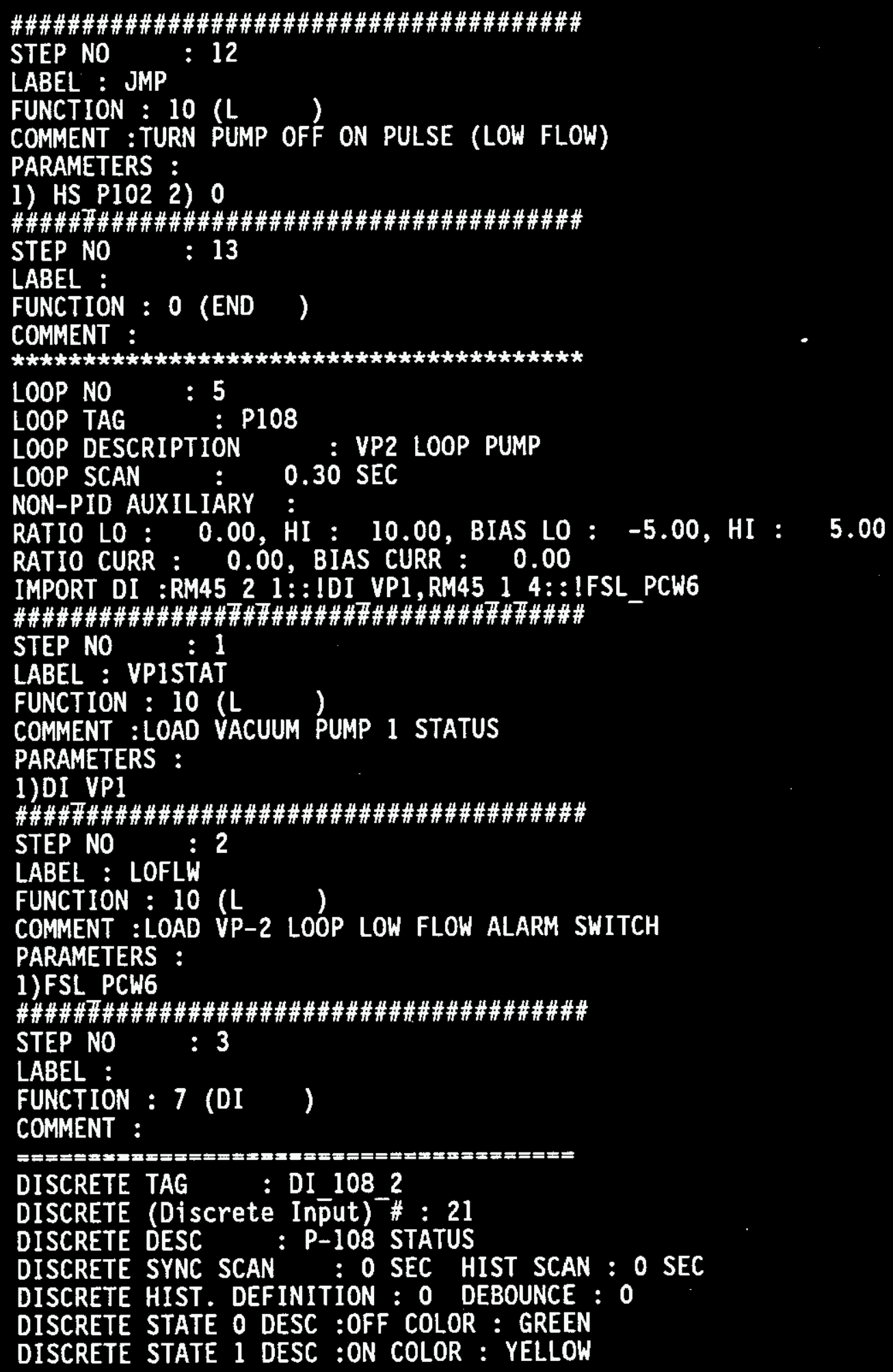




\section{WHC-SD-CP-CSWD-016 \\ Volume 3 \\ Revision 1 \\ Page 71}

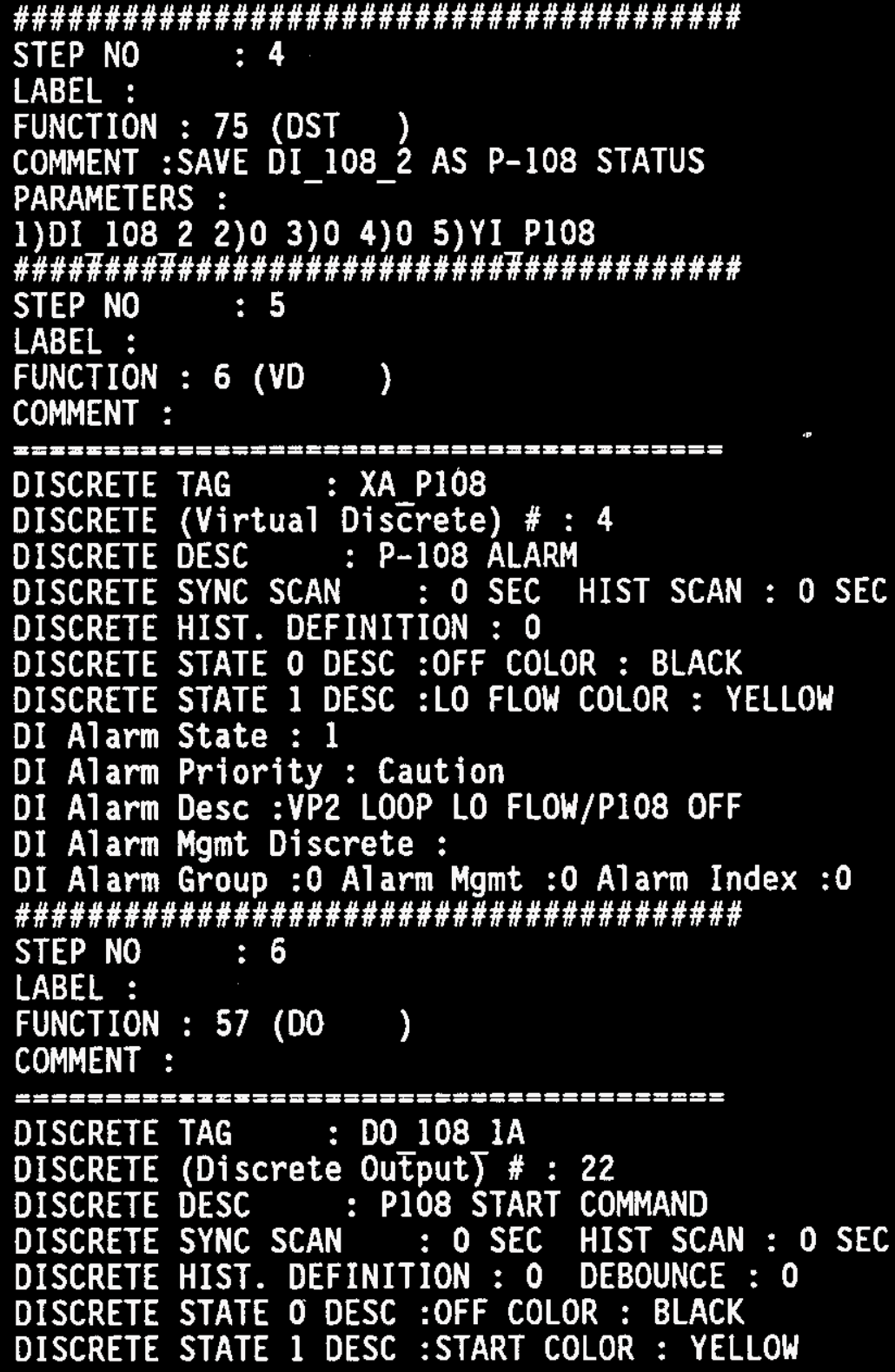


\#\#\#\#\#\#\#\#\#\#\#\#\#\#\#\#\#\#\#\#\#\#\#\#\#\#\#\#\#\#\#\#\#\#\#\#\#\#\#\#

STEP NO : 7

LABEL :

FUNCTION : $57(00)$

COMMENT :

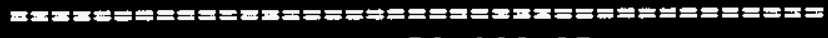

DISCRETE TAG : DO 108 1B

DISCRETE (Discrete Output) \#: 23

DISCRETE DESC : P108 STOP COMMAND

DISCRETE SYNC SCAN : O SEC HIST SCAN : O SEC

DISCRETE HIST. DEFINITION : 0 DEBOUNCE : 0

DISCRETE STATE $O$ DESC :STOP COLOR : GREEN

DISCRETE STATE 1 DESC :OFF COLOR : BLACK

\#\#\#\#\#\#\#\#\#\#\#\#\#\#\#\#\#\#\#\#\#\#\#\#\#\#\#\#\#\#\#\#\#\#\#\#\#\#\#

STEP NO

: 8

LABEL : VPSTATS

FUNCTION : 71 (ORG

COMMENT :DETERMINE COMBINED VAC PUMP STATUS $1=0 \mathrm{~N}$

PARAMETERS :

1) VP1STAT 2)DI VP2 3) 4)

\#\#\#\#\#\#\#\#\#\#\#\#\#\#男\#\#\#\#\#\#\#\#\#\#\#\#\#\#\#\#\#\#\#\#\#\#

STEP NO : 9

LABEL :

FUNCTION : 78 (MC)

COMMENT : 30 SEC START TO ALLOW TIME TO START VAC PUMP

PARAMETERS :

1)HS P108 2)DO 108_1A 3)DO_108_1B 4) 30.00 5) 1.00

6) (nü11) 7) (nuT1)

\#\#\#\#\#\#\#\#\#\#\#\#\#\#\#\#\#\#\#\#\#\#\#\#\#\#\#\#\#\#\#\#\#\#\#\#\#\#

STEP NO : 10

LABEL :

FUNCTION : 69 (LC

COMMENT : ALARM ON LOW FLOW OR PUMP OFF (EXCEPT DURING RESTART)

PARAMETERS :

1)-DO 108 1A\&(LOFLW! (HS P108\&-DI 108 2))

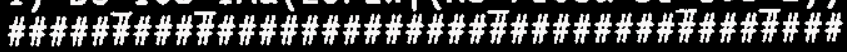

STEP NO : 11

LABEL :

FUNCTION : 75 (DST

COMMENT : SAVE AS ALARM

PARAMETERS :

1)MSV 2) 0 3) 0 4) 0 5) XA P108

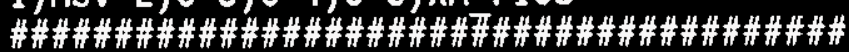

STEP NO : 12

LABEL :

FUNCTION : 69 (LC )

COMMENT : SEND PULSE ON LOW FLOW (RESTART RESETS)

PARAMETERS :

1) PULSE(!LOFLW\&-D0_108_1A) 


\title{
WHC-SD-CP-CSWD-016 \\ Volume 3 \\ Revision 1 \\ Page 73
}

\author{
\#\#\#\#\#\#\#\#\#\#列\#\#\#\#\#\#\#\#\#\#\#\#\#\#\#\#\#\#\#\#\#\#\#\#\#\#\#\#\# \\ STEP NO : 13 \\ LABEL : \\ FUNCTION : 62 (GTD \\ COMMENT :SKIP NEXT STEP IF NO PULSE \\ PARAMETERS : \\ 1) !MSV 2) Normal 3) JMPFL

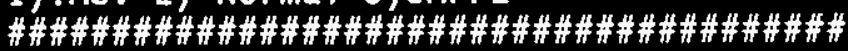 \\ STEP NO : 14 \\ LABEL : JMPFL \\ FUNCTION : 10 (L ) \\ COMMENT :TURN OFF PUMP ON PULSE (LOW FLOW)
}

PARAMETERS :

1) HS P108 2) 0

\#\#\#\#\#男\#\#\#\#\#\#\#\#\#\#\#\#\#\#\#\#\#\#\#\#\#\#\#\#\#\#\#\#\#\#\#\#\#\#

STEP NO : 15

LABEL :

FUNCTION : 71 (ORG )

COMMENT :OUTPUT=1 IF A VAC PUMP IS RUNING OR P108 IF RESTARTING

PARAMETERS :

1)VPSTATS 2)DO 108 1A 3) 4)

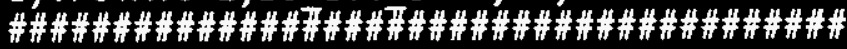

STEP NO : 16

LABEL :

FUNCTION : 62 (GTD)

COMMENT :SKIP NEXT STEP IF VP RUNNING OR P106 IN RESTART

PARAMETERS :

1)MSV 2) Off-Nomal 3) JMPVP

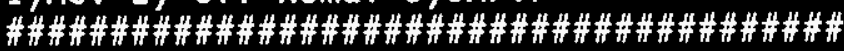

STEP NO : 17

LABEL : JMPVP

FUNCTION : 10 (L

COMMENT :TURN OFF PUMP IF BOTH VAC PUMPS OFF (EXCEPT DURING RESTART)

PARAMETERS :

1) HS P108 2) 0

\#\#\#\#\#男\#\#\#\#\#\#\#\#\#\#\#\#\#\#\#\#\#\#\#\#\#\#\#\#\#\#\#\#\#\#\#\#\#

STEP NO : 18

LABEL :

FUNCTION : 0 (END)

COMMENT :

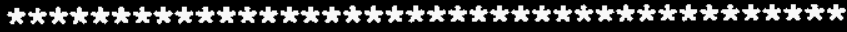

LOOP NO : 6

LOOP TAG : SP2

LOOP DESCRIPTION : FLUID COOLER 2 SPRAY PUMP

LOOP SCAN : 0.30 SEC

NON-PID AUXILIARY :

RATIO LO : 0.00 , HI : 10.00 , BIAS LO : -5.00 , HI : 5.00

RATIO CURR : 0.00 , BIAS CURR : 0.00 


\section{WHC-SD-CP-CSHD-016 \\ Volume 3 \\ Revision 1 \\ Page 74}

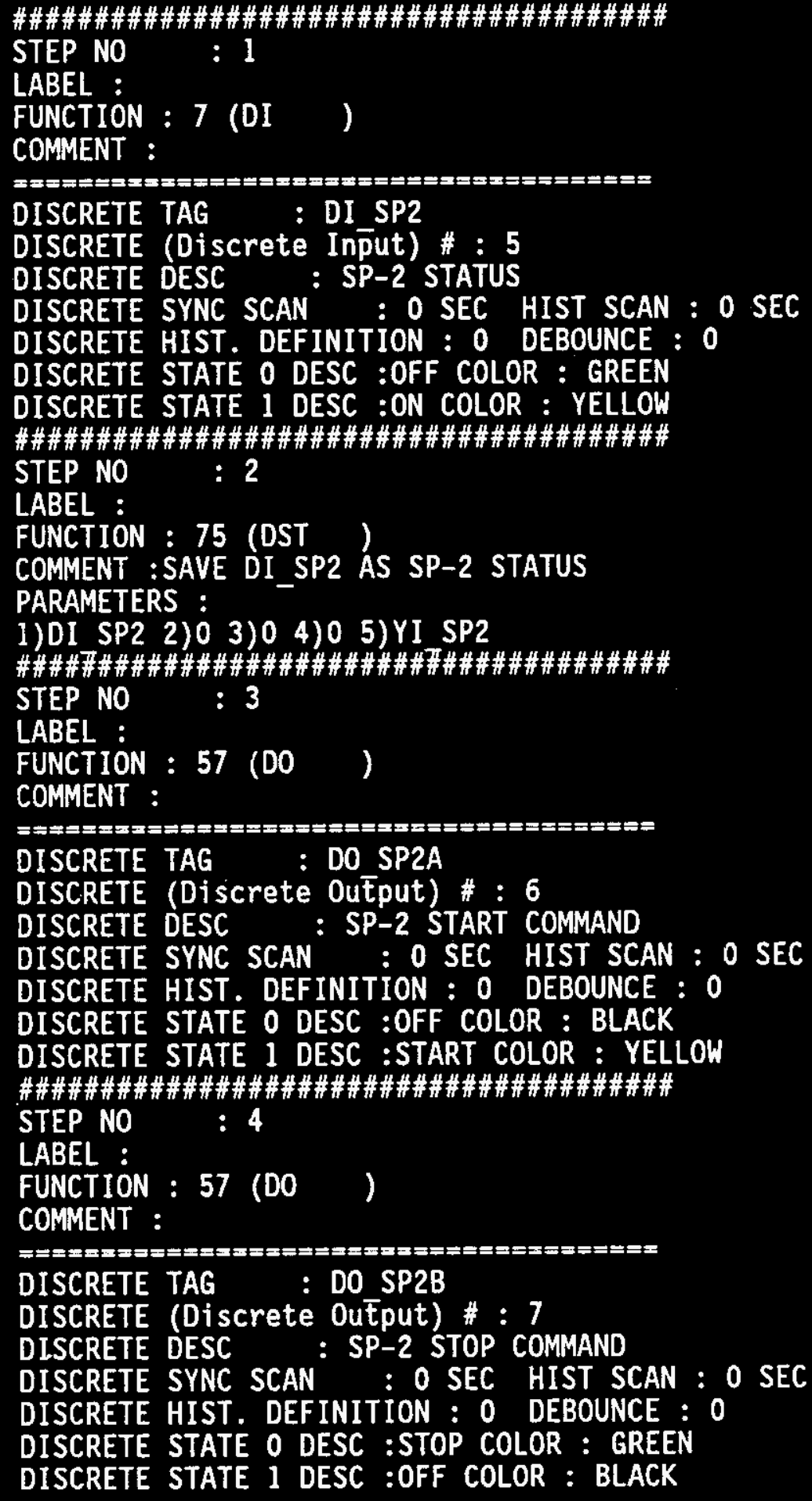


WHC-SD-CP-CSWD-016

Volume 3

Revision 1

Page 75

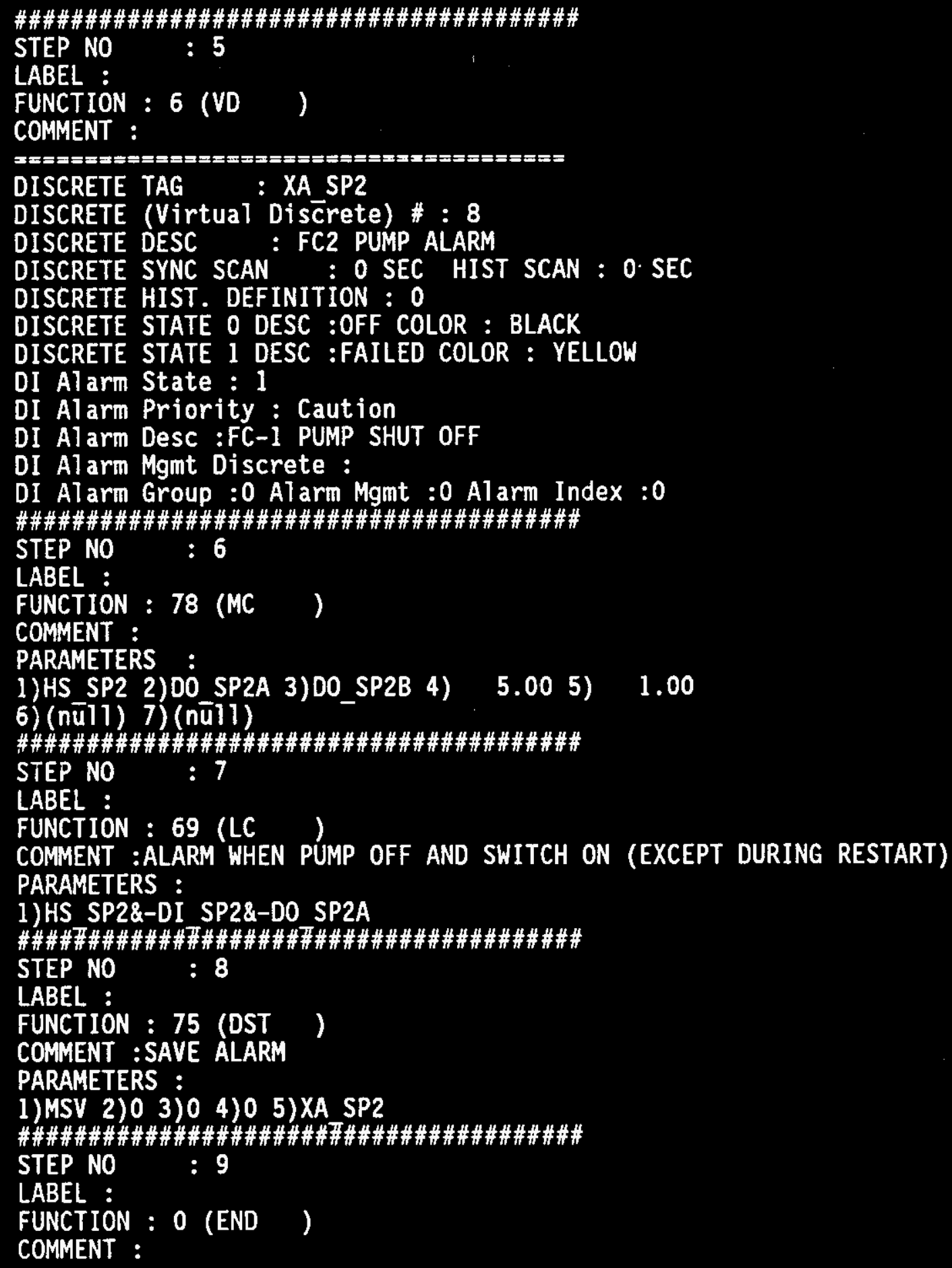


WHC-SD-CP-CSWD-016

Volume 3

Revision 1

Page 76

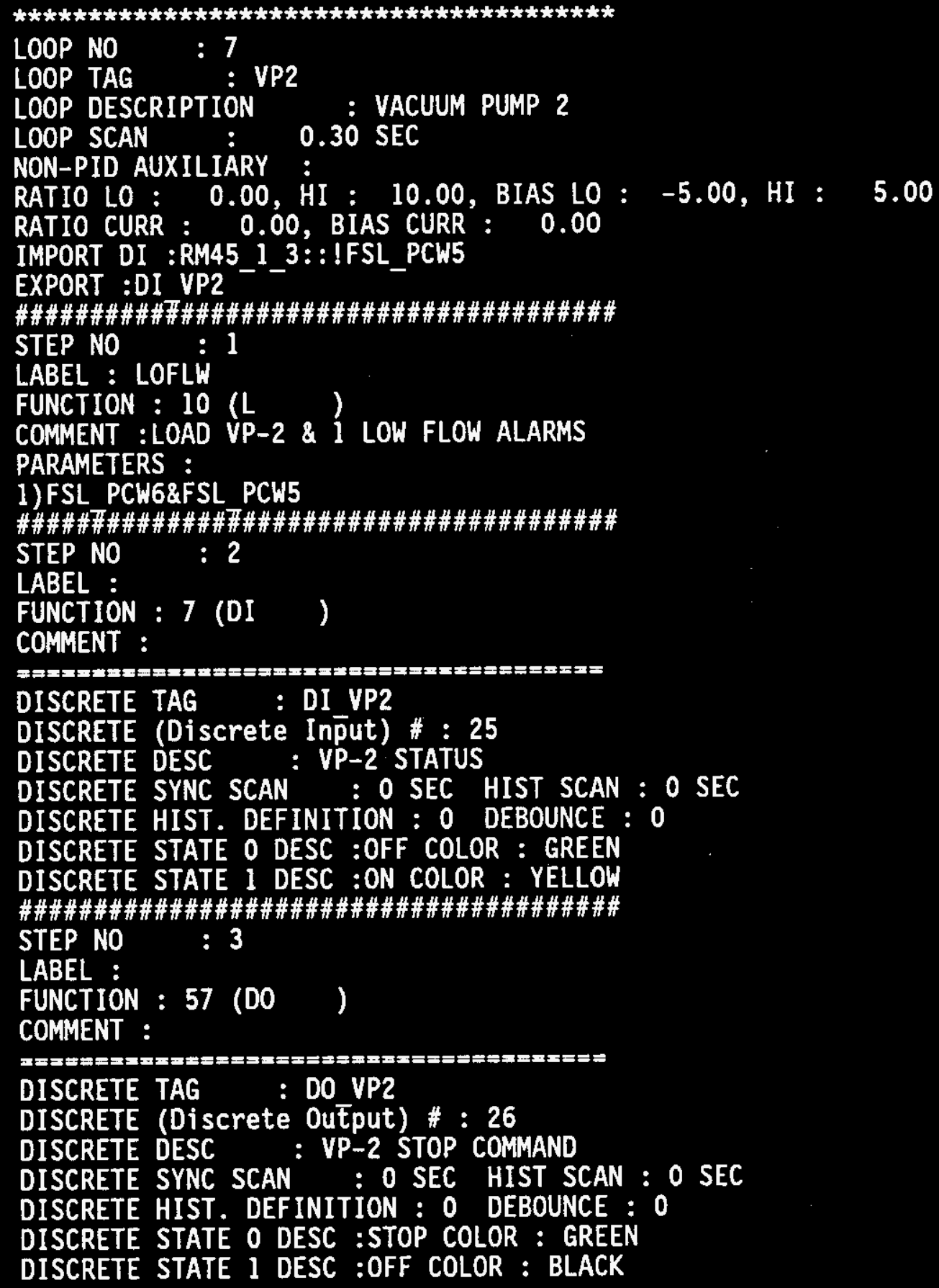


WHC-SD-CP-CSWD-016

Volume 3

Revision 1

Page 77

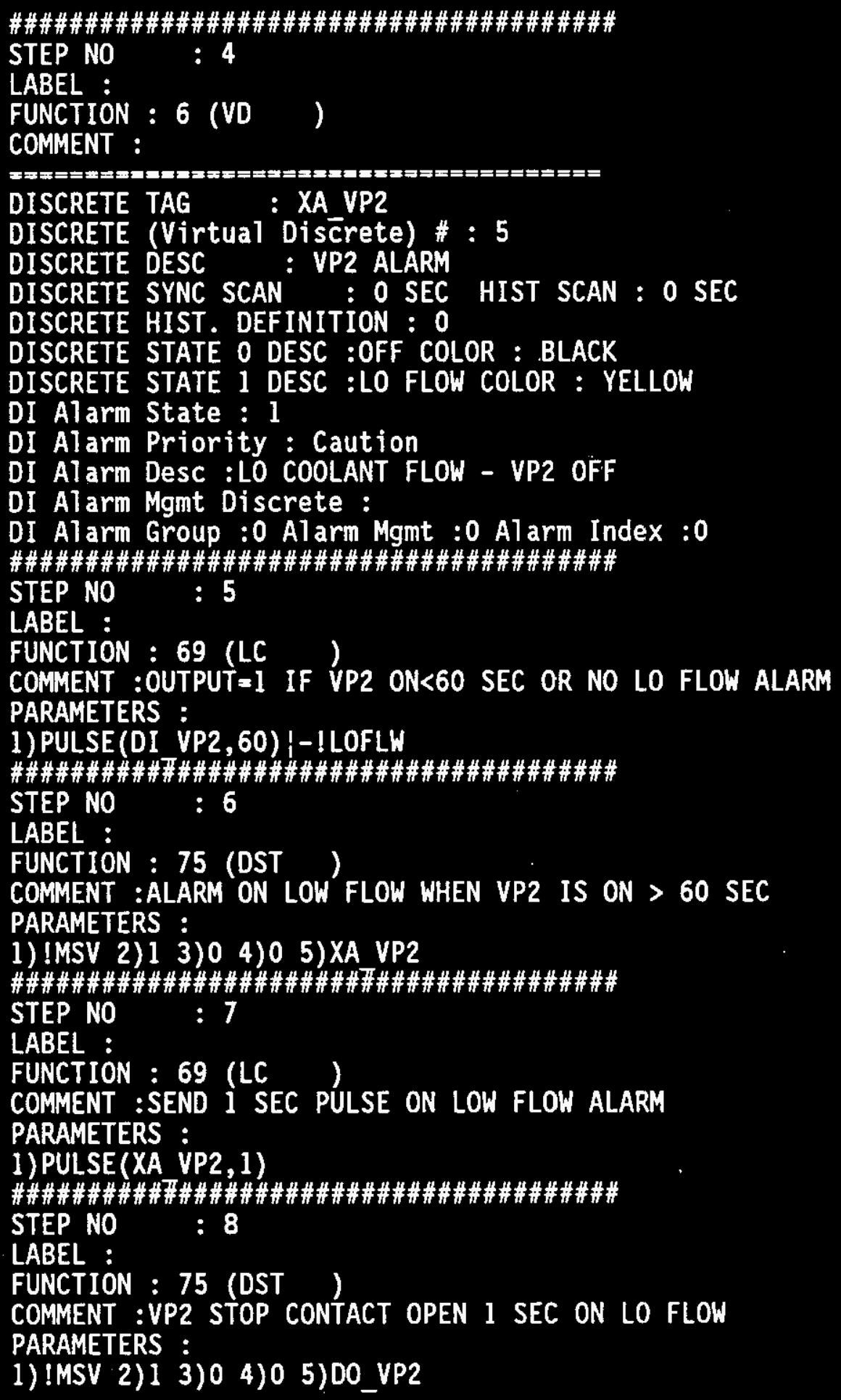




\section{WHC-SD-CP-CSWD-016 \\ Volume 3 \\ Revision 1 \\ Page 78}

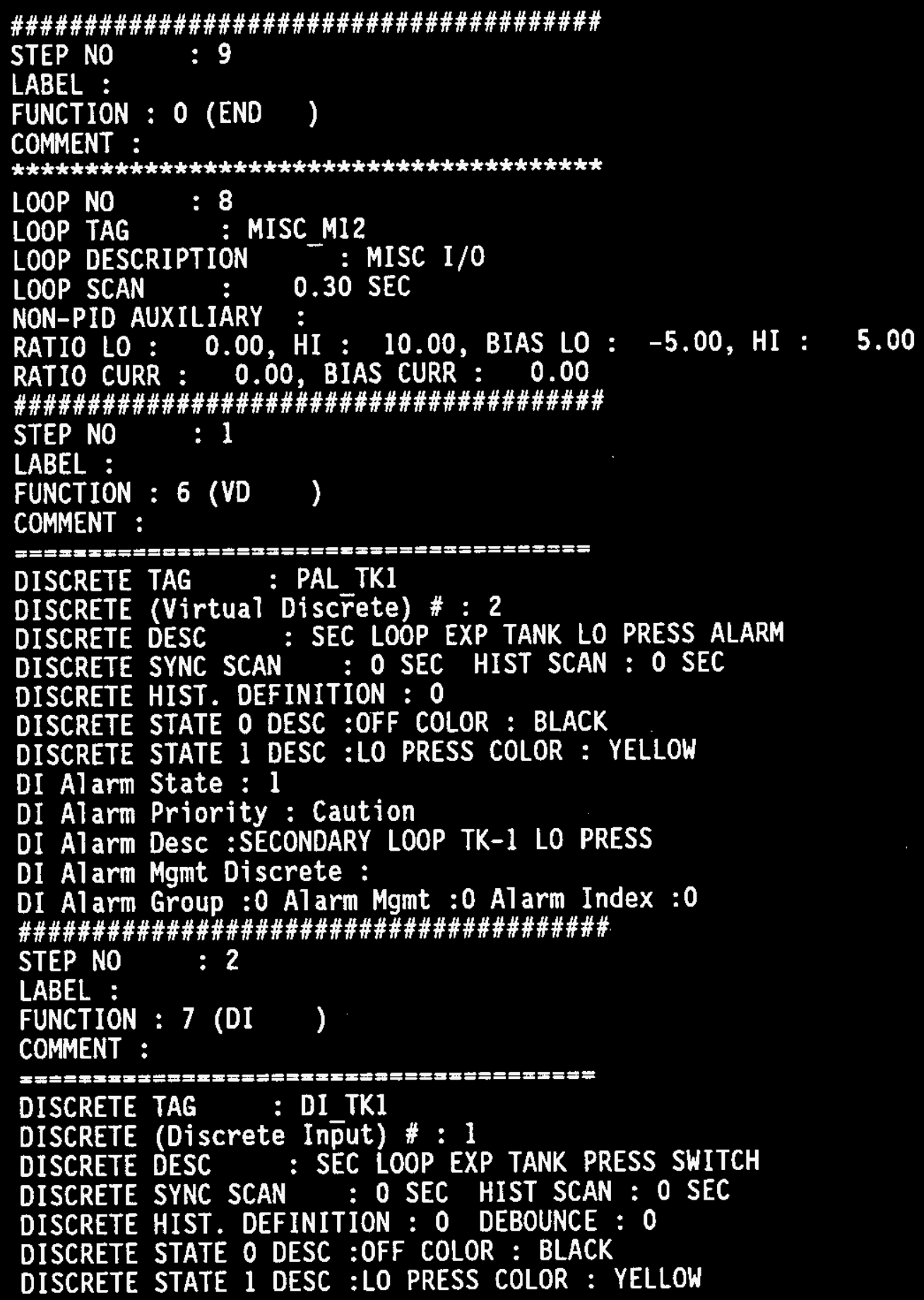


WHC-SD-CP-CSWD-016

Volume 3

Revision 1

Page 79

\#\#\#\#\#\#\#\#\#\#\#\#\#\#\#\#\#\#\#\#\#\#\#\#\#\#\#\#\#\#\#\#\#\#\#\#\#\#\#\#

STEP NO : 3

LABEL :

FUNCTION : 75 (DST)

COMMENT : SAVE DI_TK1 AS TK-1 LOW PRESSURE ALARM

PARAMETERS :

1)DI TK1 2)0 3)0 4)0 5) PAL TK1

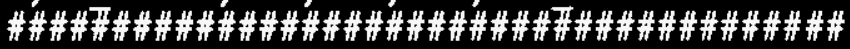

STEP NO : 4

LABEL :

FUNCTION : 0 (END)

COMMENT : 


\section{WHC-SD-CP-CSWD-016 \\ Volume 3 \\ Revision 1 \\ Page 80}

\section{MICON PROGRAM FILES}

MICON program files are created by MICON View from the configuration file listings (above). This file is the actual program that is run by the controller card. When you request a program upload from a controller card, these are the files you'll get.

These files should not be directly modified by the user. All modifications should be through the configuration file using the configure controller option on MICON View.

The programming language is a custom language developed by MICON Company. A description of this language can be found in the U-32/RCM Function Compiler Documentation available from MICON Company (Houston, TX 1-713-947-9470).

You will notice that, like the configuration file, the program file is organized into loops. With the exception of two loops, these are the same ones created by the user in the controller's configuration file. The two exceptions are loop " ZERO" at the top and loop "NINETY NINE" at the bottom. These are house-keeping loops created by MICON Viēw.

The following file listings have been edited to make them easier to read. Spaces have been put between the loops and extra long 1 ines have been indented Therefor, the actual file's format may look a little different.

NOTE: The DEF line under each loop name contains fields showing the tuning paramaters -- for example:

CGAIN $=0.50$, CRESET $=10.00$, CRATE $=0.00, M \_$RESET $=0.00$

These tuning parameters are not controlled by this document. Therefore, these numbers may be different than the ones that appear in the workstation listings. This will also be true of program files uploaded from the controllers. 


\section{WHC-SD-CP-CSWD-016}

Volume 3

Revision 1

Page 81

mic8_ro

LOOP ZERO;

DEF :TAG=AI SCW1A., AI SCW1 1.,AI SCW1_2., AI PCW5_1., AI PCW5 2., AI PCW5 3., AI PCW5 4.;

DEF :TAG=CIR PCW5.,FIR PCH5.,FIR_SCWI-,PIR_PCW5.,TIR_PCW5.,TIR_SCW1.,TIRSCWIA.;

DEF : TAG $=$ IFSL PCW5., IFSL_SCW1.;

DEF : TAG=PERIODD=1;

END;

LOOP MISC M8.;

DEF : $T A G=\bar{P} E R I O D=0.30$, SP RATE $=10000.00$, SPLOLM $=0.00$, SPHILM $=100.00$, SPANLO $=0.00$, SPANHI $=100.00$, RATI $10=0.00$, BIAS $=0.00$, CGAIN $=0.20$, CRESET $=10.00$, CRATE $=0.00, M$ RESET $=0.00 ;$

AI :TAG $=A I$ SCW1A.:CHAN $=1: S P A N L O=0.00: S P A N H I=100.00$;

LET :TAG=TIRSCW1A.=AI SCW1A.;/*SAVE AI SCW1A AS FC-1 OUTLET TEMP*/

AI :TAG $=A I$ SCW1 $1 .:$ CHAN $=2: S P A N L O=0.00:$ SPANHI $=100.00$;

LET :TAG=TIR SCW1. =AI SCW1 $1 . ; / *$ SAVE INPUT AS FC-1 INLET TEMP*/

AI :TAG=AI SCW1 2.:CHAN=3:SPANLO=0.00:SPANHI $=100.00$;

LET :TAG=FIR SCW1.=AI SCW1 2.;/*SAVE AI SCW1_2 AS FC-1 OUTLET FLOW*/

AI :TAG=AI PCW5 1.:CHAN=4:SPANLO=0.00:SPANHI $=100.00$;

LET :TAG=TIR PCW5,=AI PCW5 $1 . ; / *$ SAVE AI PCW5 1 AS VP-1 LOOP TEMP*/

AI :TAG $=A I$ PCW5 2.:CHAN $=5:$ SPANLO $=0.00:$ SPANHI $=20.00$;

LET :TAG=PIR PCW5.=AI PCW5 2.;/*SAVE AI PCW5 2 AS VP-1 LOOP PRESS*/

AI $: T A G=A I$ PCW5 3.: CHAN $=6: \overline{S P A N L O}=0.00: S \overline{P A N H I}=1000.00$;

LET $: T A G=C T R$ PCW5. =AI PCW5 $3 . ; / *$ SAVE AI PCH5 3 AS VP-1 LOOP CONDUCTIVITY*I

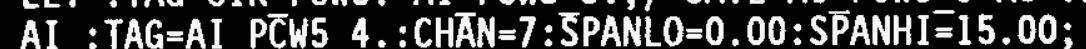

LET :TAG=FIR PCW5.=AI PCW5 4.;/*SAVE AI_PCW5_4 AS VP-1 LOOP FLOW*/

EXPORT FSL_PCW5, FSL_SCW1;

END; 
WHC-SD-CP-CSWD-016

Volume 3

Revision 1

Page 82

LOOP NINETY NINE;

ALARM :TAG=CIR PCH5. :REF=CIR PCH5. :TYPE $=1: P R I=149796:$ LIMITS $=\{?-, ?-, ?-, ?+, ?+, ?+\}: D E A D B A N D=10.00$;

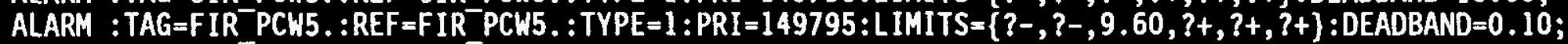

LET :TAG=FSL PCH5=FIR PCW5EXM1;

ALARM :TAG=FIR SCW1.:REF=FIR SCW1.:TYPE=1:PRI=149731:LIMITS=[?-, 15.00, 25.00,?+,?+,?+]:DEADBAND=1.00;

LET $\quad:$ TAG $=F S L$ SCH1 $=F I R$ SCW1OXM4;

ALARM :TAG=PIR PCW5.:REF= IR PCW5.:TYPE $=1:$ PRI $=149795:$ LIMITS $=\{?-, ?-, 2.00, ?+, ?+, ?+\}:$ DEADBAND=0.20;

ALARH :TAG=TIR PCW5.: REF $=$ TIR PCW5.: TYPE $=1:$ PRI $=149780:$ LIMITS $=\{?-, ?-, ?-, 90.00, ?+, ?+\}:$ DEADBAND=1.00;

ALARM :TAG =TIRSCW1A.:REF =TIRSCW1A.:TYPE $=1:$ PRI $=149778:$ LIMITS $=\{?-, ?-, 40.00,76.00, ?+, ?+\}: D E A D B A N D=1.00$;

DEF :TAG $=$ PERIOD $=1$;

END; 


\section{WHC-SD-CP-CSWD-016 \\ Volume 3}

Revision

Page 83

\section{micg_ro}

LOOP ZERO;

DEF :TAG=AI SCW2A.,AI SCW2_1.,AI SCW2_2.,AI PCW6_1.,AI_PCW6_2.,AI_PCW6_3.,AI_PCW6_4.,AI_SCW1_3., AI PCW1 1.,AI PCW1 2-,AI PCW1 3.,AI PCW1 4\%;

DEF :TAG=CIR PCW1., CIR PCW6.,CIR SCW1.,FIR_PCWT.,FIR_PCW6.,FIR_SCW2.,PIR_PCW1.,PIR_PCW6.,TIR_PCW1., TIR PCW6., TIR_SCW2., TIRSCW' 2 A.;

DEF :TAG=TFSL PCH6., IFSL_SCW2.;

DEF : TAG $=$ PERIOD $=1$;

END; 


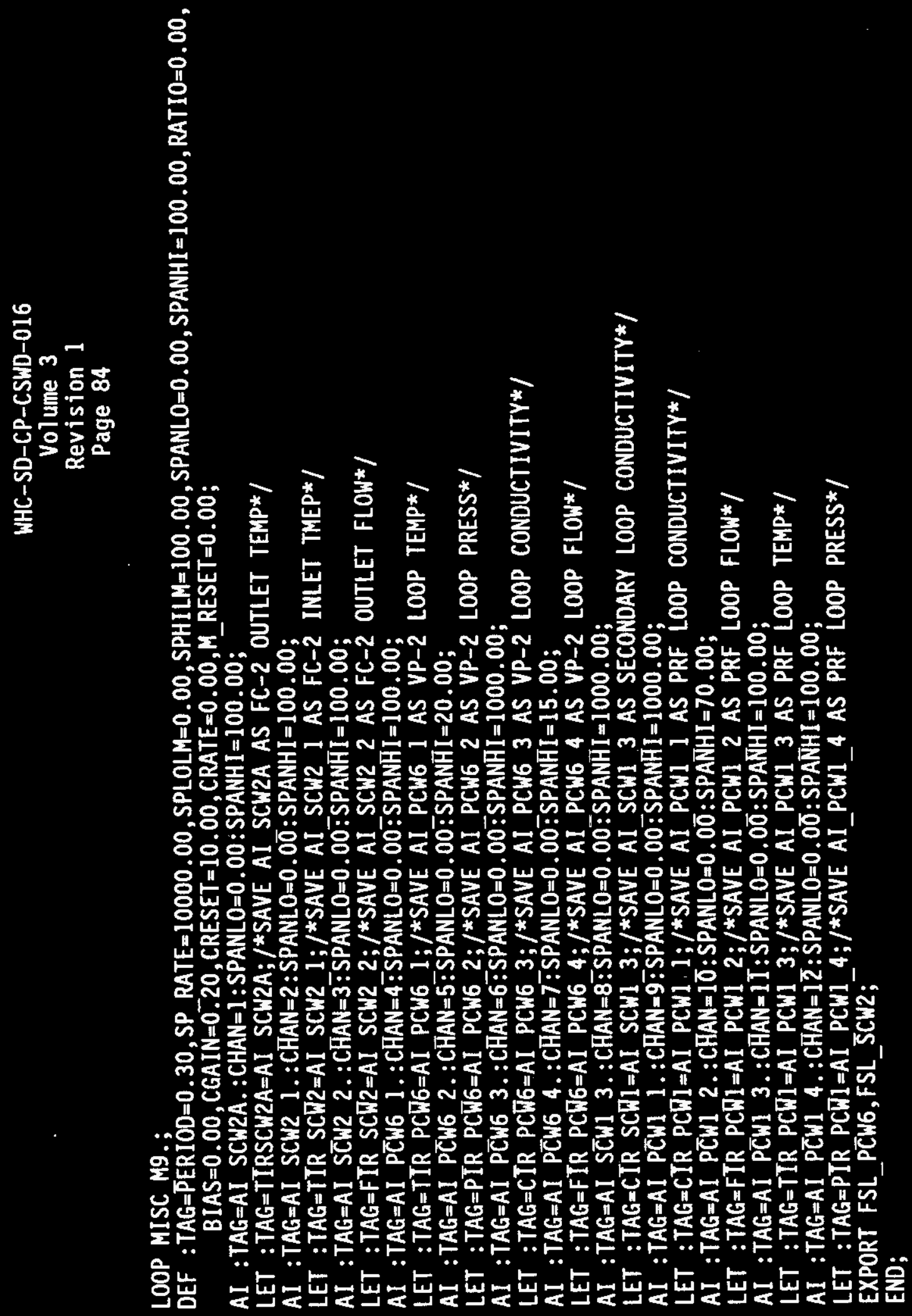




\section{WHC-SD-CP-CSWD-016}

Volume 3

Revision 1

Page 85

\section{LOOP NINETY NINE;}

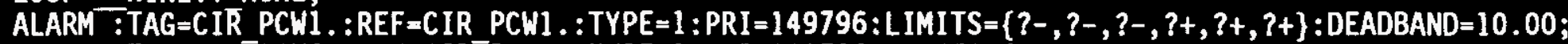
ALARM :TAG=CIR PCW6.: REF=CIR PCW6. :TYPE=1:PRI $=149796:$ LIMITS= $\{-, ?-, ?-, ?+, ?+, ?+\}:$ DEADBAND=10.00;

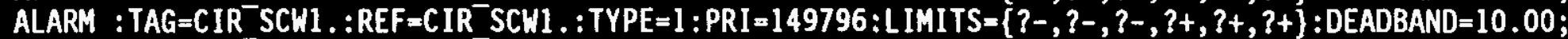
ALARM :TAG=FIR PCW1.:REF=FIR PCW1.: TYPE $=1: P R I=149795:$ LIMITS $=\{?-, ?-, 25.00, ?+, ?+, ?+\}: D E A D B A N D=0.70$;

ALARM :TAG=FIR PCW6.:REF=FIR PCW6. :TYPE=1:PRI =149795:LIMITS= $\{?-, ?-, 9.60, ?+, ?+, ?+\}: D E A D B A N D=0.10$; LET $\quad: T A G=F S L$ PCW6=FIR PCW6CXM1;

ALARM :TAG=FIR SCW2.:REF=FIR SCW2.:TYPE $=1:$ PRI $=149731:$ LIMITS $=\{?-, 15.00,25.00, ?+, ?+, ?+\}:$ DEADBAND=1.00; LET $\quad: T A G=F S L$ SCW2=FIR SCW2OXM4:

ALARM :TAG=PIR_PCW1.:REF $=$ PIR_PCW1.:TYPE $=1: P R I=149794:$ LIMITS $=\{?-, ?-, 50.00, ?+, ?+, ?+\}:$ DEADBAND=1.00;

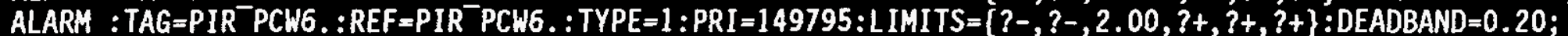

ALARM :TAG=TIR PCW1.:REF=TIR PCW1.:TYPE $=1: P R I=149780:$ LIMITS $=\{?-, ?-, ?-, 75.00, ?+, ?+\}: D E A D B A N D=1.00$; ALARM :TAG=TIR PCW6. :REF=TIR PCW6. :TYPE $=1: P R I=149780:$ LIMITS $=\{?-, ?-, ?-, 90.00, ?+, ?+\}: D E A D B A N D=1.00 ;$ ALARM :TAG=TIRSCW2A. :REF $=$ TIRSCW2A. $:$ TYPE $=1:$ PRI $=149778:$ LIMITS $=\{?-, ?-, 40.00,76.00, ?+, ?+\}: D E A D B A N D=1.00$; DEF : TAG $=$ PERIOD $=1$;

END; 

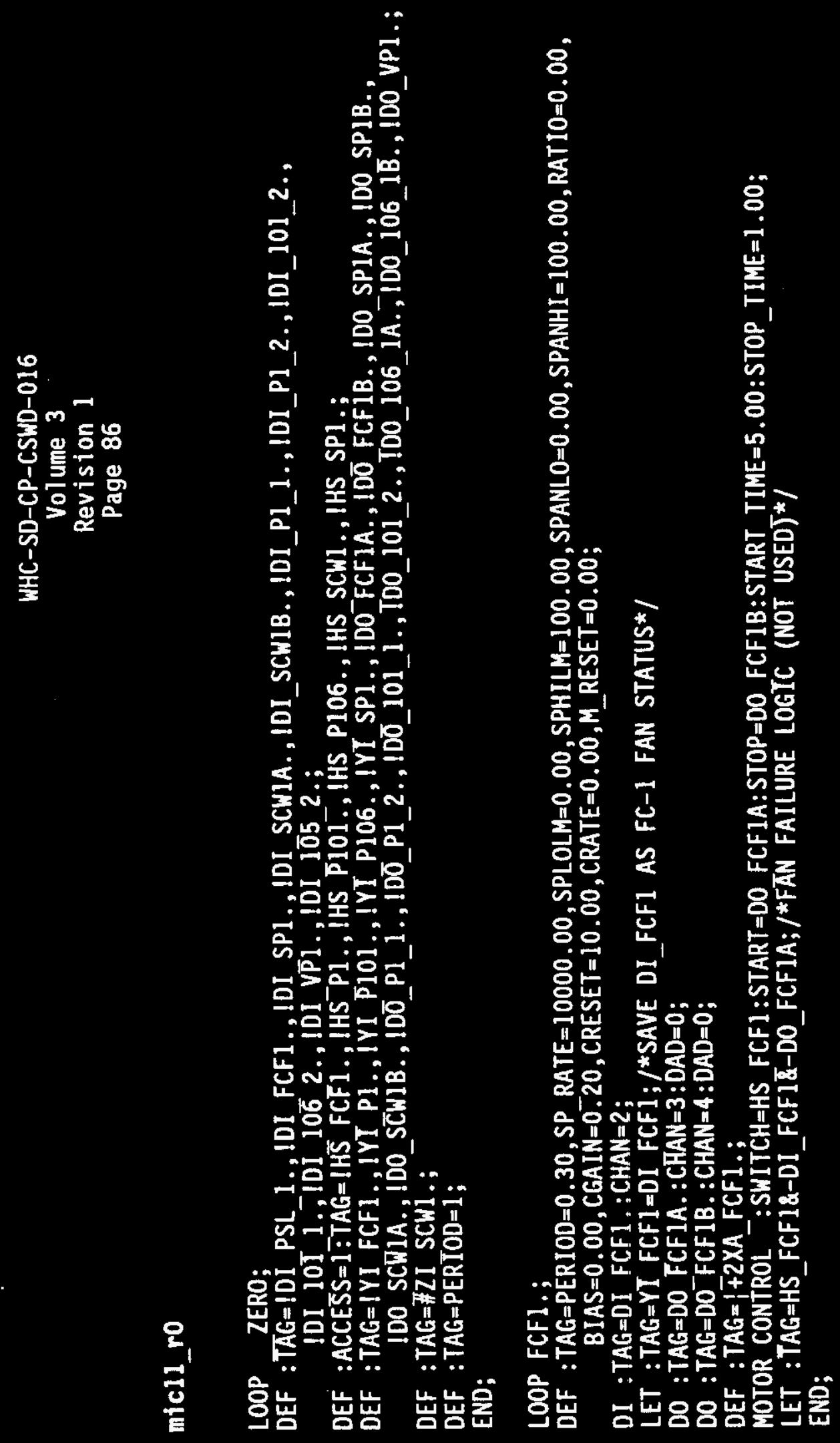


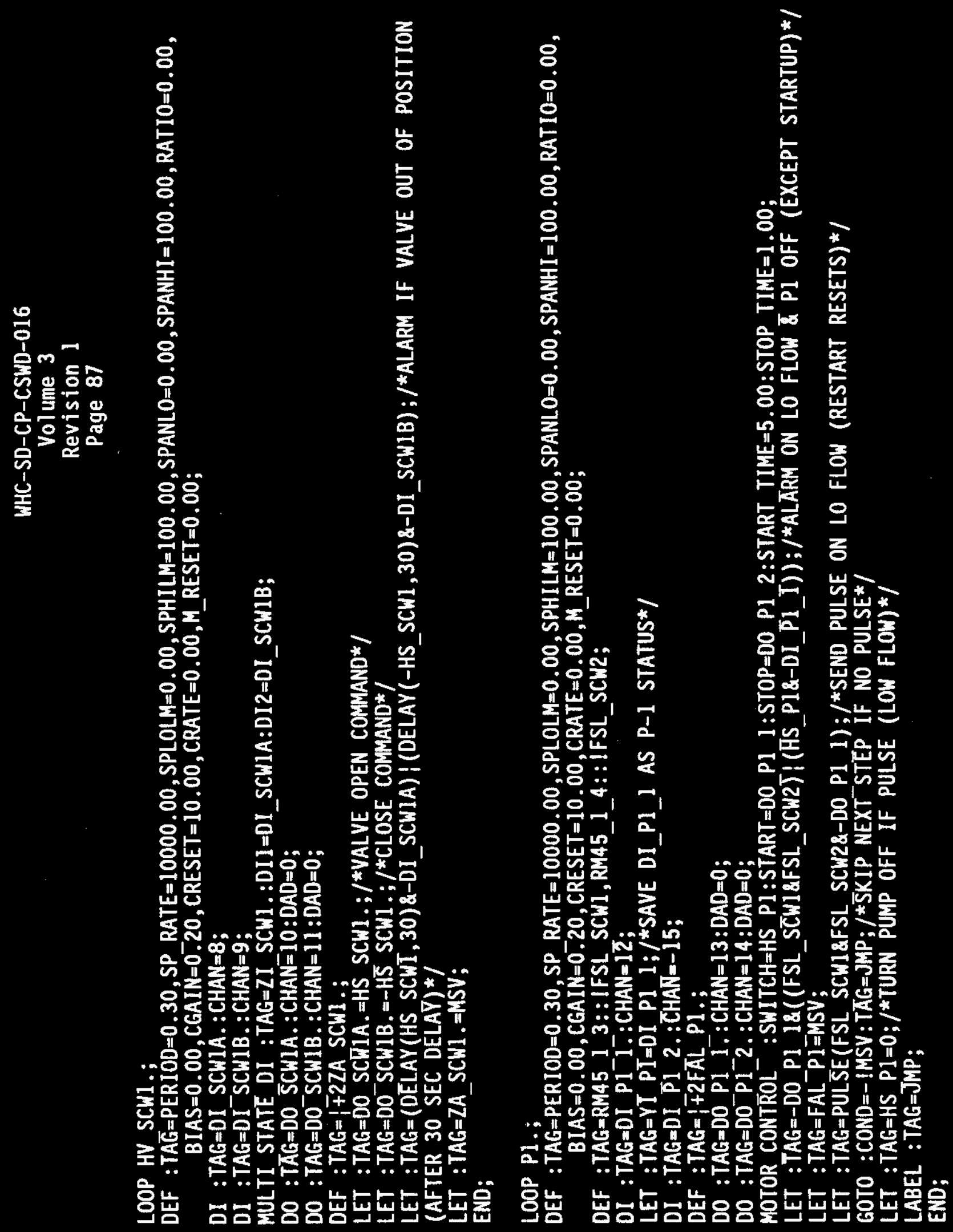




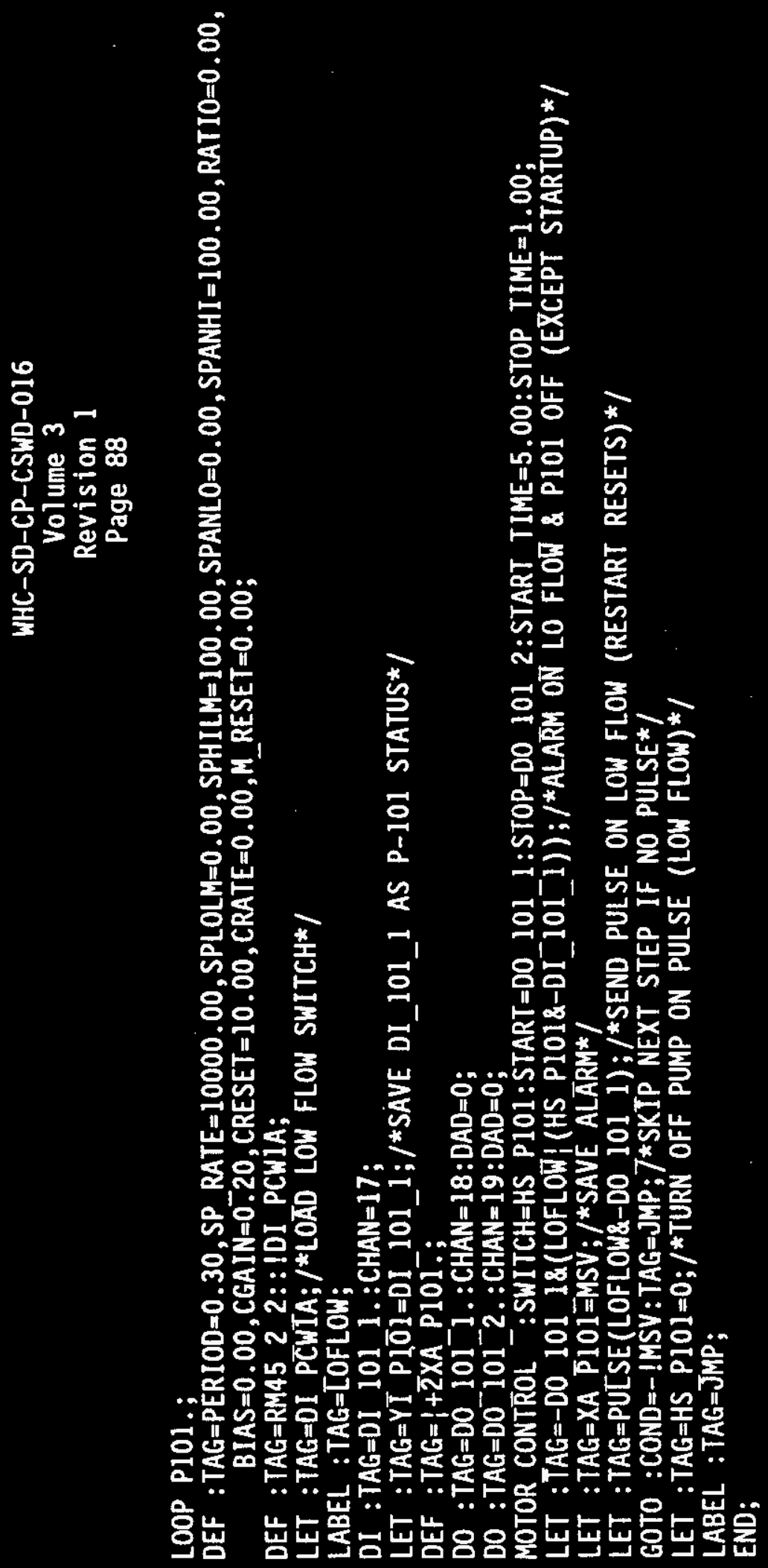




\section{WHC-SD-CP-CSWD-016}

Volume 3

Revision 1

Page 89

LOOP P106.;

DEF : TAG $=$ PERIOD $=0.30$, SP RATE $=10000.00, S P L O L M=0.00, S P H I L M=100.00, S P A N L 0=0.00, S P A N H I=100.00, R A T 10=0.00$, $B I A S=0.00, C G A I N=0.20, C R E S E T=10.00, C R A T E=0.00, M$ RESET $=0.00$;

DEF :TAG $=$ RM45 2 2:: !DI VP2, RM45 $13:$ :! IFSL PCW5;

LET :TAG $=0 I$ V $\bar{P} 2 \bar{j} / *$ LOAD VACUUM PUMP 2 STATUS*/

LABEL : TAG $=$ VP2STAT;

LET :TAG=FSL PCW5; $/ *$ LOAD VP-1 LOOP LOW FLOW ALARM SWITCH*/

LABEL :TAG=LDFLW;

DI : TAG=DI 106 2.: CHAN=21;

LET : TAG $=Y I$ P106=DI $1062 ; / \star S A V E$ DI 1062 AS P-106 STATUS*/

DEF : TAG $=1+\overline{2} \times A$ P106.;

DO :TAG $=D O$ O 106 1A.: $\mathrm{CHAN}=22: D A D=0$;

DO : TAG $=$ DO $106-18 .:$ CHAN $=23: D A D=0$;

LET :TAG=DI VPI!UP2STAT $1010 ; / * D E T E R M I N E$ COMBINED VAC PUMP STATUS $1=0 N^{*} /$

LABEL :TAG=VPSTATS;

MOTOR CONTROL :SWITCH=HS P106:START=DO 106 1A:STOP=DO 106 1B:START TIME=30.00:STOP TIME=1.00; $* 30$ SECOND

START TO ALLOW TIME TO START VAC PUMP*/

LET :TAG $=-D 0106$ 1A\&(LOFLW! (HS P106\&-DI 1062 ));/*ALARM ON LO FLOW OR PUMP OFF (EXCEPT DURING RESTART) $/$

LET :TAG $=X A$ P106=MSV;

LET :TAG=PULSE (!LOFLW\&-DO 106 1A);/*SEND PULSE ON LOW FLOW (RESTART RESETS)*/

GOTO : COND $=-$ IMSV:TAG $=$ JMPF[; $/$ \$SKIP NEXT STEP IF NO PULSE*/

LET : TAG $=H S$ P106=0;/*TURN OFF PUMP ON PULSE (LON FLOW) $* /$

LABEL : TAG $=$ JMPFL;

LET :TAG=VPSTATSIDO 1061 1A;0;0;/*OUTPUT=1 IF A VAC PUMP IS RUNNING OR PI06 IS RESTARTING*/

GOTO :COND=MSV:TAG=JMPVP; /*SKIP NEXT STEP IF VP'S RUNING OR P106 IN RESTART*/

LET :TAG $=$ HS P106=0;/*TURN OFF PUMP IF BOTH VAC PUMPS OFF (EXCEPT DURING RESTART) /

LABEL :TAG=JMPVP;

END; 
WHC-SD-CP-CSWD-016

Volume 3

Revision 1

Page 90

LOOP SP1.;

DEF : TAG $=P E R I O D=0.30, S P$ RATE $=10000.00, S P L O L M=0.00, S P H I L M=100.00, S P A N L 0=0.00, S P A N H I=100.00, R A T I 0=0.00$, $B I A S=0.00$, CGAIN $=0.20$, CRESET $=10.00$, CRATE $=0.00, M$ RESET $=0.00$; DI :TAG=0I SP1.:CHAN $=5$;

LET :TAG=YI SP1=DI SP1; $/$ *SAVE DI SP1 AS SP-1 STATUS*/

$D O: T A G=D O$ SP 1 A. $:$ CHAN $=6: D A D=0$;

$D O: T A G=D 0^{-} S P 1 B .: C H A N=7: D A D=0$;

DEF :TAG $=1 \mp 2 \times A$ SP1.:

MOTOR CONTROL - :SWITCH=HS SP1:START=DO SP1A:STOP=DO SP1B:START TIME=5.00:STOP TIME=1.00;

LET :TAG=HS SP1\&-DI SP1\&-DO SP1A; /*ALARM WHEN PUMP OFF AND SWITCH ON (EXCEPT DURING RESTART)*/

LET :TAG $=X A^{-} S P 1=M S V$;

END;

LOOP YP1.;

DEF : TAG $=$ PERIOD $=0.30$, SP RATE $=10000.00, S P L O L M=0.00, S P H I L M=100.00, S P A N L O=0.00$, SPANHI $=100.00$, RATI0=0.00, BIAS $=0.00$, CGAIN $=0.20$, CRESET $=10.00$, CRATE $=0.00, \mathrm{M}$ RESET $=0.00$;

DEF :TAG=RM45 1 4::IFSL PCW6;

LET :TAG=FSL PCT5\&FSL PCW6; $/ *$ LOAD VP-1 \& 2 LOW FLOW ALARMS* /

LABEL :TAG=LOFLW;

DI :TAG=DI VP1.:CHAN=25;

$D 0$ : TAG $=00^{-} V P 1 .:$ CHAN $=26: D A D=0$;

DEF :TAG $=1 \overline{+} 2 \times A$ VP1.;

LET :TAG=PULSE(DI VP1,60) $\{-!$ LOFLW; $/ * 0 U T P U T=1$ IF VP-1 ON<6OSEC OR NO LO FLOW ALARM*/

LET :TAG $=X A$ VPI $=-$ TMSV; $/ * A L A R M$ ON LOW FLON WHEN VP-1 IS ON > $60 \mathrm{SEC} * /$

LET :TAG=PULSE(XA VP1,1); $/$ SENO 1 SECOND PULSE ON LOW FLON ALARM*

LET :TAG=DO VP1=-TMSV; $/ *$ VP1 STOP CONTACT OPENS 1 SEC ON LO FLON*/

EXPORT DI_VP1;

END; 


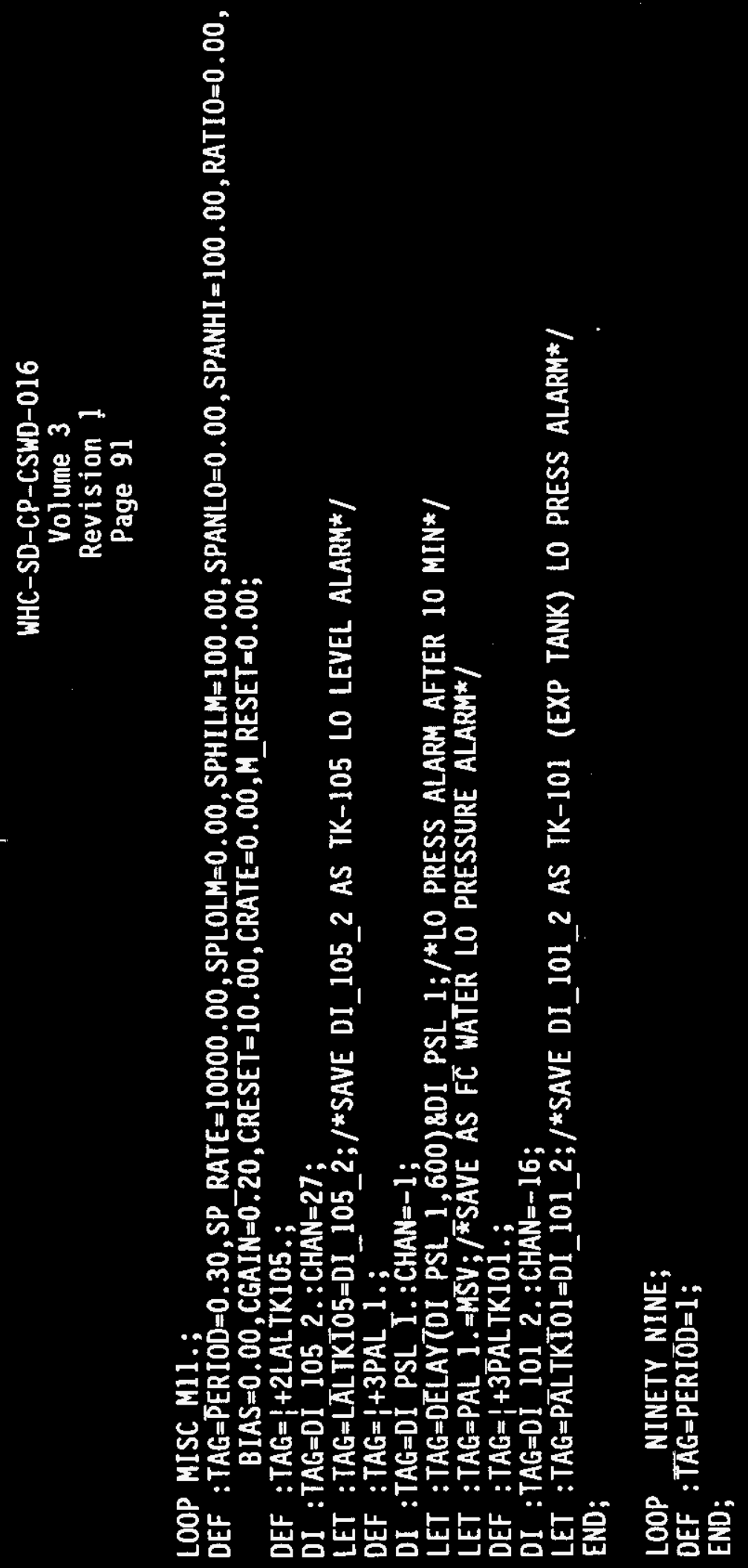



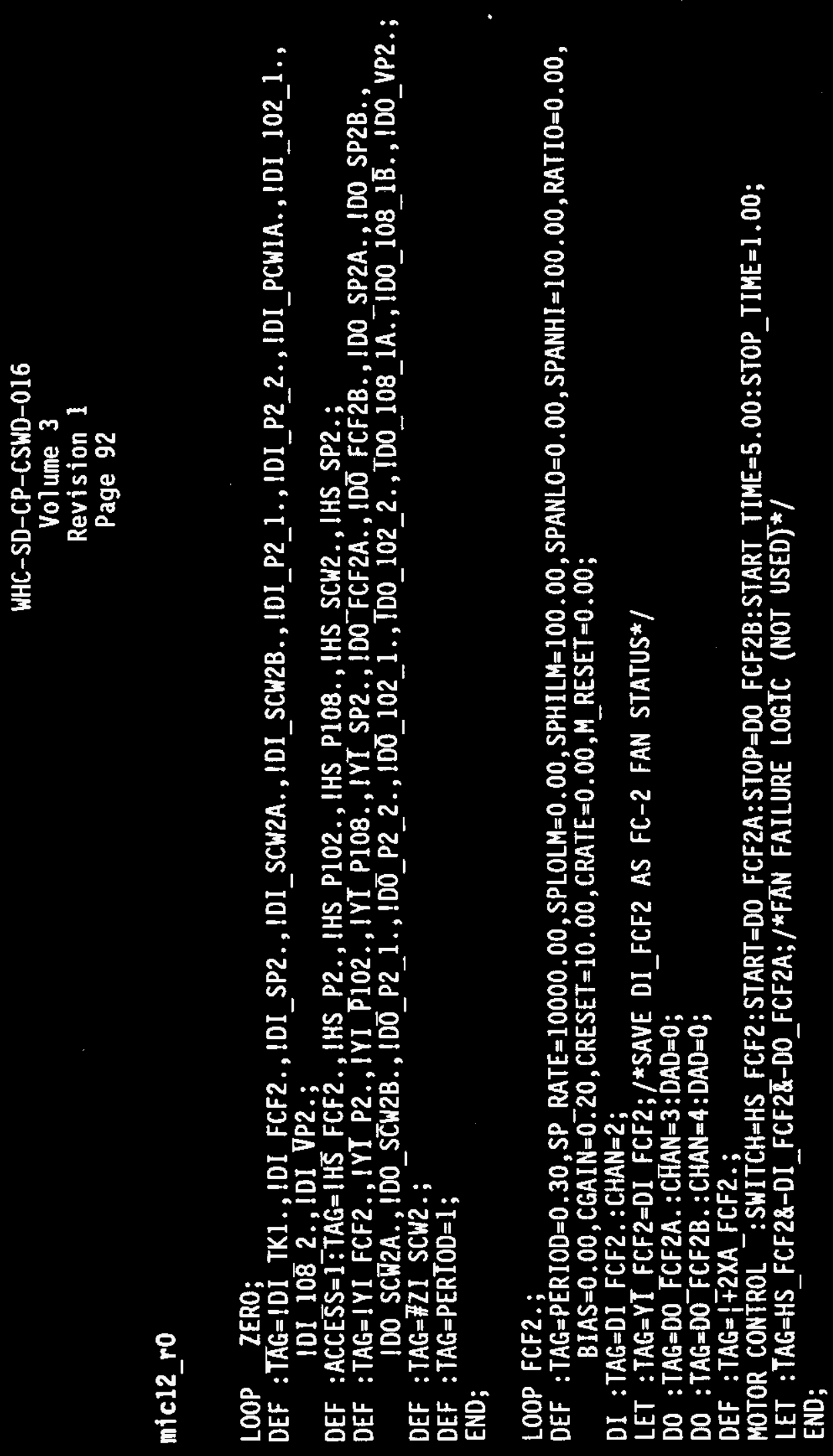
WHC-SD-CP-CSWD-016

Volume 3

Revision 1

Page 93

LOOP HV SCW2.;

DEF :TAG $=P E R I O D=0.30, S P$ RATE $=10000.00, S P L O L M=0.00, S P H I L M=100.00, S P A N L 0=0.00, S P A N H I=100.00, R A T I 0=0.00$, $B I A S=0.00$, CGAIN $=0.20$, CRESET $=10.00$, CRATE $=0.00, M$ RESET $=0.00$;

DI :TAG=DI SCW2A. :CHAN=8;

DI : TAG $=\mathrm{DI}^{-} \mathrm{SCW} 2 \mathrm{~B}$. : $\mathrm{CHAN}=9$;

MULTI STATE DI :TAG=ZI SCW2.:DI1=DI_SCW2A:DI2=DI_SCW2B;

DO : TA $\bar{A} G=00$ SCW2A. :CHAN $=10: D A D=0$;

$D 0: T A G=D 0^{-}$SCW2B . : CHAN $=11: D A D=0$;

DEF :TAG $=1 \mp 2 Z A$ SCW2.;

LET :TAG $=00$ SCW2A. $=$ HS SCW2 $; / *$ OPEN COMMAND*/

LET :TAG $=00^{-}$SCW2B. $=-H \bar{S}$ SCW2.; $/ *$ CLOSE COMMAND $* /$

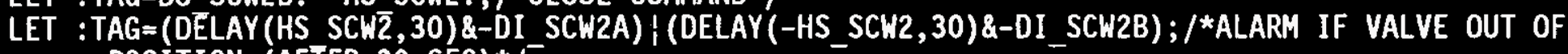
POSITION (AFTER $30 \mathrm{SEC}$ ) $I^{-}$

LET :TAG=ZA SCW2. = MSV;

END; 


\section{WHC-SD-CP-CSWD-016 \\ Volume 3 \\ Revision 1 \\ Page 94}

LOOP P2.;

DEF : TAG $=$ PERIOD $=0.30$, SP RATE $=10000.00$, SPLOLM $=0.00, S P H I L M=100.00$, SPANL $0=0.00$, SPANHI $=100.00$, RATI $10=0.00$, BIAS $=0.00$, CGAIN $=0.20$, CRESET $=10.00$, CRATE $=0.00, \mathrm{H}$ RESET $=0.00 ;$

DEF :TAG=RM45_1_4::IFSL SCW2,RM45_1_3::IFSL_SCW1;

DI :TAG $=01$ P2 1 .: CHAN $=1 \overline{2}$;

LET :TAG $=Y$ I $P \bar{Z}=D I$ P2 $1 ; / *$ SAVE DI_P2_1 AS P-2 STATUS*/

DI :TAG=DI P2 2.: $\bar{C} H A \bar{N}=-15$;

DEF : TAG $=1+2 F \bar{A} L P 2$;

$D 0: T A G=00$ P2 1.:CHAN $=13: D A D=0$;

$D O: T A G=D 0^{-} P 2^{-}$2.: $C H A N=14: D A D=0$;

MOTOR CONTROL ${ }^{-}$:SWITCH=HS P2:START=DO P2 1:STOP=DO P2 2:START FIME=5.00:STOP TIME=1.00;

LET :TAG=-D0 P2 18((FSL SCW2\&FSLSCW1)!(RS_P28-DI_P2_I));/*ALARM ON LO FLOW OR P2 OFF

(EXCEPT DURING RESTART)*/

LET :TAG $=F A L P 2=M S V ; / * S A V E$ TO ALARM* $/$

LET :TAG=PULSE(FSL SCW2\&FSL SCW1\&-DO P2 1 ); $/$ *SEND PULSE ON LO FLOW ALARM (RESTART RESETS)*/

GOTO :COND $=-$ !MSV:TAG $=$ JMP; $/ *$ SKIP NEXT STEP IF NO PULSE*/

LET : TAG $=$ HS P2 $=0 ; / *$ TURN OFF PUMP IF PULSE (LOW FLOW) $* /$

LABEL :TAG=JMP;

END; 


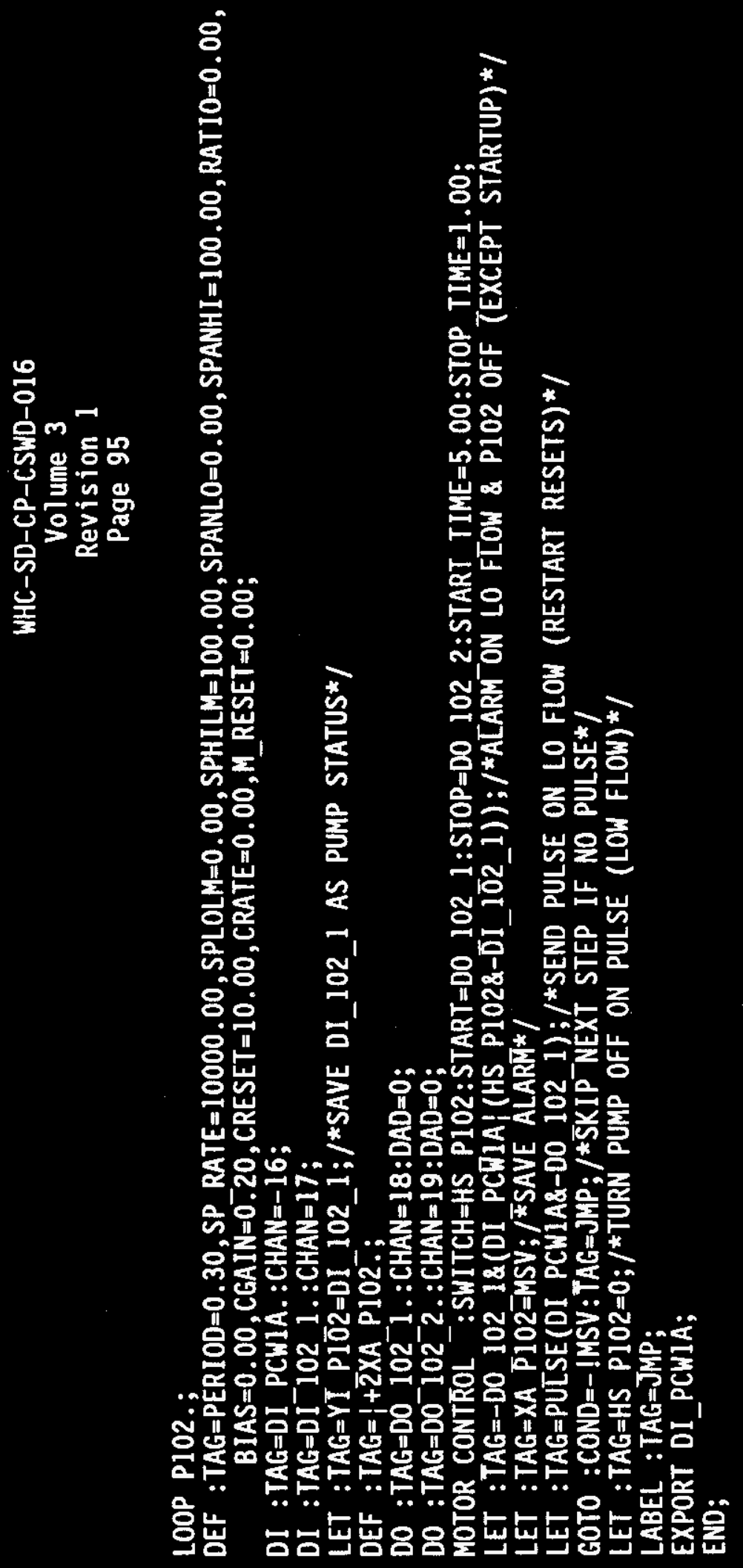




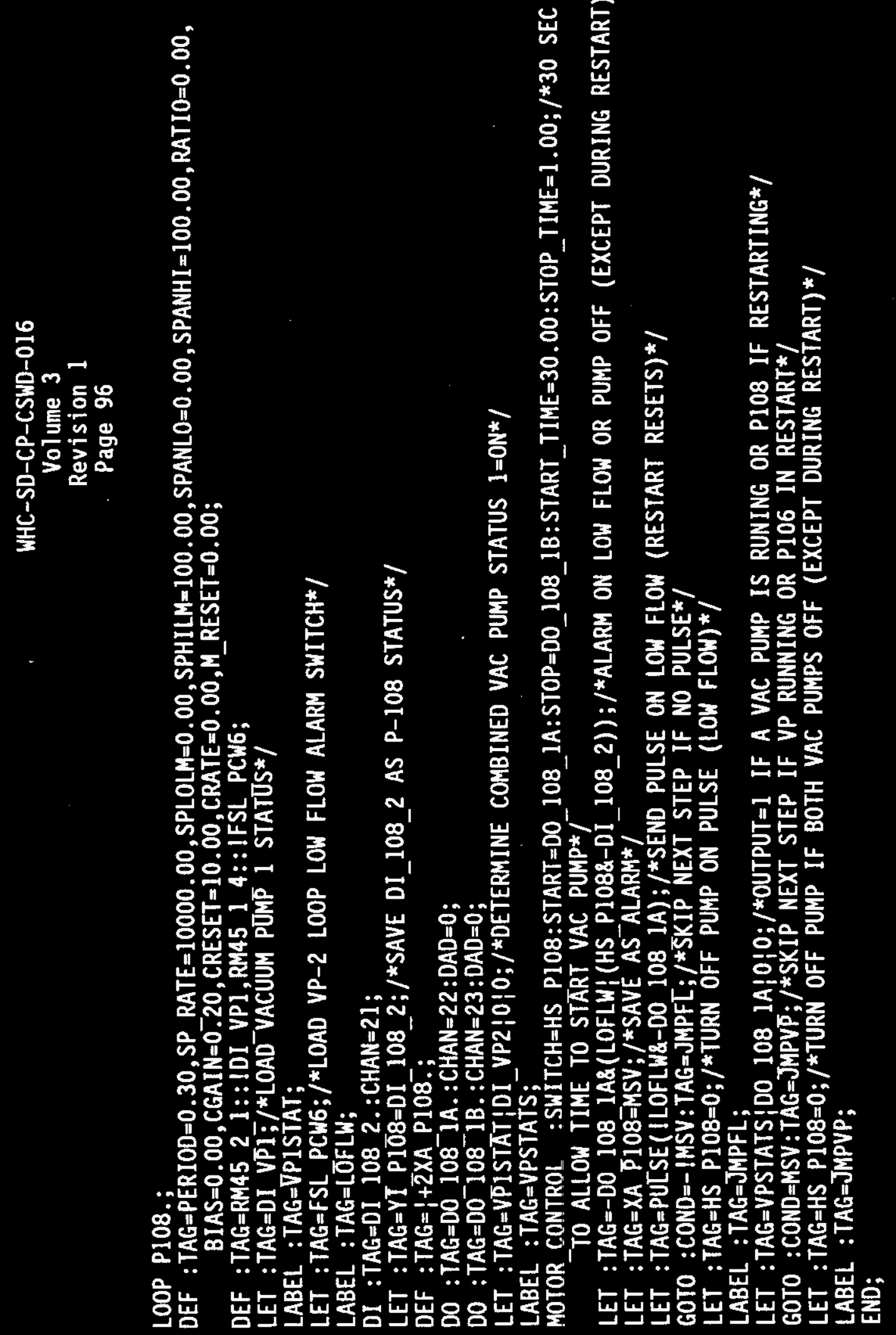




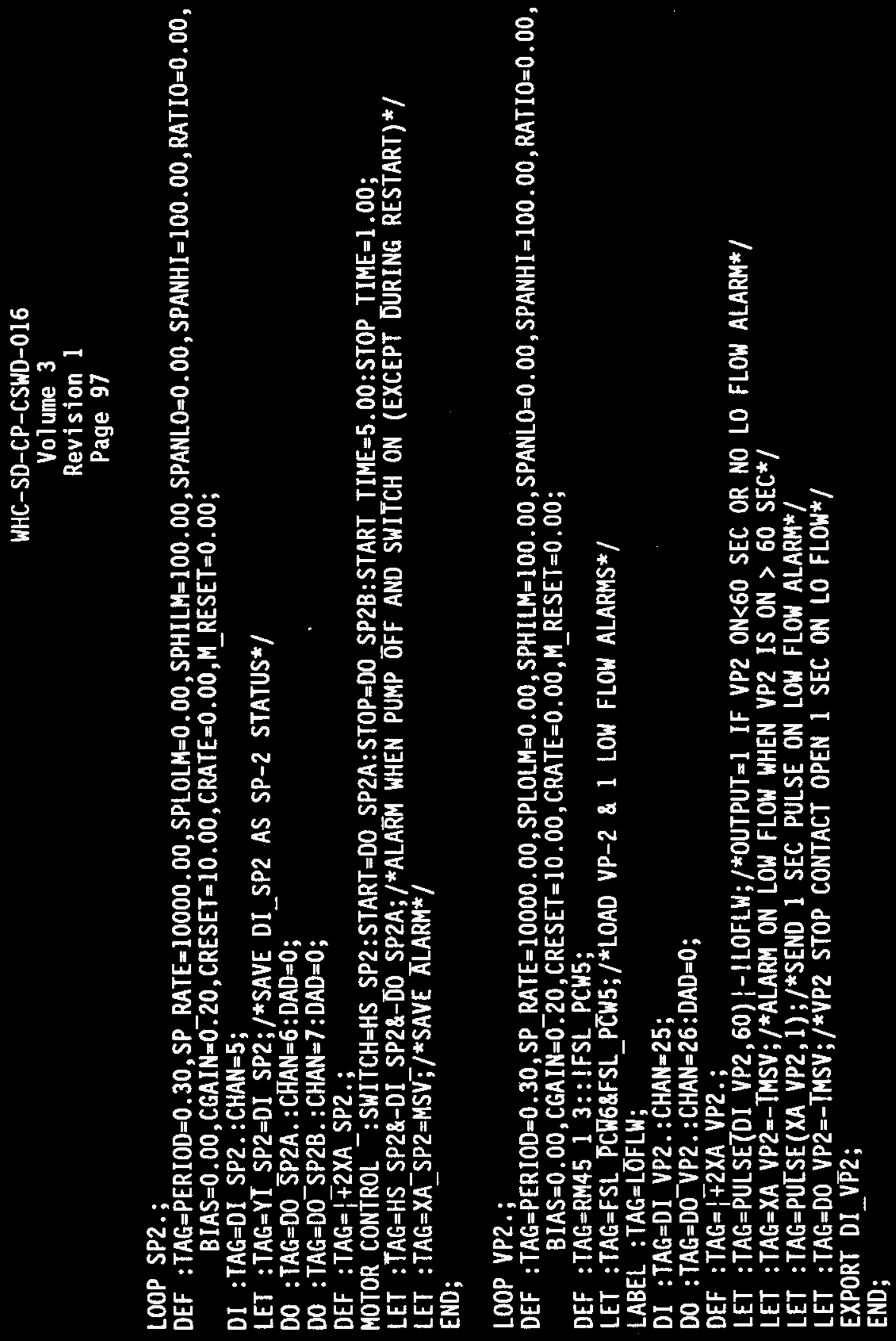




\section{WHC-SD-CP-CSWD-016 \\ Volume 3 \\ Revision 1 \\ Page 98}

LOOP MISC M12.;

DEF :TAG $=\bar{P} E R I O D=0.30$, SP RATE $=10000.00, S P L O L M=0.00, S P H I L M=100.00, S P A N L 0=0.00$, SPANHI $=100.00$, RAT 10=0.00, BIAS $=0.00$, CGAIN $=0.20$, CRESET $=10.00$, CRATE $=0.00, \mathrm{M} \_$RESET $=0.00$;

DEF : TAG $=+2 P A L$ TK1 ;

DI :TAG=DI TK1. :CHAN=-1;
LET :TAG=PAL TK1=DI TK1; $/$ SAVE DI_TK1 AS TK-1 LOW PRESSURE ALARM*/

END;

LOOP NINETY NINE;

DEF : TAG $=$ PERIŌD=1;

END; 
WHC-SD-CP-CSWD-016

Volume 3

Revision 1

Page 99

\section{APPENDIX A: MICON INPUT/OUTPUT}

Analog inputs and discrete Input/Outputs can be set up in different ways. Analog inputs can be set to accept one of three types of signals: a 4-20 miliamp input with the MICON powering the 100p, a 4-20 miliamp input with an external power supply, or a voltage input. Twentyfour volt discrete input/outputs can be set up as an input only, an output only, or as both an input and output. Since the hardware requirements are different for each of these setups, the controller cards have on-board jumpers for each analog input or discrete. These jumpers must be set to the desired type of analog input or discrete for the card to work properly.

Analog outputs are 4-20 miliamp internally powered only. Since they can't be changed they don't have any jumpers.

Before replacing an existing card with a new one or installing a backup card, you must make sure the jumpers in the new card are set the same as the jumpers in the old card.

The following tables show the Input/Output types for each controller card in the 243-Z cabinet. See the RCM-32 Remote Control Module User Manual (Vendor Information file 22068) for a description of the $1 / 0$ jumper placement. 
MICON Number: 8 Tag Name:RM45_1_3

Location: 236-Z Room 45 Rack Number: 1

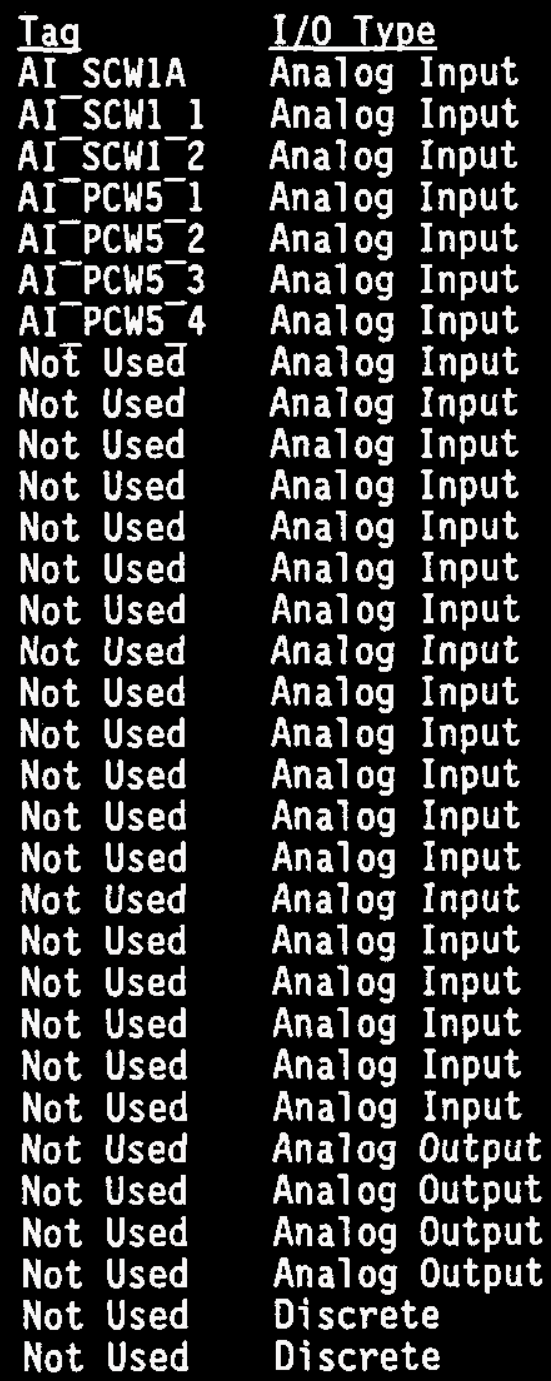

Type: $\mathrm{RCM}-32 \mathrm{~A}$

Slot Number: 3

Jumper Assignment
4-20 mA MICON Powered
$4-20 \mathrm{~mA}$ MICON Powered
4-20 mA MICON Powered
4-20 mA External Power
4-20 mA External Power
4-20 mA MICON Powered
4-20 mA External Power
Voltage
Voltage
Voltage
Voltage
Voltage
Voltage
Voltage
Voltage
Voltage
Voltage
Voltage
Voltage
Voltage
Voltage
Voltage
Voltage
Voltage
Voltage
Voltage
N/A
N/A
N/A
N/A
Input \& Output
Input \& Output

${ }^{1}$ See RCM-32 Remote Control Module User Manual for description of I/0 jumper placement. 


\section{WHC-SD-CP-CSWD-016 \\ Volume 3 \\ Revision 1 \\ Page 101}

MICON Number: 9 Tag Name:RM45_1_4 Type: RCM-32A

Location: 236-Z Room 45 Rack Number: 1 Slot Number: 4

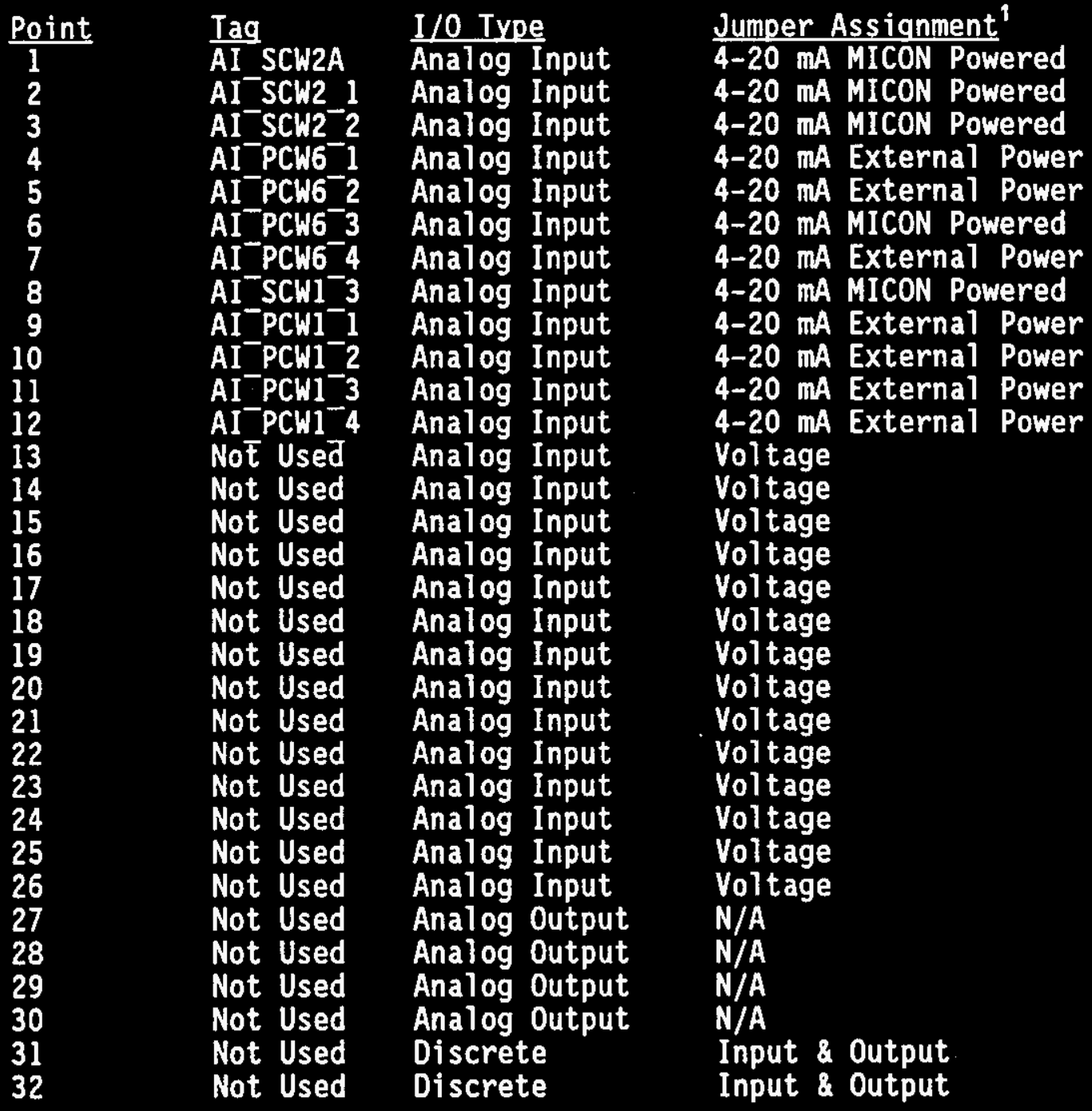

${ }^{1}$ See RCM-32 Remote Control Module User Manual for description of I/0 jumper placement. 


\section{WHC-SD-CP-CSWD-016 \\ Volume 3 \\ Revision 1 \\ Page 102}

MICON Number: 11 Tag Name:RM45 2

Location: 236-Z Room 45 Rack Number: 2

Point

1

3

4

5

6

7

8

9

10

11

12

13

14

15

16

17

18

19

20

21

22

23

24

25

26

27

28

29

30

31

32

DI FCFI Discrete Input N/A

DOFCF1A Discrete Output N/A

DOFCF1B Discrete Output N/A

DI SP1 Discrete Input N/A

DO SP1A Discrete Output N/A

DO SP1B Discrete Output N/A

DO-SCW1B Discrete Output N/A

DI P1_1 Discrete Input N/A

$\mathrm{DO}^{-} \mathrm{P1} 1^{-} \mathrm{D}$ Discrete Output N/A

$D^{-} \mathrm{P1}^{-2}$ Discrete Output N/A

DI $I^{-} 2$ Discrete Input $N / A$

DI $10 \mathrm{I} 2$ Discrete Input $\mathrm{N} / \mathrm{A}$

DI 1011 Discrete Input N/A

DO 101 1 Discrete Output N/A

DO 101_2 Discrete Output N/A

DI 1062 Discrete Input N/A

D0 $106^{-1}$ Discrete Output N/A

D0 $106^{-2}$ Discrete Output N/A

Not Usëd Discrete N/A

DI VP1 Discrete Input N/A

DOVP1 Discrete Output N/A

DI 1052 Discrete Input N/A

Not Used Discrete

Not Used

Not Used Discrete N/A

\begin{tabular}{|c|c|c|c|}
\hline Tag & I/0 Type & & Jumper Assianment \\
\hline DI PSL & Discrete & Input & $N / A$ \\
\hline $\mathrm{DI}^{-} \mathrm{FCFI}$ & Discrete & Input & $N / A$ \\
\hline DO_FCFIA & Discrete & Output & N/A \\
\hline $\begin{array}{l}\text { DOFCF } 1 \\
D^{-} I^{-S P 1}\end{array}$ & Discrete & Output & $\begin{array}{l}N / A \\
N / A\end{array}$ \\
\hline $\begin{array}{l}\text { DI SP1 } \\
\text { Do-SP1A }\end{array}$ & $\begin{array}{l}\text { Discrete } \\
\text { Discrete }\end{array}$ & $\begin{array}{l}\text { Input } \\
\text { Output }\end{array}$ & $\begin{array}{l}N / A \\
N / A\end{array}$ \\
\hline DO-SP1B & Discrete & Output & $N / A$ \\
\hline DI_SCWIA & Discrete & Input & $N / A$ \\
\hline DI_SCW1B & Discrete & Input & $N / A$ \\
\hline DO_SCW1A & Discrete & Output & $N / A$ \\
\hline D0_SCW1B & Discrete & Output & $N / A$ \\
\hline DI-P1_1 & Discrete & Input & $N / A$ \\
\hline $\mathrm{DO}^{-} \mathrm{P} 1^{-1}$ & Discrete & Output & $N / A$ \\
\hline DO-P1-2 & Discrete & Output & $N / A$ \\
\hline DIP12 & Discrete & Input & $N / A$ \\
\hline $\mathrm{DI}^{-10 I_{2}}$ & Discrete & Input & $N / A$ \\
\hline DI $101-1$ & Discrete & Input & $\begin{array}{l}\mathrm{N} / \mathrm{A} \\
\mathrm{N} / \mathrm{A}\end{array}$ \\
\hline $\begin{array}{l}\text { DO-101 } \\
\mathrm{DO}^{-1} 101^{-1}\end{array}$ & $\begin{array}{l}\text { Discrete } \\
\text { Discrete }\end{array}$ & $\begin{array}{l}\text { Output } \\
\text { Output }\end{array}$ & $\begin{array}{l}N / A \\
N / A\end{array}$ \\
\hline Not Usēed & Discrete & & $N / A$ \\
\hline DI 10622 & Discrete & Input & $\begin{array}{l}N / A \\
N / A\end{array}$ \\
\hline $00^{-1} 106^{-2}$ & Discrete & Output & $N / A$ \\
\hline Not Usēd & Discrete & & $N / A$ \\
\hline DI VP1 & Discrete & Input & $N / A$ \\
\hline DO-VP1 & Discrete & Output & $N / A$ \\
\hline $\mathrm{DI}^{-} 10$ & Discrete & Input & $N / A$ \\
\hline ot Usēd & Discrete & & $N / A$ \\
\hline Not Used & Discrete & & $N / A$ \\
\hline ot Used & Discrete & & $N / A$ \\
\hline Not Use & Discrete & & $N / A$ \\
\hline Not Use & Discrete & & $N / A$ \\
\hline
\end{tabular}

Type: RCM-32Dr

Slot Number: 1

Jumper Assignment ${ }^{1}$

OISCWIA Discrete Input N/A

Discrete Output N/A

Not Used Discrete N/A

${ }^{1}$ See RCM-32 Remote Control Module User Manual for description of I/0 jumper placement. 
WHC-SD-CP-CSWD-016

Volume 3

Revision 1

Page 103

MICON Number: 12 Tag Name:RM45_2_2 Type: RCM-32Dr

Location: 236-Z Room 45 Rack Number: 2 . STot Number: 2

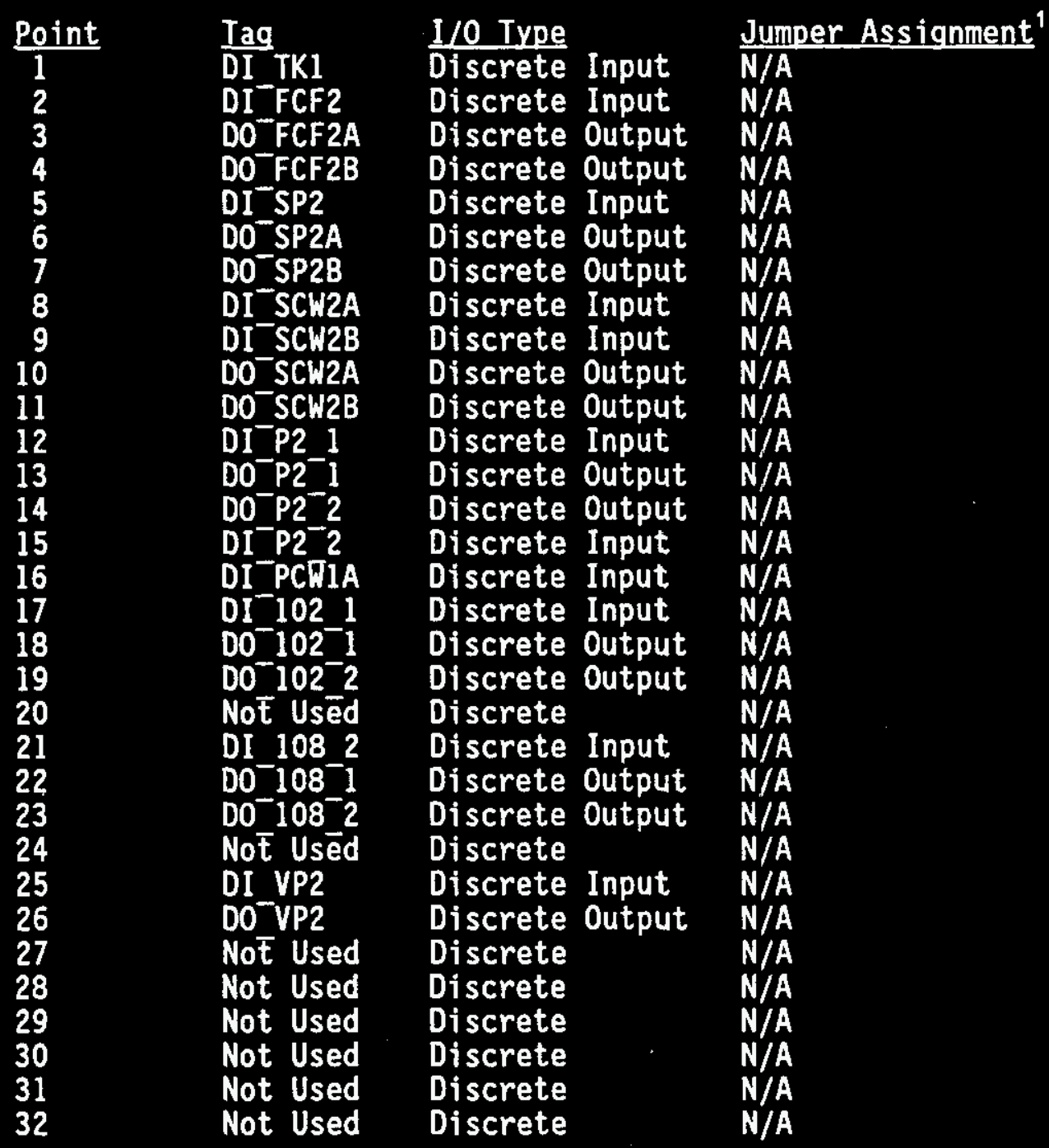

${ }^{1}$ See RCM-32 Remote Control Module User Manual for description of I/0 jumper placement. 
WHC-SD-CP-CSWD-016

Volume 4

Revision 1

Page 1

TABLE OF CONTENTS

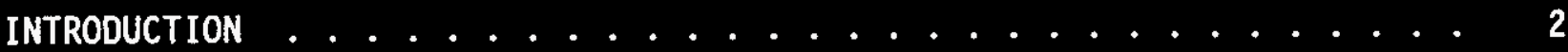

MICON CONFIGURATION FILES $\ldots \ldots \ldots$

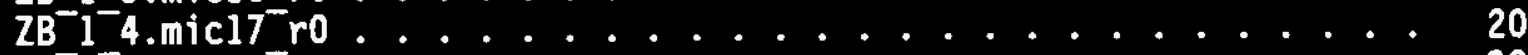

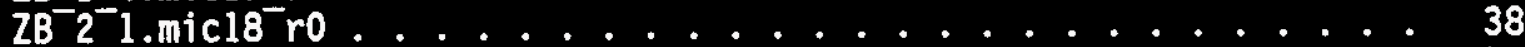

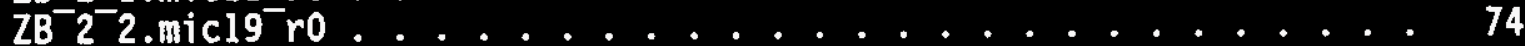

$\mathrm{ZB}_{2}{ }^{3}$.mic20 ro ....................... 110

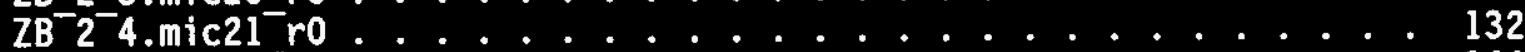

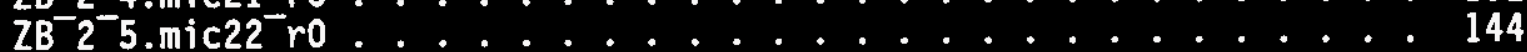

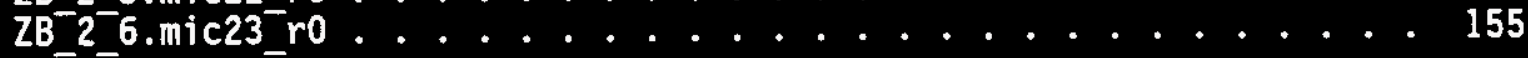

MICON PROGRAM FILES................... 168

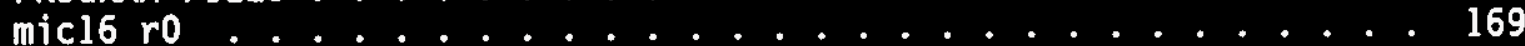

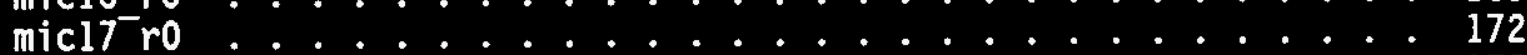

micls $r 0 \ldots \ldots \ldots \ldots . \ldots \ldots$

$\operatorname{mic19} r 0 \ldots \ldots . \ldots . . . \ldots 183$

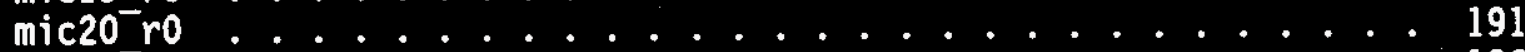

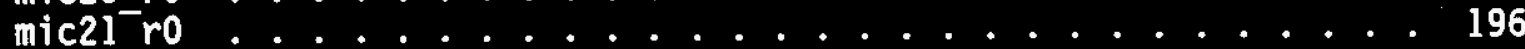

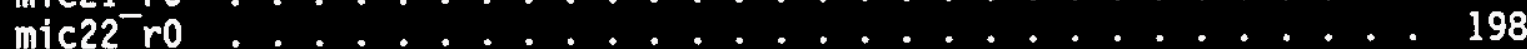

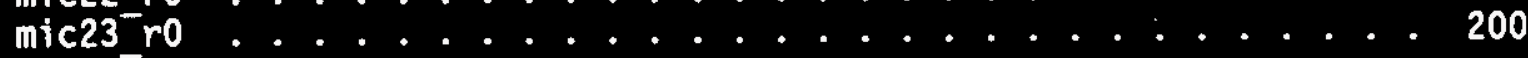

APPENDIX A: MICON INPUT/OUTPUT ............... 202

MICON Number: 16 . . . . . . . . . . . . . . 203

MICON Number: 17 ................ 204

MICON Number: 18 ................. 205

MICON Number: 19 ................. 206

MICON Number: 20 ................. 207

MICON Number: 21 . . . . . . . . . . . . . 208

MICON Number: 22 . . . . . . . . . . . . 209

MICON Number: $23 \ldots \ldots . . . \ldots 210$ 
WHC-SD-CP-CSWD-016

Volume 4

Revision 1

Page 2

\section{INTRODUCTION}

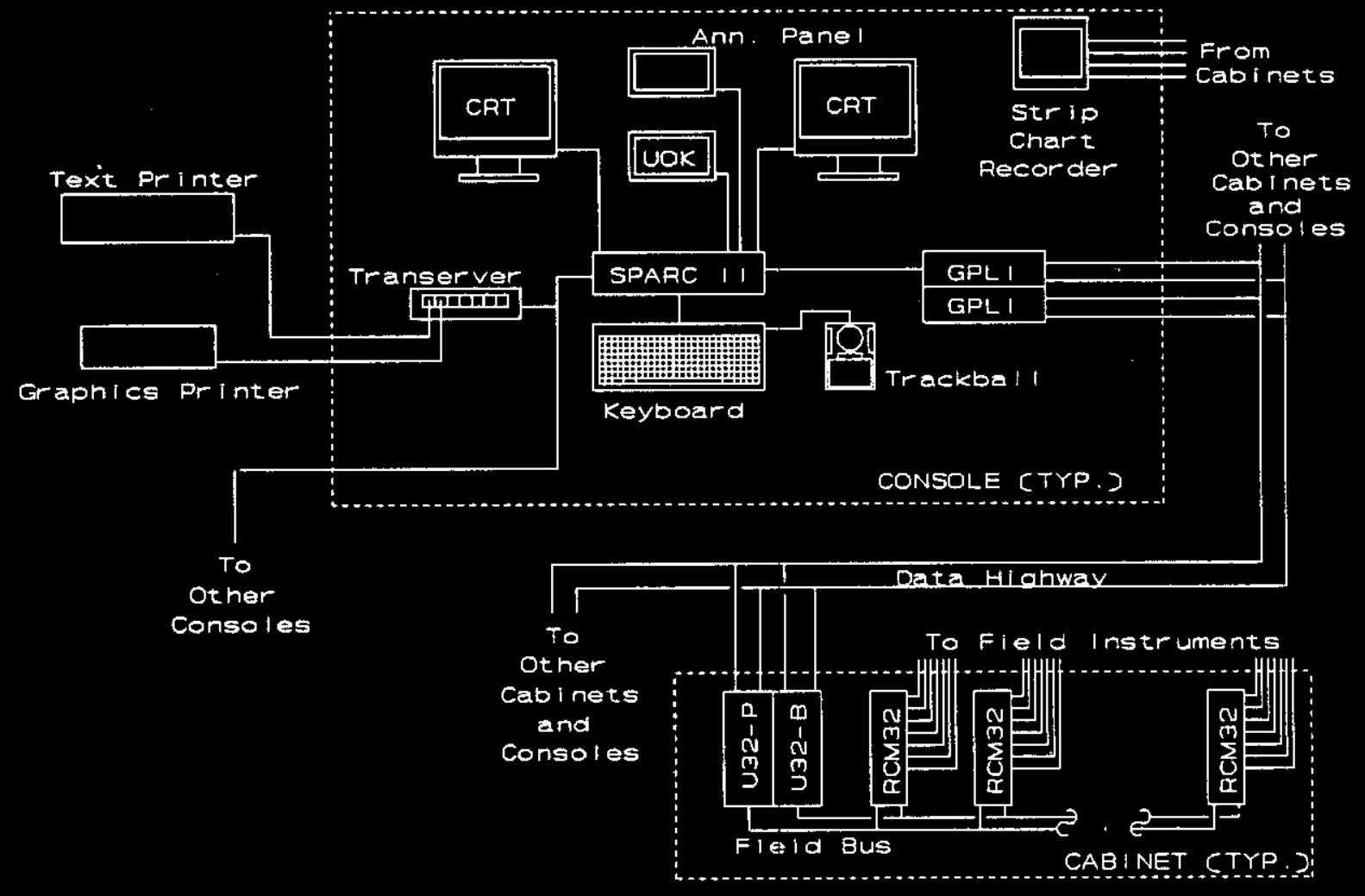

The MICON A/S distributed control system consists of four consoles or workstations (2 operator, 1 supervisor, and 1 engineer), a data highway, and eight process controller cabinets.

Each console is based on a Sun Microsystem's SPARC 20 workstation. The SPARC 20 workstation is based on a Scalable Process Architecture (SPARC) micro-processor. Each workstation contains 64 megabytes of memory, a one gigabyte hard drive, a 150 megabyte tape drive, a keyboard, and a trackball. Select SARC statations also contain an additional one gigabyte hard drive, a CD-ROM drive, and a 5 gigabyte tape drive. In addition, each SPARC 20

${ }^{1}$ For a list of Trademarks, see Volume 1, APPENDIX C 


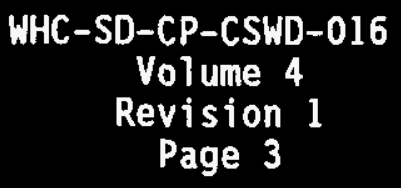

provides two video outputs, two RS-232 serial ports, and two ethernet ports (a thick-net and a thin-net). The video ports are connected to two high resolution color monitors. The RS-232 ports are connected to two Emerald model FPXT single board IBM XT compatible computers with touchscreen. One of the FPXTs is designated as an annunciator panel and is unused. The other is used as a Universal Operator Keyboard (UOK). One of the two ethernet ports -the thin-net - - is connected to the data highway via a MICON Company General Purpose LAN Interface (GPLI). The thicknet provides a Local Area Network (LAN) between the four consoles. It also connects each SPARC 20 with a Rockwe11/CMC TranServer. The TranServer provides eight additional RS-232 serial ports for each workstation. One of these serial ports is connected to an Output Technology Corporation 800 series text printer. Another port is connected to a Hewlett-Packard PaintJet color graphics printer.

The Data Highway is the main data link between the consoles and the process control cabinets. It consists of two redundant coax cables that run between all four consoles and six cabinets. The Data Highway is a local area network using a high speed token ring passing protocol. The Data Highway is also redundant. This means that if one of the two cables fail, communication is automatically passed to the other.

The process control cabinets consist of two redundant main controllers, called U-32s, and several process controllers called RCM-32s. The U-32s and RCM-32s communicate via a small local area network called a Field Bus. The U-32 is a MICON built controller based on the Motorola 68020 processor. Although it can be used to directly control instruments and devices in the field, its primary purpose is to pass information between the consoles and the RCM-32s Each U-32 has a redundant backup. That way if one fails, the other can automatically take over. The RCM-32s are also built by MICON and are based on a Motorola 68030 processor. These controllers provide input and output directly to field instruments and other devices.

There are four types of RCM-32s used in this system: A, C, D, and Dr. The A and $C$ cards are used primarily for analog signals. They provide hardware for any standard analog instrument input (4-20 mA, 10-50 mA, and DC voltages) and provide a standard 4-20 mA output signal. In addition, they provide two 28 Volt DC discrete (on/off) channels. These may be used for input, output, or both. An A card provides 26 analog inputs and 4 analog outputs. Each C card provides 18 analog inputs and 12 analog outputs. The $D$ card is used only for discrete contro1. It provides 32 on/off type channels that can be used as inputs, outputs, or both. Both inputs and outputs are 28 volt DC. The Dr cards are similar to the $D$ cards except inputs are 120 volt $A C$ or $D C$ and the outputs are 3 amp (120 VAC) dry contact relays.

Since MICON A/S systems are installed by engineers rather than computer programmers, they are designed to minimize the need for programming. All of the software needed to monitor and control a process is already programmed into the system be MICON Company. The engineer need only "configure" the system to a specific application. 


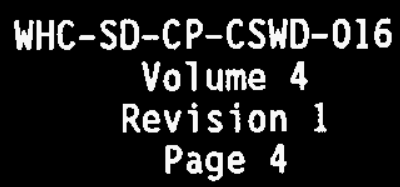

The process is very similar to the method used to install an older analog instrument system. First, a set of Pipe and instrument Diagrams (P\&ID) are created. These drawings show a schematic of the chemical process and the instrumentation to be installed to control it. With an analog system, the engineer would purchase the necessary individual components and wire them together to create the control strategy. With the MICON A/S system, the engineer would call function blocks to invoke preprogrammed modules and create the control strategy. The only difference between the two is that one uses hardware devices wired together, and the other uses software modules connected via a data base.

The MICON configuration is done in two parts. First the RCM-32 and, if needed the U-32 controllers are configured. Next, the workstation displays are created.

The RCM-32 and U-32 configuration consists of both the controller "program" and all display information for each "tag". Tags consists of all inputs and outputs from the controller, PID control loops, and any other displayed value. This information is requested by the MICON View software as the engineer creates the controller configuration.

Since all the display information (colors, ranges, etc.) was input during the controller configuration, the workstation configuration consists only of display creation. These are created by calling up a form and filling in the blanks. The only exception to this are the custom created graphics displays. Graphics are created by drawing a picture of the process and overlaying this with tag information. This is done with a special drawing program supplied with MICON View.

To keep size manageable, this document has been divided into several volumes. Volume 1 contains the display configuration for the four workstations. The other volumes each contain the configuration for one process Controller cabinet.

This volume (number 4 ) contains the configuration files for the cabinet located in 2736-ZB Room 602. This cabinet consists of six controllers:
1. U-32 (with backup)
2. $\mathrm{RCM}-32 \mathrm{C}$
3. $\mathrm{RCM}-32 \mathrm{~A}$
4. RCM-32 Dr
5. RCM-32 Dr
6. RCM-32 Dr
7. RCM-32 D
8. RCM-32 D
Controller Number: 15
Tagname: ZB MAIN
Controller Number: 16
Controller Number: 17
Tagname: $\mathrm{ZB}^{-1} 3$
Controller Number: 18
Tagname: $Z^{-}{ }^{-1} 4$
Controller Number: 19
Tagname: $\mathrm{ZB}^{-}{ }_{2}^{-} 1$
Tagname: $\mathrm{ZB}^{-}{ }^{-}-2$
Controller Number: 20
Controller Number: 21
Controller Number: 22
9. RCM-32 D
Controller Number: 23
Tagname: $\mathrm{ZB}_{2}-3$
Tagname: $\mathrm{ZB}^{-2}-4$
Tagname: $\mathrm{ZB}^{-2}-5$
Tagname: $\mathrm{ZB}_{-} 2-6$

ZB MAIN is being used for communications only and does not contain a configuration file. 


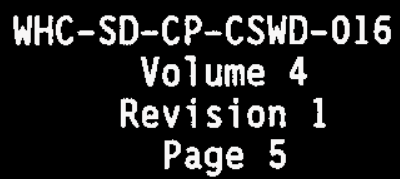

\section{MICON CONFIGURATION FILES}

The MICON configuration file is a text file that the MICON View software creates as the user configures or "programs" each MICON controller. A MICON controller is configured by selecting preprogrammed functions and filling in the required information. As this is done, MICON View places this information into the configuration file. This file contains both display and program information. For a detailed description see $U-32$ and $R C H-32$ Controller/RTU Function Description published by MICON Company (Vendor Information file 22068).

This file is named by MICON View based on input from the user. The file name's format is TAG.file where TAG is the controllers tag name and file is the program file name. The first eight lines of the file are the information on the controller itself. These lines are:

MICON TYPE - The type of MICON being programmed (A, $C$, or $D)$. In this case, D and Dr cards are considered the same.

MICON UNIT NO - The controller card's number (user assigned).

MICON TAG - The controller card's tag name (user assigned). For consistency, we have assigned tags in the following format:

$$
\text { CABINET_RACK_SLOT }
$$

CABINET is the MICON controller cabinet location. RACK is the rack number ( 1 to 3 ). Rack 1 is at the top of the cabinet. SLOT is the slot number ( 1 to 6$)$. Slot 1 is at the far left of the rack. Therefor, a controller tagged WTF_1 3 is the card in rack 1 slot 3 of the cabinet located in the Low Lével Waste Treatment Facility $(243-Z)$.

MICON DESCRIPTION - A brief description of the controller card

MICON CONFIGURATION FILE - The name of the file that contains the controller card's program (user assigned). 
WHC-SD-CP-CSWD-016

Volume 4

Revision 1

Page 6

POINT ALLOCATION - The memory allocation for the controller. Memory allocation is based on the types of inputs and outputs $(I / 0)$ and the number of storage locations desired. The types of $I / 0$ and storage locations are:
VAI - Virtual Analog (User assigned). An analog value storage location.
VDI - Virtual Discrete (User assigned). A Discrete (on/off) storage location with alarm capability.
ISW - Internal Switch (User assigned). A Discrete storage location without alarm capability.
LOOPS - Loops (User Assigned). Loops are used to hold a series of program steps. If one of those steps is a PID controller function, The loop tag name becomes the controller tag name.
MSD - Multi-State Discrete (User assigned). This is a special sixteen position indicator.
AI - Analog Inputs (Assigned based on card type used).
AO - Analog Outputs (Assigned based on card type used).
DI - Discrete Inputs (Assigned based on card type used).
DO - Discrete Outputs (Assigned based on card type used).

The remaining information is the actual controller configuration. Right at first, you will notice several blocks of information titled VIRTUAL ANALOG and DISCRETE TAG. These blocks are the configuration information for the virtual analog and internal switch type storage locations. These must always be configured first. After that are the controller loops. Each loop starts with a block of information titled LOOP NO followed by several steps. The last step is always a function 0 (END). Everything except virtual analogs and internal switches are configured inside of a loop.

Loops are a way of organizing the controller configuration. Although most controllers could be programmed using only one loop, using several loops makes the program more understandable. Loops are generally invisible to the operator. The exception to this rule is when a PID function (function 20) is used in the loop. When this happens, the loop name becomes the controller name. Otherwise, the loop name is used as an internal reference only

NOTE: Each loop using a PID function has two lines showing the tuning paramaters -- for example:

TUNING PARAMETER :

P(GAIN) : $1.00, \mathrm{I}$ (REP/MIN) : 5.00, D(RATE) : 0.00, MANUAL RESET : 0.00

These tuning parameters are not controlled by this document. Therefore, these numbers may be different than the ones that appear in the workstation listings. 
ZB_1_3.mic16_ro

MICON TYPE :RCM-C

MICON UNIT NO : 16

MICON TAG : ZB_1 3

MICON DESCRIPTION $\div$ 2736-ZB RACK 1 SLOT 3

MICON CONFIGURATION FILE : micl6_ro

POINT ALLOCATION :

VAI : 32, VDI : 32, ISH : 32, LOOPS : 12, MSD : 1 ,

$\mathrm{AI}: 18, \mathrm{AO}: 12, \mathrm{DI}: 2, \mathrm{DO}: 2$

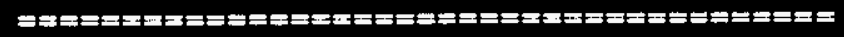

VIRTUAL ANALOG : PDRC_1

INPUT \# : 1

INPUT DESC : ADM AREA PRESS ZN IV

INPUT UNIT : IN WG

INPUT SYNC SCAN : 1 SEC HIST SCAN : 30 SEC

INPUT HIST. DEFINITION : 1

INPUT PLOT LO : 0.00 PLOT HI : 0.15 BAR LO : 0.00 BAR HI : 0.15

VIRTUAL ANALOG : PDRC_2

INPUT \# : 2

INPUT DESC : PRCS AREA PRESS ZN II

INPUT UNIT : -IN WG

INPUT SYNC SCAN : 1 SEC HIST SCAN : 30 SEC

INPUT HIST. DEFINITION : 1

INPUT PLOT LO : 0.00 PLOT HI : 0.50 BAR LO : 0.00 BAR HI $: \quad 0.50$

VIRTUAL ANALOG : PDRC_3

INPUT \# : 3

INPUT DESC : NDA AREA PRESS ZN II

INPUT UNIT : -IN WG

INPUT SYNC SCAN : 1 SEC HIST SCAN : 30 SEC

INPUT HIST. DEFINITION : 1

INPUT PLOT LO: 0.00 PLOT HI : 0.50 BAR LO : 0.00 BAR HI : 0.50

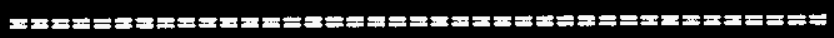

VIRTUAL ANALOG : PDRC_4

INPUT \#: 4

INPUT DESC : GBX PRESS ZN I

INPUT UNIT : IN WG

INPUT SYNC SCAN : 1 SEC HIST SCAN : 30 SEC

INPUT HIST. DEFINITION : 1

INPUT PLOT LO : $\quad 0.00$ PLOT HI : 2.00 BAR LO : 0.00 BAR HI $: \quad 2.00$ 
WHC-SD-CP-CSWD-016

Volume 4

Revision 1

Page 8

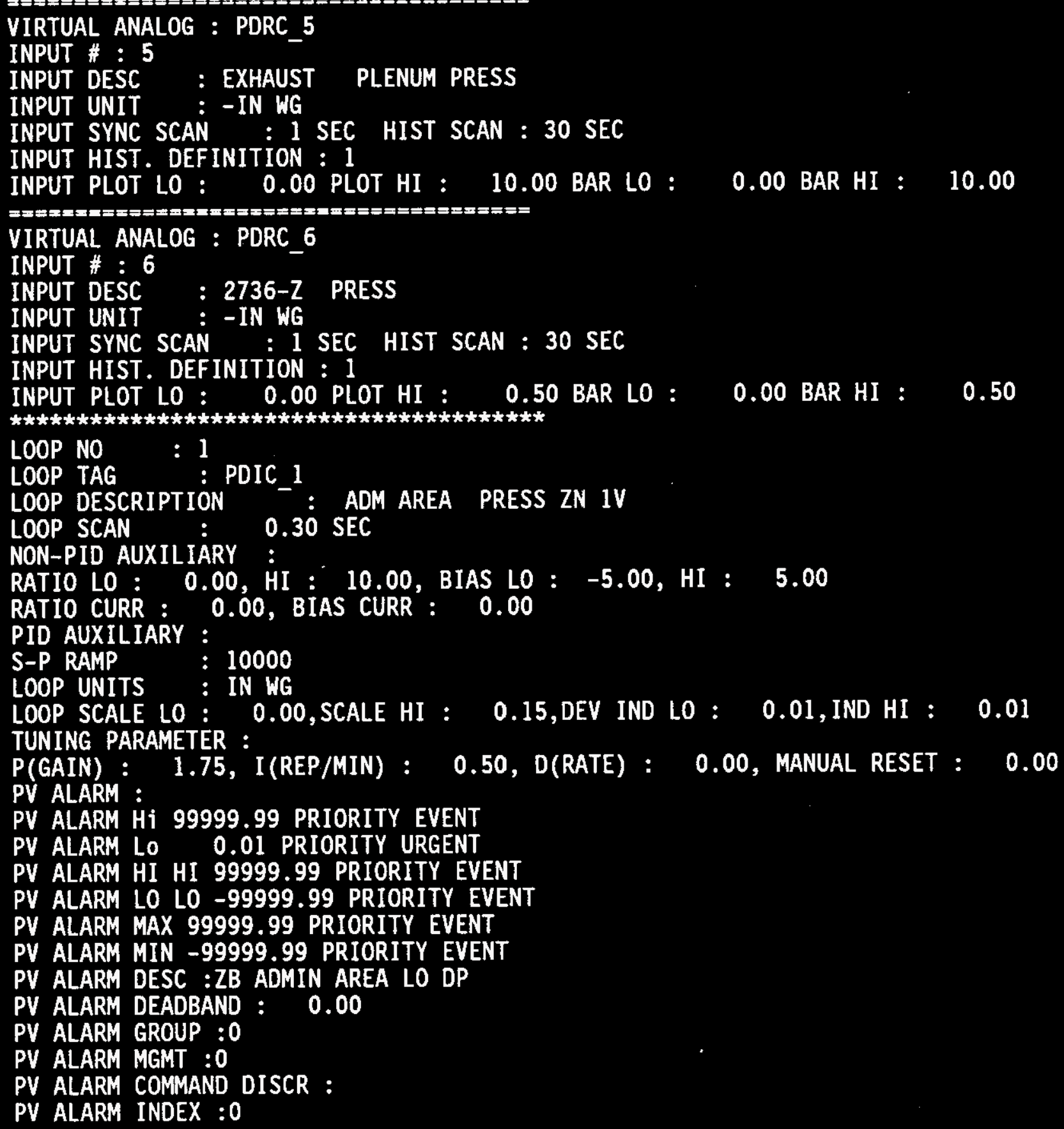


\#\#\#\#\#\#\#\#\#\#\#\#\#\#\#\#\#\#\#\#\#\#\#\#\#\#\#\#\#\#\#\#\#\#\#\#\#\#\#\#

STEP NO : 1

LABEL :

FUNCTION : 11 (AI )

COMMENT :

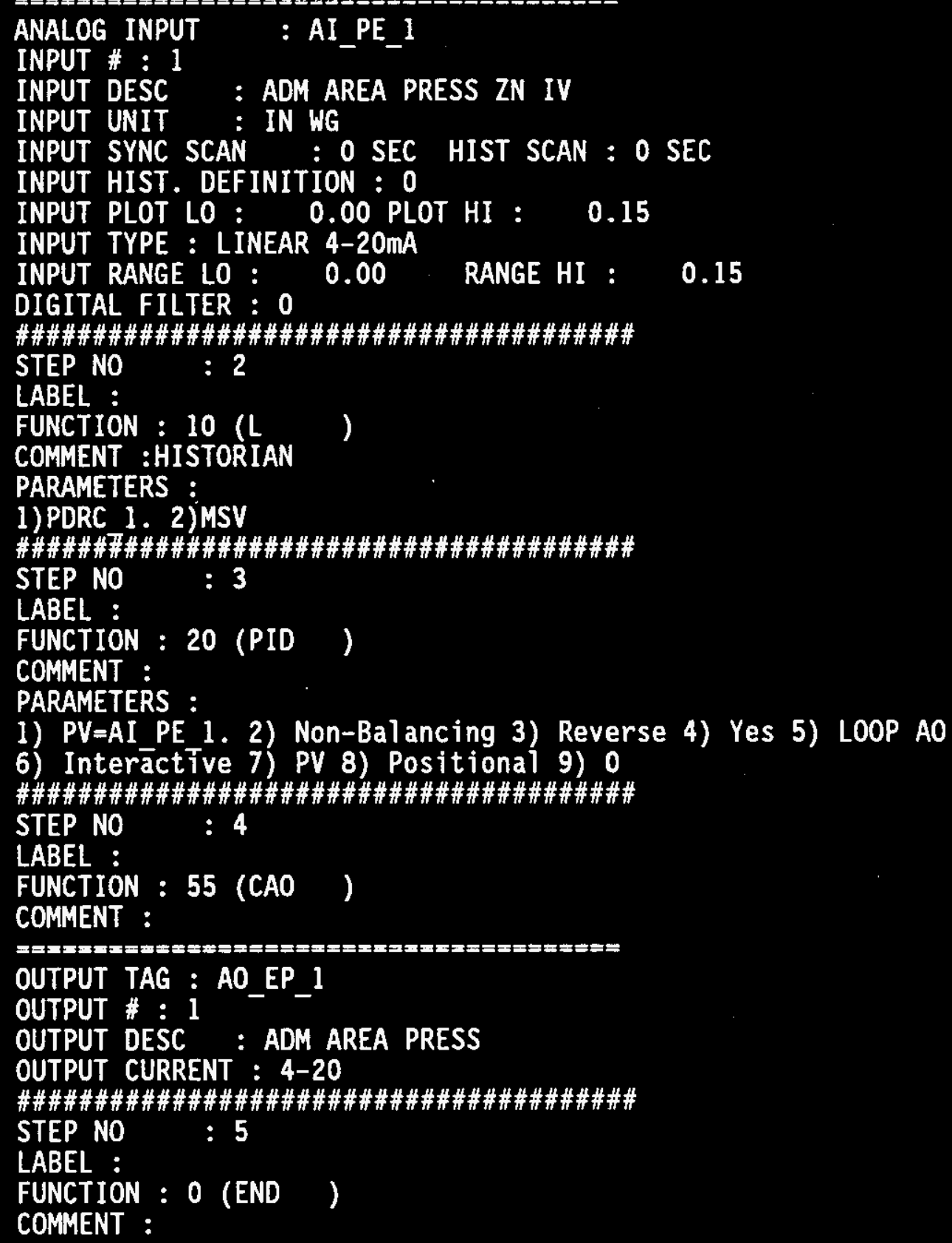




\section{WHC-SD-CP-CSWD-016 \\ Volume 4 \\ Revision 1 \\ Page 10}

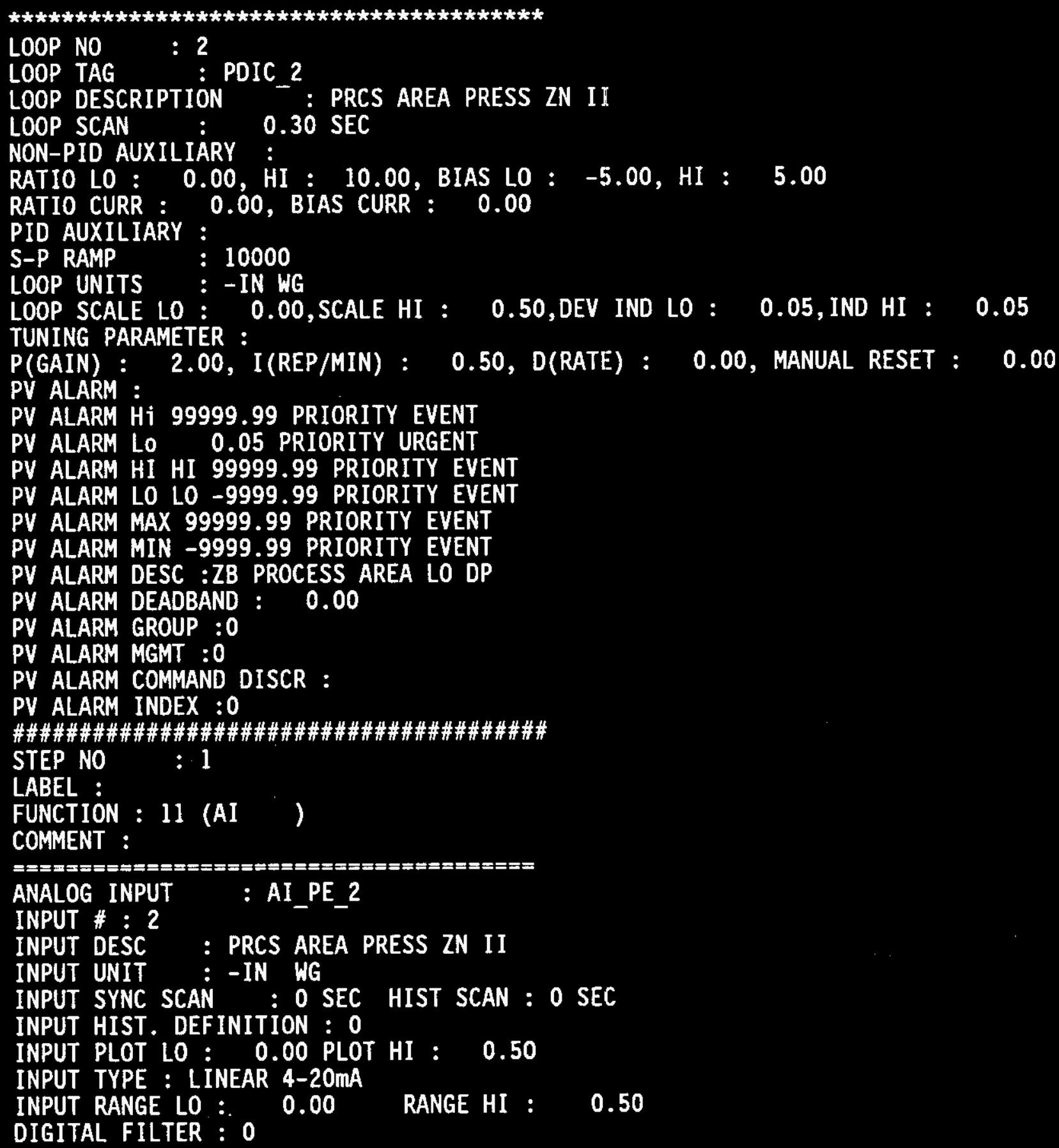




\section{WHC-SD-CP-CSWD-016 \\ Volume 4 \\ Revision 1 \\ Page 11}

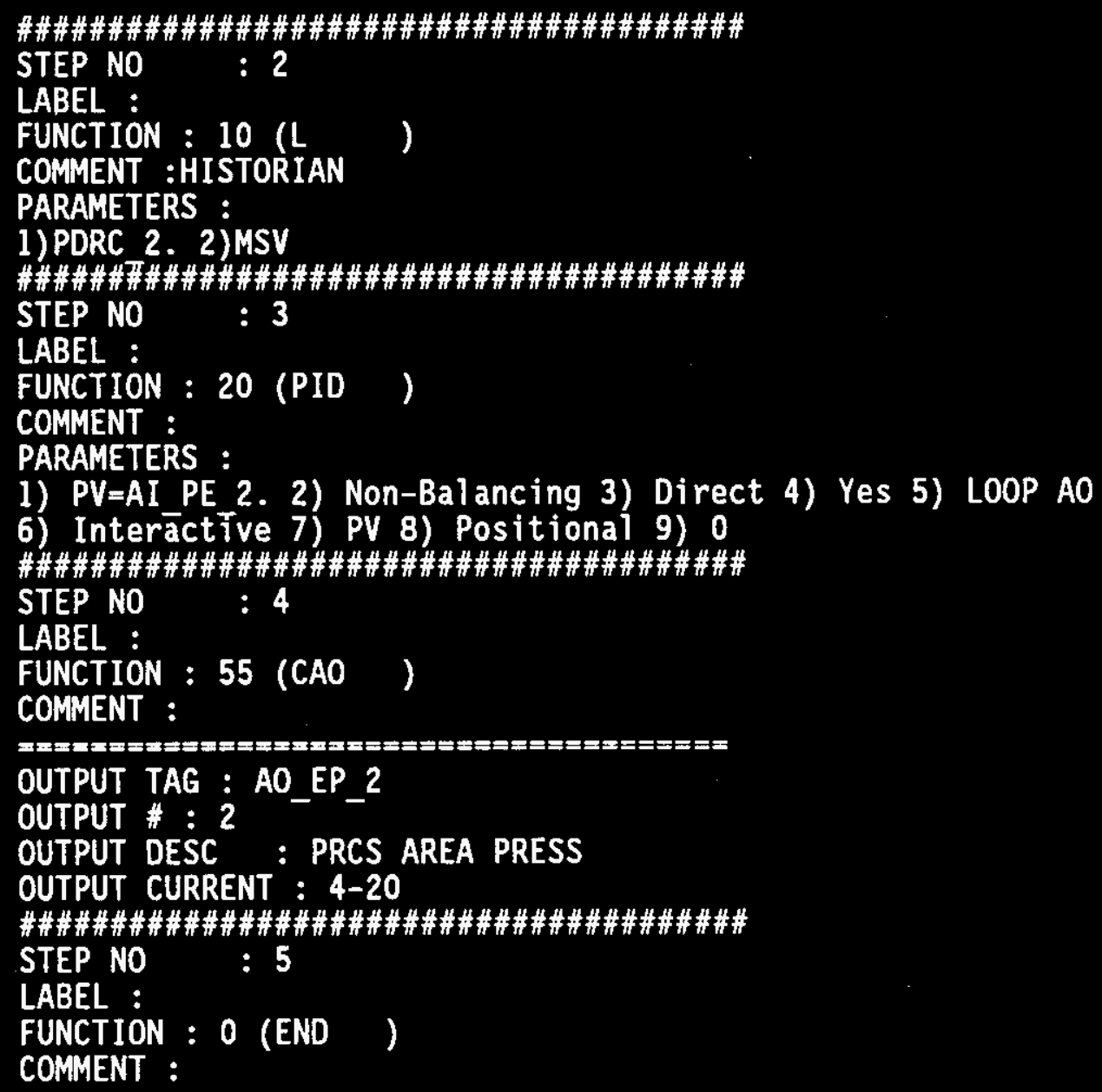


WHC-SD-CP-CSWD-016

Volume 4

Revision 1

Page 12

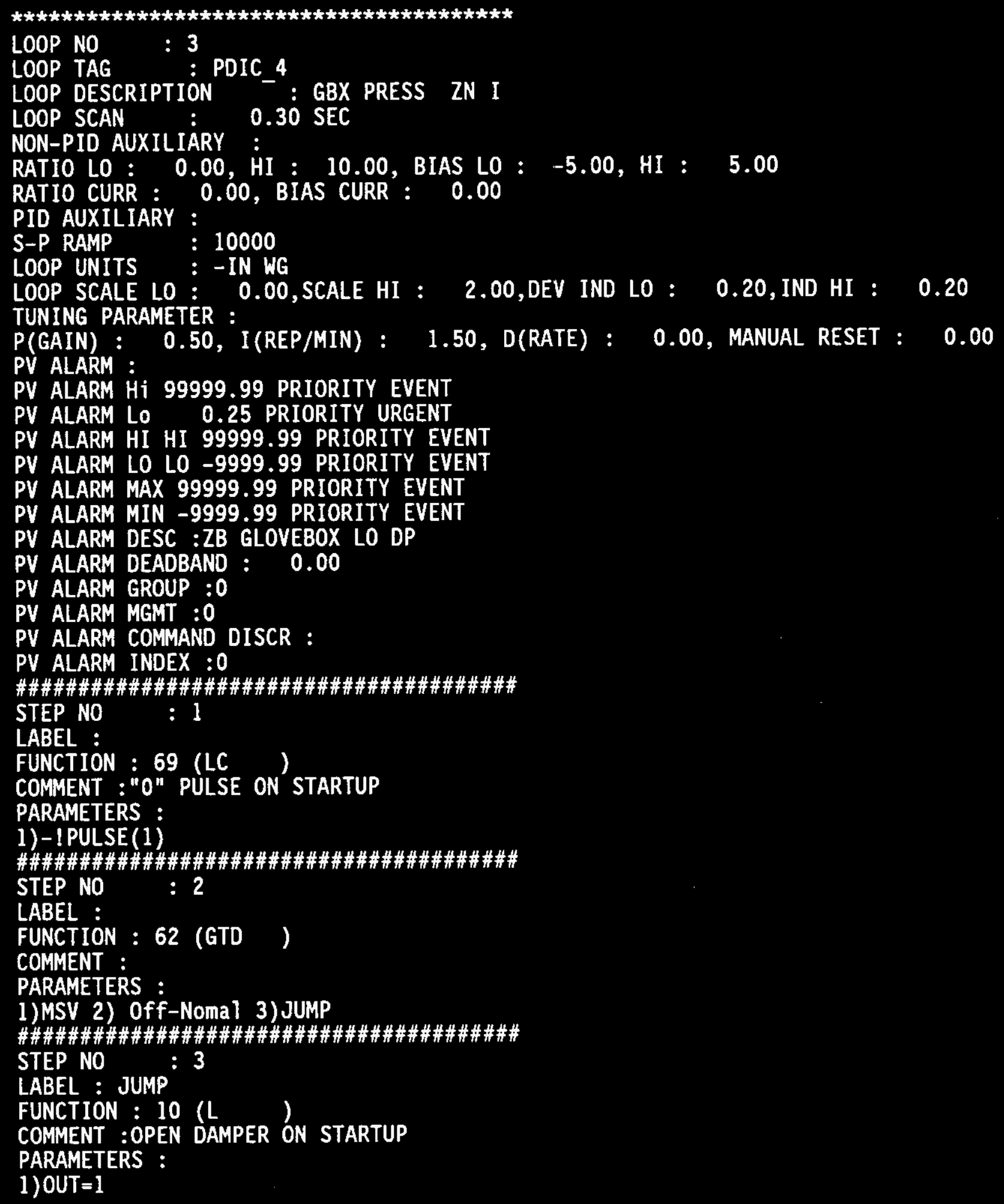


\#\#\#\#\#\#\#\#\#\#\#\#\#\#\#\#\#\#\#\#\#\#\#\#\#\#\#\#\#\#\#\#\#\#\#\#\#\#\#\#

STEP NO : 4

LABEL :

FUNCTION : 11 (AI )

COMMENT :

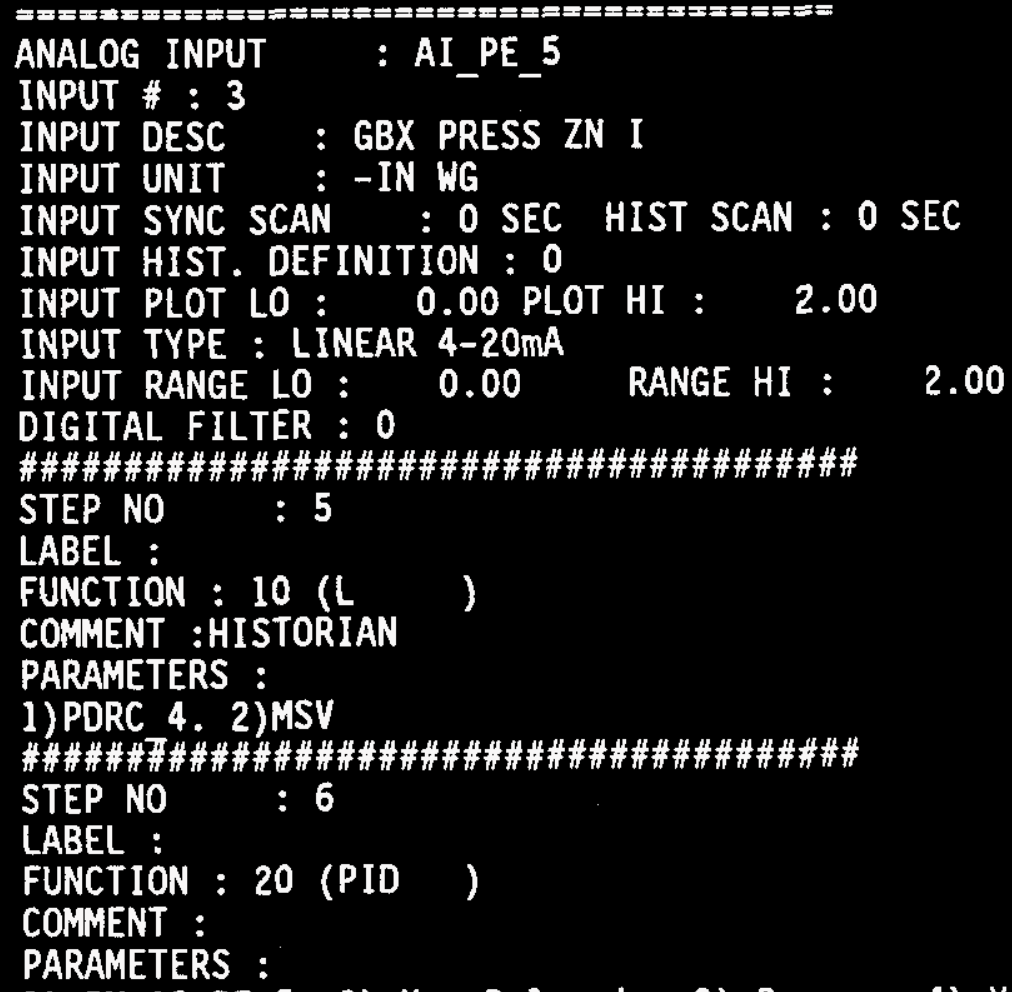


WHC-SD-CP-CSWD-016

Volume 4

Revision 1

Page 14

\#\#\#\#\#\#\#\#\#\#\#\#\#\#\#\#\#\#\#\#\#\#\#\#\#\#\#\#\#\#\#\#\#\#\#\#\#\#\#\#

STEP NO : 8

LABEL :

FUNCTION : 0 (END)

COMMENT :

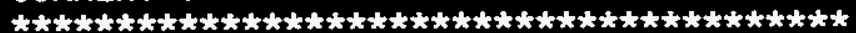

LOOP NO : 4

LOOP TAG : PDIC_5

LOOP DESCRIPTION - : EXH PRESS

LOOP SCAN : 0.30 SEC

NON-PID AUXILIARY :

RATIO LO : 0.00 , HI : 10.00 , BIAS LO : -5.00 , HI : 5.00

RATIO CURR : 0.00 , BIAS CURR : 0.00

PID AUXILIARY :

S-P RAMP : 10000

LOOP UNITS : -IN WG

LOOP SCALE LO : 0.00, SCALE HI : 10.00 , DEV IND L0 : 1.00 , IND HI : 1.00

TUNING PARAMETER :

P(GAIN) : 2.00, I (REP/MIN) : 0.75, D(RATE) : 0.00 , MANUAL RESET : 0.00

PV ALARM :

PV ALARM Hi 99999.99 PRIORITY EVENT

PV ALARM LO 1.00 PRIORITY URGENT

PV ALARM HI HI 99999.99 PRIORITY EVENT

PV ALARM LO LO -9999.99 PRIORITY EVENT

PV ALARM MAX 99999.99 PRIORITY EVENT

PV ALARM MIN - 9999.99 PRIORITY EVENT

PV ALARM DESC :ZB EXHAUST PLENUM LO DP

PV ALARM DEADBAND : 0.00

PV ALARM GROUP :0

PV ALARM MGMT :0

PV ALARM COMMAND DISCR :

PV ALARM INDEX :0

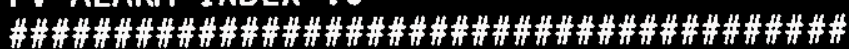

STEP NO : 1

LABEL :

FUNCTION : 11 (AI)

COMMENT :

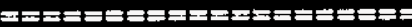

ANALOG INPUT

: AI_PE_3

INPUT \#: 4

INPUT DESC : EXH PRESS

INPUT UNIT : -IN WG

INPUT SYNC SCAN : 0 SEC HIST SCAN : O SEC

INPUT HIST. DEFINITION : 0

INPUT PLOT LO : 0.00 PLOT HI : 10.00

INPUT TYPE : LINEAR 4-20mA

INPUT RANGE LO :

0.00

RANGE HI : $\quad 10.00$

DIGITAL FILTER : 0 

WHC-SD-CP-CSWD-016 Volume 4 Revision 1 Page 15

\#\#\#\#\#\#\#\#\#\#\#\#\#\#\#\#\#\#\#\#\#\#\#\#\#\#\#\#\#\#\#\#\#\#\#\#\#\#\#\#

STEP NO : 2

LABEL :

FUNCTION : $10(\mathrm{~L} \quad)$

COMMENT :HISTORIAN

PARAMETERS :

1)PDRC 5. 2) MSV

\#\#\#\#\#\#而\#\#\#\#\#\#\#\#\#\#\#\#\#\#\#\#\#\#\#\#\#\#\#\#\#\#\#\#\#\#\#\#\#

STEP NO : 3

LABEL :

FUNCTION : 20 (PID )

COMMENT :

PARAMETERS :

1) $P V=A I P E$ 3. 2) Non-Balancing 3) Reverse 4) Yes 5) LOOP AO

6) Interāctīve 7) PV 8) Positional 9) 0

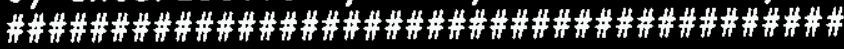

STEP NO

$: 4$

LABEL :

FUNCTION : 55 (CAO)

COMMENT :

OUTPUT TAG : AO EP 5

OUTPUT \# : 4

OUTPUT DESC : EXHAUST PLENUM PRESS

OUTPUT CURRENT : 4-20

\#\#\#\#\#\#\#\#\#\#\#\#\#\#\#\#\#\#\#\#\#\#\#\#\#\#\#\#\#\#\#\#\#\#\#\#\#\#\#

STEP NO : 5

LABEL :

FUNCTION : 0 (END )

COMMENT : 
WHC-SD-CP-CSWD-016

Volume 4

Revision 1

Page 16

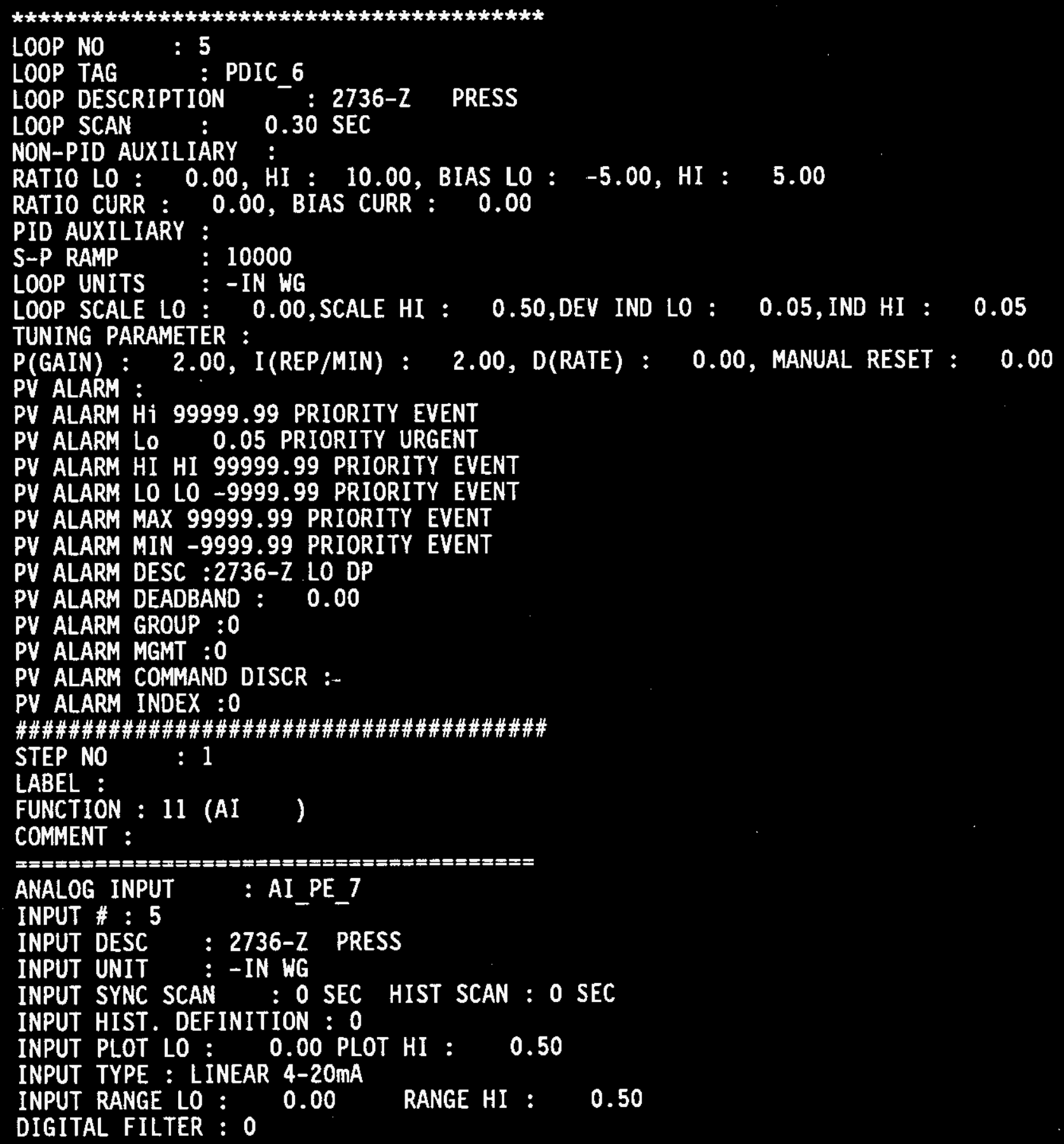


WHC-SD-CP-CSWD-016

Volume 4

Revision 1

Page 17

\#\#\#\#\#\#\#\#\#\#\#\#\#\#\#\#\#\#\#\#\#\#\#\#\#\#\#\#\#\#\#\#\#\#\#\#\#\#\#\#

STEP NO : 2

LABEL :

FUNCTION : 10 (L )

COMMENT :HISTORIAN

PARAMETERS :

1) PDRC 6. 2) MSV

\#\#\#\#\#\#界\#\#\#\#\#\#\#\#\#\#\#\#\#\#\#\#\#\#\#\#\#\#\#\#\#\#\#\#\#\#\#\#

STEP NO : 3

LABEL :

FUNCTION : 20 (PID)

COMMENT :

PARAMETERS :

1) PV=AI PE 7. 2) Non-Balancing 3) Direct 4) Yes 5) LOOP AO

6) Interactive 7) PV 8) Positional 9) 0

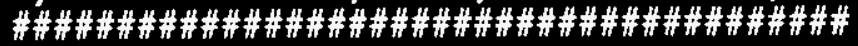

STEP NO : 4

LABEL :

FUNCTION : 55 (CAO )

COMMENT :

OUTPUT TAG : AO_EP 6

OUTPUT \# : 5

OUTPUT DESC : 2736-Z PRESS

OUTPUT CURRENT : $4-20$

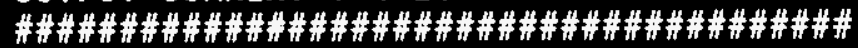

STEP NO : 5

LABEL :

FUNCTION : 0 (END)

COMMENT : 
WHC-SD-CP-CSWD-016

Volume 4

Revision 1

Page 18

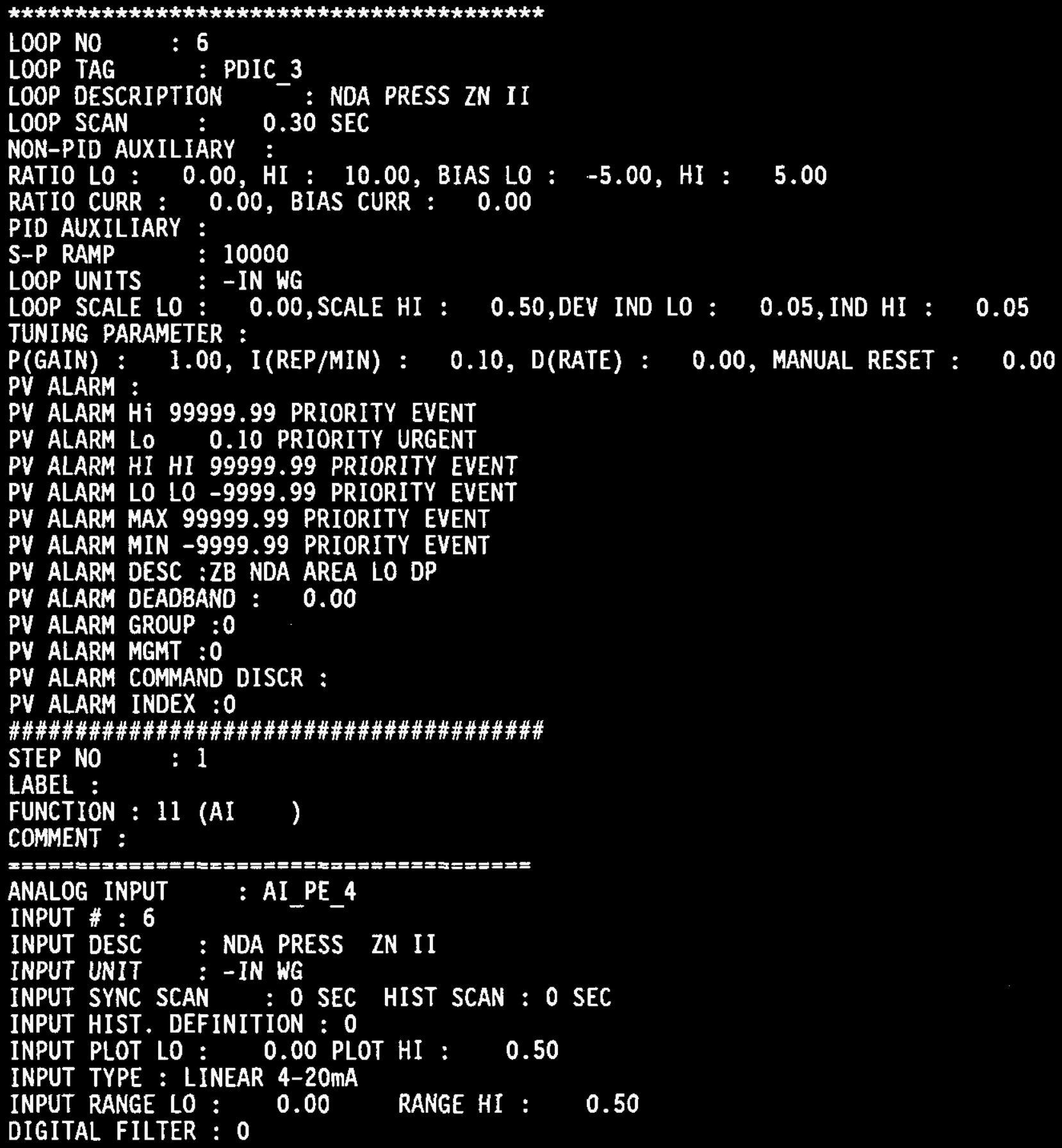


WHC-SD-CP-CSWD-016

Volume 4

Revision 1

Page 19

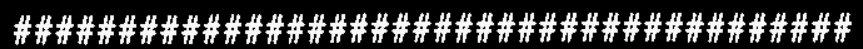

STEP NO : 2

LABEL :

FUNCTION : 10 (L )

COMMENT :HISTORIAN

PARAMETERS :

1) PDRC 3. 2) MSV

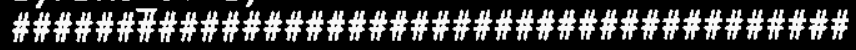

STEP NO : 3

LABEL :

FUNCTION : 20 (PID )

COMMENT :

PARAMETERS :

1) $P V=A I P E$ 4. 2) Non-Balancing 3) Direct 4) Yes 5) LOOP AO

6) Interāctioue 7) PV 8) Positional 9) 0

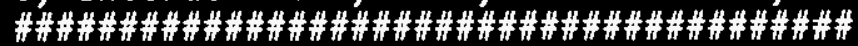

STEP NO : 4

LABEL :

FUNCTION : 55 (CAO)

COMMENT :

OUTPUT TAG : AO_EP_3

OUTPUT \#: 6

OUTPUT DESC : NDA PRESS ZN II

OUTPUT CURRENT : 4-20

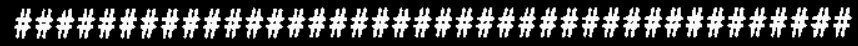

STEP NO : 5

LABEL :

FUNCTION : 0 (END )

COMMENT : 
WHC-SD-CP-CSWD-016

Volume 4

Revision 1

Page 20

ZB_1_4.micl7_ro

MICON TYPE :RCM-A

MICON UNIT NO : 17

MICON TAG : ZB_1 4

MICON DESCRIPTION $\overline{2}$-736-ZB RACK 1 SLOT 4

MICON CONFIGURATION FILE : mic17_ro

POINT ALLOCATION :

VAI : 32, VDI : 32, ISW : 32, LOOPS : 6, MSD : 1 ,

$A I: 26, A O: 4, D I: 2, D 0: 2$

VIRTUAL ANALOG : II_1_1

INPUT \# : 1

INPUT DESC : EF-1-1

INPUT UNIT : AMPS

INPUT SYNC SCAN : 1 SEC HIST SCAN : 30 SEC

INPUT HIST. DEFINITION : 1

INPUT PLOT LO : $\quad 0.00$ PLOT HI : 50.00 BAR LO : 0.00 BAR HI : 50.00

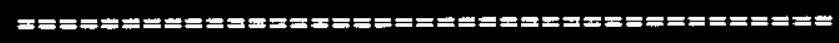

VIRTUAL ANALOG : II_1_2

INPUT \# : 2

INPUT DESC : EF-1-2

INPUT UNIT : AMPS

INPUT SYNC SCAN : 1 SEC HIST SCAN : 30 SEC

INPUT HIST. DEFINITION : 1

INPUT PLOT LO : 0.00 PLOT HI : 50.00 BAR LO : 0.00 BAR HI : 50.00

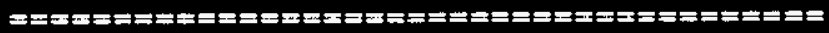

VIRTUAL ANALOG : II AC 1

INPUT \# : 3

INPUT DESC : ZB AC-1

INPUT UNIT : AMPS

INPUT SYNC SCAN : 1 SEC HIST SCAN : 30 SEC

INPUT HIST. DEFINITION : 1

INPUT PLOT LO: $\quad 0.00$ PLOT HI : 40.00 BAR LO : 0.00 BAR HI : 40.00

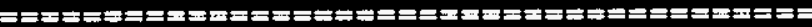

VIRTUAL ANALOG : II_AC_2

INPUT \# : 4

INPUT DESC : ZB AC-2

INPUT UNIT : AMPS

INPUT SYNC SCAN : I SEC HIST SCAN : 30 SEC

INPUT HIST. DEFINITION : 1

INPUT PLOT LO : 0.00 PLOT HI : 40.00 BAR LO : 0.00 BAR HI : 40.00 
WHC-SD-CP-CSWD-016

Volume 4

Revision 1

Page 21

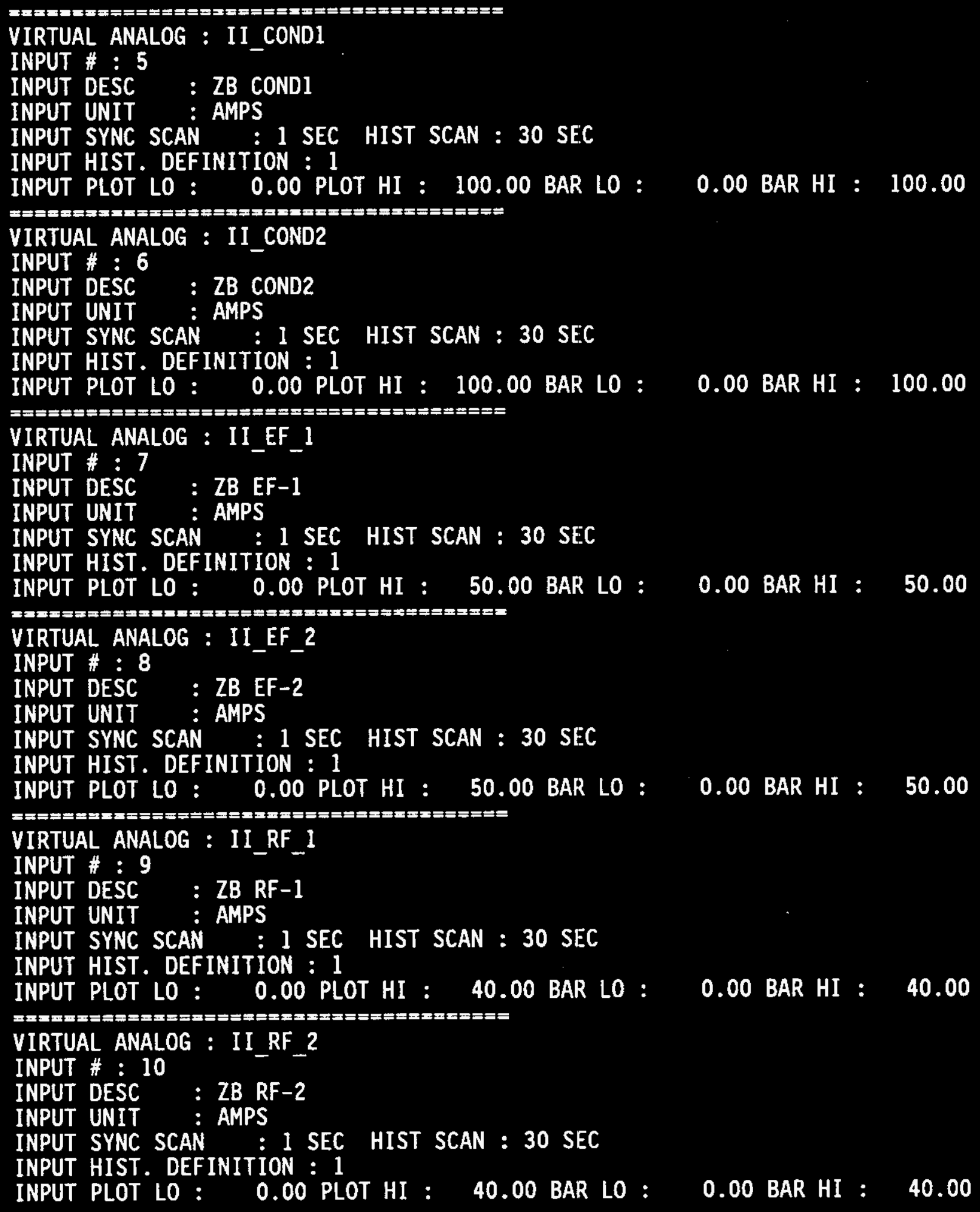


WHC-SD-CP-CSWD-016

Volume 4

Revision 1

Page 22

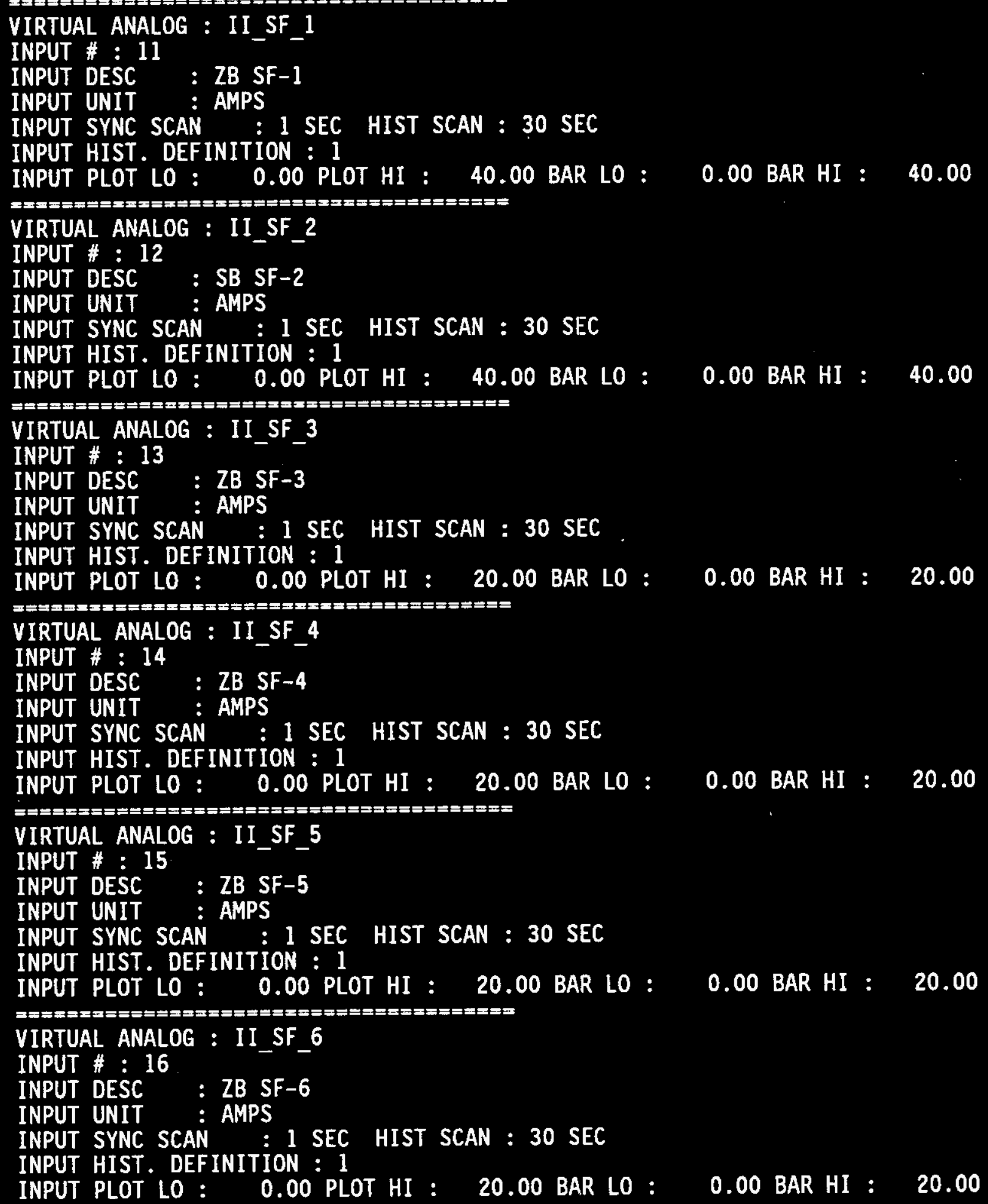


WHC-SD-CP-CSWD-016

Volume 4

Revision 1

Page 23

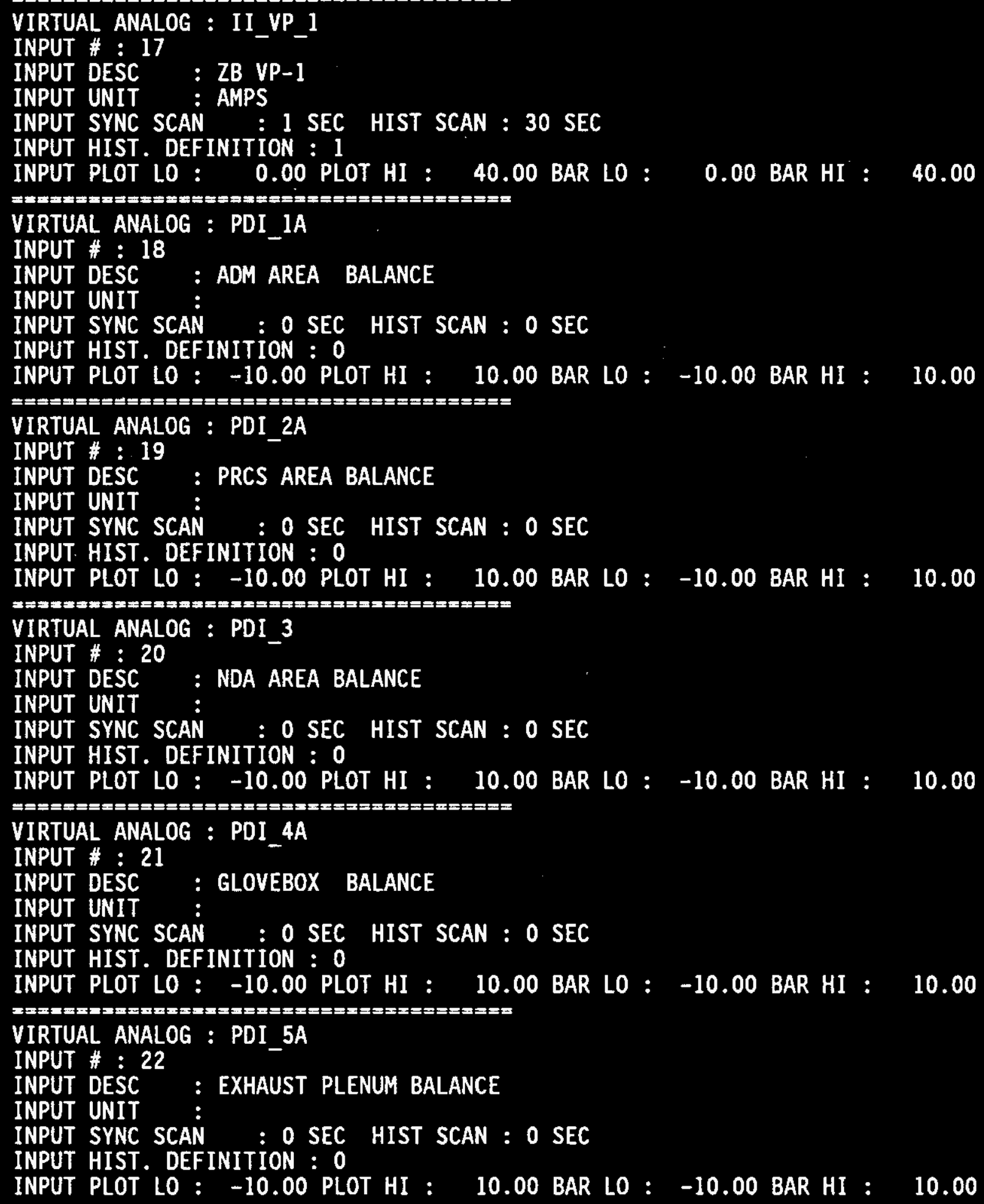


WHC-SD-CP-CSWD-016

Volume 4

Revision 1

Page 24

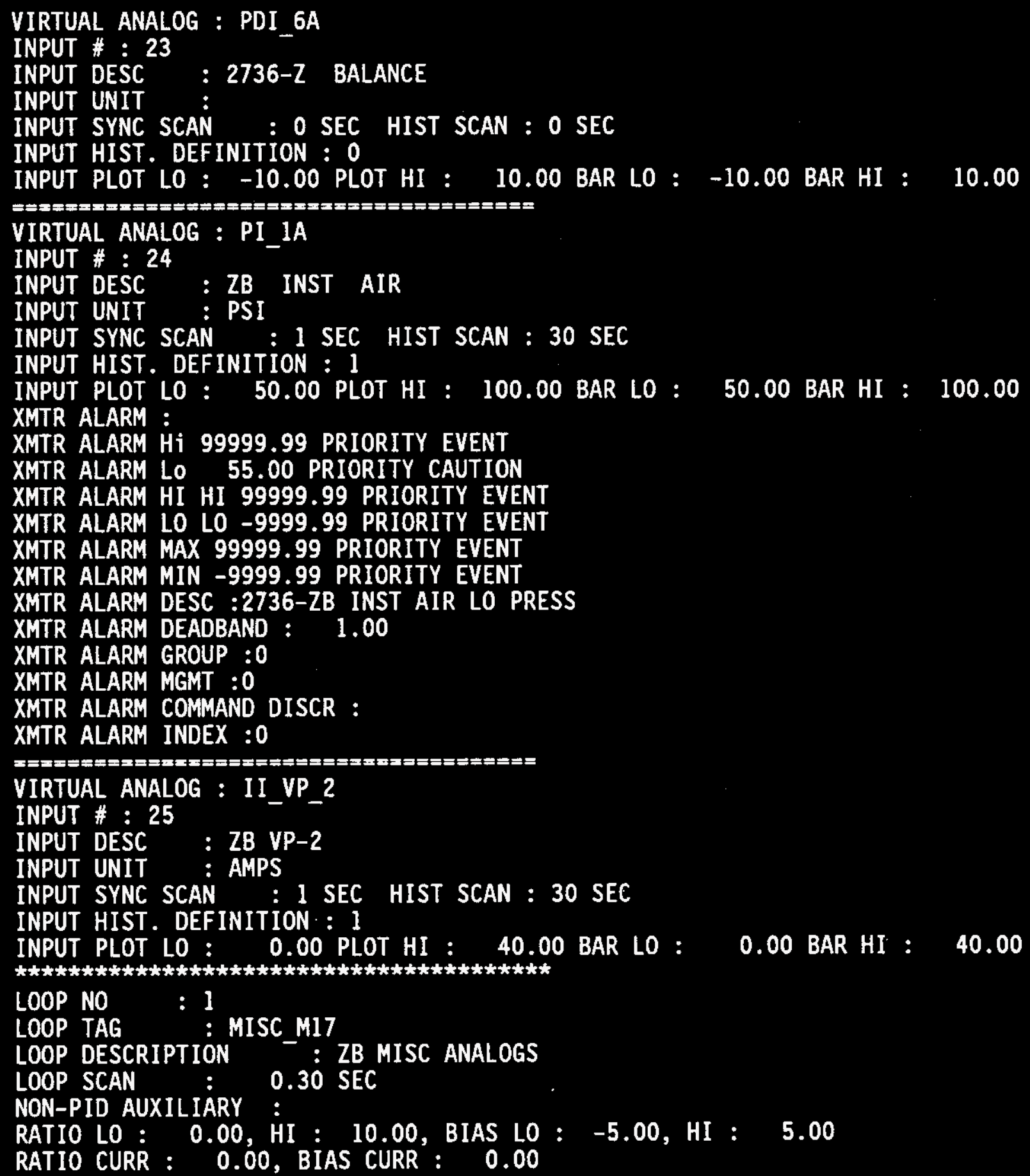


\#\#\#\#\#\#\#\#\#\#\#\#\#\#\#\#\#\#\#\#\#\#\#\#\#\#\#\#\#\#\#\#\#\#\#\#\#\#\#\#

STEP NO : 1

LABEL :

FUNCTION : 11 (AI)

COMMENT :

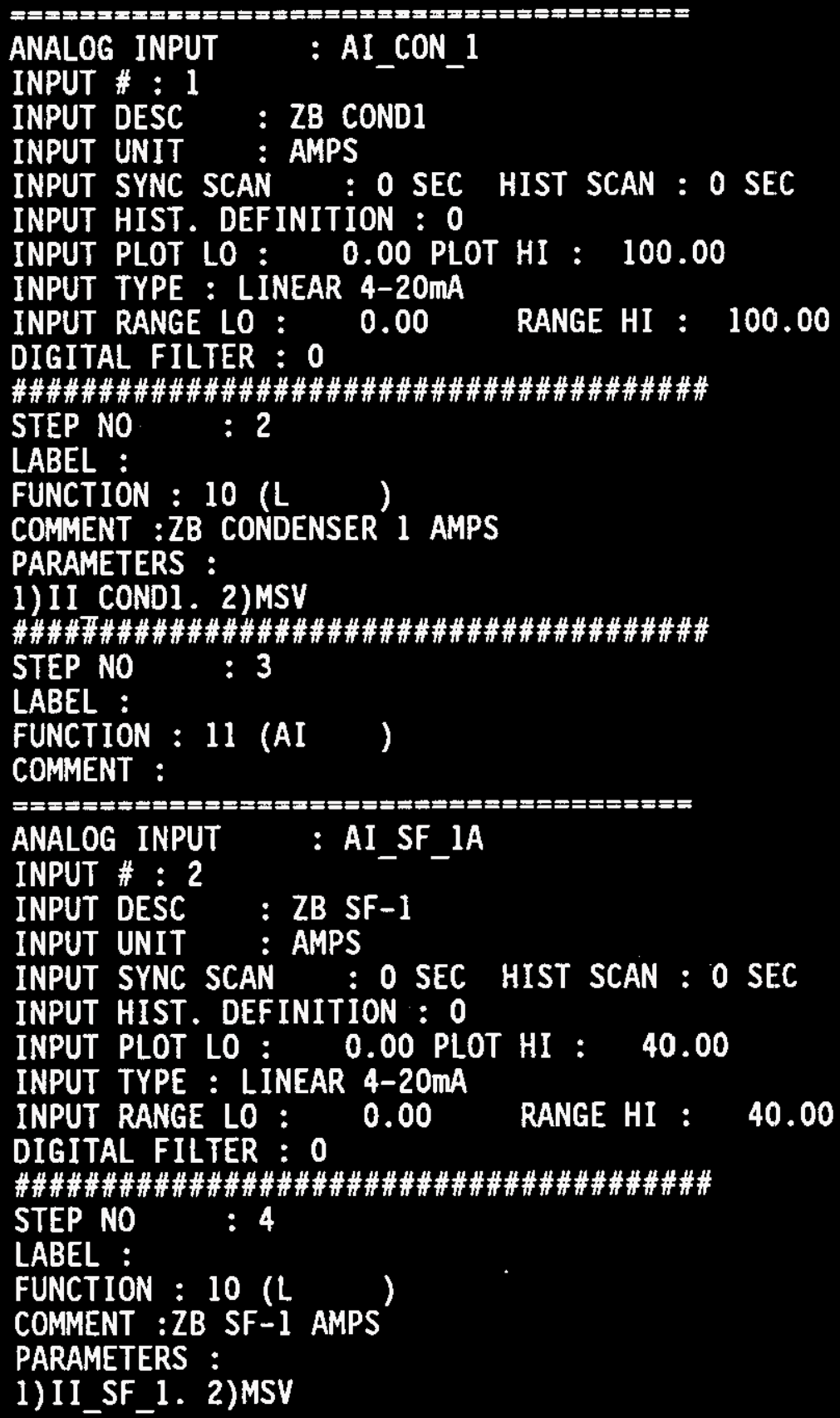


WHC-SD-CP-CSWD-016

Volume 4

Revision 1

Page 26

\#\#\#\#\#\#\#\#\#\#\#\#\#\#\#\#\#\#\#\#\#\#\#\#\#\#\#\#\#\#\#\#\#\#\#\#\#\#\#\#

STEP NO : 5

LABEL :

FUNCTION : 11 (AI)

COMMENT :

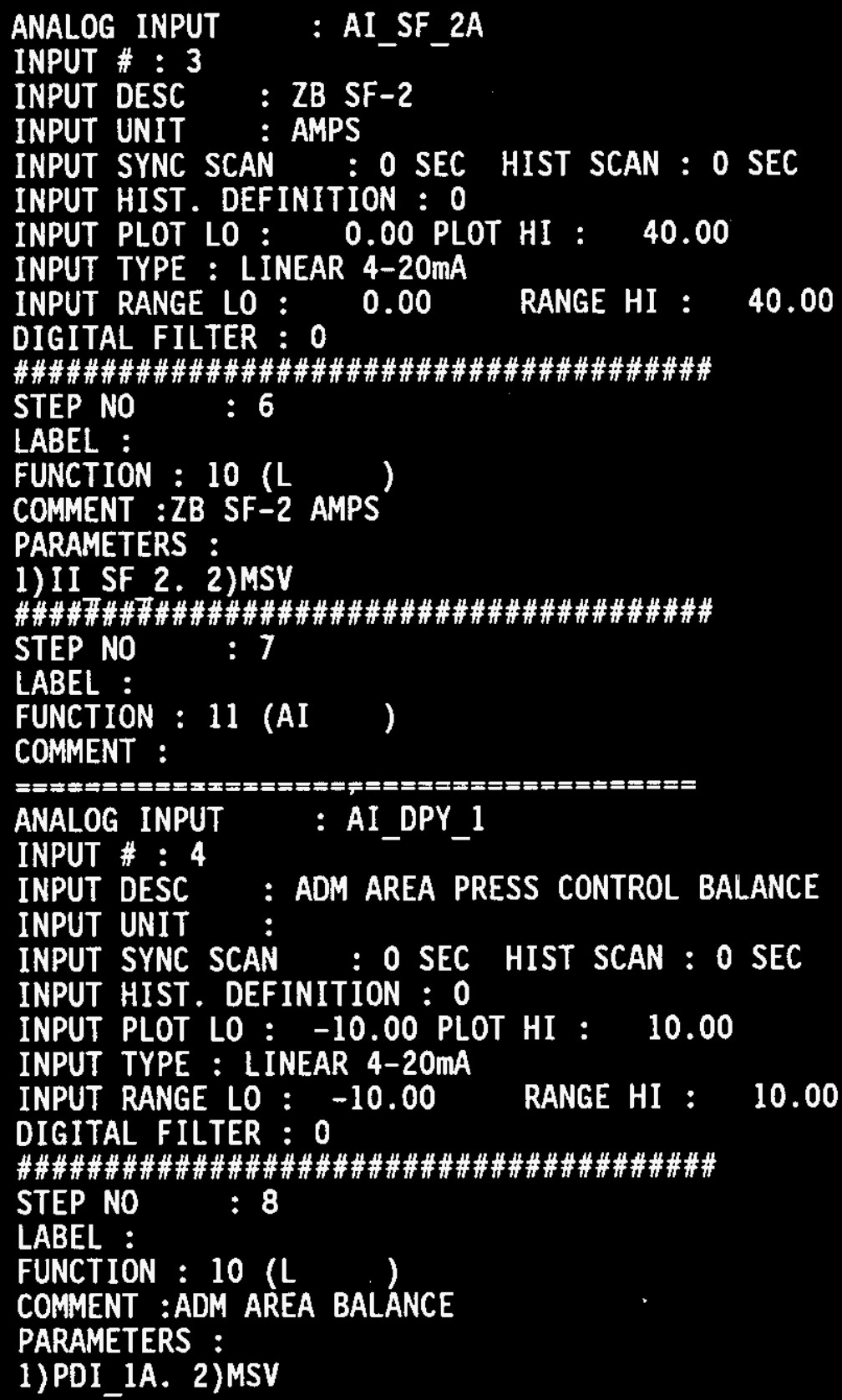




\section{WHC-SD-CP-CSWD-016 \\ Vol ume 4 \\ Revision 1 \\ Page 27}

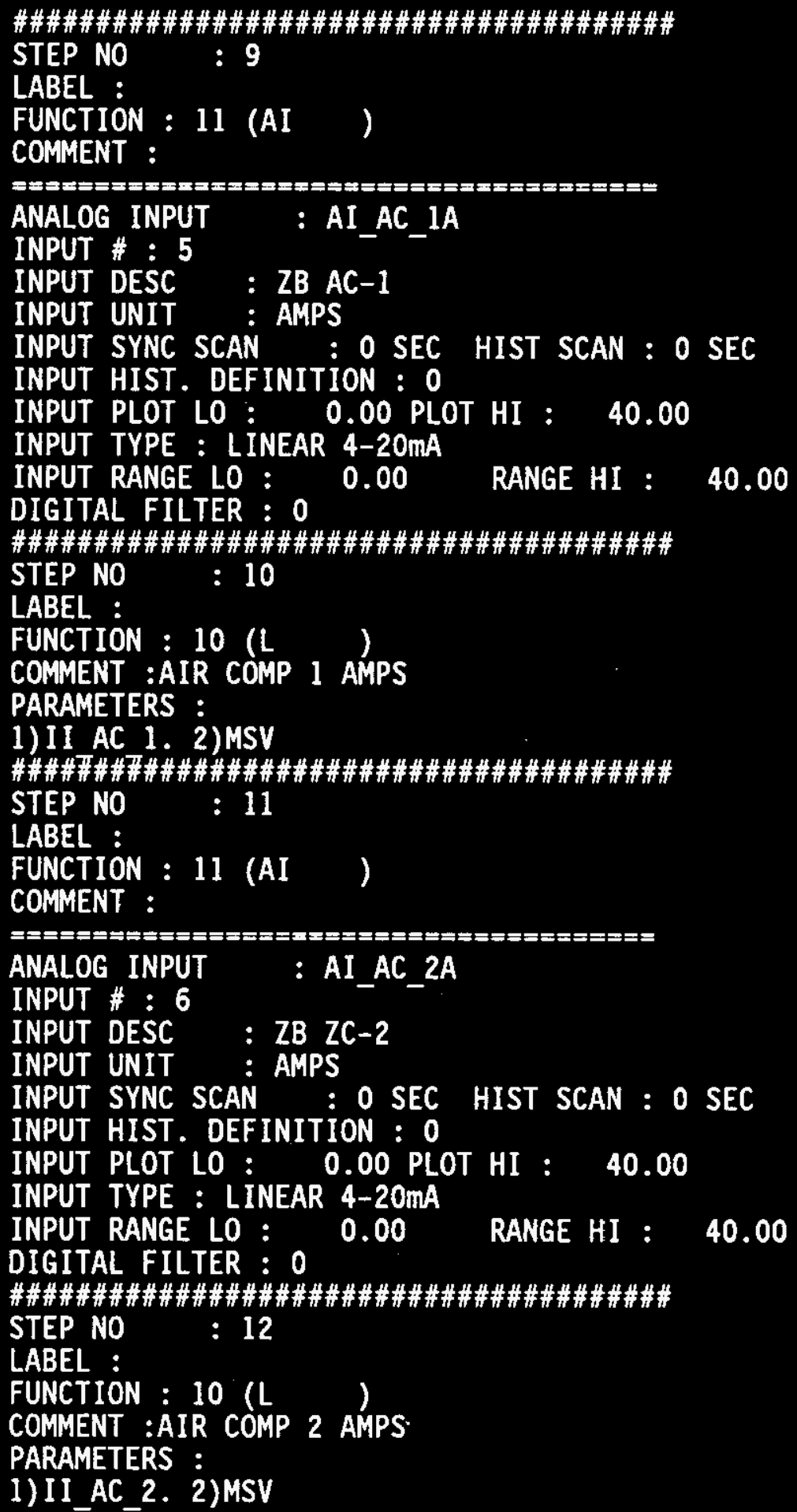


\#\#\#\#\#\#\#\#\#\#\#\#\#\#\#\#\#\#\#\#\#\#\#\#\#\#\#\#\#\#\#\#\#\#\#\#\#\#\#\#

STEP NO : 13

LABEL :

FUNCTION : 11 (AI)

COMMENT :

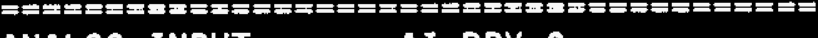

ANALOG INPUT

: AI_DPY_2

INPUT \# : 7

INPUT DESC : PRCS AREA BALANCE

INPUT UNIT :

INPUT SYNC SCAN : O SEC HIST SCAN : O SEC

INPUT HIST. DEFINITION : O

INPUT PLOT LO : -10.00 PLOT HI : 10.00

INPUT TYPE : LINEAR 4-20mA

INPUT RANGE LO : -10.00 RANGE HI : 10.00

DIGITAL FILTER : 0

\#\#\#\#\#\#\#\#\#\#\#\#\#\#\#\#\#\#\#\#\#\#\#\#\#\#\#\#\#\#\#\#\#\#\#\#\#\#\#\#

STEP NO : 14

LABEL :

FUNCTION : 10 (L )

COMMENT :PROCESS AREA BALANCE

PARAMETERS :

1)PDI 2A. 2) MSV

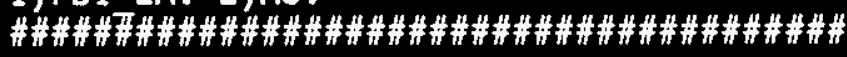

STEP NO : 15

LABEL :

FUNCTION : 11 (AI )

COMMENT :

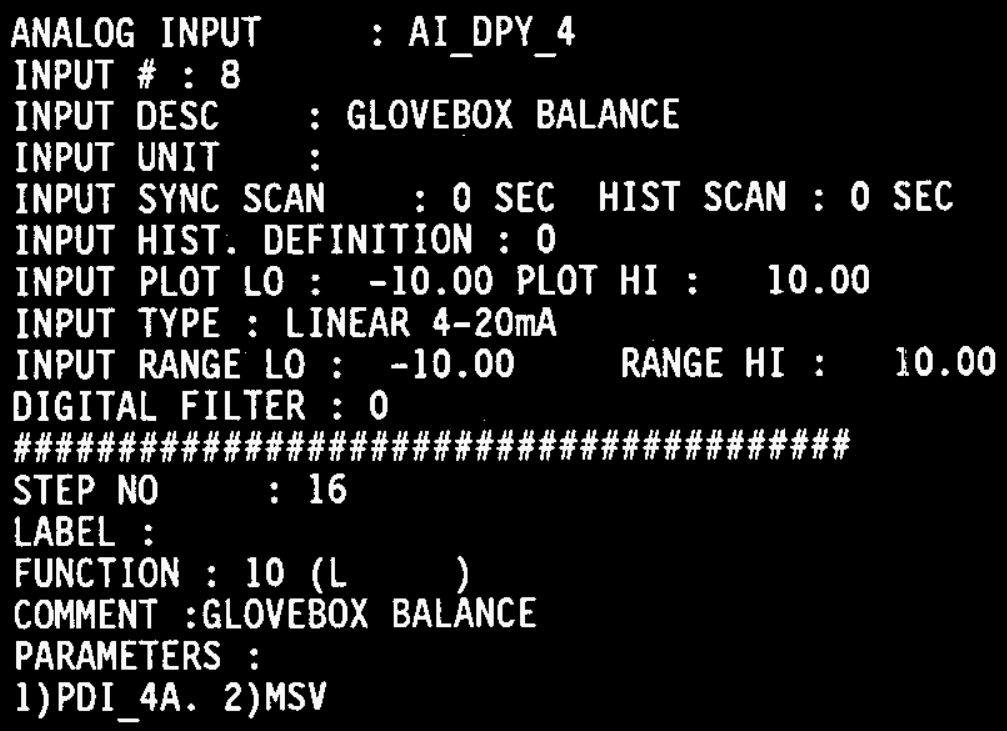




\section{WHC-SD-CP-CSWD-016 \\ Volume 4 \\ Revision 1 \\ Page 30}

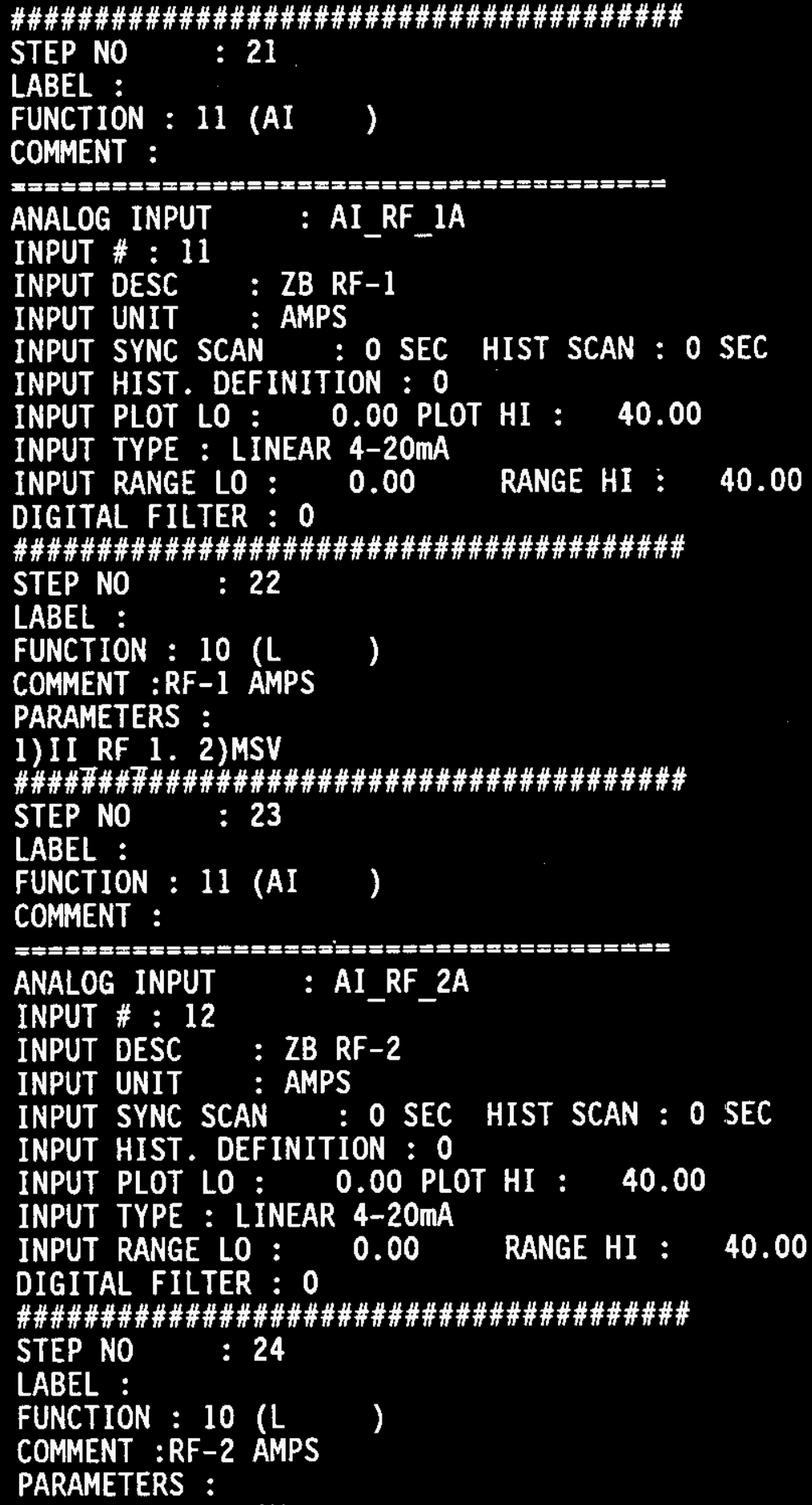


WHC-SD-CP-CSWD-016

Volume 4

Revision 1

Page 31

\#\#\#\#\#\#\#\#\#\#\#\#\#\#\#\#\#\#\#\#\#\#\#\#\#\#\#\#\#\#\#\#\#

STEP NO : 25

LABEL :

FUNCTION : 11 (AI) )

COMMENT :

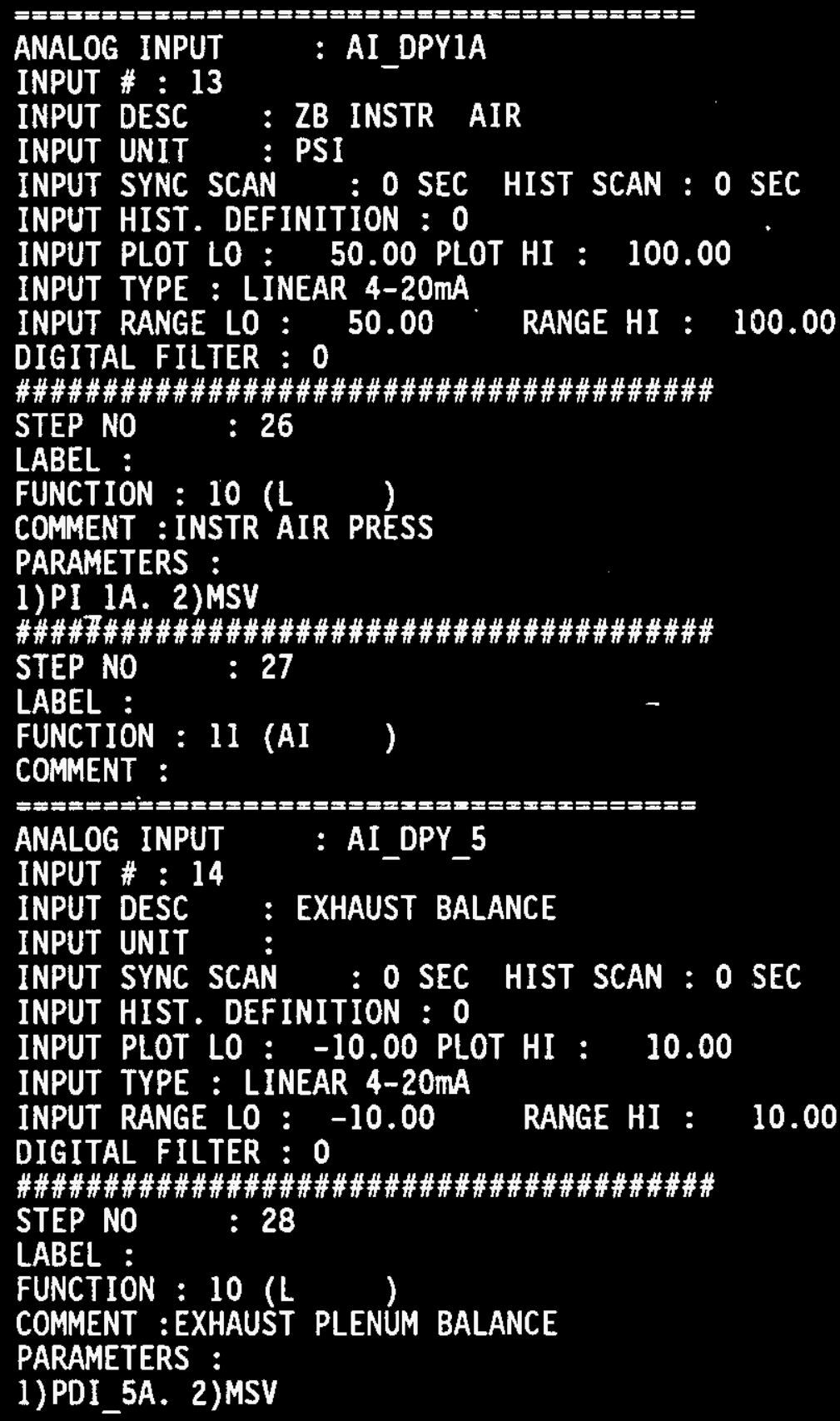




\section{WHC-SD-CP-CSWD-016 \\ Volume 4 \\ Revision 1 \\ Page 32}

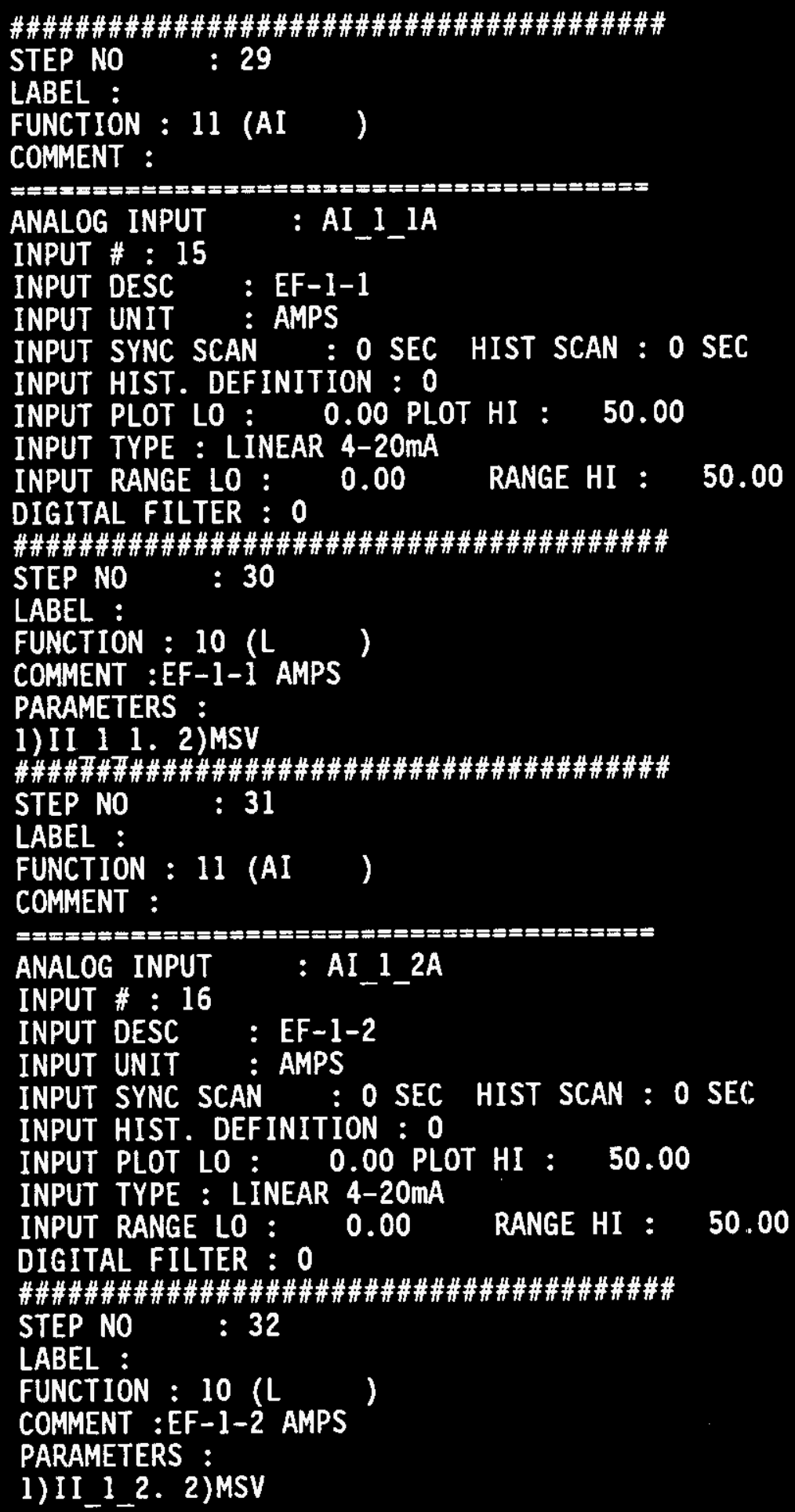




\section{WHC-SD-CP-CSWD-016 \\ Volume 4 \\ Revision 1 \\ Page 33}

\#\#\#\#\#\#\#\#\#\#\#\#\#\#\#\#\#\#\#\#\#\#\#\#\#\#\#\#\#\#\#\#\#\#\#\#\#\#\#\#

STEP NO : 33

LABEL :

FUNCTION : 11 (AI)

COMMENT :

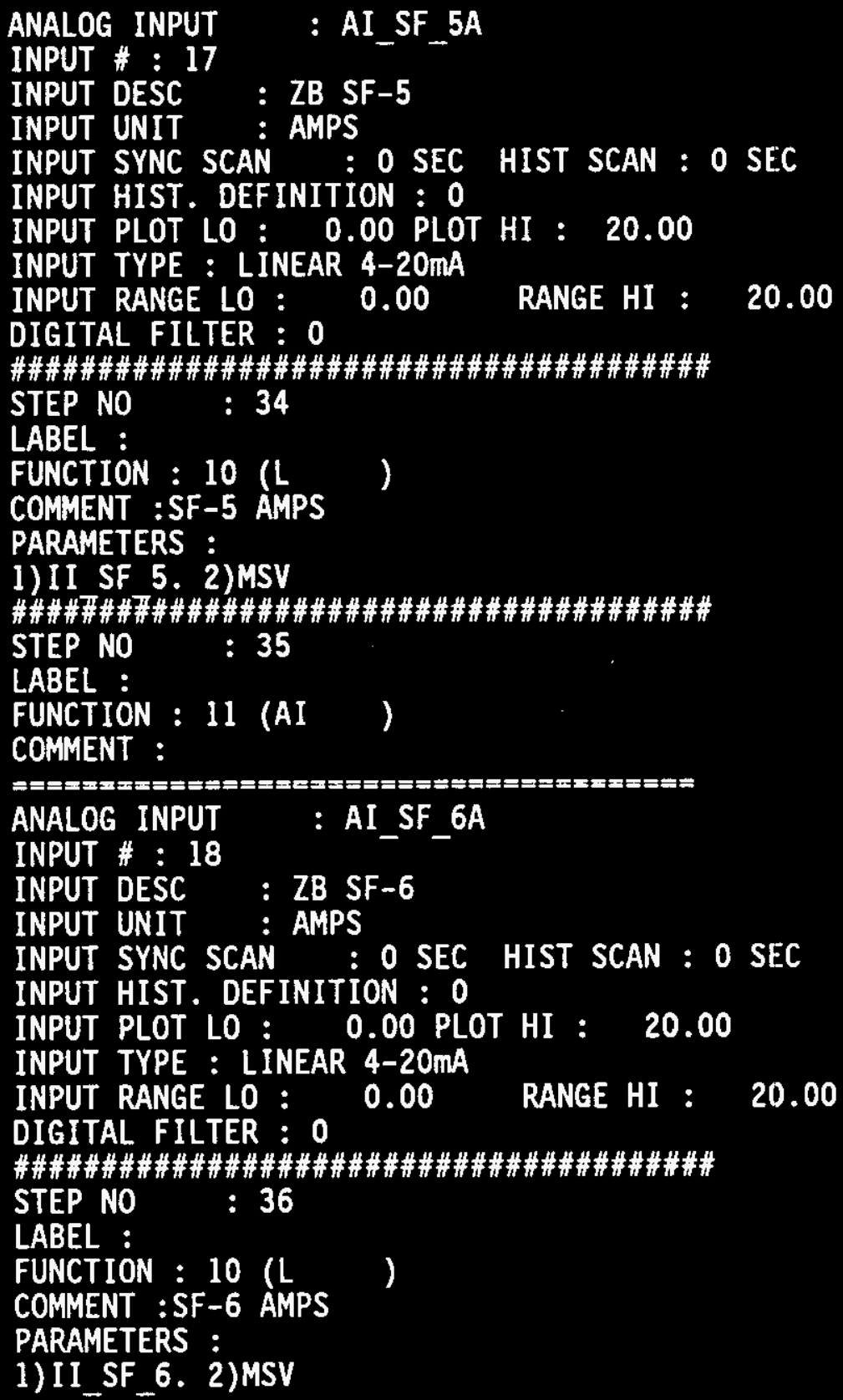




\section{WHC-SD-CP-CSWD-016 \\ Volume 4 \\ Revision 1 \\ Page 34}

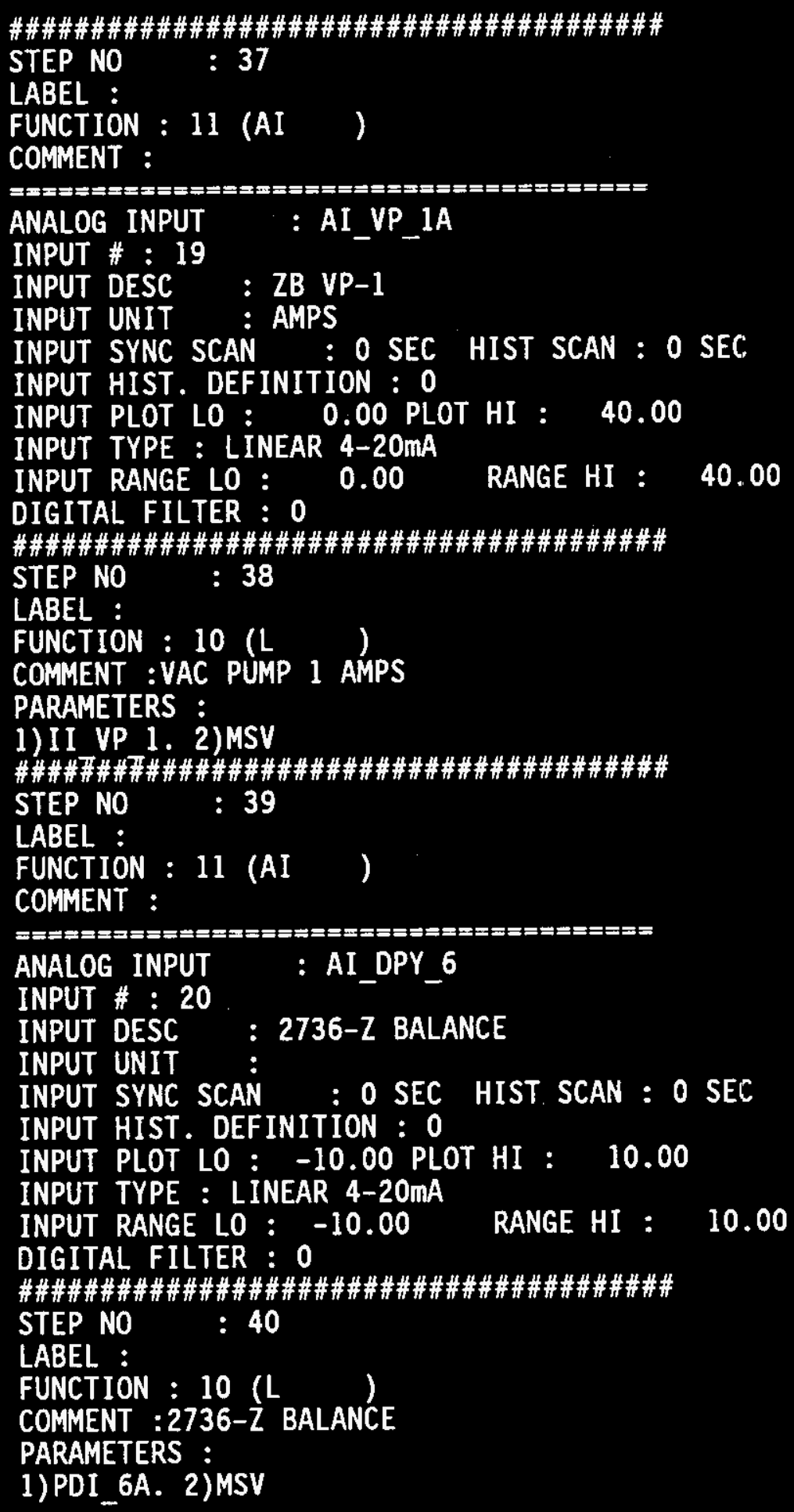


WHC-SD-CP-CSWD-016

Volume 4

Revision 1

Page 35

\#\#\#\#\#\#\#\#\#\#\#\#\#\#\#\#\#\#\#\#\#\#\#\#\#\#\#\#\#\#\#\#\#\#\#\#\#\#\#\#

STEP NO : 41

LABEL :

FUNCTION : 11 (AI)

COMMENT :

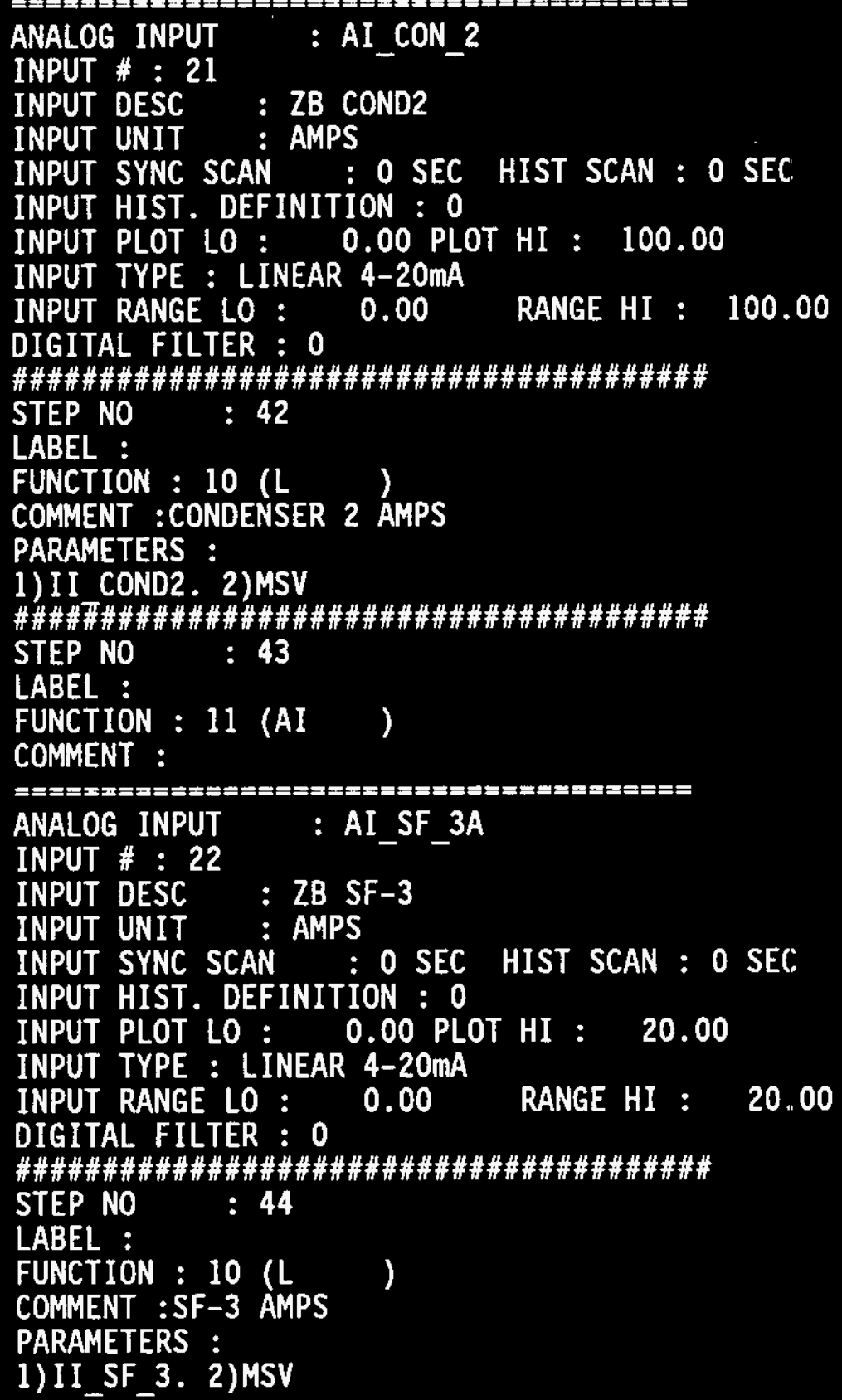




\section{WHC-SD-CP-CSWD-016 \\ Volume 4 Revision 1 \\ Page 36}

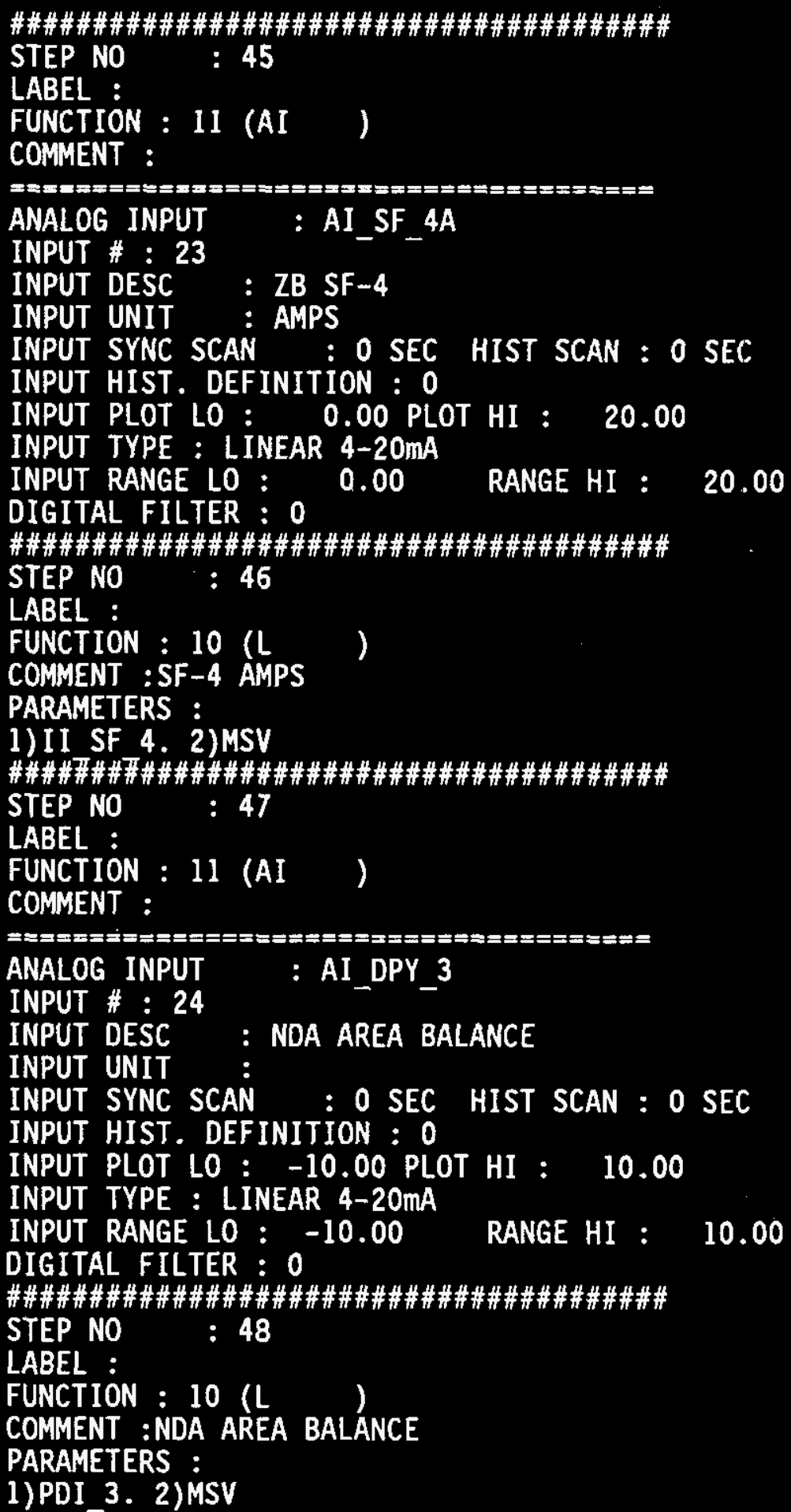


WHC-SD-CP-CSWD-016

Volume 4

Revision 1

Page 37

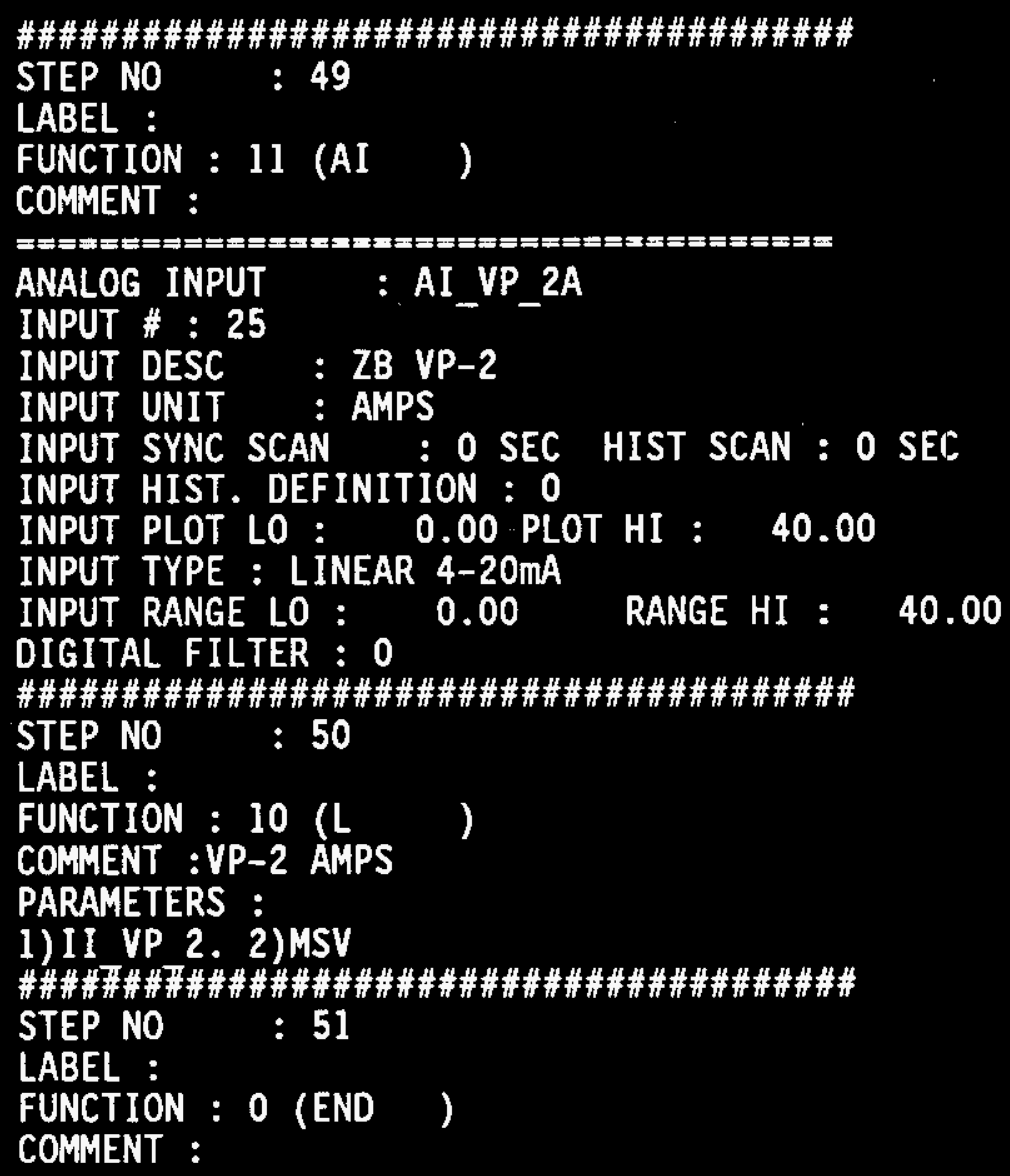


WHC-SD-CP-CSWD-016

Volume 4

Revision 1

Page 38

ZB_2_1.mic18_ro

MICON TYPE :RCM-D

MICON UNIT NO : 18

MICON TAG : ZB 21

MICON DESCRIPTION $\div$ ₹736-ZB RACK 2 SLOT 1

MICON CONFIGURATION FILE : mic18_ro

POINT ALLOCATION :

VAI : 32, VDI : 32 , ISW : 32, LOOPS : 12 , MSD : 16 ,

AI : $0, \mathrm{AO}: 0, \mathrm{DI}: 32, \mathrm{DO}: 32$

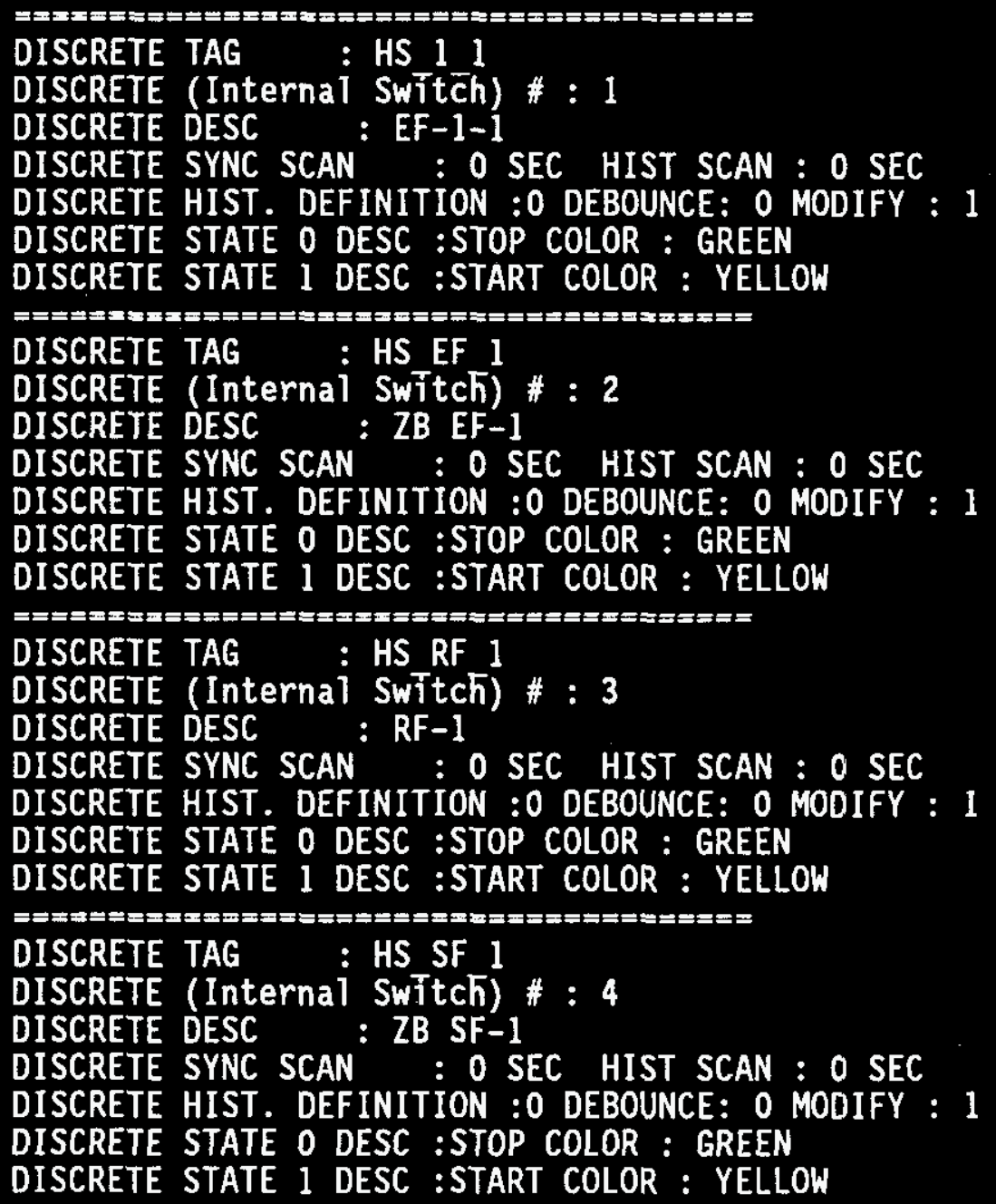


WHC-SD-CP-CSWD-016

Volume 4

Revision 1

Page 39

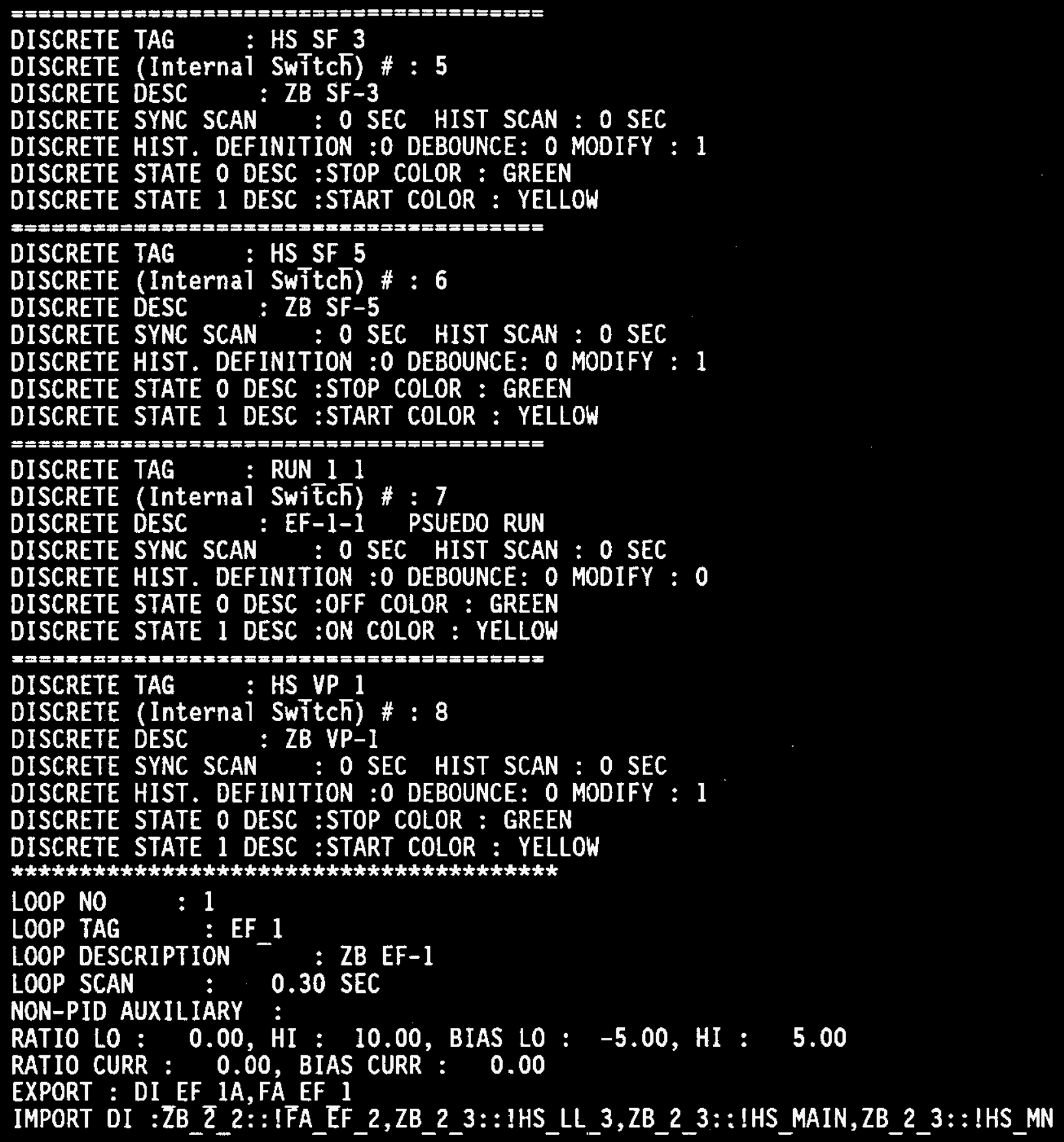


\#\#\#\#\#\#\#\#\#\#\#\#\#\#\#\#\#\#\#\#\#\#\#\#\#\#\#\#\#\#\#\#\#\#\#\#\#\#\#\#

STEP NO : 1

LABEL :

FUNCTION : 6 (VD )

COMMENT :

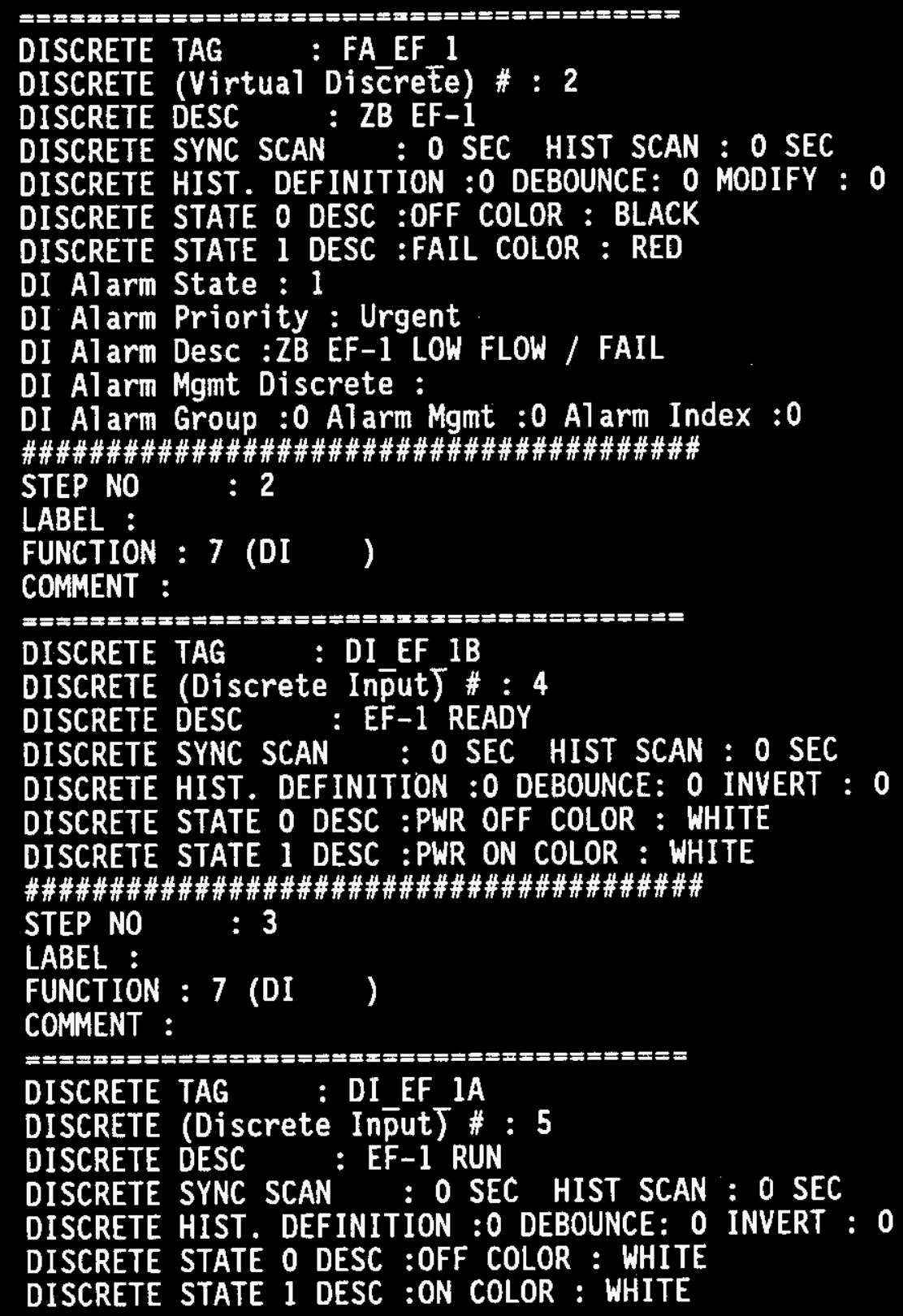




\section{WHC-SD-CP-CSWD-016 \\ Volume 4 \\ Revision 1 \\ Page 41}

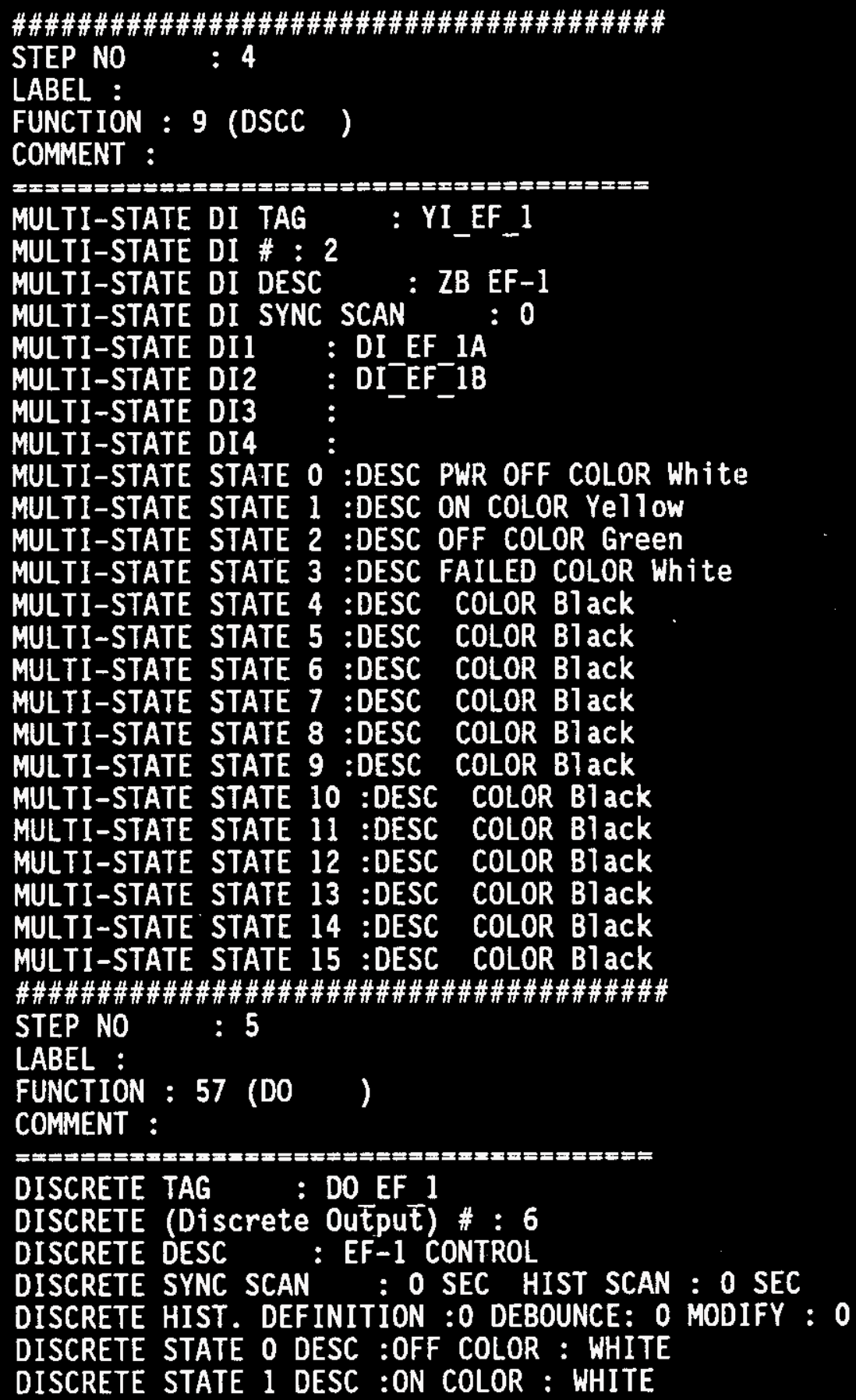




$$
\begin{gathered}
\text { WHC-SD-CP-CSWD-016 } \\
\text { Volume } 4 \\
\text { Revision } 1 \\
\text { Page } 42
\end{gathered}
$$

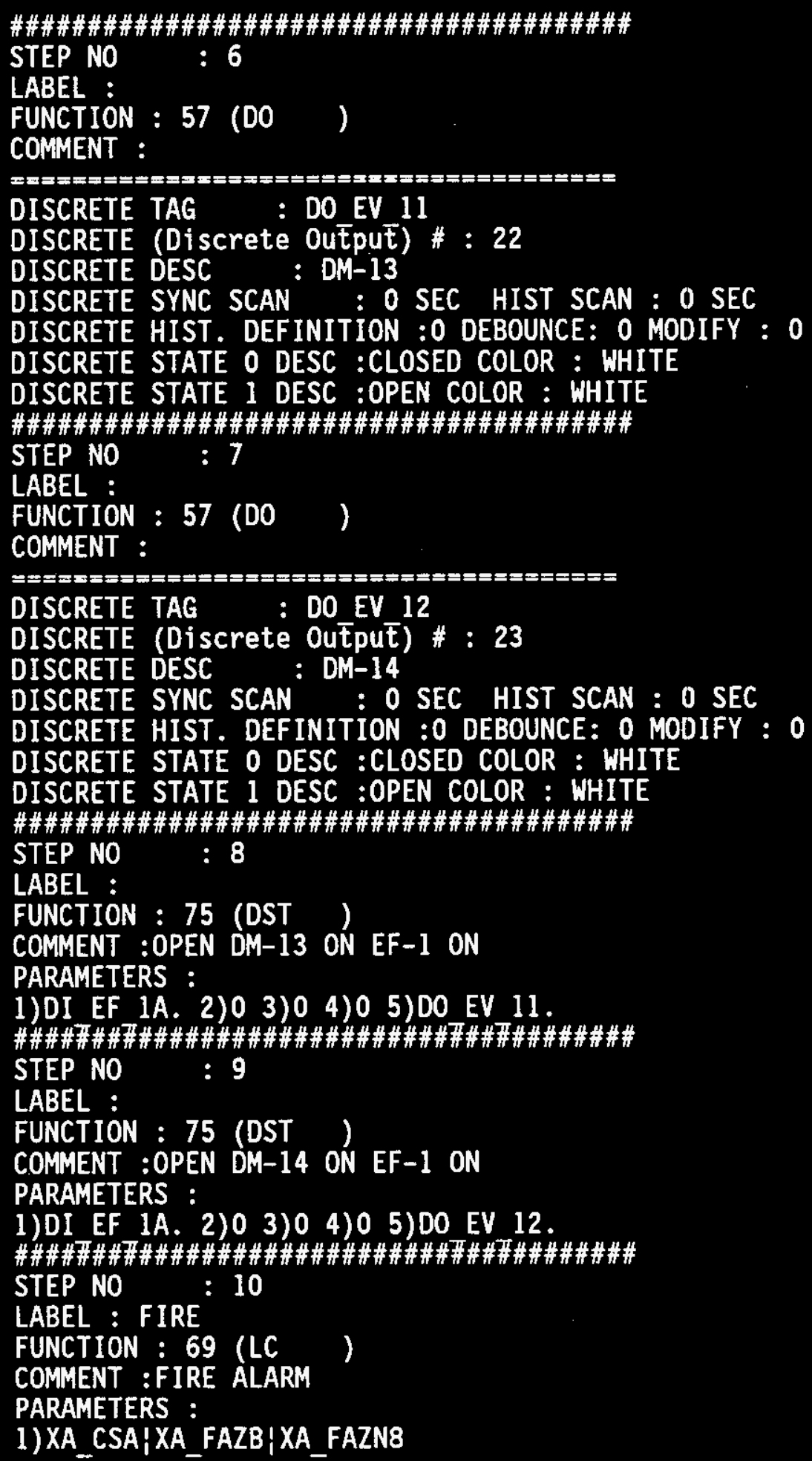




$$
\begin{gathered}
\text { WHC-SD-CP-CSWD-016 } \\
\text { Volume } 4 \\
\text { Revision } 1 \\
\text { Page } 43
\end{gathered}
$$

\#\#\#\#\#\#\#\#\#\#\#\#\#\#\#\#\#\#\#\#\#\#\#\#\#\#\#\#\#\#\#\#\#\#\#\#\#\#\#\#

STEP NO

$: 11$

LABEL :

FUNCTION : 69 (LC )

COMMENT : "0" PULSE ON AUTO START OR EF-2 FAIL.

PARAMETERS :

1)-! PULSE(-! HS LL 3\&HS MAIN)\&-! PULSE(HS LL_3\&(FA_EF_2 PULSE(-!DI EF

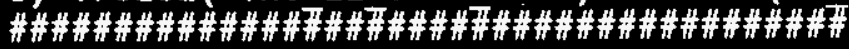

STEP NO : 12

LABEL :

FUNCTION : 62 (GTD

COMMENT :EF-1 "START" JUMP

PARAMETERS :

1) MSV 2) Off-Nomal 3) JUMPA

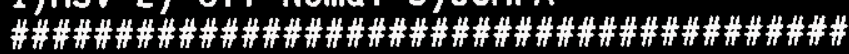

STEP NO : 13

LABEL : JUMPA

FUNCTION : $10(\mathrm{~L}$,

COMMENT :START EF-1

PARAMETERS :

1)HS EF 1. 2)1

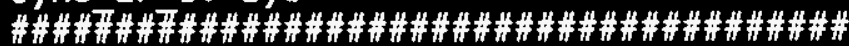

STEP NO : 14

LABEL :

FUNCTION : 69 (LC

COMMENT : PREVENT FALSE START ON RESET

PARAMETERS :

1)-! PULSE(1)

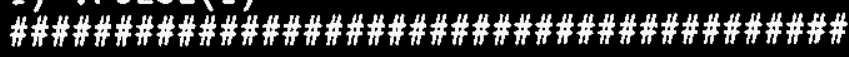

STEP NO : 15

LABEL :

FUNCTION : 62 (GTD )

COMMENT :

PARAMETERS :

1) MSV 2) Off-Nomal 3) JUMPST

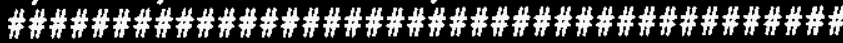

STEP NO

LABEL : JUMPST

FUNCTION : $10(\mathrm{~L} \quad)$

COMMENT :

PARAMETERS :

1) HS EF 1. 2)0

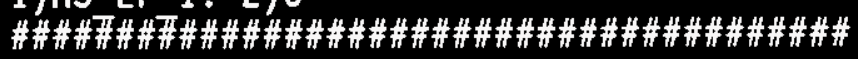

STEP NO : 17

LABEL :

FUNCTION : 69 (LC

COMMENT : "O" PULSE ON MAIN STOP

PARAMETERS :

1)-! PULSE(HS_MN_S,5) 


\section{WHC-SD-CP-CSWD-016 \\ Volume 4 \\ Revision 1 \\ Page 44}

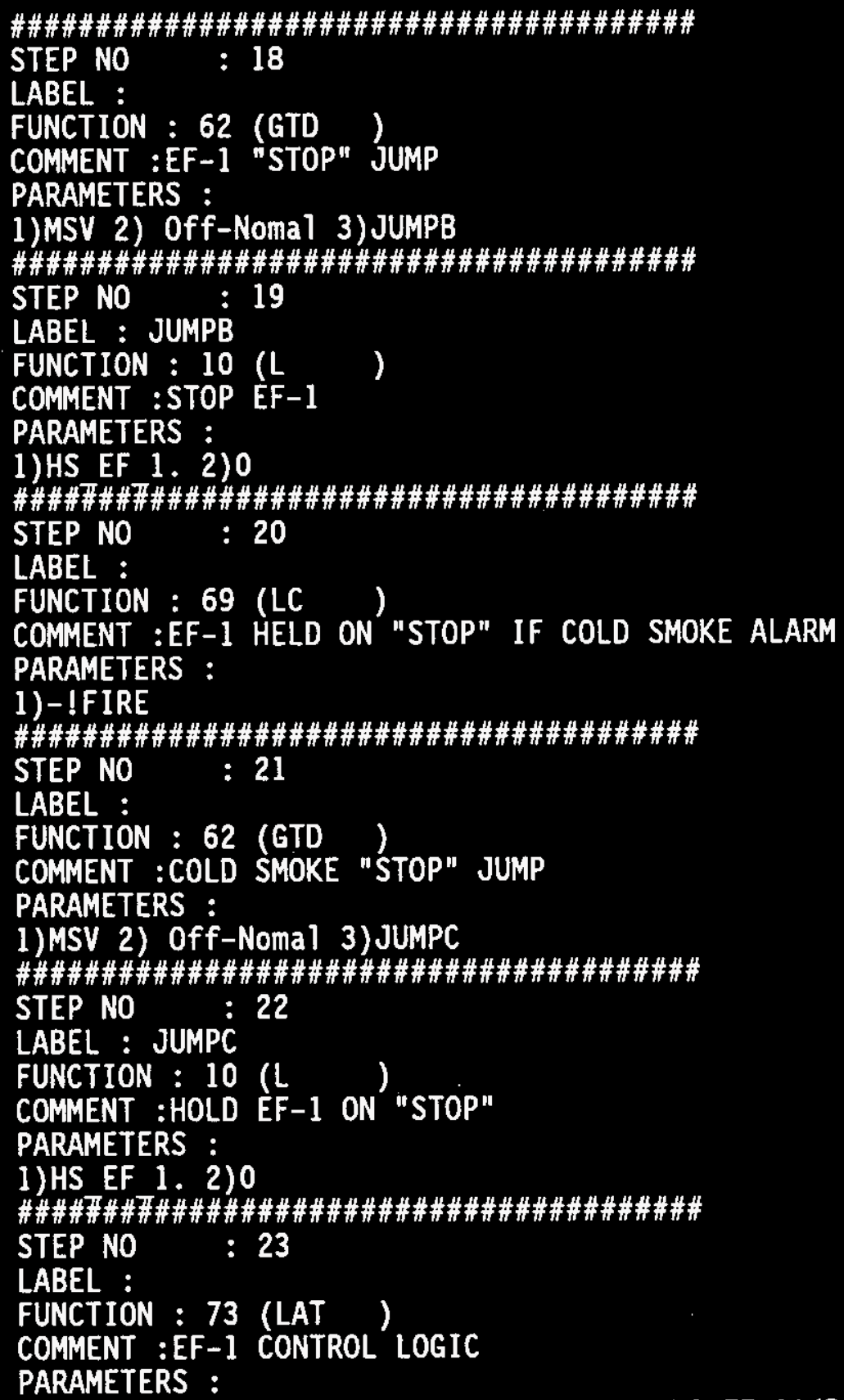


WHC-SD-CP-CSWD-016

Volume 4

Revision 1

Page 45

\#\#\#\#\#\#\#\#\#\#\#\#\#\#\#\#\#\#\#\#\#\#\#\#\#\#\#\#\#\#\#\#\#\#\#\#\#\#\#\#

STEP NO : 24

LABEL :

FUNCTION : 69 (LC

COMMENT : ALARM ON NO FLOW OR OFF WHEN HS IS ON (EXCEPT STARTUP)

PARAMETERS :

1)DELAY (HS EF 1,15)\&-(!DI EF 1A\&!DI FS 5)

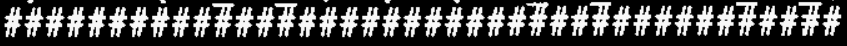

STEP NO : 25

LABEL :

FUNCTION : 75 (DST )

COMMENT :

PARAMETERS :

1)MSV 2)0 3)0 4)0 5)FA EF 1.

\#\#\#\#\#\#\#\#\#\#\#\#\#\#\#\#\#\#\#界\#\#界\#\#\#\#\#\#\#\#\#\#\#\#

STEP NO : 26

LABEL :

FUNCTION : 0 (END)

COMMENT :

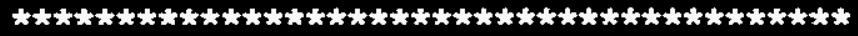

LOOP NO : 2

LOOP TAG : EF 1 1

LOOP DESCRIPTION - : EF-1-1

LOOP SCAN : 0.30 SEC

NON-PID AUXILIARY :

RATIO LO : 0.00 , HI : 10.00 , BIAS LO : -5.00 , HI : 5.00

RATIO CURR : 0.00 , BIAS CURR : 0.00

EXPORT :HS 11 , RUN 11 , FA 11

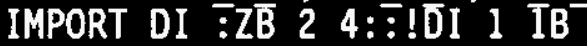

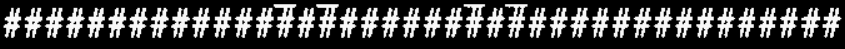

STEP NO : 1

LABEL :

FUNCTION : 6 (VD)

COMMENT :

DISCRETE TAG : FA 1 1

DISCRETE (Virtual Disçrēte) \# : 1

DISCRETE DESC : EF-1-1

DISCRETE SYNC SCAN : O SEC HIST SCAN : O SEC

DISCRETE HIST. DEFINITION :0 DEBOUNCE: 0 MODIFY : 0

DISCRETE STATE 0 DESC :OFF COLOR : BLACK

DISCRETE STATE I DESC :FAIL COLOR : RED

DI Alarm State : 1

DI Alarm Priority : Urgent

DI Alarm Desc :EF-1-1 LOW FLOW / FAIL

DI Alarm Mgmt Discrete :

DI Alarm Group :0 Alarm Mgmt :0 Alarm Index :0 
WHC-SD-CP-CSWD-016

Volume 4

Revision 1

Page 46

\#\#\#\#\#\#\#\#\#\#\#\#\#\#\#\#\#\#\#\#\#\#\#\#\#\#\#\#\#\#\#\#\#\#\#\#\#\#\#\#

STEP NO : 2

LABEL :

FUNCTION : 7 (DI )

COMMENT :

DISCRETE TAG : DI $11 \mathrm{~A}$
DISCRETE (DiScrete InpUt) \# 10
DISCRETE DESC : EF-1-1 READY
DISCRETE SYNC SCAN : 0 SEC HIST SCAN : O SEC

DISCRETE HIST. DEFINITION :0 DEBOUNCE: 0 INVERT : 0

DISCRETE STATE O DESC :PWR OFF COLOR : WHITE

DISCRETE STATE 1 DESC :PWR ON COLOR : WHITE

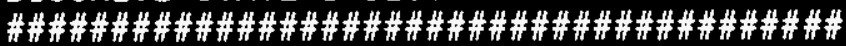

STEP NO : 3

LABEL :

FUNCTION : 9 (DSCC)

COMMENT :

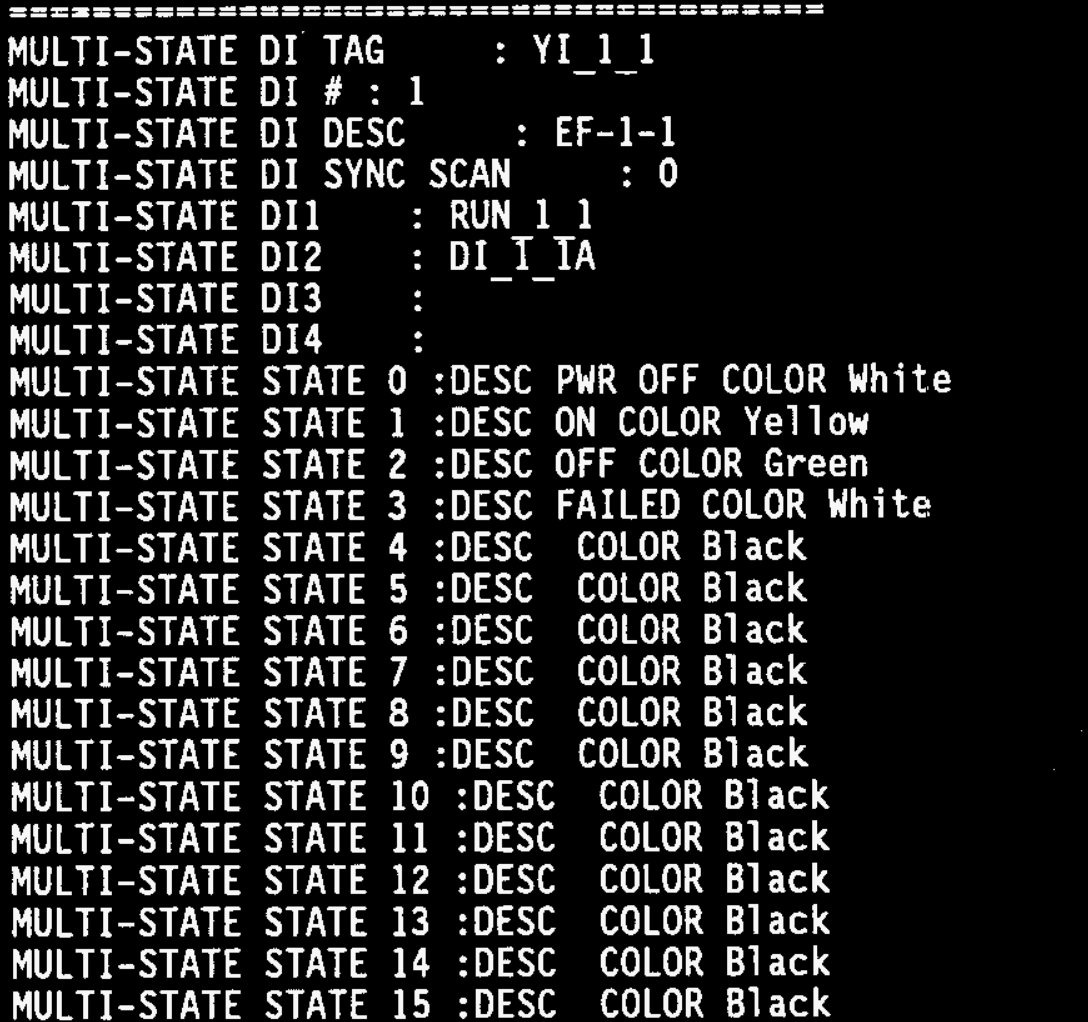


WHC-SD-CP-CSWD-016

Volume 4

Revision 1

Page 47

\#\#\#\#\#\#\#\#\#\#\#\#\#\#\#\#\#\#\#\#\#\#\#\#\#\#\#\#\#\#\#\#\#\#\#\#\#\#\#\#

STEP NO : 4

LABEL :

FUNCTION : 7 (DI )

COMMENT :

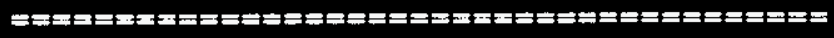

DISCRETE TAG : DI_KFS21

DISCRETE (Discrete Inp̄ut) \# : 18

DISCRETE DESC : EF-1-1 FLOW SWITCH

DISCRETE SYNC SCAN : 0 SEC HIST SCAN : O SEC

DISCRETE HIST. DEFINITION :0 DEBOUNCE: 0 INVERT : 0

DISCRETE STATE O DESC :OFF COLOR : WHITE

DISCRETE STATE 1 DESC :FLOW ON COLOR : WHITE

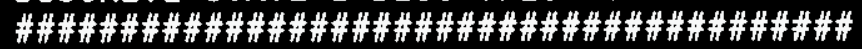

STEP NO : 5

LABEL :

FUNCTION : $57(00)$

COMMENT :

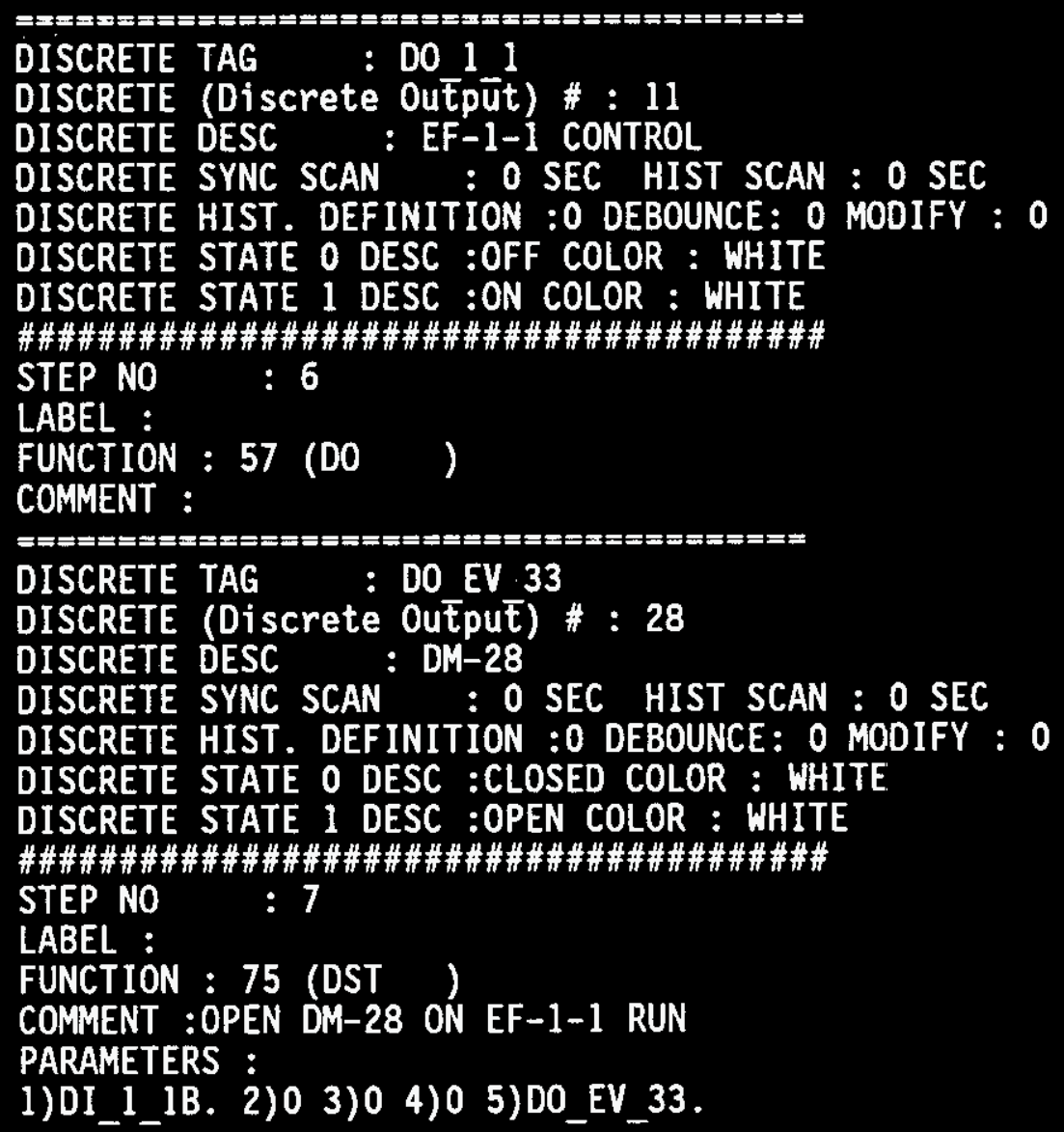




WHC-SD-CP-CSWD-016
Volume 4
Revision 1
Page 48

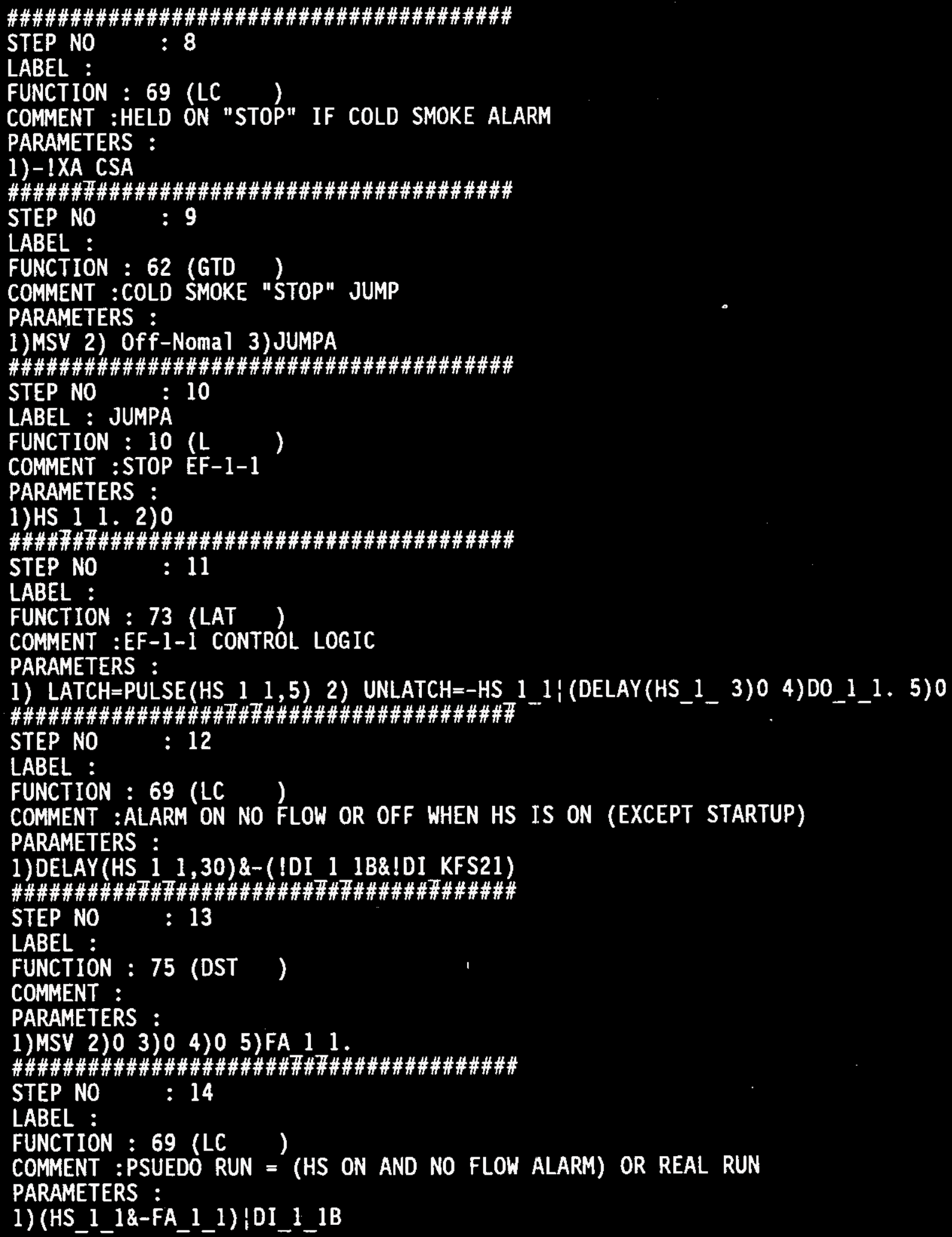


WHC-SD-CP-CSWD-016

Volume 4

Revision 1

Page 49

\#\#\#\#\#\#\#\#\#\#\#\#\#\#\#\#\#\#\#\#\#\#\#\#\#\#\#\#\#\#\#\#\#\#\#\#\#\#\#\#\#

STEP NO : 15

LABEL :

FUNCTION : 75 (DST )

COMMENT :

PARAMETERS :

1)MSV 2)0 3)0 4)0 5)RUN 11 .

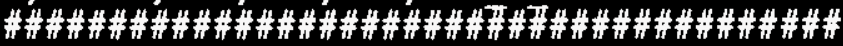

STEP NO $: 16$

LABEL :

FUNCTION : 0 (END)

COMMENT :

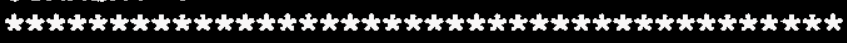

LOOP NO : 3

LOOP TAG : RF 1

LOOP DESCRIPTION - : RF-1

LOOP SCAN : 0.30 SEC

NON-PID AUXILIARY :

RATIO LO : 0.00 , HI : 10.00 , BIAS LO : -5.00 , HI : 5.00

RATIO CURR : 0.00 , BIAS CURR : 0.00

EXPORT :DI RF 1A,FA RF I

IMPORT DI $: \mathrm{ZB}^{-1} 22::$ TFA RF $2, \mathrm{ZB} 23::$ !HS LL_4,ZB_2 $3::$ !HS_NDA, ZB_2 $3::$ !HS_NDA

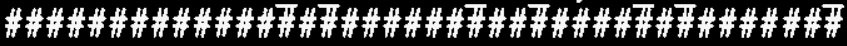

STEP NO : 1

LABEL :

FUNCTION : 6 (VD )

COMMENT :

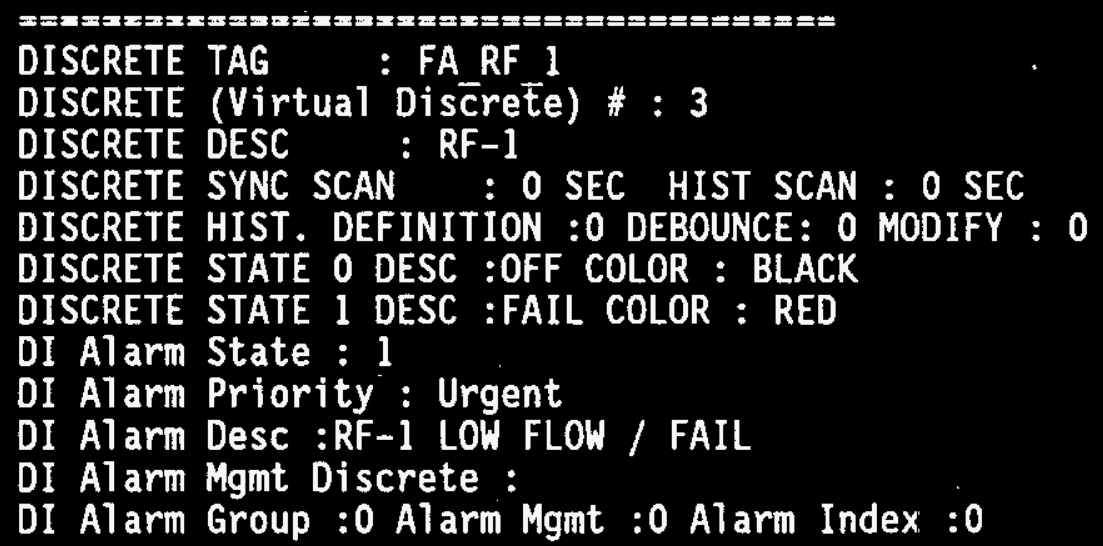


WHC-SD-CP-CSWD-016

Volume 4

Revision 1

Page 50

\#\#\#\#\#\#\#\#\#\#\#\#\#\#\#\#\#\#\#\#\#\#\#\#\#\#\#\#\#\#\#\#\#\#\#\#\#\#

STEP NO : 2

LABEL :

FUNCTION : 7 (DI )

COMMENT :

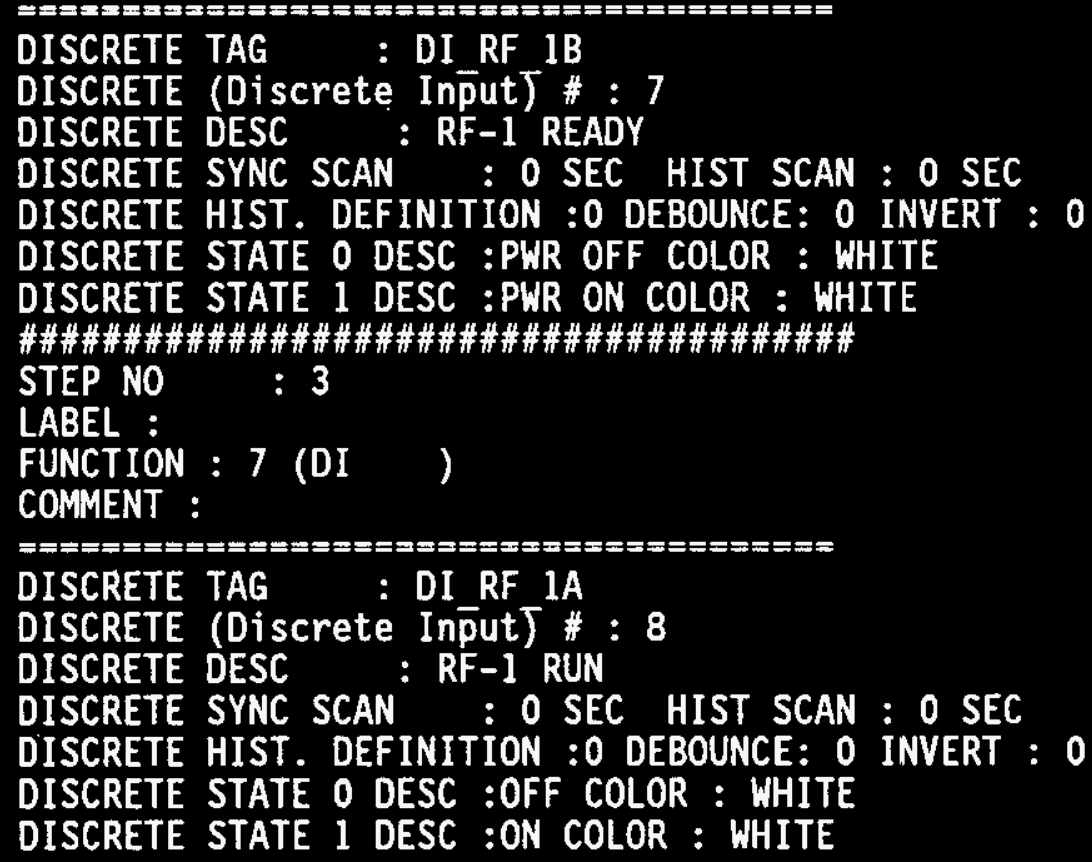


\#\#\#\#\#\#\#\#\#\#\#\#\#\#\#\#\#\#\#\#\#\#\#\#\#\#\#\#\#\#\#\#\#\#\#\#\#\#\#\#

STEP NO : 4

LABEL :

FUNCTION : 9 (DSCC)

COMMENT :

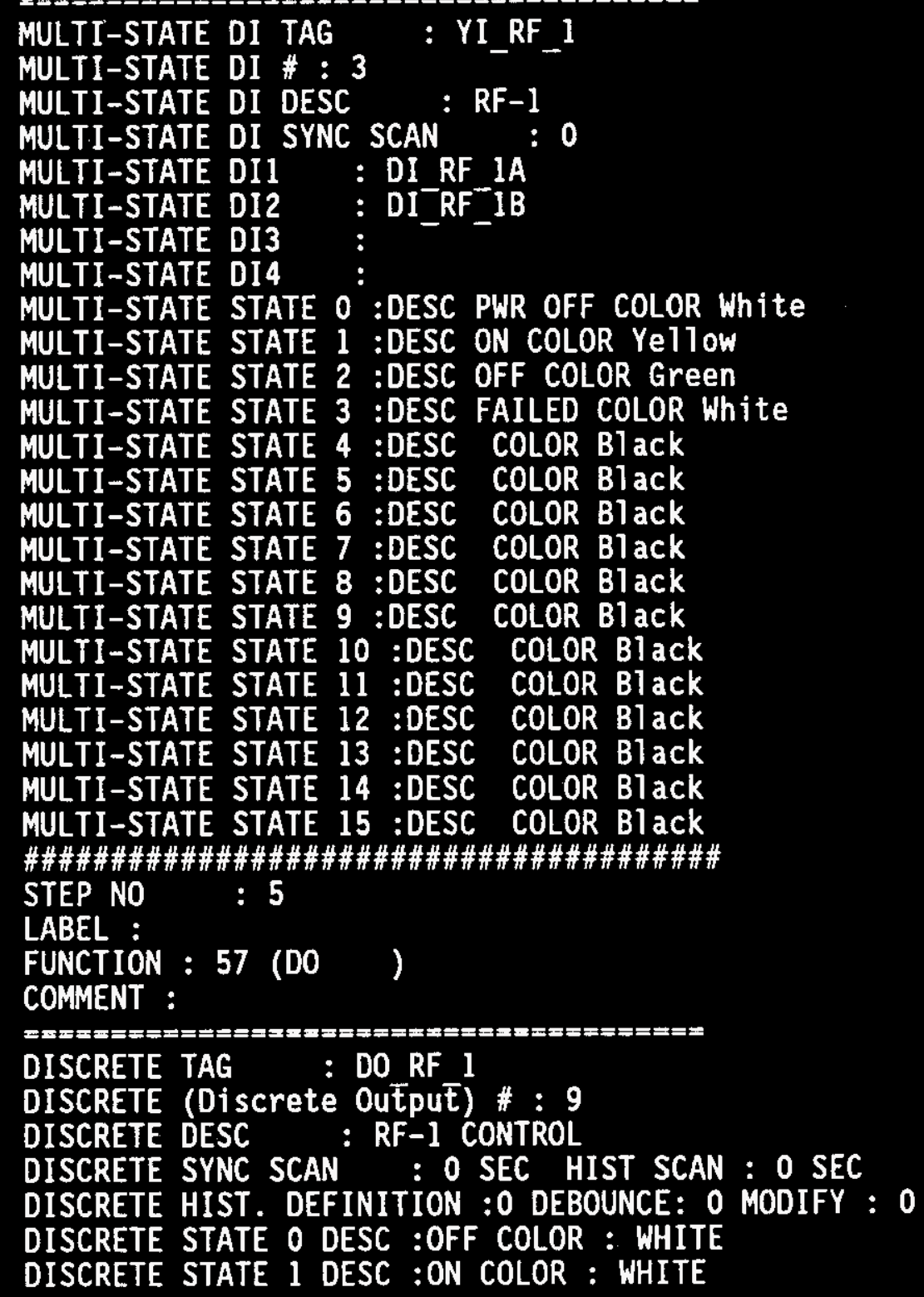


WHC-SD-CP-CSWD-016

Volume 4

Revision 1

Page 52

\#\#\#\#\#\#\#\#\#\#\#\#\#\#\#\#\#\#\#\#\#\#\#\#\#\#\#\#\#\#\#\#\#\#\#\#\#\#\#\#

STEP NO : 6

LABEL :

FUNCTION : 57 (DO)

COMMENT :

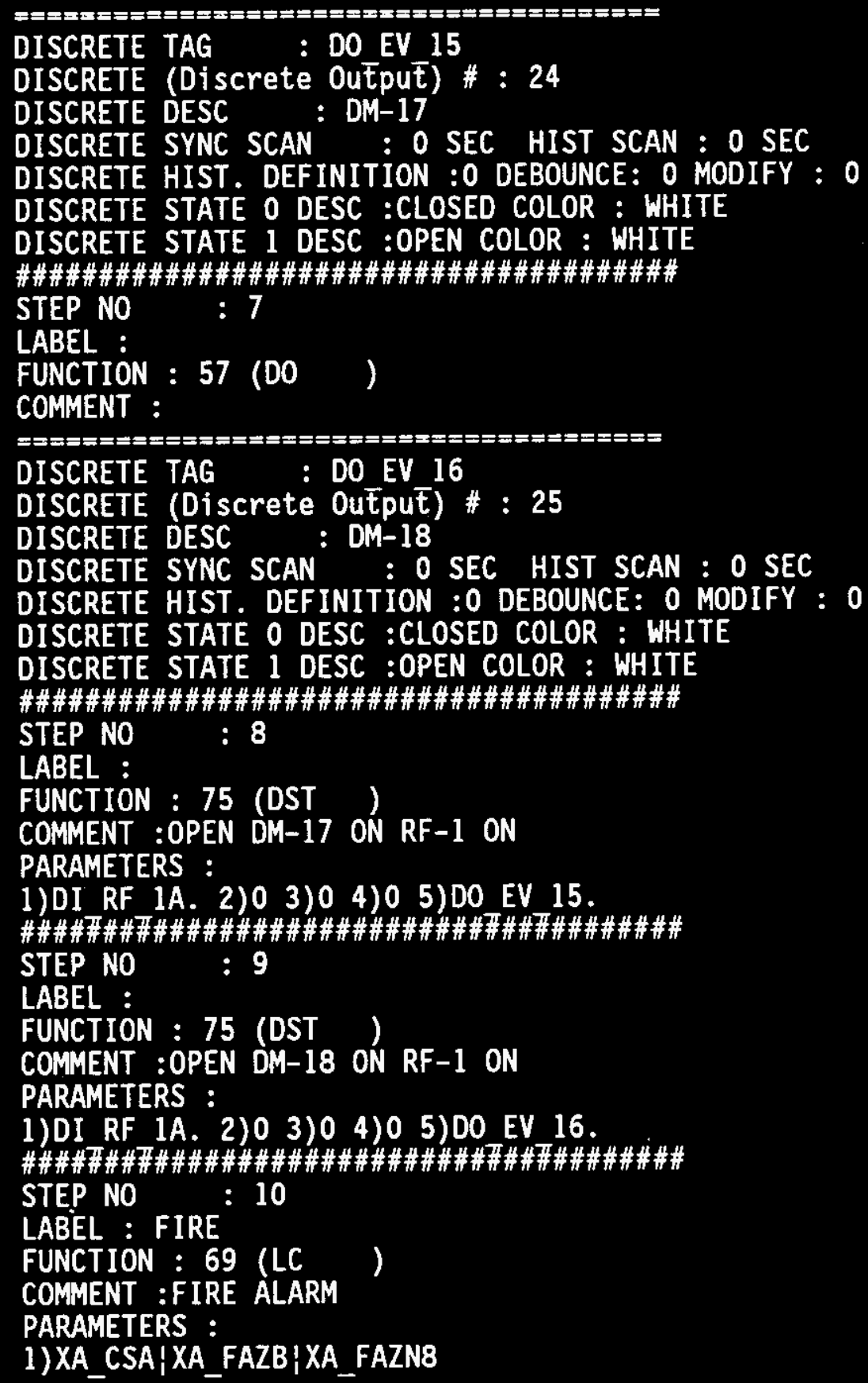




\section{WHC-SD-CP-CSWD-016 \\ Volume 4 \\ Revision 1 \\ Page 53}

\#\#\#\#\#\#\#\#\#\#\#\#\#\#\#\#\#\#\#\#\#\#\#\#\#\#\#\#\#\#\#\#\#\#\#\#\#\#\#\#

STEP NO

$: 11$

LABEL :

FUNCTION : 69 (LC )

COMMENT : PULSE " 0 " ON AUTO START OR RF-2 FAIL

PARAMETERS : 1)-! PULSE(-!HS LL 4\&HS NDA)\&-! PULSE(HS LL_4\&(FA_RF_2!PULSE(-!DI_RF_

STEP NO : 12

LABEL :

FUNCTION : 62 (GTD)

COMMENT :RF-1 START JUMP

PARAMETERS :

1)MSV 2) Off-Nomal 3) JUMPA

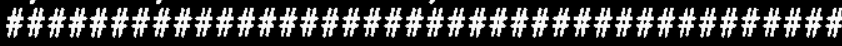

STEP NO

: 13

LABEL : JUMPA

FUNCTION : $10(\mathrm{~L})$

COMMENT :

PARAMETERS :

1)HS RF 1.2)1

\#\#\#\#界\#\#男\#\#\#\#\#\#\#\#\#\#\#\#\#\#\#\#\#\#\#\#\#\#\#\#\#\#\#\#\#\#\#\#

STEP NO : 14

LABEL :

FUNCTION : 69 (LC)

COMMENT : PREVENT FALSE START ON RESET

PARAMETERS :

1) - ! PULSE (1)

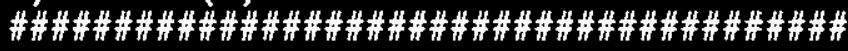

STEP NO : 15

LABEL :

FUNCTION : 62 (GTD)

COMMENT :

PARAMETERS :

1)MSV 2) Off-Nomal 3) JUMPST

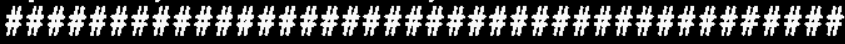

STEP NO

LABEL : JUMPST

FUNCTION : $10(\mathrm{~L})$

COMMENT :

PARAMETERS :

1) HS RF 1. 2) 0

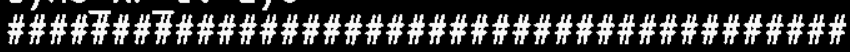

STEP NO : 17

LABEL :

FUNCTION : 69 (LC )

COMMENT : PULSE "O" ON NDA STOP

PARAMETERS :

1)-! PULSE(HS_NDA_S,5) 


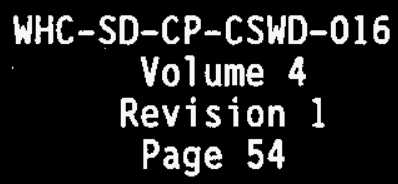

\#\#\#\#\#\#\#\#\#\#\#\#\#\#\#\#\#\#\#\#\#\#\#\#\#\#\#\#\#\#\#\#\#\#\#\#\#\#\#\#

STEP NO : 18

LABEL :

FUNCTION : 62 (GTD )

COMMENT :RF-1 STOP JUMP

PARAMETERS :

1)MSV 2) Off-Nomal 3) JUMPB

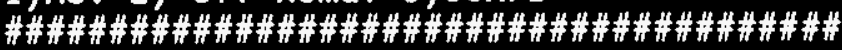

STEP NO : 19

LABEL : JUMPB

FUNCTION : $10(\mathrm{~L} \quad)$

COMMENT :

PARAMETERS :

1)HS RF 1. 2)0

\#\#\#\#界\#\#罚\#\#\#\#\#\#\#\#\#\#\#\#\#\#\#\#\#\#\#\#\#\#\#\#\#\#\#\#\#\#\#\#

STEP NO : 20

LABEL :

FUNCTION : 69 (LC

COMMENT :HOLD ON "STOP" IF FIRE ALARM

PARAMETERS :

1) - ! F IRE

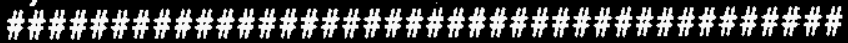

STEP NO : 21

LABEL :

FUNCTION : 62 (GTD ")
COMMENT :RF-1 FIRE "STOP" JUMP

PARAMETERS :

1)MSV 2) Off-Nomal 3) JUMPC

\#\#\#\#\#\#\#\#\#\#\#\#\#\#\#\#\#\#\#\#\#\#\#\#\#\#\#\#\#\#\#\#\#\#\#\#

STEP NO : 22

LABEL : JUMPC

FUNCTION : $10(\mathrm{~L} \quad)$

COMMENT :

PARAMETERS :

1)HS RF 1. 2)0

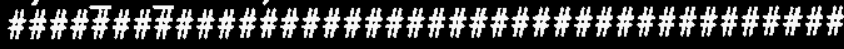

STEP NO : 23

LABEL :

FUNCTION : 73 (LAT)

COMMENT :RF-1 CONTROL LOGIC

PARAMETERS :

1) LATCH=PULSE(HS RF 1,5) 2) UNLATCH=-HS_RF_1 (DELAY(HS_R 3)0 4)00_RF_1. 5)0

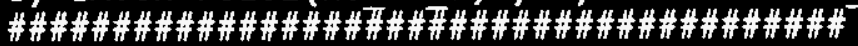

STEP NO : 24

LABEL :

FUNCTION : 69 (LC

COMMENT : ALARM ON NO FLOW OR OFF WHEN HS IS ON (EXCEPT STARTUP)

PARAMETERS :

1) DELAY (HS_RF_1,15)\&-(!DI_RF_1A\&!DI_FS_7) 


\section{WHC-SD-CP-CSWD-016 \\ Volume 4 \\ Revision 1 \\ Page 55}

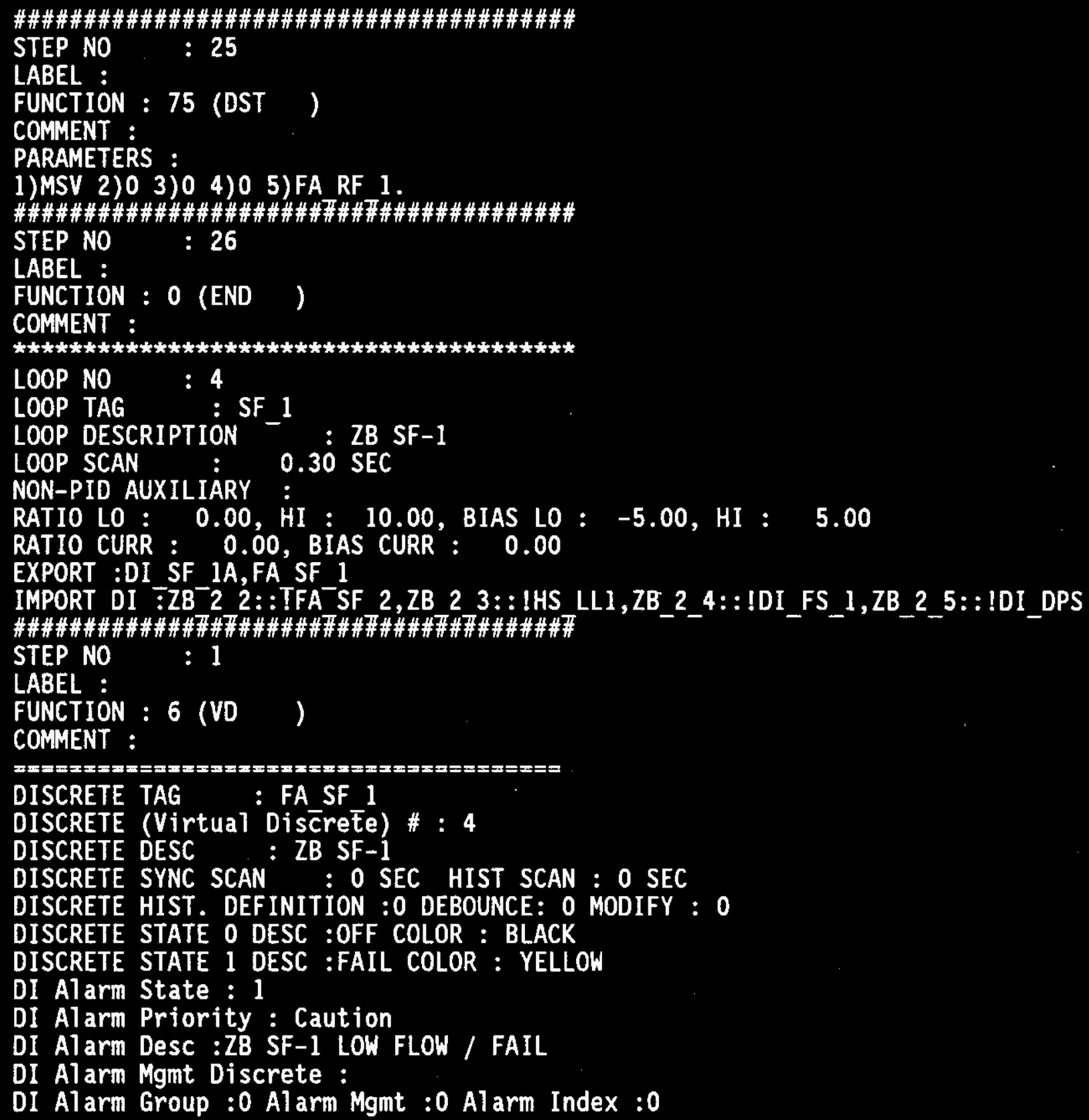


WHC-SD-CP-CSWD-016

Volume 4

Revision 1

Page 56

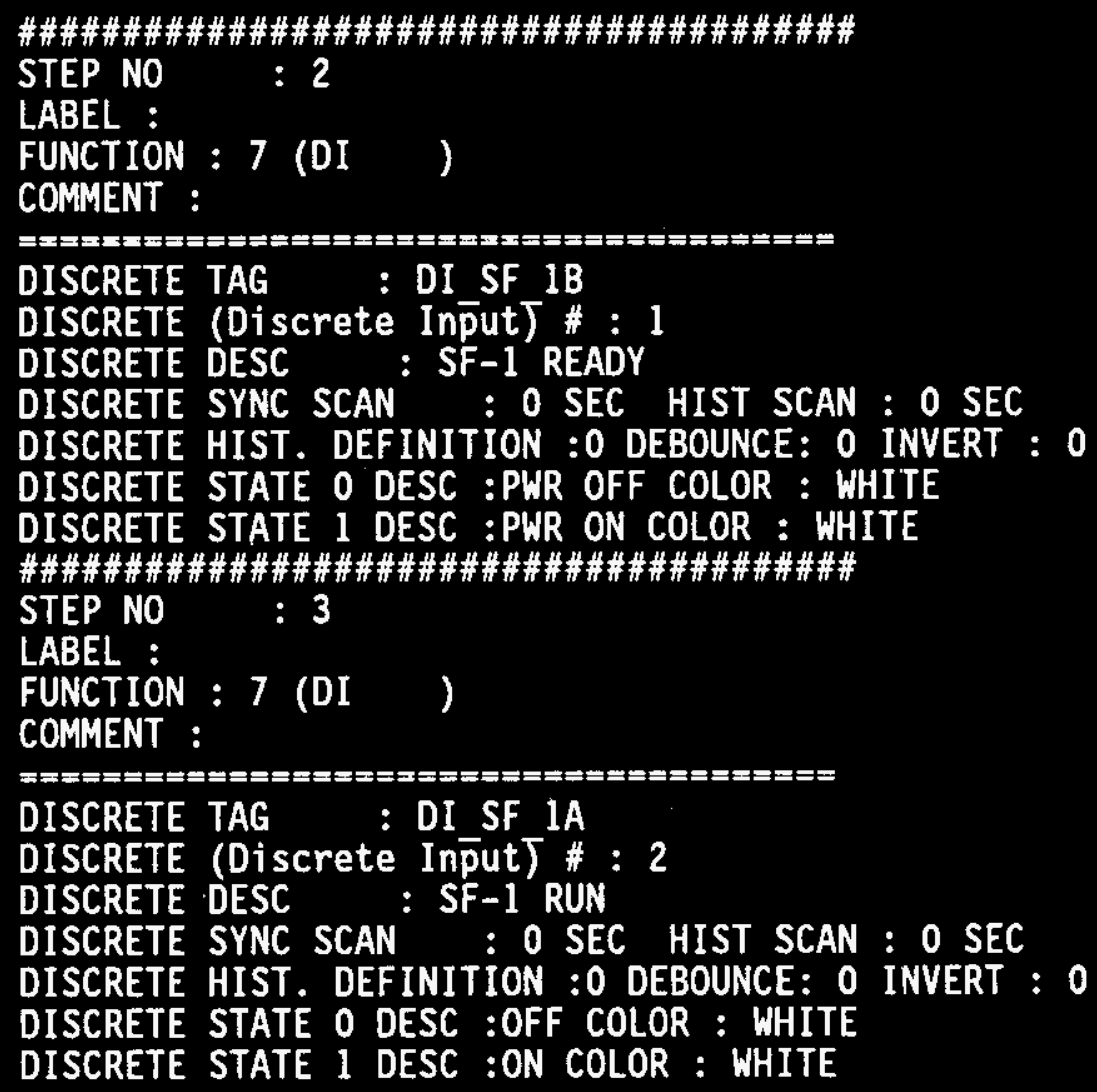


WHC-SD-CP-CSWD-016

Volume 4

Revision 1

Page 57

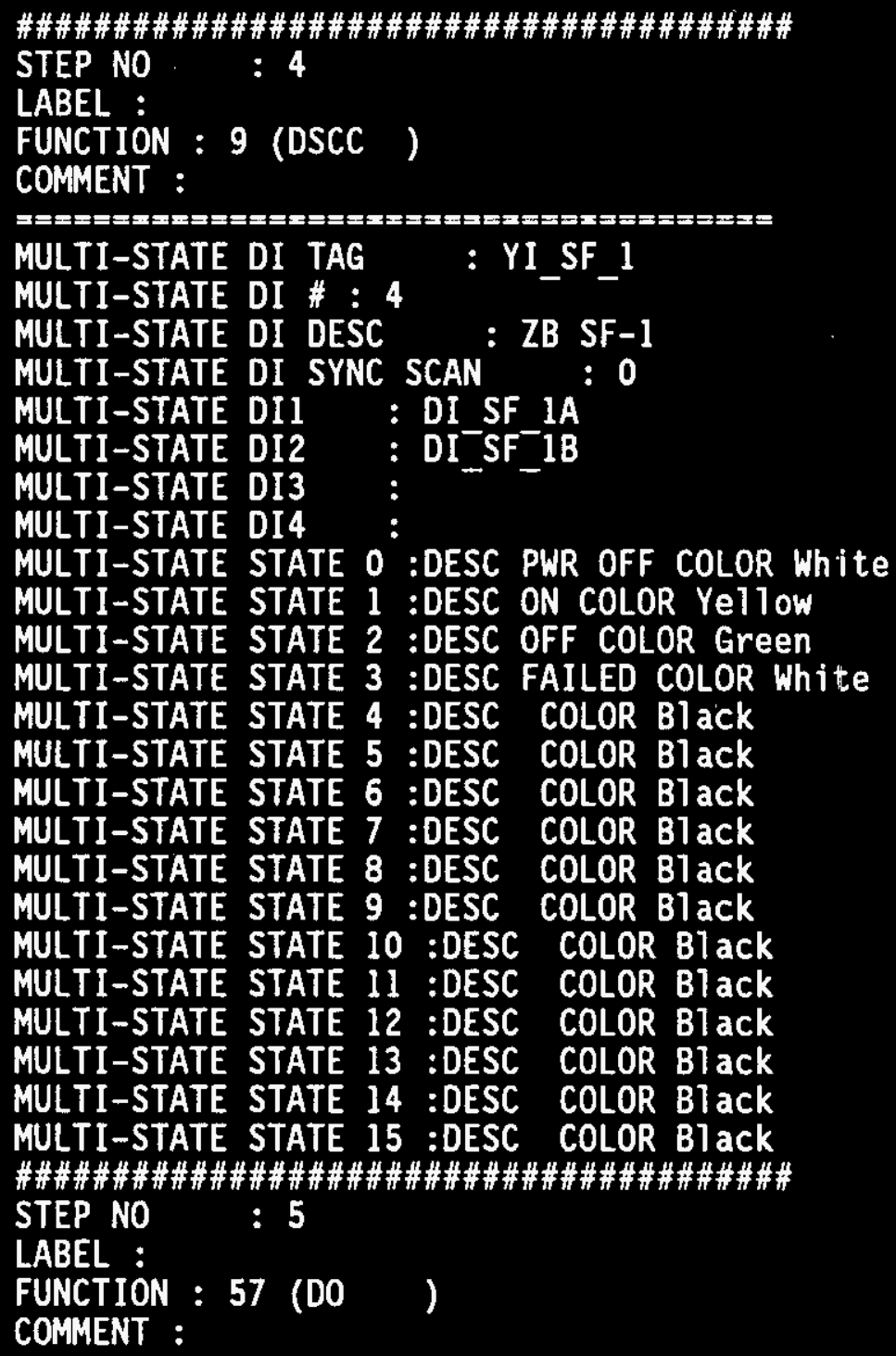


\#\#\#\#\#\#\#\#\#\#\#\#\#\#\#\#\#\#\#\#\#\#\#\#\#\#\#\#\#\#\#\#\#\#\#\#\#\#\#\#

STEP NO : 6

LABEL :

FUNCTION : 57 (DO )

COMMENT :

DISCRETE TAG : DO EV 1
DISCRETE (Discrete OUtpUE) \# : 20
DISCRETE DESC : DM-1
DISCRETE SYNC SCAN :0 SEC HIST SCAN : O SEC

DISCRETE HIST. DEFINITION :0 DEBOUNCE: 0 MODIFY : 0

DISCRETE STATE 0 DESC :CLOSED COLOR : WHITE

DISCRETE STATE 1 DESC :OPEN COLOR : WHITE

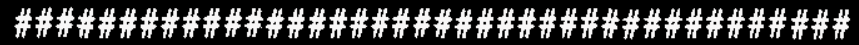

STEP NO : 7

LABEL :

FUNCTION : 57 (DO)

COMMENT :

DISCRETE TAG : DO EV 2

DISCRETE (Discrete OutpuE) \# : 21

DISCRETE DESC : DM-3

DISCRETE SYNC SCAN : O SEC HIST SCAN : O SEC

DISCRETE HIST. DEFINITION :0 DEBOUNCE: 0 MODIFY : 0

DISCRETE STATE O DESC :CLOSED COLOR : WHITE

DISCRETE STATE 1 DESC :OPEN COLOR : WHITE

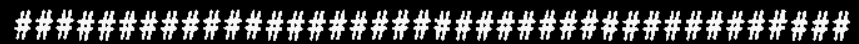

STEP NO : 8

LABEL :

FUNCTION : 75 (DST)

COMMENT :OPEN DM-1 ON SF-1 ON

PARAMETERS :

1)DI SF 1A. 2)0 3)0 4)0 5)DO EV 1.

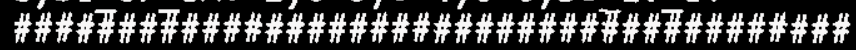

STEP NO : 9

LABEL :

FUNCTION : 75 CDST

COMMENT :OPEN DM-3 ON SF-1 ON

PARAMETERS :

1)DI SF 1A. 2)0 3)0 4)0 5)DO EV 2.

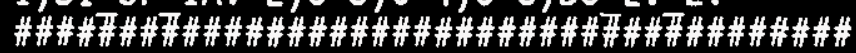

STEP NO $: 10$

LABEL : FIRE

FUNCTION : 69 (LC

COMMENT : FIRE ALARM

PARAMETERS :

1)XA_CSA|XA_FAZB |XA_FAZNB 


$$
\begin{gathered}
\text { WHC-SD-CP-CSWD-016 } \\
\text { Volume } 4 \\
\text { Revision } 1 \\
\text { Page } 59
\end{gathered}
$$

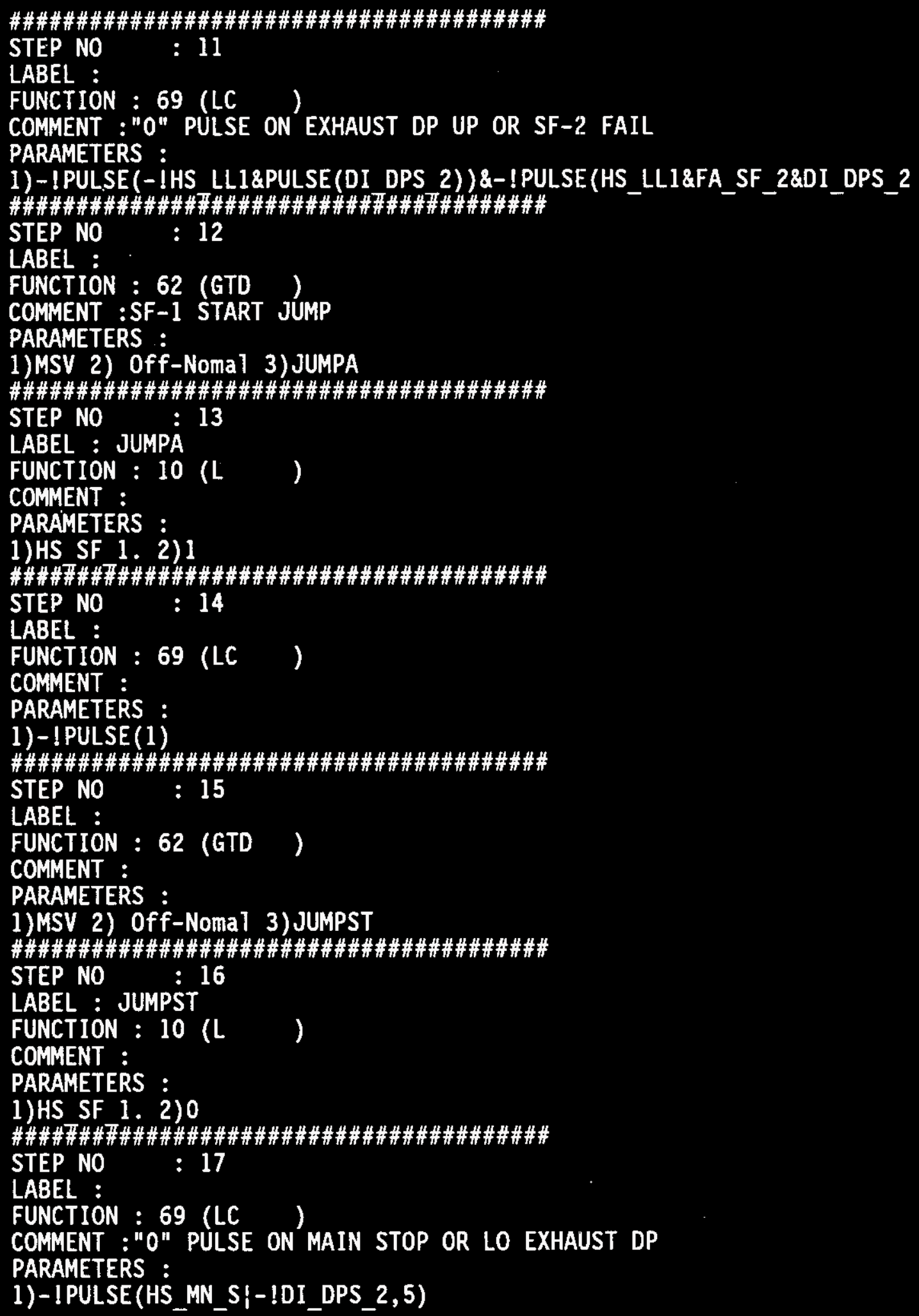


WHC-SD-CP-CSWD-016

Volume 4

Revision 1

Page 60

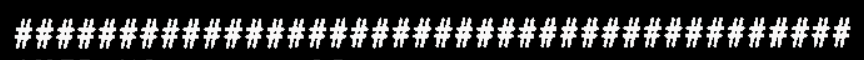

STEP NO : 18

LABEL :

FUNCTION : 62 (GTD)

COMMENT :STOP JUMP

PARAMETERS :

1) MSV 2) Off-Nomal 3) JUMPB

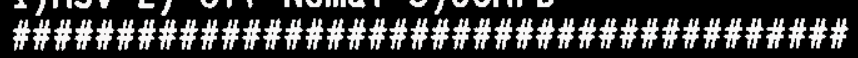

STEP NO

: 19

LABEL : JUMPB

FUNCTION : $10(\mathrm{~L})$

COMMENT :

PARAMETERS :

1) HS SF 1. 2)0

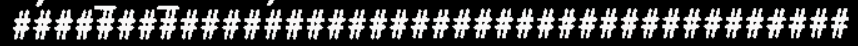

STEP NO : 20

LABEL :

FUNCTION : 69 (LC

COMMENT :HOLD ON "STOP" IF FIRE ALARM

PARAMETERS :

1)-IFIRE

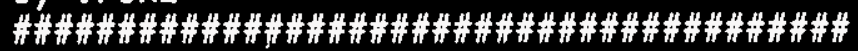

STEP NO : 21

LABEL :

FUNCTION : 62 (GTD )

COMMENT :SF-1 FIRE ALARM STOP JUMP

PARAMETERS :

1)MSV 2) Off-Nomal 3) JUMPC

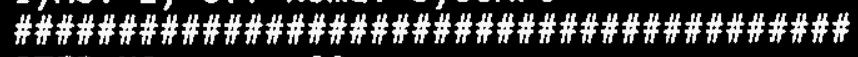

STEP NO

: 22

LABEL : JUMPC

FUNCTION : $10(\mathrm{~L} \cdot)$

COMMENT :

PARAMETERS :

1) HS SF 1. 2)0

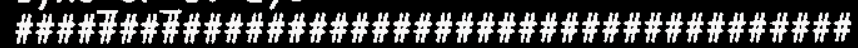

STEP NO : 23

LABEL :

FUNCTION : 73 (LAT)

COMMENT :SF-1 CONTROL LOGIC

PARAMETERS :

1) LATCH=PULSE(HS SF 1,5) 2) UNLATCH=-HS_SF_1! (DELAY(HS_S 3)0 4)DO_SF_1. 5)0

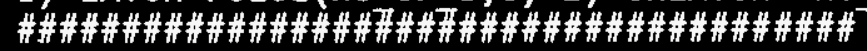

STEP NO : 24

LABEL :

FUNCTION : 69 (LC

COMMENT : ALARM ON NO FLOW OR OFF WHEN HS IS ON (EXCEPT STARTUP)

PARAMETERS :

1)DELAY(HS_SF_1, 15)\&-(1DI_SF_1A\&!DI_FS_1) 
WHC-SD-CP-CSWD-016

Volume 4

Revision 1

Page 61

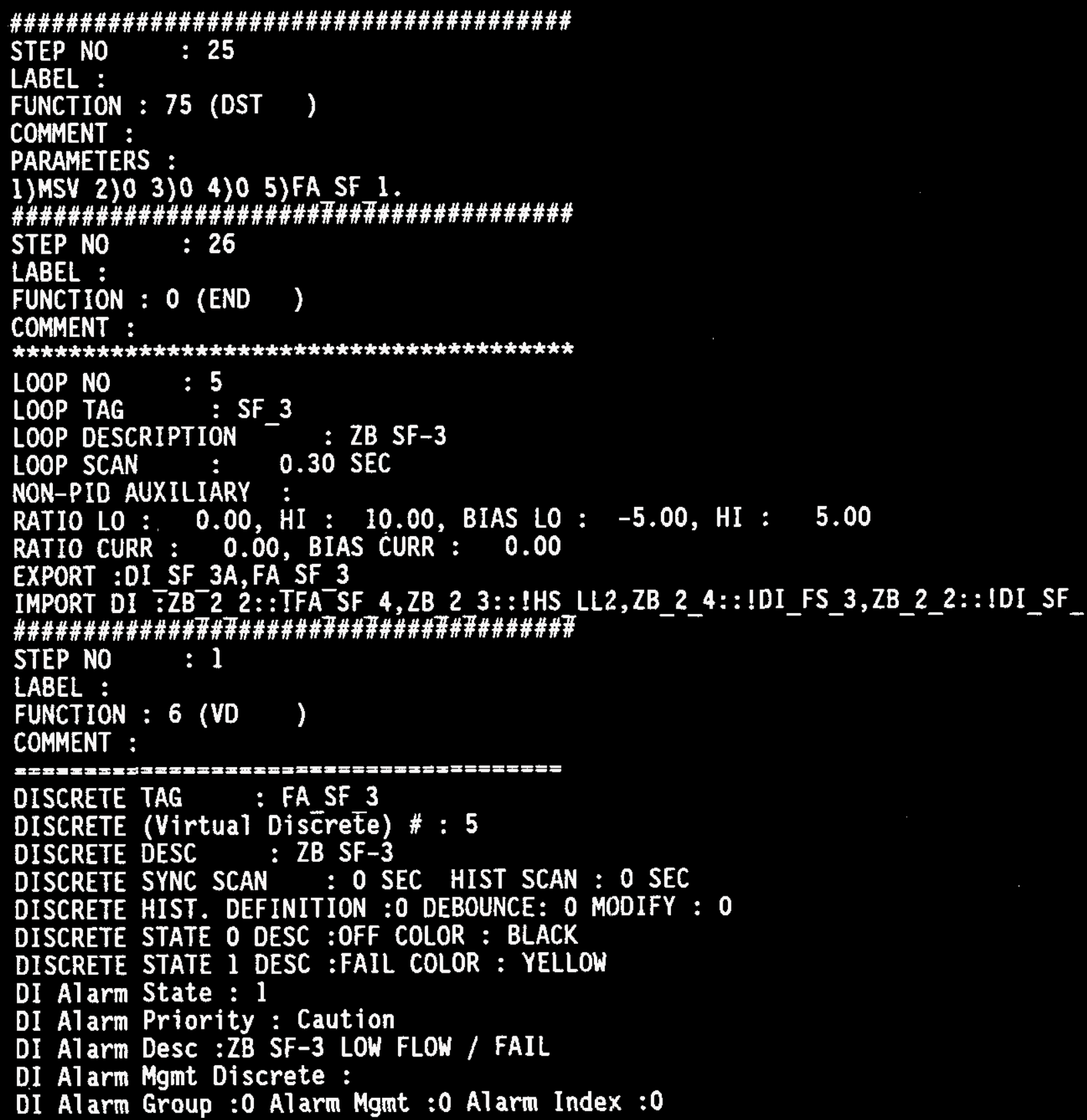


WHC-SD-CP-CSWD-016

Volume 4

Revision 1

Page 62

\#\#\#\#\#\#\#\#\#\#\#\#\#\#\#\#\#\#\#\#\#\#\#\#\#\#\#\#\#\#\#\#\#\#\#\#\#\#\#\#

STEP NO : 2

LABEL :

FUNCTION : 7 (DI )

COMMENT :

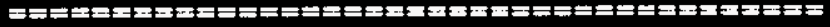

DISCRETE TAG : DI SF 3B

DISCRETE (Discrete Input) \#: 15

DISCRETE DESC : SF-3 READY

DISCRETE SYNC SCAN : O SEC HIST SCAN : O SEC

DISCRETE HIST. DEFINITION :0 DEBOUNCE: 0 INVERT : 0

DISCRETE STATE $O$ DESC :PWR OFF COLOR : WHITE

DISCRETE STATE 1 DESC :PWR ON COLOR : WHITE

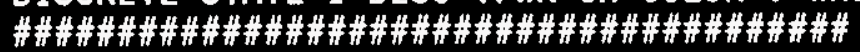

STEP NO

$: 3$

LABEL :

FUNCTION : 7 (DI )

COMMENT :

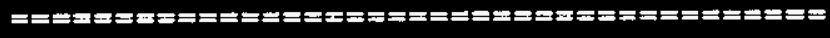

DISCRETE TAG : DI SF 3A

DISCRETE (Discrete Input) \# : 16

DISCRETE DESC : SF-3 RUN

DISCRETE SYNC SCAN : 0 SEC HIST SCAN : O SEC

DISCRETE HIST. DEFINITION :0 DEBOUNCE: 0 INVERT : 0

DISCRETE STATE 0 DESC :OFF COLOR : WHITE

DISCRETE STATE 1 DESC :ON COLOR : WHITE 
WHC-SD-CP-CSWD-016

Volume 4

Revision 1

Page 63

\#\#\#\#\#\#\#\#\#\#\#\#\#\#\#\#\#\#\#\#\#\#\#\#\#\#\#\#\#\#\#\#\#\#\#\#\#\#\#\#

STEP NO : 4

LABEL :

FUNCTION : 9 (DSCC)

COMMENT :

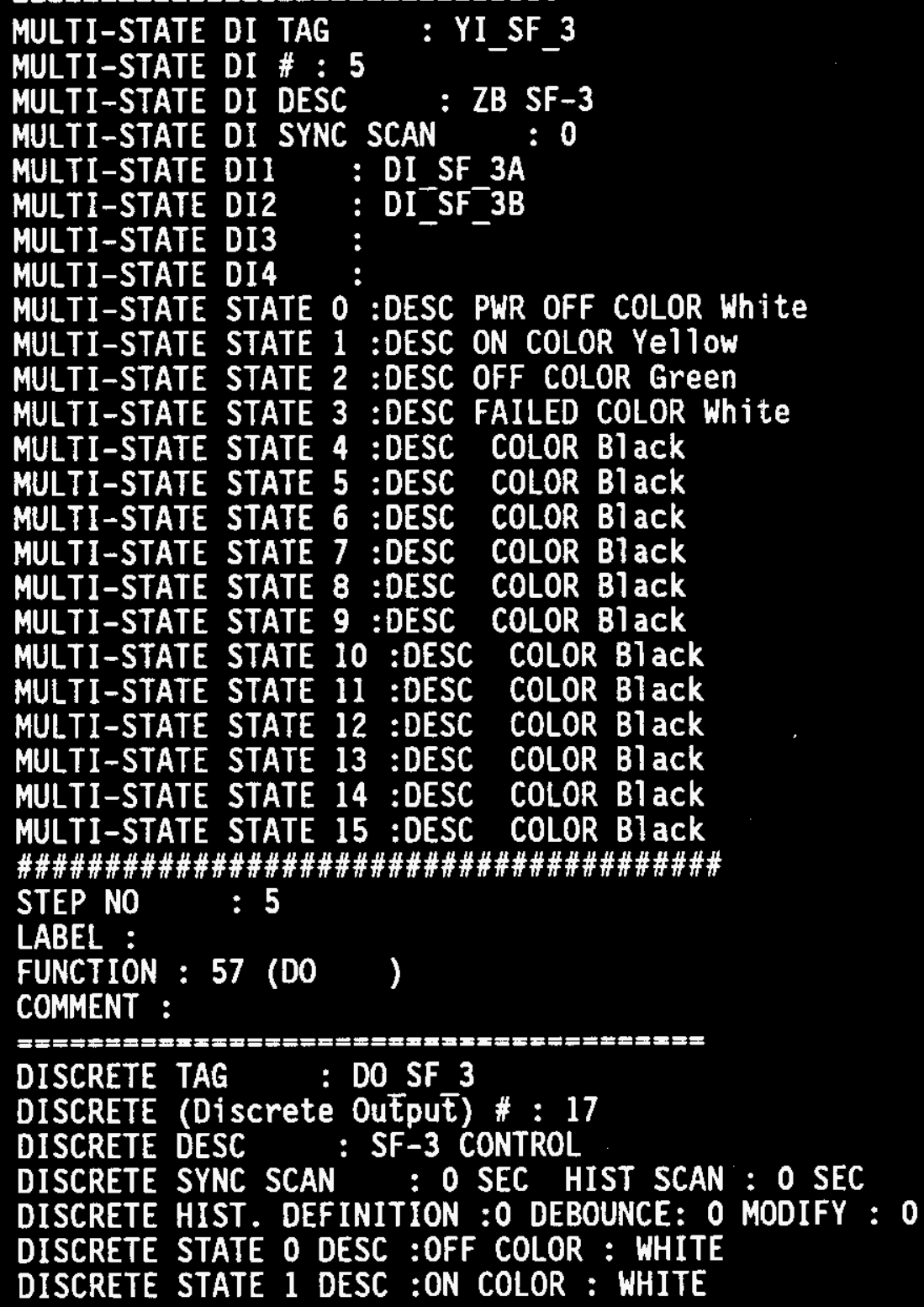


\#\#\#\#\#\#\#\#\#\#\#\#\#\#\#\#\#\#\#\#\#\#\#\#\#\#\#\#\#\#\#\#\#\#\#\#\#\#\#\#

STEP NO : 6

LABEL :

FUNCTION : 57 (DO)

COMMENT :

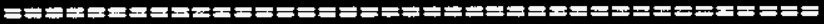

DISCRETE TAG : DO EV 5

DISCRETE (Discrete Output) \# : 29

DISCRETE DESC : DM-7

DISCRETE SYNC SCAN : O SEC HIST SCAN : O SEC

DISCRETE HIST. DEFINITION :0 DEBOUNCE: 0 MODIFY : 0

DISCRETE STATE 0 DESC :CLOSED COLOR : WHITE

DISCRETE STATE 1 DESC :OPEN COLOR : WHITE

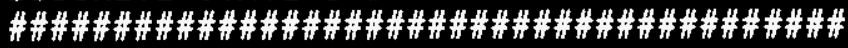

STEP NO $: 7$

LABEL :

FUNCTION : 57 (DO )

COMMENT :

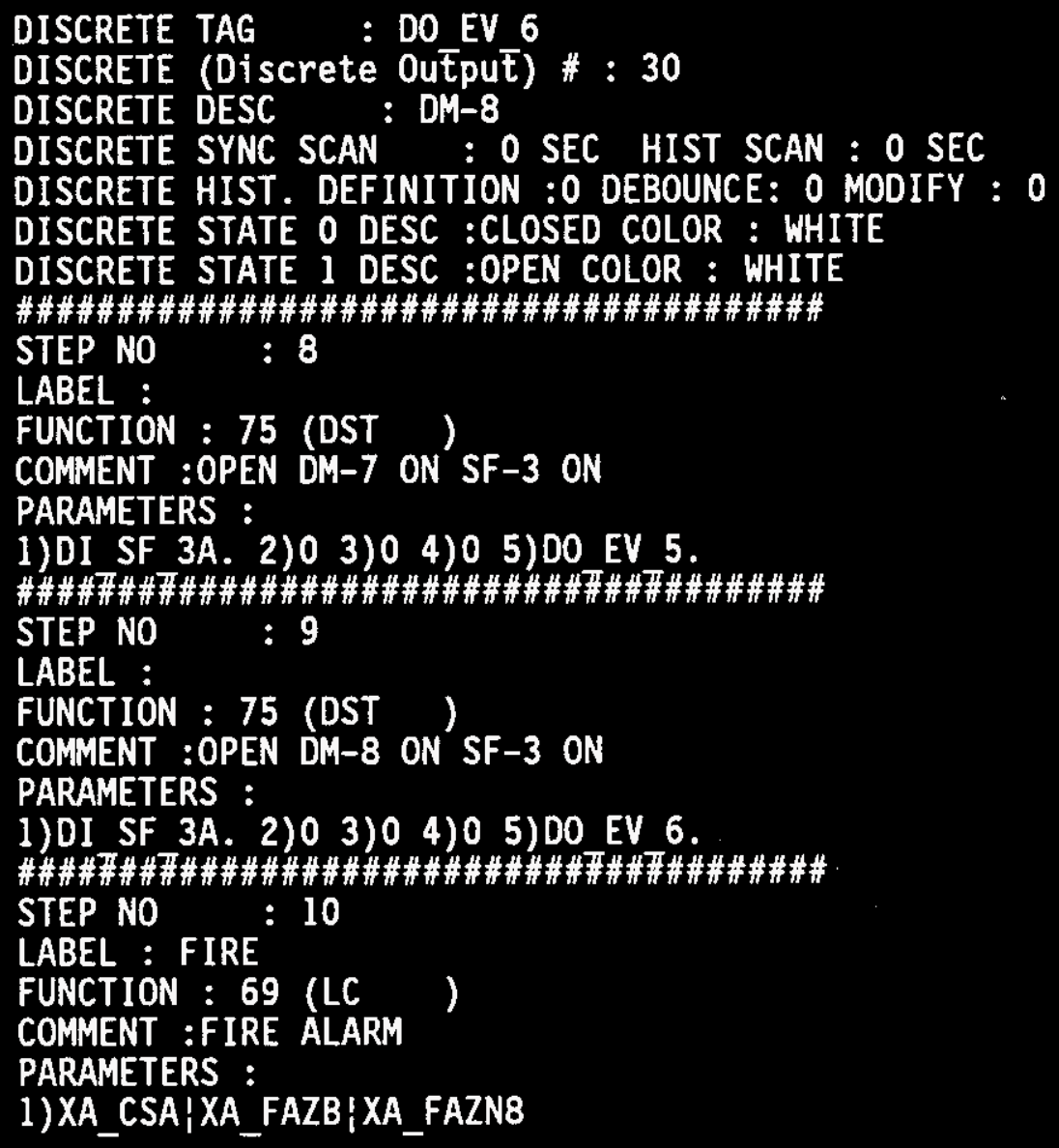




\section{WHC-SD-CP-CSWD-016 \\ Volume 4 \\ Revision 1 \\ Page 65}

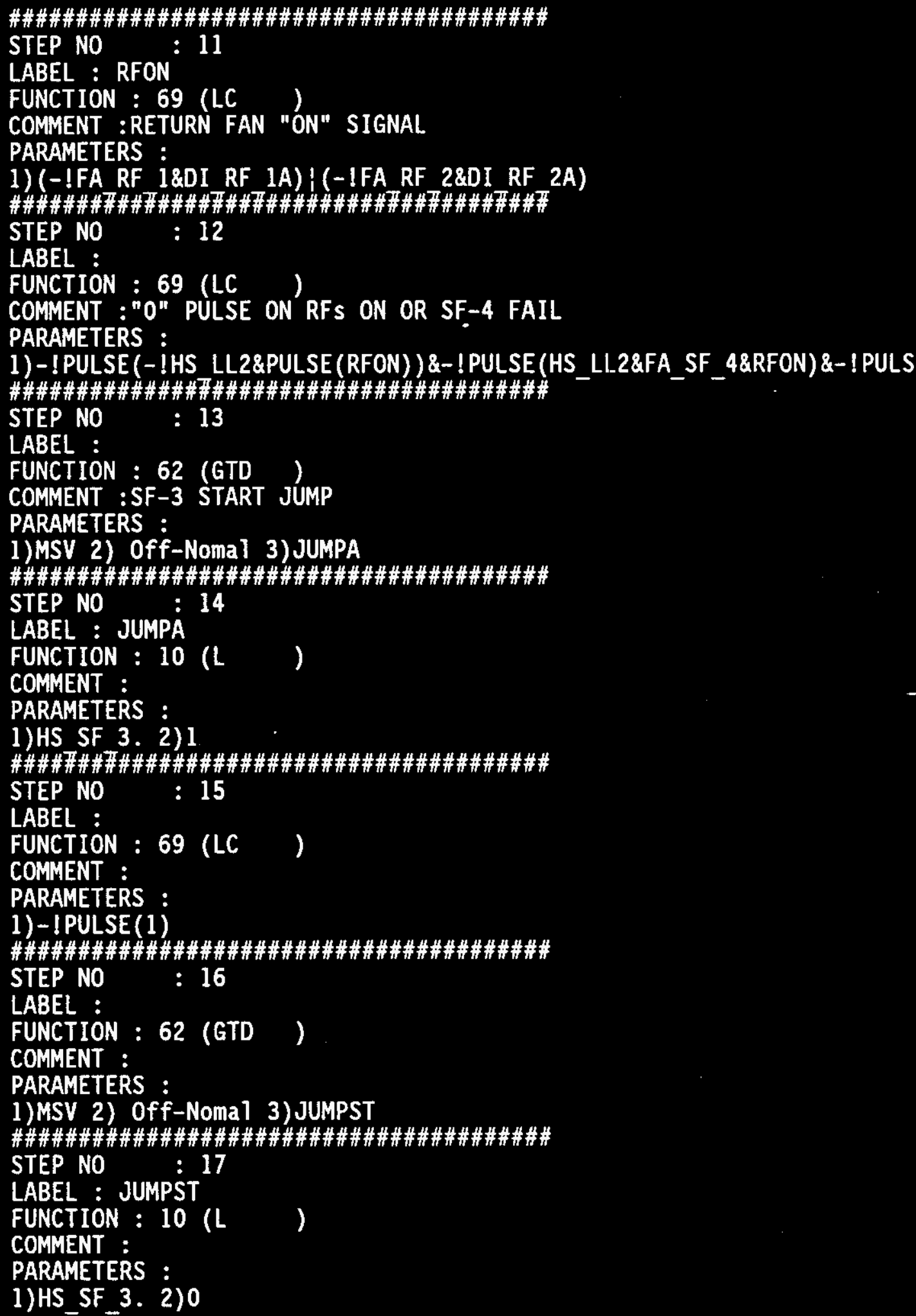


\#\#\#\#\#\#\#\#\#\#\#\#\#\#\#\#\#\#\#\#\#\#\#\#\#\#\#\#\#\#\#\#\#\#\#\#\#\#\#\#

STEP NO : 18

LABEL :

FUNCTION : 69 (LC

COMMENT : "O" PULSE ON NDA STOP OR RFS OFF

PARAMETERS :

1)-! PULSE(HS NOA 5,5$) \&-$ ! PULSE (-! RFON)

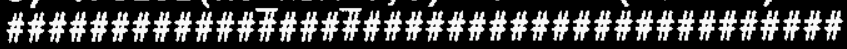

STEP NO : 19

LABEL :

FUNCTION : 62 (GTD )

COMMENT :STOP JUMP

PARAMETERS :

1)MSV 2) Off-Nomal 3) JUMPB

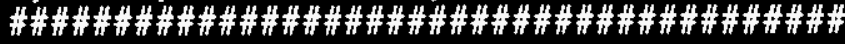

STEP NO

$: 20$

LABEL : JUMPB

FUNCTION : $10(\mathrm{~L})$

COMMENT :

PARAMETERS :

1)HS SF 3, 2)0

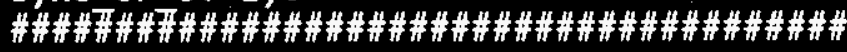

STEP NO : 21

LABEL :

FUNCTION : 69 (LC

COMMENT :HOLD ON "STOP" IF FIRE ALARM

PARAMETERS :

1) - ! FIRE

\#\#\#\#\#\#\#\#\#\#\#\#\#\#\#\#\#\#\#\#\#\#\#\#\#\#\#\#\#\#\#\#\#\#\#\#\#\#

STEP NO : 22

LABEL :

FUNCTION : 62 (GTD )

COMMENT :FIRE ALARM STOP JUMP

PARAMETERS :

1)MSV 2) Off-Nomal 3) JUMPC

\#\#\#\#\#\#\#\#\#\#\#\#\#\#\#\#\#\#\#\#\#\#\#\#\#\#\#\#\#\#\#\#\#\#\#\#\#\#\#

STEP NO : 23

LABEL : JUMPC

FUNCTION : $10(\mathrm{~L} \quad)$

COMMENT :

PARAMETERS :

1)HS SF 3. 2)0

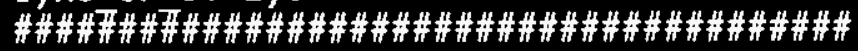

STEP NO : 24

LABEL :

FUNCTION : 73 (LAT)

COMMENT : SF-3 CONTROL LOGIC

PARAMETERS :

1) LATCH $=$ PULSE (HS_SF_3,5) 2) UNLATCH=-HS_SF_3! (DELAY(HS_S 3)0 4)00_SF_3. 5)0 

WHC-SD-CP-CSWD-016
Volume 4
Revision 1
Page 67

\#\#\#\#\#\#\#\#\#\#\#\#\#\#\#\#\#\#\#\#\#\#\#\#\#\#\#\#\#\#\#\#\#\#\#\#\#\#\#\#

STEP NO

$: 25$

LABEL :

FUNCTION : 69 (LC)

COMMENT : ALARM ON NO FLOW OR OFF WHEN HS IS ON (EXCEPT STARTUP)

PARAMETERS :

1) DELAY (HS SF 3, 15)\&-(!DI SF 3A\&!DI FS 3)

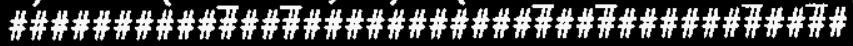

STEP NO : 26

LABEL :

FUNCTION : 75 (DST )

COMMENT :

PARAMETERS :

1)MSV 2)0 3)0 4)0 5)FA SF 3.

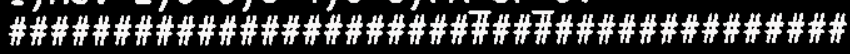

STEP NO : 27

LABEL :

FUNCTION : 0 (END )

COMMENT :

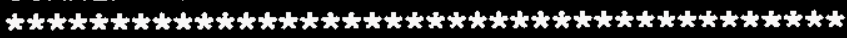

LOOP NO : 6

LOOP TAG : SF_5

LOOP DESCRIPTION - : ZB SF-5

LOOP SCAN : 0.30 SEC

NON-PID AUXILIARY :

RATIO LO: $0.00, \mathrm{HI}: 10.00$, BIAS LO : -5.00 , HI : 5.00

RATIO CURR : 0.00 , BIAS CURR : 0.00

EXPORT :DI SF 5A,FA SF 5

IMPORT DI $: Z^{-}{ }^{-2} 4::$ DI FS 10

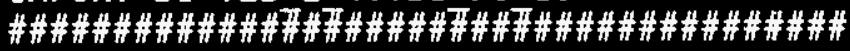

STEP NO : 1

LABEL :

FUNCTION : 6 (VD )

COMMENT :

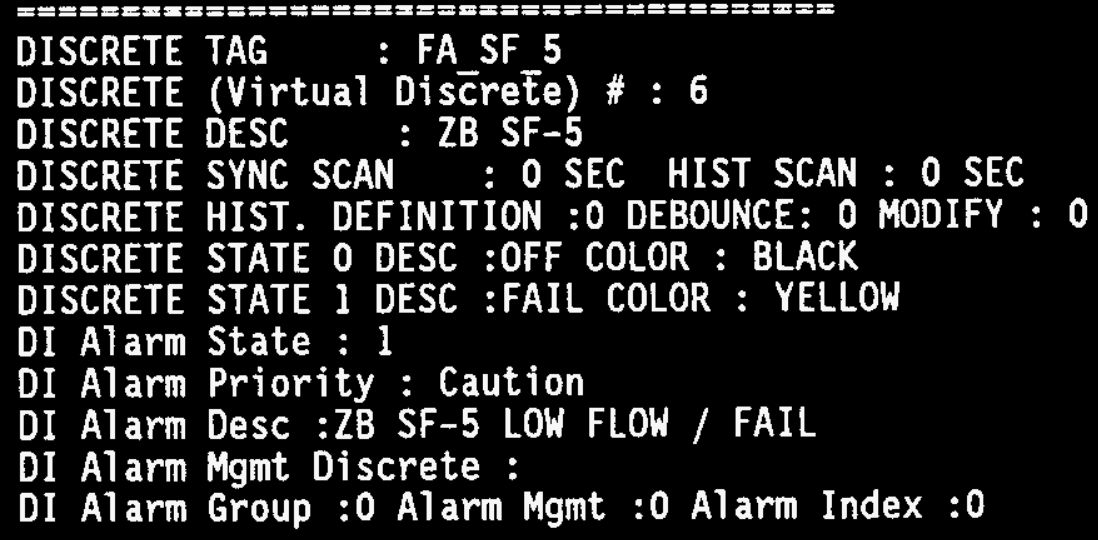




\section{WHC-SD-CP-CSWD-016 \\ Vol ume 4 \\ Revision 1 \\ Page 68}

\#\#\#\#\#\#\#\#\#\#\#\#\#\#\#\#\#\#\#\#\#\#\#\#\#\#\#\#\#\#\#\#\#\#\#\#\#\#\#

STEP NO : 2

LABEL :

FUNCTION : 7 (DI )

COMMENT :

DISCRETE TAG : DI SF $5 B$

DISCRETE (Discrete Input) \# : 12

DISCRETE DESC : SF-5 READY

DISCRETE SYNC SCAN : O SEC HIST SCAN : O SEC

DISCRETE HIST. DEFINITION :0 DEBOUNCE: 0 INVERT : 0

DISCRETE STATE $O$ DESC :PWR OFF COLOR : WHITE

DISCRETE STATE 1 DESC :PWR ON COLOR : WHITE

\#\#\#\#\#\#\#\#\#\#\#\#\#\#\#\#\#\#\#\#\#\#\#\#\#\#\#\#\#\#\#\#\#\#\#\#\#\#\#

STEP NO : 3

LABEL :

FUNCTION : 7 (DI )

COMMENT :

DISCRETE TAG : DI_SF $5 A$

DISCRETE (Discrete Input) \# : 13

DISCRETE DESC : SF-5 RUN

DISCRETE SYNC SCAN : O SEC HIST SCAN : O SEC

DISCRETE HIST. DEFINITION :0 DEBOUNCE: 0 INVERT : 0

DISCRETE STATE O DESC :OFF COLOR : WHITE

DISCRETE STATE 1 DESC :ON COLOR : WHITE 
WHC-SD-CP-CSWD-016

Volume 4

Revision 1

Page 69

\#\#\#\#\#\#\#\#\#\#\#\#\#\#\#\#\#\#\#\#\#\#\#\#\#\#\#\#\#\#\#\#\#\#\#\#\#\#\#\#

STEP NO : 4

LABEL :

FUNCTION : 9 (DSCC)

COMMENT :

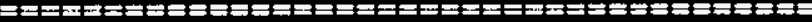

MULTI-STATE DI TAG

MULTI-STATE DI \#: 6

MULTI-STATE DI DESC

: YI_SF_5

MULTI-STATE DI SYNC SCAN : 0

MULTI-STATE DII : DI SF 5A

MULTI-STATE DI2 : DI_SF $5 B$

MULTI-STATE DI3 :

MULTI-STATE DI4 :

MULTI-STATE STATE 0 :DESC PWR OFF COLOR White

MULTI-STATE STATE 1 :DESC ON COLOR YeTlOW

MULTI-STATE STATE 2 :DESC OFF COLOR Green

MULTI-STATE STATE 3 :DESC FAILED COLOR White

MULTI-STATE STATE 4 :DESC COLOR B1 ack

MULTI-STATE STATE 5 :DESC COLOR B1ack

MULTI-STATE STATE 6 :DESC COLOR Black

MULTI-STATE STATE 7 :DESC COLOR Black

MULTI-STATE STATE 8 :DESC COLOR Black

MULTI-STATE STATE 9 :DESC COLOR Black

MULTI-STATE STATE 10 :DESC COLOR Black

MULTI-STATE STATE 11 :DESC COLOR Black

MULTI-STATE STATE 12 :DESC COLOR Black

MULTI-STATE STATE 13 :DESC COLOR Black

MULTI-STATE STATE 14 :DESC COLOR Black

MULTI-STATE STATE 15 :DESC COLOR BTack

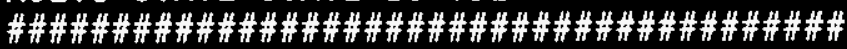

STEP NO : 5

LABEL :

FUNCTION : $57(00)$

COMMENT :

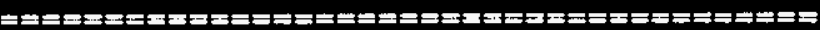

DISCRETE TAG : DO SF 5

DISCRETE (Discrete Output) \#: 14

DISCRETE DESC : SF-5 CONTROL

DISCRETE SYNC SCAN : 0 SEC HIST SCAN : 0 SEC

DISCRETE HIST. DEFINITION :0 DEBOUNCE: 0 MODIFY : 0

DISCRETE STATE O DESC :OFF COLOR : WHITE

DISCRETE STATE 1 DESC :ON COLOR : WHITE 
WHC-SD-CP-CSWD-016

Vol ume 4

Revision 1

Page 70

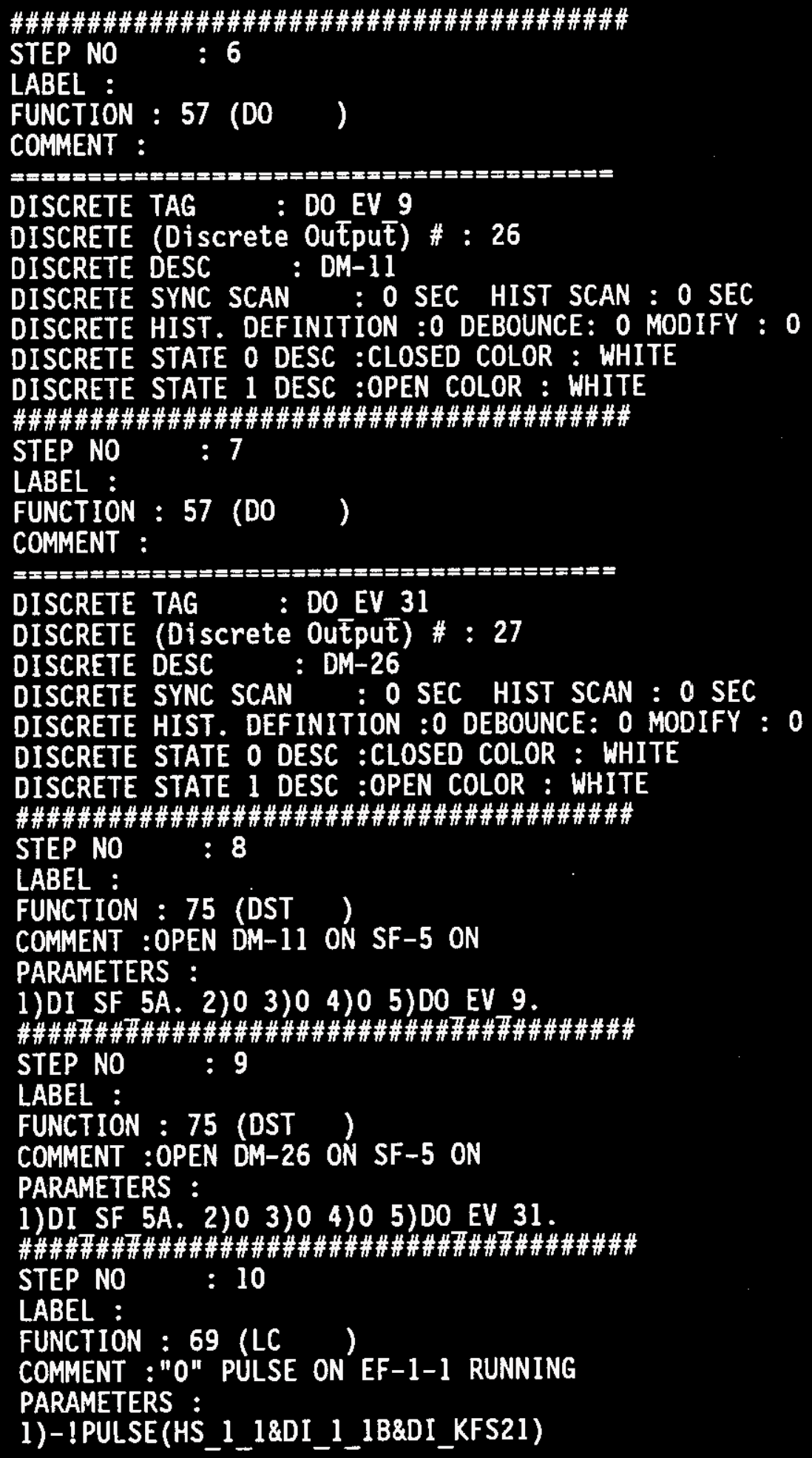


\#\#\#\#\#\#\#\#\#\#\#\#\#\#\#\#\#\#\#\#\#\#\#\#\#\#\#\#\#\#\#\#\#\#\#\#\#\#\#\#

STEP NO : 11

LABEL :

FUNCTION : 62 (GTD)

COMMENT :SF-5 START JUMP

PARAMETERS :

1)MSV 2) Off-Nomal 3) JUMPA

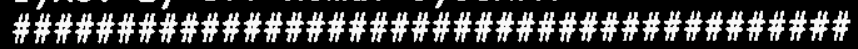

STEP NO : 12

LABEL : JUMPA

FUNCTION : $10(\mathrm{~L})$

COMMENT :

PARAMETERS :

1)HS SF 5. 2) 1

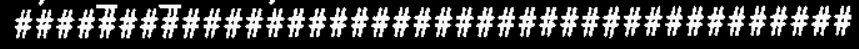

STEP NO : 13

LABEL :

FUNCTION : 69 (LC

COMMENT : "0" PULSE OF EF-1-1 FAIL OR OFF PARAMETERS :

1)-! PULSE(FA 11 1!-RUN 11 1)

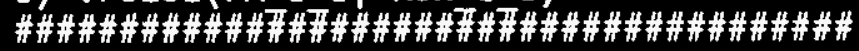

STEP NO : 14

LABEL :

FUNCTION : 62 (GTD)

COMMENT :SF-5 STOP JUMP

PARAMETERS :

1)MSV 2) Off-Nomal 3) JUMPB

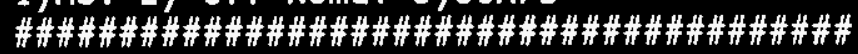

STEP NO : 15

LABEL : JUMPB

FUNCTION : $10(\mathrm{~L})$

COMMENT :

PARAMETERS :

1)HS SF 5. 2)0

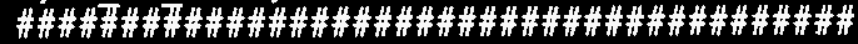
STEP NO : 16

LABEL :

FUNCTION : 69 (LC )

COMMENT :HOLD ON STOP IF COLD SMOKE ALARM

PARAMETERS :

1) - IXA CSA

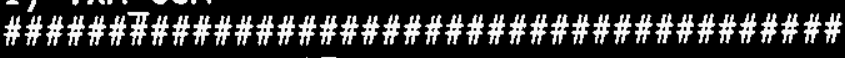

STEP NO : 17

LABEL :

FUNCTION : 62 (GTD )

COMMENT :SF-5 STOP JUMP

PARAMETERS :

1)MSV 2) Off-Nomal 3) JUMPC 


\section{WHC-SD-CP-CSWD-016 \\ Volume 4 \\ Revision 1 \\ Page 72}

\#\#\#\#\#\#\#\#\#\#\#\#\#\#\#\#\#\#\#\#\#\#\#\#\#\#\#\#\#\#\#\#\#\#\#\#\#\#\#\#

STEP NO

$: 18$

LABEL : JUMPC

FUNCTION : $10(\mathrm{~L})$

COMMENT :

PARAMETERS :

1)HS SF 5. 2) 0

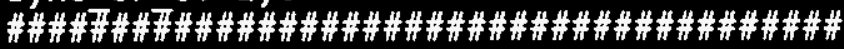

STEP NO : 19

LABEL :

FUNCTION : 73 (LAT)

COMMENT :SF-5 CONTROL LOGIC

PARAMETERS :

1) LATCH=PULSE(HS SF 5,5) 2) UNLATCH=-HS_SF_5 3)0 4)D0_SF_5. 5)0

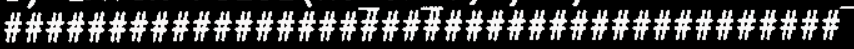

STEP NO : 20

LABEL :

FUNCTION : 69 (LC

COMMENT : ALARM ON NO FLOW OR OFF WHEN HS IS ON (EXCEPT STARTUP)

PARAMETERS :

1)HS SF 5\&-! PULSE(HS SF 5, 15)\&-(!DI SF 5A\&!DI_FS_10)

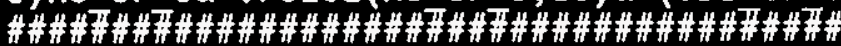

STEP NO : 21

LABEL :

FUNCTION : 75 (DST)

COMMENT :

PARAMETERS :

1)MSV 2)0 3)0 4)0 5) FA SF 5.

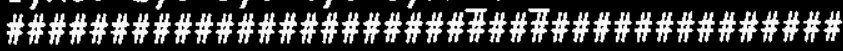

STEP NO : 22

LABEL :

FUNCTION : 0 (END)

COMMENT :

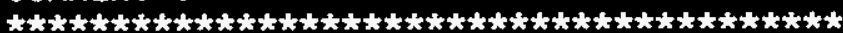

LOOP NO : 7

LOOP TAG : MISC M18

LOOP DESCRIPTION - MICON 18 MISC.

LOOP SCAN : 0.30 SEC

NON-PID AUXILIARY :

RATIO LO : 0.00 , HI : 10.00 , BIAS LO : -5.00 , HI : 5.00

RATIO CURR : 0.00 , BIAS CURR : 0.00 
WHC-SD-CP-CSWD-016

Vol ume 4

Revision 1

Page 73

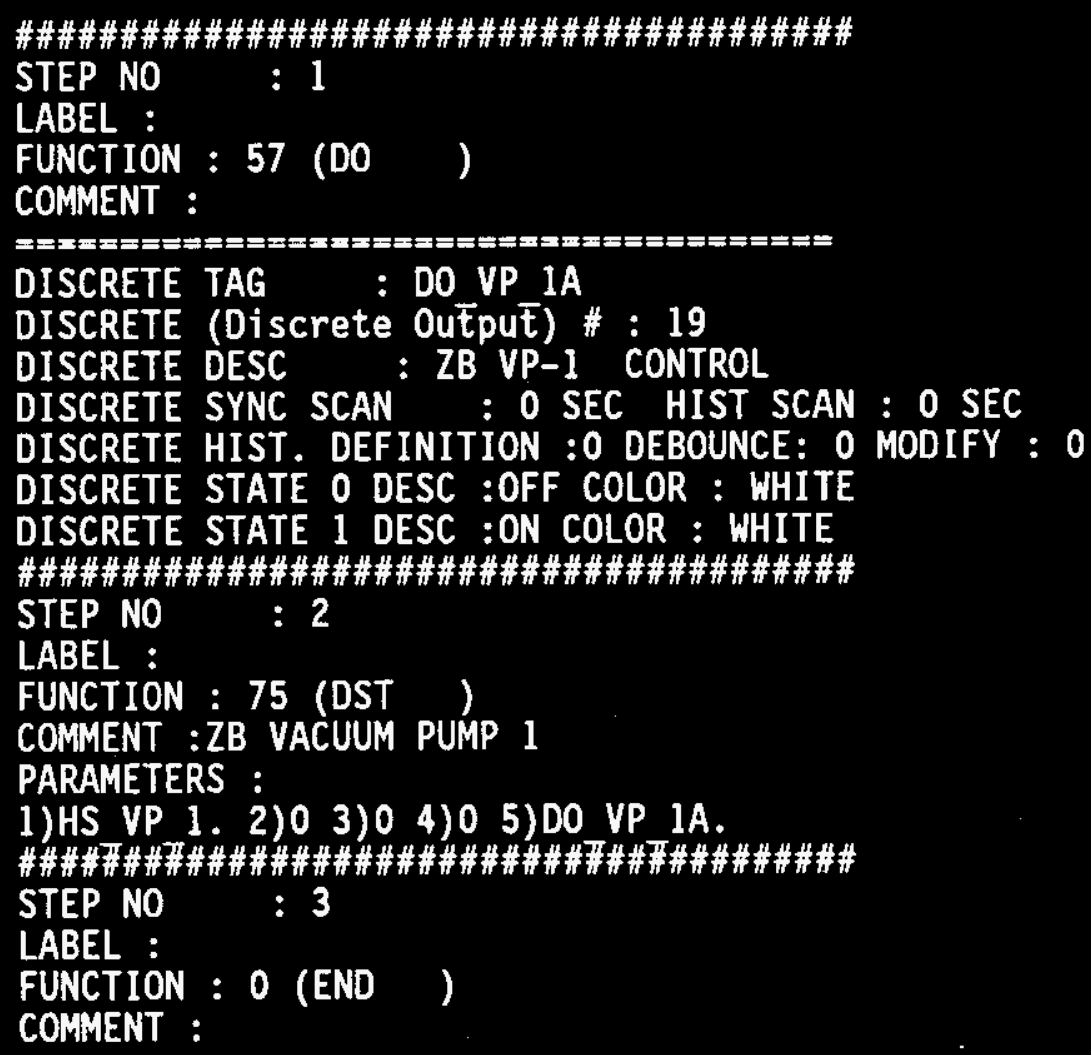


WHC-SD-CP-CSWD-016

Vol ume 4

Revision 1

Page 74

ZB_2_2.mic19_ro

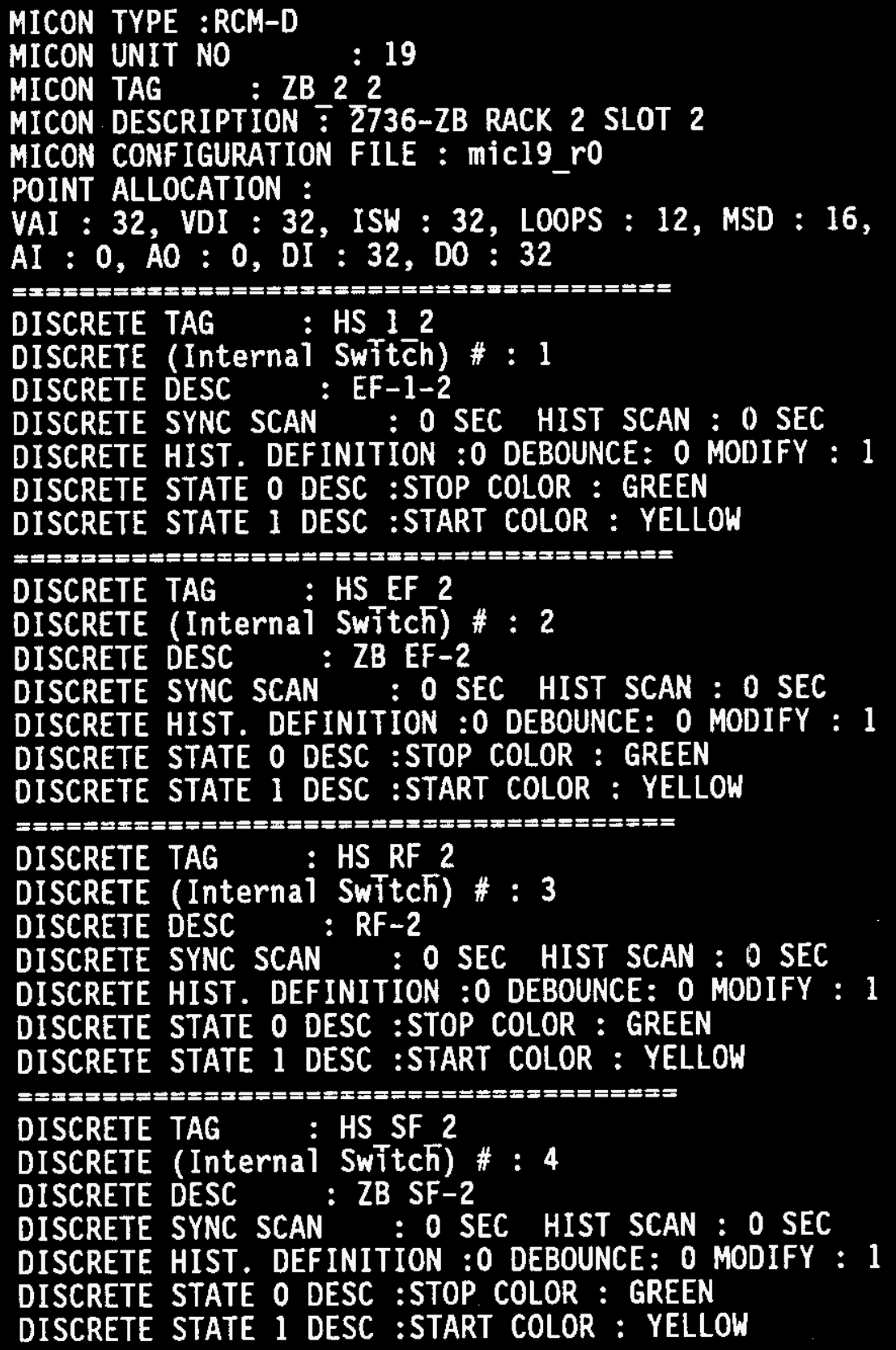


WHC-SD-CP-CSWD-016

Volume 4

Revision 1

Page 75

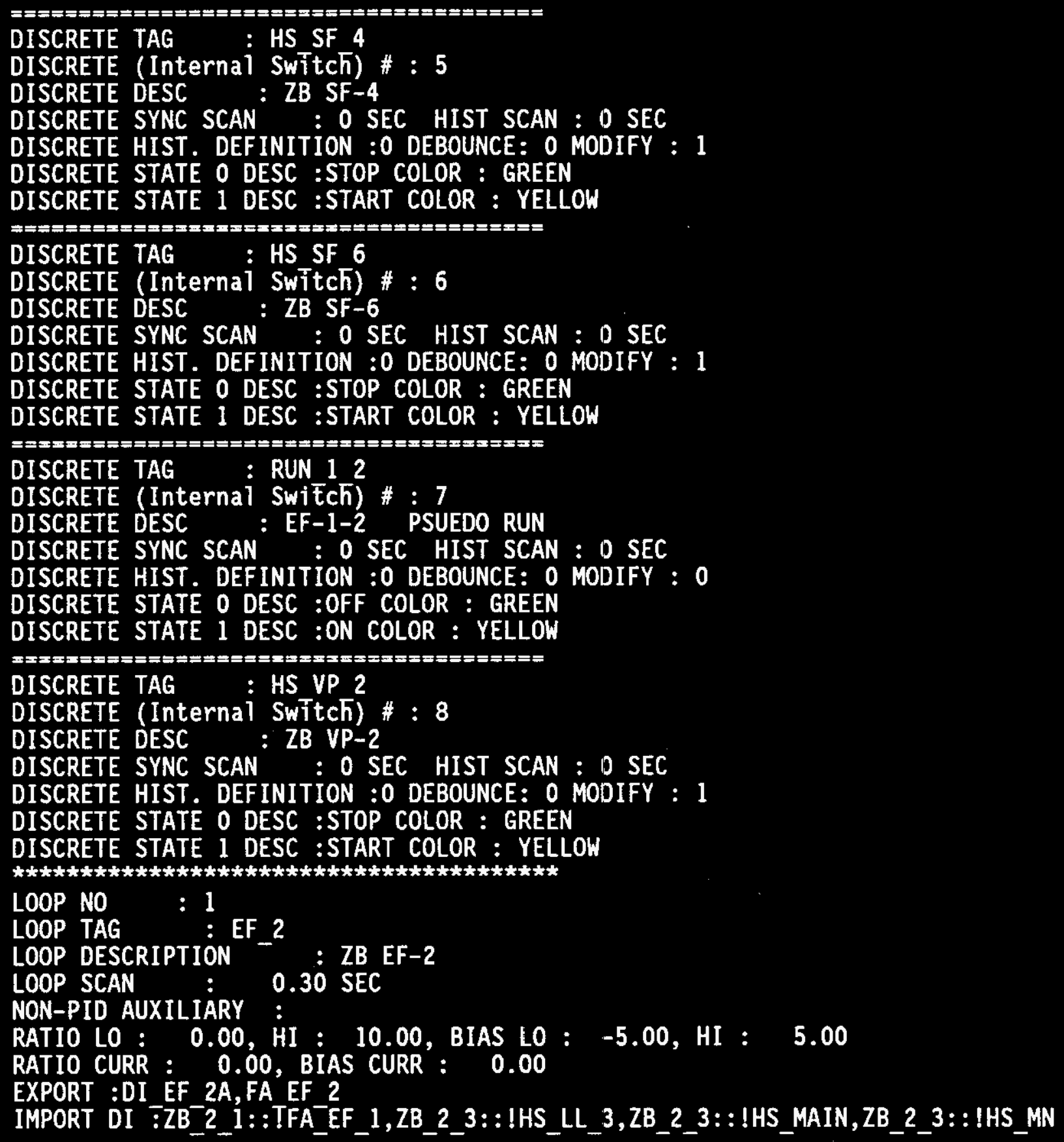




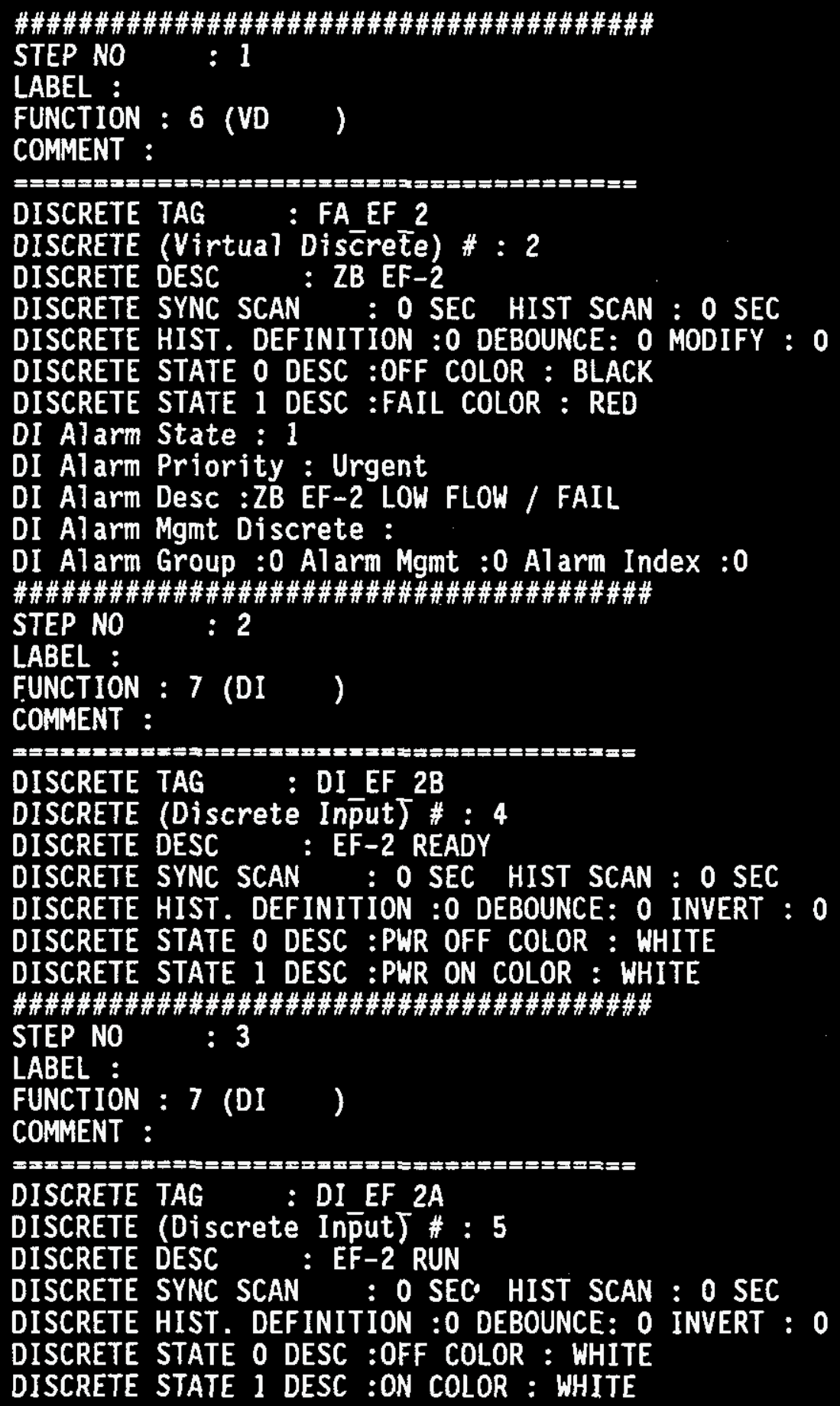


WHC-SD-CP-CSWD-016

Volume 4

Revision 1

Page 77

\#\#\#\#\#\#\#\#\#\#\#\#\#\#\#\#\#\#\#\#\#\#\#\#\#\#\#\#\#\#\#\#\#\#\#\#\#\#\#\#

STEP NO

$: 4$

LABEL :

FUNCTION : 9 (DSCC)

COMMENT :

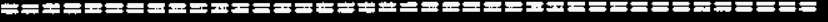

MULTI-STATE DI TAG

MULTI-STATE DI \# : 2

MULTI-STATE DI DESC : ZB EF-2

MULTI-STATE DI SYNC SCAN : 0

MULTI-STATE DI1 : DI EF_2A

MULTI-STATE DI2 : DI_EF_2B

MULTI-STATE DI3 :

MULTI-STATE DI4 :

MULTI-STATE STATE 0 :DESC PWR OFF COLOR White

MULTI-STATE STATE 1 :DESC ON COLOR Yellow

MULTI-STATE STATE 2 :DESC OFF COLOR Green

MULTI-STATE STATE 3 :DESC FAILED COLOR White

MULTI-STATE STATE 4 :DESC COLOR Black

MULTI-STATE STATE 5 :DESC COLOR Black

MULTI-STATE STATE 6 :DESC COLOR Black

MULTI-STATE STATE 7 :DESC COLOR Black

MULTI-STATE STATE 8 :DESC COLOR B1ack

MULTI-STATE STATE 9 :DESC COLOR B1ack

MULTI-STATE STATE 10 :DESC COLOR BTack

MULTI-STATE STATE 11 :DESC COLOR Black

MULTI-STATE STATE 12 :DESC COLOR Black

MULT.I-STATE STATE 13 :DESC COLOR Black

MULTI-STATE STATE 14 :DESC COLOR Black

MULTI-STATE STATE 15 :DESC COLOR Black

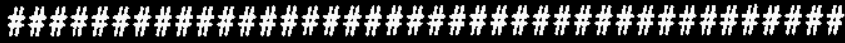

STEP NO : 5

LABEL :

FUNCTION : 57 (DO )

COMMENT :

DISCRETE TAG : DO EF 2

DISCRETE (Discrete Output) \#: 6

DISCRETE DESC : EF-2 CONTROL

DISCRETE SYNC SCAN : O SEC HIST SCAN : O SEC

DISCRETE HIST. DEFINITION :0 DEBOUNCE: 0 MODIFY : 0

DISCRETE STATE O DESC :OFF COLOR : WHITE

DISCRETE STATE 1 DESC :ON COLOR : WHITE 
WHC-SD-CP-CSWD-016

Volume 4

Revision 1

Page 78

\#\#\#\#\#\#\#\#\#\#\#\#\#\#\#\#\#\#\#\#\#\#\#\#\#\#\#\#\#\#\#\#\#\#\#\#\#\#\#

STEP NO : 6

LABEL :

FUNCTION : $57($ DO )

COMMENT :

=

DISCRETE TAG : DO EV 13

DISCRETE (Discrete Output) \# : 22

DISCRETE DESC : DM-15

DISCRETE SYNC SCAN : O SEC HIST SCAN : O SEC

DISCRETE HIST. DEFINITION :0 DEBOUNCE: 0 MODIFY : 0

DISCRETE STATE 0 DESC :CLOSED COLOR : WHITE

DISCRETE STATE 1 DESC :OPEN COLOR : WHITE

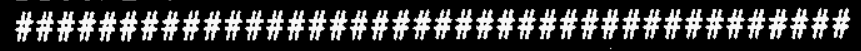

STEP NO : 7

LABEL :

FUNCTION : $57(00)$

COMMENT :

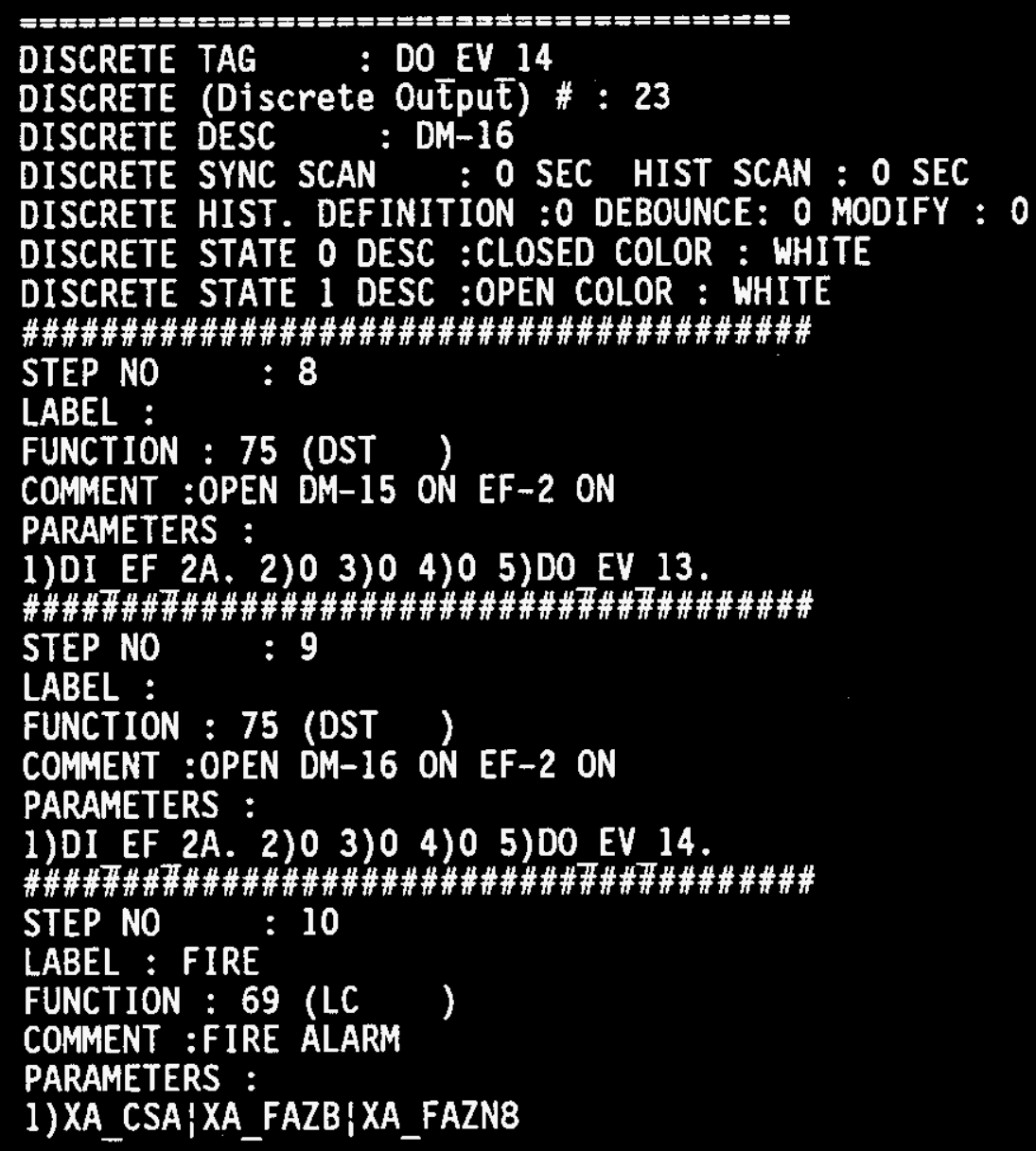



WHC-SD-CP-CSWD-016
Volume 4
Revision 1
Page 79

\#\#\#\#\#\#\#\#\#\#\#\#\#\#\#\#\#\#\#\#\#\#\#\#\#\#\#\#A\#\#\#\#\#\#\#\#\#\#\#

STEP NO : 11

LABEL :

FUNCTION : 69 (LC

COMMENT : "0" PULSE ON AUTO START OR EF-1 FAIL

PARAMETERS :

1)-! PULSE(HS LL 3\&HS MAIN)\&-! PULSE(-!HS_LL_3\&(FA_EF_1!PULSE(-! DI_EF

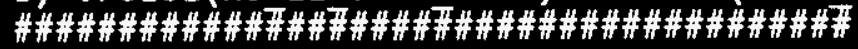

STEP NO : 12

LABEL :

FUNCTION : 62 (GTD)

COMMENT :EF-2 "START" JUMP

PARAMETERS :

1)MSV 2) Off-Nomal 3) JUMPA

\#\#\#\#\#\#\#\#\#\#\#\#\#\#\#\#\#\#\#\#\#\#\#\#\#\#\#\#\#\#\#\#\#\#\#\#\#\#\#

STEP NO : 13

LABEL : JUMPA

FUNCTION : 10 (L )

COMMENT :START EF-2

PARAMETERS :

1) HS EF 2. 2)1

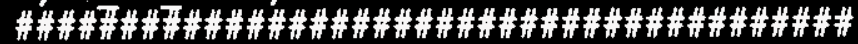

STEP NO : 14

LABEL :

FUNCTION : 69 (LC

COMMENT : PREVENT FALSE START ON RESET

PARAMETERS :

1) - ! PULSE (1)

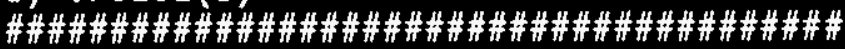

STEP NO : 15

LABEL :

FUNCTION : 62 (GTD )

COMMENT :

PARAMETERS :

1)MSV 2) Off-Nomal 3) JUMPST

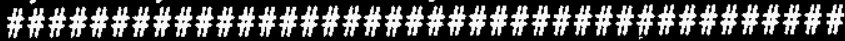

STEP NO : 16

LABEL : JUMPST

FUNCTION : 10 (L )

COMMENT :

PARAMETERS :

1) HS EF 2. 2)O

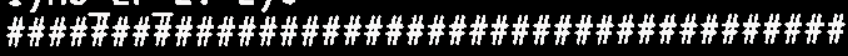

STEP NO

: 17

LABEL :

FUNCTION : 69 (LC)

COMMENT : "O" PULSE ON MAIN STOP

PARAMETERS :

1)-! PULSE(HS_MN_S, 5) 
WHC-SD-CP-CSWD-016

Volume 4

Revision 1

Page 80

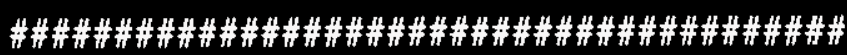

STEP NO : 18

LABEL :

FUNCTION : 62 (GTD )

COMMENT :EF-2 "STOP" JUMP

PARAMETERS :

1)MSV 2) Off-Nomal 3) JUMPB

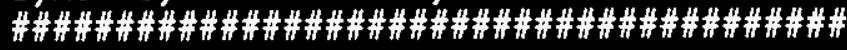

STEP NO : 19

LABEL : JUMPB

FUNCTION : $10(\mathrm{~L} \quad)$

COMMENT : STOP EF-2

PARAMETERS :

1)HS EF 2. 2) 0

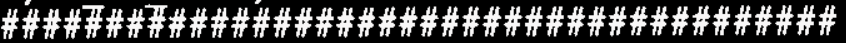

STEP NO : 20

LABEL :

FUNCTION : 69 (LC )

COMMENT :HELD ON "STOP" IF COLD SMOKE ALARM

PARAMETERS :

1)-! F IRE

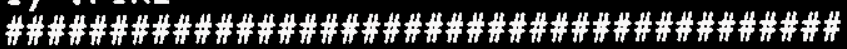

STEP NO : 21

LABEL :

FUNCTION : 62 (GTD )

COMMENT :COLD SMOKE "STOP" JUMP

PARAMETERS :

1)MSV 2) Off-Nomal 3) JUMPC

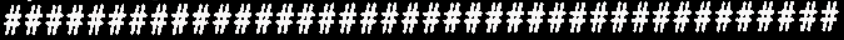

STEP NO : 22

LABEL : JUMPC

FUNCTION : 10 (L ${ }^{\prime}$ "

PARAMETERS :

1) HS EF 2. 2) 0

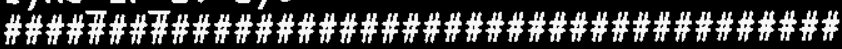

STEP NO : 23

LABEL :

FUNCTION : 73 (LAT)

COMMENT : EF-2 CONTROL LOGIC

PARAMETERS :

1) LATCH=PULSE(HS EF 2,5) 2) UNLATCH=-HS_EF_2! (DELAY (HS_E 3)0 4)DO_EF_2. 5) 0

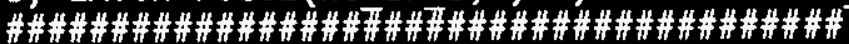

STEP NO : 24

LABEL :

FUNCTION : 69 (LC )

COMMENT : ALARM ON NO FLOW OR OFF WHEN HS IS ON (EXCEPT STARTUP)

PARAMETERS :

1)DELAY(HS_EF_2,15)\&-(IDI_EF_2A\&!DI_FS_6) 


\section{WHC-SD-CP-CSWD-016 \\ Vol ume 4 \\ Revision 1 \\ Page 81}

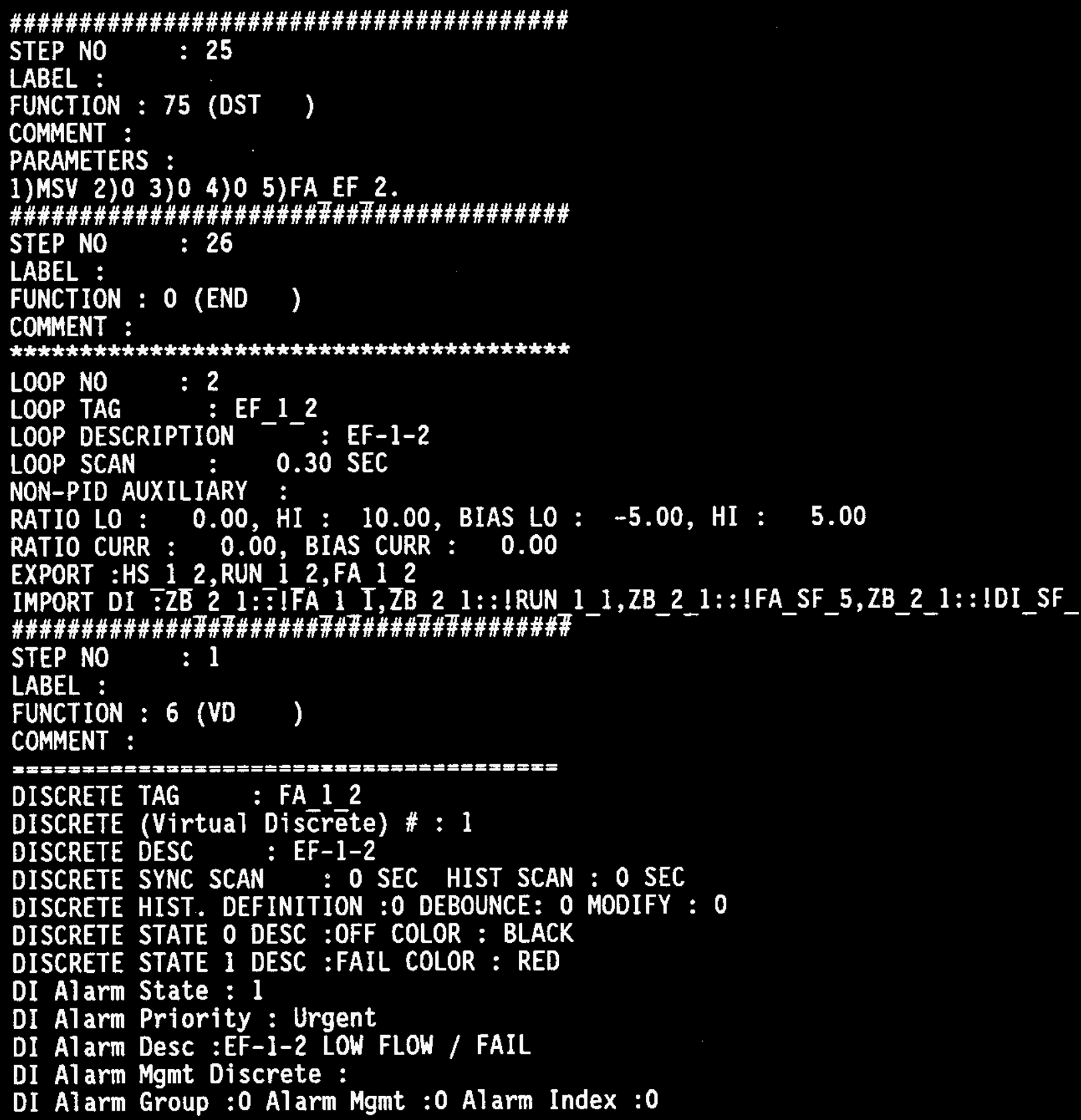




\section{WHC-SD-CP-CSWD-016 \\ Volume 4 \\ Revision 1 \\ Page 82}

\#\#\#\#\#\#\#\#\#\#\#\#\#\#\#\#\#\#\#\#\#\#\#\#\#\#\#\#\#\#\#\#\#\#\#\#\#\#\#\#

STEP NO : 2

LABEL :

FUNCTION : 7 (DI )

COMMENT :

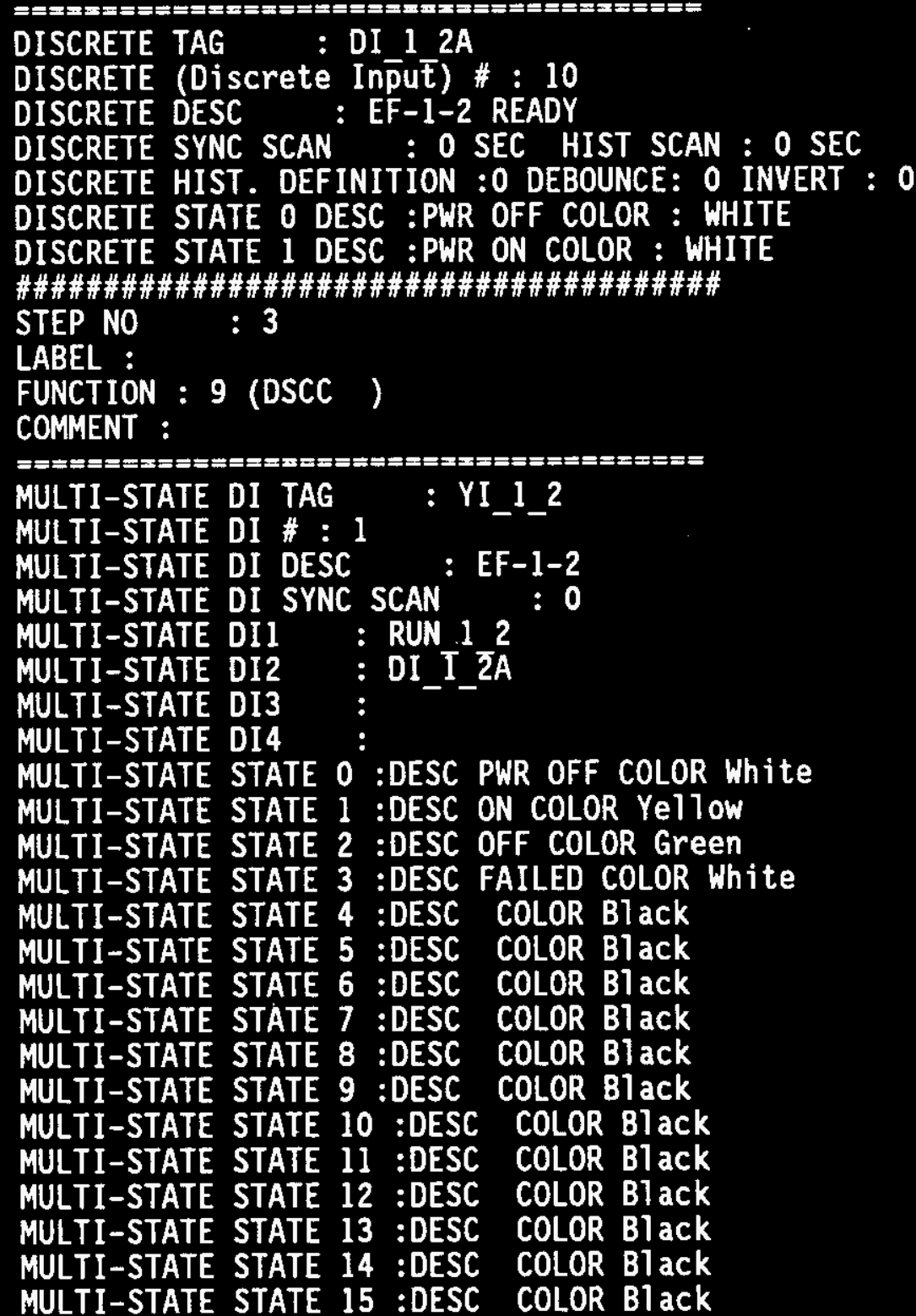




\section{WHC-SD-CP-CSWD-016 \\ Volume 4 \\ Revision 1 \\ Page 83}

\#\#\#\#\#\#\#\#\#\#\#\#\#\#\#\#\#\#\#\#\#\#\#\#\#\#\#\#\#\#\#\#\#\#\#\#\#\#\#\#

STEP NO

$: 4$

LABEL :

FUNCTION : 7 (DI )

COMMENT :

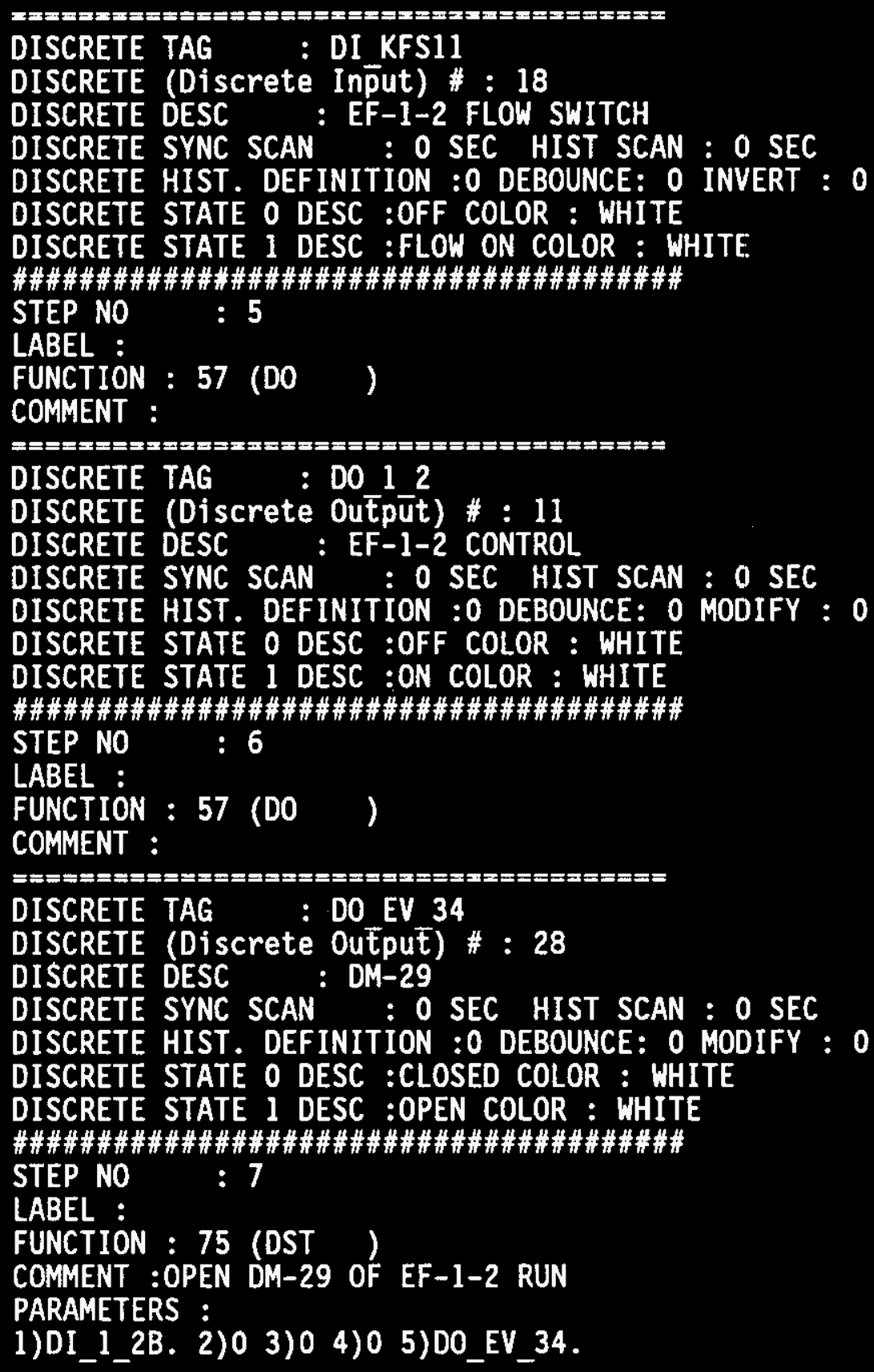




$$
\begin{gathered}
\text { WHC-SD-CP-CSWD-016 } \\
\text { Volume } 4 \\
\text { Revis ion } 1 \\
\text { Page } 84
\end{gathered}
$$

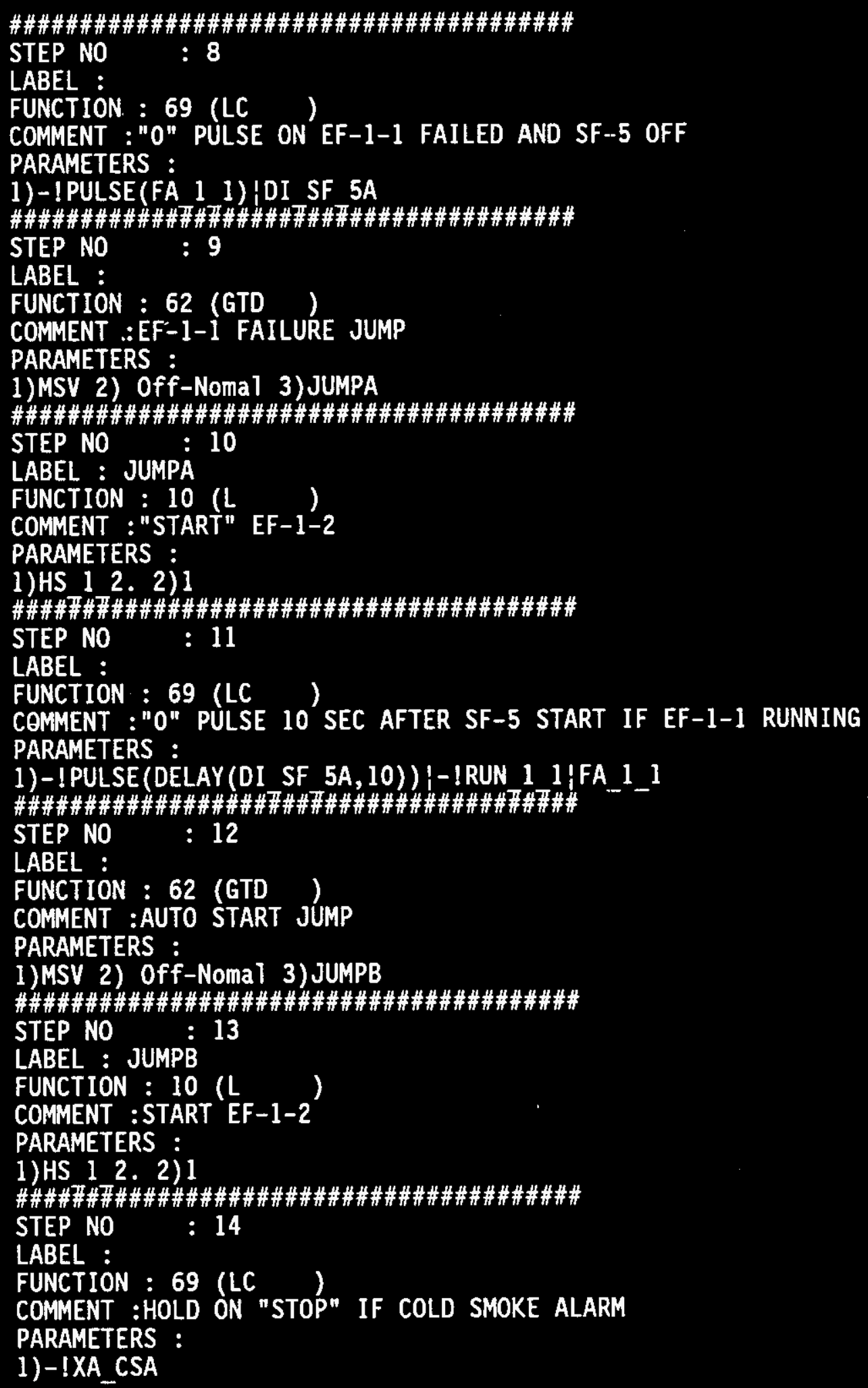




\title{
WHC-SD-CP-CSWD-016 \\ Volume 4 \\ Revision 1 \\ Page 85
}

\author{
\#\#\#\#\#\#\#\#\#\#\#\#\#\#\#\#\#\#\#\#\#\#\#\#\#\#\#\#\#\#\#\#\#\#\#\#\#\#\#\# \\ STEP NO : 15 \\ LABEL : \\ FUNCTION : 62 (GTD) \\ COMMENT : COLD SMOKE ALARM JUMP \\ PARAMETERS : \\ 1)MSV 2) Off-Nomal 3) JUMPC

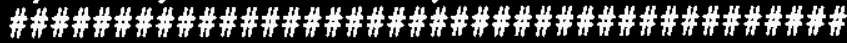 \\ STEP NO : 16 \\ LABEL : JUMPC \\ FUNCTION : $10(\mathrm{~L}$, \\ COMMENT :STOP EF-1-2 \\ PARAMETERS : \\ 1)HS 1 2. 2)0 \\ \#\#\#\#男\#开\#\#\#\#\#\#\#\#\#\#\#\#\#\#\#\#\#\#\#\#\#\#\#\#\#\#\#\#\#\#\#\#\# \\ STEP NO : 17 \\ LABEL : \\ FUNCTION : 73 (LAT) \\ COMMENT :EF-1-2 CONTROL LOGIC \\ PARAMETERS :
}

1) LATCH=PULSE(HS 1 2) 2) UNLATCH=-HS 1 2! (DELAY(HS_1 3)0 4)DO_1_2. 5) 0

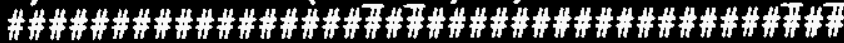

STEP NO : 18

LABEL :

FUNCTION : 69 (LC

COMMENT : ALARM ON NO FLOW OR OFF WHEN HS IS ON (EXCEPT STARTUP)

PARAMETERS :

1)HS $12 \&-$ ! PULSE(HS 12,30$) \&$-(!DI 1 2B\&!DI_KFS11)

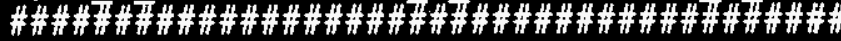

STEP NO : 19

LABEL :

FUNCTION : 75 (DST )

COMMENT :

PARAMETERS :

1)MSV 2) 0 3) 0 4)0 5)FA 12.

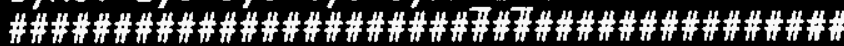

STEP NO : 20

LABEL :

FUNCTION : 69 (LC )

COMMENT : PSEUDO RUN = (HS ON AND NO FLON ALRM) OR REAL RUN ON

PARAMETERS :

1)(HS 1 2\&-FA 12 2) |DI $12 B$

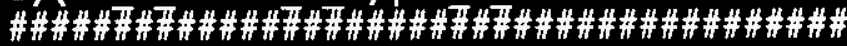

STEP NO : 21

LABEL :

FUNCTION : 75 (DST)

COMMENT :

PARAMETERS :

1)MSV 2)0 3)0 4)0 5)RUN_1_2. 


\section{WHC-SD-CP-CSWD-016 \\ Volume 4 \\ Revision 1 \\ Page 86}

\#\#\#\#\#\#\#\#\#\#\#\#\#\#\#\#\#\#\#\#\#\#\#\#\#\#\#\#\#\#\#\#\#\#\#\#\#\#\#\#

STEP NO : 22

LABEL :

FUNCTION : 0 (END)

COMMENT :

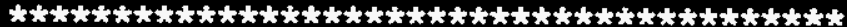

$\begin{array}{ll}\text { LOOP NO } & \text { : } \\ \text { LOOP TAG } & \text { : RF_2 }\end{array}$

LOOP DESCRIPTION - : RF-2

LOOP SCAN : 0.30 SEC

NON-PID AUXILIARY :

RATIO LO : 0.00 , HI : 10.00 , BIAS LO : -5.00 , HI : 5.00

RATIO CURR : 0.00 , BIAS CURR : 0.00

EXPORT :DI RF 2A, FA RF 2

IMPORT DI :ZB $21::$ TFA

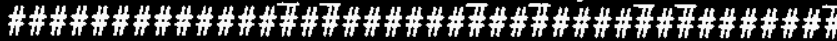

STEP NO : 1

LABEL :

FUNCTION : 6 (VD )

COMMENT :

DISCRETE TAG : FA RF 2

DISCRETE (Virtual Discrete) \# : 3

DISCRETE DESC : RF-2

DISCRETE SYNC SCAN : O SEC HIST SCAN : O SEC

DISCRETE HIST. DEFINITION :0 DEBOUNCE: 0 MODIFY : 0

DISCRETE STATE 0 DESC :OFF COLOR : BLACK

DISCRETE STATE 1 DESC :FAIL COLOR : RED

DI Alarm State : 1

DI Alarm Priority : Urgent

DI Alarm Desc :RF-2 LOW FLOW / FAIL

DI Alarm Mgmt Discrete :

DI Alarm Group :0 Alarm Mgmt :0 Alarm Index :0

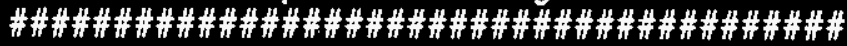

STEP NO : 2

LABEL :

FUNCTION : 7 (DI )

COMMENT :

DISCRETE TAG : DI RF $2 B$

DISCRETE (Discrete Input) \#: 7

DISCRETE DESC : RF-2 READY

DISCRETE SYNC SCAN : O SEC HIST SCAN : O SEC

DISCRETE HIST. DEFINITION :0 DEBOUNCE: 0 INVERT : 0

DISCRETE STATE 0 DESC :PWR OFF COLOR : WHITE

DISCRETE STATE 1 DESC :PWR ON COLOR : WHITE 


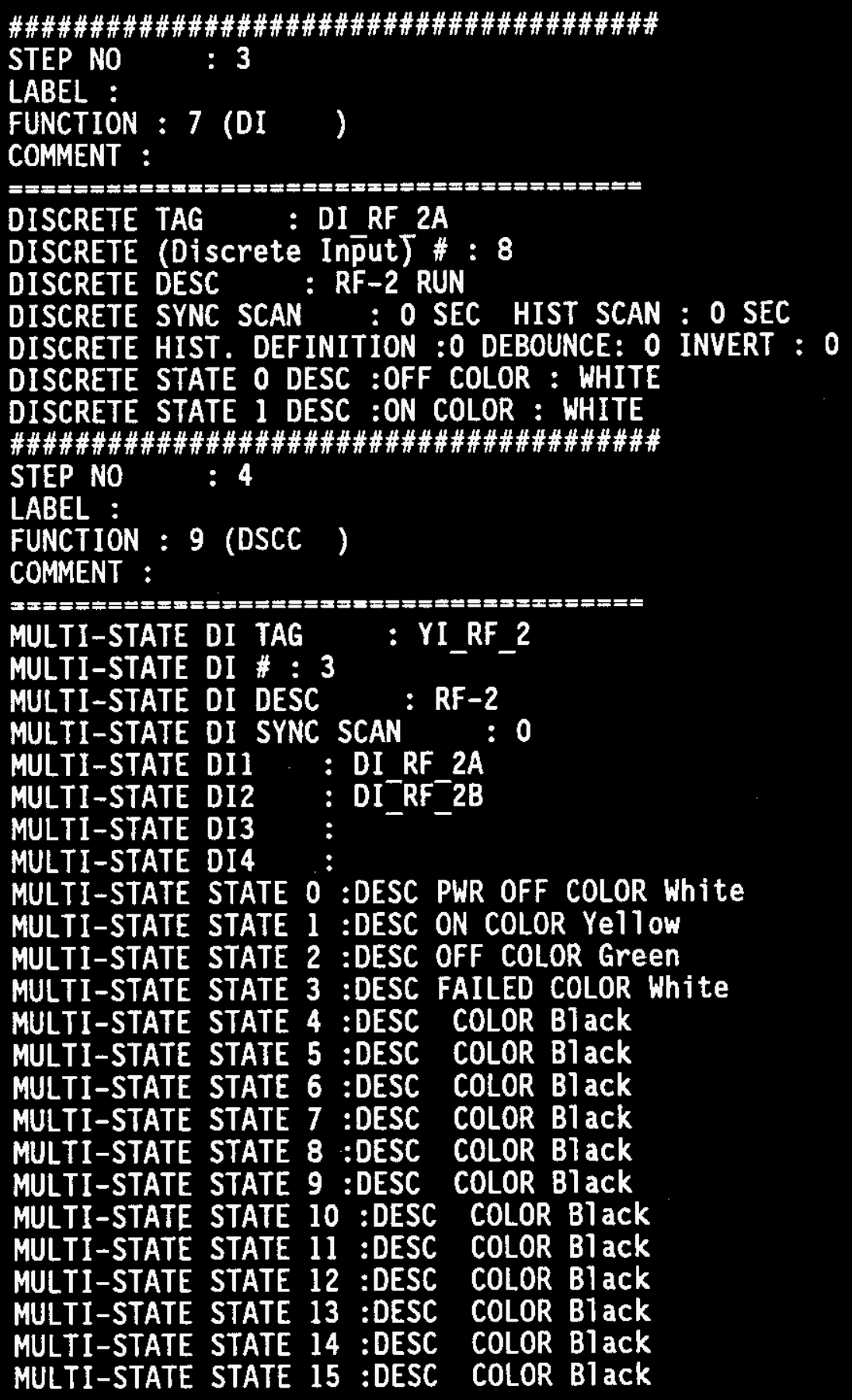


\#\#\#\#\#\#\#\#\#\#\#\#\#\#\#\#\#\#\#\#\#\#\#\#\#\#\#\#\#\#\#\#\#\#\#\#\#\#\#\#

STEP NO : 5

LABEL :

FUNCTION : $57(\mathrm{DO})$

COMMENT :

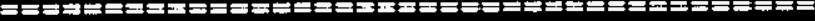

DISCRETE TAG : DO RF 2

DISCRETE (Discrete OUtput) \#: 9

DISCRETE DESC : RF-2 CONTROL

DISCRETE SYNC SCAN : O SEC HIST SCAN : O SEC

DISCRETE HIST. DEFINITION :0 DEBOUNCE: 0 MODIFY : 0

DISCRETE STATE O DESC :OFF COLOR : WHITE

DISCRETE STATE 1 DESC :ON COLOR : WHITE

\#\#\#\#\#\#\#\#\#\#\#\#\#\#\#\#\#\#\#\#\#\#\#\#\#\#\#\#\#\#\#\#\#\#\#

STEP NO : 6

LABEL :

FUNCTION : 57 (DO) )

COMMENT :

DISCRETE TAG : DO EV 17

DISCRETE (Discrete Output) \#: 24

DISCRETE DESC : DM-19

DISCRETE SYNC SCAN : O SEC HIST SCAN : O SEC

DISCRETE HIST. DEFINITION :0 DEBOUNCE: 0 MODIFY : 0

DISCRETE STATE 0 DESC :CLOSED COLOR : WHITE

DISCRETE STATE 1 DESC :OPEN COLOR : WHITE

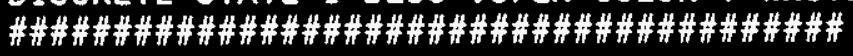

STEP NO : 7

LABEL :

FUNCTION : 57 (DO)

COMMENT :

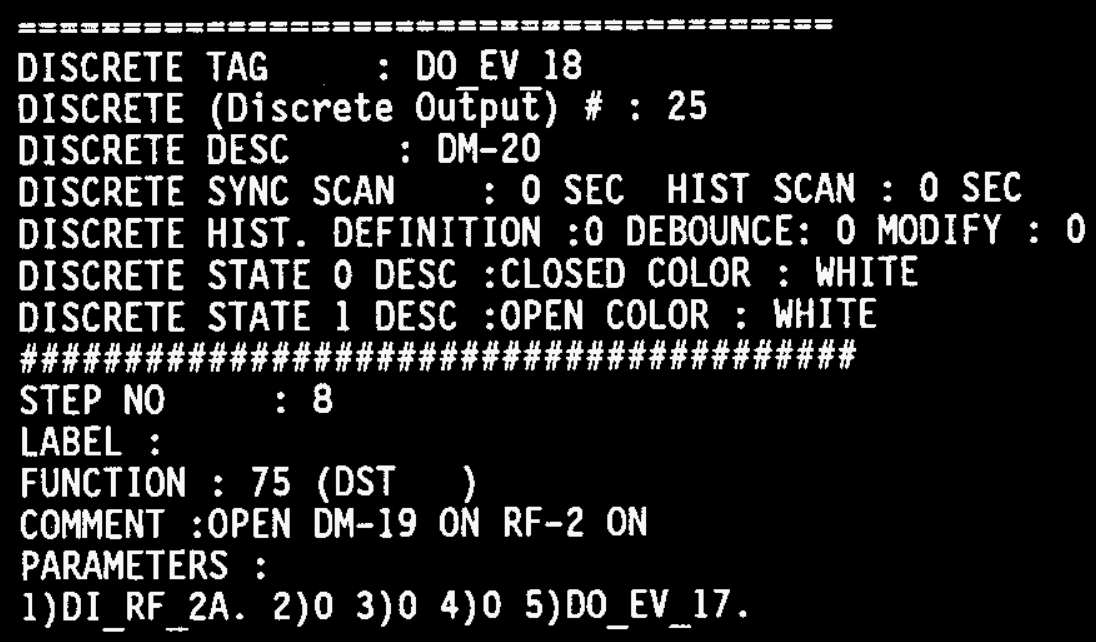


\#\#\#\#\#\#\#\#\#\#\#\#\#\#\#\#\#\#\#\#\#\#\#\#\#\#\#\#\#\#\#\#\#\#\#\#\#\#\#\#

STEP NO : 9

LABEL :

FUNCTION : 75 (DST)

COMMENT :OPEN DM-20 ON RF-2 ON

PARAMETERS :

1)DI RF 2A. 2)0 3)0 4)0 5)D0 EV 18.

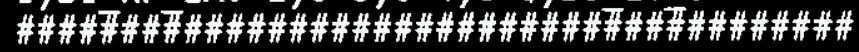

STEP NO : 10

LABEL : FIRE

FUNCTION : 69 (LC)

COMMENT :FIRE ALARM

PARAMETERS :

1) XA CSA XA FAZB $X X A$ FAZN8

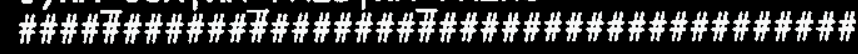

STEP NO : 11

LABEL :

FUNCTION : 69 (LC)

COMMENT : PULSE "O" ON AUTO START OR RF-1 FAIL

PARAMETERS :

1) -! PULSE(HS LL 48HS NDA)\&-! PULSE(-!HS_LL_4\&(FA_RF_1 PULSE(-1DI_RF_

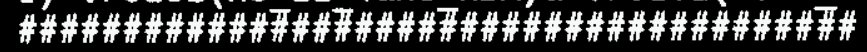

STEP NO : 12

LABEL :

FUNCTION : 62 (GTD)

COMMENT :RF-2 START JUMP

PARAMETERS :

1) MSV 2) Off-Nomal 3) JUMPA

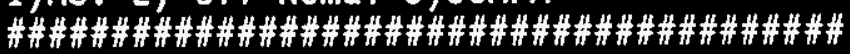

STEP NO : 13

LABEL : JUMPA

FUNCTION : $10(\mathrm{~L})$

COMNENT :

PARAMETERS :

1)HS RF 2. 2) 1

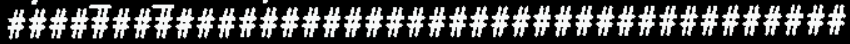

STEP NO : 14

LABEL :

FUNCTION : 69 (LC )

COMMENT :

PARAMETERS :

1) - ! PULSE (1)

\#\#\#\#\#\#\#\#\#\#\#\#\#\#\#\#\#\#\#\#\#\#\#\#\#\#\#\#\#\#\#\#\#\#\#\#\#\#\#\#

STEP NO : 15

LABEL :

FUNCTION : 62 (GTD )

COMMENT :

PARANETERS :

1)MSV 2) Off-Nomal 3) JUMPST 
WHC-SD-CP-CSWD-016

Volume 4

Revision 1

Page 90

\#\#\#\#\#\#\#\#\#\#\#\#\#\#\#\#\#\#\#\#\#\#\#\#\#\#\#\#\#\#\#\#\#\#\#\#\#\#\#\#

STEP NO : 16

LABEL : JUMPST

FUNCTION : $10(\mathrm{~L})$

COMMENT :

PARAMETERS :

1)HS RF 2. 2)0

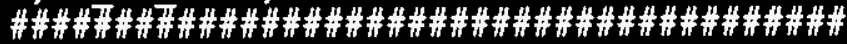

STEP NO : 17

LABEL :

FUNCTION : 69 (LC

COMMENT : PULSE "O" ON NDA STOP

PARAMETERS :

1)-1 PULSE (HS NDA $S, 5$ )

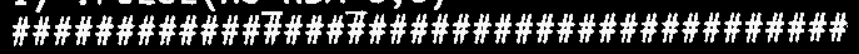

STEP NO : 18

LABEL :

FUNCTION : 62 (GTD)

COMMENT :RF-2 NDA STOP JUMP

PARAMETERS :

1)MSV 2) Off-Nomal 3) JUMPB

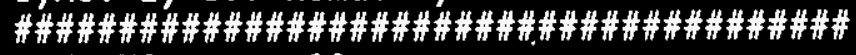

STEP NO

LABEL : JUMPB

FUNCTION : $10(\mathrm{~L})$

COMMENT :

PARAMETERS :

1)HS RF 2. 2)0

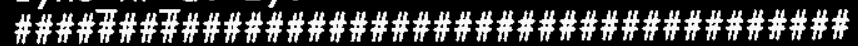

STEP NO : 20

LABEL :

FUNCTION : 69 (LC)

COMMENT :HOLD ON "STOP" IF FIRE ALARM

PARAMETERS :

1)-IFIRE

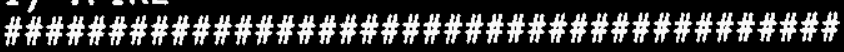

STEP NO : 21

LABEL :

FUNCTION : 62 (GTD)

COMMENT :RF-2 FIRE ALARM STOP JUMP

PARAMETERS :

1)MSV 2) Off-Nomal 3) JUMPC

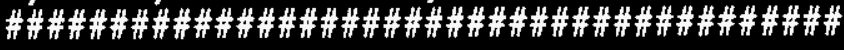

STEP NO : 22

LABEL : JUMPC

FUNCTION : $10(\mathrm{~L} \quad)$

COMMENT :

PARAMETERS :

1)HS_RF_2. 2)0 


\section{WHC-SD-CP-CSWD-016 \\ Volume 4 \\ Revision 1 \\ Page 91}

\#\#\#\#\#\#\#\#\#\#\#\#\#\#\#\#\#\#\#\#\#\#\#\#\#\#\#\#\#\#\#\#\#\#\#\#\#\#\#\#

STEP NO : 23

LABEL :

FUNCTION : 73 (LAT)

COMMENT :RF-2 CONTROL LOGIC

PARAMETERS :

1) LATCH=PULSE(HS RF 2,5) 2) UNLATCH=-HS_RF_2!(DELAY(HS_R 3)0 4)DO_RF_2. 5) 0

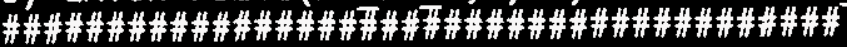

STEP NO

$: 24$

LABEL :

FUNCTION : 69 (LC

COMMENT : ALARM ON NO FLON OR OFF WHEN HS IS ON (EXCEPT STARTUP)

PARAMETERS :

1)DELAY(HS RF 2, 15)\&-(1DI RF 2A\&!DI FS 8)

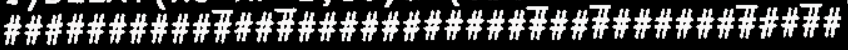

STEP NO : 25

LABEL :

FUNCTION : 75 (DST )

COMMENT :

PARAMETERS :

1) MSV 2) 0 3)0 4)0 5)FA RF 2 .

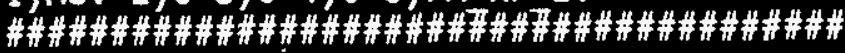

STEP NO : 26

LABEL :

FUNCTION : 0 (END)

COMMENT :

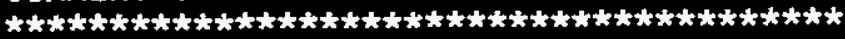

$$
\begin{aligned}
& \text { LOOP NO : } 4 \\
& \text { LOOP TAG : SF } 2 \\
& \text { LOOP DESCRIPTION - : ZB SF-2 } \\
& \text { LOOP SCAN : } 0.30 \text { SEC } \\
& \text { NON-PID AUXILIARY : } \\
& \text { RATIO LO : } 0.00 \text {, HI : } 10.00 \text {, BIAS LO : }-5.00 \text {, HI : } 5.00 \\
& \text { RATIO CURR : } 0.00 \text {, BIAS CURR : } 0.00 \\
& \text { EXPORT :DI SF 2A,FA SF_ } 2 \\
& \text { IMPORT OI :ZB_2_1::TFA_SF_1,ZB_2_3::!HS_LL1,ZB_2_5::!DI_FS_2,ZB_2_5::!DI_DPS }
\end{aligned}
$$


\#\#\#\#\#\#\#\#\#\#\#\#\#\#\#\#\#\#\#\#\#\#\#\#\#\#\#\#\#\#\#\#\#\#\#\#\#\#\#\#

STEP NO : 1

LABEL :

FUNCTION : 6 (VD)

COMMENT :

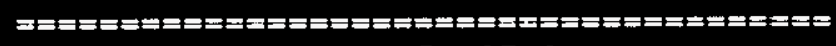

DISCRETE TAG : FA_SF 2

DISCRETE (Virtual Disçrete) \# : 4

DISCRETE DESC : ZB SF-2

DISCRETE SYNC SCAN : O SEC HIST SCAN : O SEC

DISCRETE HIST. DEFINITION :0 DEBOUNCE: 0 MODIFY : 0

DISCRETE STATE 0 DESC :OFF COLOR : BLACK

DISCRETE STATE I DESC :FAIL COLOR : YELLOW

DI Alarm State : 1

DI Alarm Priority : Caution

DI Alarm Desc :ZB SF-2 LOW FLOW / FAIL

DI Alarm Mgmt Discrete :

DI Alarm Group :0 Alarm Mgmt :0 Alarm Index :0

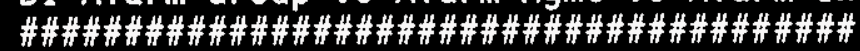

STEP NO : 2

LABEL :

FUNCTION : 7 (DI )

COMMENT :

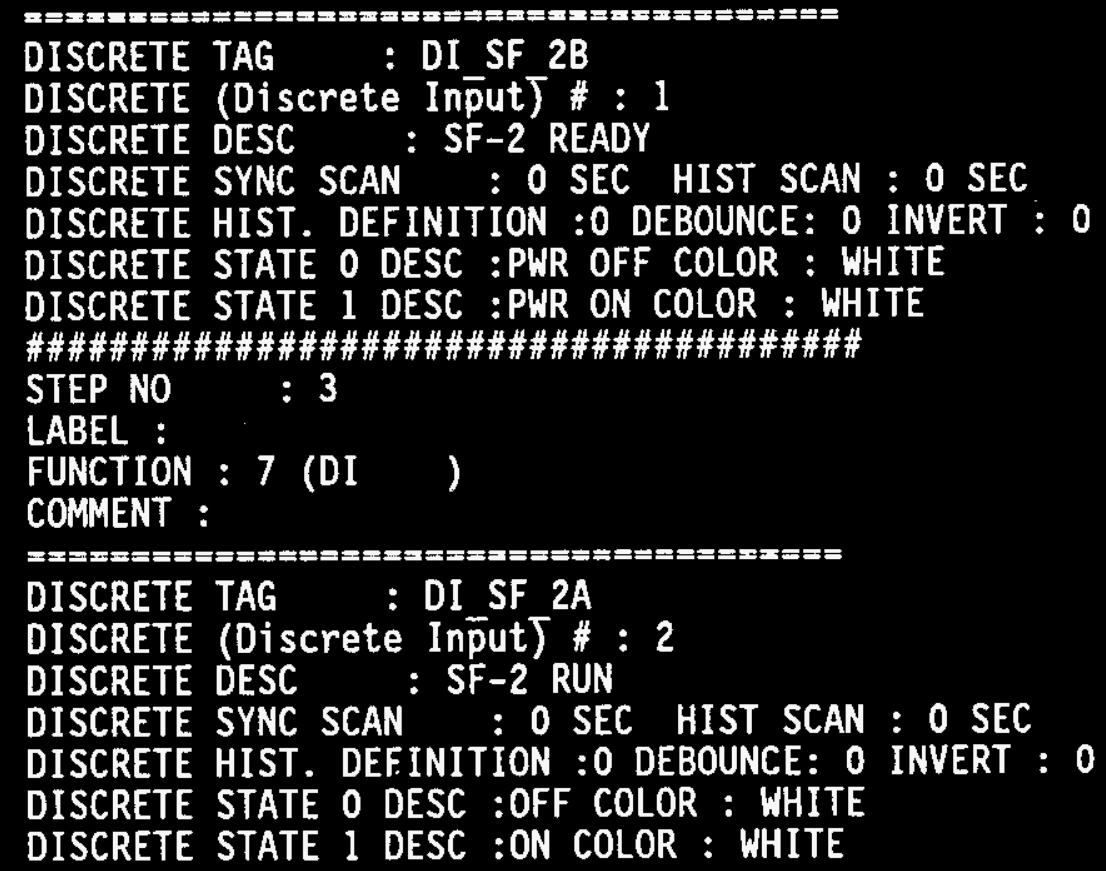


WHC-SD-CP-CSWD-016

Volume 4

Revision 1

Page 93

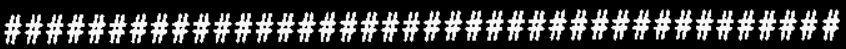

STEP NO : 4

LABEL :

FUNCTION : 9 (DSCC)

COMMENT :

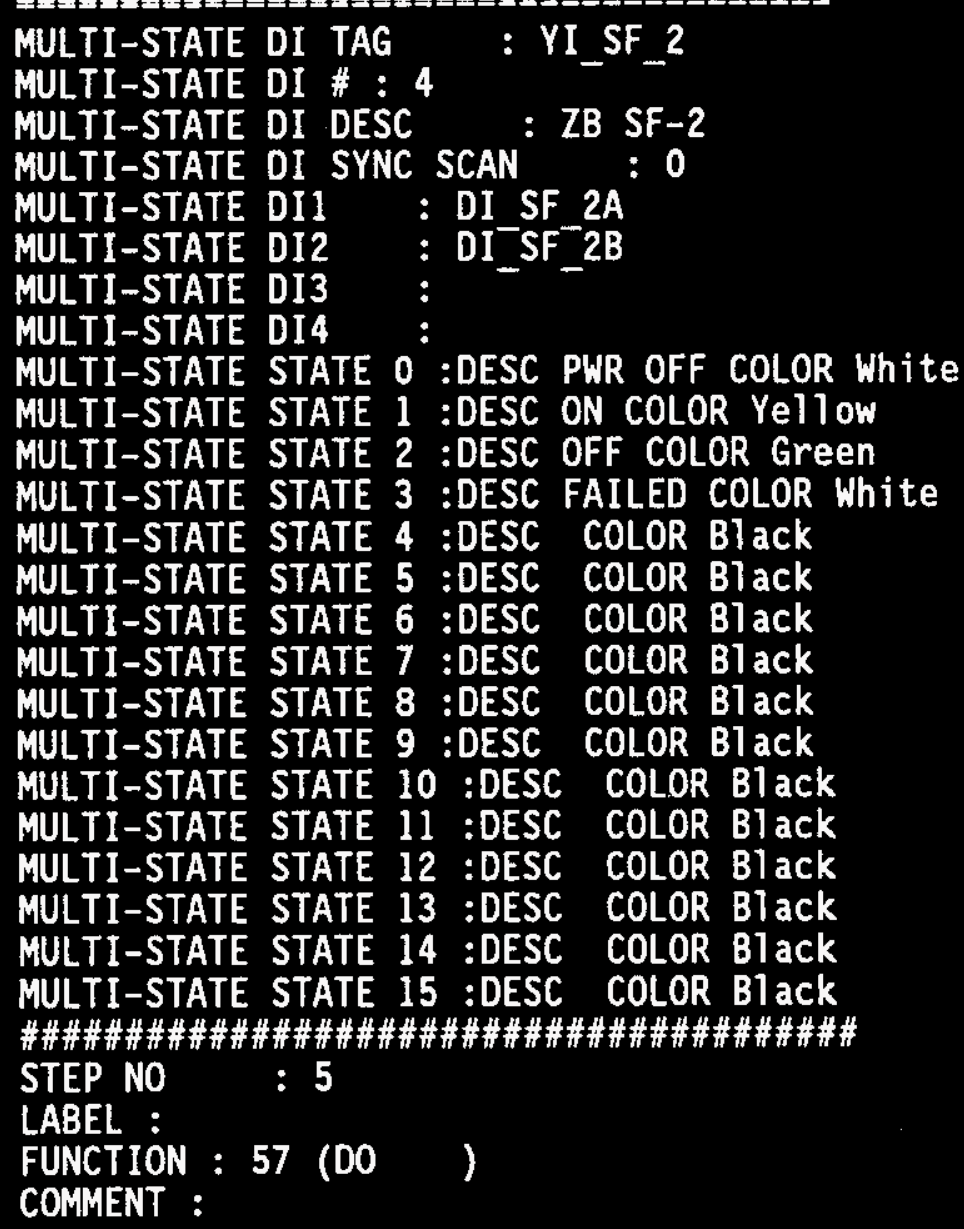


\#\#\#\#\#\#\#\#\#\#\#\#\#\#\#\#\#\#\#\#\#\#\#\#\#\#\#\#\#\#\#\#\#\#\#\#\#\#\#\#

STEP NO : 6

LABEL :

FUNCTION : $57(\mathrm{DO})$

COMMENT :

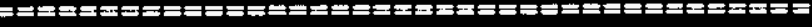

DISCRETE TAG : DO EV 3

DISCRETE (Discrete Output) \#: 20

DISCRETE DESC : DM-5

DISCRETE SYNC SCAN : O SEC HIST SCAN : O SEC

DISCRETE HIST. DEFINITION :0 DEBOUNCE: 0 MODIFY : 0

DISCRETE STATE 0 DESC :CLOSED COLOR : WHITE

DISCRETE STATE 1 DESC :OPEN COLOR : WHITE

\#\#\#\#\#\#\#\#\#\#\#\#\#\#\#\#\#\#\#\#\#\#\#\#\#\#\#\#\#\#\#\#\#\#\#\#

STEP NO : 7

LABEL :

FUNCTION : $57(00)$

COMMENT :

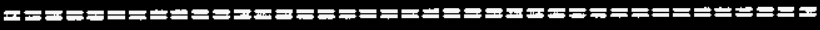

DISCRETE TAG : DO EV 4

DISCRETE (Discrete Output) \#: 21

DISCRETE DESC : DM-2

DISCRETE SYNC SCAN : O SEC HIST SCAN : O SEC

DISCRETE HIST. DEFINITION :0 DEBOUNCE: 0 MODIFY : 0

DISCRETE STATE 0 DESC :CLOSED COLOR : WHITE

DISCRETE STATE 1 DESC ;OPEN COLOR : WHITE

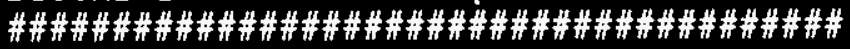

STEP NO : 8

LABEL :

FUNCTION : 75 (DST)

COMMENT :DM-5 OPEN ON SF-2 ON

PARAMETERS :

1)DI SF 2A. 2)0 3)0 4)0 5)DO EV 3.

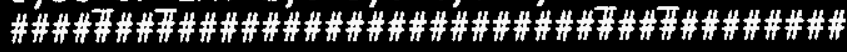

STEP NO : 9

LABEL :

FUNCTION : 75 (DST)

COMMENT :DM-2 OPEN ON SF-2 ON

PARAMETERS :

1)DI SF 2A. 2)0 3)0 4)0 5)DO EV 4.

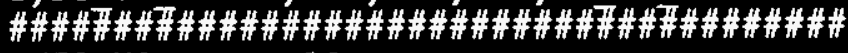

STEP NO : 10

LABEL : FIRE

FUNCTION : $69^{\circ}$ (LC )

COMMENT :FIRE ALARM

PARAMETERS :

1)XA_CSA |XA_FAZB $X X_{-}$FAZN8 
\#\#\#\#\#\#\#\#\#\#\#\#\#\#\#\#\#\#\#\#\#\#\#\#\#\#\#\#\#\#\#\#\#\#\#\#\#\#\#\#

STEP NO : 11

LABEL :

FUNCTION : 69 (LC

COMMENT : "O" PULSE ON EXHAUST DP UP OR SF-2 FAIL

PARAMETERS :

1)-! PULSE(HS LL1\&PULSE(DI DPS 2))\&-! PULSE(-!HS_LL18FA_SF_18DI_DPS_2

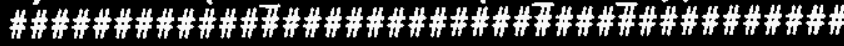

STEP NO : 12

LABEL :

FUNCTION : 62 (GTD)

COMMENT : AUTO START JUMP

PARAMETERS :

1)MSV 2) Off-Nomal 3) JUMPA

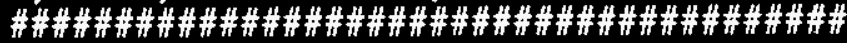

STEP NO

LABEL : JUMPA

FUNCTION : $10(\mathrm{~L})$

COMMENT :

PARAMETERS :

1)HS SF 2. 2)1

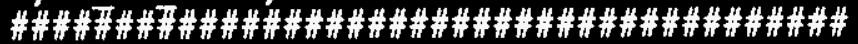

STEP NO : 14

LABEL :

FUNCTION : 69 (LC

COMMENT : PREVENT FALSE START ON RESET

PARAMETERS :

1) -! PULSE (1)

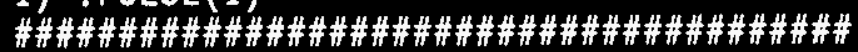

STEP NO : 15

LABEL :

FUNCTION : 62 (GTD )

COMMENT :

PARAMETERS :

1)MSV 2) Off-Nomal 3) JUMPST

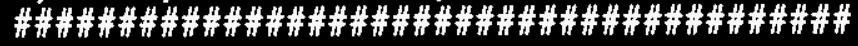

STEP NO

LABEL : JUMPST

FUNCTION : $10(\mathrm{~L})$

COMMENT :

PARAMETERS :

1)HS SF 2. 2)0

\#\#\#\#罗\#\#西\#\#\#\#\#\#\#\#\#\#\#\#\#\#\#\#\#\#\#\#\#\#\#\#\#\#\#\#\#\#

STEP NO : 17

LABEL :

FUNCTION : 69 (LC)

COMMENT : "O" PULSE ON MAIN STOP OR LO EXHAUST DP

PARAMETERS :

1) -! PULSE(HS_MN_S!-!DI_DPS_2,5) 
WHC-SD-CP-CSWD-016

Volume 4

Revision 1

Page 96

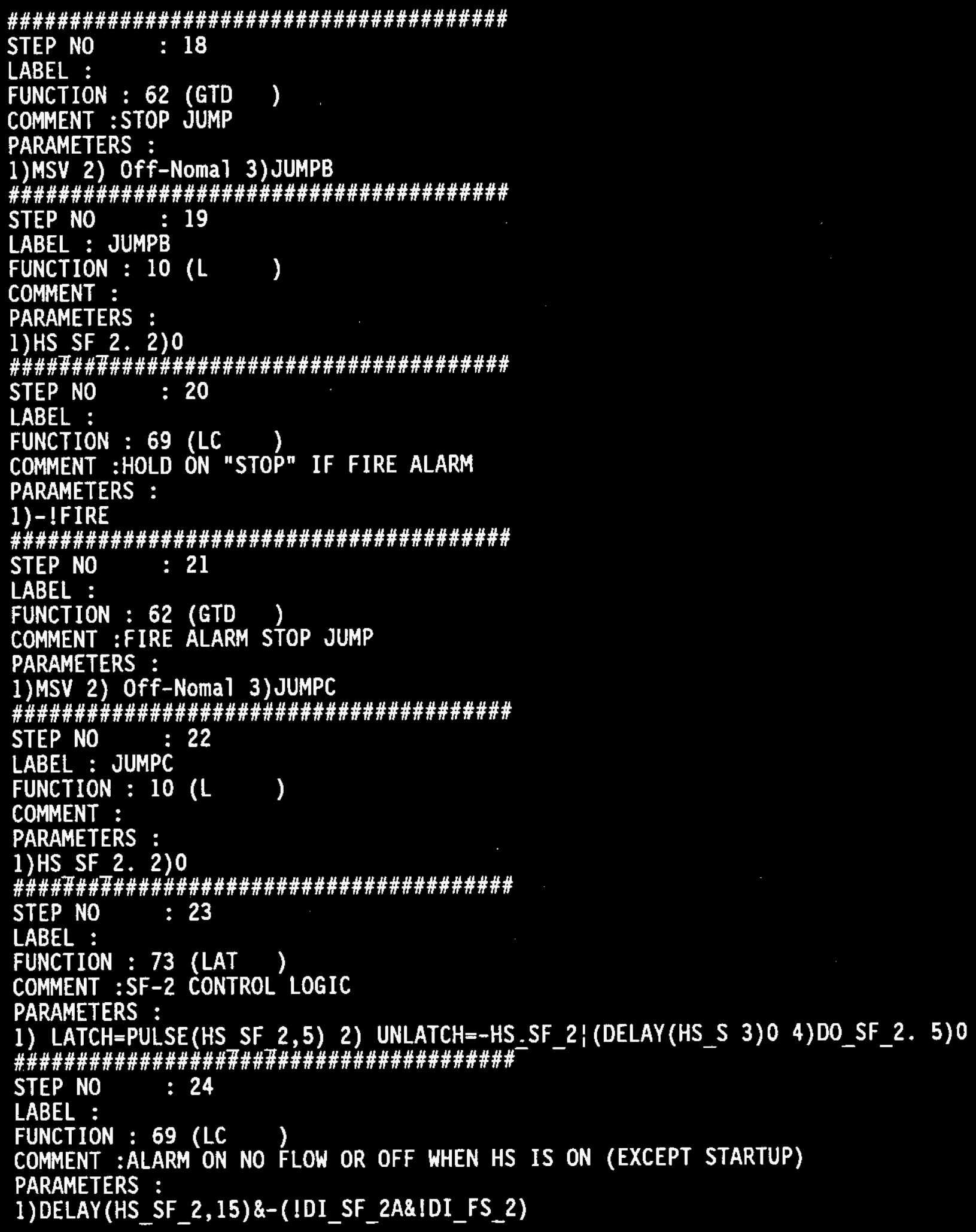




\section{WHC-SD-CP-CSWD-016 \\ Volume 4 \\ Revision 1 \\ Page 97}

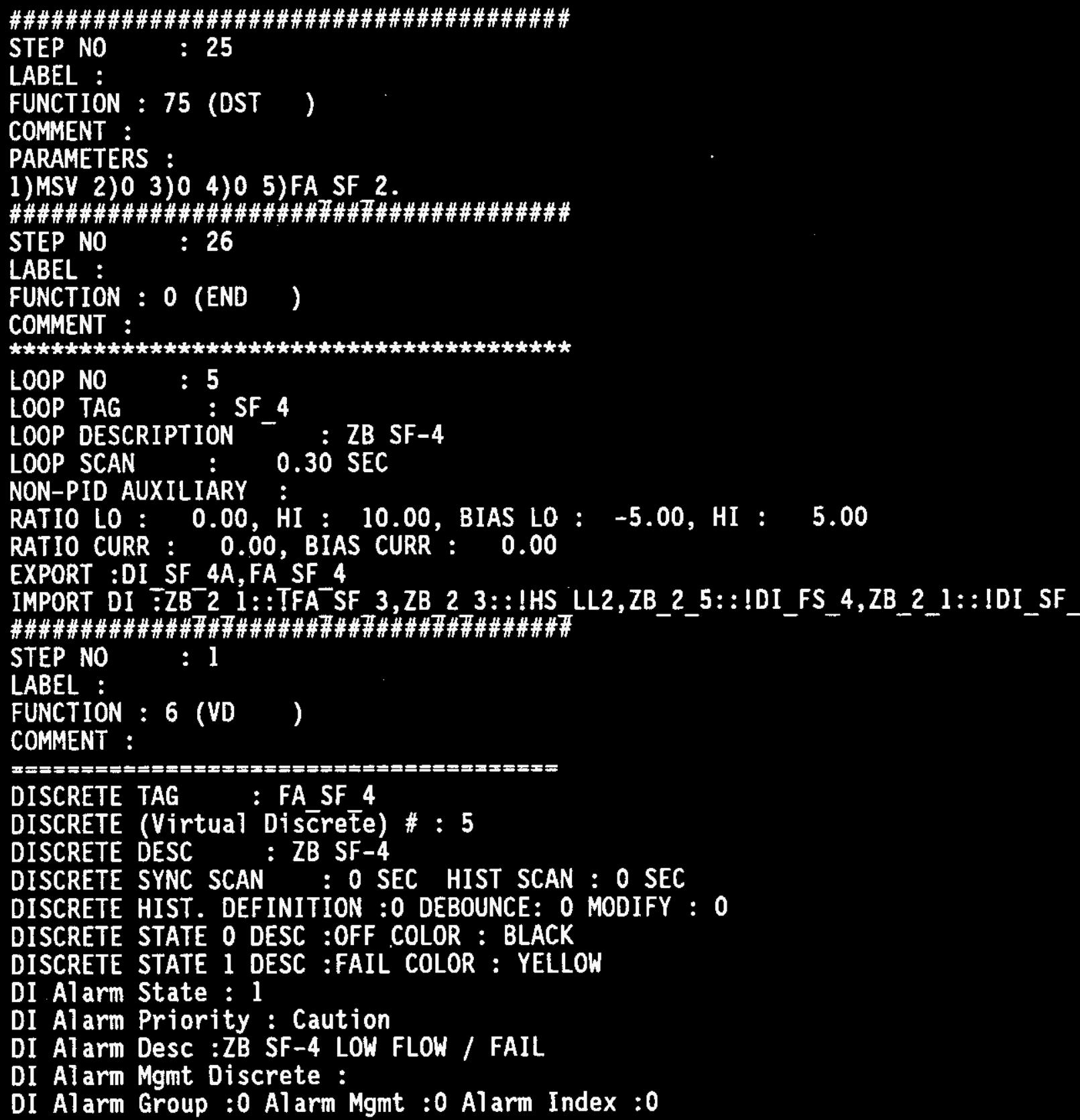


WHC-SD-CP-CSWD-016

Volume 4

Revision 1

Page 98

\#\#\#\#\#\#\#\#\#\#\#\#\#\#\#\#\#\#\#\#\#\#\#\#\#\#\#\#\#\#\#\#\#\#\#\#\#\#\#\#

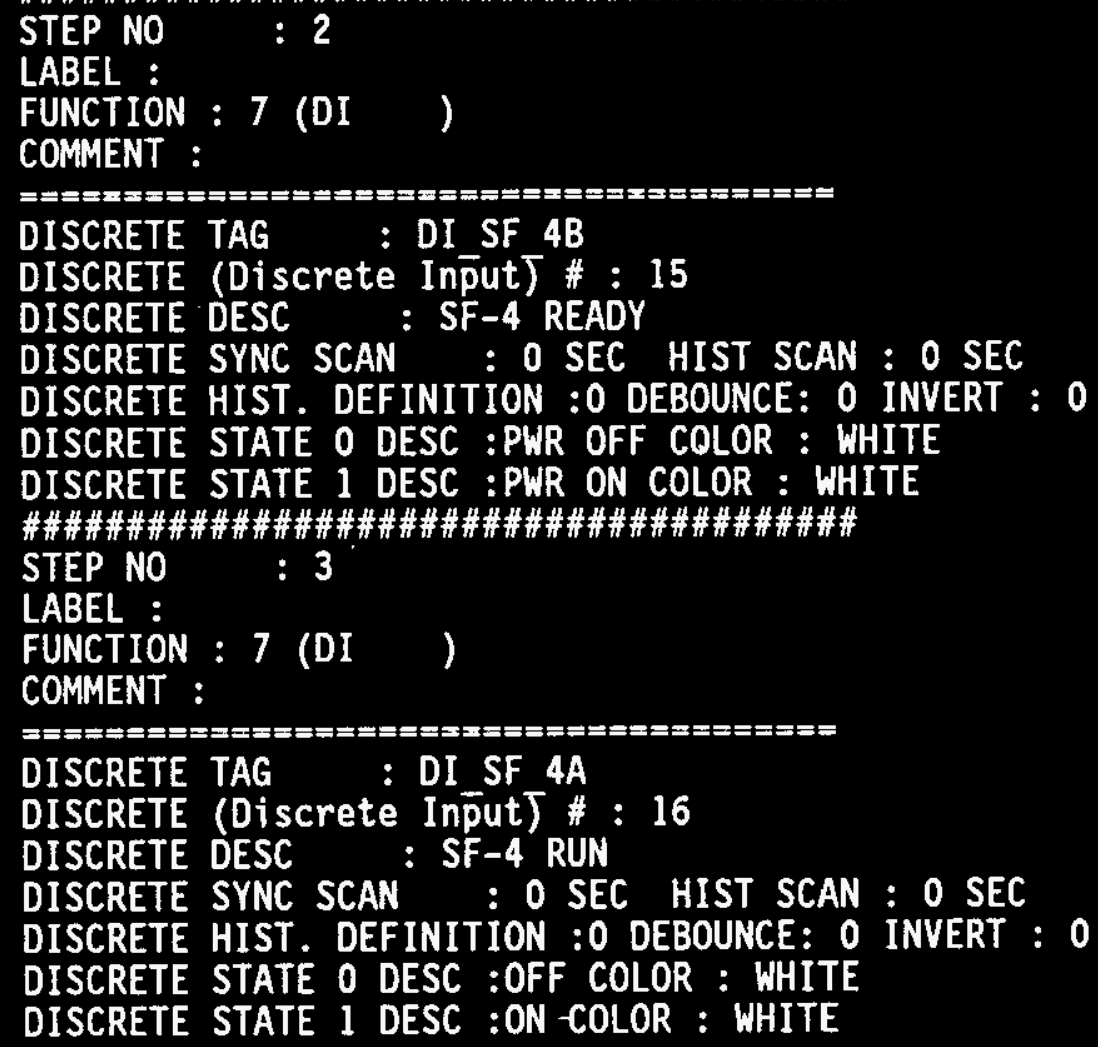


WHC-SD-CP-CSWD-016

Volume 4

Revision 1

Page 99

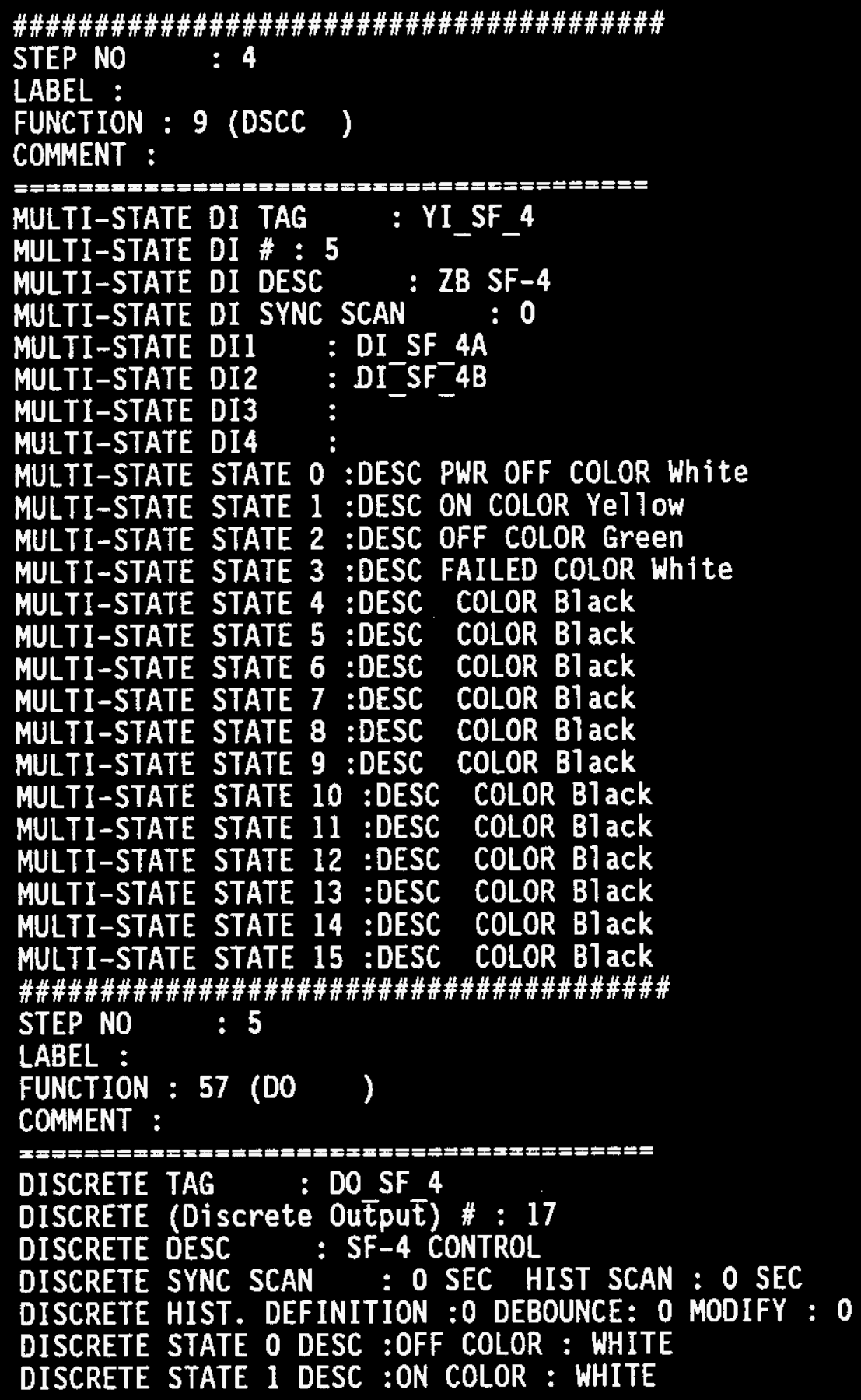


WHC-SD-CP-CSWD-016

Volume 4

Revision 1

Page 100

\#\#\#\#\#\#\#\#\#\#\#\#\#\#\#\#\#\#\#\#\#\#\#\#\#\#\#\#\#\#\#\#\#\#\#\#\#\#\#

STEP NO : 6

LABEL :

FUNCTION : $57(\mathrm{DO})$

COMMENT :

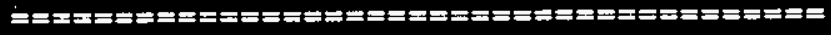

DISCRETE TAG : DO EV 7

DISCRETE (Discrete Output) \# : 29

DISCRETE DESC : DM-9

DISCRETE SYNC SCAN : O SEC HIST SCAN : O SEC

DISCRETE HIST. DEFINITION :0 DEBOUNCE: 0 MODIFY : 0

DISCRETE STATE O DESC :CLOSED COLOR : WHITE

DISCRETE STATE 1 DESC :OPEN COLOR : WHITE

\#\#\#\#\#\#\#\#\#\#\#\#\#\#\#\#\#\#\#\#\#\#\#\#\#\#\#\#\#\#\#\#\#\#\#\#\#\#

STEP NO : 7

LABEL :

FUNCTION : 57 (DO) )

COMMENT :

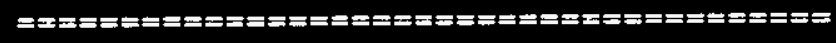

DISCRETE TAG : DO EV 8

DISCRETE (Discrete Output) \#: 30

DISCRETE DESC : DM-10

DISCRETE SYNC SCAN : 0 SEC. HIST SCAN : O SEC

DISCRETE HIST. DEFINITION :0 DEBOUNCE: 0 MODIFY : 0

DISCRETE STATE 0 DESC :CLOSED COLOR : WHITE

DISCRETE STATE 1 DESC :OPEN COLOR : WHITE

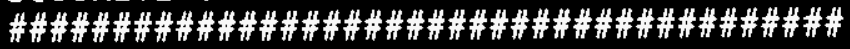

STEP NO : 8

LABEL :

FUNCTION : 75 (DST)

COMMENT :OPEN DM-9 ON SF-4 ON

PARAMETERS :

1)DI SF 4A. 2)0 3)0 4)0 5)DO EV 7.

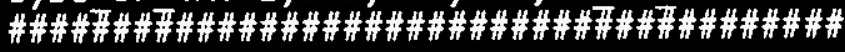

STEP NO

$: 9$

LABEL :

FUNCTION : 75 (DST)

COMMENT :OPEN DM-10 ON SF-4 ON

PARAMETERS :

1)DI SF 4A. 2)0 3)0 4)0 5)DO EV 8.

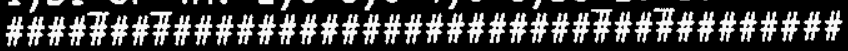

STEP NO : 10

LABEL : FIRE

FUNCTION : 69 (LC

COMMENT :FIRE ALARM

PARAMETERS :

1) XA_CSA XA_FAZB $X A$ FAZN 8 
WHC-SD-CP-CSWD-016

Volume 4

Revision 1

Page 101

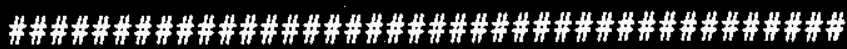

STEP NO : 11

LABEL : RFON

FUNCTION : 69 (LC )

COMMENT : RETURN FAN "ON" SIGNAL

PARAMETERS :

1)(-!FA RF 18DI RF 1A) | (-!FA RF 28DI RF 2A)

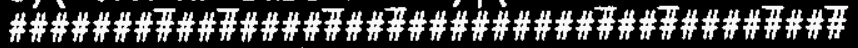

STEP NO : 12

LABEL :

FUNCTION : 69 (LC

COMMENT : "0" PULSE ON RFS ON OR SF-3 FAIL

PARAMETERS :

1)-! PULSE(HS LL2\&PULSE(RFON))\&-! PULSE(-!HS_LL28FA_SF_3\&RFON)\&-!PULS

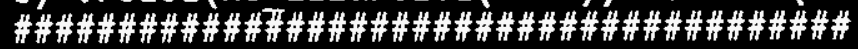

STEP NO : 13

LABEL :

FUNCTION : 62 (GTD )

COMMENT :SF-4 START JUMP

PARAMETERS :

1) MSV 2) Off-Nomal 3) JUMPA

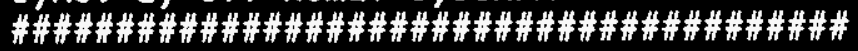

STEP NO

$: 14$

LABEL : JUMPA

FUNCTION : $10(\mathrm{~L})$

COMMENT :

PARAMETERS :

1)HS SF 4. 2)1

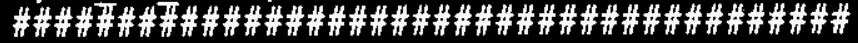

STEP NO : 15

LABEL :

FUNCTION : 69 (LC

COMMENT : PREVENT FALSE START ON RESET

PARAMETERS :

1) - IPULSE (1)

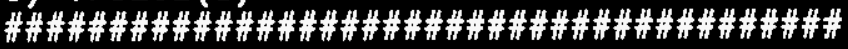

STEP NO : 16

LABEL :

FUNCTION : 62 (GTD )

COMMENT :

PARAMETERS :

1)MSV 2) Off-Nomal 3) JUMPST

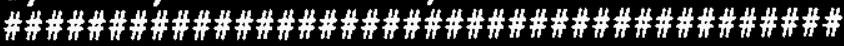

STEP NO : 17

LABEL : JUMPST

FUNCTION : $10(\mathrm{~L})$

COMMENT :

PARAMETERS :

1)HS_SF_4. 2)0 


\section{WHC-SD-CP-CSWD-016 \\ Volume 4 \\ Revision 1 \\ Page 102}

\#\#\#\#\#\#\#\#\#\#\#\#\#\#\#\#\#\#\#\#\#\#\#\#\#\#\#\#\#\#\#\#\#\#\#\#\#\#\#\#

STEP NO : 18

LABEL :

FUNCTION : 69 (LC

COMMENT : "O" PULSE ON NDA STOP OR RFS OFF PARAMETERS :

1)-! PULSE(HS NDA S, 5)\&-! PULSE (-! RFON)

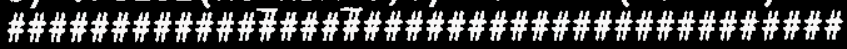

STEP NO : 19

LABEL :

FUNCTION : 62 (GTD )

COMMENT :STOP JUMP

PARAMETERS :

1)MSV 2) Off-Nomal 3) JUMPB

\#\#\#\#\#\#\#\#\#\#\#\#\#\#\#\#\#\#\#\#\#\#\#\#\#\#\#\#\#\#\#\#\#\#\#\#\#\#\#\#

STEP NO

$: 20$

LABEL : JUMPB

FUNCTION : $10(\mathrm{~L})$

COMMENT :

PARAMETERS :

1) HS SF 4. 2)0

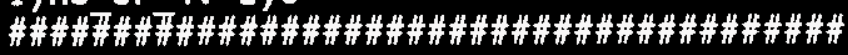

STEP NO : 21

LABEL :

FUNCTION : 69 (LC)

COMMENT :HOLD ON STOP IF FIRE ALARM

PARAMETERS :

1) - ! FIRE

\#\#\#\#\#\#\#\#\#\#\#\#\#\#\#\#\#\#\#\#\#\#\#\#\#\#\#\#\#\#\#\#\#\#\#\#\#\#\#

STEP NO : 22

LABEL :

FUNCTION : 62 (GTD)

COMMENT : FIRE ALARM STOP JUMP

PARAMETERS :

1)MSV 2) Off-Nomal 3) JUMPC

\#\#\#\#\#\#\#\#\#\#\#\#\#\#\#\#\#\#\#\#\#\#\#\#\#\#\#\#\#\#\#\#\#\#\#\#\#\#

STEP NO : 23

LABEL : JUMPC

FUNCTION : $10(\mathrm{~L})$

COMMENT :

PARAMETERS :

1)HS SF 4. 2)0

\#\#\#\#男\#界\#\#\#\#\#\#\#\#\#\#\#\#\#\#\#\#\#\#\#\#\#\#\#\#\#\#\#\#\#\#\#

STEP NO : 24

LABEL :

FUNCTION : 73 (LAT)

COMMENT :SF-4 CONTROL LOGIC

PARAMETERS :

1) LATCH=PULSE(HS_SF_4,5) 2) UNLATCH=-HS_SF_4! (DELAY(HS_S 3)0 4)DO_SF_4. 5)0 
WHC-SD-CP-CSWD-016

Volume 4

Revision 1

Page 103

\#\#\#\#\#\#\#\#\#\#\#\#\#\#\#\#\#\#\#\#\#\#\#\#\#\#\#\#\#\#\#\#\#\#\#\#\#\#\#\#

STEP NO : 25

LABEL : :

FUNCTION : 69 (LC) )

COMMENT : ALARM ON NO FLON OR OFF WHEN HS IS ON (EXCEPT STARTUP)

PARAMETERS :

1)DELAY (HS SF 4,15)\&-(!DI SF 4A\&!DI FS 4)

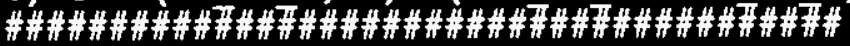

STEP NO : 26

LABEL :

FUNCTION : 75 (DST )

COMMENT :

PARAMETERS :

1)MSV 2)0 3)0 4)0 5)FA SF 4.

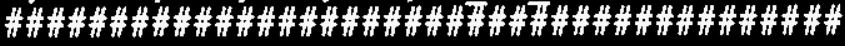

STEP NO : 27

LABEL :

FUNCTION : 0 (END)

COMMENT :

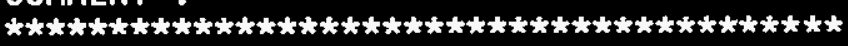

LOOP NO : 6

LOOP TAG : SF_ 6

LOOP DESCRIPTION - : ZB SF-6

LOOP SCAN : 0.30 SEC

NON-PID AUXILIARY :

RATIO LO : 0.00 , HI : 10.00 , BIAS LO : -5.00 , HI : 5.00

RATIO CURR : 0.00 , BIAS CURR : 0.00

EXPORT :DI SF 6A, FA SF 6

IMPORT DI $\mathbf{Z Z B}^{-}{ }^{-}$5::TOI ${ }^{-}$FS 9

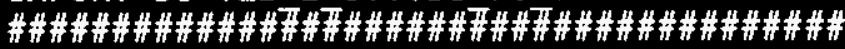

STEP NO : 1

LABEL :

FUNCTION : 6 (VD)

COMMENT :

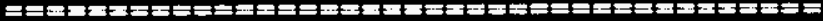

DISCRETE TAG : FA SF 6

DISCRETE (Virtual Disçrēe) \#: 6

DISCRETE DESC : ZB SF-6

DISCRETE SYNC SCAN : O SEC HIST SCAN : O SEC

DISCRETE HIST. DEFINITION :0 DEBOUNCE: 0 MODIFY : 0

DISCRETE STATE O DESC :OFF COLOR : BLACK

DISCRETE STATE 1 DESC :FAIL COLOR : YELLOW

DI Alarm State : 1

DI Alarm Priority : Caution

DI Alarm Desc :ZB SF-6 LOW FLOW / FAIL

DI Alarm Mgmt Discrete :

DI Alarm Group :0 Alarm Mgmt :0 Alarm Index :0 
WHC-SD-CP-CSWD-016 Volume 4 Revision 1 Page 104

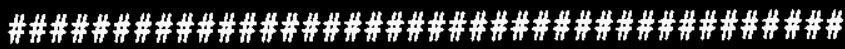

STEP NO : 2

LABEL :

FUNCTION : 7 (DI )

COMMENT :

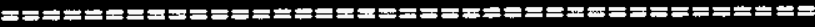

DISCRETE TAG : DI SF 6B

DISCRETE (Discrete Input) \# : 12

DISCRETE DESC : SF-6 READY

DISCRETE SYNC SCAN : O SEC HIST SCAN : O SEC

DISCRETE HIST. DEFINITION :0 DEBOUNCE: 0 INVERT : 0

DISCRETE STATE O DESC :PWR OFF COLOR : WHITE

DISCRETE STATE 1 DESC :PWR ON COLOR : WHITE

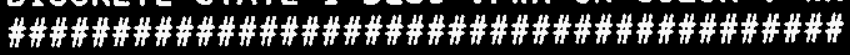

STEP NO

: 3

LABEL :

FUNCTION : 7 (DI )

COMMENT :

DISCRETE TAG : DI SF 6A

DISCRETE (Discrete Input) \#: 13

DISCRETE DESC : SF-6 RUN

DISCRETE SYNC SCAN : O SEC HIST SCAN : O SEC

DISCRETE HIST. DEFINITION :0 DEBOUNCE: 0 INVERT : 0

DISCRETE STATE O DESC :OFF COLOR : WHITE

DISCRETE STATE 1 DESC :ON COLOR : WHITE 
WHC-SD-CP-CSWD-016

Volume 4

Revision 1

Page 105

\#\#\#\#\#\#\#\#\#\#\#\#\#\#\#\#\#\#\#\#\#\#\#\#\#\#\#\#\#\#\#\#\#\#\#\#\#\#\#\#

STEP NO : 4

LABEL :

FUNCTION : 9 (DSCC)

COMMENT :

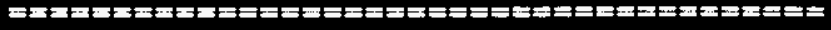

MULTI-STATE DI TAG : YI_SF_6

MULTI-STATE DI \# : 6

MULTI-STATE DI DESC : ZB SF-6

MULTI-STATE DI SYNC SCAN : 0

MULTI-STATE DI1 : DI SF 6A

MULTI-STATE DI2 : DI_SF_6B

MULTI-STATE DI3 :

MULTI-STATE DI4:

MULTI-STATE STATE 0 :DESC PWR OFF COLOR White

MULTI-STATE STATE 1 :DESC ON COLOR Yellow

MULTI-STATE STATE 2 :DESC OFF COLOR Green

MULTI-STATE STATE 3 :DESC FAILED COLOR White

MULTI-STATE STATE 4 :DESC COLOR Black

MULTI-STATE STATE 5 :DESC COLOR Black

MULTI-STATE STATE 6 :DESC COLOR Black

MULTI-STATE STATE 7 :DESC COLOR Black

MULTI-STATE STATE 8 :DESC COLOR Black

MULTI-STATE STATE 9 :DESC COLOR BTaCk

MULTI-STATE STATE 10 :DESC COLOR Black

MULTI-STATE STATE 11 :DESC COLOR BTack

MULTI-STATE STATE 12 :DESC COLOR Black

MULTI-STATE STATE 13 :DESC COLOR B1ack

MULTI-STATE STATE 14 :DESC COLOR B1ack

MULTI-STATE STATE 15 :DESC COLOR B1ack

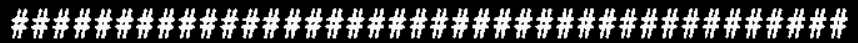

STEP NO : 5

LABEL :

FUNCTION : 57 (DO)

COMMENT :

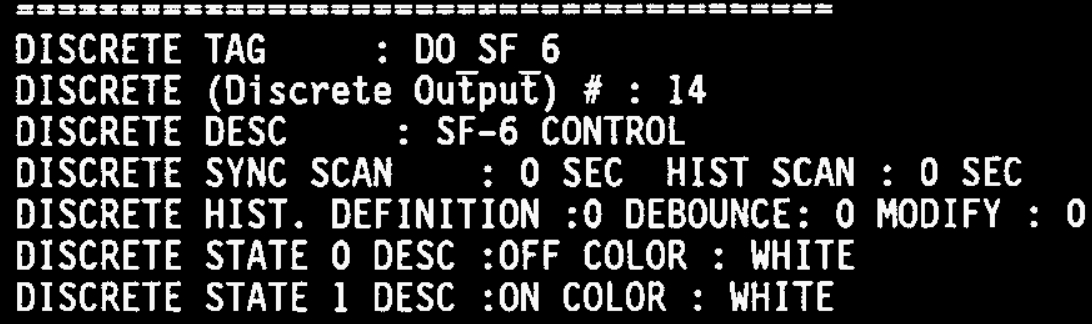


WHC-SD-CP-CSWD-016

Volume 4

Revision 1

Page 106

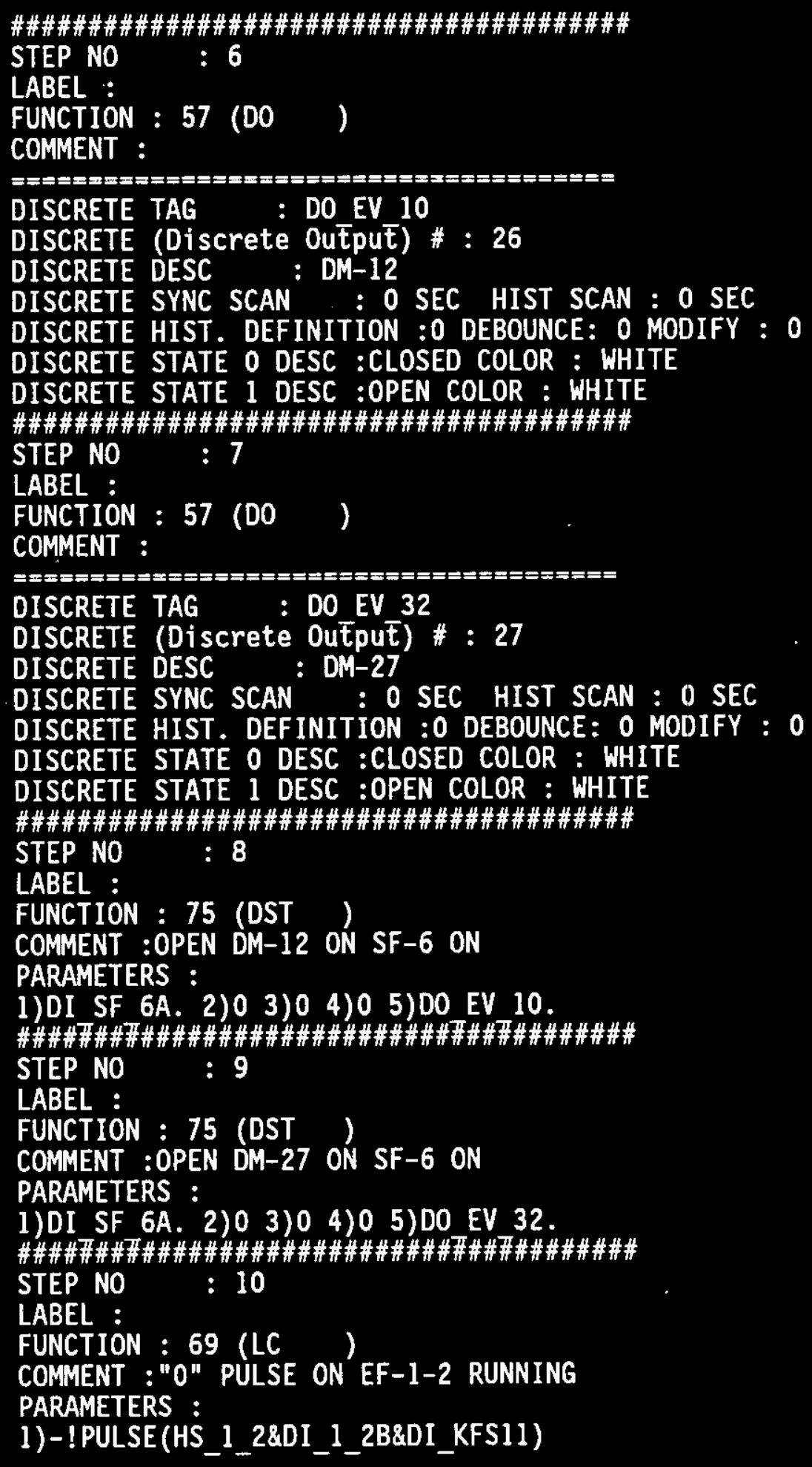




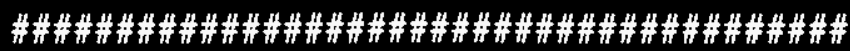

STEP NO : 11

LABEL :

FUNCTION : 62 (GTD

COMMENT :SF-6 START JUMP

PARAMETERS :

1) MSV 2) Off-Nomal 3) JUMPA

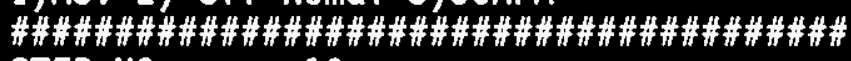

STEP NO : 12

LABEL : JUMPA

FUNCTION : $10(\mathrm{~L})$

COMMENT :

PARAMETERS :

1)HS SF 6. 2)1

\#\#\#\#男\#\#界\#\#\#\#\#\#\#\#\#\#\#\#\#\#\#\#\#\#\#\#\#\#\#\#\#\#\#\#\#\#\#\#

STEP NO : 13

LABEL :

FUNCTION : 69 (LC )

COMMENT : "0" PULSE ON EF-1-2 FAIL OR OFF

PARAMETERS :

1)-! PULSE(FA 12 2:-RUN 12 2)

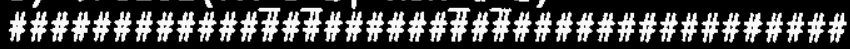

STEP NO : 14

LABEL :

FUNCTION : 62 (GTD )

COMMENT :STOP JUMP

PARAMETERS :

1) MSV 2) Off-Nomal 3) JUMPB

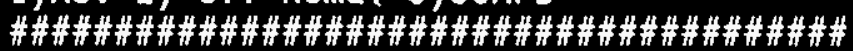

STEP NO : 15

LABEL : JUMPB

FUNCTION : $10(\mathrm{~L})$

COMMENT :

PARAMETERS :

1)HS SF 6. 2)0

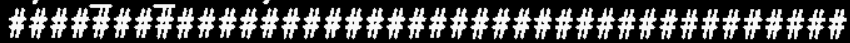

STEP NO : 16

LABEL :

FUNCTION : 69 (LC )

COMMENT :HOLD ON STOP IF COLD SMOKE ALARM

PARAMETERS :

1) - IXA CSA

\#\#\#\#\#\#畀\#\#\#\#\#\#\#\#\#\#\#\#\#\#\#\#\#\#\#\#\#\#\#\#\#\#\#\#\#\#\#\#

STEP NO : 17

LABEL :

FUNCTION : 62 (GTD)

COMMENT :COLD SMOKE ALARM STOP JUMP

PARAMETERS :

1)MSV 2) Off-Noma1 3) JUMPC 
WHC-SD-CP-CSWD-016

Volume 4

Revision 1

Page 108

\#\#\#\#\#\#\#\#\#\#\#\#\#\#\#\#\#\#\#\#\#\#\#\#\#\#\#\#\#\#\#\#\#\#\#\#\#\#\#

STEP NO : 18

LABEL : JUMPC

FUNCTION : $10(\mathrm{~L})$

COMMENT :

PARAMETERS :

1)HS SF 6. 2) 0

\#\#\#\#界\#邢\#\#\#\#\#\#\#\#\#\#\#\#\#\#\#\#\#\#\#\#\#\#\#\#\#\#\#\#\#\#\#

STEP NO : 19

LABEL :

FUNCTION : 73 (LAT)

COMMENT :SF-6 CONTROL LOGIC

PARAMETERS :

1) $L A T C H=P U L S E(H S$ SF 6,5) 2) UNLATCH=-HS_SF 6 3)0 4)D0_SF_6. 5)0

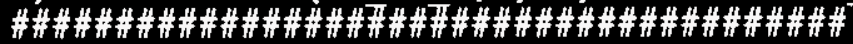

STEP NO : 20

LABEL :

FUNCTION : 69 (LC

COMMENT :ALARM ON NO FLOW OR OFF WHEN HS IS ON (EXCEPT STARTUP)

PARAMETERS :

1)HS SF 6\&-! PULSE(HS SF 6,15)\&-(10I SF 6A\&!DI_FS_9)

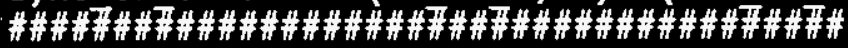

STEP NO : 21

LABEL :

FUNCTION : 75 (DST)

COMMENT :

PARAMETERS :

1)MSV 2)0 3)0 4)0 5) FA SF 6.

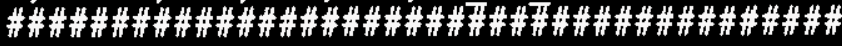

STEP NO : 22

LABEL :

FUNCTION : 0 (END )

COMMENT :

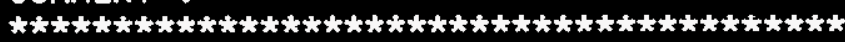

LOOP NO : 7

LOOP TAG : MISC M19

LOOP DESCRIPTION - : MICON 19 MISC

LOOP SCAN : 0.30 SEC

NON-PID AUXILIARY :

RATIO LO : 0.00 , HI : 10.00 , BIAS LO : -5.00 , HI : 5.00

RATIO CURR : 0.00 , BIAS CURR : 0.00 


\section{WHC-SD-CP-CSWD-016 \\ Volume 4 Revision 1 \\ Page 109}

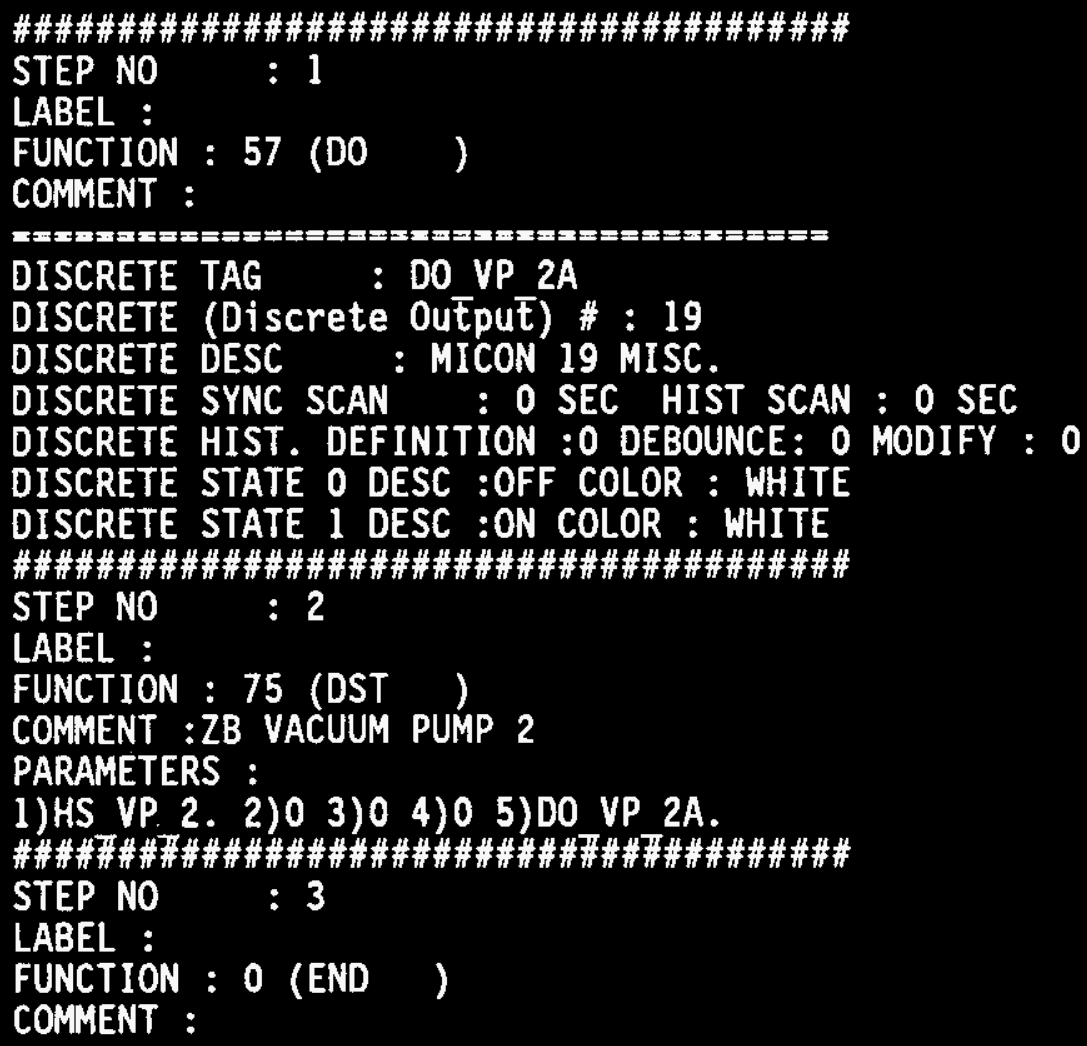


WHC-SD-CP-CSWD-016

Volume 4

Revision 1

Page 110

ZB_2_3.mic20_ro

MICON TYPE :RCM-D

MICON UNIT NO $: 20$

MICON TAG : ZB 23

MICON DESCRIPTION $\div$ 2736-ZB RACK 2 SLOT 3

MICON CONFIGURATION FILE : mic20_ro

POINT ALLOCATION :

VAI : 32, VDI : 32, ISW : 32, LOOPS : 12, MSD : 16,

$A I: 0, A O: 0, D I: 32, D 0: 32$

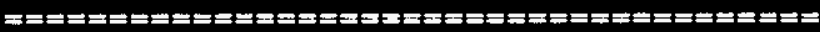

DISCRETE TAG : HS 1

DISCRETE (Internal Switch) \#: I

DISCRETE DESC : ADM AREA

DISCRETE SYNC SCAN : O SEC HIST SCAN : O SEC

DISCRETE HIST. DEFINITION :0 DEBOUNCE: 0 MODIFY : 1

DISCRETE STATE 0 DESC :AUTO COLOR : CYAN

DISCRETE STATE 1 DESC :MANUAL COLOR : YELLOW

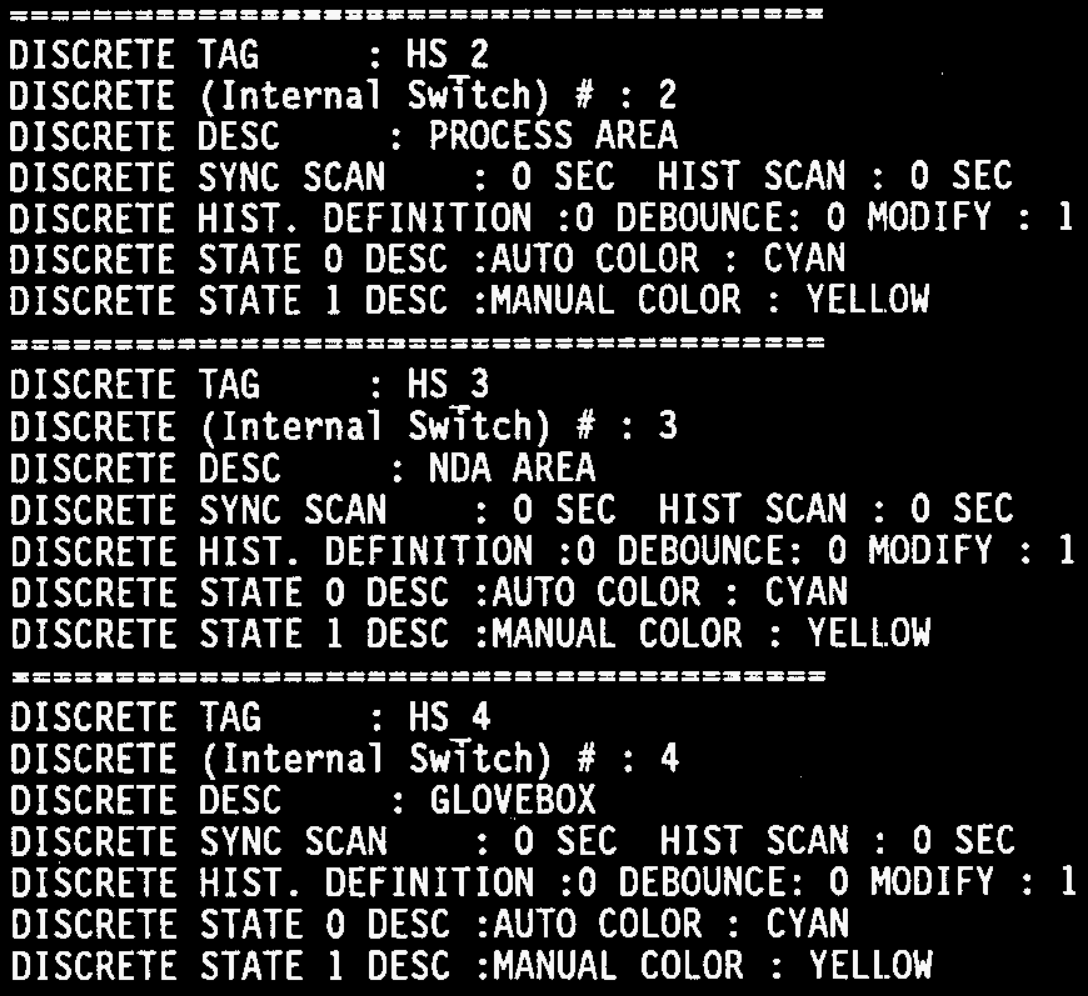


WHC-SD-CP-CSWD-016

Volume 4

Revision 1

Page 111

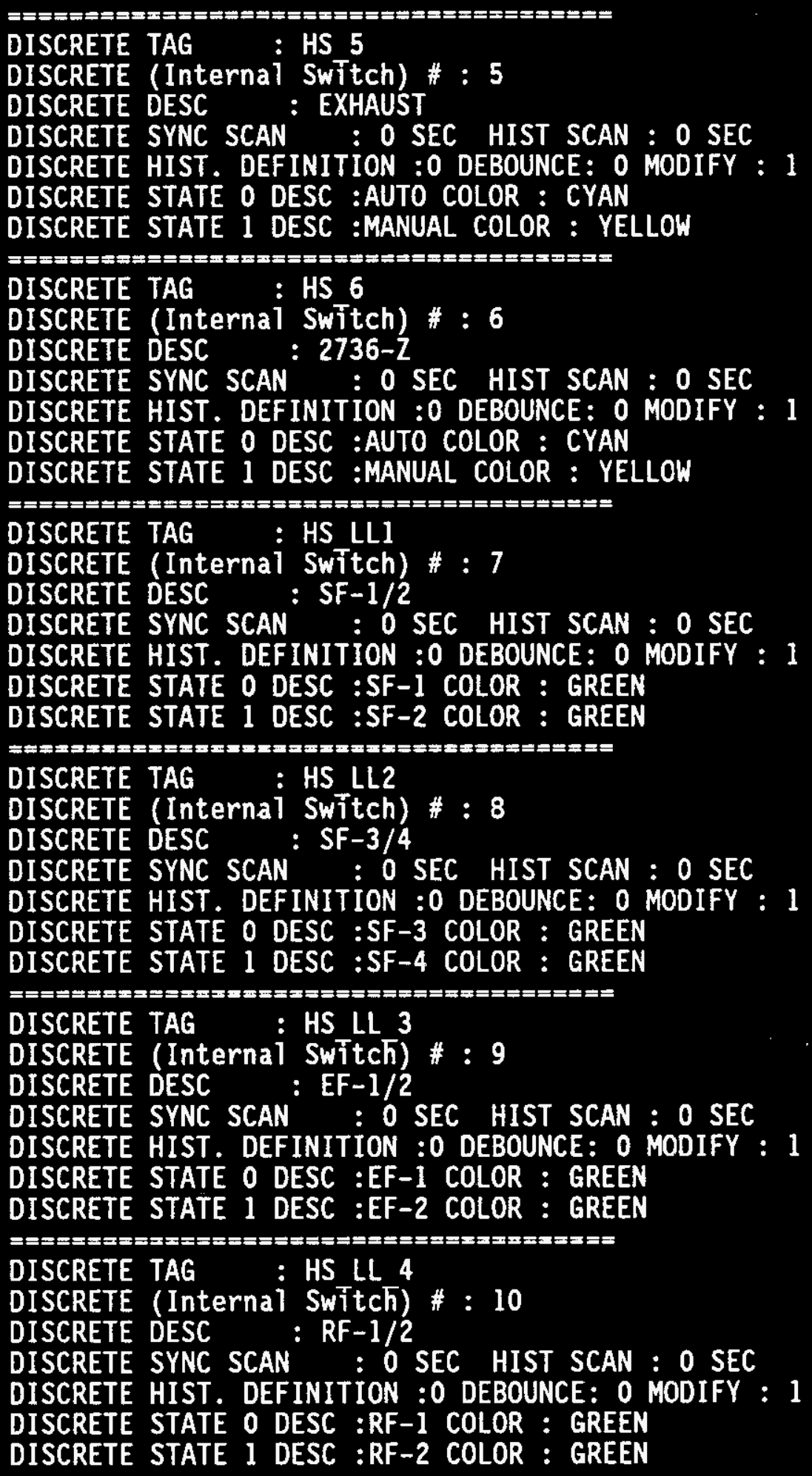


WHC-SD-CP-CSWD-016

Volume 4

Revision 1

Page 112

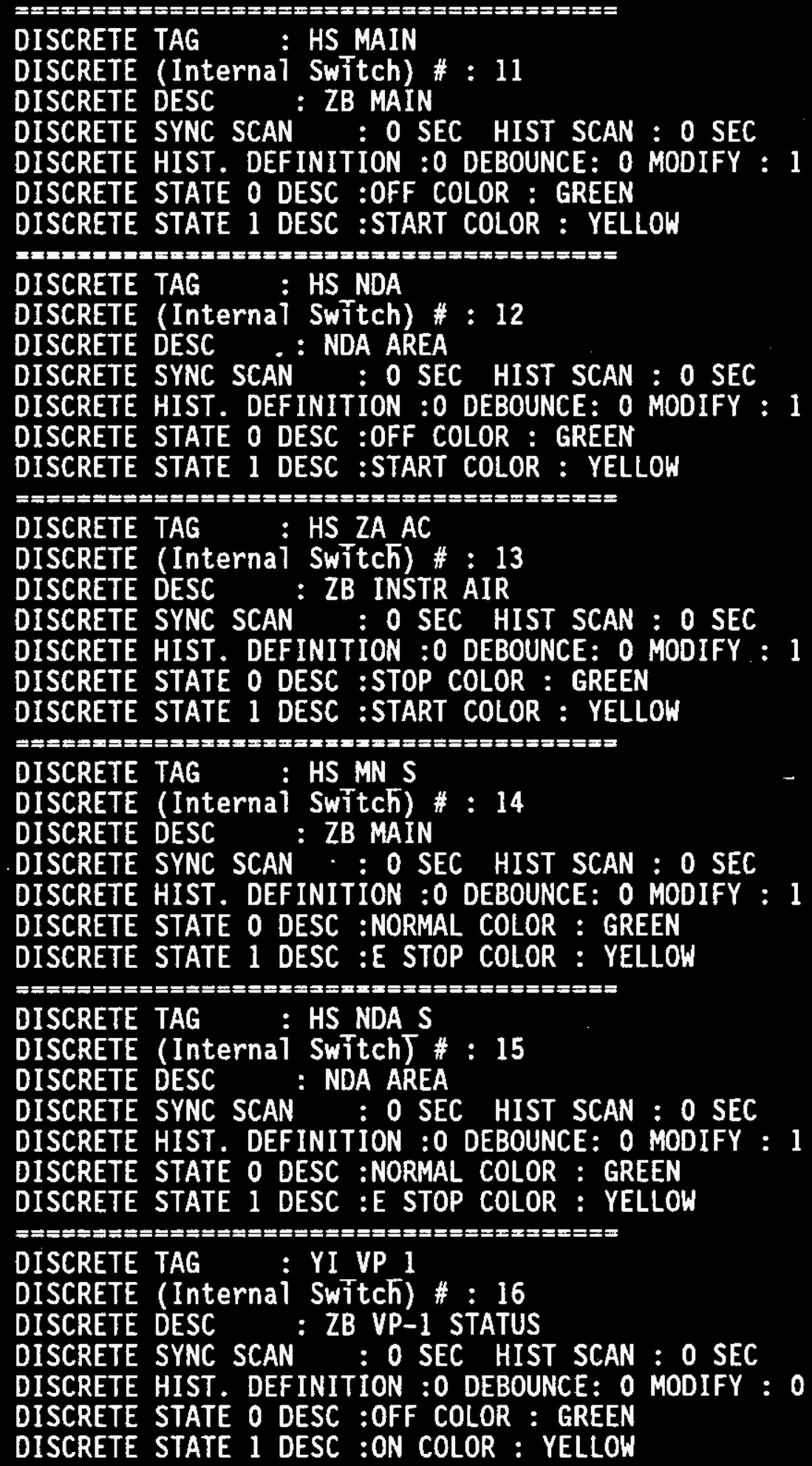




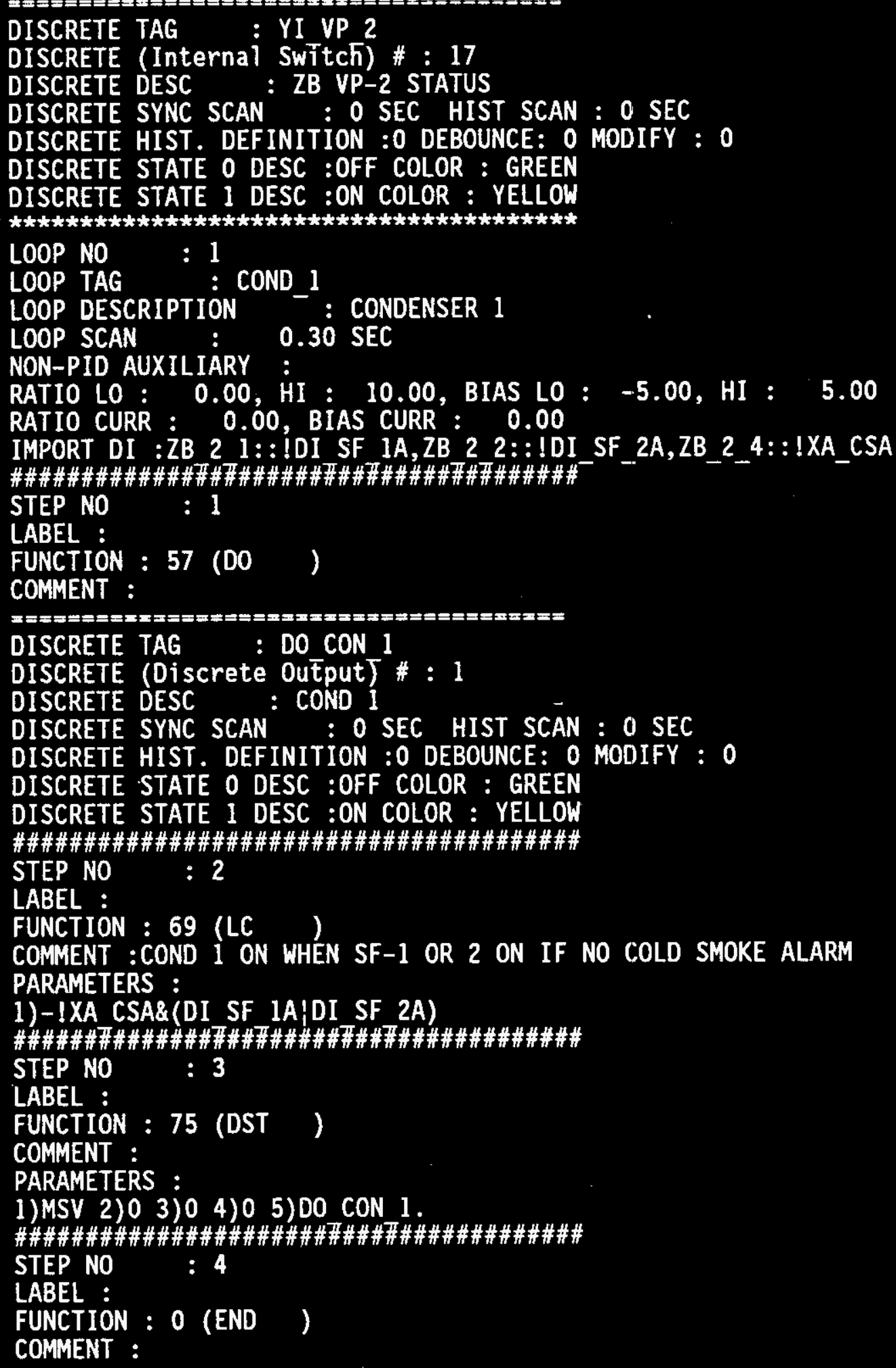


WHC-SD-CP-CSWD-016

Volume 4

Revision 1

Page 114

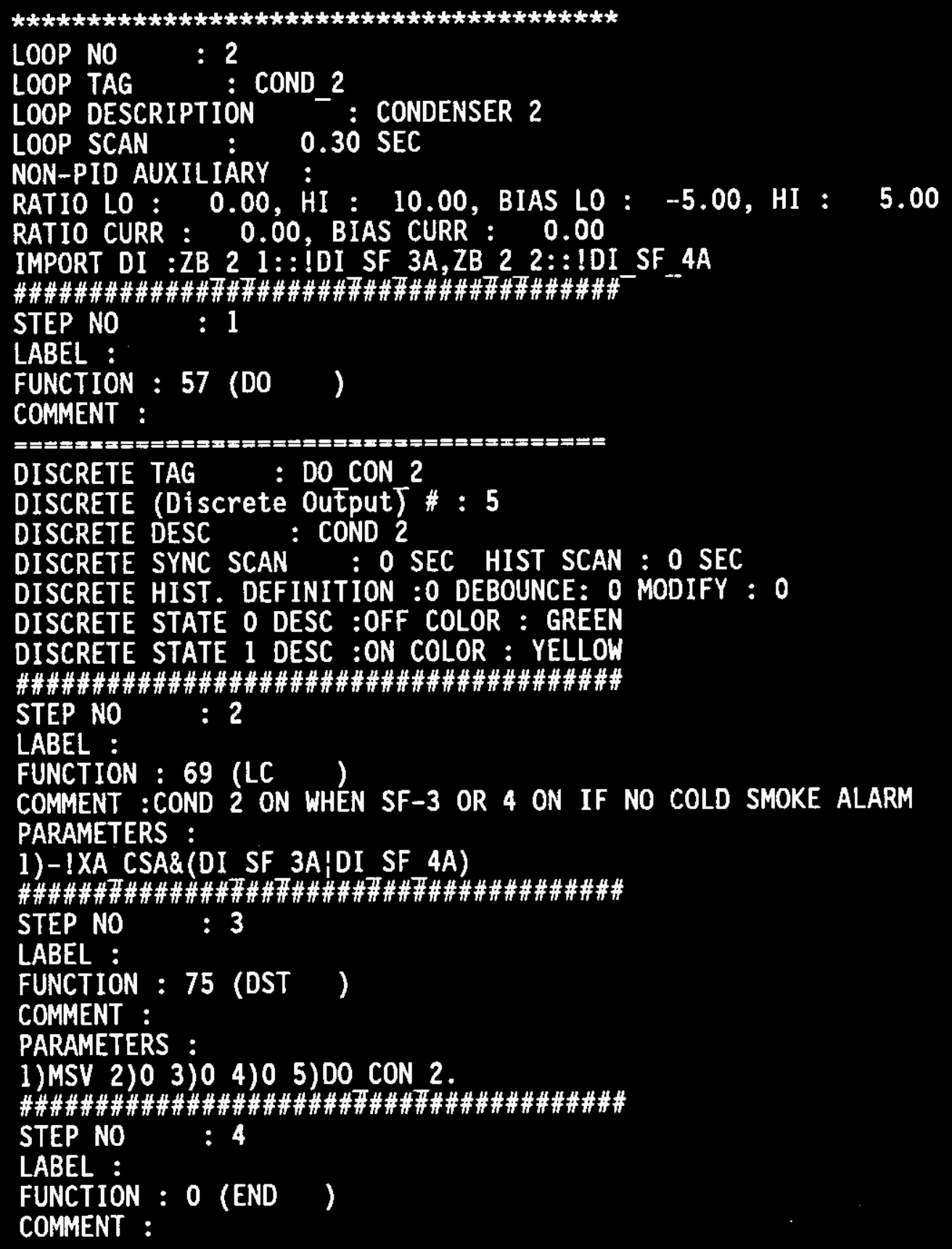




$$
\begin{aligned}
& \text { WHC-SD-CP-CSWD-016 } \\
& \text { Volume } 4 \\
& \text { Revision } 1 \\
& \text { Page } 115
\end{aligned}
$$

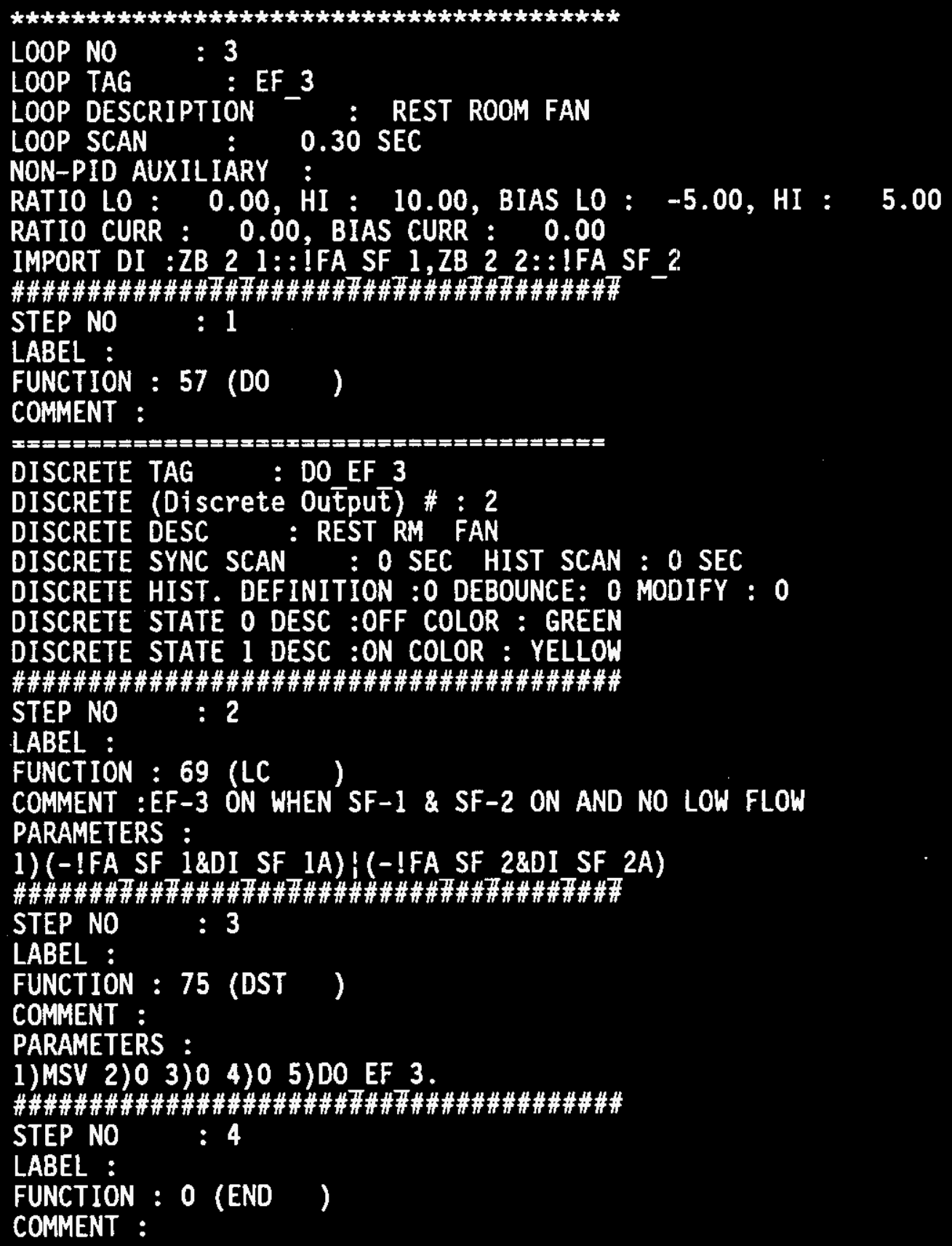


WHC-SD-CP-CSWD-016

Volume 4

Revision 1

Page 116

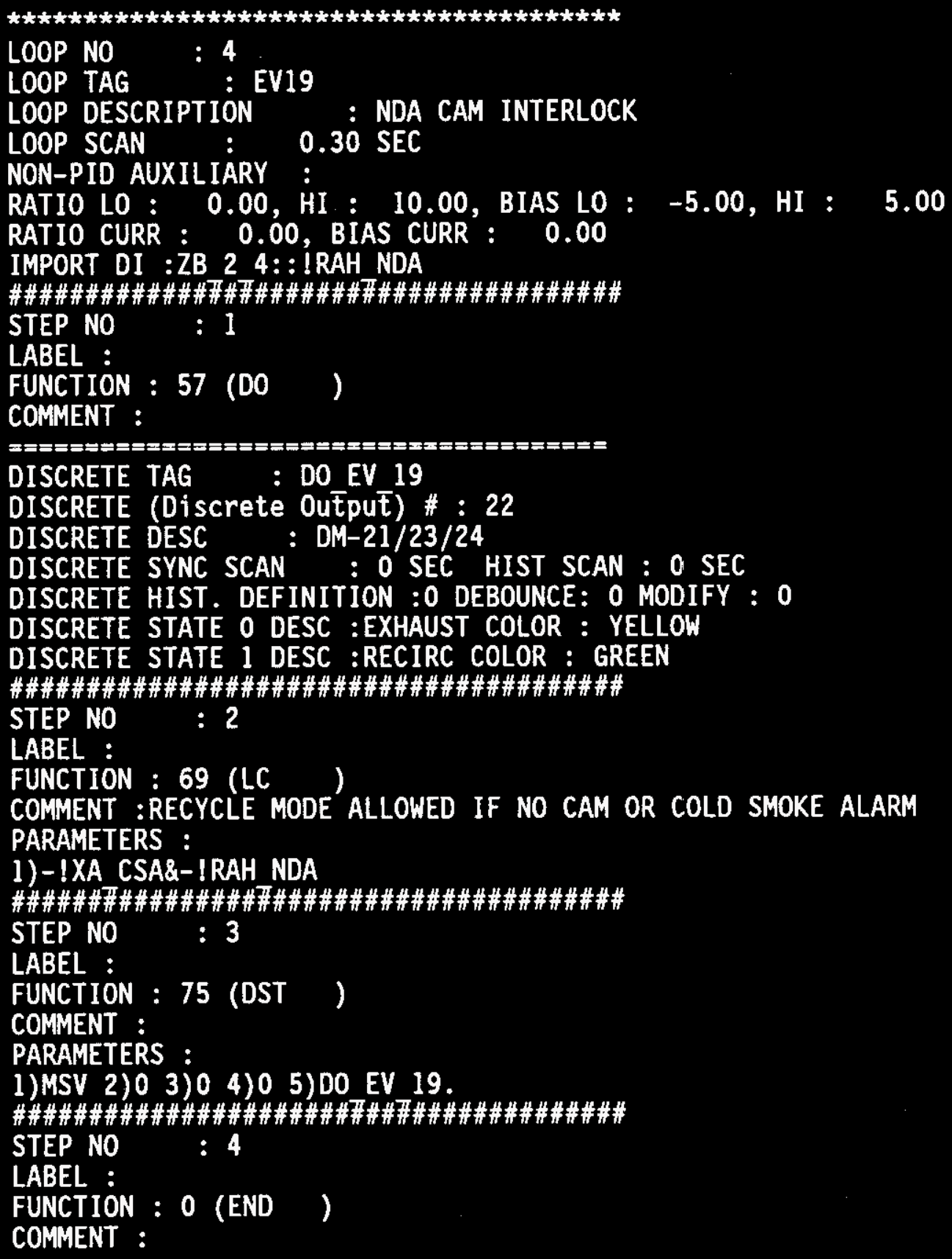


WHC-SD-CP-CSWD-016

Volume 4

Revision 1

Page 117

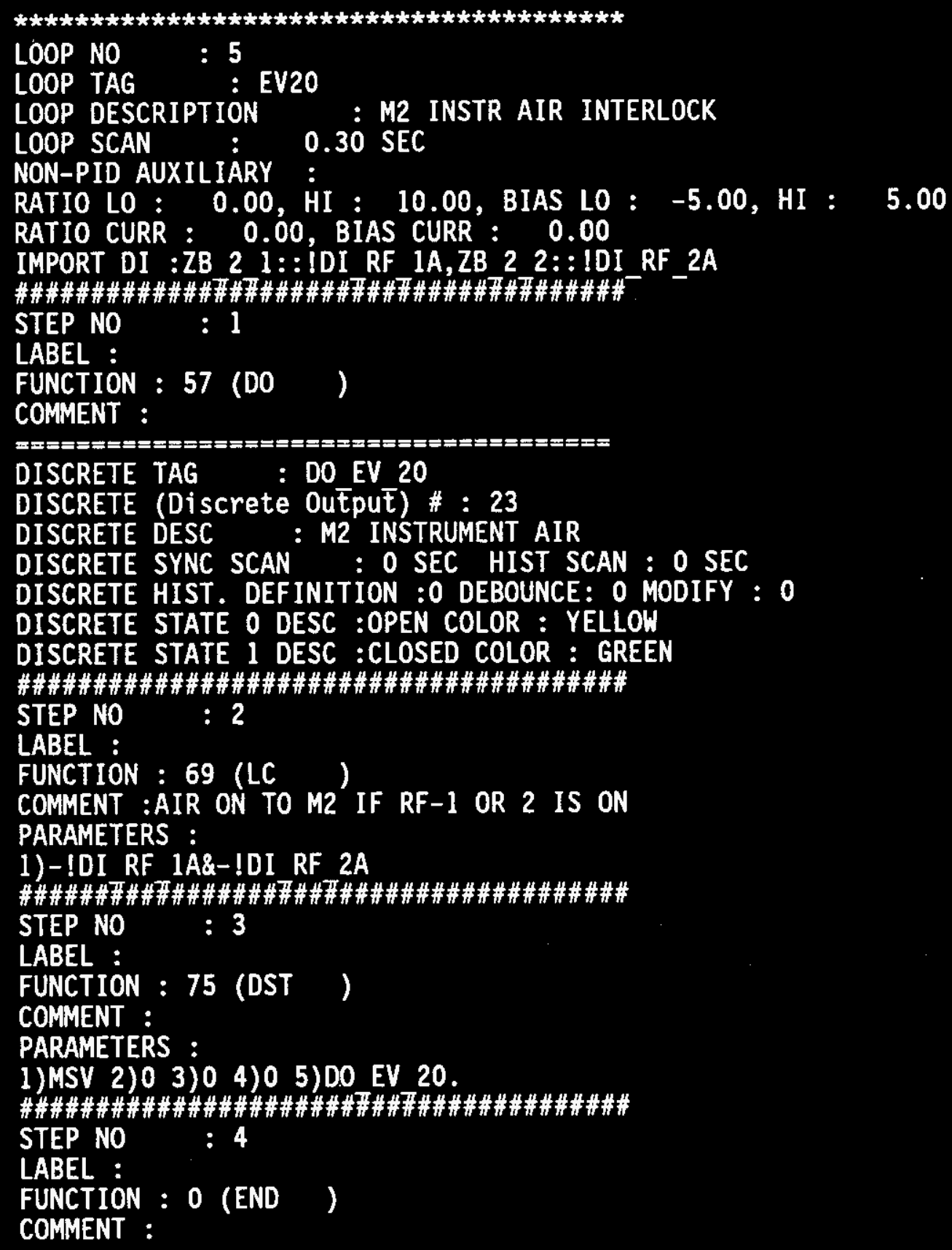


WHC-SD-CP-CSWD-016

Vol ume 4

Revision 1

Page 118

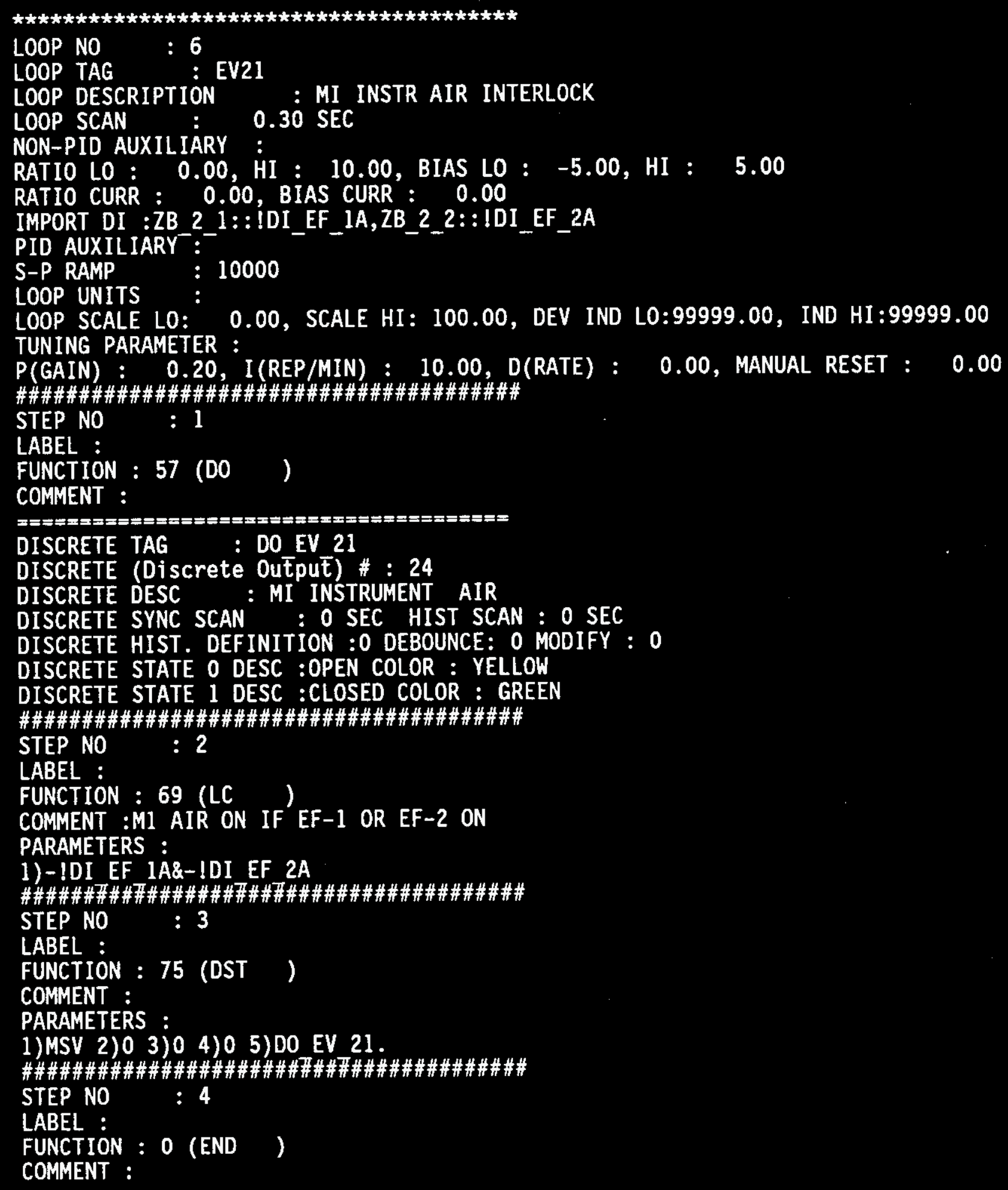


WHC-SD-CP-CSWD-016

Volume 4

Revision 1

Page 119

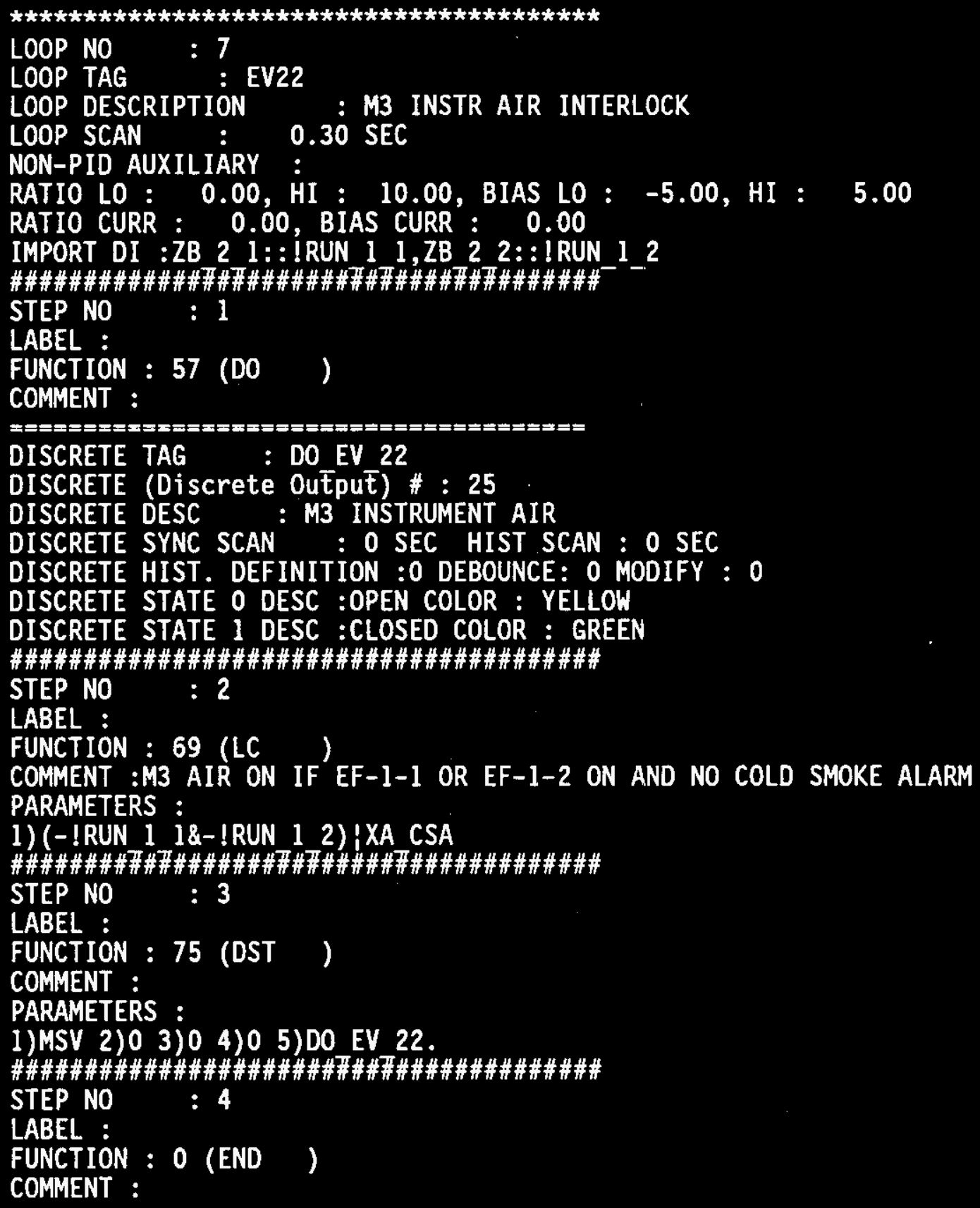


WHC-SD-CP-CSWD-016

Volume 4

Revision 1

Page 120

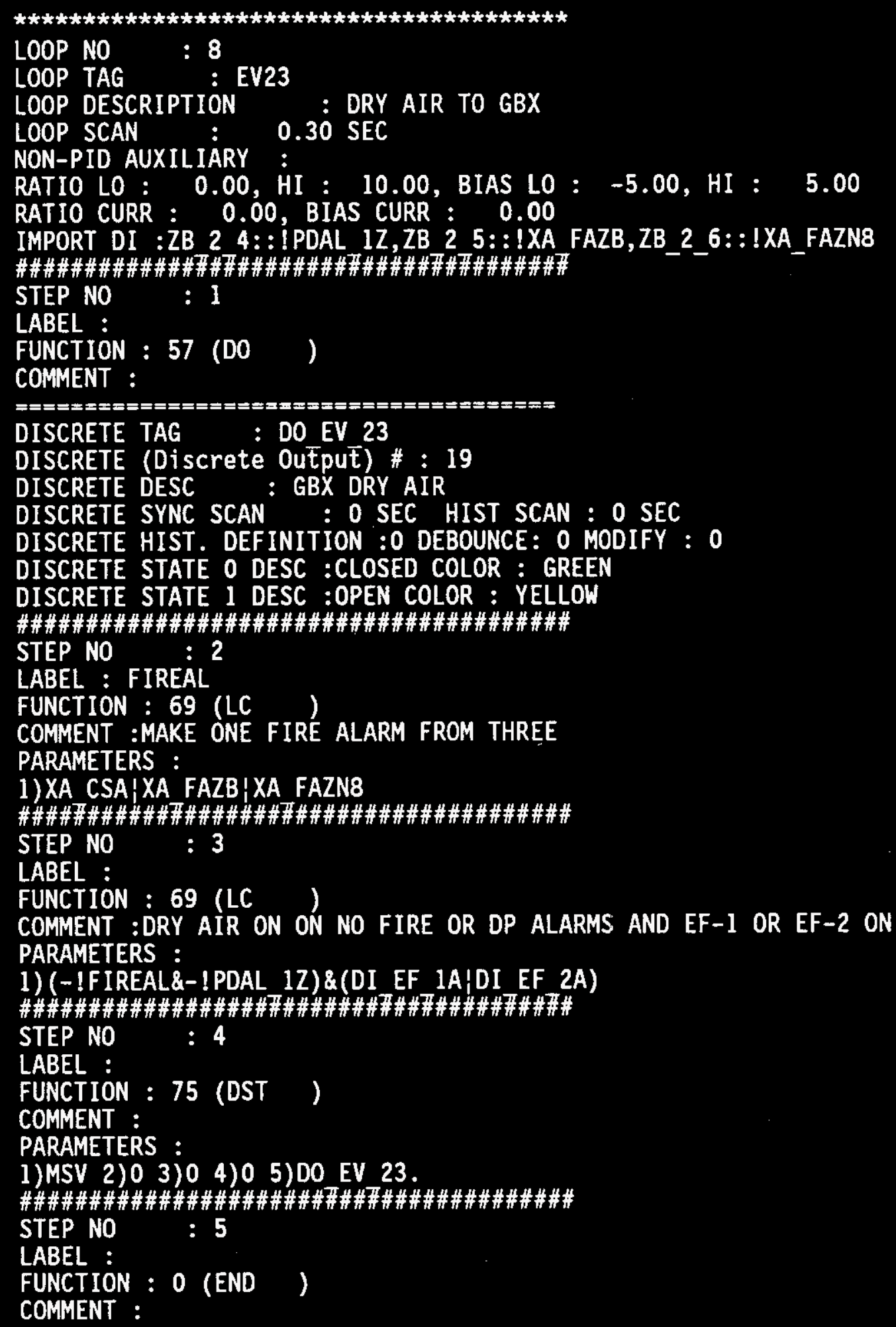


WHC-SD-CP-CSWD-016

Volume 4

Revision 1

Page 121

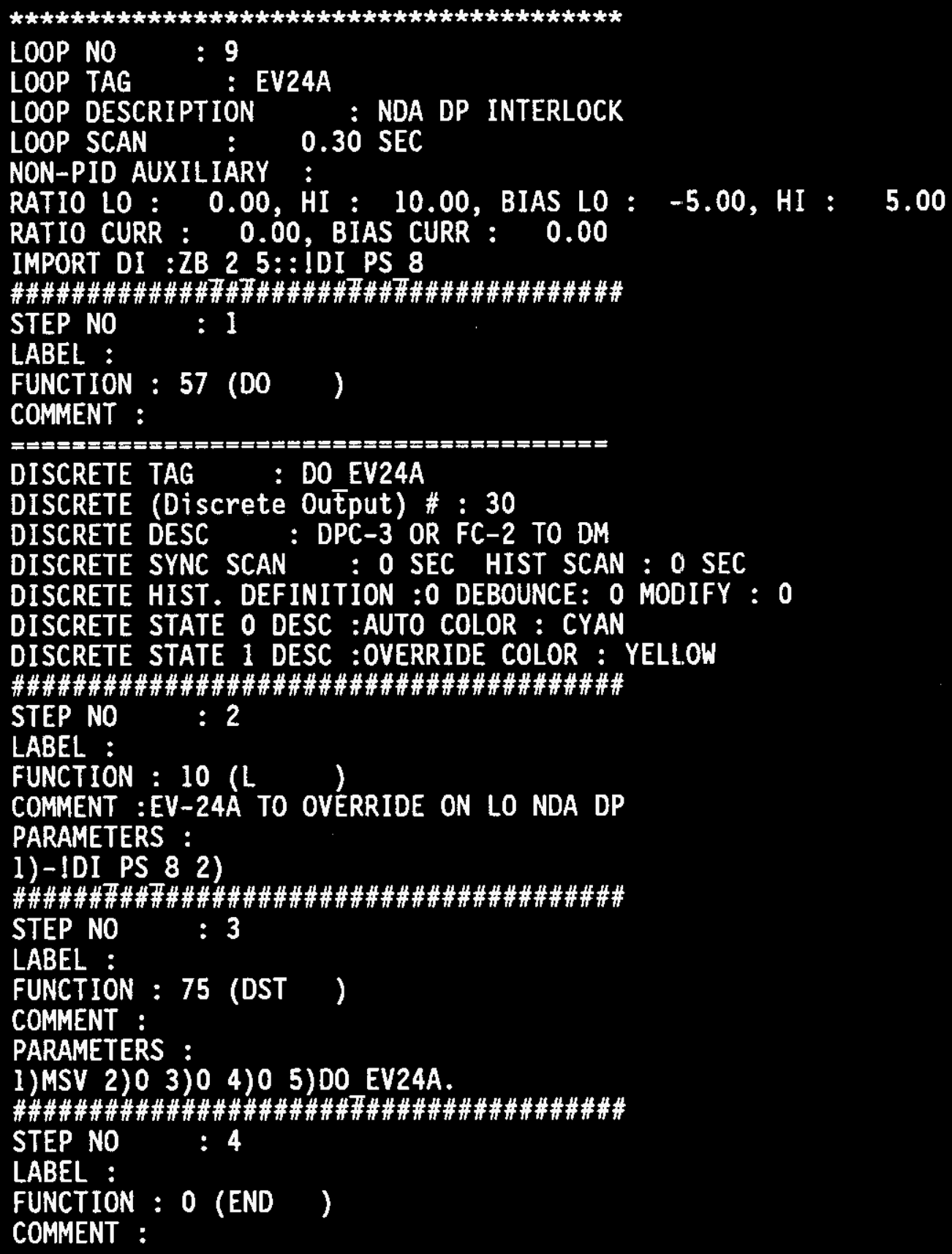



WHC-SD-CP-CSWD-016
Volume 4
Revision 1
Page 122

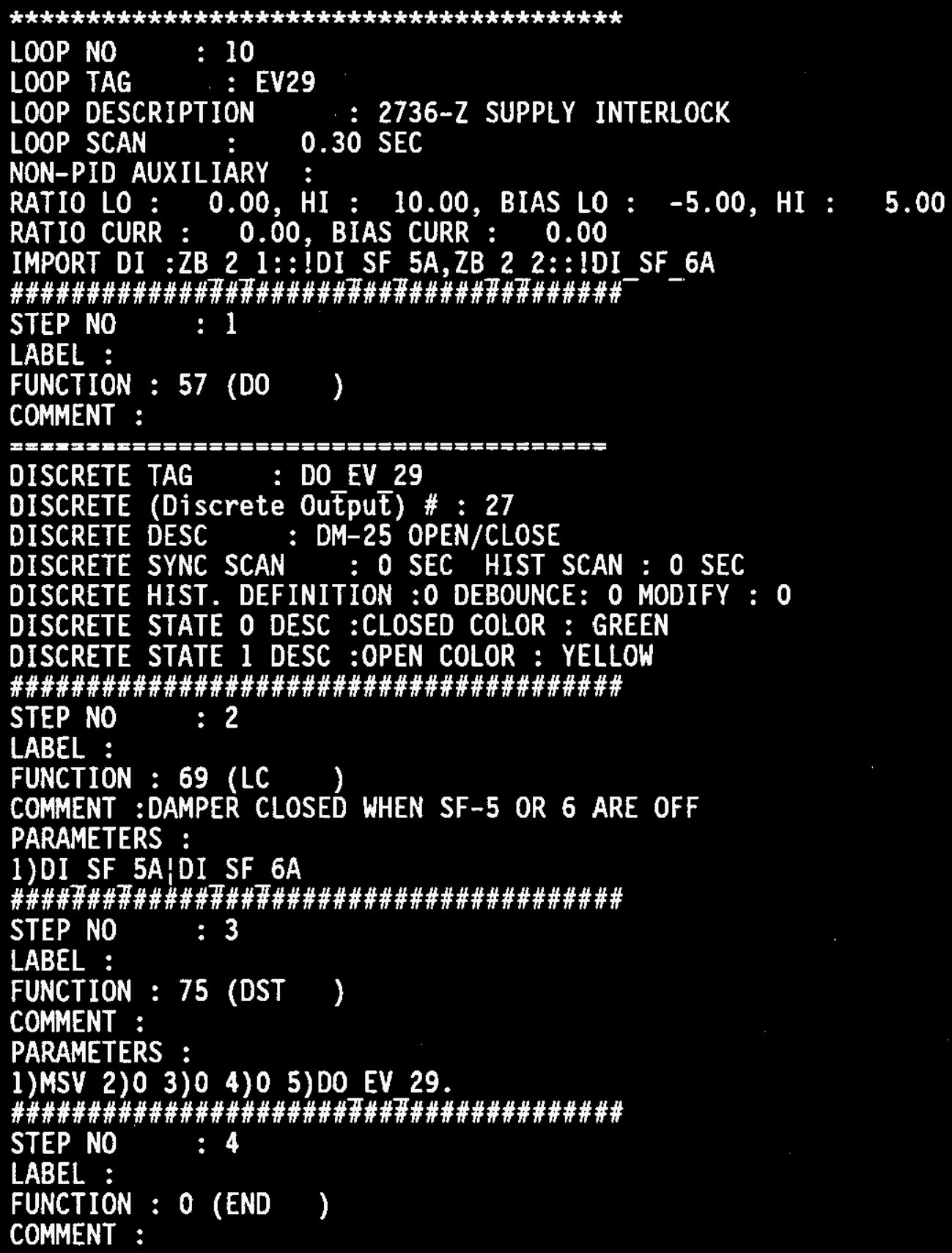


WHC-SD-CP-CSWD-016

Volume 4

Revision 1

Page 123

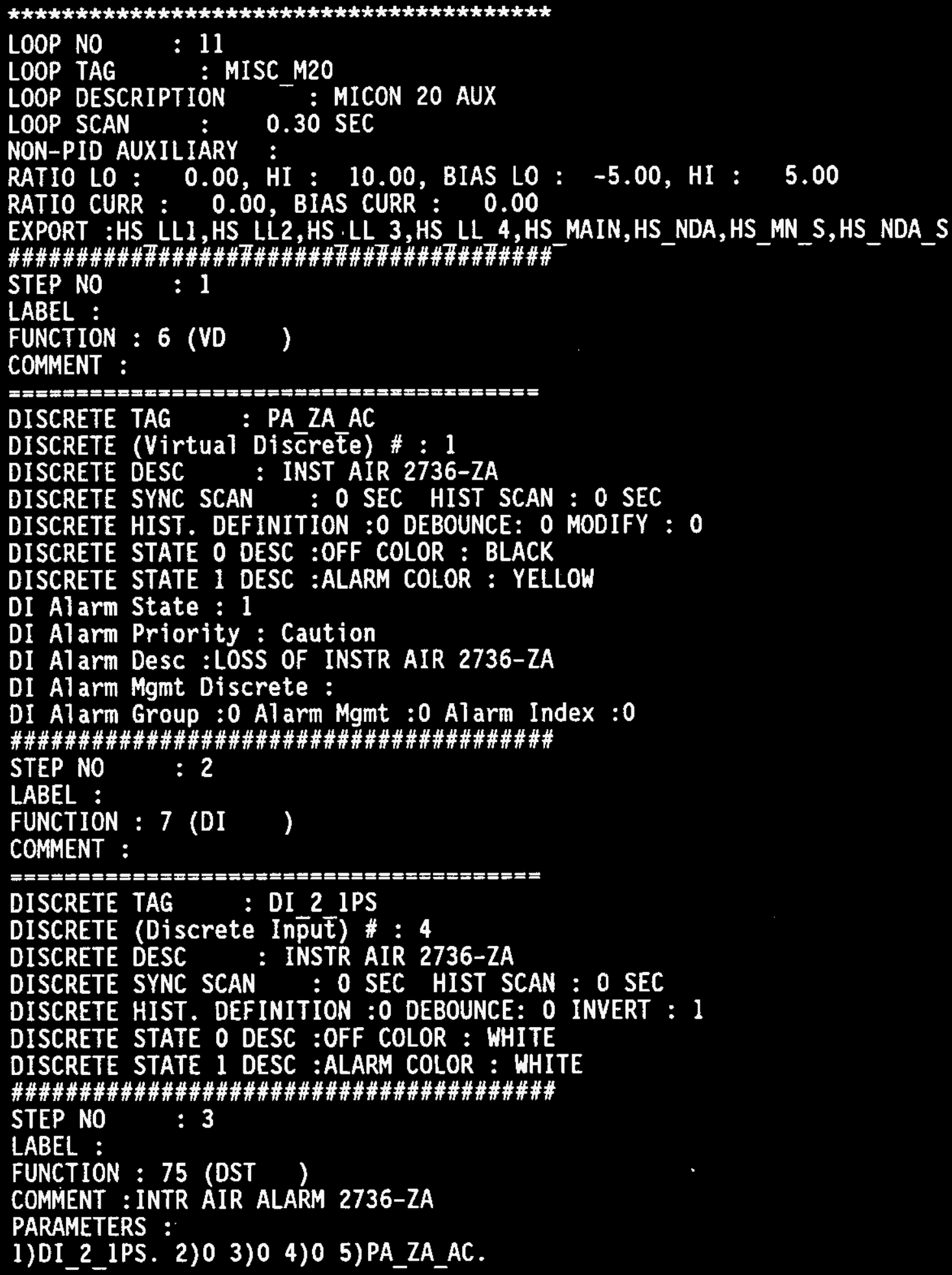


WHC-SD-CP-CSWD-016

Volume 4

Revision 1

Page 124

\#\#\#\#\#\#\#\#\#\#\#\#\#\#\#\#\#\#\#\#\#\#\#\#\#\#\#\#\#\#\#\#\#\#\#\#\#\#\#\#

STEP NO : 4

LABEL :

FUNCTION : $57(\mathrm{DO})$

COMMENT :

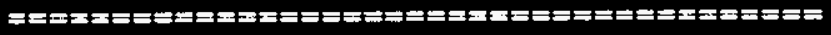

DISCRETE TAG : DO ZA

DISCRETE (Discrete Output) \#: 3

DISCRETE DESC : COMRESSOR 2736-ZA

DISCRETE SYNC SCAN : O SEC HIST SCAN : O SEC

DISCRETE HIST. DEFINITION :0 DEBOUNCE: 0 MODIFY : 0

DISCRETE STATE O DESC :OFF COLOR : GREEN

DISCRETE STATE 1 DESC :ON COLOR : YELLOW

\#\#\#\#\#\#\#\#\#\#\#\#\#\#\#\#\#\#\#\#\#\#\#\#\#\#\#\#\#\#\#\#\#\#

STEP NO : 5

LABEL :

FUNCTION : 75 (DST)

COMMENT : INSTR AIR COMPR 2736-ZA

PARAMETERS :

1)HS ZA AC. 2)0 3)0 4)0 5)D0 ZA.

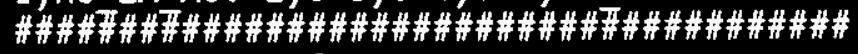

STEP NO : 6

LABEL :

FUNCTION : $57(00)$

COMMENT :

DISCRETE TAG : DO EV 27

DISCRETE (Discrete Output̄) \# : 18

DISCRETE DESC : ADM AREA ZN IV

DISCRETE SYNC SCAN : O SEC HIST SCAN : O SEC

DISCRETE HIST. DEFINITION :0 DEBOUNCE: 0 MODIFY : 0

DISCRETE STATE 0 DESC :AUTO COLOR : CYAN

DISCRETE STATE 1 DESC :MANUAL COLOR : YELLOW

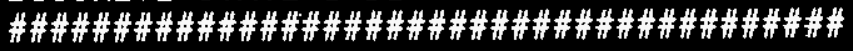

STEP NO : 7

LABEL :

FUNCTION : 75 (DST

COMMENT : ADMIN AREA ZN IV AUTO/MANUAL

PARAMETERS :

1)HS_1. 2)0 3)0 4)0 5)DO_EV_27. 


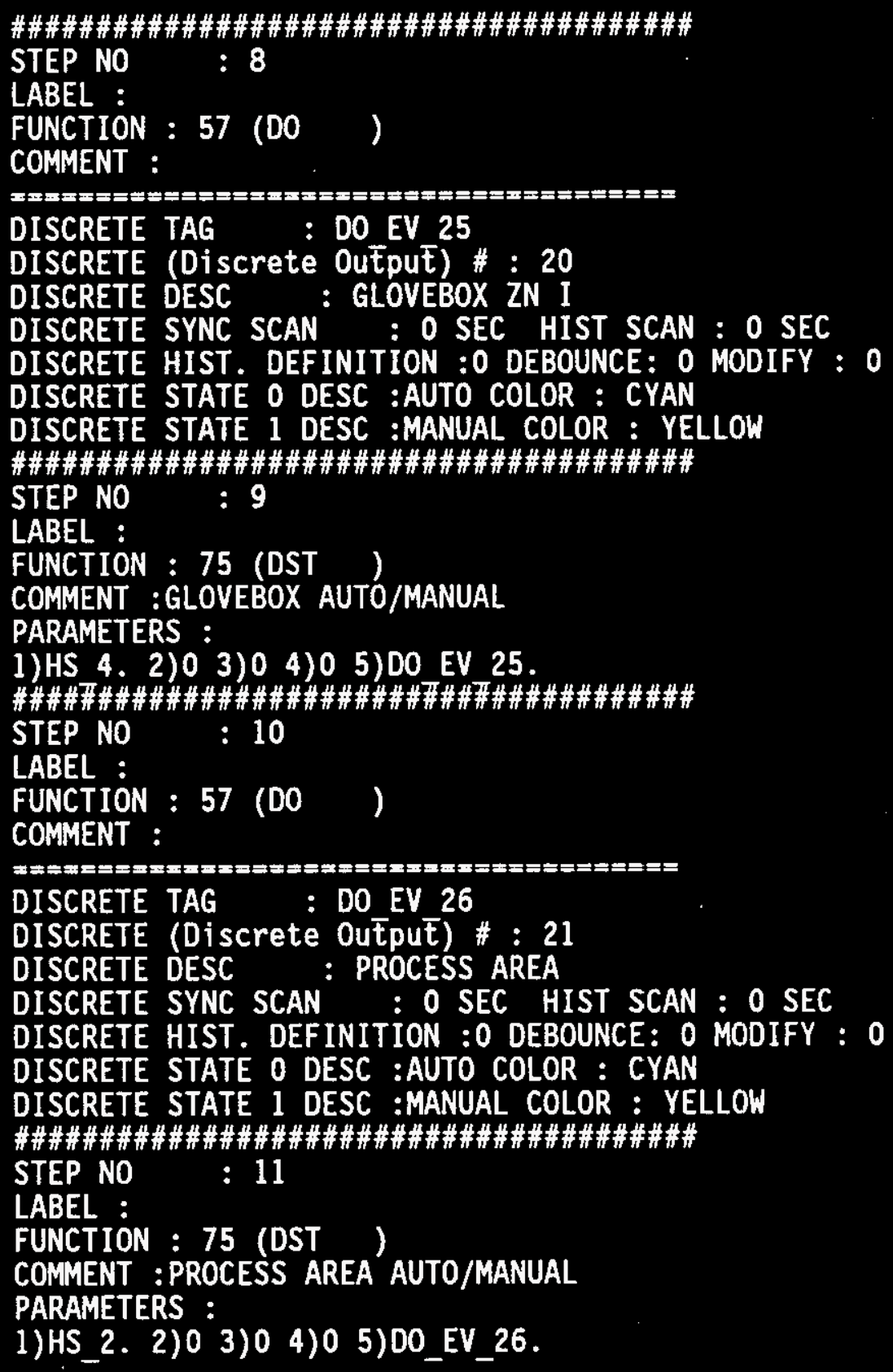




\section{WHC-SD-CP-CSWD-016 \\ Volume 4 \\ Revision 1 \\ Page 126}

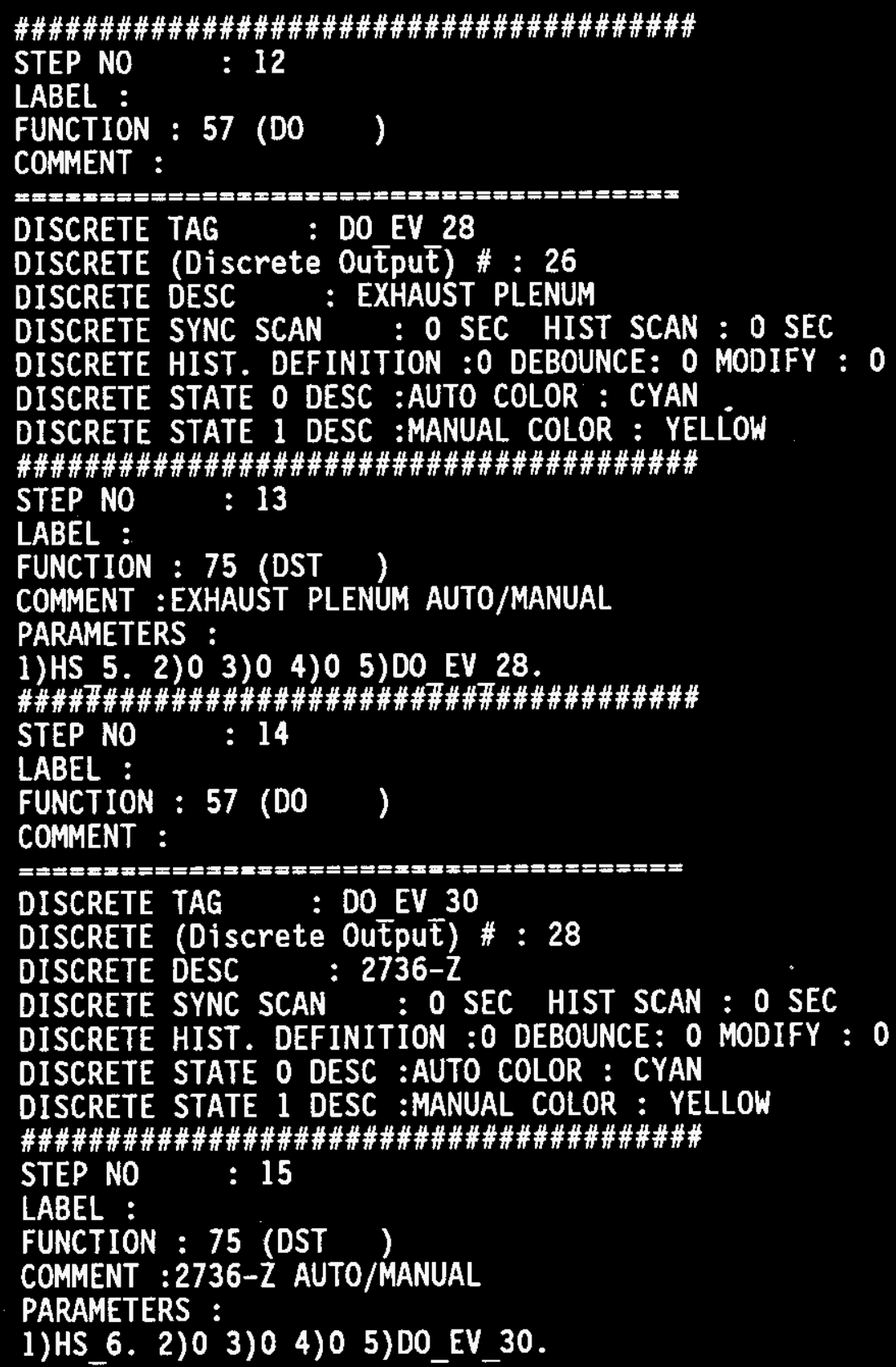


WHC-SD-CP-CSWD-016

Volume 4

Revision 1

Page 127

\#\#\#\#\#\#\#\#\#\#\#\#\#\#\#\#\#\#\#\#\#\#\#\#\#\#\#\#\#\#\#\#\#\#\#\#\#\#\#\#

STEP NO : 16

LABEL :

FUNCTION : 57 (DO)

COMMENT :

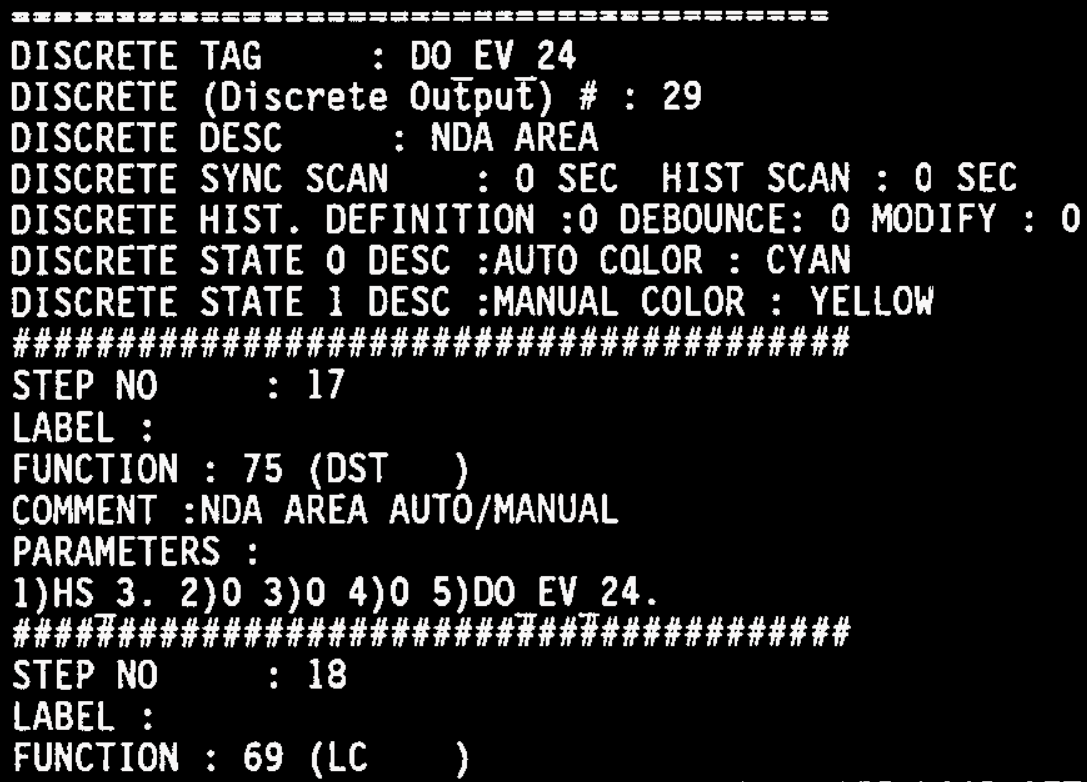


\#\#\#\#\#\#\#\#\#\#\#\#\#\#\#\#\#\#\#\#\#\#\#\#\#\#\#\#\#\#\#\#\#\#\#\#\#\#\#\#

STEP NO : 22

LABEL :

FUNCTION : 62 (GTD )

COMMENT :

PARAMETERS :

1)MSV 2) Off-Nomal 3)BUTNDA

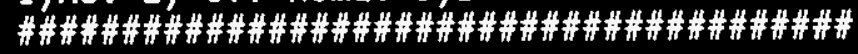

STEP NO

$: 23$

LABEL : BUTNDA

FUNCTION : 10 (L

COMMENT :NDA AUTOSTART SWITCH

PARAMETERS :

1)HS NDA. 2) 0

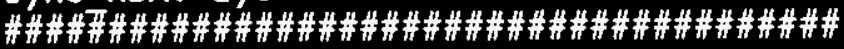

STEP NO : 24

LABEL :

FUNCTION : 69 (LC )

COMMENT :SIMULATE BUTTON

PARAMETERS :

1)-! PULSE(DELAY (HS MN S,5))

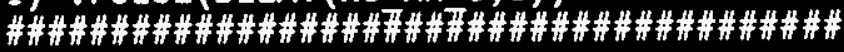

STEP NO

: 25

LABEL :

FUNCTION : 62 (GTD )

COMMENT :

PARAMETERS :

1)MSV 2) Off-Nomal 3)BUTMNS

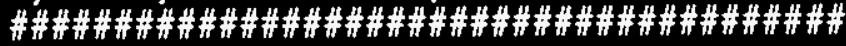

STEP NO $: 26$

LABEL : BUTMNS

FUNCTION : 10 (L

COMMENT :MAIN EMERG STOP

PARAMETERS :

1)HS MN S. 2)0

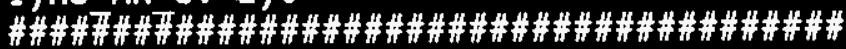

STEP NO : 27

LABEL :

FUNCTION : 69 (LC

COMMENT : SIMULATE BUTTON

PARAMETERS :

1)-! PULSE(DELAY (HS NDA S, 5))

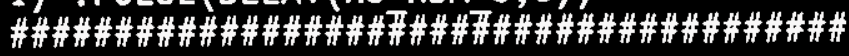

STEP NO : 28

LABEL :

FUNCTION : 62 (GTD )

COMMENT :

PARAMETERS :

1)MSV 2) Off-Nomal 3) BUTNDAS 
\#\#\#\#\#\#\#\#\#\#\#\#\#\#\#\#\#\#\#\#\#\#\#\#\#\#\#\#\#\#\#\#\#\#\#\#\#\#\#\#

STEP NO : 29

LABEL : BUTNDAS

FUNCTION : 10 (L

COMMENT :NDA EMERG SHUTDOWN

PARAMETERS :

1)HS NDA S. 2)0

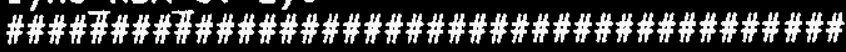

STEP NO : 30

LABEL :

FUNCTION : 7 (DI)

COMMENT :

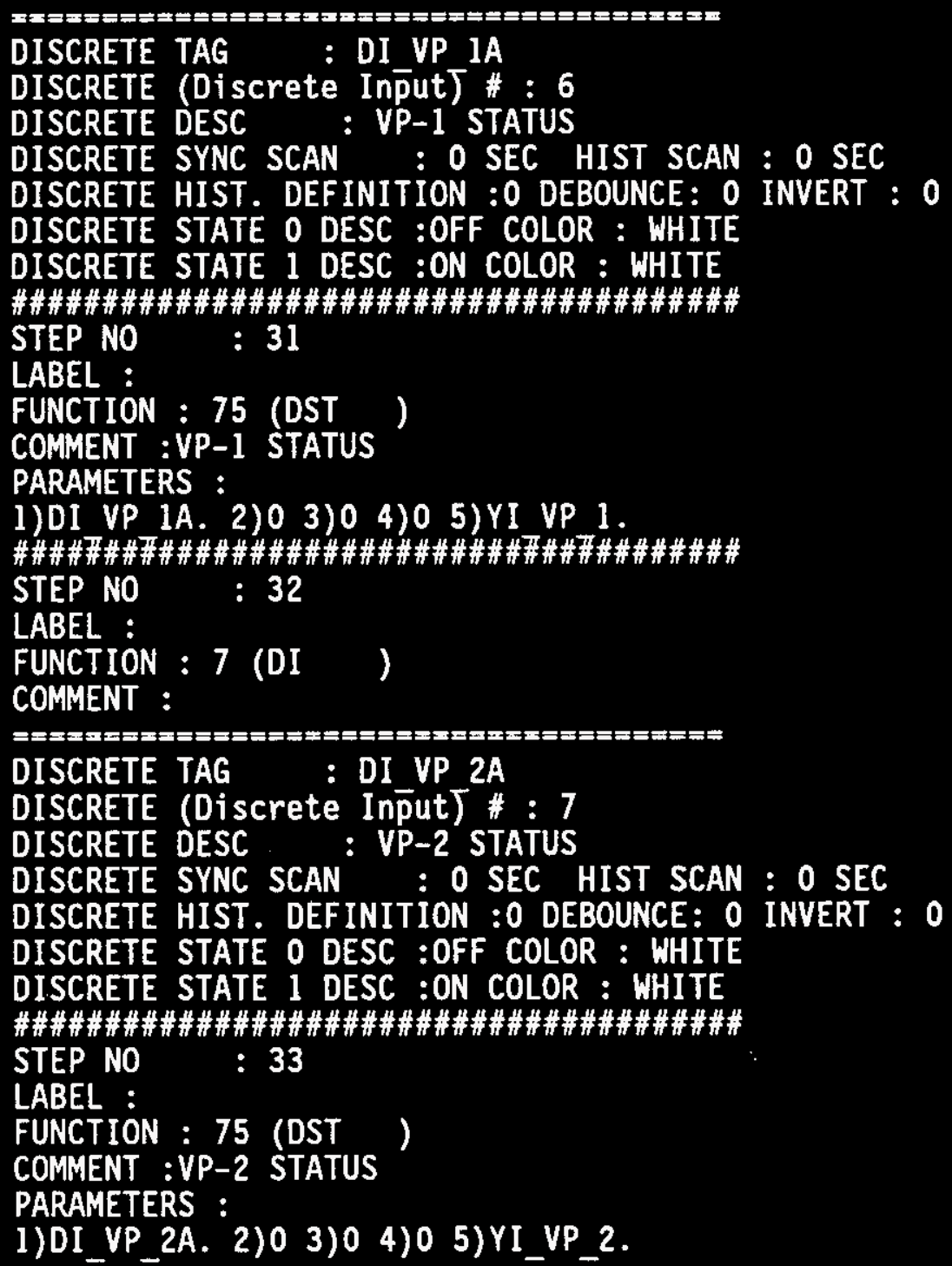




\section{WHC-SD-CP-CSWD-016 \\ Volume 4 \\ Revision 1 \\ Page 130}

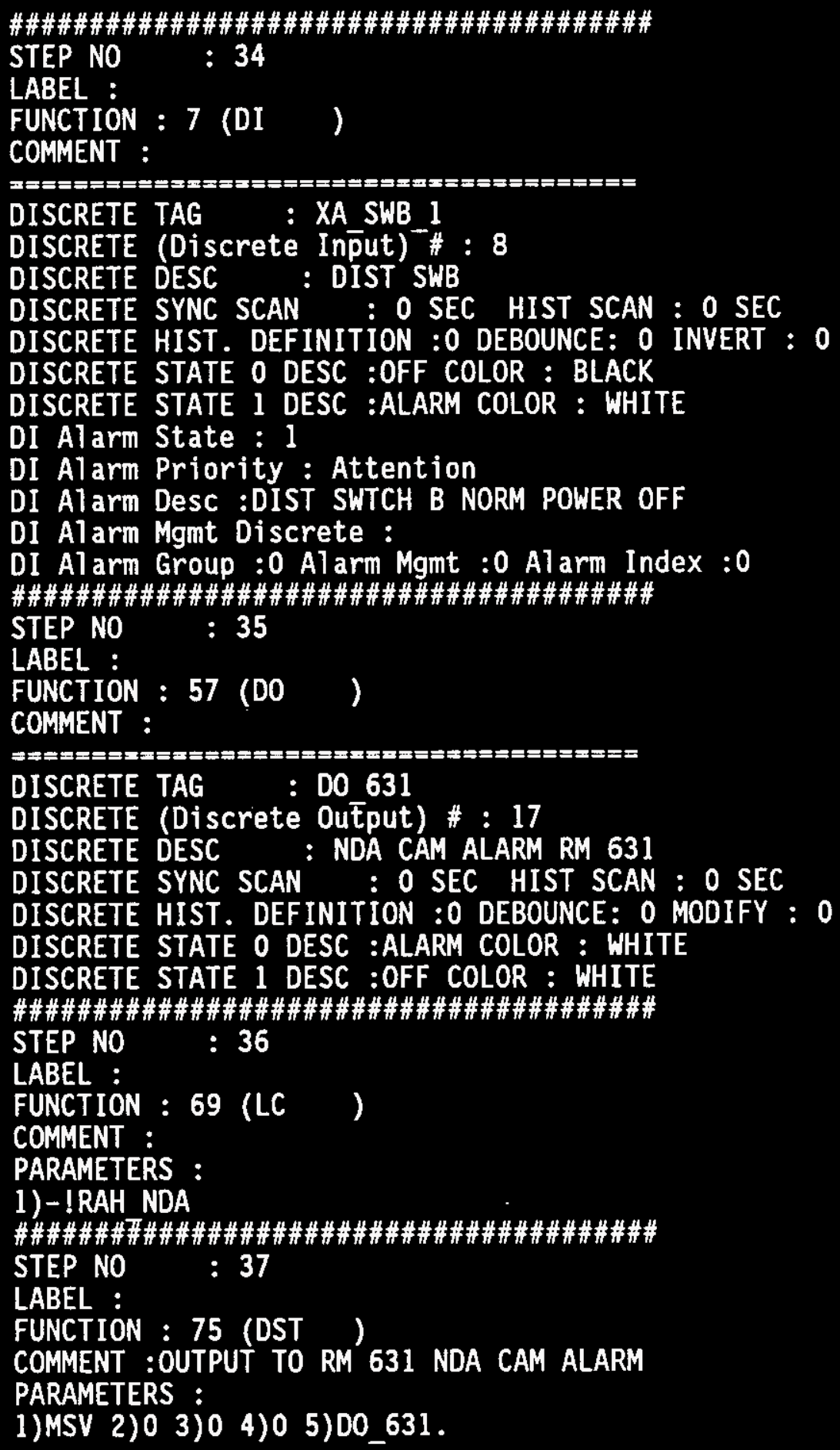


WHC-SD-CP-CSWD-016 Volume 4 Revision 1

Page 131

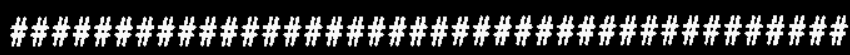

STEP NO : 38

LABEL :

FUNCTION : 0 (END )

COMMENT : 
WHC-SD-CP-CSWD-016

Volume 4

Revision 1

Page 132

ZB_2_4.mic21_ro

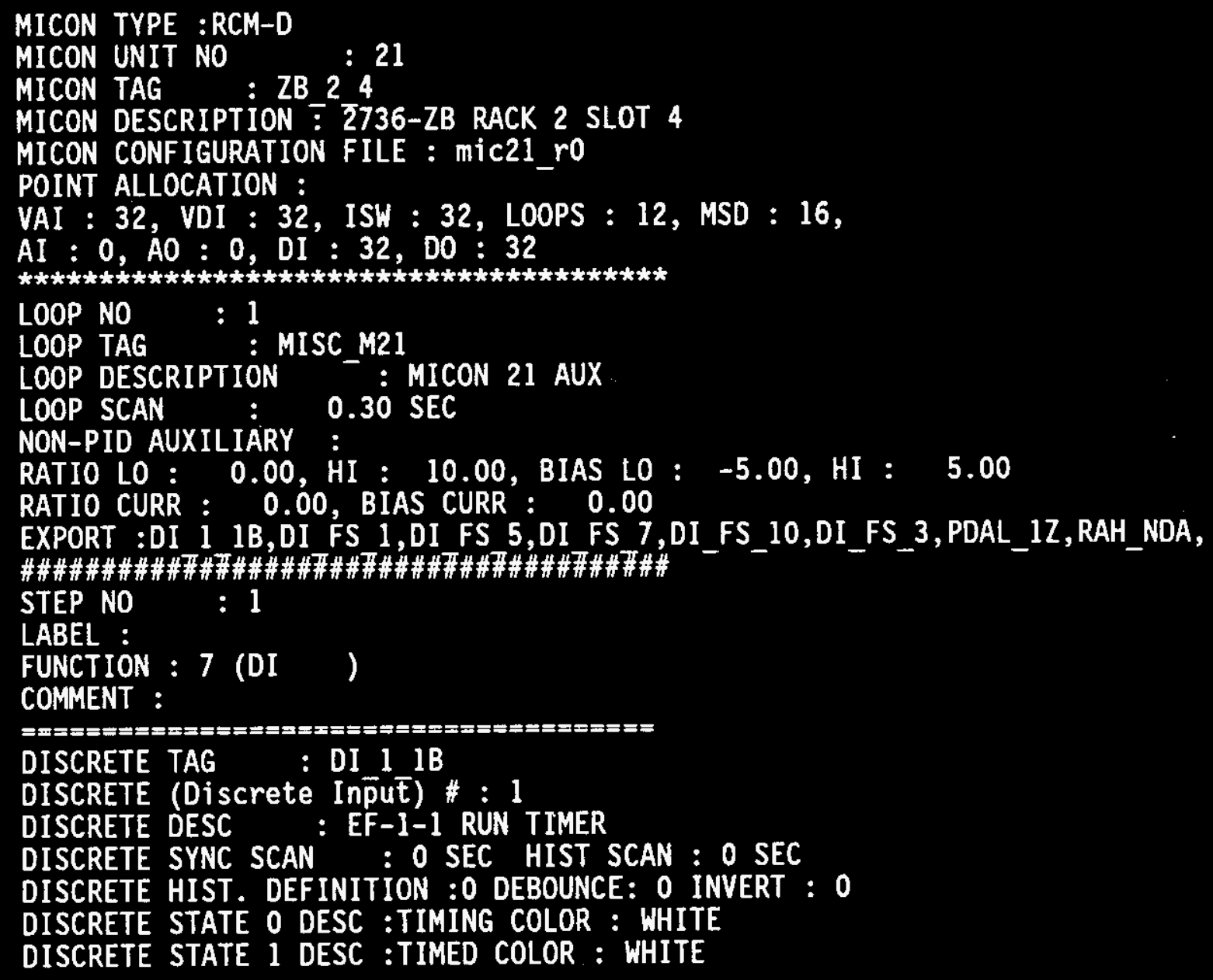


WHC-SD-CP-CSWD-016

Volume 4

Revision 1

Page 133

\#\#\#\#\#\#\#\#\#\#\#\#\#\#\#\#\#\#\#\#\#\#\#\#\#\#\#\#\#\#\#\#\#\#

STEP NO : 2

LABEL :

FUNCTION : 6 (VD)

COMMENT :

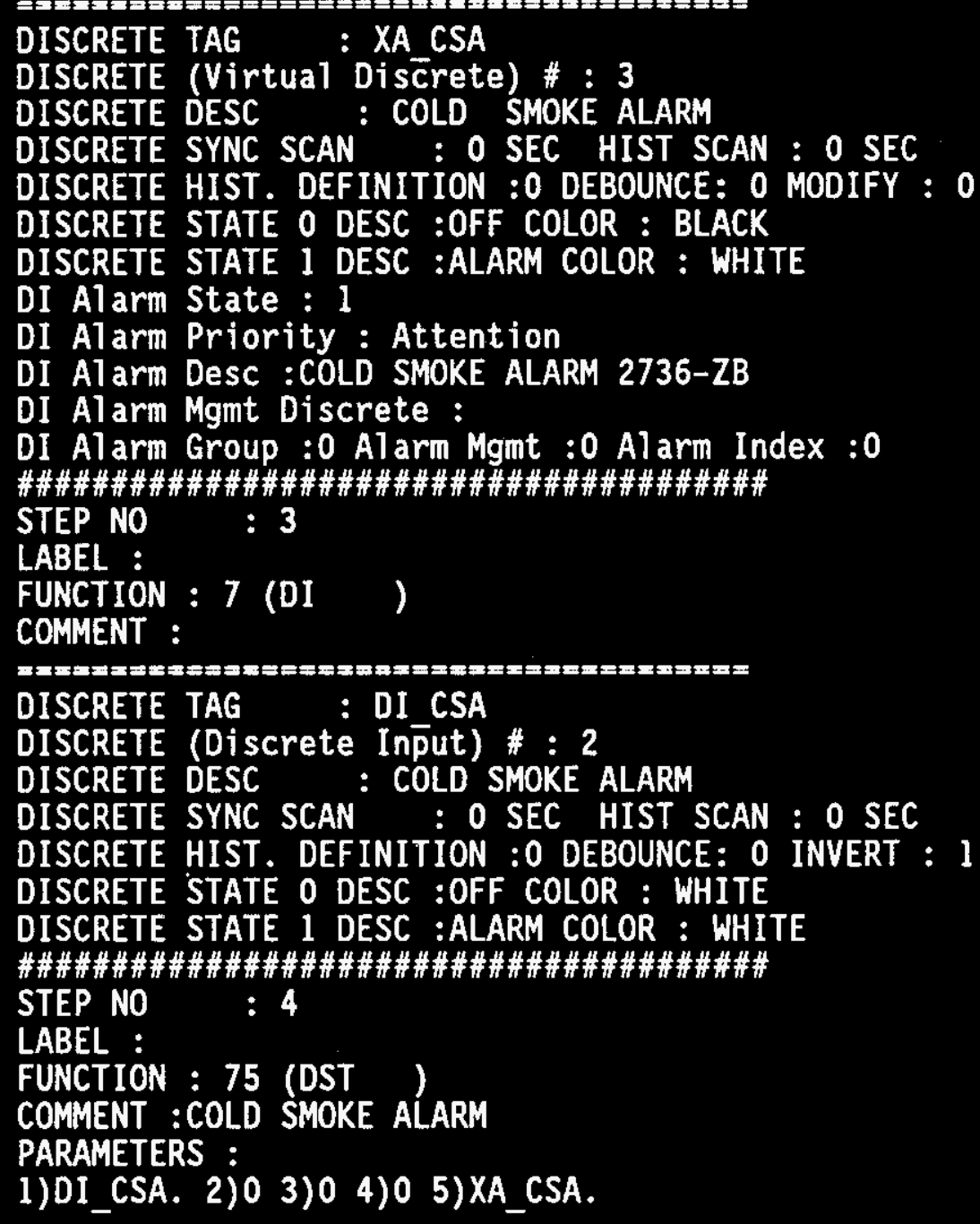




\section{WHC-SD-CP-CSWD-016 \\ Volume 4 \\ Revision 1 \\ Page 134}

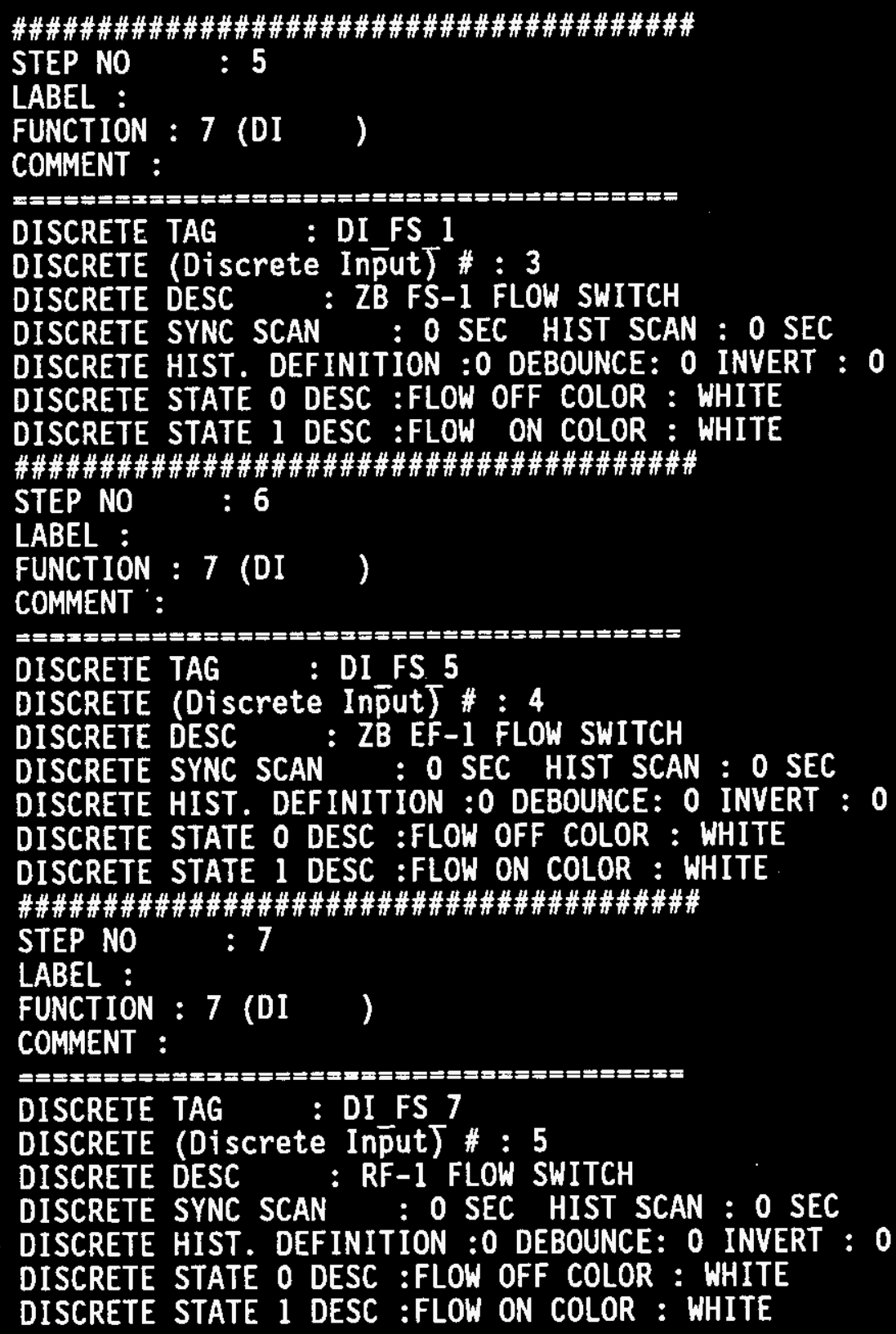


WHC-SD-CP-CSWD-016

Volume 4

Revision 1

Page 135

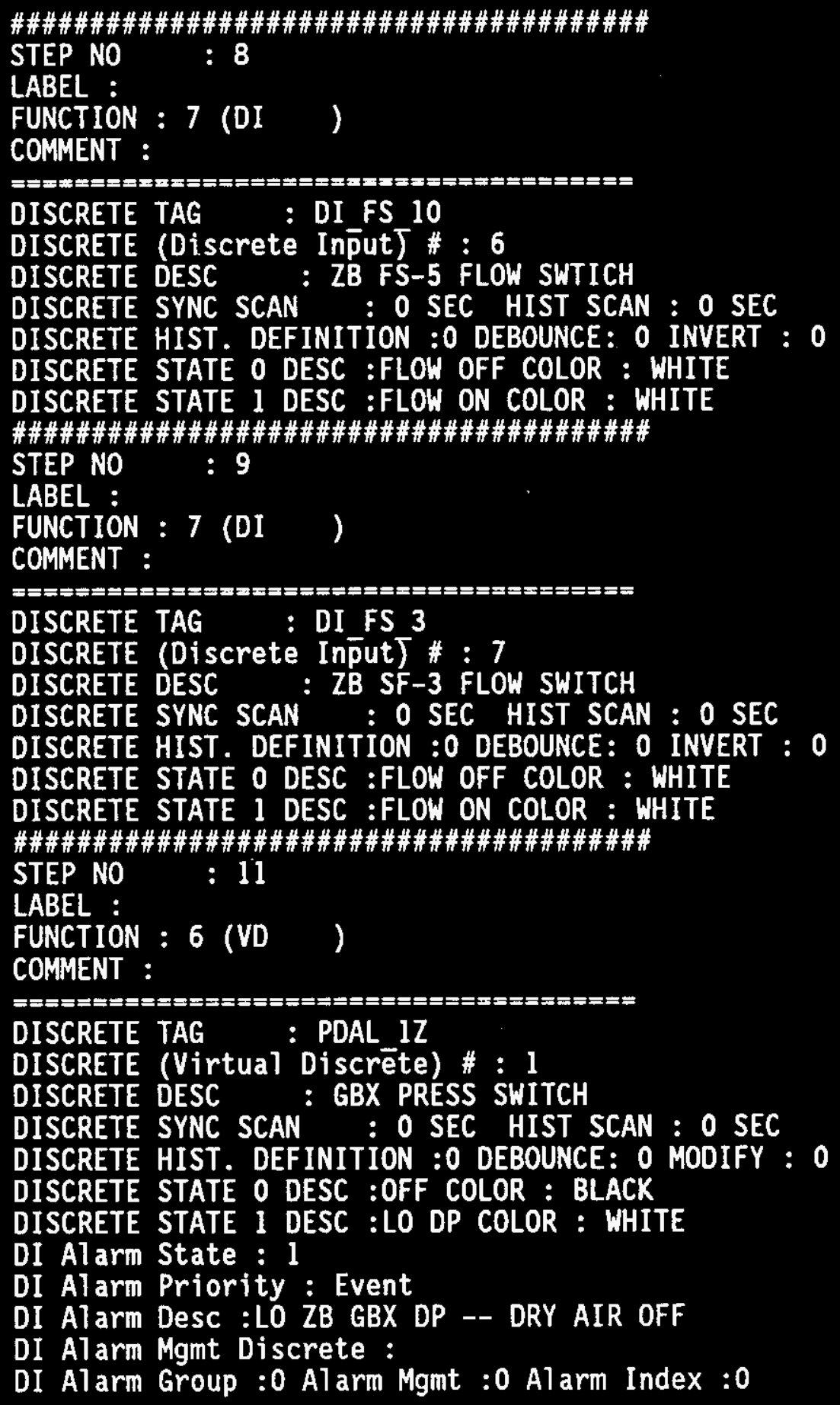




\section{WHC-SD-CP-CSWD-016 \\ Volume 4 \\ Revision 1 \\ Page 136}

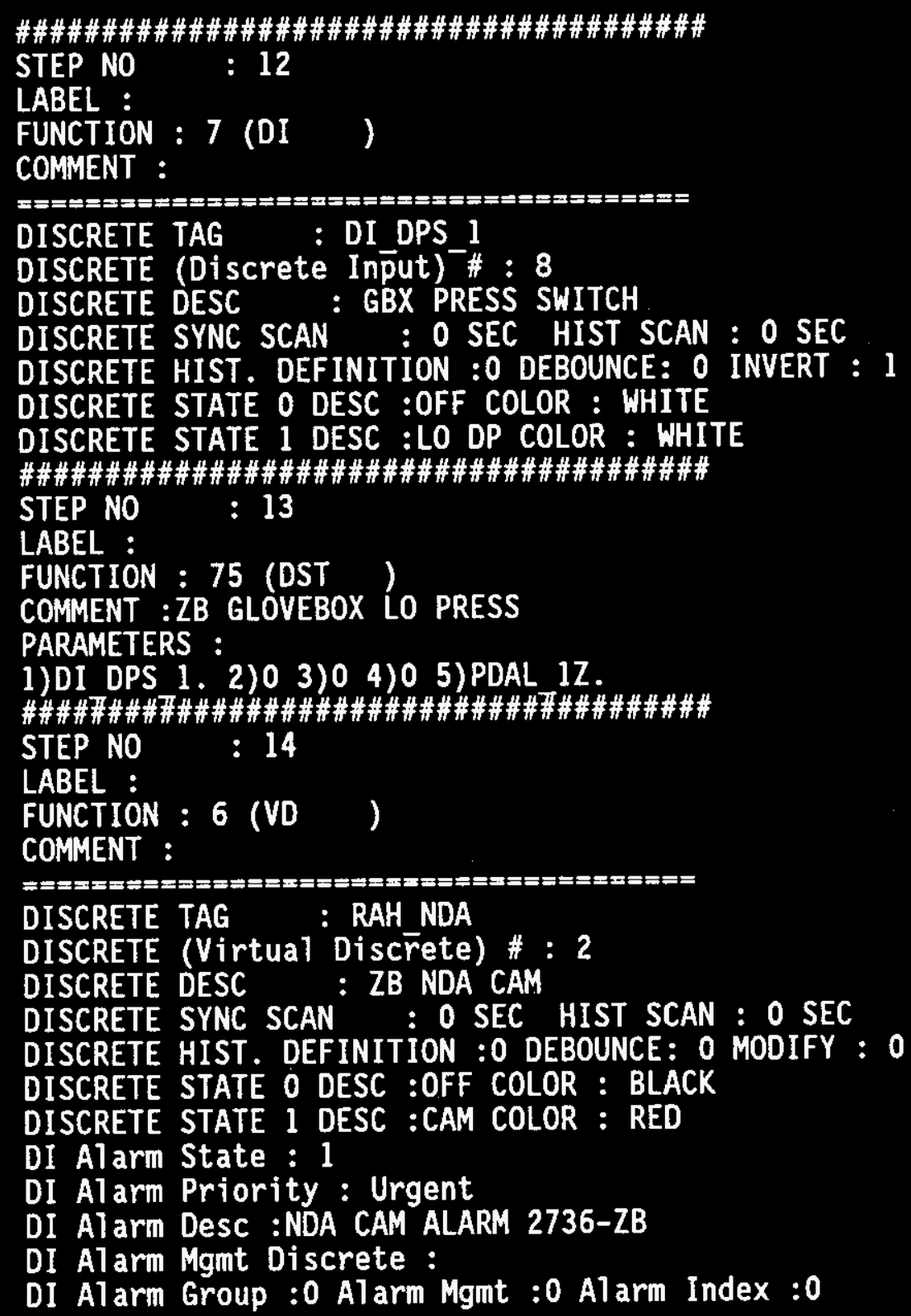


WHC-SD-CP-CSWD-016

Volume 4

Revision 1

Page 137

\#\#\#\#\#\#\#\#\#\#\#\#\#\#\#\#\#\#\#\#\#\#\#\#\#\#\#\#\#\#\#\#\#\#\#

STEP NO : 15

LABEL :

FUNCTION : 7 (DI )

COMMENT :

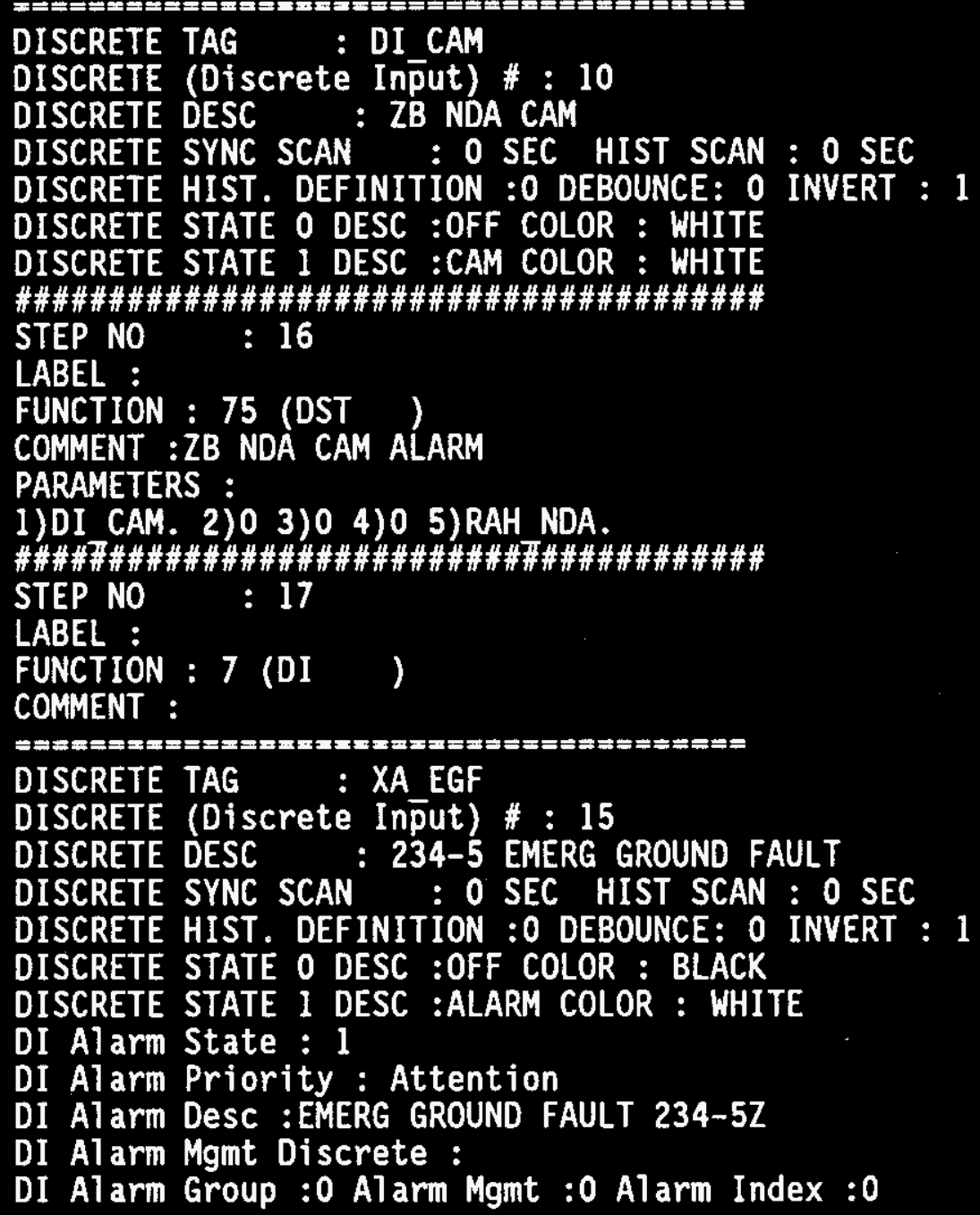


WHC-SD-CP-CSWD-016

Volume 4

Revision 1

Page 138

\#\#\#\#\#\#\#\#\#\#\#\#\#\#\#\#\#\#\#\#\#\#\#\#\#\#\#\#\#\#\#\#\#\#\#\#\#\#\#\#

STEP NO : 18

LABEL :

FUNCTION : 7 (DI )

COMMENT :

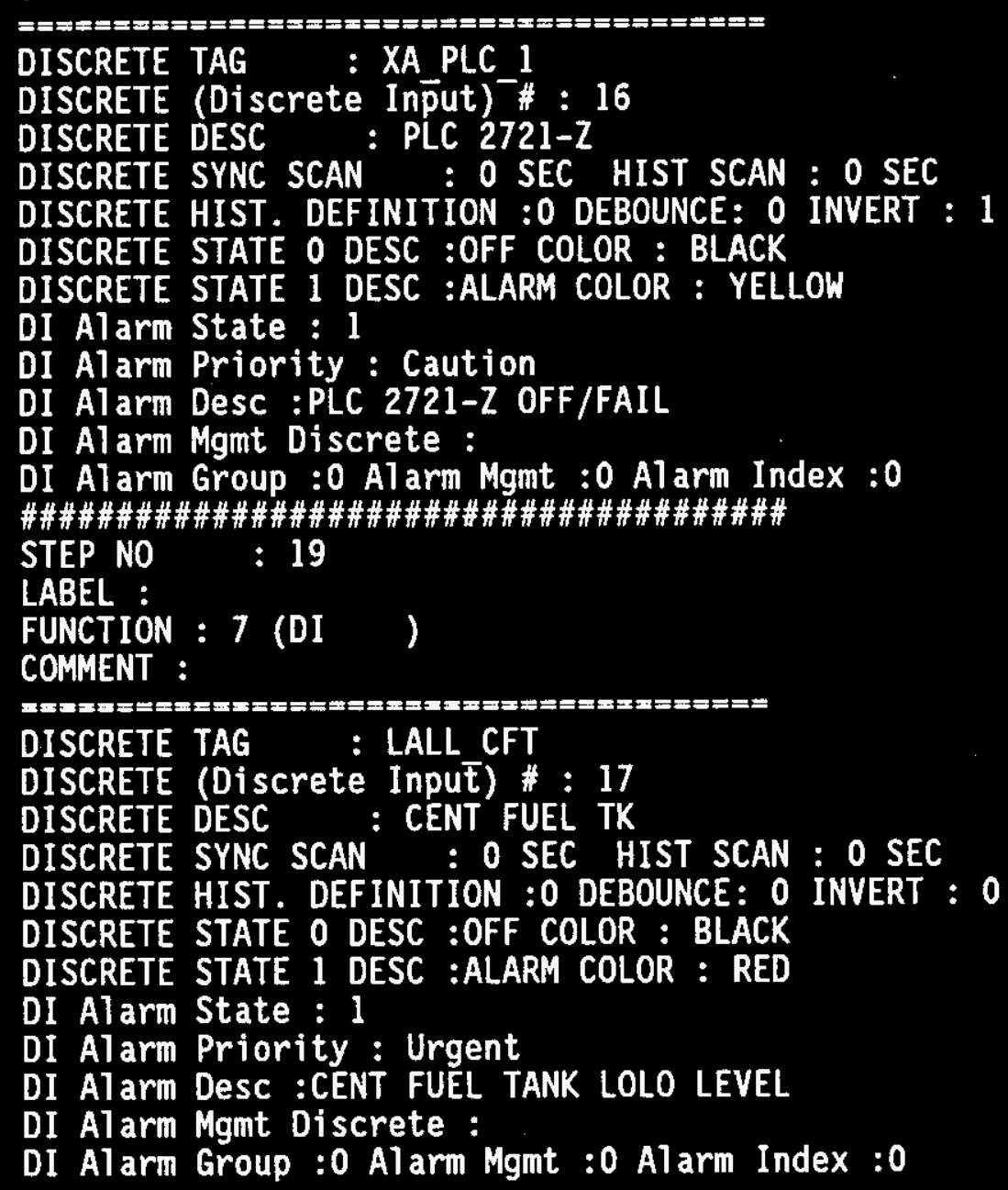




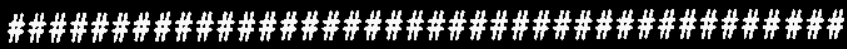

STEP NO : 20

LABEL :

FUNCTION : 7 (DI )

COMMENT :

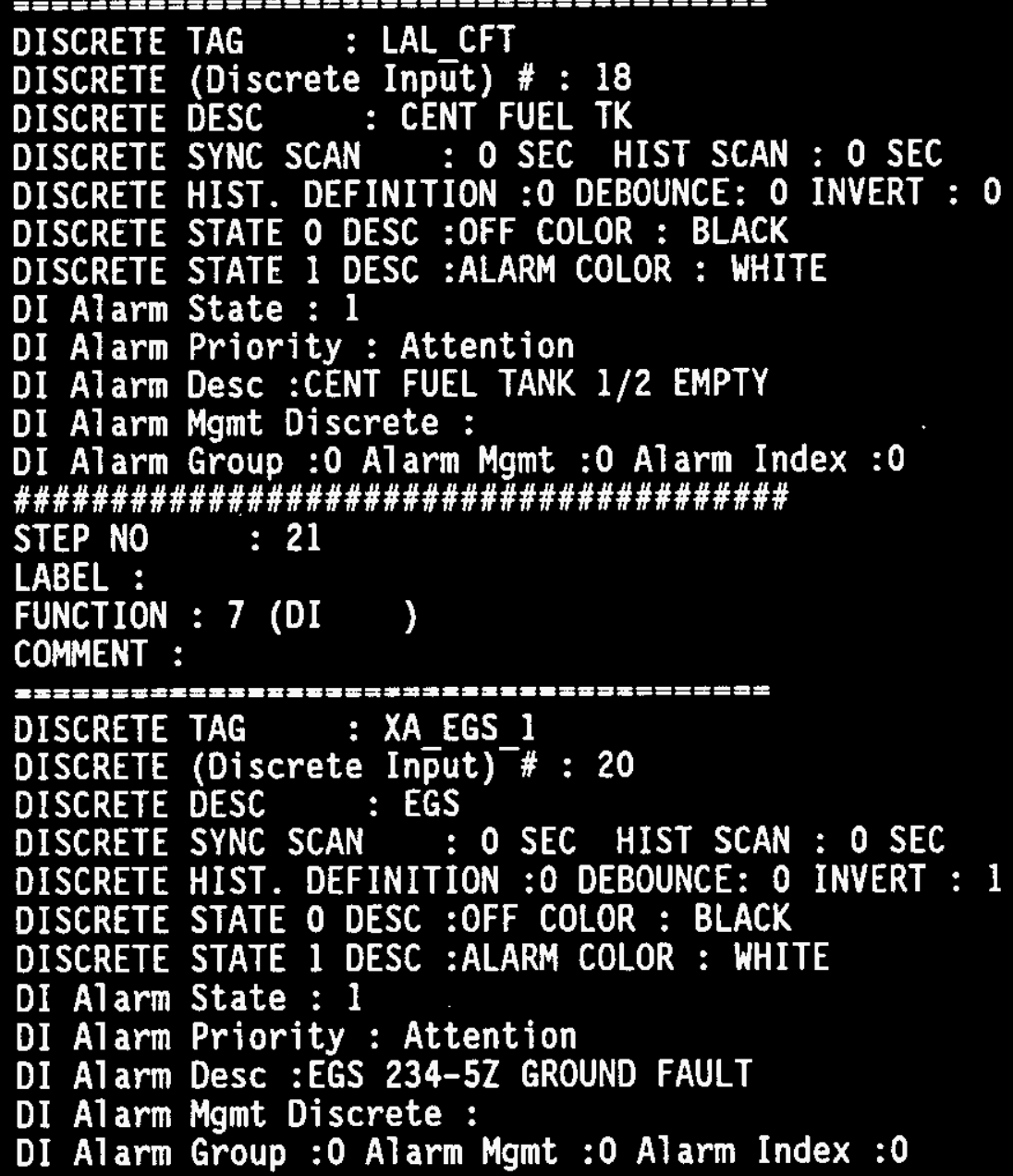




\section{WHC-SD-CP-CSWD-016 \\ Volume 4 \\ Revision 1 \\ Page 140}

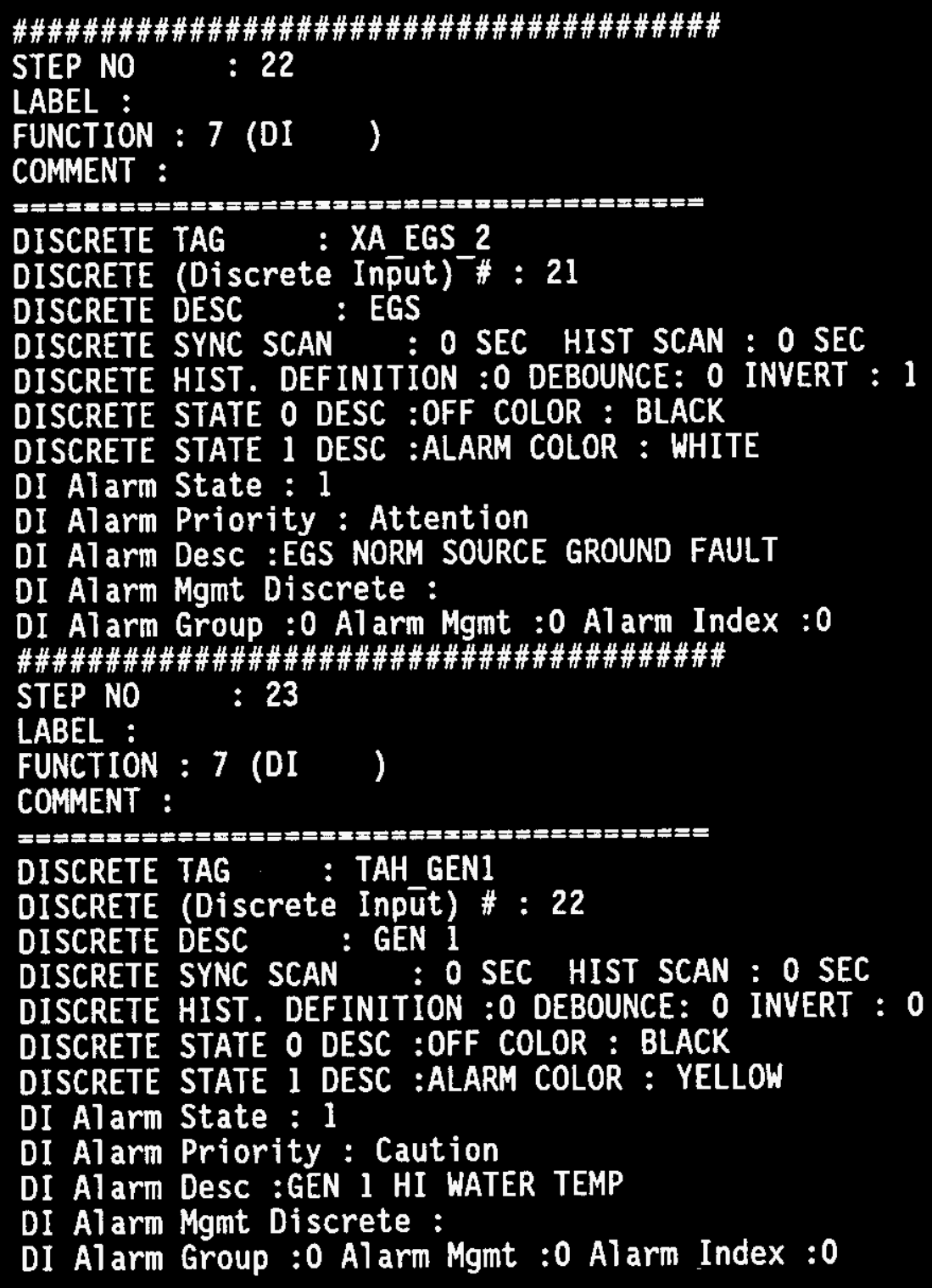




\section{WHC-SD-CP-CSWD-016 \\ Volume 4 \\ Revision 1 \\ Page 141}

\#\#\#\#\#\#\#\#\#\#\#\#\#\#\#\#\#\#\#\#\#\#\#\#\#\#\#\#\#\#\#\#\#\#\#\#\#\#\#

STEP NO : 24

LABEL :

FUNCTION : 7 (DI )

COMMENT :

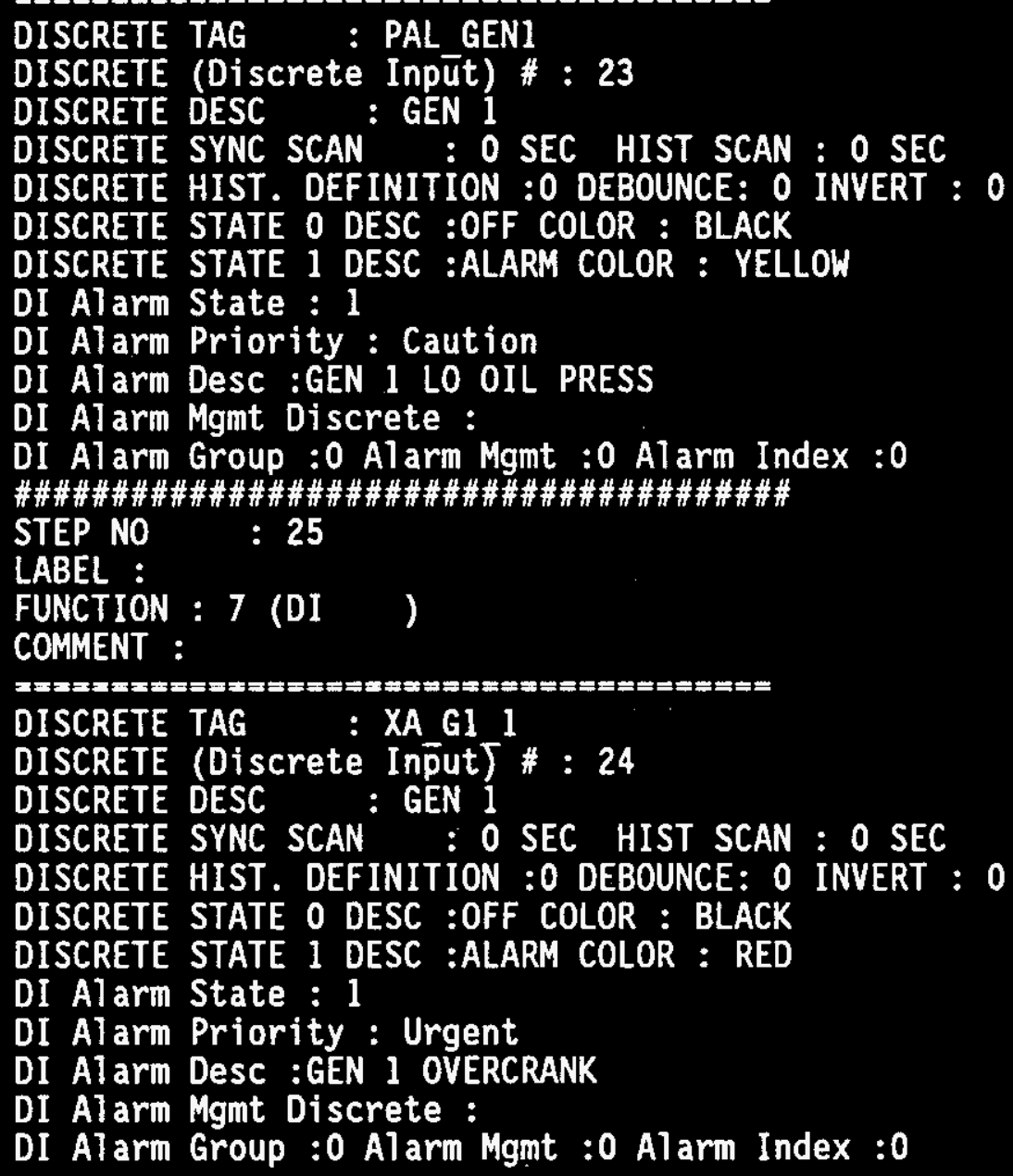


WHC-SD-CP-CSWD-016

Volume 4

Revision 1

Page 142

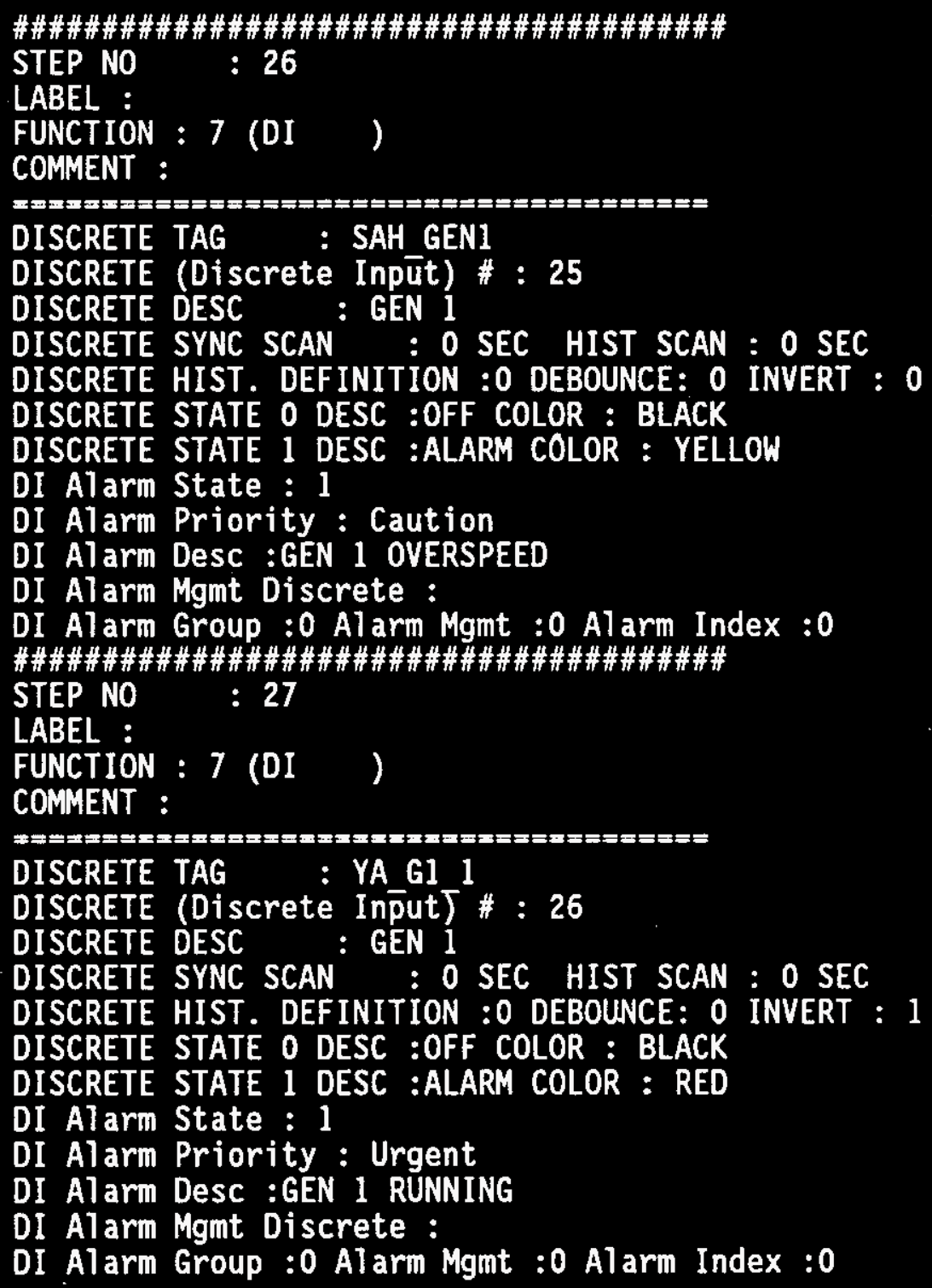


WHC-SD-CP-CSWD-016

Volume 4

Revision 1

Page 143

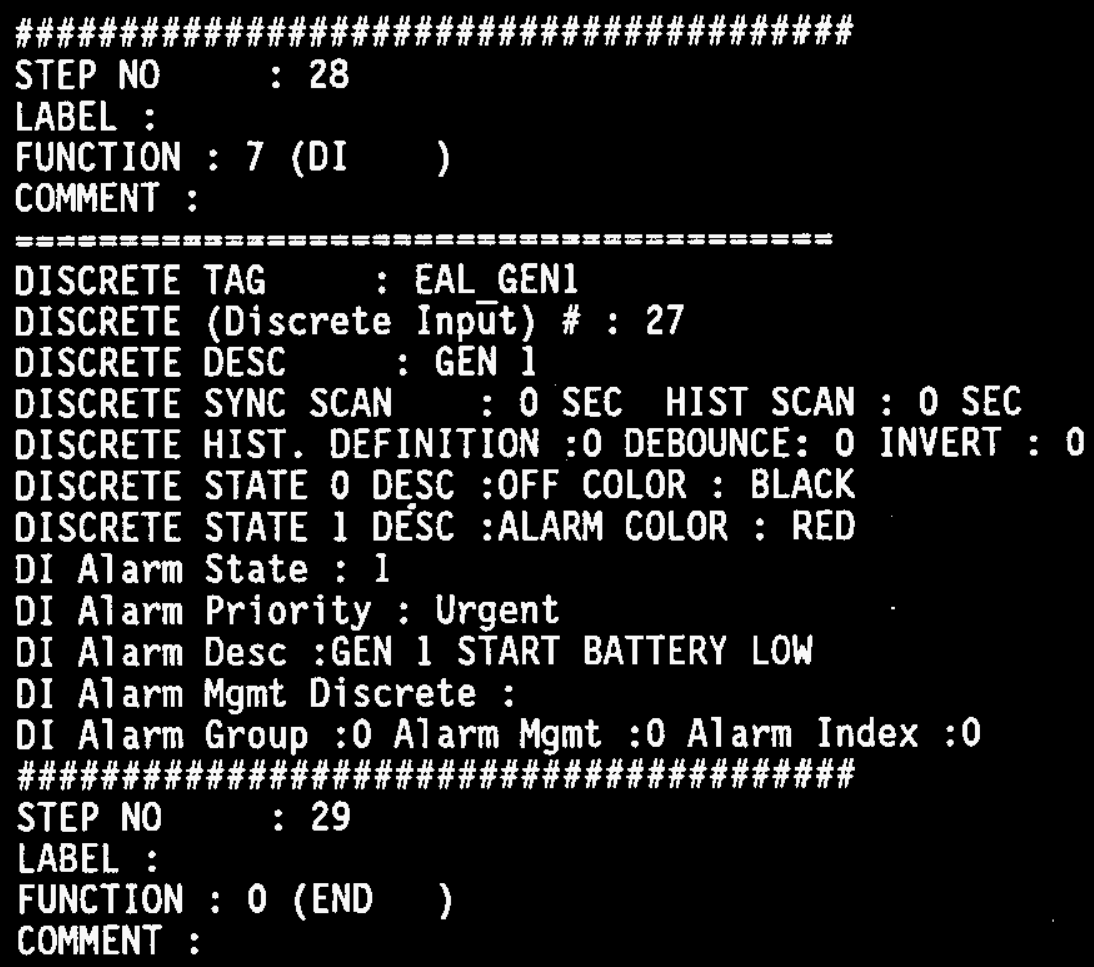


WHC-SD-CP-CSWD-016

Volume 4

Revision 1

Page 144

ZB_2_5.mic22_ro

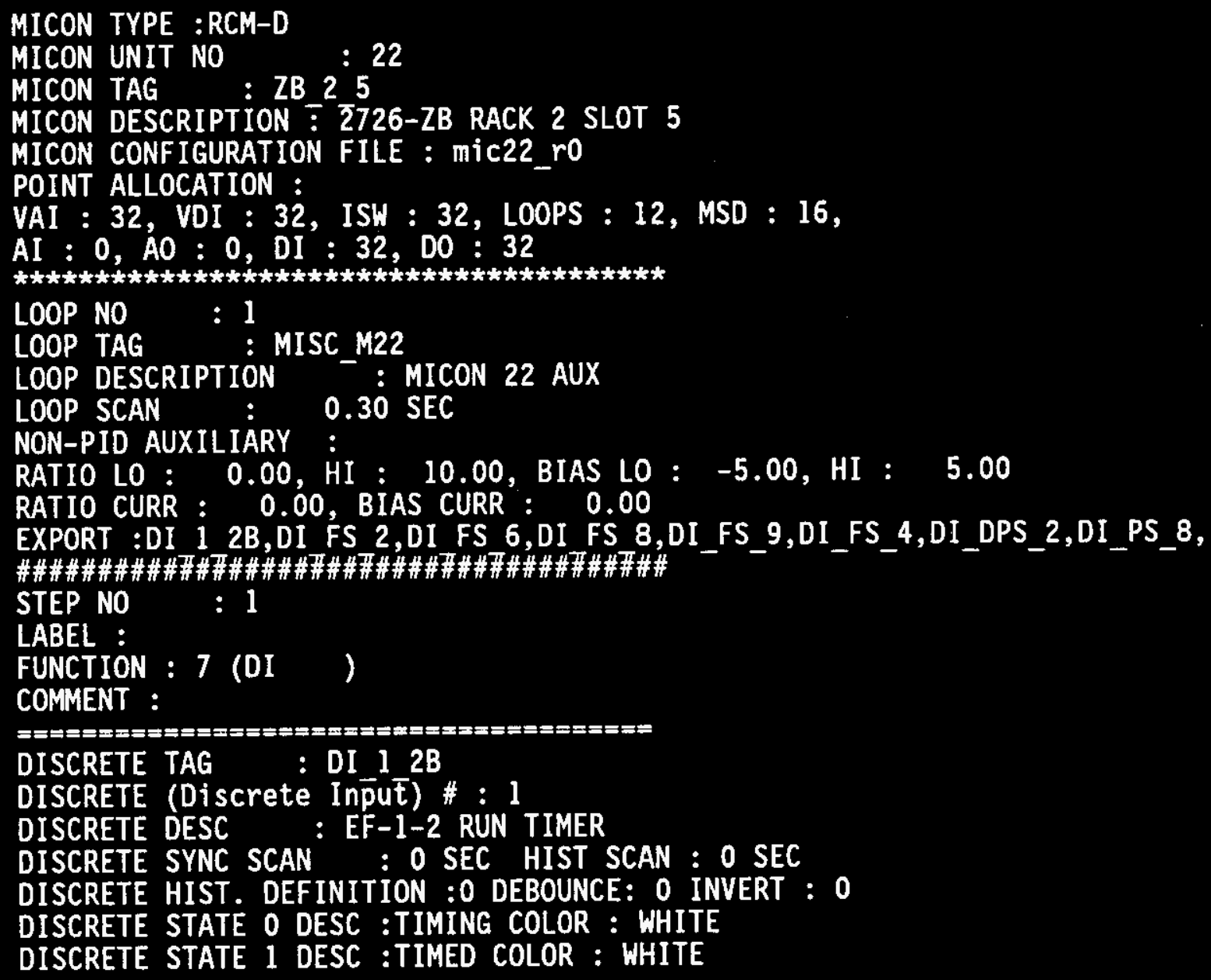


WHC-SD-CP-CSWD-016

Volume 4

Revision 1

Page 145

\#\#\#\#\#\#\#\#\#\#\#\#\#\#\#\#\#\#\#\#\#\#\#\#\#\#\#\#\#\#\#\#\#\#\#\#\#\#\#\#

STEP NO : 2

LABEL :

FUNCTION : 6 (VD )

COMMENT :

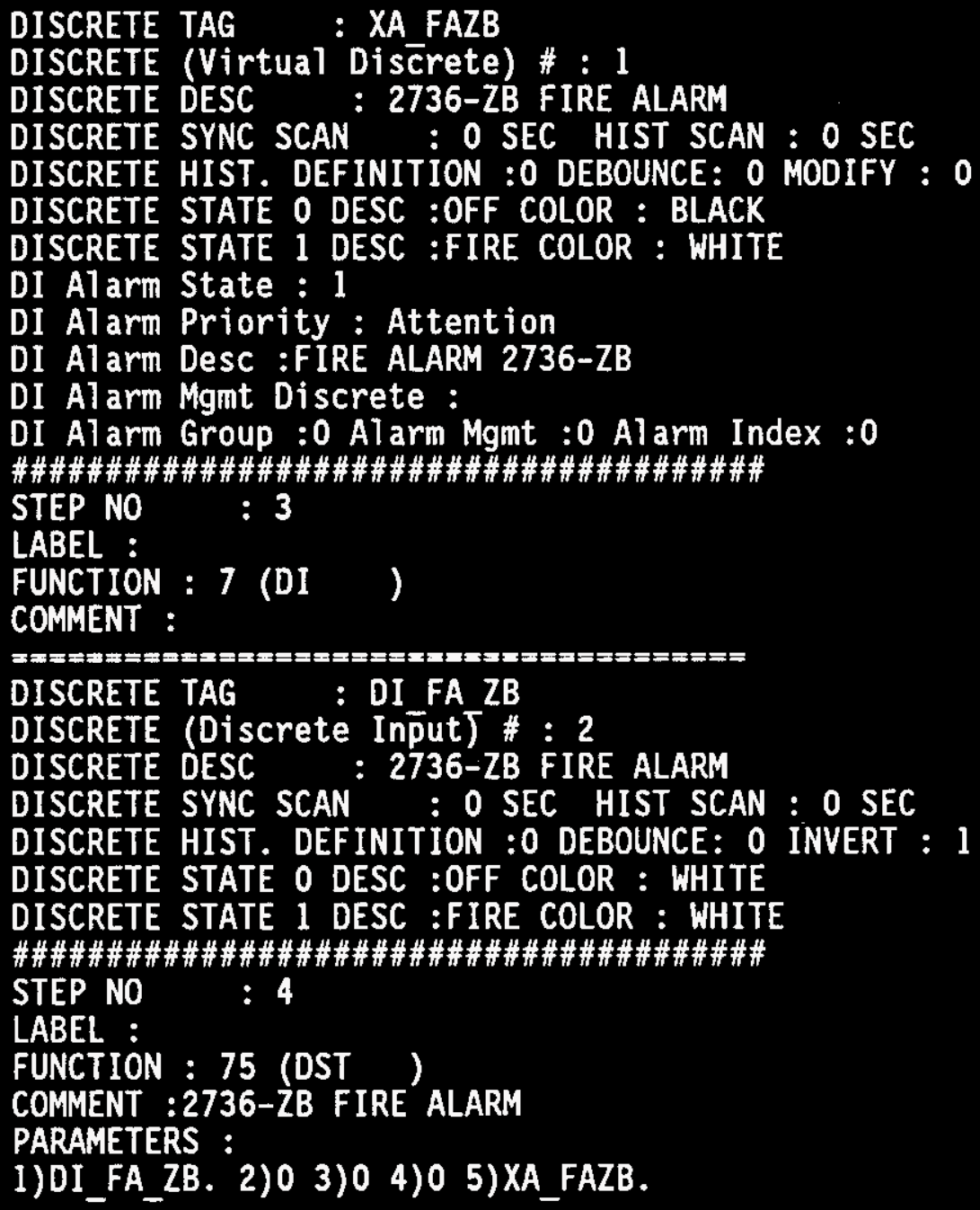


WHC-SD-CP-CSWD-016

Volume 4

Revision 1

Page 146

\#\#\#\#\#\#\#\#\#\#\#\#\#\#\#\#\#\#\#\#\#\#\#\#\#\#\#\#\#\#\#\#\#\#\#\#\#\#\#\#

STEP NO : 5

LABEL :

FUNCTION : 7 (DI)

COMMENT :

.

DISCRETE TAG : DI FS 2

DISCRETE (Discrete Input) \#: 3

DISCRETE DESC : SF-2 FLOW SWITCH

DISCRETE SYNC SCAN : O SEC HIST SCAN : O SEC

DISCRETE HIST. DEFINITION :0 DEBOUNCE: 0 INVERT : 0

DISCRETE STATE O DESC :FLOW OFF COLOR : WHITE

DISCRETE STATE 1 DESC :FLON ON COLOR : WHITE

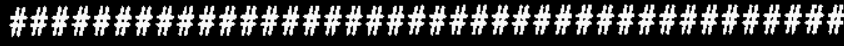

STEP NO : 6

LABEL :

FUNCTION : 7 (DI )

COMMENT :

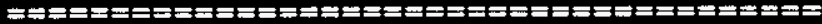

DISCRETE TAG : DI FS 6

DISCRETE (Discrete Input) \# : 4

DISCRETE DESC : EF-2 FLOW SWITCH

DISCRETE SYNC SCAN : O SEC HIST SCAN : O SEC

DISCRETE HIST. DEFINITION :0 DEBOUNCE: 0 INVERT : 0

DISCRETE STATE O DESC :FLOW OFF COLOR : WHITE

DISCRETE STATE 1 DESC :FLOW ON COLOR : WHITE

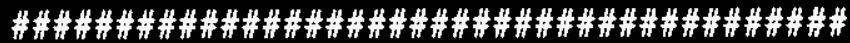

STEP NO : 7

LABEL :

FUNCTION : 7 (DI )

COMMENT :

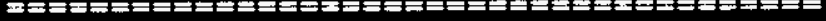

DISCRETE TAG : DI FS 8

DISCRETE (Discrete Input) \#: 5

DISCRETE DESC : RF-2 FLOW SWITCH

DISCRETE SYNC SCAN :O SEC HIST SCAN : O SEC

DISCRETE HIST. DEFINITION :0 DEBOUNCE: 0 INVERT : 0

DISCRETE STATE 0 DESC :FLON OFF COLOR : WHITE

DISCRETE STATE 1 DESC :FLOW ON COLOR : WHITE 
WHC-SD-CP-CSWD-016

Volume 4

Revision 1

Page 147

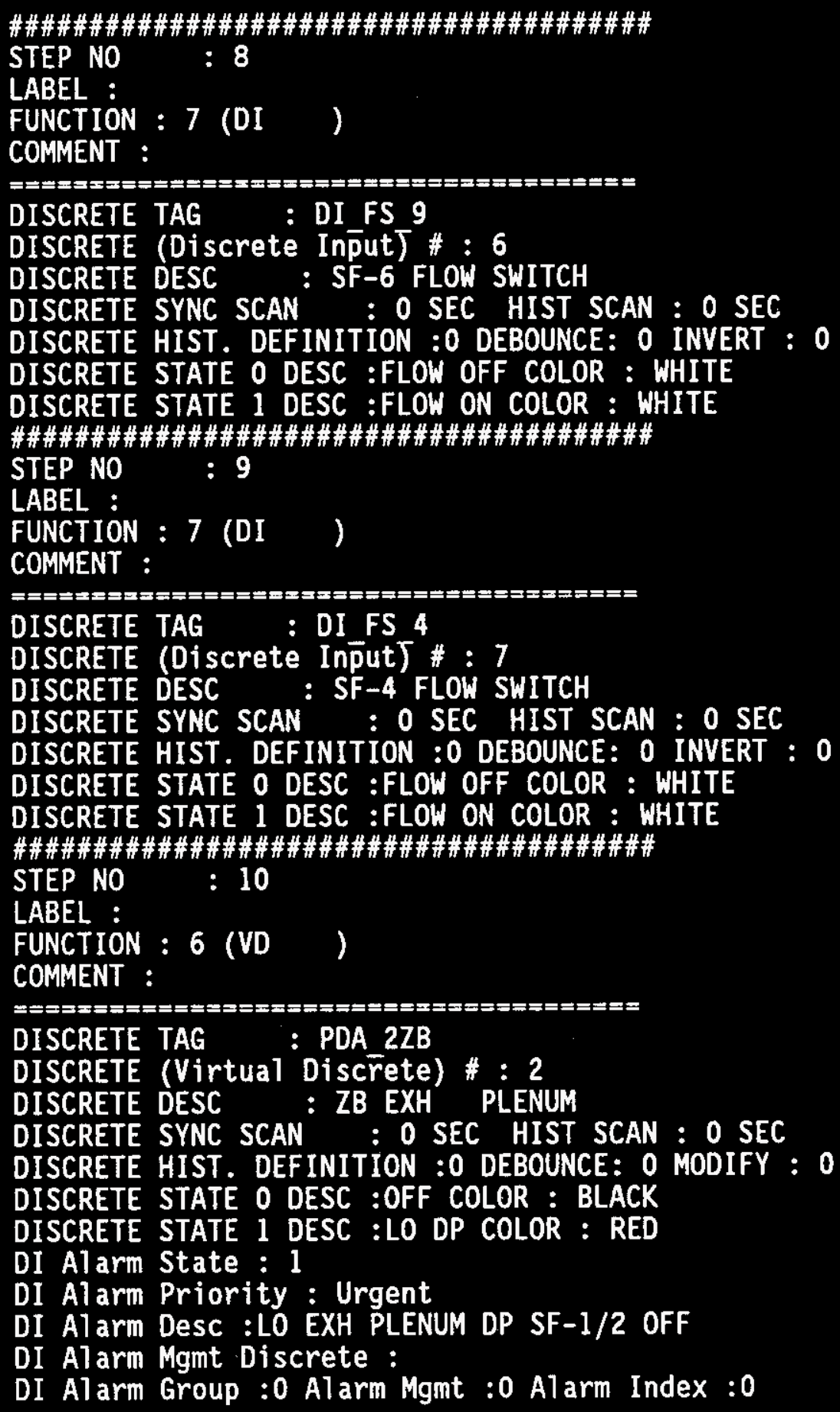




\section{WHC-SD-CP-CSWD-016 \\ Volume 4 \\ Revision 1 \\ Page 148}

\#\#\#\#\#\#\#\#\#\#\#\#\#\#\#\#\#\#\#\#\#\#\#\#\#\#\#\#\#\#\#\#\#\#\#\#\#

STEP NO : 11

LABEL :

FUNCTION : 7 (DI)

COMMENT :

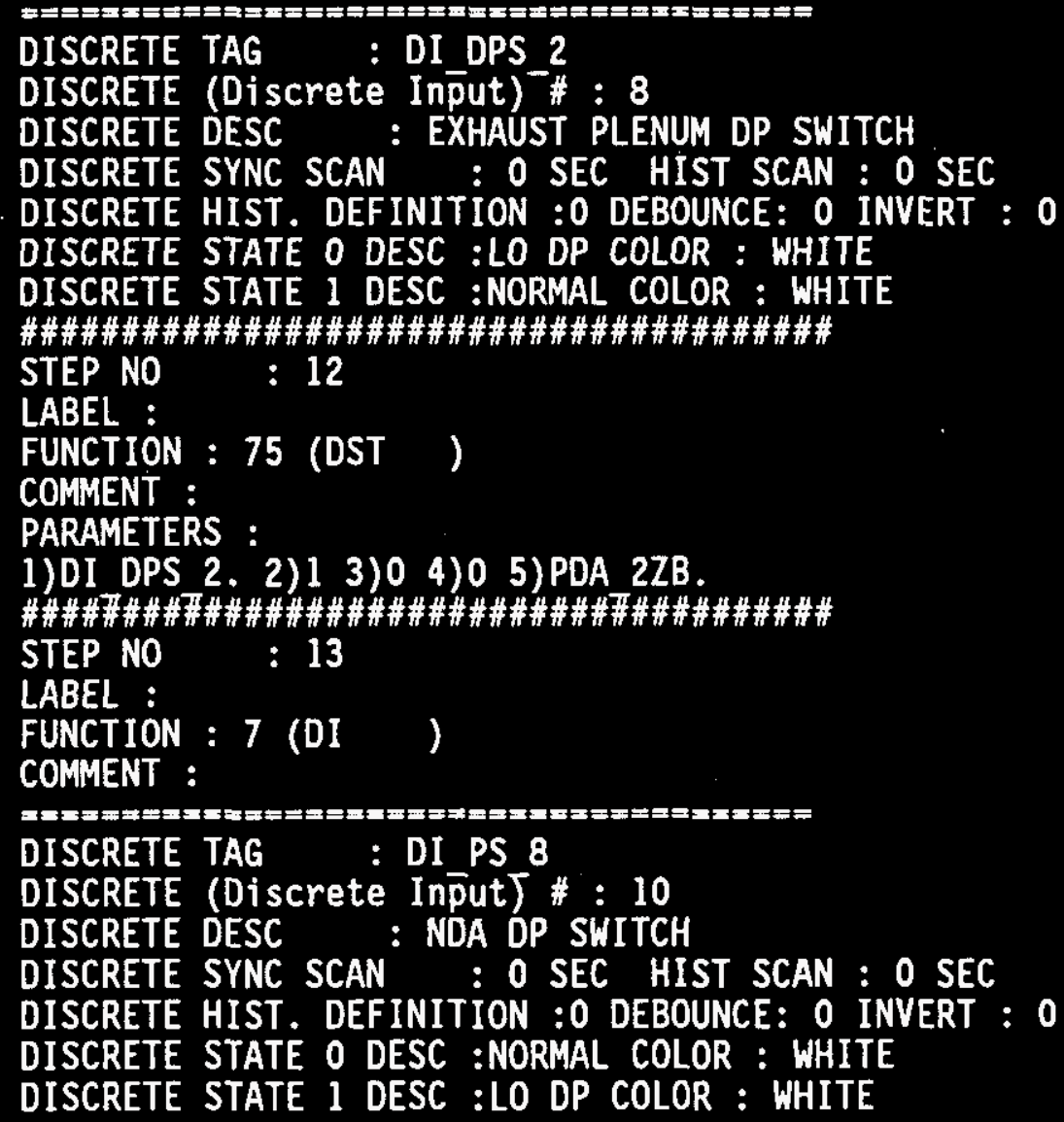


WHC-SD-CP-CSWD-016

Volume 4

Revision 1

Page 149

\#\#\#\#\#\#\#\#\#\#\#\#\#\#\#\#\#\#\#\#\#\#\#\#\#\#\#\#\#\#\#\#\#\#\#\#\#\#\#\#

STEP NO : 14

LABEL :

FUNCTION : 7 (DI )

COMMENT :

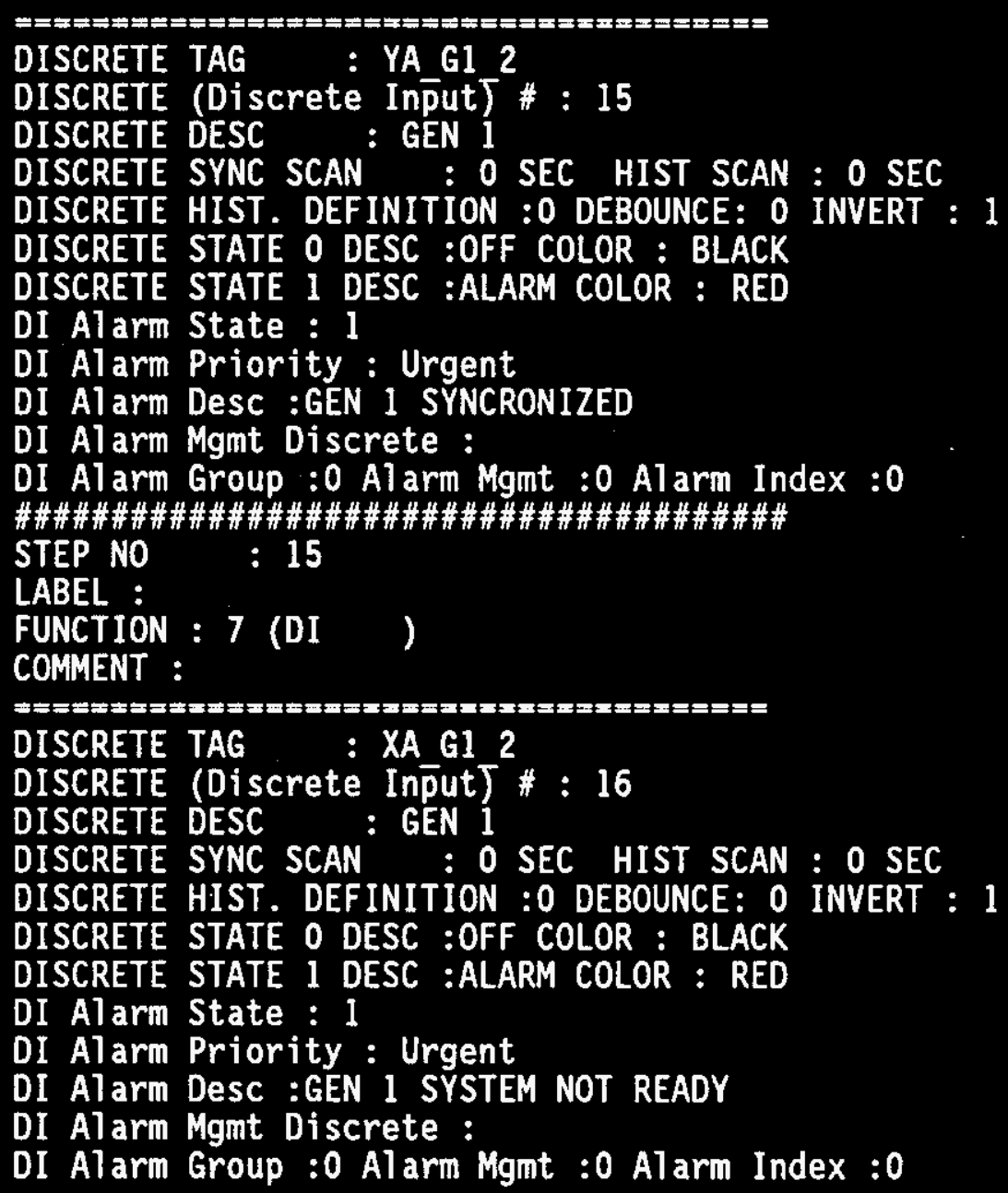




\section{WHC-SD-CP-CSWD-016 \\ Volume 4 \\ Revision 1 \\ Page 150}


WHC-SD-CP-CSWD-016

Volume 4

Revision 1

Page 151

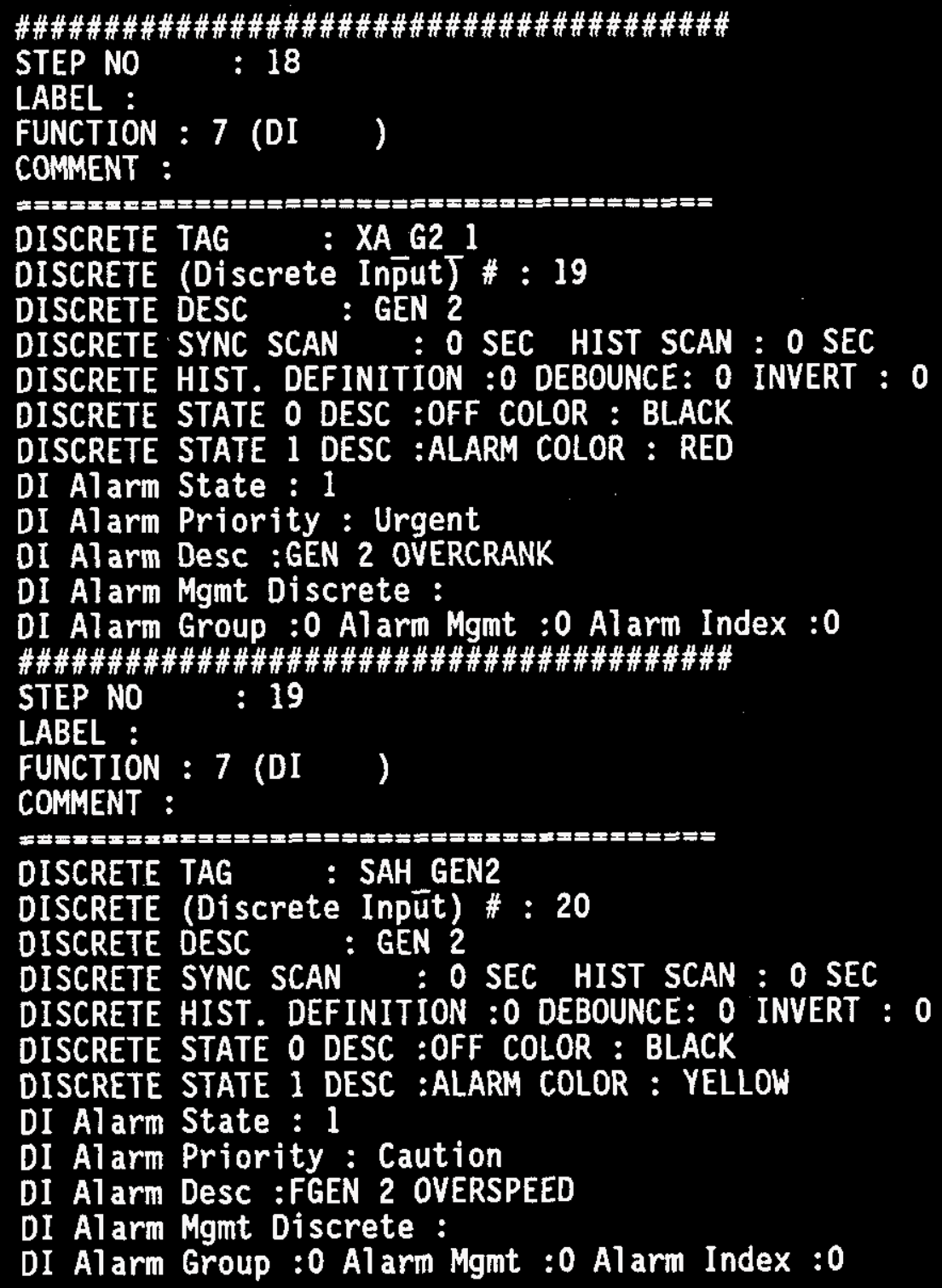


WHC-SD-CP-CSWD-016

Volume 4

Revision 1

Page 152

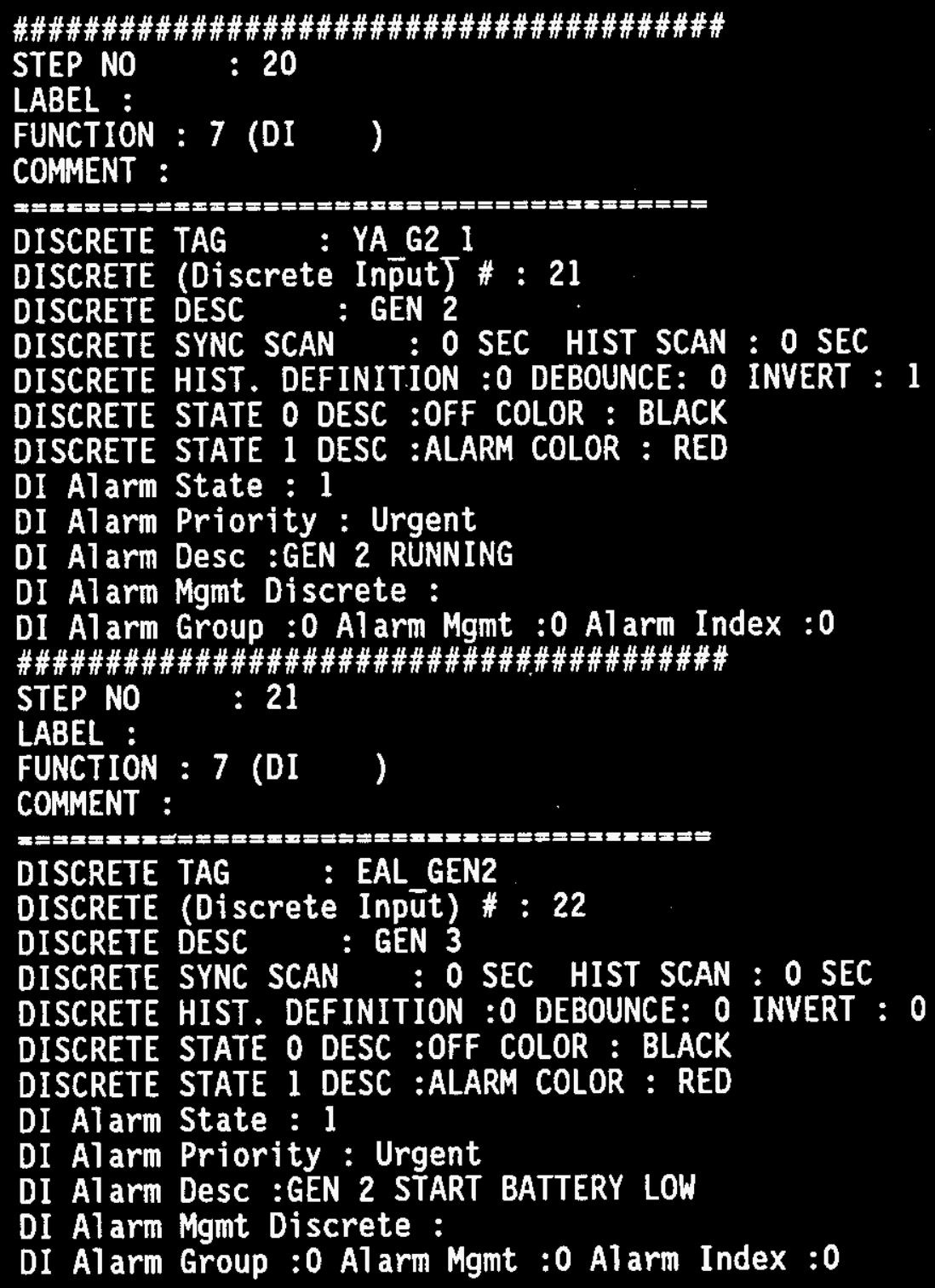




\section{WHC-SD-CP-CSWD-016 \\ Volume 4 \\ Revision 1 \\ Page 153}

\#\#\#\#\#\#\#\#\#\#\#\#\#\#\#\#\#\#\#\#\#\#\#\#\#\#\#\#\#\#\#\#\#\#\#\#\#\#\#\#

STEP NO : 22

LABEL :

FUNCTION : 7 (DI )

COMMENT :

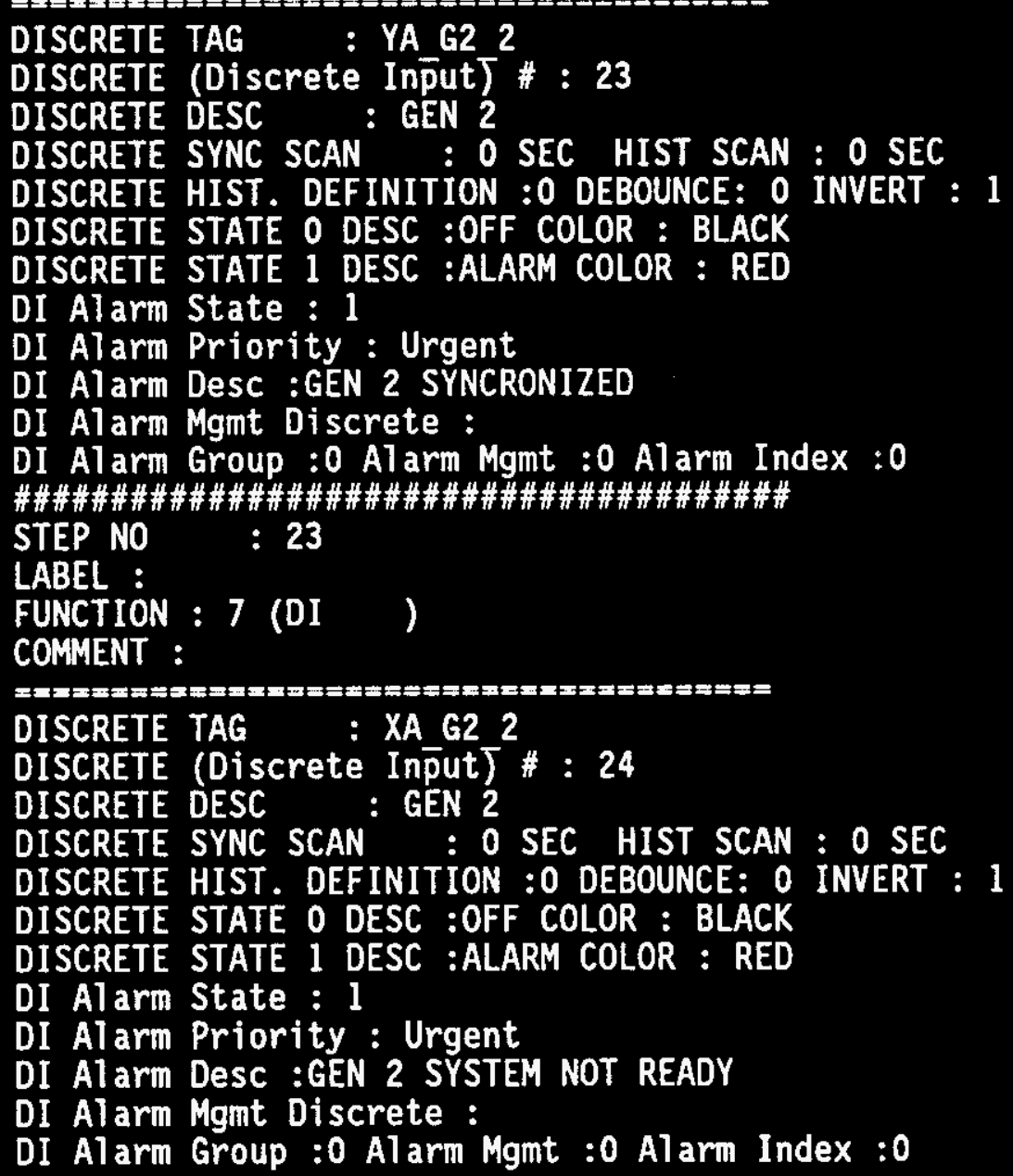


\#\#\#\#\#\#\#\#\#\#\#\#\#\#\#\#\#\#\#\#\#\#\#\#\#\#\#\#\#\#\#\#\#\#\#\#\#\#\#\#

STEP NO : 24

LABEL :

FUNCTION : 7 (DI )

COMMENT :

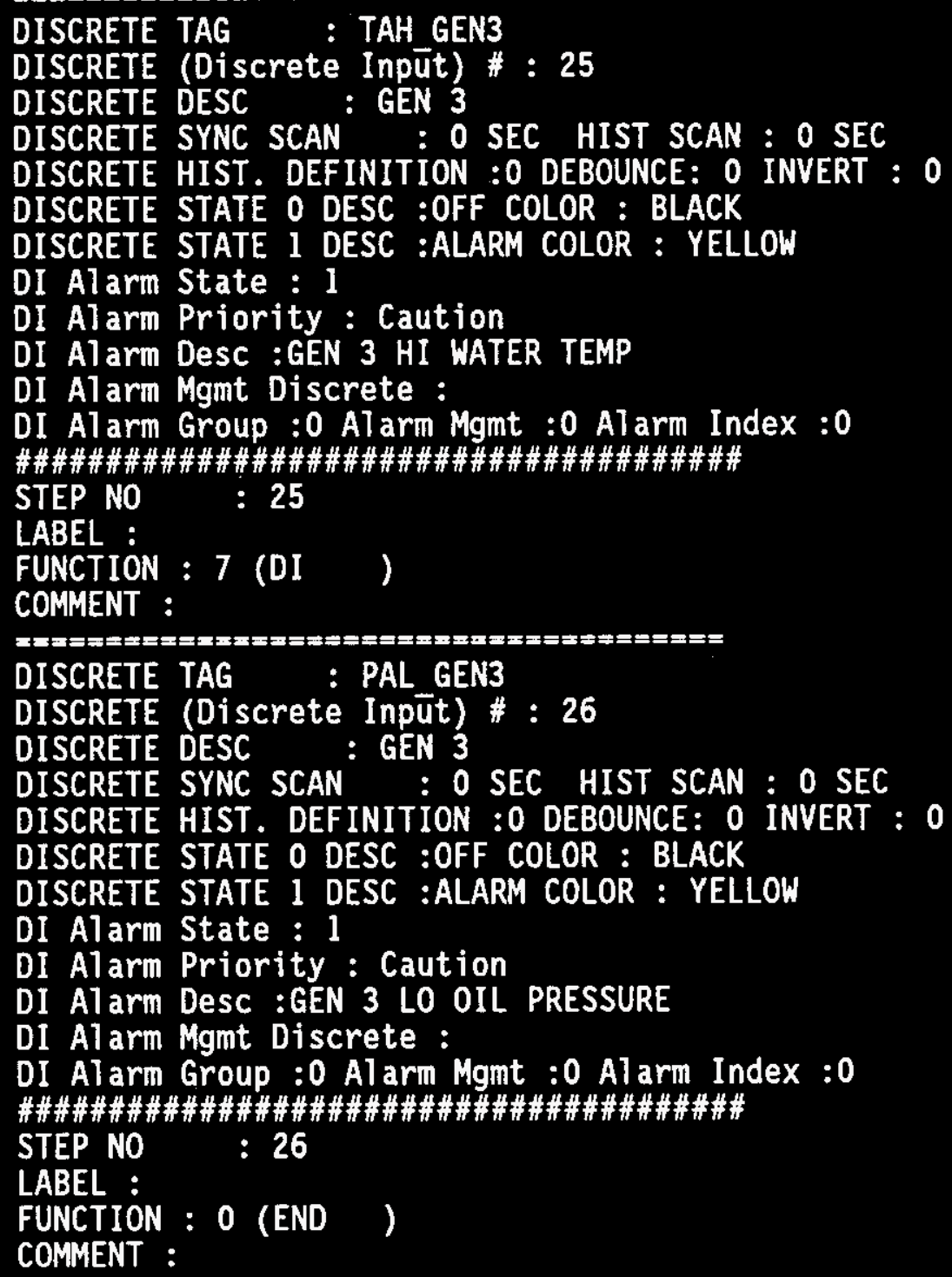


WHC-SD-CP-CSWD-016

Volume 4

Revision 1

Page 155

ZB 2 6.mic23_ro

MICON TYPE :RCM-D

MICON UNIT NO : 23

MICON TAG : ZB 26

MICON DESCRIPTION $\overline{2} \overline{2} 736-Z B$ RACK 2 SLOT 6

MICON CONFIGURATION FILE : mic23_ro

POINT ALLOCATION :

VAI : 32, VDI : 32, ISW : 32, LOOPS : 12, MSD : 16,

AI : $0, A 0: 0$, DI : 32, DO : 32

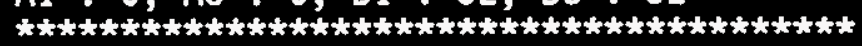

LOOP NO : 1

LOOP TAG : MISC M23

LOOP DESCRIPTION - : MICON 23 AUX

LOOP SCAN : 0.30 SEC

NON-PID AUXILIARY :

RATIO LO: $0.00, \mathrm{HI}: 10.00$, BIAS LO : $-5.00, \mathrm{HI}: 5.00$

RATIO CURR : 0.00 , BIAS CURR : 0.00

EXPORT :XA FAZN8

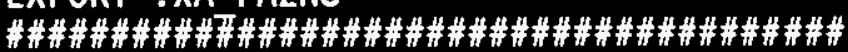

STEP NO : 1

LABEL :

FUNCTION : 7 (DI)

COMMENT :

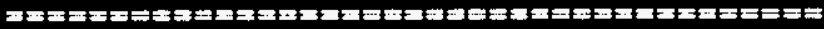

DISCRETE TAG : DI $12 \mathrm{C}$

DISCRETE (Discrete Inpūt) \#: 1

DISCRETE DESC : EF-1-2 AUTO/MAN

DISCRETE SYNC SCAN : 0 SEC HIST SCAN : 0 SEC

DISCRETE HIST. DEFINITION :0 DEBOUNCE: 0 INVERT : 0

DISCRETE STATE 0 DESC :MANUAL COLOR : WHITE

DISCRETE STATE 1 DESC :AUTO COLOR : WHITE 


\section{WHC-SD-CP-CSWD-016 \\ Volume 4 \\ Revision 1 \\ Page 156}

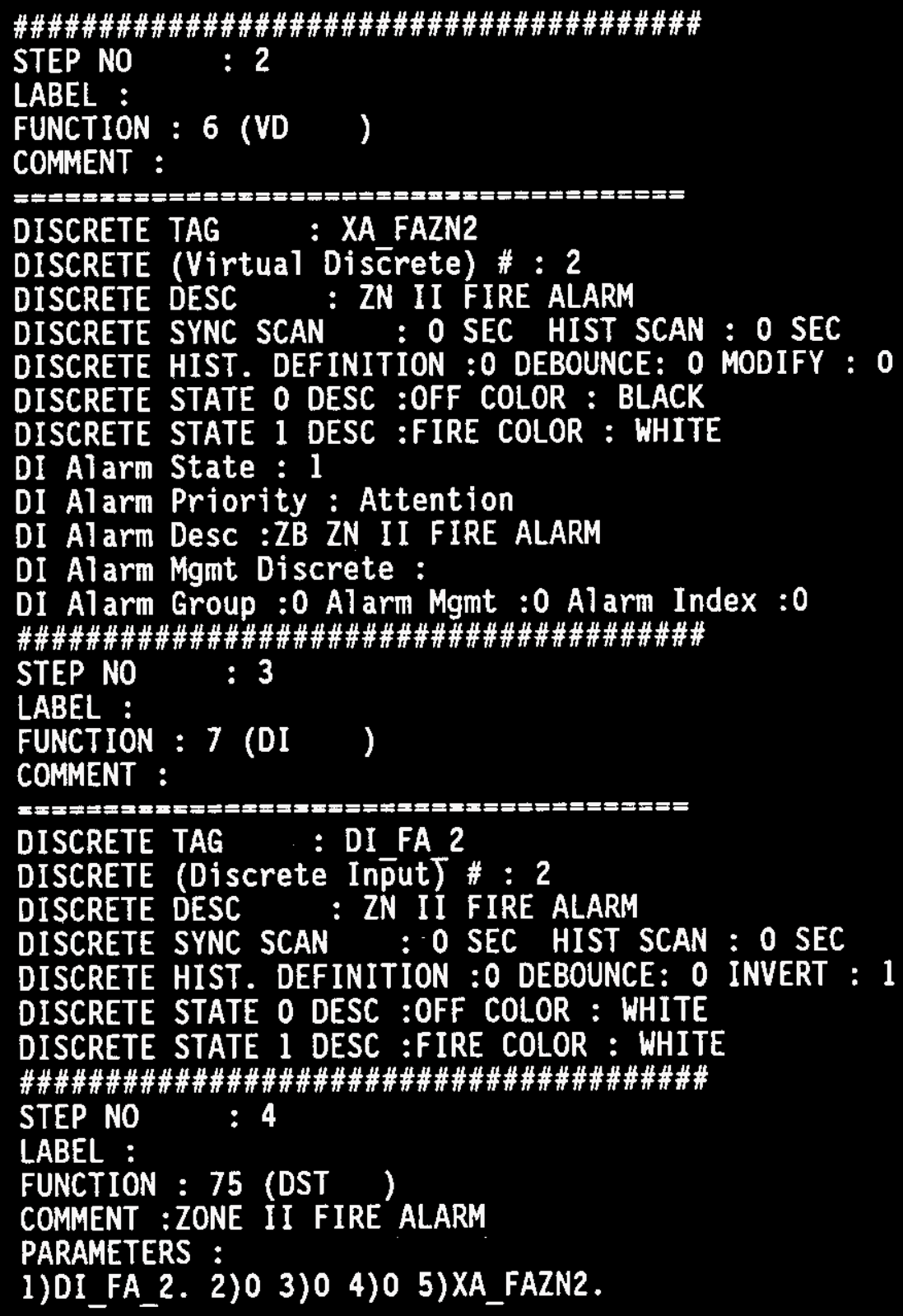


\#\#\#\#\#\#\#\#\#\#\#\#\#\#\#\#\#\#\#\#\#\#\#\#\#\#\#\#\#\#\#\#\#\#

STEP NO : 5

LABEL :

FUNCTION : 6 (VD)

COMMENT :

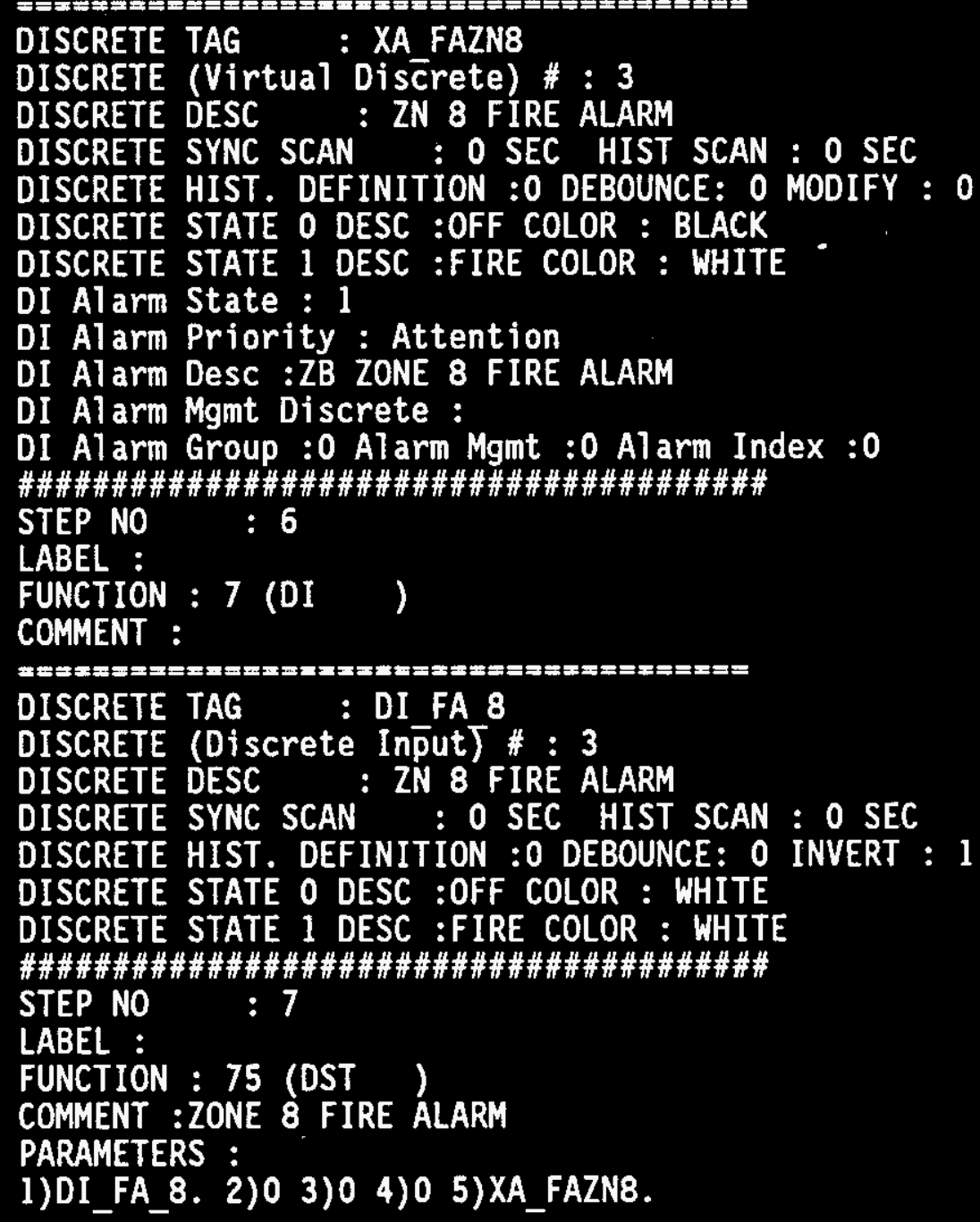




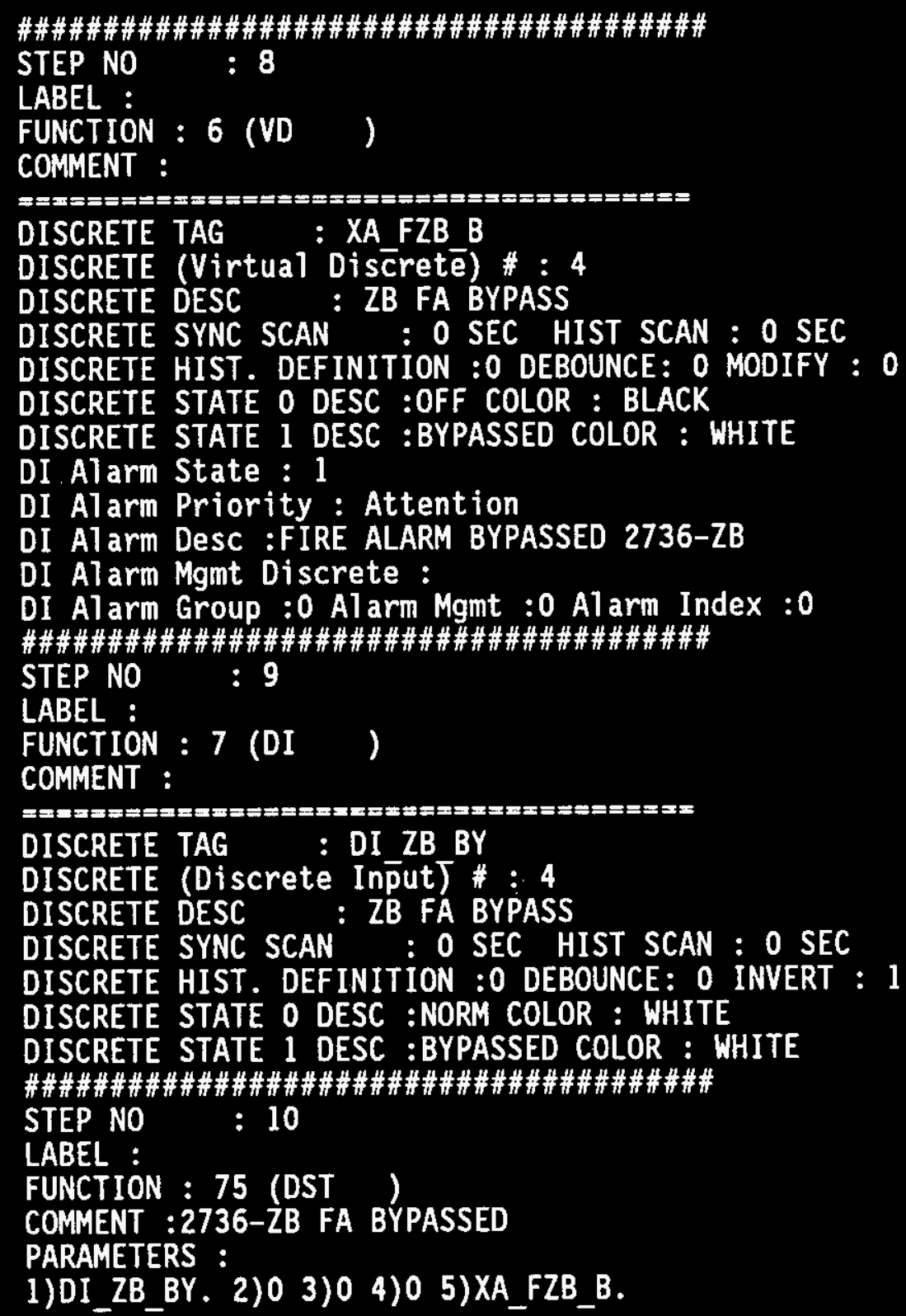


WHC-SD-CP-CSWD-016

Volume 4

Revision 1

Page 159

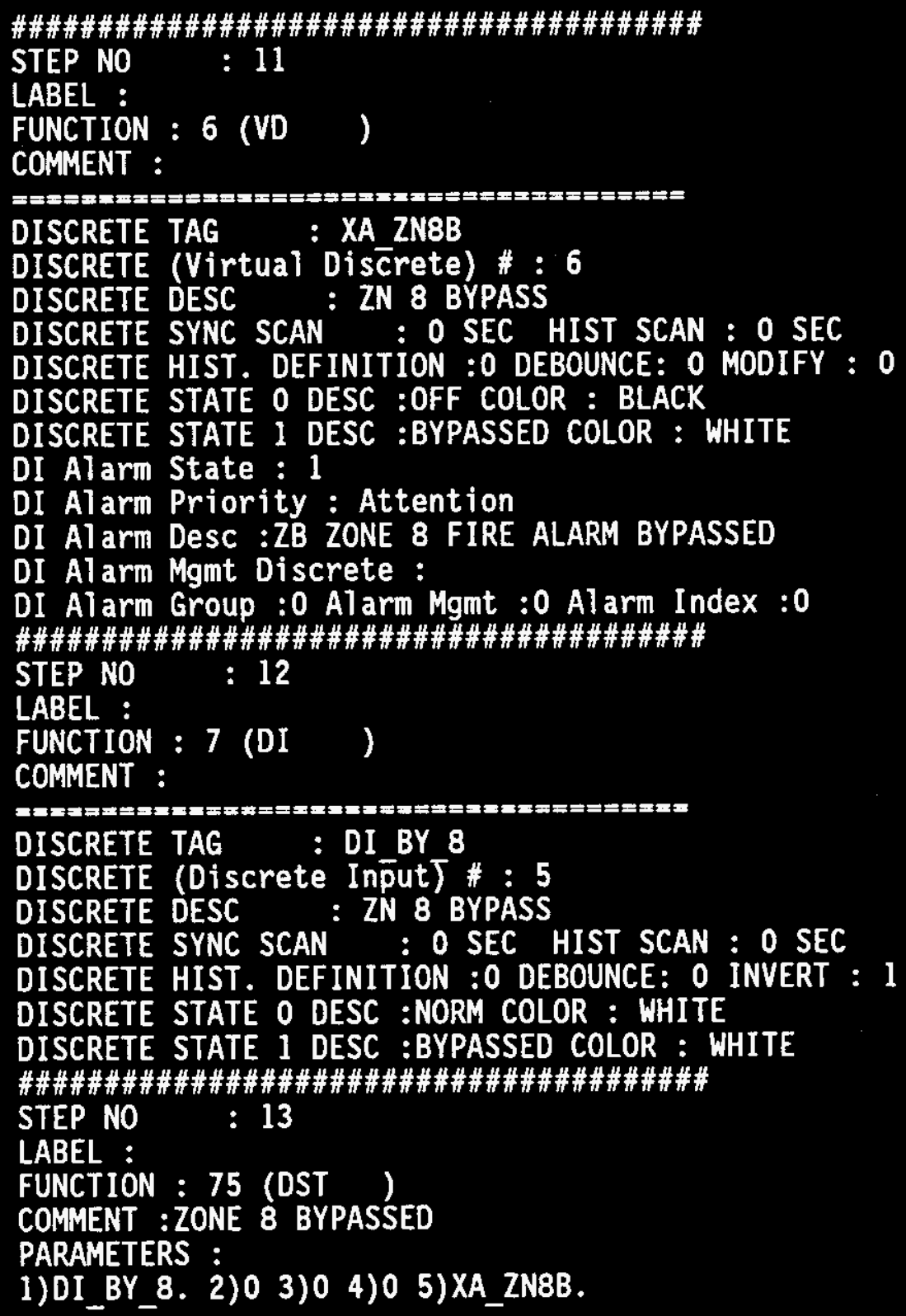


WHC-SD-CP-CSWD-016

Vol ume 4

Revision 1

Page 160

\#\#\#\#\#\#\#\#\#\#\#\#\#\#\#\#\#\#\#\#\#\#\#\#\#\#\#\#\#\#\#\#\#\#\#\#\#\#\#\#

STEP NO : 14

LABEL :

FUNCTION : 6 (VD )

COMMENT :

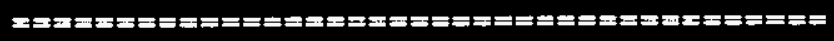

DISCRETE TAG : XA_ZN2B

DISCRETE (Virtual Disçrete) \#: 5

DISCRETE DESC : ZN II BYPASS

DISCRETE SYNC SCAN : O SEC HIST SCAN : O SEC

DISCRETE HIST. DEFINITION :0 DEBOUNCE: 0 MODIFY : 0

DISCRETE STATE O DESC :OFF COLOR : BLACK

DISCRETE STATE 1 DESC :BYPASSED COLOR : WHITE

DI Alarm State : 1

DI Alarm Priority : Attention

DI Alarm Desc :ZB ZONE II FIRE ALARM BYPASSED

DI Alarm Mgmt Discrete :

DI Alarm Group :0 Alarm Mgmt :0 Alarm Index :0

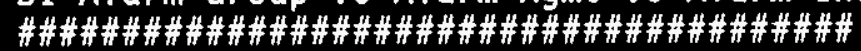

STEP NO : 15

LABEL :

FUNCTION : 7 (DI )

COMMENT :

DISCRETE TAG : DI BY 2

DISCRETE (Discrete Input) \# : 6

DISCRETE DESC : ZN II BYPASS

DISCRETE SYNC SCAN : O SEC HIST SCAN : O SEC

DISCRETE HIST. DEFINITION :0 DEBOUNCE: 0 INVERT : 1

DISCRETE STATE O DESC :NORM COLOR : WHITE

DISCRETE STATE 1 DESC :BYPASSED COLOR : WHITE

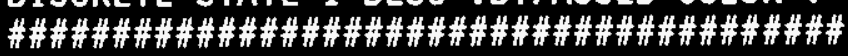

STEP NO : 16

LABEL :

FUNCTION : 75 (DST)

COMMENT :ZONE II BYPASSED

PARAMETERS :

1)DI_BY_2. 2)0 3)0 4)0 5)XA_ZN2B. 
\#\#\#\#\#\#\#\#\#\#\#\#\#\#\#\#\#\#\#\#\#\#\#\#\#\#\#\#\#\#\#\#\#\#\#\#\#\#\#\#

STEP NO : 17

LABEL :

FUNCTION : 6 (VD)

COMMENT :

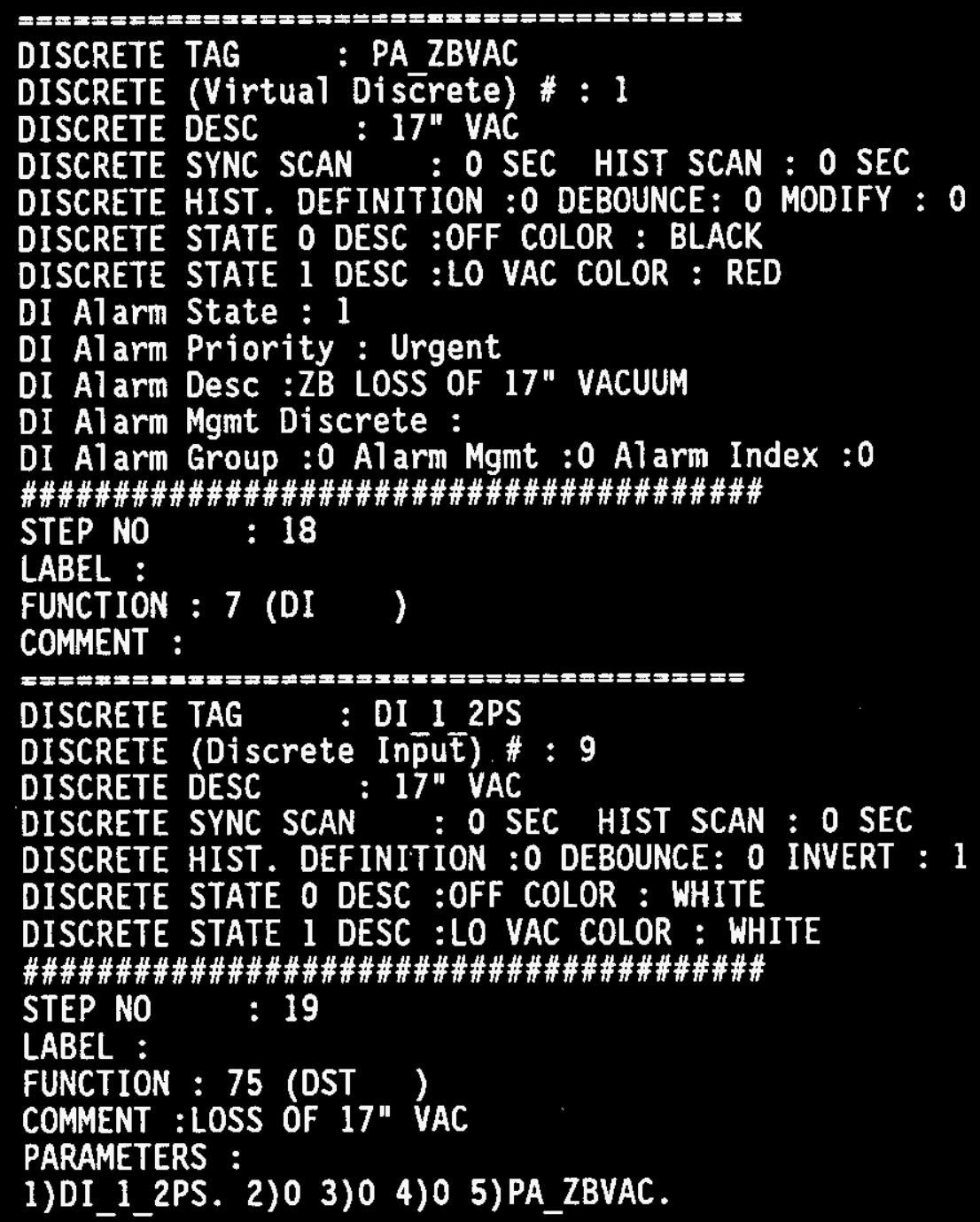




\section{WHC-SD-CP-CSWD-016 \\ Volume 4 \\ Revision 1 \\ Page 162}

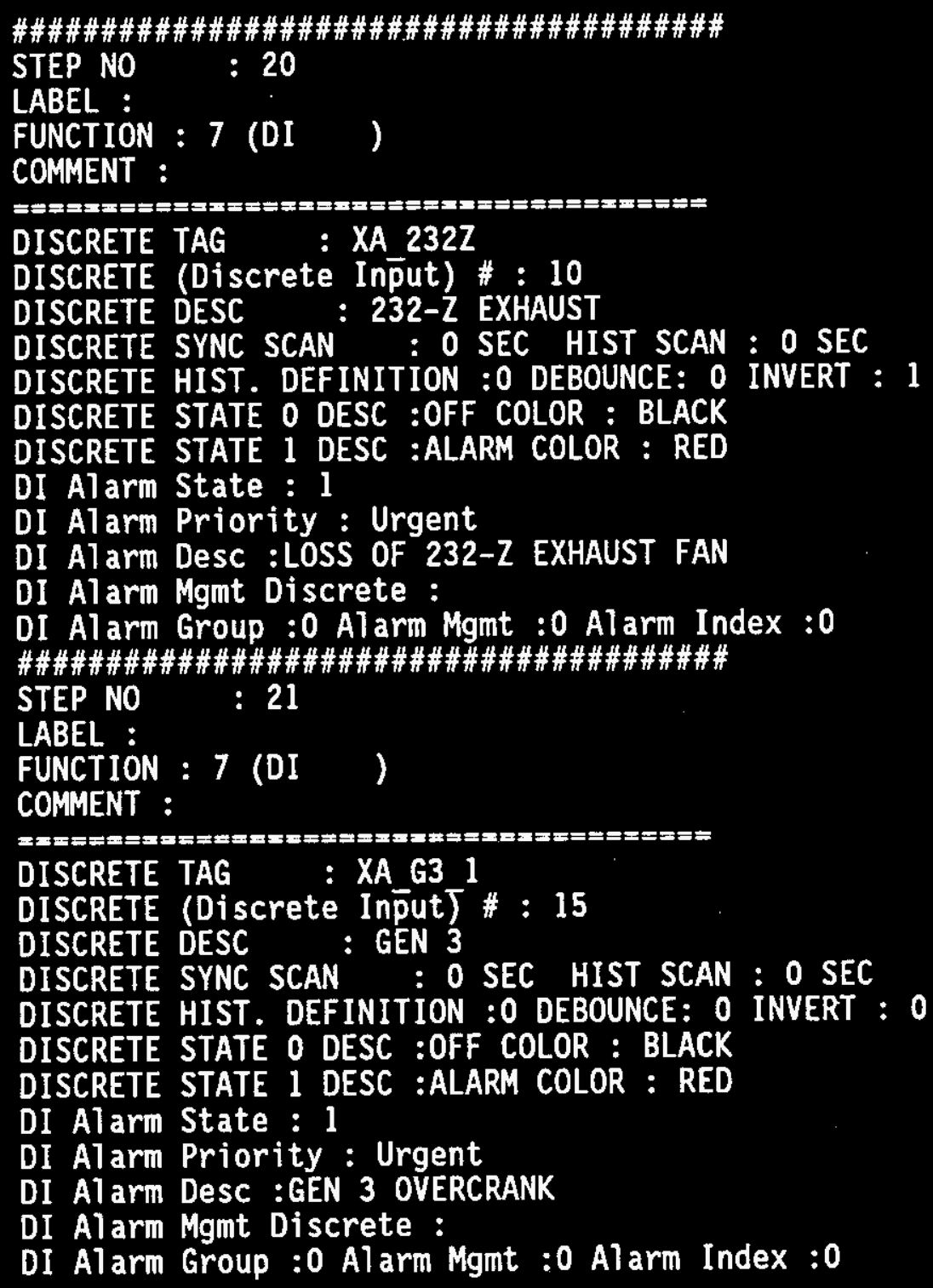


WHC-SD-CP-CSWD-016

Volume 4

Revision 1

Page 163

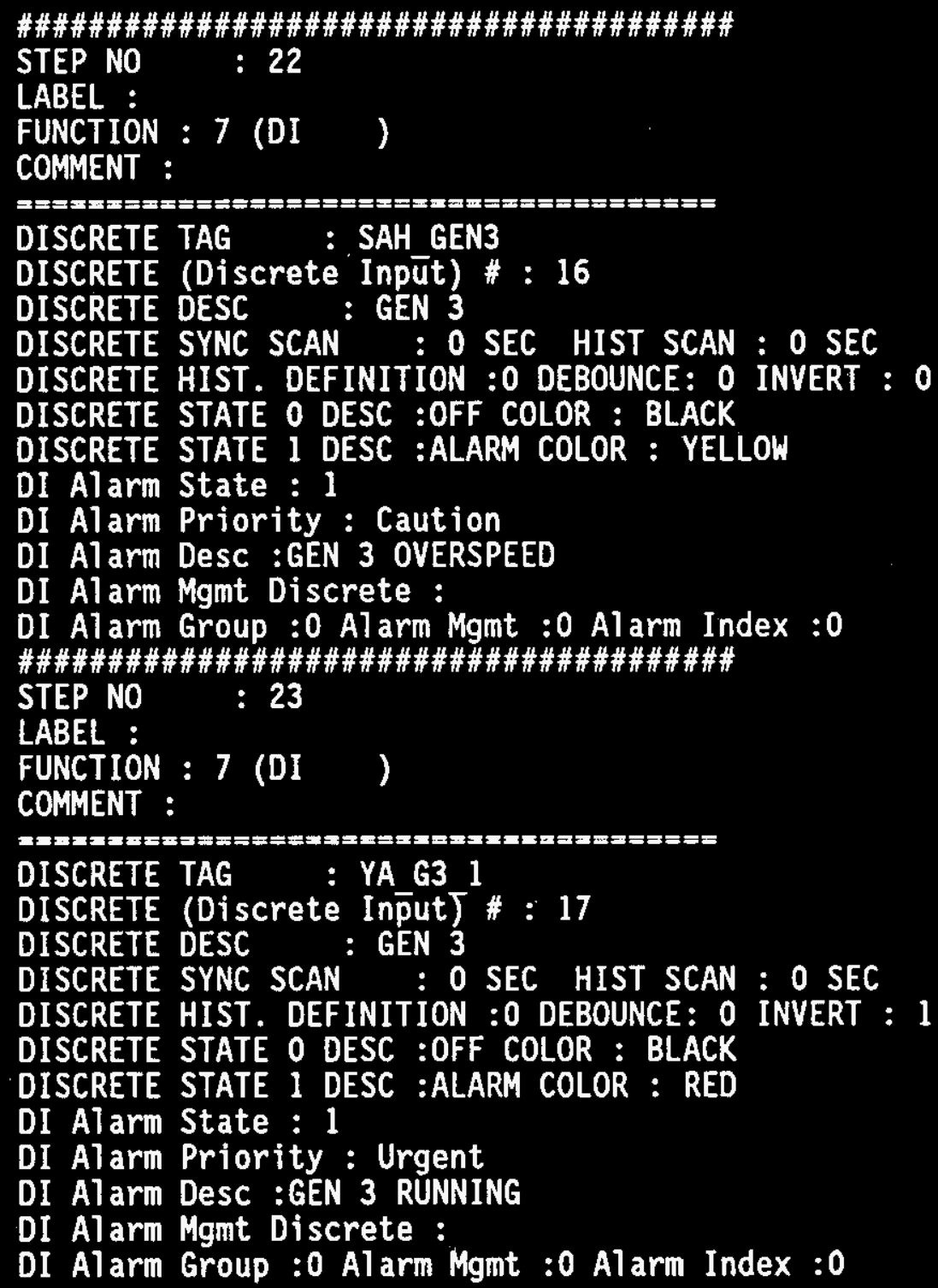




\section{WHC-SD-CP-CSWD-016 \\ Volume 4 \\ Revision 1 \\ Page 164}

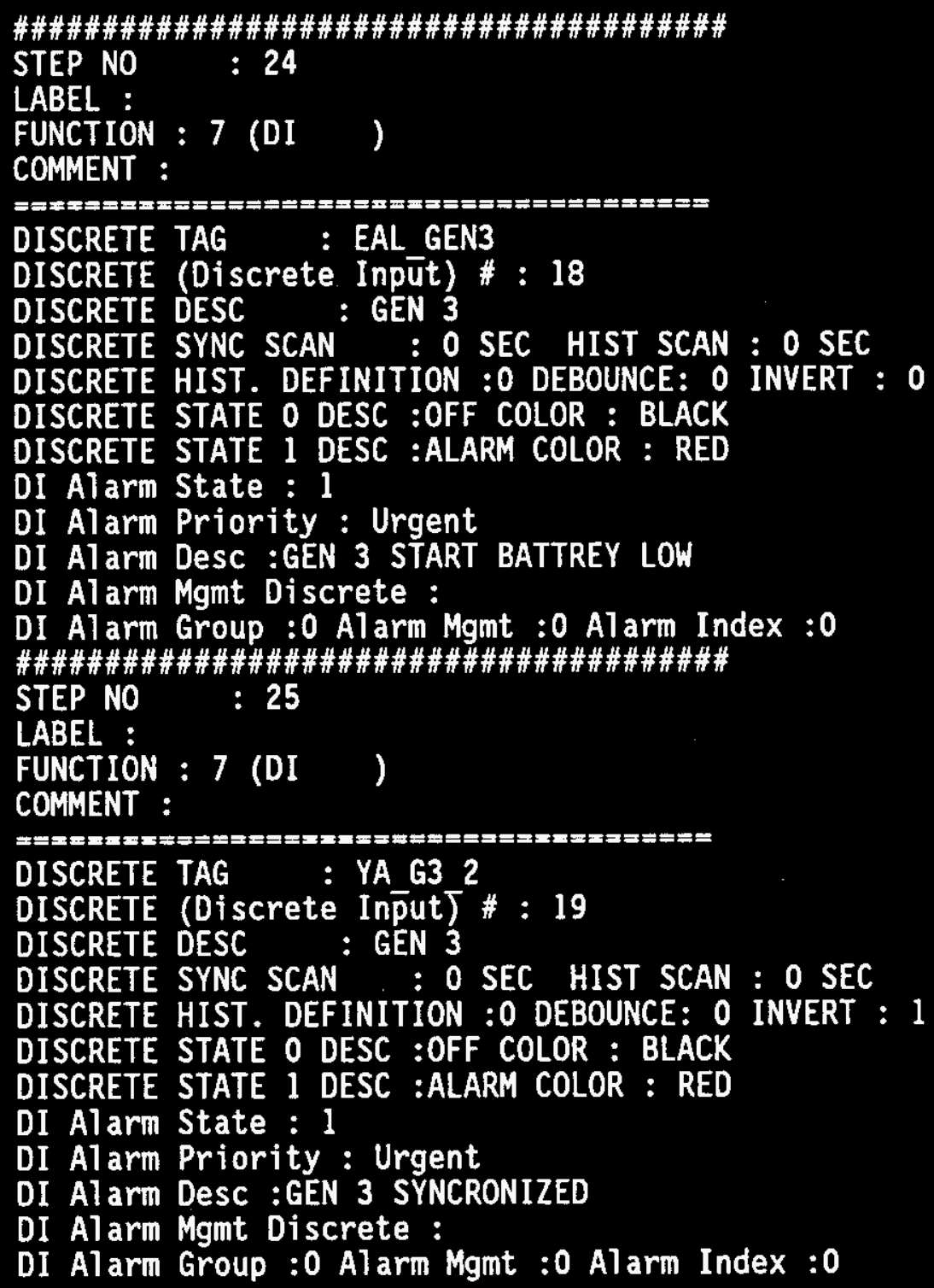


WHC-SD-CP-CSWD-016

Volume 4

Revision 1

Page 165

\#\#\#\#\#\#\#\#\#\#\#\#\#\#\#\#\#\#\#\#\#\#\#\#\#\#\#\#\#\#\#\#\#\#\#\#\#\#\#\#

STEP NO : 26

LABEL :

FUNCTION : 7 (DI )

COMMENT :

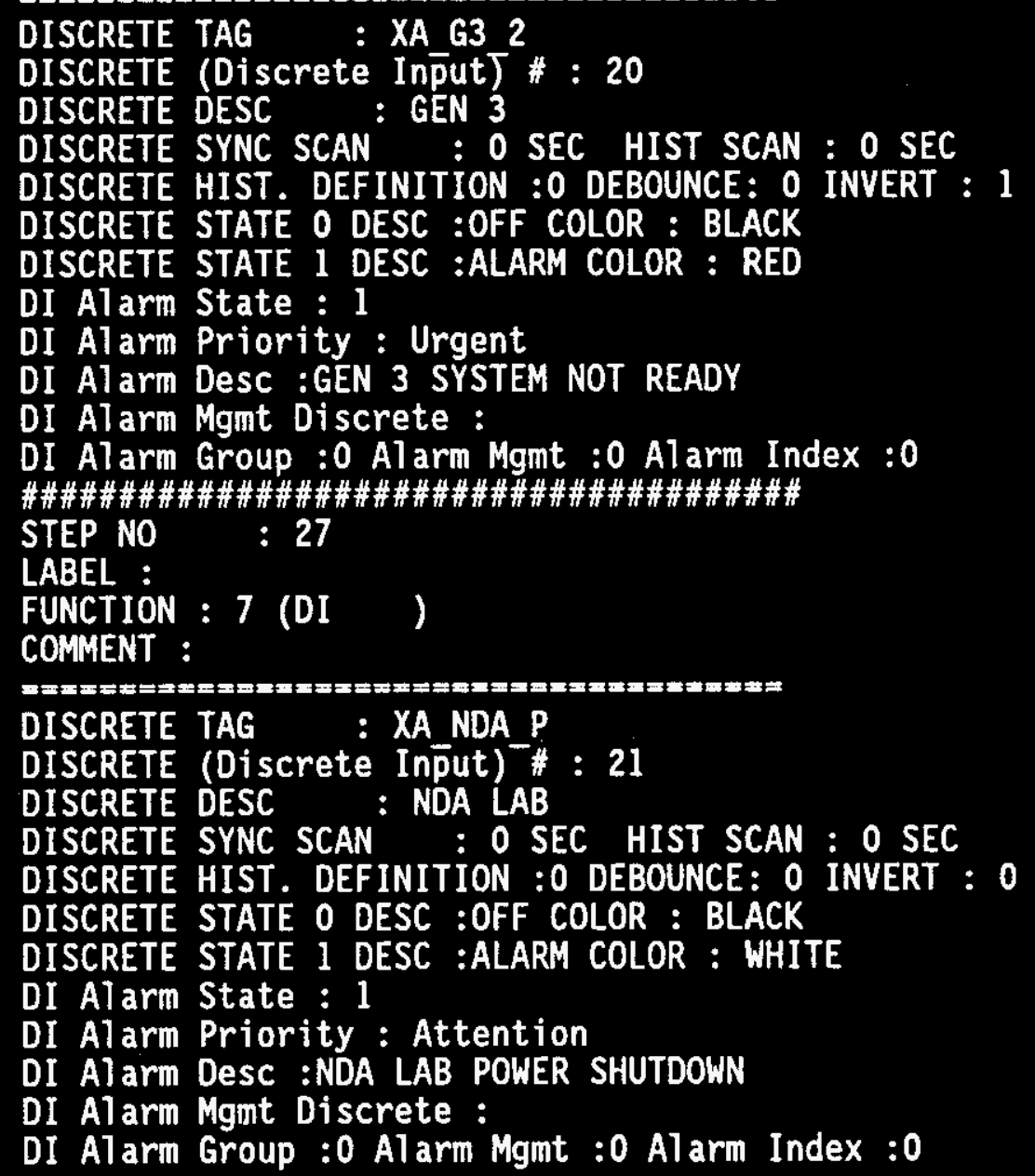




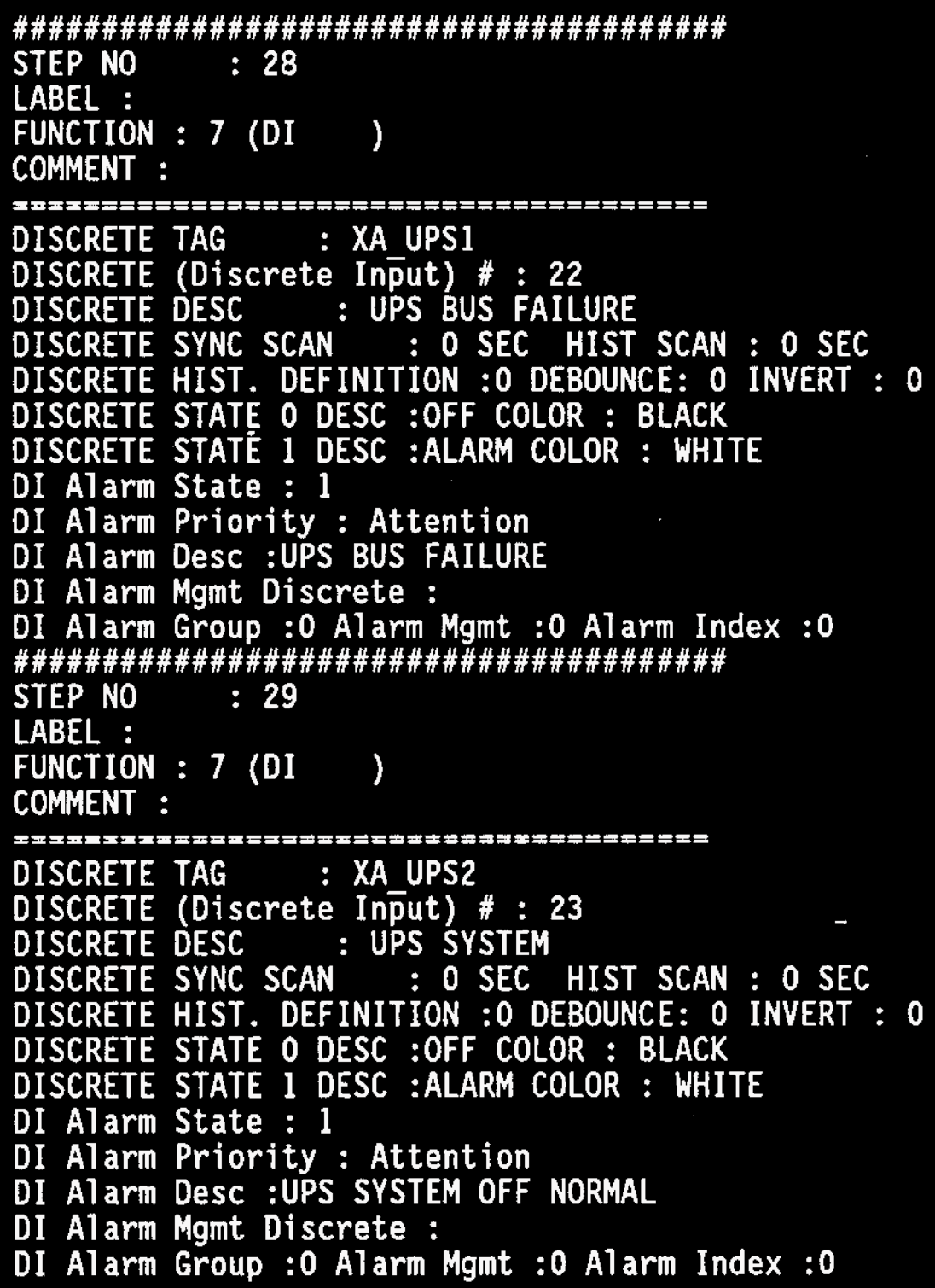


WHC-SD-CP-CSWD-016

Volume 4

Revision 1

Page 167

\#\#\#\#\#\#\#\#\#\#\#\#\#\#\#\#\#\#\#\#\#\#\#\#\#\#\#\#\#\#\#\#\#\#\#\#\#\#\#\#

STEP NO : 30

LABEL :

FUNCTION : 7 (DI )

COMMENT :

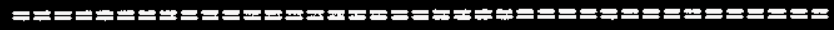

DISCRETE TAG : XA UPS3

DISCRETE (Discrete Input) \# : 24

DISCRETE DESC : UPS SYSTEM

DISCRETE SYNC SCAN : O SEC HIST SCAN : O SEC

DISCRETE HIST. DEFINITION :0 DEBOUNCE: 0 INVERT : 0

DISCRETE STATE O DESC :OFF COLOR : BLACK

DISCRETE STATE 1 DESC :ALARM COLOR : WHITE

DI Alarm State : 1

DI Alarm Priority : Attention

DI A1 arm Desc :UPS XFER SHITCH BYPASSED

DI Alarm Mgmt Discrete :

DI Alarm Group :0 Alarm Mgmt :0 Alarm Index :0

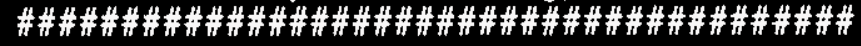

STEP NO : 31

LABEL :

FUNCTION : 0 (END)

COMMENT : 
WHC-SD-CP-CSWD-016

Volume 4

Revision 1

Page 168

\section{MICON PROGRAM FILES}

MICON program files are created by MICON View from the configuration file listings (above). This file is the actual program that is run by the controller card. When you request a program upload from a controller card, these are the files you'll get.

These files should not be directly modified by the user. All modifications should be through the configuration file using the configure controller option on MICON View.

The programming language is a custom language developed by MICON Company. A description of this language can be found in the U-32/RCH Function Compiler Documentation available from MICON Company (Houston, TX 1-713-947-9470).

You will notice that, like the configuration file, the program file is organized into loops. With the exception of two loops, these are the same ones created by the user in the controller's configuration file. The two exceptions are loop "ZERO" at the top and loop " NINETY NINE" at the bottom. These are house-keeping loops created by JICON View.

The following file listings have been edited to make them easier to read. Spaces have been put between the loops and extra long lines have been indented Therefor, the actual file's format may look a little different.

NOTE: The DEF line under each loop name contains fields showing the tuning paramaters -- for example:

CGAIN $=0.50$, CRESET $=10.00$, CRATE $=0.00, M \_$RESET $=0.00$

These tuning parameters are not controlled by this document. Therefore, these numbers may be different than the ones that appear in the workstation listings. This will also be true of program files uploaded from the controllers. 
WHC-SD-CP-CSWD-016

Volume 4

Revision 1

Page 169

\section{mic16_ro}

LOOP ZERO;

DEF :TTG=AI PE 1.,AI PE 2.,AI PE 5, AI PE 3.,AI PE 7.,AI_PE_4.;

DEF :TAG=PDRC $1 .$, PDRC_2-,PDRC_3.,PDRC_4 ., PDRC_5.,PDRC_6.;

DEF : TAG $=$ PERIOD $=1$;

END;

LOOP PDIC 1.;

DEF :TAG $=$ PERIOD $=0.30$, SP RATE $=10000.00$, SPLOLM $=0.00$, SPHILM $=0.15$, SPANL $0=0.00$, SPANHI $=0.15$, RATIO $=0.00$, BIAS $=0.00$, CGAIN $=1.75$, CRESET $=0.50$, CRATE $=0.00, M$ RESET $=0.00 ;$

AI : TAG=AI PE 1.: CHAN=1:SPANLO=0.00:SPANHI=0.15;

LET :TAG=PDRC $1 .=$ MSV; /*HISTORIAN*/

PID :PV=AI PE-1.:RECALL=1;

AO :TAG=A0EPP $1 .:$ CHAN=19:STEP $=M S V: O P L O L M=0.00: 0 P H I L M=100.00: 0 P N R L M=1000.00: 0 P P R L M=1000.00 ;$

ALARM : TAG=PDIC_1.PV:TYPE $=3:$ PRI $=149793:$ LIMITS $=\{?-, ?-, 0.01, ?+, ?+, ?+\}:$ DEADBAND $=0.00$;

END;

LOOP PDIC 2.;

DEF :TAG $=\bar{P} E R I O D=0.30, S P$ RATE $=10000.00, S P L O L M=0.00, S P H I L M=0.50, S P A N L O=0.00, S P A N H I=0.50$, RATIO $=0.00$, $B I A S=0.00$, CGAIN $=2.00$, CRESET $=0.50$, CRATE $=0.00, \mathrm{M}$ RESET $=0.00$;

AI : TAG $=A I$ PE_2.: CHAN $=2:$ SPANLO $=0.00:$ SPANHI $=0.50$;

LET :TAG=PDRC 2. =MSV; /*HISTORIAN*/

PID :PV=AI_PE_2.:DIRECT=1:RECALL $=1$;

$A 0: T A G=A 0^{-} E P-2 .: C H A N=20: S T E P=M S V: O P L O L M=0.00: 0 P H I L M=100.00: 0 P N R L M=1000.00: 0 P P R L M=1000.00$;

ALARM :TAG=PDIC_2.PV:TYPE=3:PRI $=149793:$ LIMITS $=\{?-, ?-, 0.05, ?+, ?+, ?+\}:$ DEADBAND=0.00;

END; 
WHC-SD-CP-CSWD-016

Volume 4

Revision 1

Page 170

LOOP PDIC 4;

DEF :TAG $=\bar{P} E R I O D=0.30$, SP RATE $=10000.00$, SPLOLM $=0.00$, SPHILM $=2.00$, SPANLO $=0.00$, SPANHI $=2.00$, RATIO=0.00, $B I A S=0.00$, CGAIN $=0.50$, CRESET $=1.50$, CRATE $=0.00, M$ RESET $=0.00$;

LET :TAG $=-$ !PULSE(1); $/ *$ "0" PULSE ON STARTUP*/

GOTO :COND=MSV:TAG=JUMP;

LET : TAG $=0 U T=1 ; / * O P E N$ DAMPER ON STARTUP*/

LABEL :TAG $=$ JUMP;

AI :TAG=AI PE 5.: CHAN=3:SPANL0=0.00:SPANHI=2.00;

LET :TAG=PDRC 4.=MSV; / *HISTORIAN*/

PID :PV $=A I$ PE $5 .:$ RECALL $=1$;

AO :TAG $=A 0^{-}$EP $4 .:$ CHAN $=21:$ REVERSE $=1: S T E P=M S V: 0 P L O L M=0.00: 0 P H I L M=100.00: 0 P N R L M=1000.00: 0 P P R L M=1000.00$

ALARM :TAG $=$ PDIC_4.PV:TYPE $=3: P R I=149793:$ LIMITS $=\{?-, ?-, 0.25, ?+, ?+, ?+\}:$ DEADBAND $=0.00$;

END;

LOOP PDIC 5.;

DEF :TAG $=\bar{P} E R I O D=0.30, S P$ RATE $=10000.00, S P L O L M=0.00, S P H I L M=10.00$, SPANLO $=0.00, S P A N H I=10.00$, RAT10=0.00, BIAS $=0.00, C G A I N=2.00, C R E S E T=0.75, C R A T E=0.00, M \_$RESET $=0.00$;

AI :TAG=AI PE 3.: CHAN $=4:$ SPANLO $=0.00:$ SPANHI $=10.00$;

LET :TAG=PDORC $5 .=$ HSV; /*HISTORIAN*/

PID :PV=AI PE 3.:RECALL $=1$;

$A 0: T A G=A 0^{-} E P-5 .: C H A N=22: S T E P=N S V: O P L O L M=0.00: 0 P H I L M=100.00: 0 P N R L M=1000.00: 0 P P R L M=1000.00$

ALARM :TAG $=$ PDIC 5.PV:TYPE $=3:$ PRI $=149793:$ LIMITS $=\{?-, ?-, 1.00, ?+, ?+, ?+\}:$ DEADBAND $=0.00$;

END;

LOOP PDIC 6.;

DEF :TAG=PERIOD $=0.30, S P$ RATE $=10000.00, S P L O L M=0.00, S P H I L M=0.50, S P A N L O=0.00, S P A N H I=0.50, R A T I 0=0.00$, $B I A S=0.00$, CGAIN $=2.00$, CRESET $=2.00$, CRATE $=0.00, \mathrm{M}$ RESET $=0.00$;

AI :TAG=AI PE $7 .:$ CHAN $=5:$ SPANL0 $=0.00:$ SPANHI $=0.50$;

LET :TAG=PDRC $6 .=M S V ; / * H I S T O R I A N * /$

PID : PV=AI PE $7 .:$ DIRECT $=1:$ RECALL $=1$;

$A 0: T A G=A 0^{-} E P-6 .: C H A N=23: S T E P=M S V: O P L O L M=0.00: 0 P H I L M=100.00: 0 P N R L M=1000.00: 0 P P R L M=1000.00 ;$

ALARM :TAG $=$ PDIC 6. PV:TYPE $=3:$ PRI $=149793:$ LIMITS $=\{?-, ?-, 0.05, ?+, ?+, ?+\}:$ DEADBAND $=0.00$;

END; 

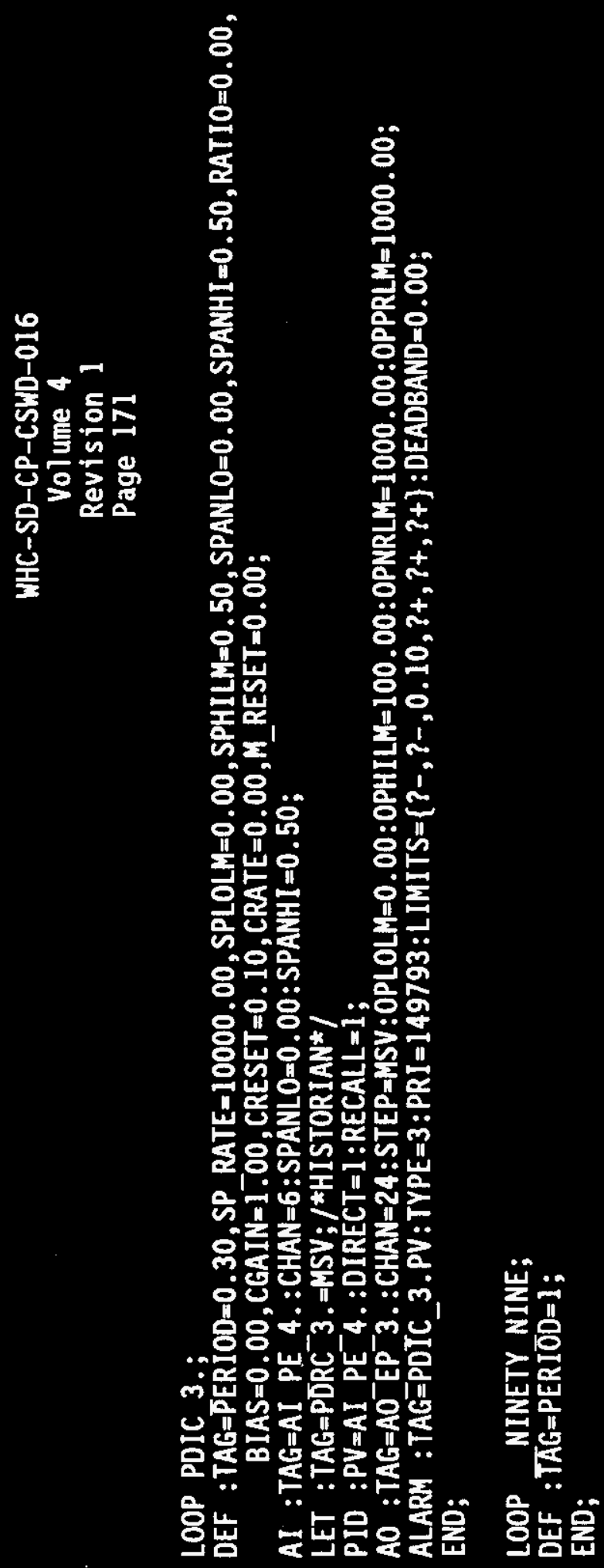

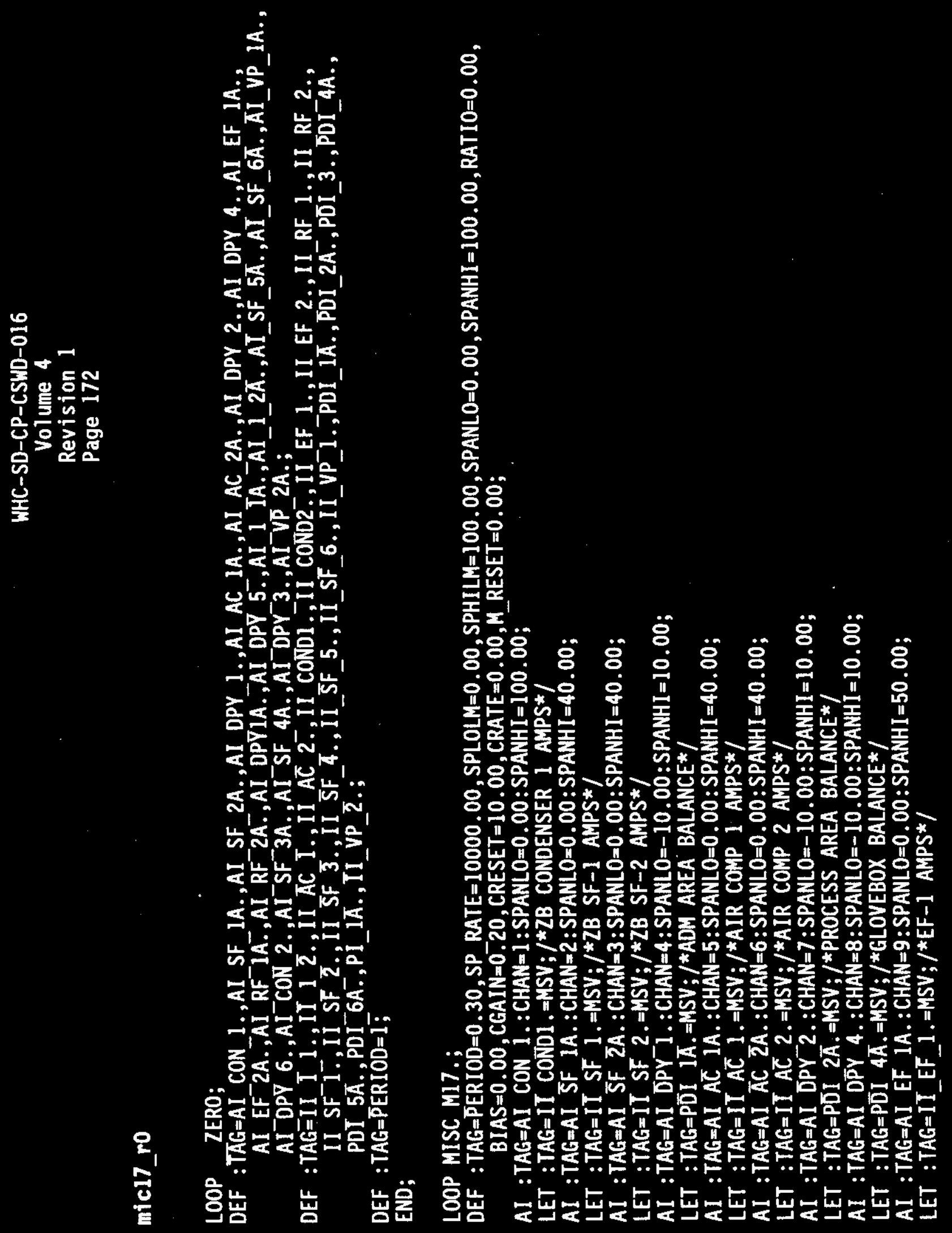


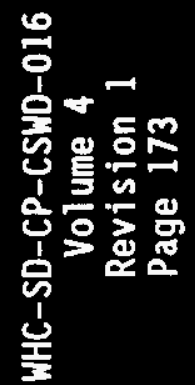

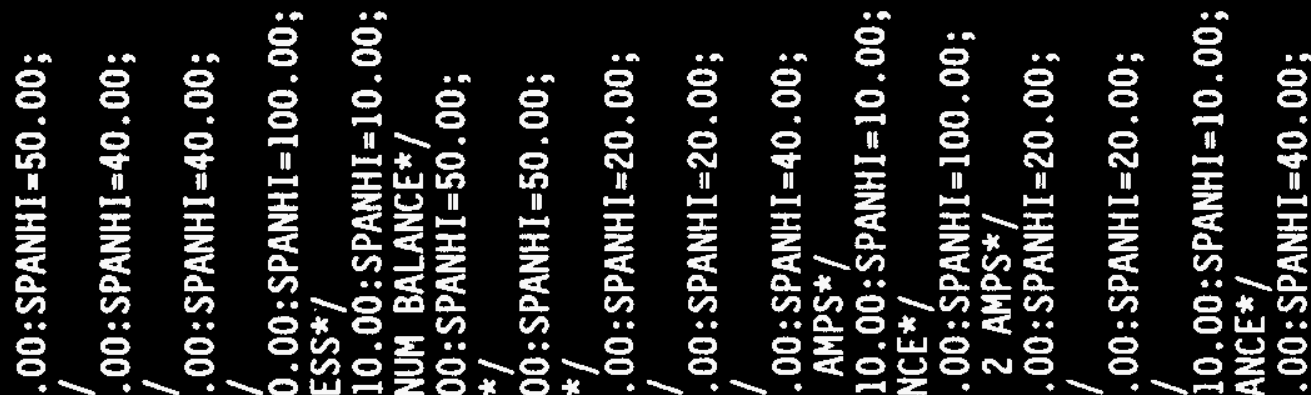

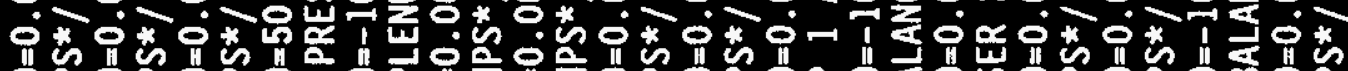

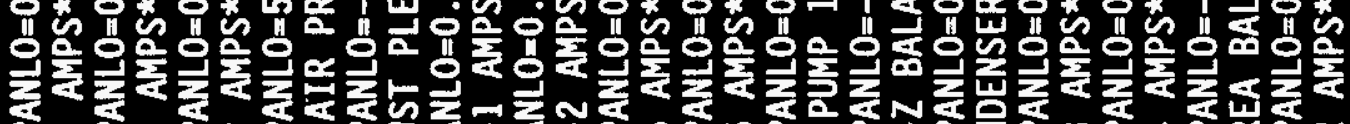

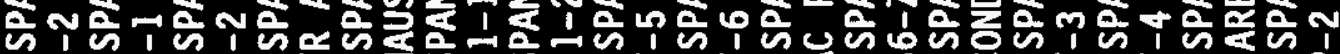

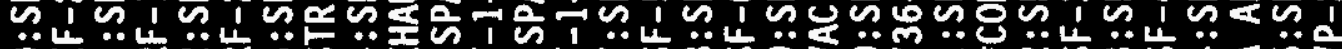
$\ddot{0}$ 光 *

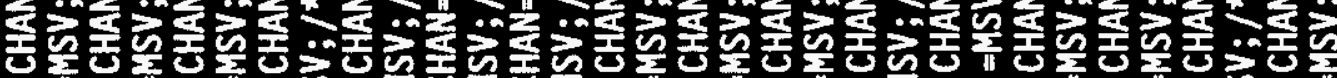

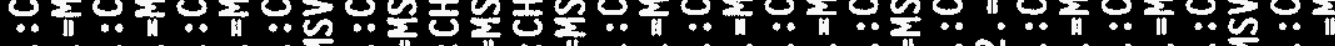

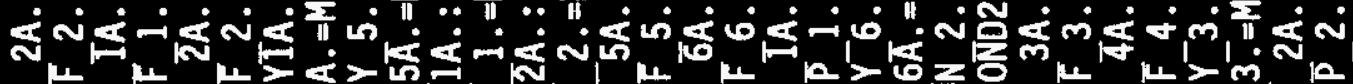

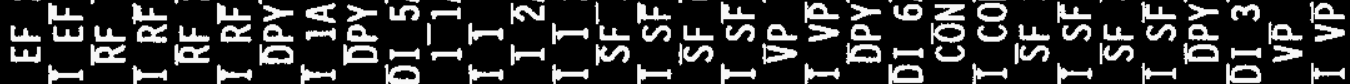

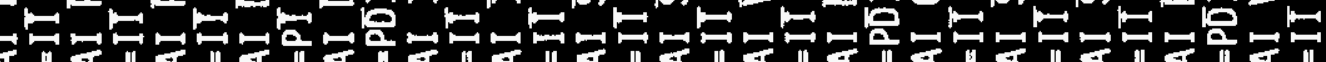

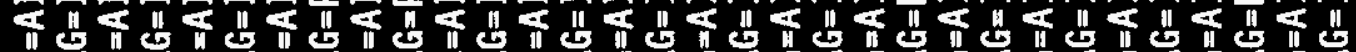

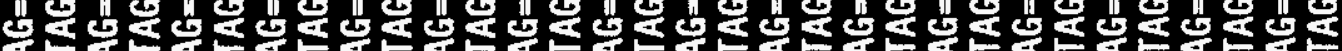

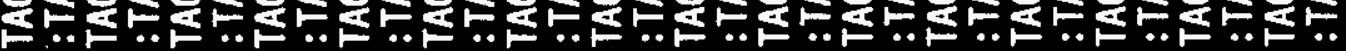

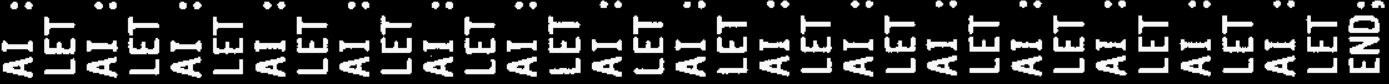




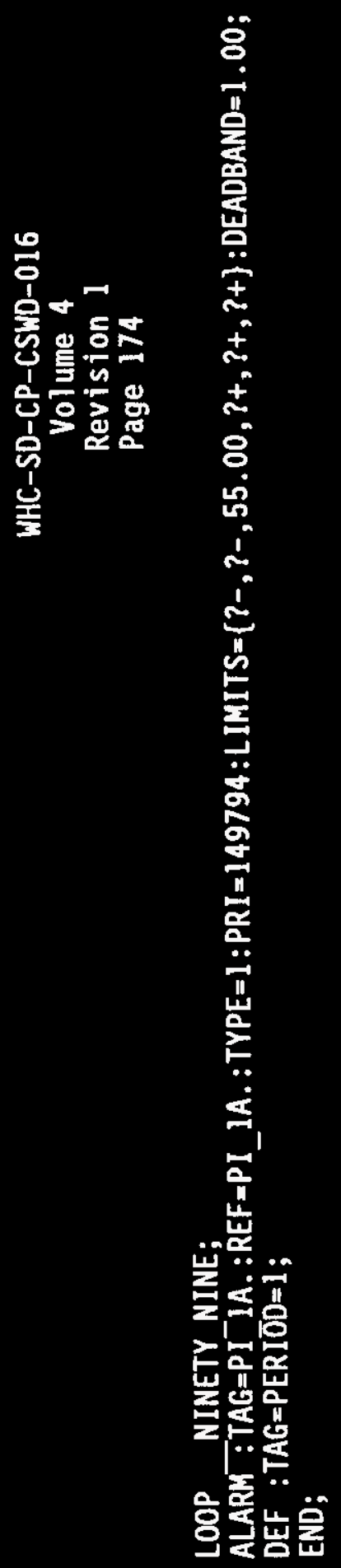



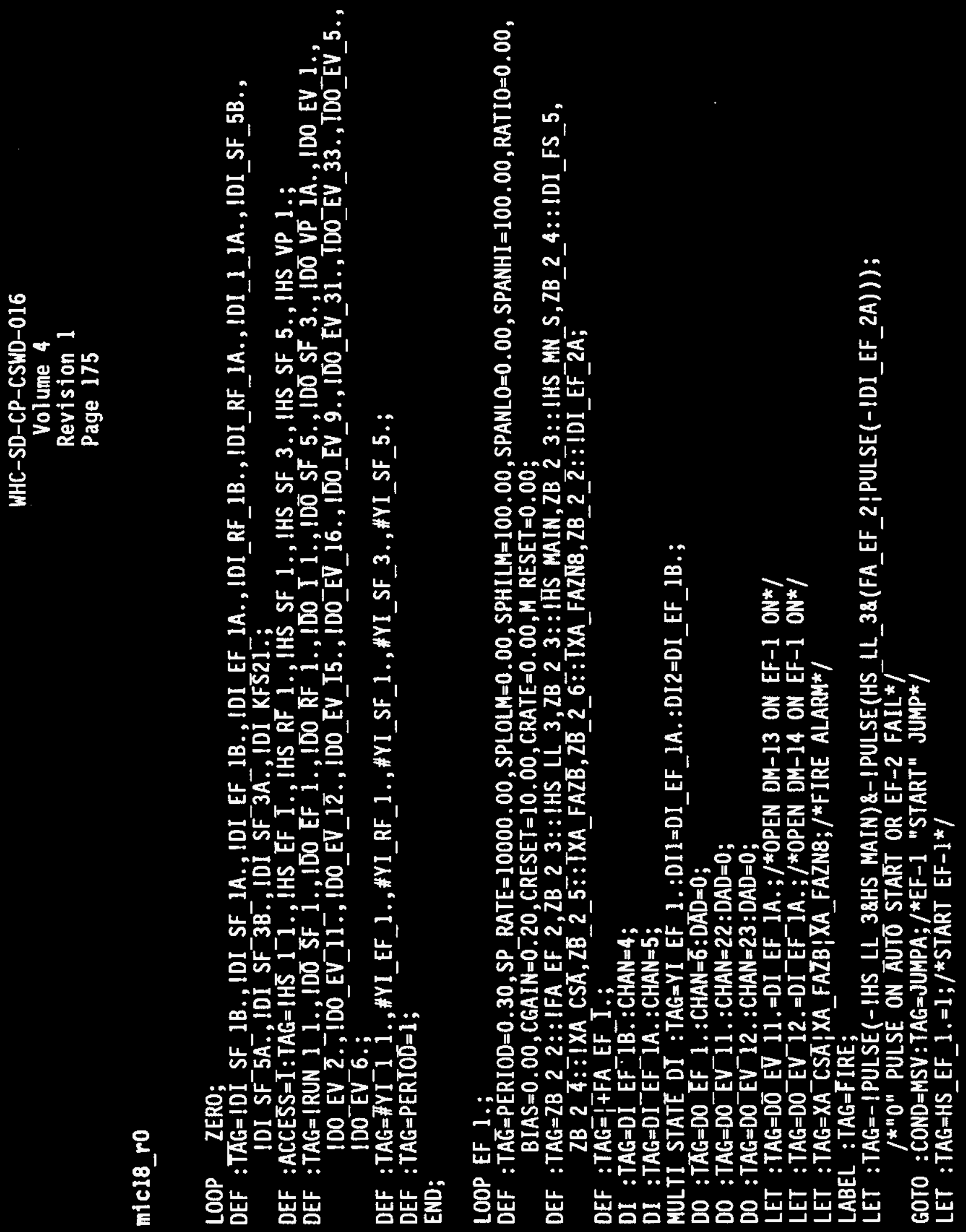
Volume 4

Revision 1

Page 176

LABEL :TAG=JUMPA:

LET :TAG=-IPULSE(1);/*PREVENT FALSE START ON RESET*/

GOTO :COND=MSV:TAG=JUMPST;

LET : TAG=HS EF $1 .=0$;

LABEL :TAG=JUMPST;

LET :TAG $=-$ IPULSE(HS MN $S, 5) ; / * 0 "$ PULSE ON MAIN STOP*/

GOTO :COND=NSV:TAG=JUMPB; $/ * E F-1$ "STOP" JUMP*/

LET :TAG $=$ HS EF $1 .=0 ; / * S T O P$ EF $-1 * 1$

LABEL :TAG=JUMPB;

LET :TAG=-! IFIRE; $/ * E F-1$ HELD ON "STOP" IF COLD SMOKE ALARM*/

GOTO :COND=MSV:TAG=JUMPC; / COLD SMOKE "STOP" JUMP*/

LET :TAG=HS EF $1 .=0 ; / * H O L D$ EF-1 ON "STOP"*/

LABEL :TAG= JUMPC;

LATCH :TAG=DO EF 1.:LATCH=PULSE(HS EF_1,5):UNLATCH=-HS_EF_1 (DELAY(HS_EF_1, 15)\&-1DI_FS_5\&-!HS_LL_3): RECALL $=\overline{0} ; /^{\bar{\star}} \mathrm{EF}-1$ CONTROL LOGIC $\star$

LET :TAG=DELAY (HS_EF 1,15$) \&-($ DI_EF_1A\&!DI_FS_5); $/ \star A L A R M$ ON NO FLOW OR OFF WHEN HS IS ON (EXCEPT STARTUP) $* /$

LET :TAG=FA EF 1. $=M S V$;

EXPORT DI_EF_IA,FA_EF_1;

END;

LOOP EF 11 .;

DEF :TAG $=$ PERIOD $=0.30$, SP RATE $=10000.00$, SPLOLM $=0.00$, SPHILM $=100.00$, SPANLO $=0.00, S P A N H I=100.00$, RAT 10 $=0.00$, BIAS $=0.00$, CGAIN $=0.20$, CRESET $=10.00$, CRATE $=0.00, M$ RESET $=0.00 ;$

DEF :TAG=ZB 24 : : : IDI 1 1B;

DEF : TAG $=1+\mathrm{FA}^{-} 1 \mathrm{1}$.;

DI :TAG $=$ DI 1 TA.: $C H A N=10$;

MULTI STATE DI :TAG=YI_1 1 : :DI1=RUN_1_1.:DI2=0I_1_1A.;

DI :TAG $=0$ I KFS21.:CHAN=18;

DO :TAG $=00^{-1} 1$ 1.:CHAN $=11: D A D=0$;

DO :TAG $=D 0^{-} E V$ 33.:CHAN $=28: D A D=0$;

LET :TAG=DO EV 33.=DI $11 \mathrm{~B} . ; / *$ OPEN DH-28 ON EF-1-1 RUN*/

LET :TAG=-IXA CSA;/*HELD ON" "STOP" IF COLD SMOKE ALARN*/

GOTO :COND $=M S V: T A G=J U M P A ; / * C O L D$ SMOKE "STOP" JUMP*/

LET :TAG=HS_1_1.=0;/*STOP EF-1-1*/ 


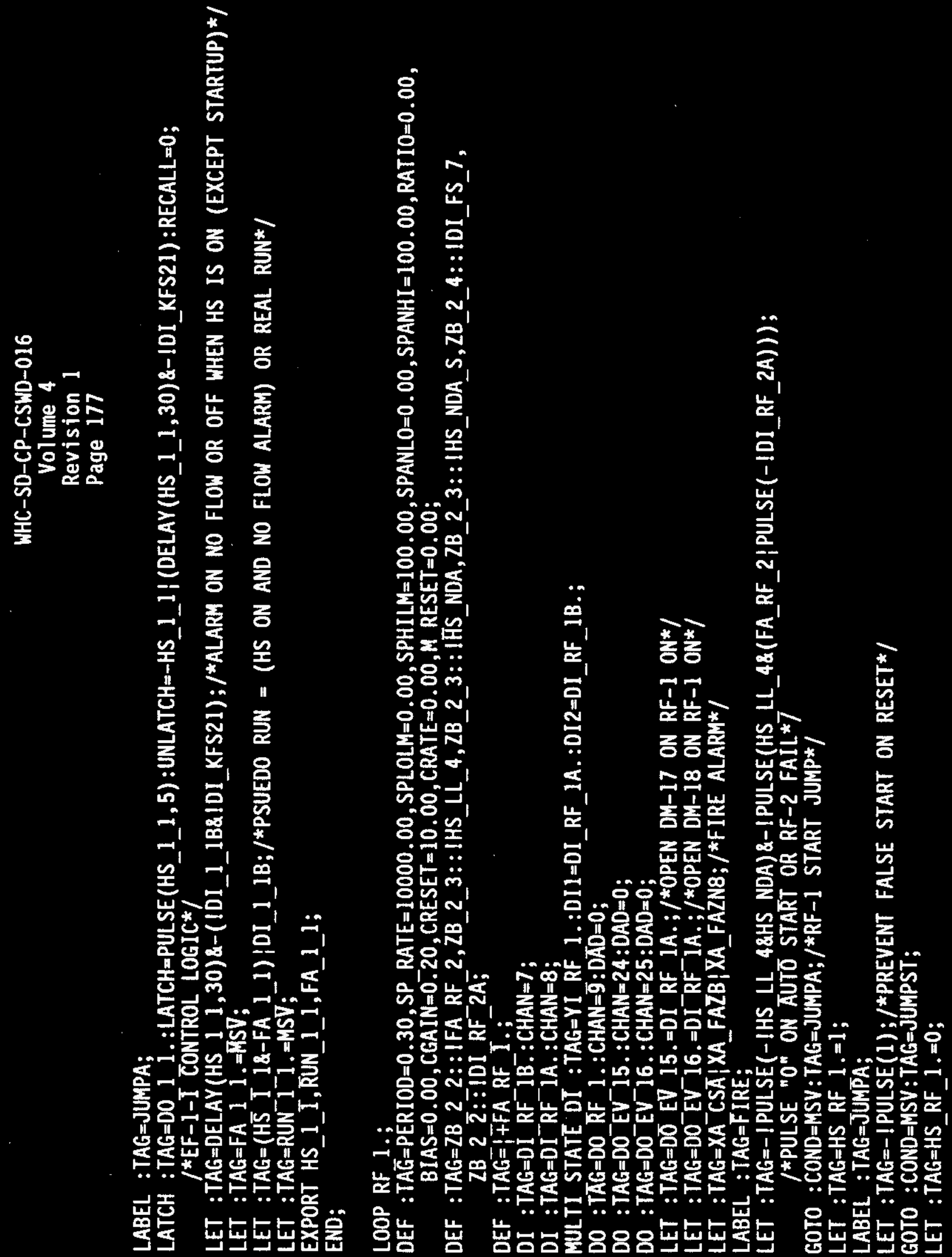



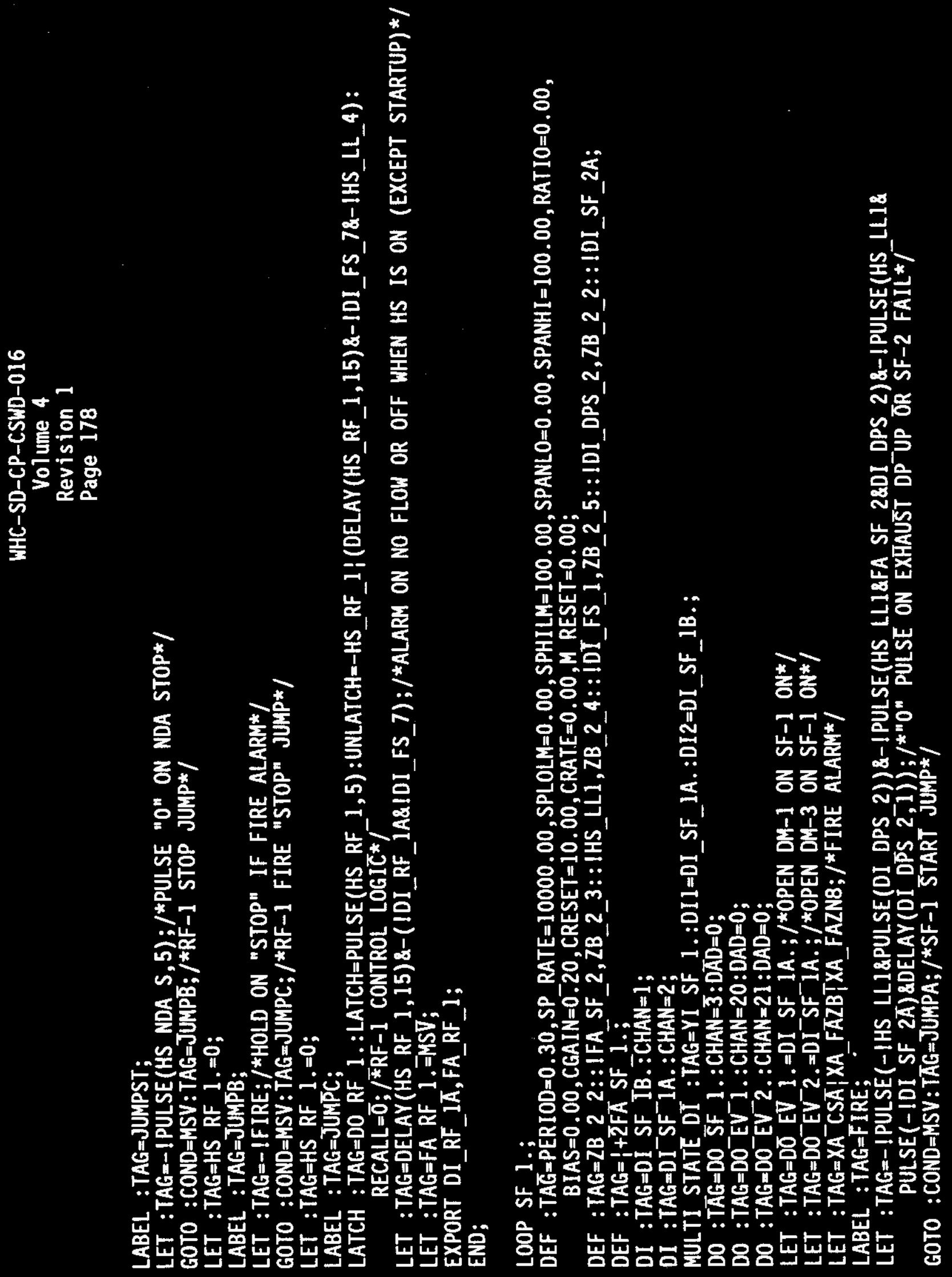

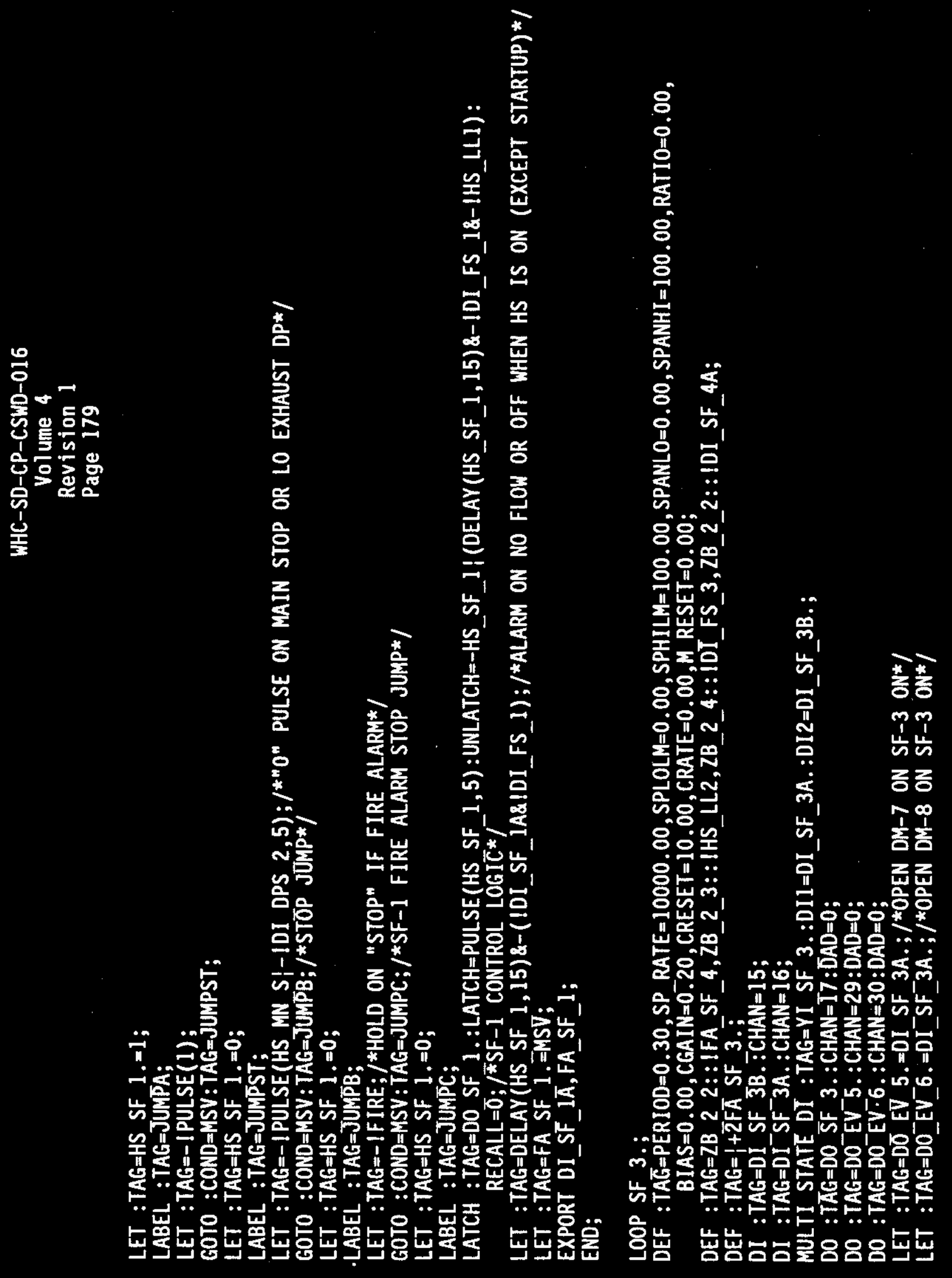

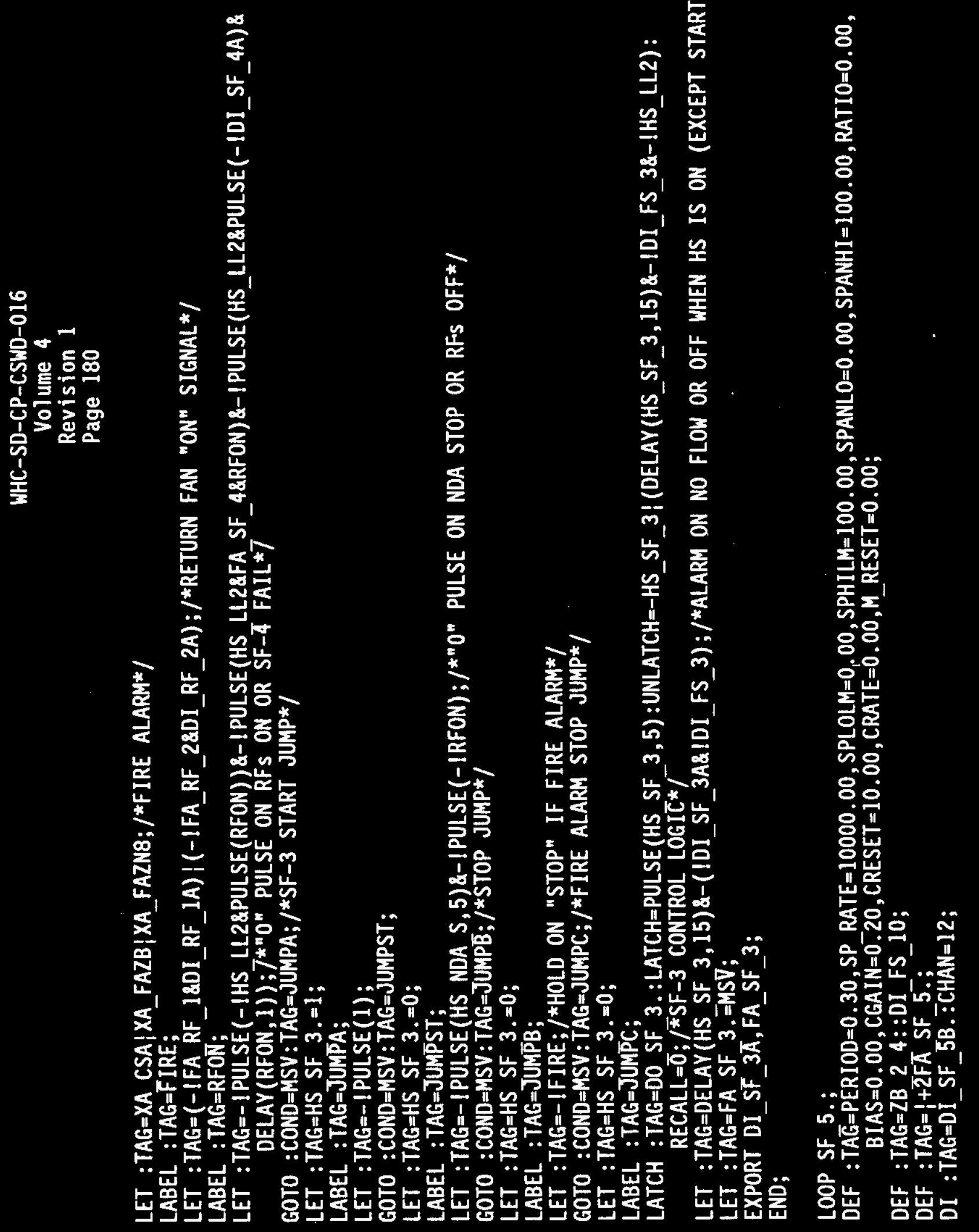

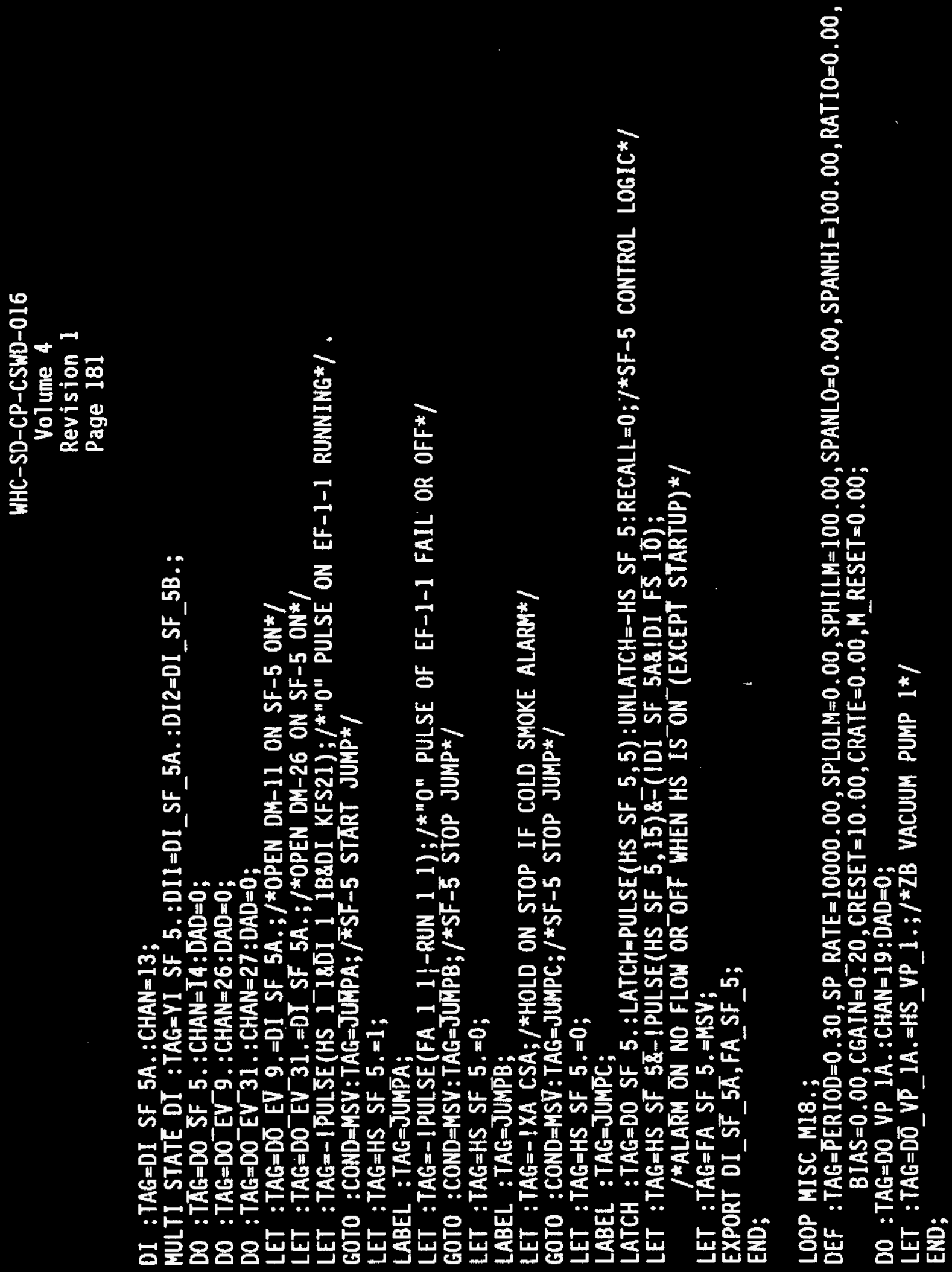
WHC-SD-CP-CSWD-016

Volume 4

Revision 1

Page 182

LOOP NINETY NINE;

DEF : TAG $=$ PERI $\bar{D} D=1$;

END; 

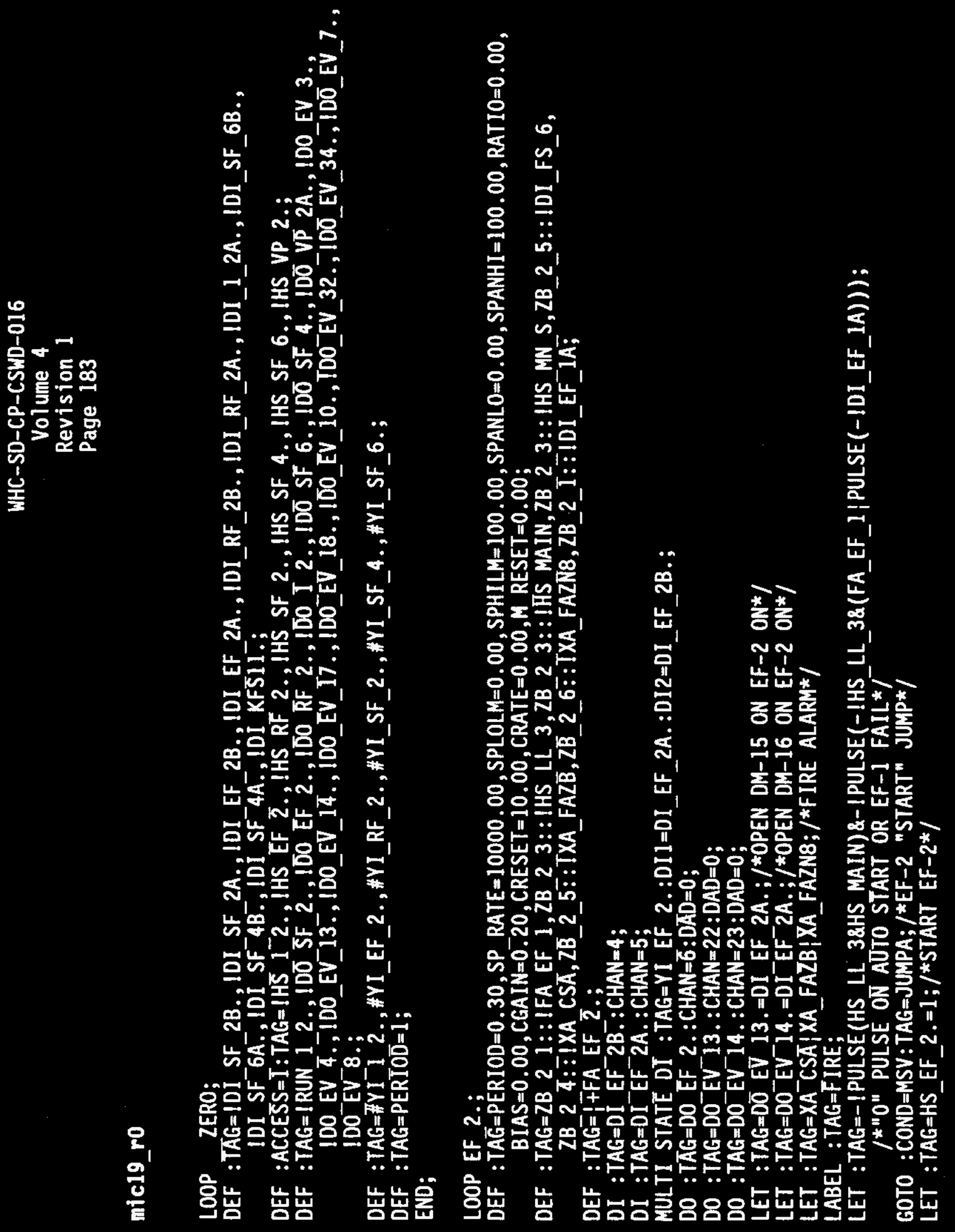

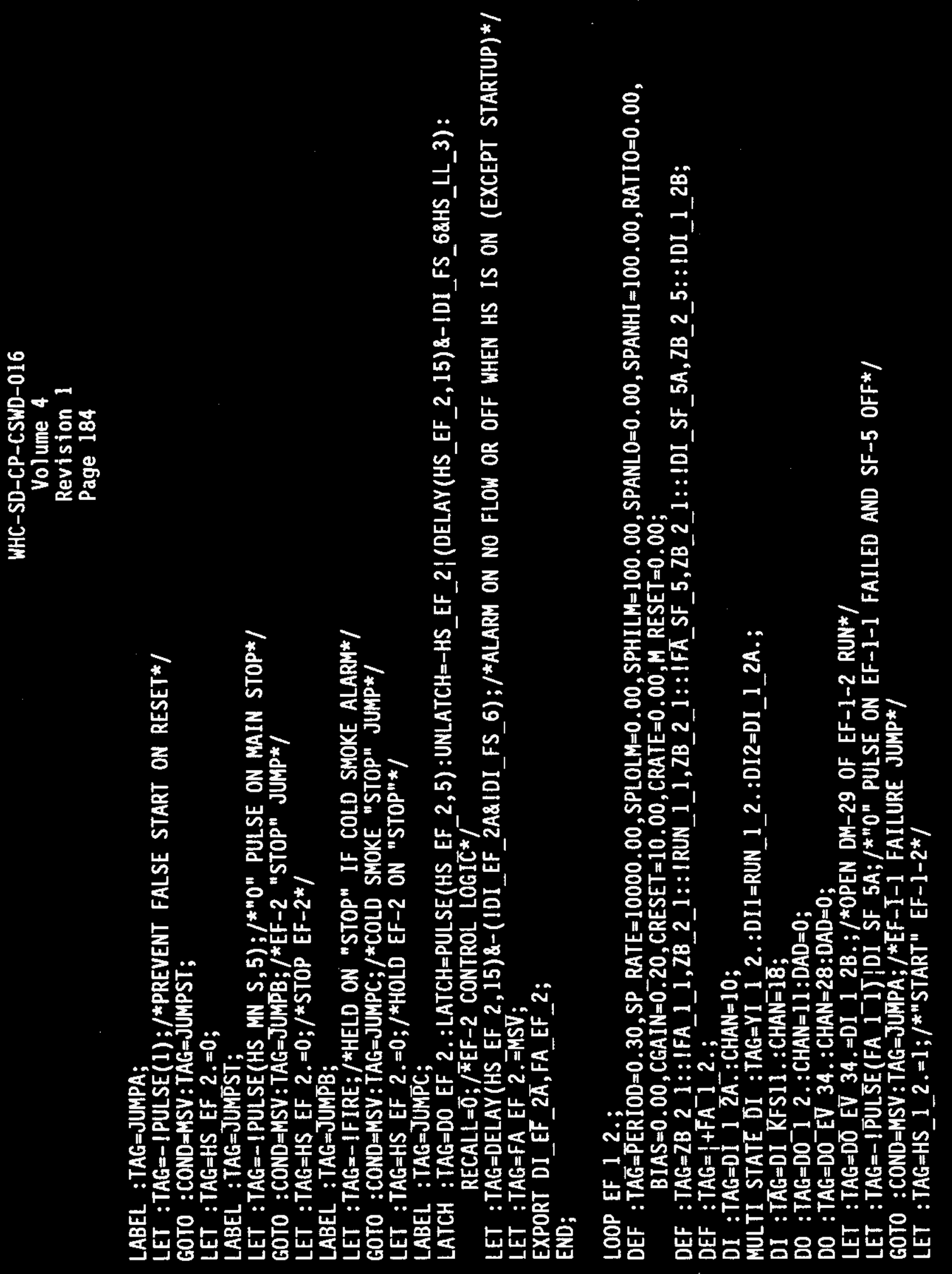
WHC-SD-CP-CSWD-016

Volume 4

Revision 1

Page 185

LABEL : TAG=JUMPA;

LET :TAG=-I PULSE(DELAY (DI SF 5A, 10)) |-IRUN 1 1 IFA_1_1;

$1 *$ "0" PULSE 10 SEC AFTER SF-5 START IF EF-1-1-RUNNING*/

GOTO :COND=MSV:TAG=JUMPB; /*AUTO START JUMP*/

LET :TAG=HS $12 .=1 ; / * S T A R T$ EF-1-2*/

LABEL :TAG=JUMPB;

LET :TAG $=-1$ IXA CSA; /*HOLD ON "STOP" IF COLD SHOKE ALARM*/

GOTO :COND=MSV:TAG=JUMPC; $/ *$ COLD SMOKE ALARM JUMP*/

LET :TAG $=$ HS $12 .=0 ; / \star S T O P$ EF $-1-2 * /$

LABEL :TAG=JURPC;

LATCH :TAG=DO $12 .:$ LATCH=PULSE(HS 1 2):UNLATCH=-HS_1_2! (DELAY $(H S$ 1_2,30)\&-IDI_KFS11\&RUN_1_1): RECALL $=\overline{0} ; 7 * E F-1-2$ CONTROL LOTGIC

LET :TAG=HS 1 2\&-! PULSE(HS 12,30$) \&-($ IDI 1 28\&!DI KFS11);

$l^{*}$ ALATRM ON NO FLOW OR OFF WHEN HS IS ON (EXCEPT STARTUP)*/

LET :TAG $=F A$ 1 2.=MSV;

LET :TAG $=\left(H \bar{S}\right.$ I_28-FA 1_2) $\mid D I \_1 \_2 B ; / * P S E U D O$ RUN $=$ (HS ON AND NO FLOW ALRM) OR REAL RUN ON*/

LET :TAG $=$ RUN $^{-1}-2 .=M S V ;$

EXPORT HS_1 2 , RUN_1_2,FA_1_2;

END;

LOOP RF 2.;

DEF :TAG $=$ PERIOD $=0.30, S P$ RATE $=10000.00, S P L O L M=0.00, S P H I L M=100.00, S P A N L 0=0.00, S P A N H I=100.00, R A T I 0=0.00$, BIAS $=0.00$, CGAIN $=0.20$, CRESET $=10.00$, CRATE $=0.00, \mathrm{M}$ RESET $=0.00 ;$

DEF :TAG=ZB 2 $1::$ !FA_RF_1,ZB_2_3:: IHS_LL_4,ZB_2_3::IHS_NDA,ZB_2_3::IHS_NDA_S,ZB_2_5::!DI FS_8, ZB 2 I: :IDI RF 1 iA;

DEF :TAG $=1$ +FA_RF 2 .;

DI :TAG=DI RF 2B.: CHAN=7;

DI :TAG=DI ${ }^{-} F^{-} 2 A .: C H A N=8$;

MULTI STATE DI :TAG=YI RF 2.:DI1=DI_RF_2A.:DI2=0I_RF 2B.;

$D O: T A G=D O \quad \overline{R F} 2$ 2.:CHAN $=\overline{9}: D A D=0$;

DO :TAG $=D^{-} \mathrm{EV}^{-} 17 .: \mathrm{CHAN}=24: \mathrm{DAD}=0$;

DO : TAG $=D 0^{-} \mathrm{EV}^{-} 18 .: \mathrm{CHAN}=25: \mathrm{DAD}=0$;

LET :TAG=DO EV 17.=DI RF 2A.;/*OPEN DM-19 ON RF-2 ON*/

LET :TAG $=$ DO_EV_18.=DI_RF_2A.;/*OPEN DM-20 ON RF-2 ON*/ 

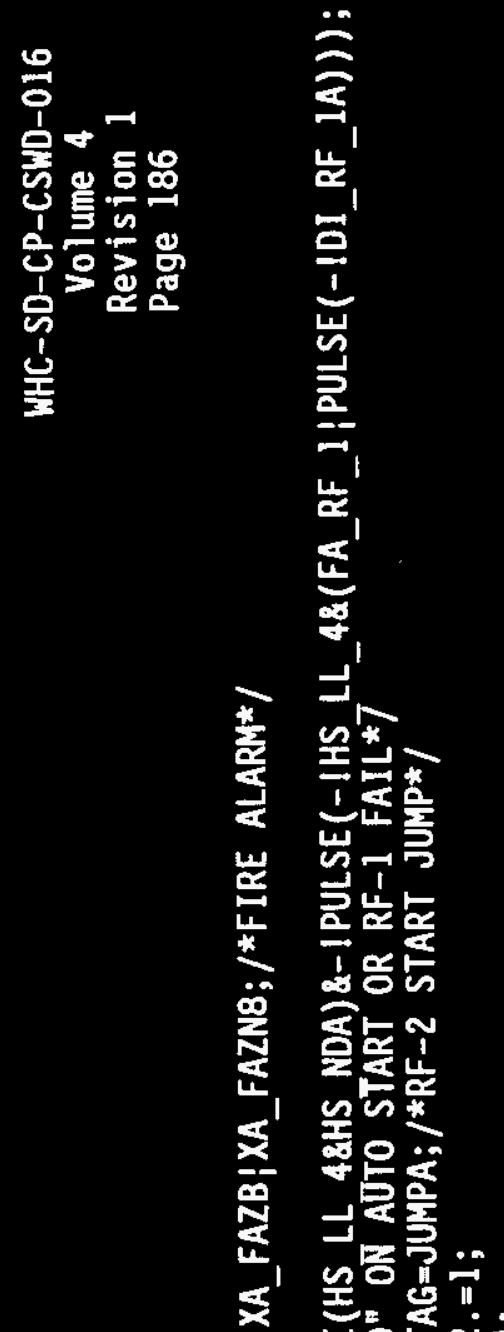

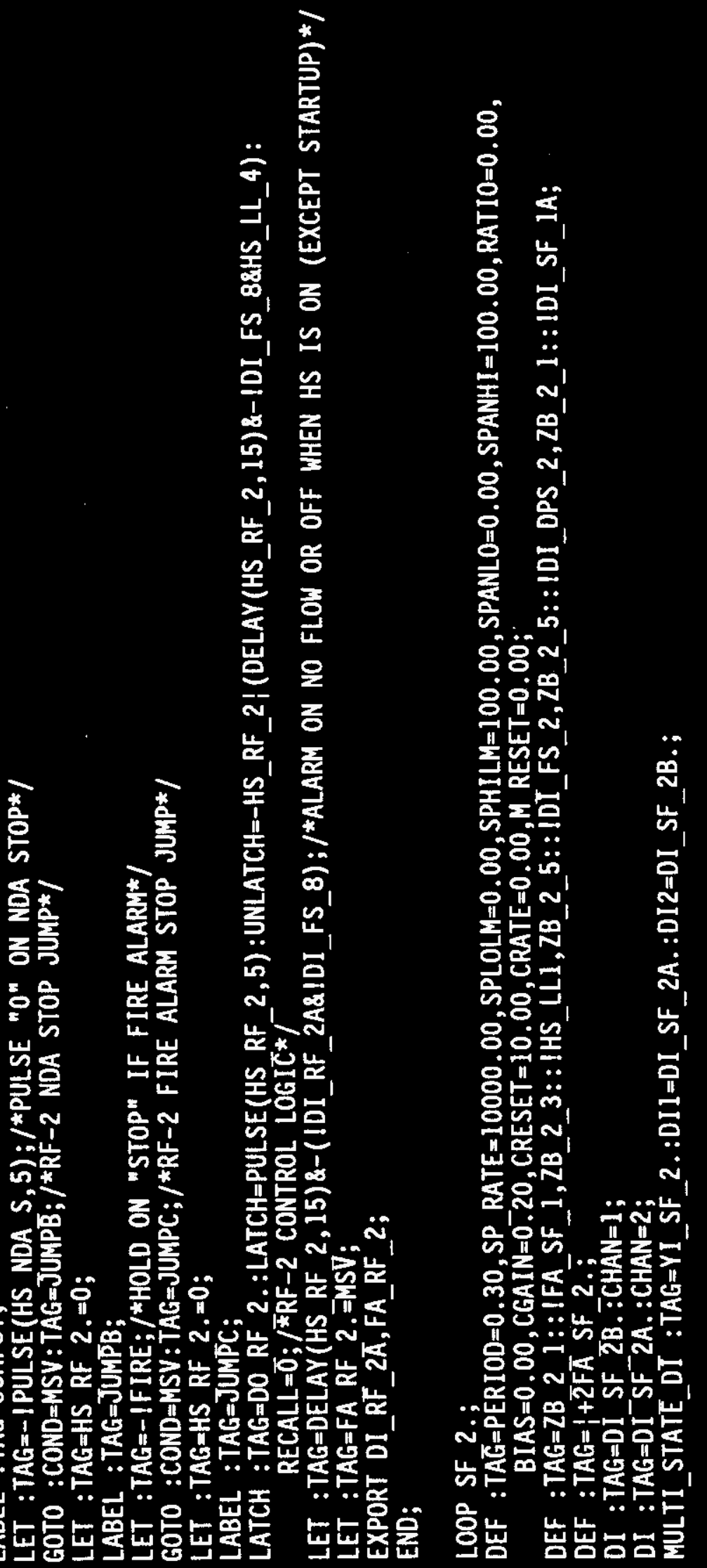


WHC-SD-CP-CSWD-016

Volume 4

Revision 1

Page 187

DO : TAG $=$ DO SF 2.: $\mathrm{CHAN}=3: \mathrm{DAD}=0$;

DO : $T A G=D O E V-3 .: C H A N=20: D A D=0$;

DO : TAG $=00^{-} \mathrm{EV}^{-} 4 .: \mathrm{CHAN}=21: \mathrm{DAD}=0$;

LET :TAG=DO EV 3.=DI SF_2A.; /*DM-5 OPEN ON SF-2 ON*/

LET :TAG=DO-EV 4.=DI SF $2 A . ; / * D H-2$ OPEN ON SF-2 ON*/

LET :TAG $=X A-C S A \mid X A \_F A Z B T X A \_F A Z N 8 ; / * F I R E$ ALARM*/

LABEL :TAG=FIRE;

LET :TAG=-! PULSE(HS LL1\&PULSE(DI DPS 2))\&-! PULSE(-IHS LL1\&FA SF 18DI DPS 2)\&-IPULSE(-IHS LLI\& PULSE (-IDI SF 1A)\&DELAY(DI DPS 2,1$)$ ); $/ * 0$ " PULSE ON EXHAUST DP-UP OR SF-2 FAIL*/

GOTO :COND=MSV:TAG=JUMPA; $/ * A U T O$ START JUMP*/

LET :TAG $=$ HS SF 2. $=1$;

LABEL :TAG=JUMPA;

LET :TAG $=-$ ! PULSE $(1) ; / \star$ PREVENT FALSE START ON RESET*/

GOTO :COND=MSV:TAG=JUMPST;

LET : TAG $=H S$ SF $2 .=0$;

LABEL :TAG=JUMPST;

LET : TAG $=-$ ! PULSE(HS MN $S \mid-1$ I DPS 2,5$) ; / * 0 "$ PULSE ON MAIN STOP OR LO EXHAUST DP*/

GOTO : COND=MSV:TAG=JUMPB; $/$ *STOP JUMP*/

LET : TAG=HS SF 2.=0;

LABEL :TAG=JUMPB;

LET :TAG=-IFIRE;/*HOLD ON "STOP" IF FIRE ALARM*/

GOTO :COND=MSV:TAG=JUMPC; /*FIRE ALARM STOP JUMP*/

LET :TAG $=$ HS SF 2. $=0$;

LABEL :TAG=JUMPC;

LATCH :TAG=DO SF_2.:LATCH=PULSE(HS SF_2,5):UNLATCH=-HS_SF_2 (DELAY(HS_SF_2, 15)\&-1DI_FS_28HS_LL1): RECALL $=\overline{0} ; / \overline{\text { FSF}}-2$ CONTROL LOGIC $* /$

LET :TAG=DELAY (HS_SF 2,15)\&-(10I_SF_2A\&IDI_FS_2); $/^{\star}$ ALARM ON NO FLOW OR OFF WHEN HS IS ON (EXCEPT STARTUP)*/

LET :TAG=FA SF 2. $=$ MSV;

EXPORT DI_SF_2A,FA_SF_2;

END;

LOOP SF 4 ; ;

DEF :TAG $=P E R I O D=0.30, S P$ RATE $=10000.00, S P L O L M=0.00, S P H I L=100.00, S P A N L 0=0.00, S P A N H I=100.00$, RATI0=0.00, $B I A S=0.00$, CGAIN $=0.20$, CRESET $=10.00$, CRATE $=0.00, M$ RESET $=0.00$; 
WHC-SD-CP-CSHD-016

Volume 4

Revision 1

Page 188

DEF :TAG=ZB 2 $1::$ IFA_SF_3,ZB_2_3:: IHS_LL2,ZB_2_5::1DI_FS_4,ZB_2_1::!DI_SF_3A;

DEF :TAG $=$ + $\overline{2} F \bar{F}$ SF $4 . \overline{;}$

DI :TAG=DI SF $\mathbf{4 B} . \overline{: C H A N}=15$;

DI :TAG $=D^{-}$SF $^{-} 4 A .:$ CHAN $=16$;

MULTI STATE DI :TAG=YI SF 4.:DI1=DI_SF_4A.:DI2=DI_SF_4B.;

DO :TAG $=00$ SF 4.:CHAN $=$ T7:DAD $=0$;

DO : TAG $=D 0^{-} E V-7 .: C H A N=29: D A D=0$;

DO :TAG $=D O-E V$ 8. : $C H A N=30: D A D=0$;

LET :TAG=DO EV 7.=0I SF 4A.; $/$ OPEN DH-9 ON SF 4 ON*/

LET :TAG $=00^{-} \mathrm{EV}^{-} 8 .=\mathrm{DI}^{-} \mathrm{SF}^{-} 4 \mathrm{AA} . ; / * O P E N$ DM-10 ON SF-4 ON*/

LET :TAG $=X A-C S A \mid X A \_F A Z B T X A \_F A Z N 8 ; / * F I R E$ ALARM*/

LABEL :TAG=FIRE;

LET :TAG $=(-$ !FA RF_18DI_RF_1A) $\mid(-$ IFA_RF_28DI_RF_2A); $/$ RETURN FAN "ON" SIGNAL*I

LABEL :TAG=RFOR:

LET :TAG=-! PULSE(HS_LL2\&PULSE(RFON))\&-! PULSE(-!HS LL2\&FA SF_3\&RFON)\&-! PULSE(-IHS_LL2\&PULSE(-!DI_SF_3A)\& DELAY (RFON, 1$) T ; /{ }^{*}{ }^{\circ "}$ PULSE ON RFS ON OR SF $-\overline{3}$ FAIL $* 7$

GOTO :COND=MSV:TAG=JUMPA; $/ \star S F-4$ START JUMP*/

LET :TAG=HS SF $4 .=1$;

LABEL :TAG=JUMPA;

LET :TAG=-!PULSE $(1) ; /$ *PREVENT FALSE START ON RESET*/

GOTO : COND=MSV : TAG $=$ JUMPST;

LET :TAG $=H S$ SF $4,=0$;

LABEL :TAG=JUMPST;

LET :TAG $=-$ ! PULSE(HS NDA $S, 5) \&-$ ! PULSE $(-!$ RFON); $/ *$ "On PULSE ON NDA STOP OR RFs OFF*/

GOTO :COND=MSV:TAG=JUMPB; $/$ \$STOP JUMP $/$

LET : TAG $=$ HS SF $4 .=0$;

LABEL : TAG=JUMPB;

LET :TAG=-! IFIRE; /*HOLD ON STOP IF FIRE ALARM*/

GOTO :COND=MSV:TAG=JUMPC;/*FIRE ALARM STOP JUMP*/

LET : TAG $=$ HS SF $4 .=0$;

LABEL :TAG=JUMPC;

LATCH :TAG=DO SF 4.:LATCH=PULSE(HS SF_4,5):UNLATCH=-HS_SF_4!(DELAY(HS_SF_4, 15)\&-1DI_FS_48HS_LL2): RECALL $=\overline{0} ; / \overline{\text { SF }}-4$ CONTROL LOGIC $* /$

LET :TAG=DELAY (HS_SF_4,15)\&-(!DI_SF_4A\&!DI_FS_4); $/^{\star}$ ALARM ON NO FLON OR OFF WHEN HS IS ON (EXCEPT STARTUP)*/ 


\section{WHC-SD-CP-CSWD-016 \\ Volume 4 \\ Revision 1 \\ Page 189}

LET :TAG $=F A$ SF $4 .=$ MSV;

EXPORT DI SF 4A,FA_SF_4;

END;

LOOP SF 6.

DEF :TAG $=$ PERIOD=0.30,SP_RATE $=10000.00, S P L O L M=0.00, S P H I L M=100.00, S P A N L 0=0.00, S P A N H I=100.00, R A T 10=0.00$, BIAS $=0.00$, CGAIN $=0.20$, CRESET $=10.00$, CRATE $=0.00, M$ RESET $=0.00 ;$

DEF :TAG $=$ ZB 2 5:: !DI_FS_9;

DEF :TAG $=1+2 F \overline{2}$ SF 6

DI :TAG $=D I$ SF $6 B . \overline{: C H A N}=12$;

DI :TAG=DI $S F-6 A .: C H A N=13$;

MULTI STATE DI :TAG=YI SF 6.:DI1=DI_SF_6A.:DI2=DI_SF_6B.;

$D O: T A G=D O$ SF $6 .: C H A N=T 4: \overline{D A D}=0$;

$D O: T A G=D 0^{-} E V^{-10}$. : CHAN $=26: D A D=0$;

$D O: T A G=D 0^{-} E V^{-} 32 .: C H A N=27: D A D=0$;

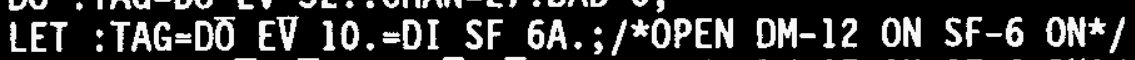

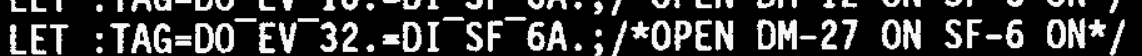

LET :TAG $=-$ ! PULSE (HS 1 28DI 1 2B\&DI KFS11); /*"O" PULSE ON EF-1-2 RUNNING*/

GOTO : COND=MSV:TAG=JUTPA; $/{ }^{\star} S F-6$ START JUMP*/

LET :TAG $=$ HS SF $6 .=1$;

LABEL : TAG=JUMPA;

LET :TAG $=-!$ PULSE(FA 12 -RUN 12$) ; / * " 0 "$ PULSE ON EF-1-2 FAIL OR OFF*/

GOTO : COND=MSV:TAG=JUNPB;/*STOP JUMP*/

LET :TAG $=$ HS SF $6 .=0$;

LABEL :TAG=JUMPB;

LET :TAG=-IXA CSA; $/$ *HOLD ON STOP IF COLD SMOKE ALARM*/

GOTO :COND=MSV:TAG=JUMPC; $/ *$ COLD SMOKE ALARM STOP JUMP*/

LET :TAG=HS SF $6 .=0$;

LABEL :TAG=JUMPC;

LATCH :TAG=DO SF $6 .:$ LATCH=PULSE (HS SF 6,5$):$ UNLATCH $=-H S$ SF $6:$ RECALL $=0 ; / * S F-6$ CONTROL LOGIC*/

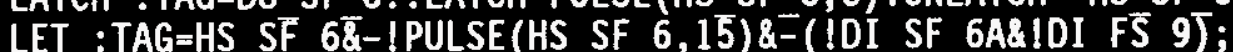

$/{ }^{*}$ ALARM ON NO FLOW OR OFF WHEN HS IS ON (EXCEPT STARTUP) */

LET :TAG=FA SF 6.=MSV;

EXPORT DI_SF 6A, FA_SF_6; 


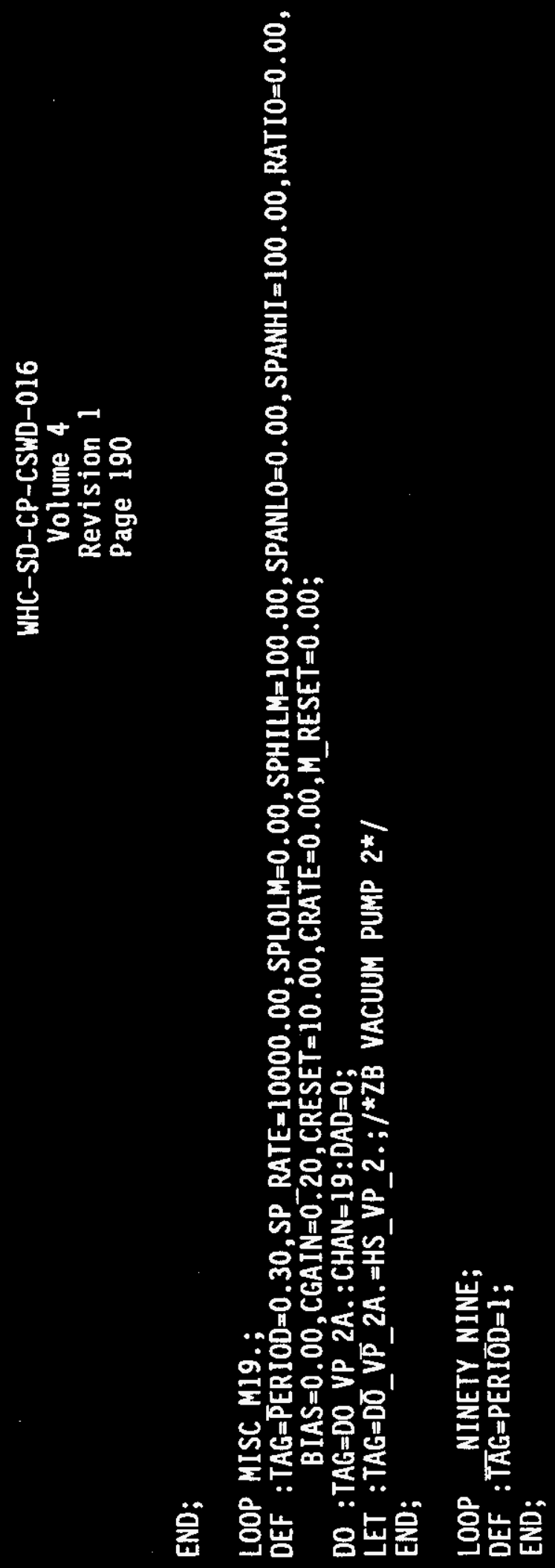




\section{WHC-SD-CP-CSWD-016 \\ Volume 4 \\ Revision 1 \\ Page 191}

mic20_ro

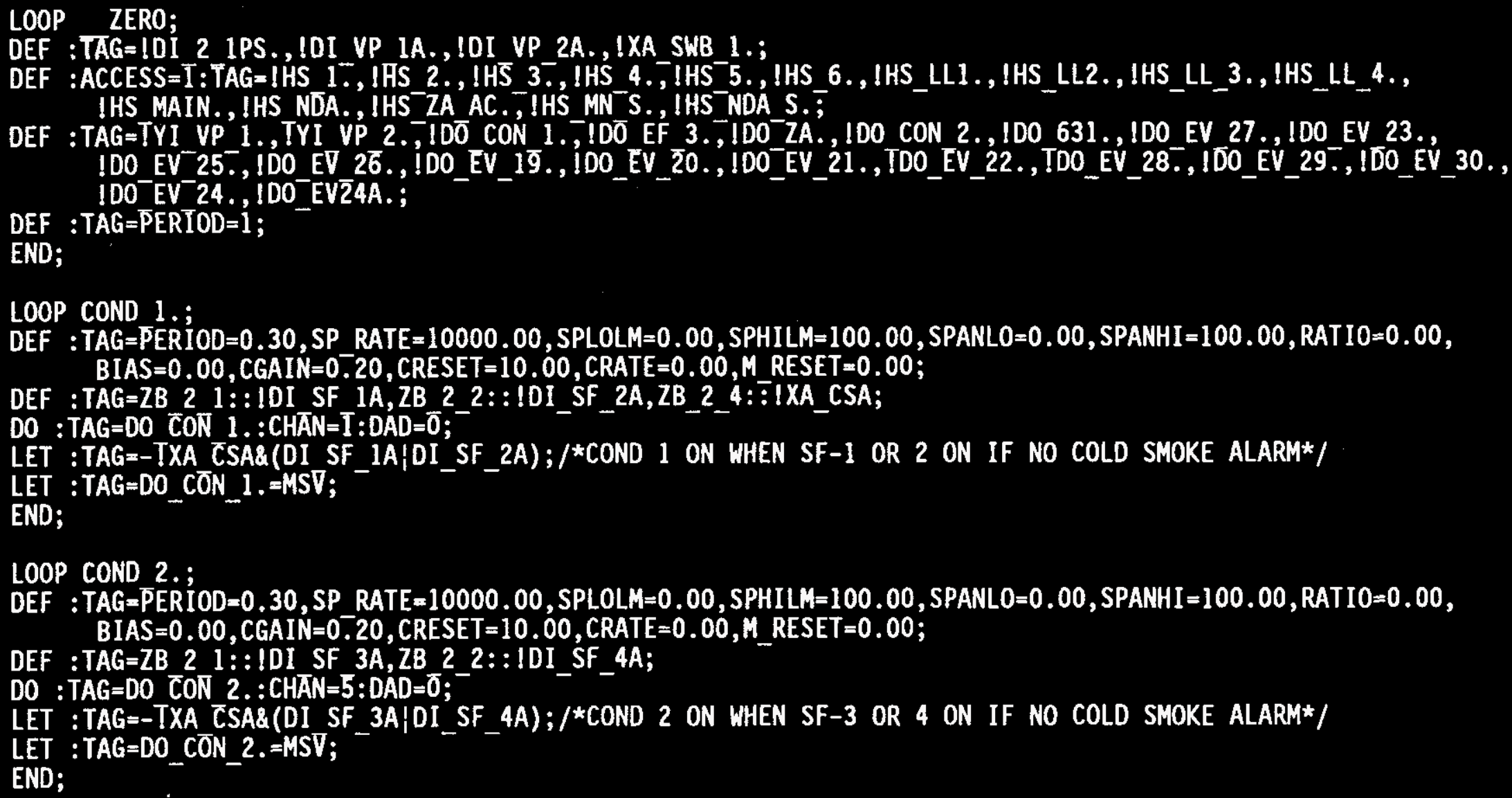




\section{WHC-SD-CP-CSWD-016 \\ Volume 4}

Revision 1

Page 192

LOOP EF 3.;

DEF :TAG $=$ PERIOD $=0.30$, SP RATE $=10000.00$, SPLOLM $=0.00$, SPHILM $=100.00$, SPANLO $=0.00$, SPANHI $=100.00$, RATIO=0.00, BIAS $=0.00$, CGAIN $=0.20$, CRESET $=10.00$, CRATE $=0.00, M$ RESET $=0.00$;

DEF :TAG=ZB 2 1::!FA SF_1, ZB 2_2::IFA_SF_2;

$D 0$ :TAG $=00$ EF $3 .: C H A N=2 \overline{D A D}=\overline{0}$;

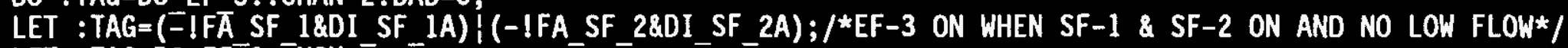

LET :TAG=00_EF_3. $=$ MSV;

END;

LOOP EV19.;

DEF :TAG=PERIOD=0.30,SP RATE $=10000.00, S P L O L M=0.00, S P H I L M=100.00, S P A N L 0=0.00, S P A N H I=100.00$, RATI0=0.00, $B I A S=0.00$, CGAIN $=0.20$, CRESET $=10.00$, CRATE $=0.00, M$ RESET $=0.00$;

DEF :TAG=ZB 2 4::IRAH NDA;

DO :TAG $=00$ EV $19 .:$ CHAN $=22: 0 A D=0$;

LET :TAG=-TXA-CSA\&-!RAH_NDA;/*RECYCLE MODE ALLOWED IF NO CAM OR COLD SMOKE ALARM*/

LET :TAG=DO EV 19.=MSV;

END;

LOOP EV20.:

DEF : TAG $=$ PERIOD $=0.30, S P$ RATE $=10000.00, S P L O L M=0.00, S P H I M=100.00, S P A N L 0=0.00, S P A N H I=100.00$, RATIO=0.00, $B I A S=0.00, C G A I N=0.20, C R E S E T=10.00, C R A T E=0.00, M$ RESET $=0.00$;

DEF :TAG=ZB 2 1::!DI RF_1A,ZB_2_2::!DI_RF_2A;

DO : TAG $=D 0$ EV 20.:CHAN $=\overline{2} 3: D A D=0 ;$

LET :TAG=-TDI RF IA\&-IDIRF_2A; $/ * A I R$ ON TO M2 IF RF-1 OR 2 IS ON*/

LET :TAG=DO_EV_20.=MSV;

END; 


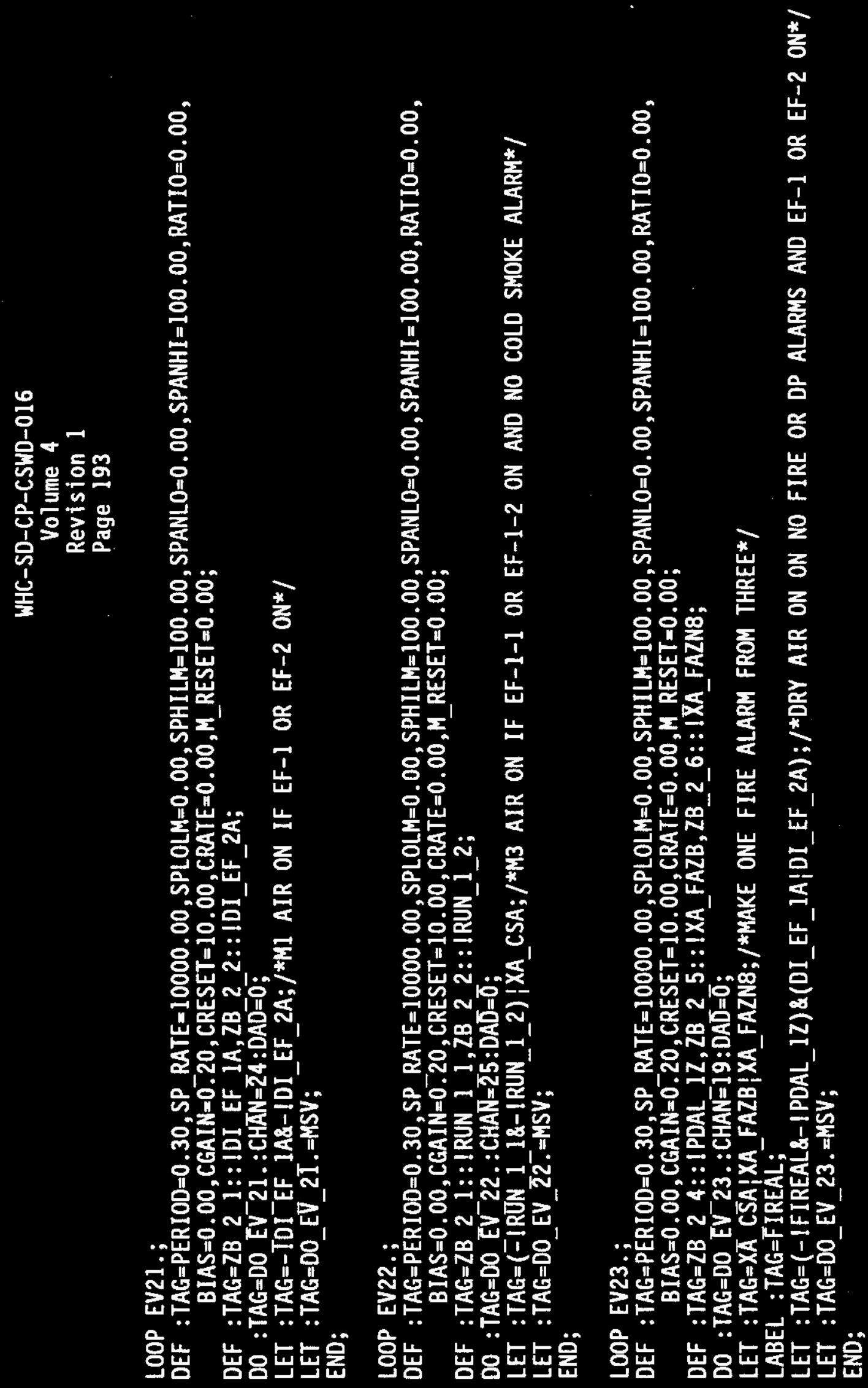




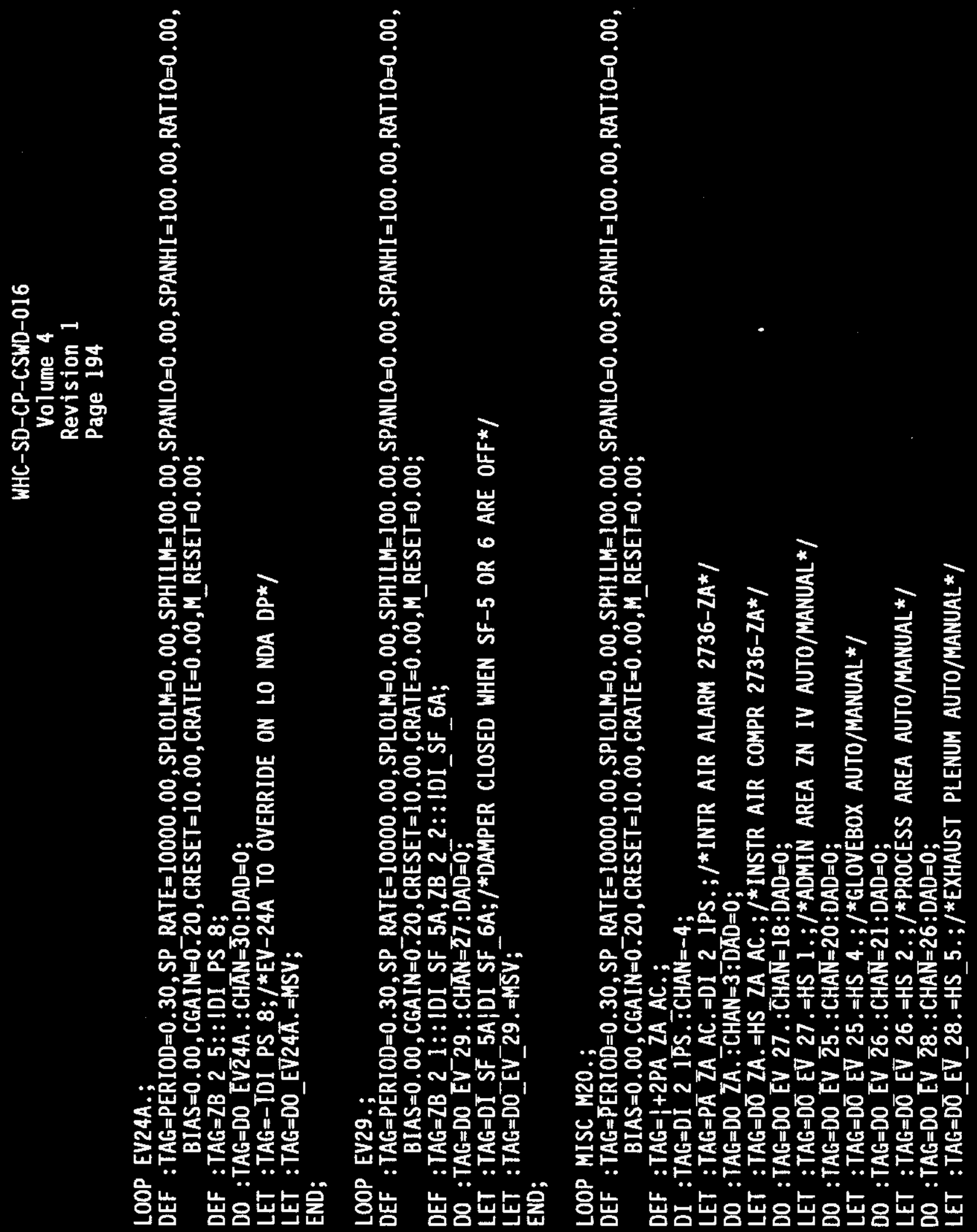


WHC-SD-CP-CSWD-016

Volume 4

Revision 1

Page 195

DO :TAG $=$ DO EV 30.: CHAN $=28: D A D=0$;

LET :TAG=DŌ EV 30. \#HS 6.;/*2736-Z AUTO/MANUAL*/

DO :TAG=DO EV $24 .:$ CHAN $=29: D A D=0$;

LET :TAG=DO EV 24.=HS 3.;/*NDA AREA AUTO/MANUAL*/

LET : TAG $=-1$ PULSE (DELAY (HS MAIN, 5));/*SIMULATE BUTTON ACTIVATE TURN OFF LOOP AFTER 5 SEC*/

GOTO :COND=MSV:TAG=BUTMN;

LET : TAG $=$ HS MAIN. $=0 ; / *$ MAIN AUTOSTART SWITCH*/

LABEL :TAG=BUTMN;

LET :TAG $=-$ ! PULSE(DELAY (HS NDA, 5)); $/$ SIMULATE BUTTON $* /$

GOTO :COND=MSV: TAG $=B U T N D A ;$

LET : TAG $=$ HS NDA. $=0 ; / *$ NDA AUTOSTART SWITCH*/

LABEL :TAG $=\overline{\mathrm{B}} U T N D A ;$

LET :TAG=-! PULSE(DELAY (HS MW $S, 5)) ; /$ SIMULATE BUTTON*/

GOTO :COND=MSV: TAG=BUTMNS;

LET :TAG $=$ HS MN $S .=0 ; / *$ MAIN EMERG STOP*/

LABEL : TAG $=\bar{B} U T$ TNNS;

LET :TAG=-! PULSE(DELAY (HS NDA $S, 5)$ ); *SIMULATE BUTTON*/

GOTO :COND=MSV: TAG=BUTNDAS;

LET :TAG=HS NDA $S .=0 ; / * N D A$ EMERG SHUTDOWN*/

LABEL :TAG= $\bar{B} U T N D A S ;$

DI :TAG=DI VP $1 A .: C H A N=6$;

LET :TAG=YI VP $1 .=0$ I VP 1 A. ; / VP-1 STATUS* $/$

DI :TAG=DI VP $\overline{2 A} .:$ CHAN $=7$;

LET :TAG $=Y$ T VP 2.=DI VP 2A.; /*VP-2 STATUS*/

DI :TAG $=X A$ SWB $1 .:$ CHAN $=\overline{8}: A L A R M=1: P R I=3$;

DO : TAG $=D 0^{-631}$. : $\mathrm{CHAN}=17: \mathrm{DAD}=0$;

LET :TAG $=-$ TRAH NDA;

LET :TAG=D0 63T.=MSV; /*OUTPUT TO RM 631 NDA CAM ALARM*/

EXPORT HS_L[1,HS_LL2,HS_LL_3,HS_LL_4,HS_MAIN,HS_NDA, HS_MN_S, HS_NDA_S;

END;

LOOP NINETY NINE;

DEF :TAG $=P E R I \bar{O} D=1$;

END; 


\section{WHC-SD-CP-CSWD-016 \\ Volume 4}

Revision 1

Page 196

mic21 ro

LOOP ZERO;

DEF :TAG $=$ !DI 1 1B., !DI CSA., !DI FS 1., IDI FS 5., IDI FS 7.,!DI FS 10., IDI FS 3.,!DI DPS 1., !DI CAM., IXA EGF.,IXA PLC 1., ILALL_CFT., ILAL_CFT., IXA_EGS_1.,IXA_EGS_2.,!TAH_GEN1.,IPAL_GEN1.,IXA_G1_1., ISAH GEN1.,! TA_GI_1.,IEAL_GEN1.;

DEF : TAG $=P E R I O D=1$;

END;

LOOP MISC M21.;

DEF : TAG $=$ PERIOD $=0.30$, SP RATE $=10000.00$, SPLOLM $=0.00$, SPHILM $=100.00$, SPANL $0=0.00$, SPANHI $=100.00$, RATI0 $=0.00$, BIAS $=0.00$, CGAIN $=0.20$, CRESET $=10.00$, CRATE $=0.00, \mathrm{M}$ RESET $=0.00$;

DI :TAG=DI 1 18.:CHAN $=1$;

DEF :TAG $=1 \overline{+} 3 \mathrm{XA}$ CSA.;

DI : TAG $=01$ CSA. : $C H A N=-2$;

LET :TAG $=X \bar{A}$ CSA. $=$ DI CSA.; $/ *$ COLD SMOKE ALARM $/$

DI :TAG $=$ DI FS $1 .:$ CHAN $=3$;

DI :TAG=DI FS 5.: $\mathrm{CHAN}=4$;

DI : TAG=DIFS 7.: $\mathrm{CHAN}=5$;

DI : $T A G=0 I^{-} F^{-10}$ : : $C H A N=6$;

DI :TAG=DIFS 3.: CHAN=7;

DEF : TAG $=1+4 \mathrm{PDAL} 12$.

DI :TAG $=0$ I DPS $1 .:$ CHAN $=-8$;

LET :TAG=PDAL IZ.=DI DPS $1 . ; / * Z B$ GLOVEBOX LO PRESS*/

DEF :TAG $=$ + + RAH NDA.;

DI :TAG $=D I$ CAM.: $C H A N=-10$;

LET :TAG $=$ RAH NDA.=DI CAM.; $* Z B$ NDA CAM ALARM*/

DI :TAG $=X A$ EGF.$:$ CHAN $=-15: A L A R M=1: P R I=3$;

DI :TAG $=X A$ PLC 1.:CHAN $=-16: A L A R M=1: P R I=2$;

DI :TAG $=$ LALL CFT.: CHAN=17:ALARM $=1:$ PRI $=1$;

DI :TAG $=$ LAL CFT.: CHAN $=18: A L A R M=1: P R I=3$;

DI :TAG $=X A$ EGS 1.:CHAN $=-20: A L A R M=1: P R I=3$;

DI :TAG $=X A \_E G S \_2 .: C H A N=-21: A L A R M=1: P R I=3$; 

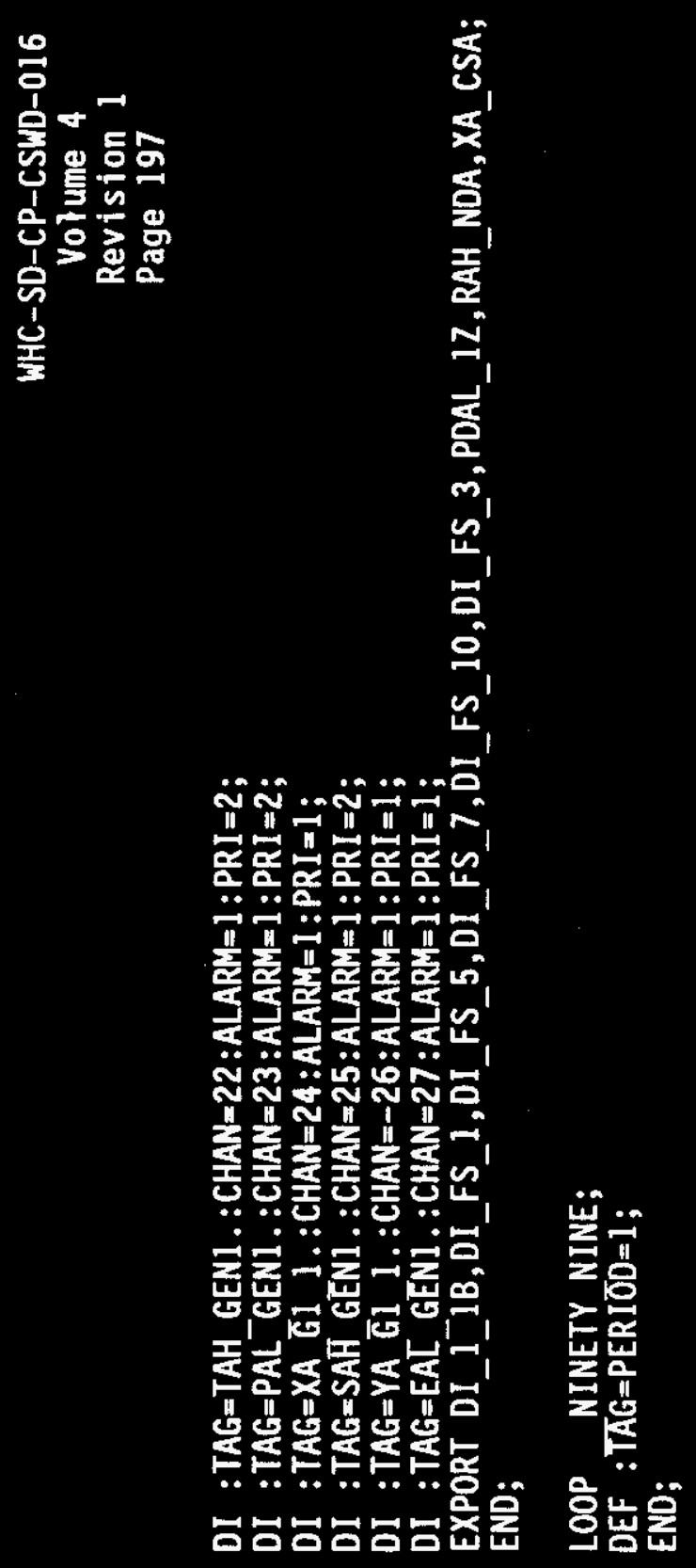

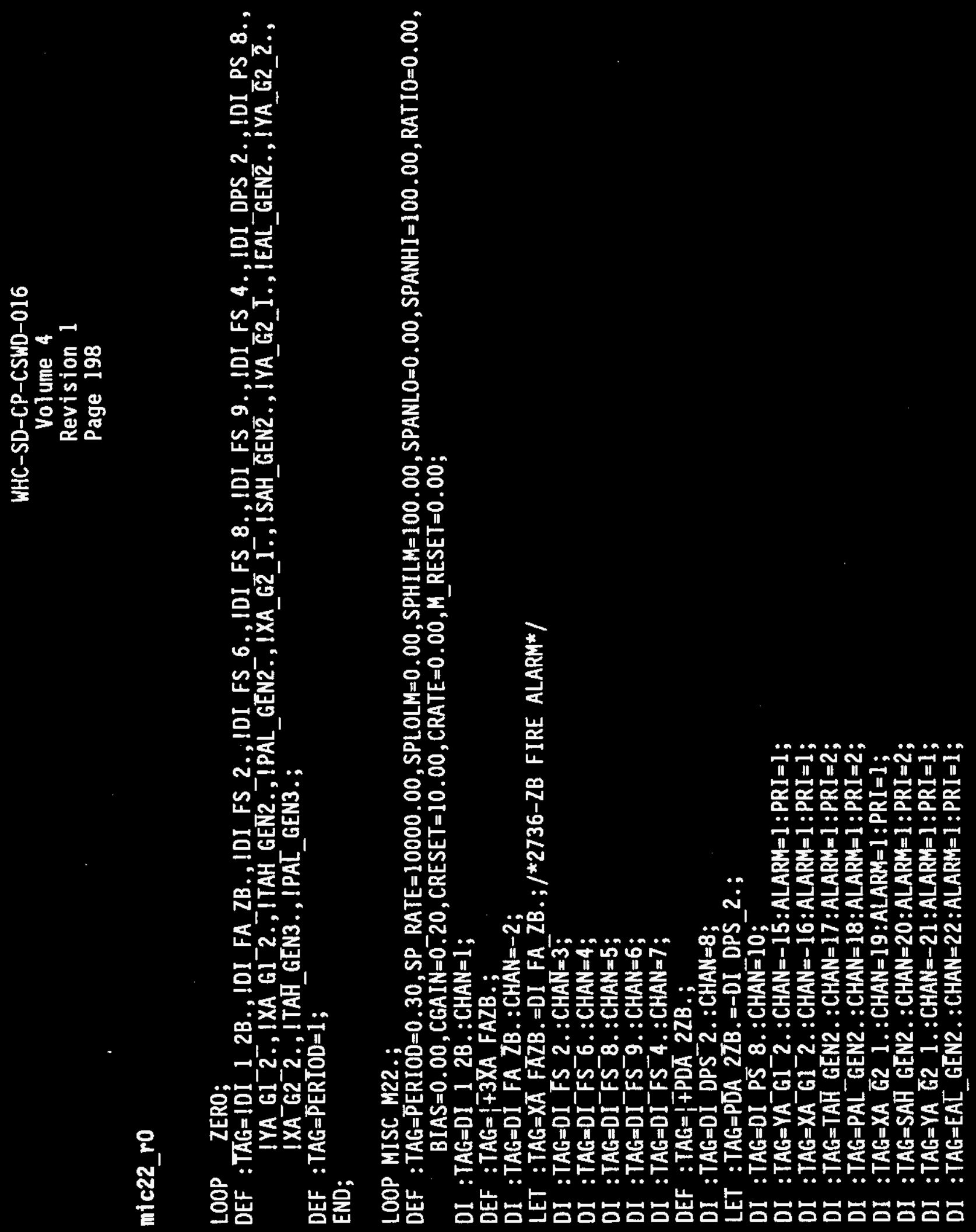

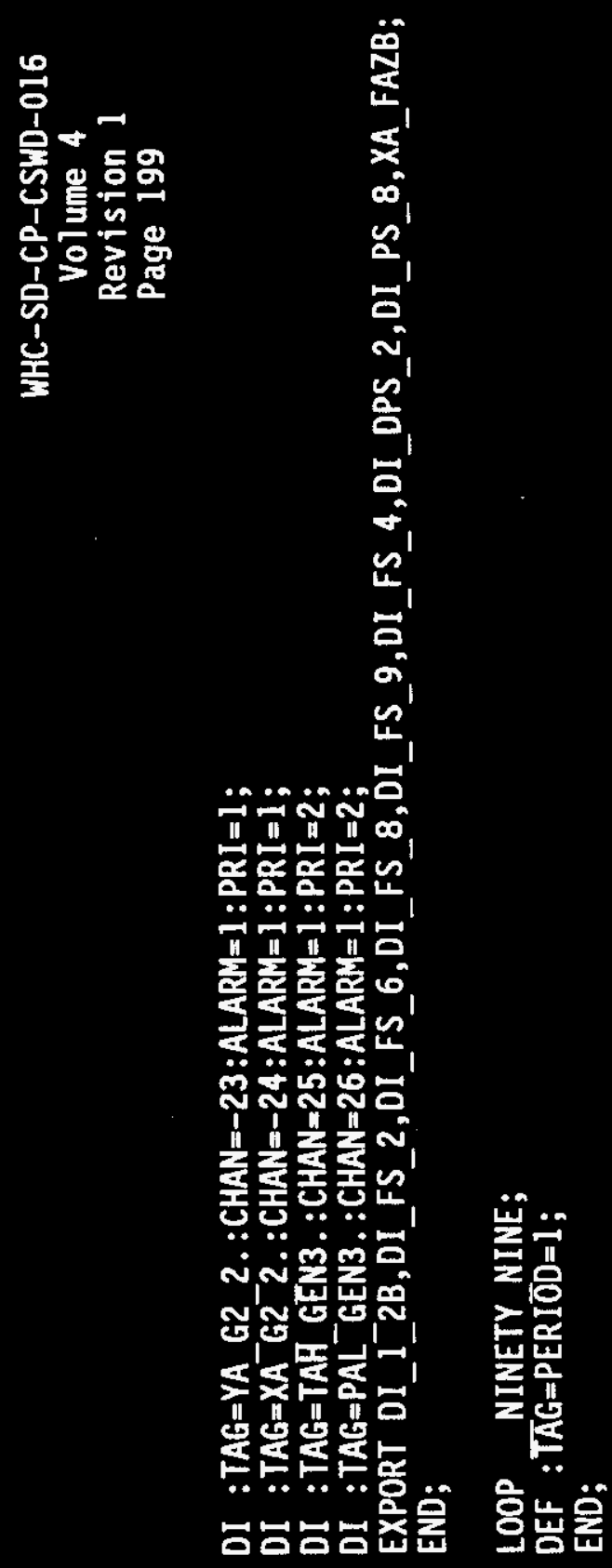

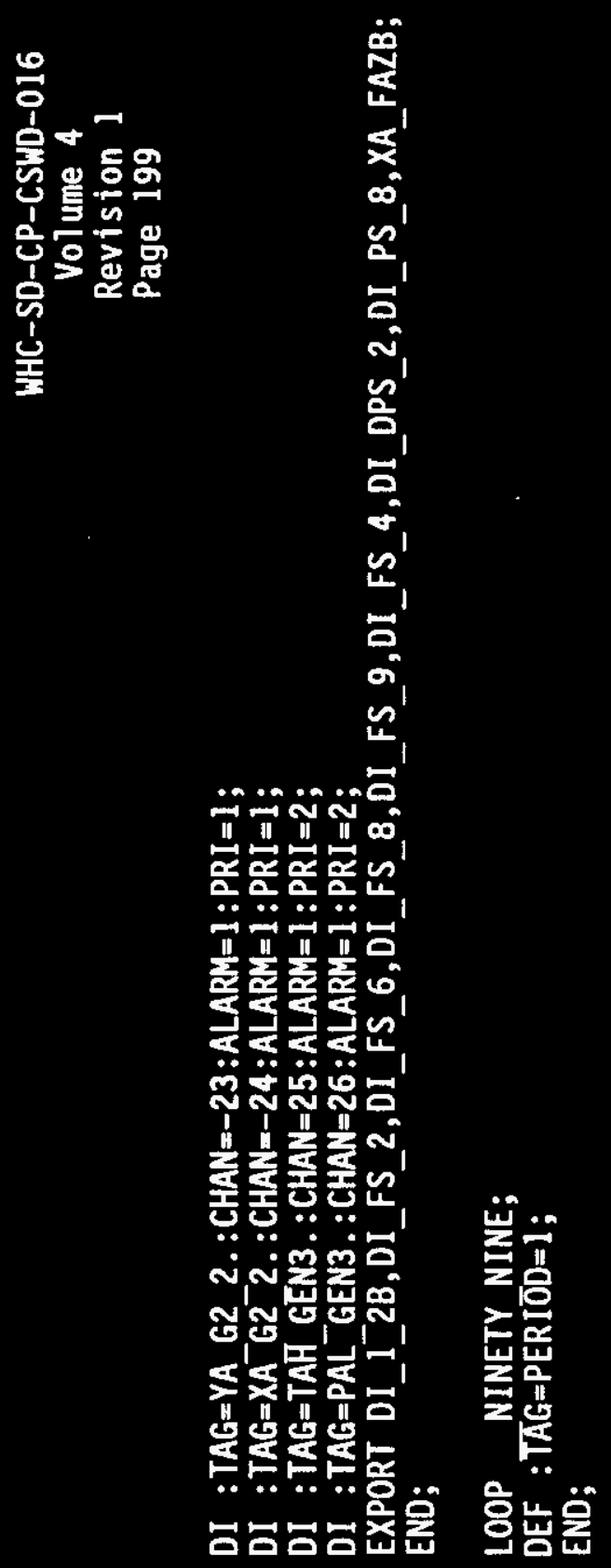

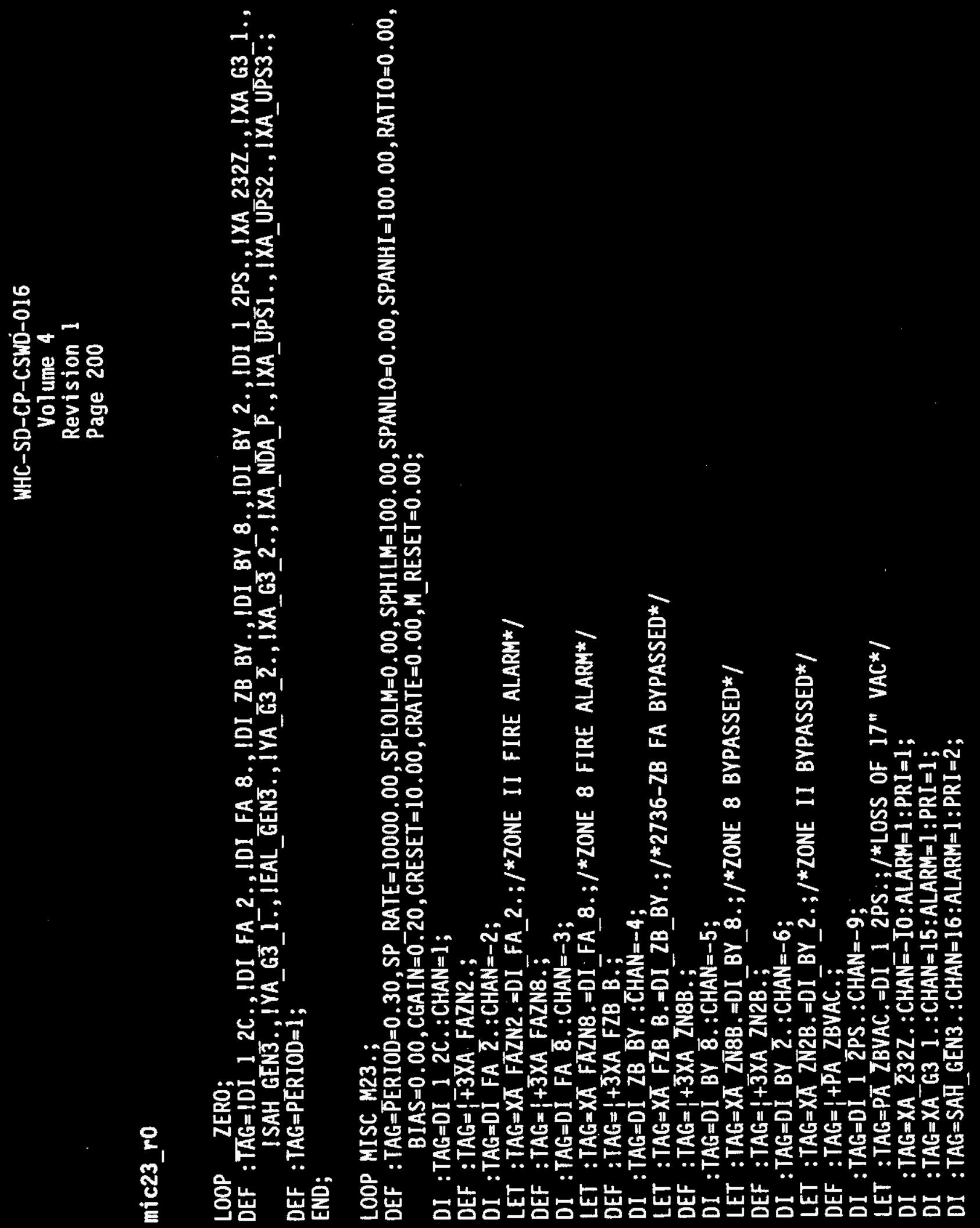

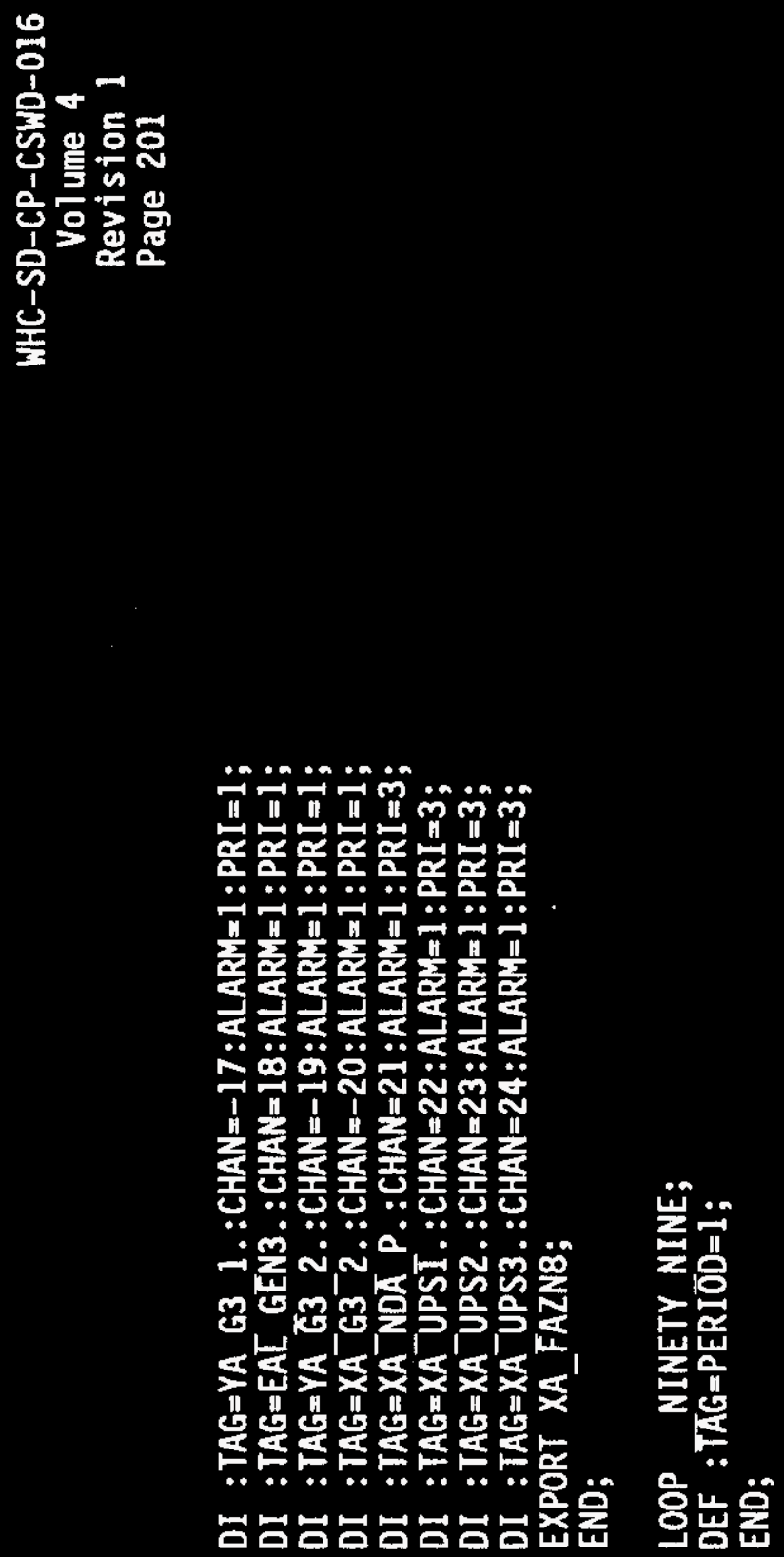


\section{WHC-SD-CP-CSWD-016 \\ Volume 4 \\ Revision 1 \\ Page 202}

\section{APPENDIX A: MICON INPUT/OUTPUT}

Analog inputs and discrete Input/Outputs can be set up in different ways. Analog inputs can be set to accept one of three types of signals: a 4-20 miliamp input with the MICON powering the loop, a 4-20 miliamp input with an external power supply, or a voltage input. Twentyfour volt discrete input/outputs can be set up as an input only, an output only, or as both an input and output. Since the hardware requirements are different for each of these setups, the controller cards have on-board jumpers for each analog input or discrete. These jumpers must be set to the desired type of analog input or discrete for the card to work properly.

Analog outputs are 4-20 miliamp internally powered only. Since they can't be changed they don't have any jumpers.

Before replacing an existing card with a new one or installing a backup card, you must make sure the jumpers in the new card are set the same as the jumpers in the old card.

The following tables show the Input/Output types for each controller card in the 243-Z cabinet. See the RCM-32 Remote Control Module User Manual (Vendor Information file 22068) for a description of the I/O jumper placement. 


\section{WHC-SD-CP-CSWD-016 \\ Volume 4 \\ Revision 1 \\ Page 203}

MICON Number: 16 Tag Name:ZB_1_3 Type: RCM-32C

Location: 2736-ZB Room 602 Rack Number: 1 Slot Number: 3

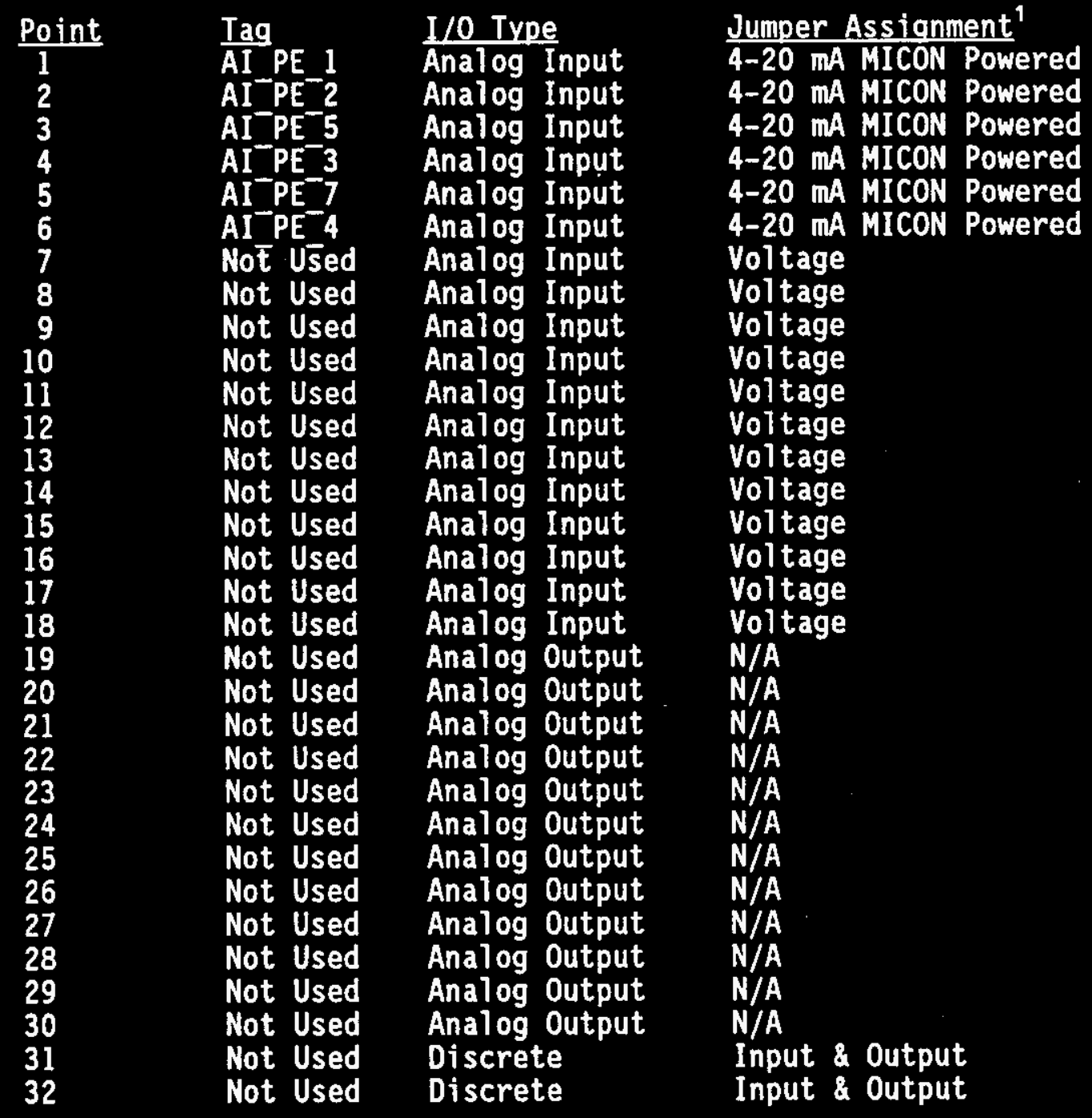

${ }^{1}$ See RCM-32 Remote Control Module User Manual for description of 1/0 jumper placement. 


\section{WHC-SD-CP-CSWD-016 \\ Volume 4 \\ Revision 1 \\ Page 204}

MICON Number: 17 Tag Name:ZB_1_4 Type: RCM-32A

Location: 2736-ZB Room 602 Rack Number: 1 Slot Number: 4

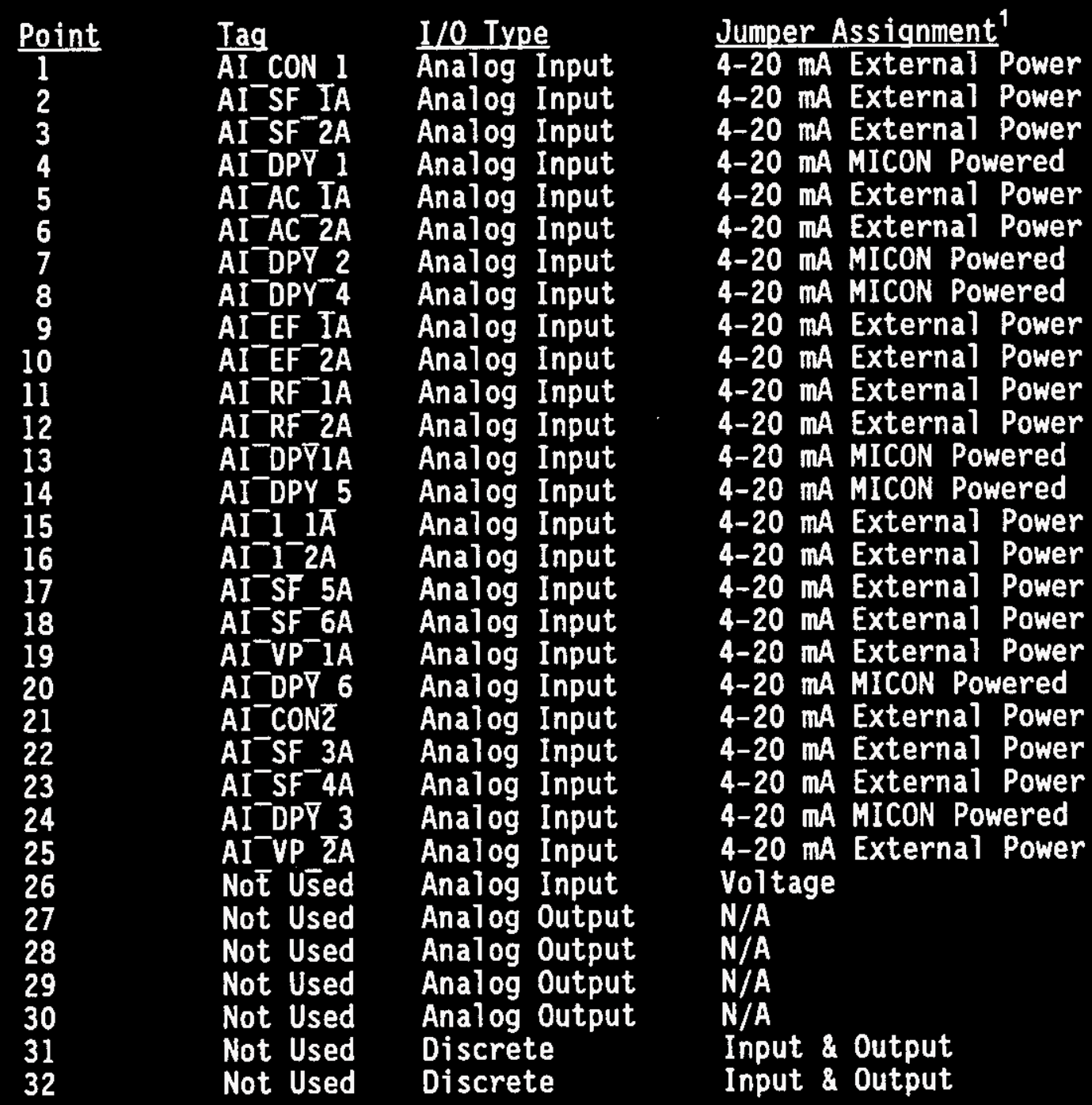

${ }^{1}$ See RCM-32 Remote Control Module User Manual for description of I/0 jumper placement. 


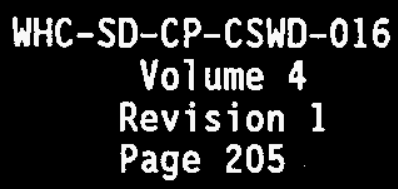

MICON Number: 18 Tag Name:ZB_2_1 Type: RCM-32Dr

Location: 2736-ZB Room 602 Rack Number: 2 Slot Number: 1

\begin{tabular}{|c|c|c|c|c|}
\hline Point & Iag & I/0 Type & & Jumper Assignment \\
\hline 1 & $\overline{D I}$ SF $1 B$ & Discrete & Input & $\mathrm{N} / \mathrm{A}$ \\
\hline 2 & $\mathrm{DI}^{-} \mathrm{SF}^{-1} \mathrm{~A}$ & Discrete & Input & $N / A$ \\
\hline $\begin{array}{l}3 \\
4\end{array}$ & $\begin{array}{l}\text { DO SF }-1 \\
D^{-}-F^{-18}\end{array}$ & Discrete & Output & $\begin{array}{l}\text { N/A } \\
N / A\end{array}$ \\
\hline 5 & $D I-E F-1 A$ & Discrete & Input & N/A \\
\hline 6 & DO-EF-1 & Discrete & Output & $N / A$ \\
\hline 7 & $\mathrm{DI}^{-} \mathrm{RF}^{-} 1 \mathrm{~B}$ & Discrete & Input & $N / A$ \\
\hline 8 & $\mathrm{DI}^{-} \mathrm{RF}^{-} 1 \mathrm{~A}$ & Discrete & Input & $N / A$ \\
\hline 9 & $\mathrm{DO}^{-} \mathrm{RF}^{-} 1$ & Discrete & Output & $N / A$ \\
\hline 10 & $\mathrm{DI}^{-} 1 \mathrm{IA}$ & Discrete & Input & $N / A$ \\
\hline 11 & & Discrete & Output & $N / A$ \\
\hline 12 & $\mathrm{DI}^{-} \mathrm{SF} 5 \mathrm{~B}$ & Discrete & Input & $N / A$ \\
\hline 13 & $\mathrm{DI}^{-} \mathrm{SF}^{-5 \mathrm{~A}}$ & Discrete & Input & $N / A$ \\
\hline 14 & $\mathrm{DO}^{-} \mathrm{SF}^{-} 5$ & Discrete & Output & $N / A$ \\
\hline 15 & $\mathrm{DI}^{-3} \mathrm{SF}^{-3} 3 \mathrm{~B}$ & Discrete & Input & $N / A$ \\
\hline 16 & $\mathrm{DI}^{-} \mathrm{SF}^{-3 A}$ & Discrete & Input & $N / A$ \\
\hline 17 & $\mathrm{DO}^{-} \mathrm{SF}^{-3}$ & Discrete & Output & $N / A$ \\
\hline 18 & $\mathrm{DI}^{-} \mathrm{KF} \overline{11}$ & Discrete & Input & N/A \\
\hline 19 & $00^{-}$VP $1 \mathrm{~A}$ & Discrete & Output & $N / A$ \\
\hline 20 & $00^{-} \mathrm{EV}^{-1} 1$ & Discrete & Output & $N / A$ \\
\hline 21 & $\mathrm{DO}^{-} \mathrm{EV}^{-} 2$ & Discrete & Output & $N / A$ \\
\hline 22 & $\mathrm{DO}^{-} \mathrm{EV}^{-} 11$ & Discrete & Output & $N / A$ \\
\hline 23 & $\mathrm{DO}^{-} \mathrm{EV}^{-} 12$ & Discrete & Output & $N / A$ \\
\hline 2 & $\mathrm{DO}^{-} \mathrm{EV}^{-} 15$ & Discrete & Output & $N / A$ \\
\hline 25 & $\mathrm{DO}^{-} \mathrm{EV}^{-} 16$ & Discrete & Output & $N / A$ \\
\hline 26 & $\mathrm{DO}^{-} \mathrm{EV}^{-} 9$ & Discrete & Output & $N / A$ \\
\hline 27 & $\mathrm{DO}^{-} \mathrm{EV}^{-} 31$ & Discrete & Output & $N / A$ \\
\hline 28 & $\mathrm{DO}^{-} \mathrm{EV}^{-} 33$ & Discrete & Output & $N / A$ \\
\hline 2 & DO-EV 5 & Discrete & Output & $N / A$ \\
\hline 3 & $00^{-} E V-6$ & Discrete & Output & $N / A$ \\
\hline 31 & NoE Used & Discrete & & $N / A$ \\
\hline 3 & Not Used & Discrete & & $N / A$ \\
\hline
\end{tabular}

${ }^{1}$ See RCM-32 Remote Control Module User Manual for description of 1/0 jumper placement. 
MICON Number: 19 Tag Name:ZB_2_2 Type: RCM-32Dr

Location: 2736-ZB Room 602 Rack Number: 2 Slot Number: 2

\begin{tabular}{|c|c|c|c|c|}
\hline Point & 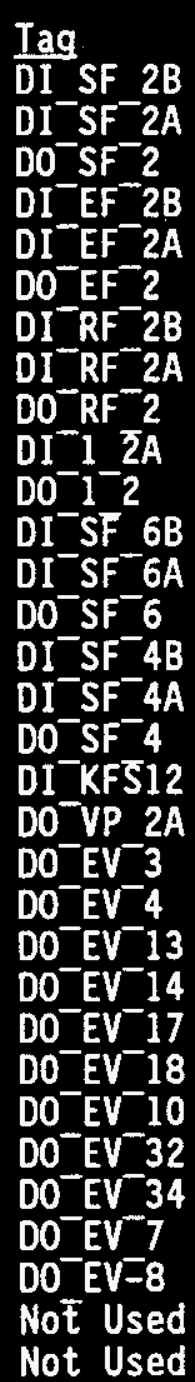 & $\begin{array}{l}\text { I/0 Type } \\
\text { Discrete } \\
\text { Discrete } \\
\text { Discrete } \\
\text { Discrete } \\
\text { Discrete } \\
\text { Discrete } \\
\text { Discrete } \\
\text { Discrete } \\
\text { Discrete } \\
\text { Discrete } \\
\text { Discrete } \\
\text { Discrete } \\
\text { Discrete } \\
\text { Discrete } \\
\text { Discrete } \\
\text { Discrete } \\
\text { Discrete } \\
\text { Discrete } \\
\text { Discrete } \\
\text { Discrete } \\
\text { Discrete } \\
\text { Discrete } \\
\text { Discrete } \\
\text { Discrete } \\
\text { Discrete } \\
\text { Discrete } \\
\text { Discrete } \\
\text { Discrete } \\
\text { Discrete } \\
\text { Discrete } \\
\text { Discrete } \\
\text { Discrete }\end{array}$ & $\begin{array}{l}\text { Input } \\
\text { Input } \\
\text { Output } \\
\text { Input } \\
\text { Input } \\
\text { Output } \\
\text { Input } \\
\text { Input } \\
\text { Output } \\
\text { Input } \\
\text { Output } \\
\text { Input } \\
\text { Input } \\
\text { Output } \\
\text { Input } \\
\text { Input } \\
\text { Output } \\
\text { Input } \\
\text { Output } \\
\text { Output } \\
\text { Output } \\
\text { Output } \\
\text { Output } \\
\text { Output } \\
\text { Output } \\
\text { Output } \\
\text { Output } \\
\text { Output } \\
\text { Output } \\
\text { Output } \\
\end{array}$ & $\begin{array}{l}\text { Jumper Assignment } \\
\text { N/A } \\
N / A \\
N / A \\
N / A \\
N / A \\
N / A \\
N / A \\
N / A \\
N / A \\
N / A \\
N / A \\
N / A \\
N / A \\
N / A \\
N / A \\
N / A \\
N / A \\
N / A \\
N / A \\
N / A \\
N / A \\
N / A \\
N / A \\
N / A \\
N / A \\
N / A \\
N / A \\
N / A \\
N / A \\
N / A \\
N / A \\
N / A\end{array}$ \\
\hline
\end{tabular}

${ }^{1}$ See RCM-32 Remote Control Module User Manual for description of I/0 jumper placement. 
WHC-SD-CP-CSWD-016

Volume 4

Revision 1

Page 207

MICON Number: 20 Tag Name:ZB_2_3 Type: RCM-32Dr

Location: 2736-ZB Room 602 Rack Number: 2 Slot Number: 3

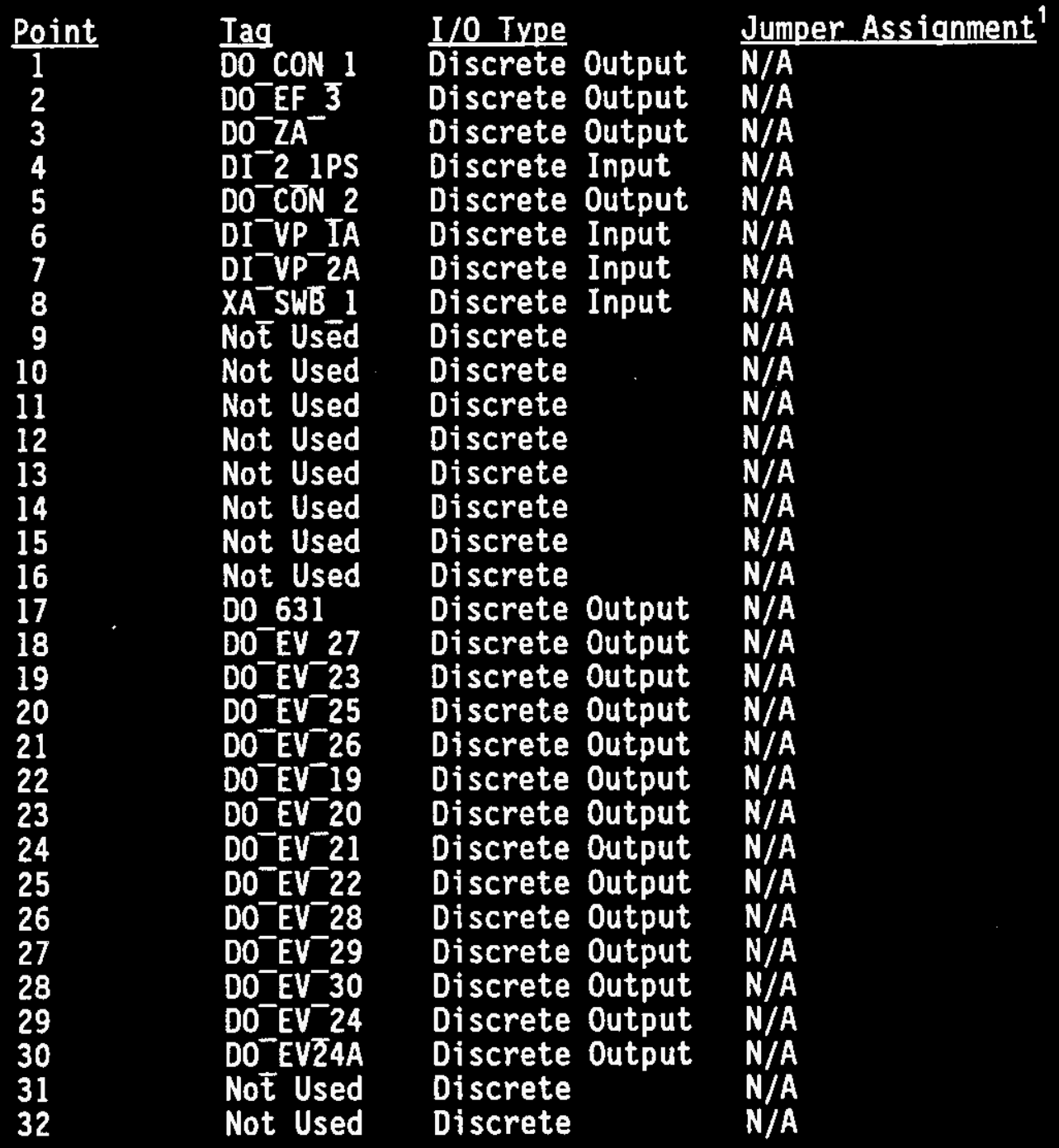

${ }^{1}$ See RCM-32 Remote Control Module User Manual for description of I/0 jumper placement. 


\section{WHC-SD-CP-CSWD-016 \\ Volume 4 \\ Revision 1 \\ Page 208}

MICON Number: 21 Tag Name:ZB_2_4 Type: RCM-32D

Location: 2736-ZB Room 602 Rack Number: 2 Slot Number: 4

\begin{tabular}{|c|c|c|c|c|c|}
\hline Point & Tag & I/0 Type & & Jumper & Iss ignme \\
\hline 1 & $\overline{\mathrm{DI} 1}$ & Discrete & Input & Input \& & Output \\
\hline 2 & $\mathrm{DI}^{-} \mathrm{CS} A$ & Discrete & Input & Input \& & Output \\
\hline 3 & DIFS 1 & Discrete & Input & Input \& & Output \\
\hline 4 & DI FS 5 & Discrete & Input & Input \& & Output \\
\hline $\begin{array}{l}5 \\
6\end{array}$ & DI FS 10 & $\begin{array}{l}\text { Uiscrete } \\
\text { Discrete }\end{array}$ & $\begin{array}{l}\text { Input } \\
\text { Input }\end{array}$ & $\begin{array}{l}\text { Input \& } \\
\text { Inout \& }\end{array}$ & $\begin{array}{l}\text { Uutpul } \\
\text { Outnut }\end{array}$ \\
\hline 7 & $\mathrm{II}^{-} \mathrm{FS}$ & Discrete & Input & Input \& & Output \\
\hline 8 & DI'DPS 1 & Discrete & Input & Input \& & Output \\
\hline 9 & Isēd & Discrete & & Input \& & Output \\
\hline 10 & & Discrete & Input & Input \& & Output \\
\hline 11 & Not Used & Discrete & & Input \& & Output \\
\hline 12 & Not Used & Discrete & & Input \& & Output \\
\hline 13 & Not Used & Discrete & & Input \& & Output \\
\hline 14 & Not Used & Discrete & & Input \& & Output \\
\hline 15 & $X A$ & Discrete & Input & Input \& & Output \\
\hline 16 & $X A$ & Discrete & Input & Input \& & Output \\
\hline 17 & & Discrete & Input & Input \& & Output \\
\hline 18 & FT & Discrete & Input & Input \& & Output \\
\hline 19 & Jsed & Discrete & & Input & Output \\
\hline 20 & GS & Discrete & Input & Input & Output \\
\hline 21 & $\mathrm{GS}^{-2}$ & Discrete & Input & Input & Output \\
\hline 22 & TAH GEN1 & Discrete & Input & Input & Output \\
\hline 23 & PAL ${ }^{-}$EN1 & Discrete & Input & Input \& & Output \\
\hline 24 & $X A \overline{G l} 1$ & Discrete & Input & Input \& & Output \\
\hline 25 & SAH GEN1 & Discrete & Input & Input \& & Output \\
\hline 26 & YA Ğ 1 & Discrete & Input & Input \& & Output \\
\hline 27 & EAL GEN1 & Discrete & Input & Input \& & Output \\
\hline 28 & Not'Used & Discrete & & Input \& & Output \\
\hline 9 & Not Used & Discrete & & Input \& & Output \\
\hline 30 & Not Used & Discrete & & Input \& & Output \\
\hline & Not Used & Discrete & & Input & Output \\
\hline 32 & Not Used & Discrete & & Input \& & Output \\
\hline
\end{tabular}

${ }^{1}$ See RCM-32 Remote Control Module User Manual for description of I/0 jumper placement. 


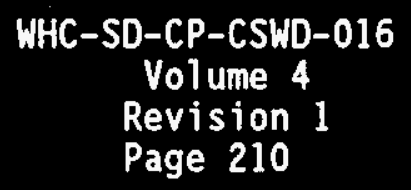

MICON Number: 23 Tag Name:ZB_2_6

Type: RCM-32D

Location: 2736-ZB Room 602 Rack Number: 2 Slot Number: 6

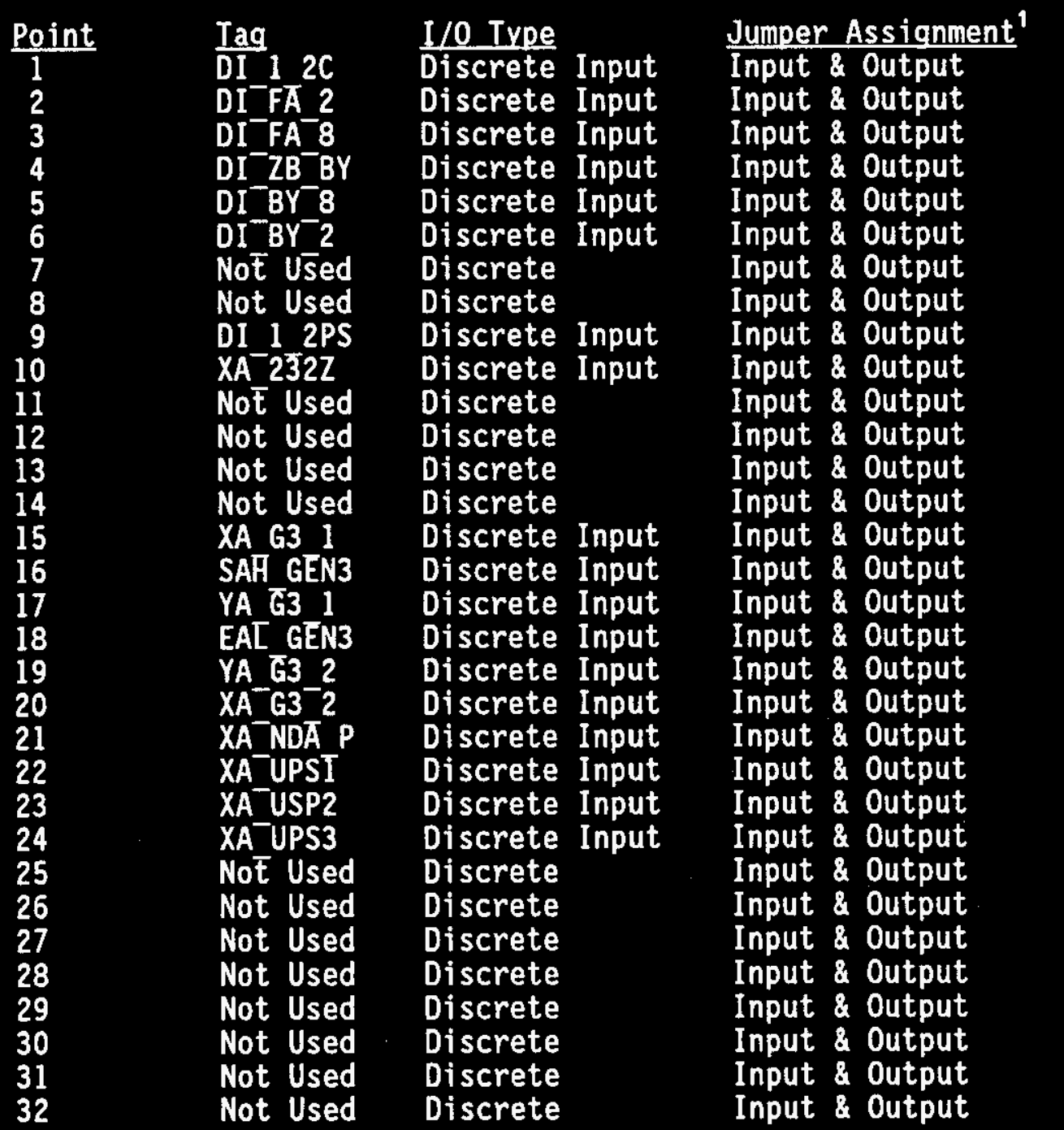

${ }^{1}$ See RCM-32 Remote Control Module User Manual for description of 1/0 jumper placement. 


\begin{tabular}{|c|c|c|c|c|c|c|}
\hline \multicolumn{7}{|c|}{ DISTRIBUTION SHEET } \\
\hline To & & \multirow{2}{*}{\multicolumn{3}{|c|}{$\begin{array}{l}\text { From } \\
\text { G. R. Silvan }\end{array}$}} & \multicolumn{2}{|c|}{ Page 1 of 1} \\
\hline DISTRIBUTION & & & & & \multicolumn{2}{|c|}{ Date 3-13-96 } \\
\hline \multirow{2}{*}{\multicolumn{5}{|c|}{$\begin{array}{l}\text { Project Title/Work Order } \\
\text { PFP Instrumentation Upgrade }\end{array}$}} & \multirow{2}{*}{\multicolumn{2}{|c|}{ 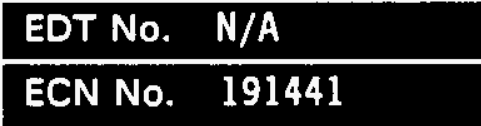 }} \\
\hline & & & & & & \\
\hline \multicolumn{2}{|c|}{ Name } & MSIN & $\begin{array}{c}\text { Text } \\
\text { With All } \\
\text { Attach. }\end{array}$ & Text Only & $\begin{array}{l}\text { Attach./ } \\
\text { Appendix } \\
\text { Only }\end{array}$ & $\begin{array}{l}\text { EDT/ECN } \\
\text { Only }\end{array}$ \\
\hline $\begin{array}{l}\text { E. Icayan } \\
\text { J. L. Wilk } \\
\text { G. R. Silvan } \\
\text { O.S.f. I f fo } \\
\text { Central Files }\end{array}$ & $A 3-88$ & $\begin{array}{l}T 4-20 \\
T 3-20 \\
T 4-18 \\
+8=07 \\
+8-04\end{array}$ & $\begin{array}{r}X \\
X \\
2 X \\
-2 X \\
X \\
\end{array}$ & & & \\
\hline
\end{tabular}

